\title{
Martin Schastak
}

\section{Bilinguale Interaktion} beim Peer-Learning in der Grundschule

Eine Mixed-Methods Studie mit bilingual türkischdeutschsprachig aufwachsenden Schüler*innen 


\section{Martin Schastak}

Bilinguale Interaktion beim Peer-Learning in der Grundschule 
Reihe

Mehrsprachigkeit und Bildung

herausgegeben von

Anke Wegner

İnci Dirim

Band 4 
Martin Schastak

\section{Bilinguale Interaktion beim Peer-Learning in der Grundschule}

Eine Mixed-Methods Studie mit bilingual türkisch-deutschsprachig aufwachsenden Schüler*innen

Verlag Barbara Budrich

Opladen • Berlin • Toronto 2020 
Bibliografische Information der Deutschen Nationalbibliothek

Die Deutsche Nationalbibliothek verzeichnet diese Publikation in der Deutschen Nationalbibliografie; detaillierte bibliografische Daten sind im Internet über https://portal.dnb.de abrufbar.

Inauguraldissertation zur Erlangung des Grades eines Doktors der Philosophie im Fachbereich 04 Erziehungswissenschaft der Johann-Wolfgang-Goethe-Universität zu Frankfurt am Main (2019).

Originaltitel der am Fachbereich 04 Erziehungswissenschaft der Johann-WolfgangGoethe-Universität zu Frankfurt am Main eingereichten Dissertationsschrift: Bilinguale Interaktion beim Peer-Learning als Angebot für bilingual türkischdeutschsprachige GrundschülerInnen der 3. und 4. Klasse. Eine Mixed-Methods Studie.

1. Gutachter: Herr Prof. Dr. Eckhard Klieme, 2. Gutachterin: Frau Prof. Dr. Dominique Rauch, Tag der mündlichen Prüfung: 11.06.2019 Siegelziffer: D.30 Affiliation des Autors: DIPF | Leibniz-Institut für Bildungsforschung und Bildungsinformation

(C) 2020 Dieses Werk ist bei der Verlag Barbara Budrich GmbH erschienen und steht unter der Creative Commons Lizenz Attribution-ShareAlike 4.0 International (CC BY-SA 4.0): https://creativecommons.org/licenses/by-sa/4.0/.

Diese Lizenz erlaubt die Verbreitung, Speicherung, Vervielfältigung und Bearbeitung bei Verwendung der gleichen CC-BY-SA 4.0-Lizenz und unter Angabe der UrheberInnen, Rechte, Änderungen und verwendeten Lizenz. www.budrich.de

\section{(c) (i) (2)}

Dieses Buch steht im Open-Access-Bereich der Verlagsseite zum kostenlosen Download bereit (https://doi.org/10.3224/84742378). Diese Open Access Veröffentlichung wurde vom Leibniz Publikationsfonds für Open-AccessMonographien gefördert.

Eine kostenpflichtige Druckversion (Print on Demand) kann über den Verlag bezogen werden. Die Seitenzahlen in der Druck- und Onlineversion sind identisch.

$$
\begin{array}{ll}
\text { ISBN } & 978-3-8474-2378-2 \text { (Paperback) } \\
\text { eISBN } & 978-3-8474-1521-3 \text { (eBook) } \\
\text { DOI } & 10.3224 / 84742378
\end{array}
$$

Umschlaggestaltung: Bettina Lehfeldt, Kleinmachnow - www.lehfeldtgraphic.de Typographisches Lektorat: Anja Borkam, Jena - kontakt@lektorat-borkam.de Druck: Books on Demand GmbH, Norderstedt Printed in Europe 


\section{Zusammenfassung}

In der Bundesrepublik Deutschland weisen Kinder mit Migrationshintergrund, die häufig bilingual aufwachsen und Deutsch als Zweitsprache erwerben (vgl. Schulz \& Tracy, 2011), seit Dekaden persistente Bildungsbenachteiligungen im Vergleich zu Kindern ohne Migrationshintergrund auf (vgl. z. B. Baumert \& Schümer, 2001; Britz, 2006; Gomolla \& Radtke, 2009; Haag et al., 2012, 2016; Kempert et al., 2016; Rauch et al., 2016; Tarelli, Schwippert \& Stubbe, 2012; Wendt \& Schwippert, 2017). Obwohl die Ursachen der Bildungsbenachteiligung dieser Schülerschaft vielfältig sowie komplex sind (vgl. z. B. Baumert \& Schümer, 2001; Britz, 2006) und zum Teil durch flexible, anforderungsbezogene Praktiken der Selektions- und Allokationsfunktion der Schule selbst erzeugt werden (vgl. Gomolla \& Radtke, 2009), stellen (bildungs)sprachliche Defizite im Deutschen einen weit verbreiteten Erklärungsansatz für die Bildungsbenachteiligung dieser Schülerschaft dar (vgl. Kempert et al., 2016). Innerhalb dieses Narrativs werden die weiteren Sprachen dieser Schülerschaft neben dem Deutschen aus einer Defizitperspektive betrachtet und problematisiert (vgl. z. B. Esser, 2009; Gomolla \& Radtke, 2009; Kersten et al., 2011; Kratzmann, et al., 2017; Tracy, 2014; Wlossek \& Rost-Roth, 2016). So wird z. B. ein Konkurrenzverhältnis zwischen der deutschen Sprache und den weiteren natürlich erworbenen Sprachen dieser Schülerschaft postuliert, in welchem die Förderung der einen Sprache auf Kosten der Förderung der anderen Sprache geht. Die Lösung der Bildungsbenachteiligung wird folglich in präventiver und kompensatorischer Sprachförderung des Deutschen gesehen.

Dieser vermeintliche Lösungsansatz zur Überwindung der Bildungsbenachteiligung von Schülerinnen und Schülern mit Migrationshintergrund stärkt den „monolingualen Habitus“ (Gogolin, 1994) der deutschen Regelschule. Der „monolinguale Habitus“ der deutschen Regelschule lässt sich historisch rekonstruieren und beeinflusst in Form einer „Wahrnehmungs-, Handlungs- und Denkmatrix" (ebd., S. 31) das Handeln und den Umgang der Lehrkräfte mit der sprachlichen Vielfalt im Unterricht. Er zeichnet sich primär dadurch aus, dass das Lernen sowie die Kommunikation in der Regelschule ausschließlich im Deutschen vollzogen wird - mit Ausnahme der Sprachen des Fremdsprachenkanons - und monolingual aufwachsende Schülerinnen und Schüler die soziale Bezugsnormorientierung für Lehrkräfte darstellt (vgl. ebd.).

Der monolinguale Habitus der deutschen Regelschule steht im Widerspruch zu theoretischen Modellen des Zusammenhangs von Bilingualität und Bildung (vgl. z. B. Cummins, 2000; Hamers \& Blanc, 2000) und einer Vielzahl empirischer Befunde der Bilingualismusforschung (vgl. z. B. Baker, 2011; Barwell, 2014; Clarkson, 2007; Dirim, 1998; García et al., 2011; García \& Kano, 2014; García \& Li Wei, 2014; Hopewell, 2011; Klingner \& Vaughn, 
2000; Rauch et al., in Vorb.; Rehbein, 2011; Rolstad, Mahoney \& Glass, 2005; Schüler-Meyer et al., 2017; Slavin \& Cheung, 2005; Swain \& Lapkin, 2000; Worthy et al., 2013), die fruchtbare Effekte und/oder Praktiken des Einbezugs beider Sprachen von bilingual aufwachsenden Schülerinnen und Schülern hinsichtlich ihrer Bildungsprozesse identifizieren. Zudem stellt diese Praxis aus anerkennungstheoretischer Perspektive (vgl. Honneth, 2003; Mecherill, 2005; Stojanov, 2006, 2011) eine Missachtung der bilingualen Disposition von Kindern mit Migrationshintergrund dar. Angesichts dieser Perspektiven stellt sich eher die Frage, wie die natürlich erworbene Bilingualität von Kindern mit Migrationshintergrund in den Unterricht der Regelschule fruchtbar integriert werden könnte? Eine Möglichkeit hierfür stellt die Sozialform des „Peer-Learning“ dar, und zwar in Form eines Angebots zur bilingualen Interaktion während der Gruppen- oder Partnerarbeit von Schülerinnen und Schülern, die über die gleichen Sprachen verfügen. Für den Schulkontext der Submersion gibt es erste Hinweise für positive Effekte durch bilinguale Interaktion beim PeerLearning (vgl. z. B. Meyer \& Prediger, 2011; Planas \& Setati, 2009; Rauch et al., in Vorb.; Rehbein, 2011; Schüler-Meyer et al. 2017), allerdings lässt die bisherige Empirie viele Fragen offen und weist diverse methodische Mängel auf. Die vorliegende Studie möchte einen Beitrag zur Schließung dieser Forschungslücke leisten, indem Peer-Learning mit bilingualem Interaktionsangebot bei türkisch-deutschsprachig aufwachsenden Schülerinnen und Schülern der dritten und vierten Klasse untersucht wird.

In der vorliegenden Studie werden anhand der Daten der „BiPeer-Studie“ die folgenden übergeordneten Fragestellungen analysiert: F1) Ist die Prävalenz bilingualer Praktiken beim Peer-Learning abhängig von der Dyade? F2) Wie hoch ist die Prävalenz bilingualer Praktiken beim Peer-Learning? F3) Bestehen Zusammenhänge individueller Eigenschaften der Probanden mit der Prävalenz bilingualer Praktiken beim Peer-Learning? F4) Welche selbstberichteten Gründe für die Annahme oder Ablehnung des bilingualen Sprachangebots nennen die Probanden? F5) Für welche Funktionen werden bilinguale Praktiken beim Peer-Learning eingesetzt? Die Forschungsfragen sind in ein „Mixed Methods Vertiefungsdesign“ (vgl. Kuckartz, 2014, S. 78ff) eingebettet, wobei F1F3 mit quantitativen Methoden unter Berücksichtigung der Dyadenstruktur (vgl. Kenny, Kashy \& Cook, 2006) und F4 sowie F5 mithilfe der qualitativen Inhaltsanalyse nach Mayring (2015) analysiert werden.

Die Stichprobe besteht aus insgesamt 114 bilingual türkisch-deutschsprachig aufwachsenden Kindern der dritten und vierten Klasse, die sich auf vier Gruppen in einem quasi-experimentellen Mixed-Methods Vertiefungsdesign verteilen, bei denen der Lerngegenstand sowie das Sprachangebot systematisch variiert werden. Das Peer-Learning ist in Dyaden mit reziproken Rollen strukturiert. Die Trainings bestehen aus 12 Sitzungen je 45 Minuten, die am Nachmittag zweimal wöchentlich stattfinden. Das bilinguale Sprachangebot 
wird explizit und standardisiert durch eine bilinguale studentische Übungsleitung vermittelt und zum Teil durch zweisprachige Materialien unterstützt. Die Datengrundlage der vorliegenden Studie besteht aus Transkripten der neunten Sitzung, einem bilingualen Wortschatztest, Selbstberichten und Fragebogenskalen.

Die zentralen Ergebnisse lassen sich folgendermaßen zusammenfassen: Die Prävalenz bilingualer Interaktion ist abhängig von der Dyade und darf somit nicht als individuelle Variable ohne Berücksichtigung der Dyadenstruktur analysiert werden. Die Prävalenz unterscheidet sich signifikant zwischen den Gruppen mit und ohne explizitem bilingualen Interaktionsangebot, aber nicht zwischen den verschiedenen Lerninhalten der Angebotsgruppen. Es konnten keine signifikanten Zusammenhänge der Prävalenz bilingualer Interaktion mit sprachlichen oder motivationalen Probandenmerkmalen identifiziert werden. Die Annahme des Sprachangebots wurde häufig damit begründet, dass es als obligatorischer Bestandteil der Intervention interpretiert wurde und weil es zu einer Erleichterung des Peer-Learnings führt. Auch affinitätsbezogene Ursachen wie das Empfinden von $\mathrm{Spa}$ bei der Nutzung des bilingualen Interaktionsangebots wurden hierbei als Begründungen angegeben, während die Dyadenpartner in den Argumentationen der Probanden kaum eine Rolle spielen. Die Ablehnung des Sprachangebots wurde vor allem mit fehlenden Sprachkompetenzen im Türkischen beziehungsweise einer Sprachasymmetrie zu Gunsten des Deutschen legitimiert. Das Empfinden von Missvergnügen beim Gebrauch des Türkischen wurde ebenfalls berichtet und die Dyadenpartner wurden auch bei der Ablehnung des bilingualen Interaktionsangebots selten als Grund für das jeweilige Sprachverhalten angeführt. Bilinguale Interaktion wird beim Peer-Learning in der vorliegenden Studie vor allem für Task-Management und zur metakognitiven (Selbst-)Regulation eingesetzt. Auch für „off-task“ Diskurse wird bilinguale Interaktion manchmal verwendet, allerdings nur sehr selten für die Aufgabenbearbeitung selbst. Insgesamt weisen die Ergebnisse der vorliegenden Studie auf das Potential bilingualer Interaktion beim Peer-Learning hin. In der Diskussion werden neben der Datenintegration der quantitativen und qualitativen Daten auch praktische Implikationen, die Limitationen der Studie sowie sich anschließende Forschungsdesiderata reflektiert. 


\section{Danksagung}

Ich möchte mich bei meinem Gutachter Herr Prof. Dr. Eckhard Klieme für die fachliche Untertützung, konstruktive Kritik sowie das Interesse an meiner Forschung bedanken. Ebenfalls danke ich Frau Prof. Dr. Dominique Rauch, die mir als meine Zweitgutachterin beratend zur Seite stand.

Besonderer Dank gilt meiner Kollegin Katja Richter, die maßgeblich an der Erstellung der BiPeer-Studienanlage und Intervention beteiligt war. Die gemeinsame Arbeit sowie den informellen Austausch über unsere Dissertationsprojekte empfand ich stets als äußerst produktiv sowie wertschätzend und die gemeinsamen Pausen haben den Projektalltag humorvoll aufgelockert. Ich möchte auch Valentina Reitenbach danken, die nach Katjas Ausscheiden aus dem Projekt ihre Stelle übernahm und mit ihrem frischen Blick auf die Studienanlage die weitere Erhebungs- und Auswertungsphase bereicherte. Zudem gilt es Frau Prof. Dr. Jasmin Decristan zu danken, die als weitere Projektleiterin neben Frau Prof. Dr. Dominique Rauch bei Problemen schnell praktikable Lösungsansätze entwickelte. Ein großes Dankeschön möchte ich an alle studentischen Hilfskräfte sowie Praktikanten und Praktikantinnen des Projektes BiPeer richten, die stets engagiert ihre Aufgaben durchführten und für eine gute Stimmung im Projekt gesorgt haben. Hierbei gilt besonderer Dank Tabea Fleps und Katharina Dees, welche mich bei den Kodierungen des Materials intensiv unterstützten.

Weiterhin danke ich meinen Kollegen und Kolleginnen der Abteilung Bildungsqualität und Evaluation des DIPF | Leibniz-Institut für Bildungsforschung und Bildungsinformation für ihr anregendes Feedback in den Kolloquien. Diesbezüglich gilt mein Dank auch dem IDeA-Verbund sowie dem Forschungsschwerpunkt „Sprachliche Bildung und Mehrsprachigkeit“, deren Angebote zum interdisziplinären wissenschaftlichen Austausch einen positiven Einfluss auf die vorliegende Dissertation nahmen.

Schließlich möchte ich mich bei meinen Eltern Ursula und Andreas Schastak bedanken, die mir den Wert von Bildung schon früh vermittelt haben und immer für mich da waren. Weiterhin möchte ich mich bei meinen Freunden Mark, Simon, Marcel, Lukas, Marius, Max, David, Daniel, Achim, Andi, Freddie und Florian für die unterhaltsame, sportive und/oder kreative Ablenkung vom wissenschaftlichen Alltag bedanken. Besonderer Dank gilt meiner Partnerin Anna Kammermann für ihre tatkräftige Unterstützung, Geduld und Humor, die mir insbesondere in der letzten Phase des Dissertationsprozesses Kraft gab und mich zum Durchhalten motivierte. Vielen lieben Dank! 



\section{Inhalt}

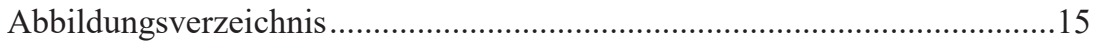

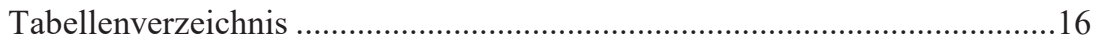

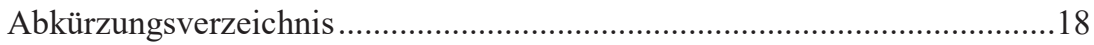

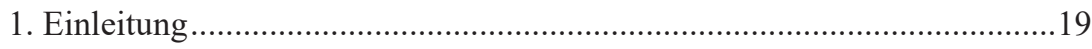

2. Eine kurze Einführung zum Phänomen Sprache .........................................33

2.1 Begriffspräzisierung und Varietäten von Sprache....................................33

2.2 Teilsysteme der Grammatik und zentrale linguistische

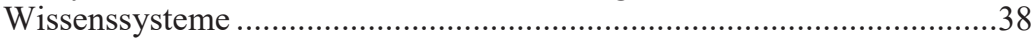

2.3 Sprachliches Wissen und Sprachkompetenz ..........................................40

2.3.1 Sprache als kognitives Werkzeug .................................................43

2.3.2 Metalinguistische Bewusstheit.....................................................44

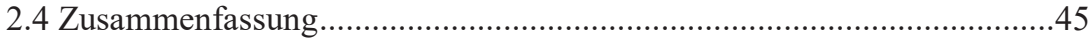

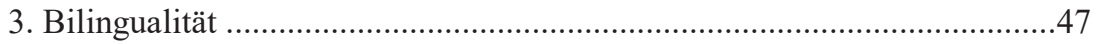

3.1 Begriffsbestimmung Bilingualität ........................................................49

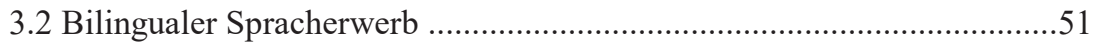

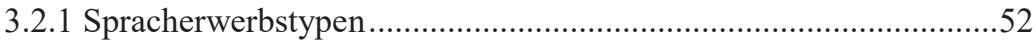

3.2.2 Gemeinsamkeiten und Unterschiede der Entwicklungsverläufe der Spracherwerbstypen im Kontext des Erwerbs des Deutschen ..54

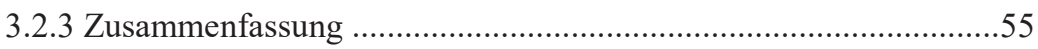

3.3 Bilinguale (Sprach-)Kompetenzen .....................................................56

3.3.1 Historischer Abriss der Bilingualismusforschung zu sprachlichen und kognitiven Kompetenzen von bilingualen Sprechern ..............57

3.3.2 (Meta-)Linguistische Kompetenzen Bilingualer .............................58

3.3.3 (Meta-)Kognitive Konsequenzen von Bilingualität .........................73

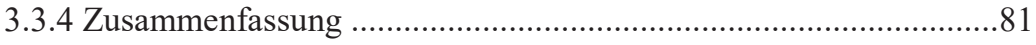

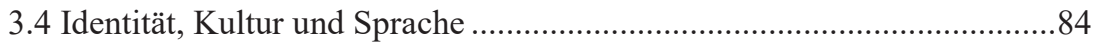

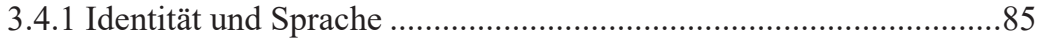

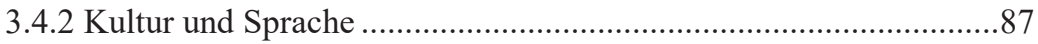


3.4.3 Beziehung der Trias von Identität, Kultur und Sprache ...................88

3.4.4 Einstellungen zu Sprache(n) und Bilingualität ..............................89

3.4.5 Die identitätsstiftende Funktion von Sprache bei bilingualen

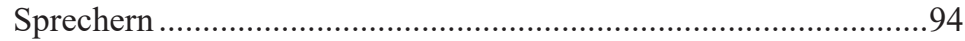

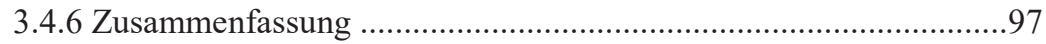

3.5 Bilingualer Sprachgebrauch ................................................................99

3.5.1 Soziolinguistisch orientierte Theorien und Modelle der Sprachwahl und des Sprachverhaltens ............................................101

3.5.2 Diskursiv-rhetorische Zwecke von Code-Switching und

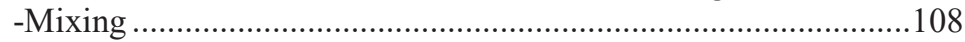

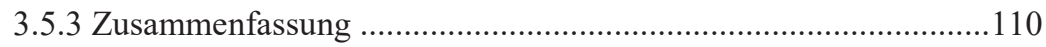

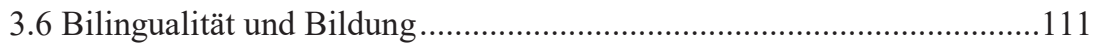

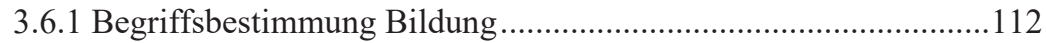

3.6.2 Grundlegende Positionen zum Zusammenhang von

Bilingualität und Bildung.........................................................114

3.6.3 Die Threshold- und Interdependenzhypothese nach Cummins

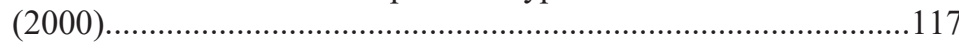

3.6.4 Mono- und bilinguale Schulformen für bilinguale Schülerinnen und

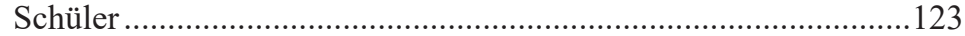

3.6.5 Bilinguale Praktiken in Bildungsprozessen ..................................134

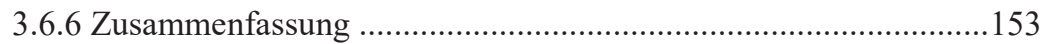

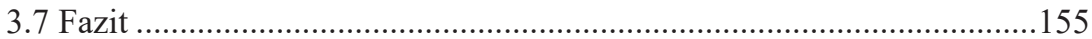

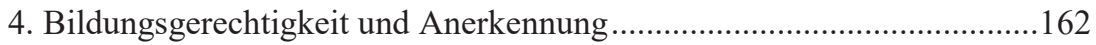

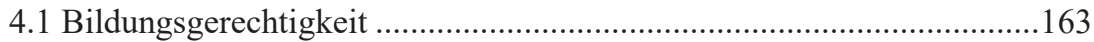

4.1.1 Auf Gleichheit basierende Verteilungsgerechtigkeit ....................165

4.1.2 Auf Menschenwürde basierende Verteilungsgerechtigkeit...........172

4.1.3 Bildungsgerechtigkeit als Anerkennungsgerechtigkeit .................173

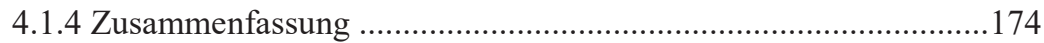

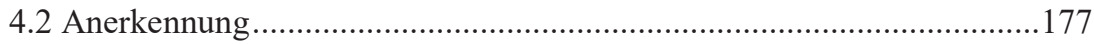

4.2.1 Bedeutungsfacetten und Dimensionen von Anerkennung ............177

4.2.2 Anerkennungstheorie nach Honneth ............................................179

4.2.3 Rezeption der Anerkennungstheorie im erziehungswissenschaftlichen/pädagogischen Diskurs .................185

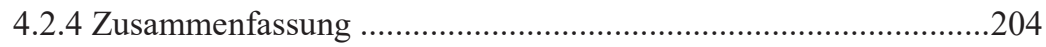

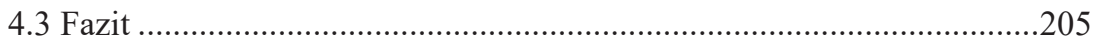




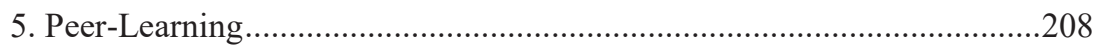

5.1 Grundlagen und Formen des Peer-Learnings ......................................208

5.1.1 Formelles und informelles Peer-Learning nach Johnson und

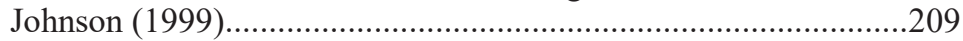

5.1.2 Subtypen des Peer-Learnings am Beispiel „Peer-Tutoring“.........210

5.1.3 Förderliche Basiselemente des Peer-Learnings nach Johnson \&

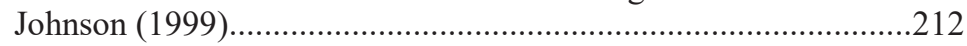

5.2 Befunde von Meta-Analysen zur Effektivität von Peer-Learning..........215

5.3 Interaktion beim Peer-Learning ........................................................218

5.3.1 Förderliche Sprachhandlungen und Diskurskulturen beim

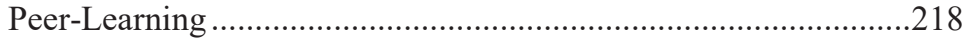

5.3.2 Task-Management und metakognitive Regulation beim Peer-Learning

5.3.3 Bedeutung und Wirkmechanismen von Interaktion beim Peer-Learning für Bildungs- und Lernprozesse..............................229

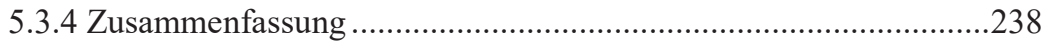

5.4 Kognitive Lernstrategien beim Peer-Learning .....................................239

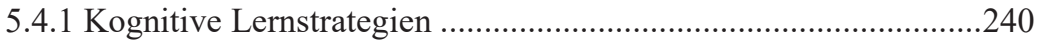

5.4.2 Erwerb und Vermittlung kognitiver Lernstrategien ......................243

5.4.3 Empirische Evidenz zur Lernwirksamkeit von kognitiven

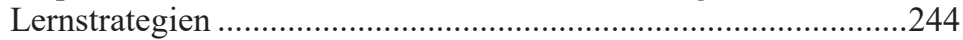

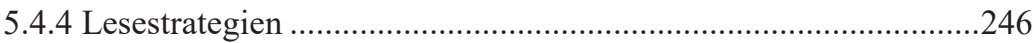

5.4.5 Halbschriftliche Rechenstrategien bei Addition und Subtraktion .250

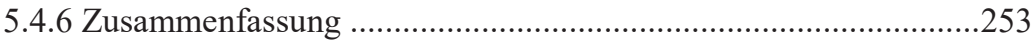

5.5 Bilinguale Interaktion als Angebot beim Peer-Learning .......................255

5.5.1 Evidenz zu bilingualer Interaktion beim Peer-Learning im Kontext bilingualer Bildung

5.5.2 Evidenz zu bilingualer Interaktion beim Peer-Learning im Kontext

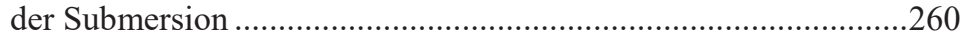

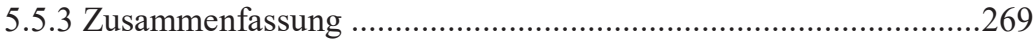

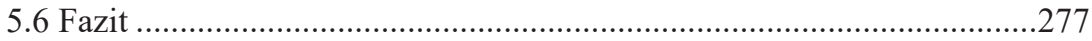

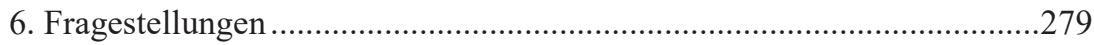

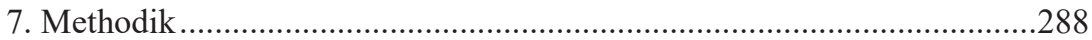

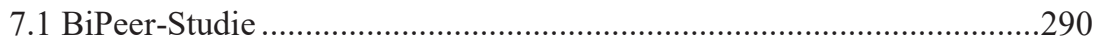




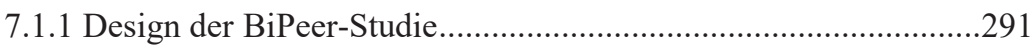

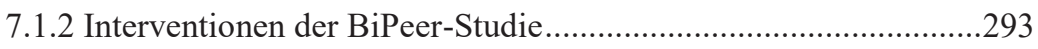

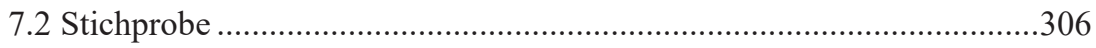

7.3 Datengrundlage und Datenaufbereitung bilingualer Praktiken ...............309

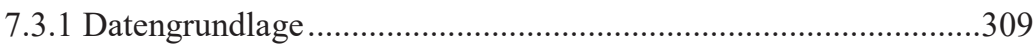

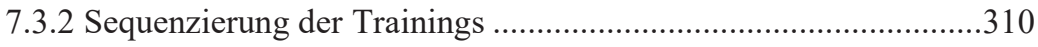

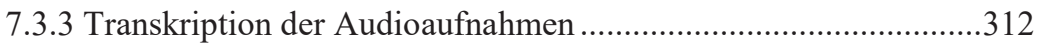

7.3.4 Operationalisierung bilingualer Praktiken ..................................313

7.3.5 Auszählung bilingualer Praktiken mit MAXQDA .........................317

7.4 Quantitative Datenanalyse ................................................................. 318

7.4.1 Prüfung der Abhängigkeit bilingualer Praktiken von der Dyade

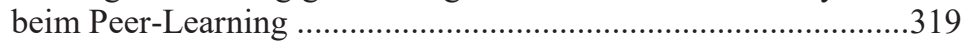

7.4.2 Prävalenz bilingualer Praktiken mit und ohne explizitem bilingualen Interaktionsangebot beim Peer-Learning

7.4.3 Der Zusammenhang sprachlicher und motivationaler Eigenschaften mit der Prävalenz bilingualer Praktiken beim

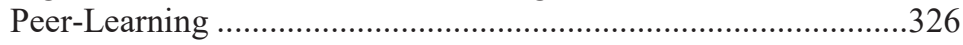

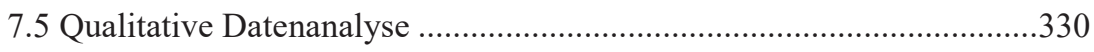

7.5.1 Selbstberichte des Sprachgebrauchs im Training und dessen

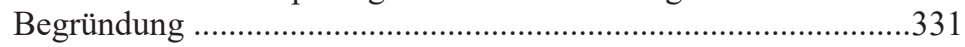

7.5.2 Funktionen bilingualer Praktiken beim Peer-Learning .................337

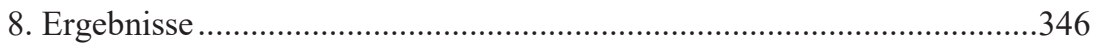

8.1 Prüfung der Abhängigkeit der Prävalenz bilingualer Praktiken von

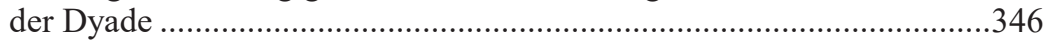

8.2 Prävalenz bilingualer Praktiken mit und ohne explizitem bilingualen Interaktionsangebot beim Peer-Learning .............................351

8.2.1 Vergleich der Prävalenz bilingualer Praktiken zwischen den Angebots- und Nichtangebotsgruppen.........................................355

8.2.2 Vergleich der Prävalenz bilingualer Praktiken zwischen den Angebotsgruppen LGT/D und RGT/D ......................................356

8.2.3 Prävalenz bilingualer Praktiken innerhalb der verschiedenen Phasen

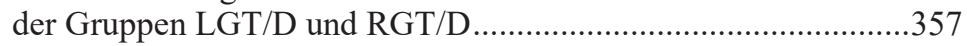

8.3 Zusammenhänge sprachlicher und motivationaler Merkmale mit der Prävalenz bilingualer Praktiken

8.4 Ergebnisse der Selbstberichte für die Annahme und Ablehnung des bilingualen Sprachangebots 
8.4.1 Ergebnisse des geschlossenen Formats der Selbstberichte für die Annahme und Ablehnung des bilingualen Sprachangebots

8.4.2 Ergebnisse des offenen Formats der Selbstberichte für die Annahme und Ablehnung des bilingualen Sprachangebots 368

8.5 Ergebnisse der Funktionsanalyse bilingualer Praktiken beim Peer-

Learning .375

8.5.1 Summative Ergebnisse der Haupt- und Unterkategorien der Funktionsanalyse bilingualer Praktiken beim Peer-Learning .375

8.5.2 Zentrale Kennwerte der Haupt- und Unterkategorien der Funktionsanalyse bilingualer Praktiken beim Peer-Learning .377

8.5.3 Nähere Beschreibung der Nutzungsweise bilingualer Praktiken für die vier Unterkategorien der Hauptkategorie „On-Task“ 379

9. Diskussion 385

9.1 Diskussion der einzelnen Ergebnisse der Studie .385

9.2 Datenintegration der quantitativen und qualitativen Ergebnisse der Studie.... 401

9.3 Praktische Implikationen der Studie 407

9.4 Limitationen der Studie und Forschungsdesiderata 413

10. Fazit 418

11. Literaturverzeichnis 424 


\section{Abbildungsverzeichnis}

Abb. 1 Soziokognitives Modell der Sprachentwicklung nach Hamers

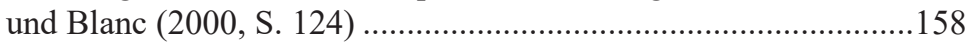

Abb. 2 Mixed-Methods-Vertiefungsdesign des Forschungsvorhabens......290

Abb. 3 Struktur einer Sitzung der Übungsphase des Lesetrainings ...........298

Abb. 4 Globalstrategie Schrittweise Rechnen .............................................300

Abb. 5 Globalstrategie Stellenweise Rechnen ..........................................300

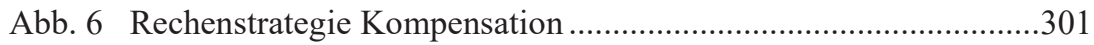

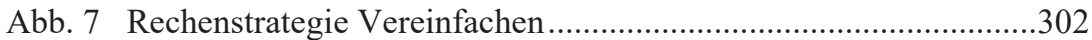

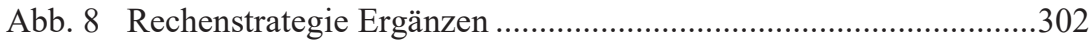

Abb. 9 Struktur einer Sitzung der Übungsphase des Rechentrainings ........304

Abb. 10 Beispiel für einen paarweise strukturierten Datensatz ....................321

Abb. 11 Mögliche paarweise Korrelationen zweier Variablen bei ununterscheidbaren Dyaden nach Griffin \& Gonzalez (1995, S. 432)

Abb. 12 Histogramm der Prävalenz bilingualer Praktiken der aggregierten Angebotsgruppen LGT/D \& RGT/D auf Individualebene

Abb. 13 Histogramm der logtransformierten Werte der Prävalenz bilingualer Praktiken der aggregierten Angebotsgruppen LGT/D \& RGT/D auf Individualebene.

Abb. 14 Streudiagramm der logtransformierten deskriptiven Werte der Prävalenz bilingualer Praktiken der Probanden der aggregierten Angebotsgruppen LGT/D \& RGT/D 


\section{Tabellenverzeichnis}

Tab. 1 Interventionsdesign der BiPeer-Studie............................................291

Tab. 2 Zentrale Kennwerte der Stichprobe auf Individualebene.................307

Tab. 3 Zentrale Kennwerte der Dyadenkonstellation der Stichprobe

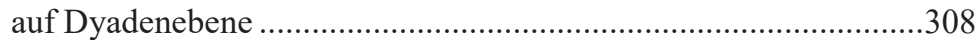

Tab. 4 Deskriptive Werte der Prävalenz bilingualer Praktiken der aggregierten Angebotsgruppen LGT/D \& RGT/D auf Individualebene

Tab. 5 Logtransformierte deskriptive Werte der Prävalenz bilingualer Praktiken der aggregierten Angebotsgruppen LGT/D \& RGT/D auf Individualebene .348

Tab. 6 Deskriptive Werte der bilingualen Sprachkompetenz, sprachbezogener Einstellungen und der Sprachpraxis auf Individualebene

Tab. 7 Deskriptive Werte der bilingualen Sprachkompetenz, sprachbezogener Einstellungen und der Sprachpraxis auf Dyadenebene

Tab. 8 Deskriptive Werte der Prävalenz bilingualer Praktiken der aggregierten Angebotsgruppen LGT/D und RGT/D sowie der aggregierten Nicht-Angebotsgruppen LGD und RGD auf Dyadenebene

Tab. 9 Deskriptive Werte der Prävalenz bilingualer Praktiken der Angebotsgruppen LGT/D \& RGT/D auf Dyadenebene

Tab. 10 Deskriptive Werte der Prävalenz bilingualer Praktiken innerhalb der verschiedenen Phasen der Gruppe LGT/D auf Dyadenebene .358

Tab. 11 Deskriptive Werte der Prävalenz bilingualer Praktiken innerhalb der verschiedenen Phasen der Gruppe RGT/D auf Dyadenebene

Tab. 12 Deskriptive Werte der motivationalen Variablen der Gruppen LGT/D und RGT/D auf Individualebene. 361

Tab. 13 „Overall correlation“ und „cross-intraclass correlation“ sprachlicher Variablen mit der logtransformierten Prävalenz bilingualer Praktiken der aggregierten Gruppen LGT/D und RGT/D auf Individualebene 
Tab. 14 „Overall correlation“ und „cross-intraclass correlation“ motivationaler Variablen mit der logtransformierten Prävalenz bilingualer Praktiken der aggregierten Gruppen LGT/D und RGT/D auf Individualebene. 366

Tab. 15 Selbstberichtete Annahme und Ablehnung des Sprachangebots im geschlossenen Format

Tab. 16 Häufigkeiten der Begründungsebenen nach Annahme und Ablehnung sowie nach Erhebungszeitpunkten 369

Tab. 17 Prävalenzen der Kategorien bei der Annahme des Angebots .370

Tab. 18 Prävalenzen der Kategorien bei der Ablehnung des Angebots 374

Tab. 19 Prävalenzen der Hauptkategorien der Funktionen bilingualer Praktiken beim Peer-Learning

Tab. 20 Prävalenzen der On-Task-Unterkategorien der Funktionen bilingualer Praktiken beim Peer-Learning

Tab. 21 Zentrale Kennwerte der Hauptkategorien der Funktionen bilingualer Praktiken beim Peer-Learning .378

Tab. 22 Zentrale Kennwerte der Unterkategorien der Hauptkategorie „On-Task“ der Funktionen bilingualer Praktiken beim PeerLearning 379 


\section{Abkürzungsverzeichnis}

2L1a $=$ Bilingualer Erstspracherwerb

L1 = Erstsprache

L1a $=$ Erstspracherwerb

L2 = Zweitsprache

L2a = Zweitspracherwerb

$\mathrm{MH}=$ Migrationshintergrund

PL = Peer-Learning 


\section{Einleitung}

Bilingualität - verstanden als Aufwachsen mit und/oder als alltäglicher Gebrauch von zwei Sprachen - stellt aus internationaler Perspektive den linguistischen Normalfall dar, während Monolingualität eher als eine Ausnahmeerscheinung zu bezeichnen ist (vgl. z. B. Gogolin, 2015, S. 293; Tracy, 2011, S. 72). Diverse einwohnerreiche Staaten wie z. B. China, Indien, Pakistan, Nigeria, Mexiko oder Kanada haben entweder mehr als eine offizielle Amtssprache und/oder erkennen je nach Region weitere Sprachen als legitime Amtssprachen neben der allgemeingültigen Verkehrssprache an. Selbst wenn Staaten lediglich eine offizielle Amtssprache anerkennen, kann dennoch kaum von Monolingualität innerhalb von Staaten gesprochen werden: „Der weitaus größte Teil der Staaten der Welt beheimatet mehr als eine Sprache“ (Gogolin, 2015, S. 293). Dieses Postulat stellt angesichts der Sprachenvielfalt von ca. 7000 verschiedenen Sprachen (vgl. Lewis, Simons \& Fennig, 2015) bei weltweit ungefähr 195 Staaten keine Überraschung dar. Insbesondere Kolonialismus und Migrationsbewegungen tragen Sprachen über Staatsgrenzen hinaus, sodass mehrsprachige Gemeinschaften in Teilen der Welt entstehen, in denen die jeweiligen Sprachen zuvor nicht gesprochen sowie gelebt wurden. Unabhängig davon, ob Bilingualität kollektiv im Selbstverständnis eines Staates implementiert ist oder individuell durch eigene oder familiäre Migrationsbewegungen entsteht (Edwards, 2013, S. 6), stellt der Gebrauch von mehr als einer Sprache im Alltag die Realität für eine Vielzahl von Menschen dar.

Nichtsdestotrotz vertreten einige Staaten wie z. B. die Bundesrepublik Deutschland vehement ein monolinguales Selbstverständnis, selbst wenn sie faktisch Einwanderungsgesellschaften sind (vgl. Gogolin, 2015, S. 292f). Das monolinguale Selbstverständnis Deutschlands lässt sich historisch rekonstruieren, ist in zentralen Institutionen wie z. B. der Regelschule verankert (vgl. Gogolin, 1994) und wird auch von Teilen der Gesellschaft vertreten, obwohl Deutschland spätestens mit der Gastarbeiterbewegung ab den 1950er Jahren als Einwanderungsland bezeichnet werden kann. Auch diverse gesetzliche Grundlagen zur Zuwanderung unterstützen eine Kategorisierung Deutschlands als Einwanderungsland. So regeln z. B. das Asylrecht, der „Aufenthaltstitel“ oder die „Personenfreizügigkeit“ die Zuwanderung der Bundesrepublik Deutschlands auf gesetzlicher Ebene. Insbesondere die gesetzlichen Grundlagen der Personenfreizügigkeit, welchen allen Bürgern der Europäischen Union (EU) prinzipiell eine dauerhafte Niederlassung in der Bundesrepublik Deutschland samt Arbeitserlaubnis gewährt, sprechen deutlich für den Status Deutschlands als Einwanderungsland. Ein monolinguales Selbstverständnis erscheint dementsprechend unangemessen.

Während der Migration und auch in den Generationen danach werden die jeweilige, von der ursprünglichen Herkunft geprägte Identität und Kultur nicht 
aufgegeben, sondern innerhalb diverser Anpassungsprozesse bewahrt und/oder adaptiert (vgl. z. B. Bohnsack \& Nohl, 2001; Hu, 2007; Keim, 2012; Stojanov, 2006, 2011). Hierbei handelt es sich aber nicht zwingend nur um unidirektionale Anpassungsprozesse einer gesellschaftlichen Minderheit an eine gesellschaftliche Mehrheit, da z. B. bei dem Fusionsmodell der Akkulturation (vgl. Arends-Tóth \& van de Vijver, 2006) auch reziproke, bidirektionale Anpassungsprozesse der Mehrheits- und Minderheitsgesellschaft im Kontext der Einwanderung beobachtet werden können. Identität und Kultur sind eng mit Sprache verbunden, da Sprache sowohl zur Konstituierung von Identität und Kultur beiträgt als auch für beide Phänomene als salienter Marker fungieren kann (vgl. Hu, 2007). Migranten in Europa geben ihre Erstsprache (L1) nach zwei Generationen nicht auf, wie es z. B. im Kontext der Vereinigten Staaten von Amerika der Fall ist (vgl. Gogolin, 2015, S. 293). Hierbei erwerben Kinder und Jugendliche mit Migrationshintergrund (MH) in Europa häufig die L1 ihrer Eltern früher als oder parallel zur Verkehrssprache des jeweiligen Einwanderungslandes. Folglich kommunizieren bilingual aufwachsende Kinder und Jugendliche mit MH in Deutschland im familiären Umfeld (vgl. Reich, 2009) und in ihrer bilingualen Peer-Group (vgl. Keim, 2007, 2012) in ihren beiden Sprachen. Hierbei befinden sie sich in einem „bilingualen Sprachmodus“ (Grosjean, 2008), in welchem es zu intensiven Formen von Sprachwechseln und -mischungen kommen kann (vgl. z. B. Auer, 2009; Fuller, 2009; GardnerChloros, 2009; Potowski, 2009).

Dementsprechend herrscht auch in der Bundesrepublik Deutschland faktisch eine Sprachenvielfalt, die allerdings ähnlich wie dessen Status als Einwanderungsland von Teilen der Politik, Medien und Gesellschaft nicht anerkannt wird. Dies spiegelt sich auch in der vorherrschenden Sprachideologie wider, die als ,assimilation ideology“ (vgl. Bourhis, 2001, S. 13-14) bezeichnet werden kann. Diese Sprachideologie greift zwar nicht direkt in die Privatsphäre von gesellschaftlichen Minderheiten ein, allerdings drückt sie implizit die Erwartungshaltung aus, dass sich gesellschaftliche Minderheiten im Lauf der Zeit freiwillig an die Mehrheitsgesellschaft anpassen und somit deren Werte, kulturelle Praktiken und Sprache übernehmen. Sprachideologien operieren auch über die sprachliche Gestaltung staatlicher Institutionen. So herrscht z. B. in der deutschen Regelschule ein „monolingualer Habitus“, in welchem das Lernen und die Kommunikation stets einsprachig - mit Ausnahme des Fremdsprachenunterrichts - in der Verkehrssprache Deutsch erfolgt und die Lehrkräfte sich an der ,monolingualen Norm“ ihrer Schülerschaft orientieren (vgl. Gogolin, 1994). Weiterhin tragen die in der Bundesrepublik Deutschland weit verbreiteten und regelmäßig auftretenden Mythen über Mehrsprachigkeit ebenfalls zur Problematisierung und Diffamierung von migrationsbedingter Bilingualität bei. So zeichnen Prämissen wie z. B. „Mehrsprachigkeit ist eine Überforderung für die Kinder“, ,zunächst muss ein Kind seine 
Muttersprache beherrschen bevor es eine andere Sprache lernen kann“, „,mehrsprachige Kinder lernen keine Sprache richtig“ oder ,auch nicht-deutsche Eltern sollten mit ihren Kindern mehr Deutsch sprechen“ (vgl. z. B. Kersten et al., 2011), welche alle auf Basis empirischer Evidenz als falsch zu beurteilen sind, ein inadäquates Bild von Bilingualität und Mehrsprachigkeit. Insbesondere die ersten beiden zitierten Mythen weisen auf den selbst in der Bilingualismusforschung verbreiteten „monolingual bias“ (vgl. Butler, 2013, S. 119f) hin, welcher Monolingualität und den monolingualen Spracherwerb als sprachliche Norm definiert und aus dieser Perspektive Bilingualität betrachtet. Der „monolingual bias“ (vgl. ebd.) spricht Bilingualität und bilingualem Spracherwerb stets eine gewisse Devianz beziehungsweise Andersartigkeit zu.

Mehrsprachigkeit stellt allerdings ein zentrales Bildungsziel in einer globalisierten Welt dar, welches auf verschiedenen politisch-gesellschaftlichen Ebenen gefördert wird. So wird Mehrsprachigkeit z. B. von der EU in der Charter zum Schutz und zur Förderung von linguistischen Minderheiten wertgeschätzt und im multilingualen Spracherwerbsziel „mother tongue plus two“ gefördert (vgl. Yağmur, 2016). In Deutschland stellt Mehrsprachigkeit sowohl durch die langfristige Etablierung des Fremdsprachenunterrichts in den schulischen Curricula der Länder als auch im vorschulischen Bereich in den Bildungs- und Erziehungsplänen ein zentrales Bildungsziel dar (vgl. Viernickel \& Schwarz, 2009). Dementsprechend gilt Mehrsprachigkeit als eine relevante Fähigkeit zur Teilhabe an einer globalisierten Gesellschaft sowie zur internationalen Zusammenarbeit, über welche auch hinsichtlich der verschiedenen politischen Ebenen Konsens als zentrales Bildungsziel herrscht.

Diese in der Bundesrepublik Deutschland vorliegende Paradoxie eines monolingualen Selbstverständnisses trotz des Status als Einwanderungsland bei gleichzeitiger Bestrebung des Bildungsziels Mehrsprachigkeit kann damit erklärt werden, dass nicht alle Arten von Bilingualität und Mehrsprachigkeit gleichermaßen anerkannt, wertgeschätzt und gefördert werden. Hinsichtlich der individuellen und gesellschaftlichen Wahrnehmung von Sprachen lässt sich eine gewisse Sprachenhierarchie konstatieren, die einigen Sprachen und Sprachkombinationen ein höheres Prestige zuspricht als anderen (vgl. Hélot, 2006). Für die Bundesrepublik Deutschland werden neben dem Deutschen primär Sprachen des Fremdsprachenkanons wie Englisch, Französisch und Latein präferiert und priorisiert, während Sprachen von gesellschaftlichen Minderheiten mit MH wie Türkisch, Polnisch, Italienisch oder Russisch kaum wertgeschätzt und gefördert werden (vgl. Tracy, 2014, S. 20). So tragen z. B. die letztgenannten Sprachen in der Institution Schule weder den Status als legitime Sprachen im Unterricht noch - mit wenigen Ausnahmen einzelner Bundesländer (vgl. Schröder \& Küppers, 2016) - als versetzungsrelevante Sprachen des Fremdsprachenkanons. Mit Ausnahme des Herkunftssprachlichen Unterrichts (HSU), der weder an allen Schulen noch für alle Sprachen angebo- 
ten wird und auch nicht mit dem Regelunterricht verbunden sowie gleichgestellt ist (vgl. ebd.), stehen mehrsprachigen SuS keine institutionalisierten Angebote vom Staat zum Ausbau ihrer (bildungs-)sprachlichen Fähigkeiten in ihrer anderen Sprache neben dem Deutschen zur Verfügung. Zudem sehen ein nicht zu unterschätzender Anteil an Lehrkräften (vgl. Wlossek \& Rost-Roth, 2016) und pädagogischen Fachkräften (vgl. Kratzmann et al., 2017) zwischen der deutschen Sprache und Sprachen von gesellschaftlichen Minderheiten ein Konkurrenzverhältnis, in welchem die Förderung der einen Sprache auf Kosten der anderen geht. Diese Prämisse eröffnet eine Defizitperspektive auf die mehrsprachige Disposition von Schülerinnen und Schülern (SuS) mit MH, die keine Ressource darstellt, sondern ein Hindernis für die Entwicklung deutschsprachiger Fähigkeiten. Dementsprechend können medial diskutierte Forderungen der sprachlichen Fokussierung auf das Deutsche (vgl. z. B. Reimann, 2014; Seibel, 2016) wie der oben bereits angesprochene Mythos, Eltern mit MH sollen zuhause mit ihren Kindern Deutsch sprechen (vgl. Kersten et al., 2011, S. 90f), oder die „Deutschpflicht auf dem Schulhof" sowohl als Formen eines impliziten Assimilationsdrucks als auch einer Hierarchisierung von Sprachen interpretiert werden.

Die Krux an dem monolingualen Habitus und der Sprachenhierarchisierung der deutschen Regelschule ist nicht, dass bestimmten Sprachen eine höhere Relevanz zugesprochen wird als anderen. Angesichts der Bedeutung der deutschen Sprache für die Teilhabe an der Gesellschaft in der Bundesrepublik Deutschland stellt der Erwerb hoher (bildungs-)sprachlicher Fähigkeiten im Deutschen ein zentrales Bildungsziel dar, dem auch Bürger mit MH zustimmen (Gogolin, 2015, S. 294). Auch die Relevanz einer Fremdsprache wie Englisch als internationale „lingua franca“ in einer globalisierten Welt erscheint absolut nachvollziehbar und verständlich. Vielmehr liegt die zentrale Problematik des monolingualen Habitus (vgl. Gogolin, 1994) und der Sprachenhierarchisierung der deutschen Regelschule in den unterschiedlichen Narrativen der rechtlichen Anerkennung und sozialen Wertschätzung der vorliegenden Mehrsprachigkeit abhängig von dem jeweiligen Sprachenprofil. So wird z. B. die L1 Englisch von SuS, die Deutsch als Zweitsprache (L2) erwerben, aufgrund ihres Status als Sprache des Fremdsprachenkanons im Gegensatz zum Türkischen, Polnischen, Russischen etc. nicht problematisiert, sondern eher wertgeschätzt. Diese Form der Sprachenhierarchisierung dürfte zu einem nicht zu unterschätzenden Anteil durch den empirisch robusten Zusammenhang der Einstellungen zu einer Sprache in Abhängigkeit von der Wahrnehmung des Status der prototypischen Gruppe der Sprecher dieser Sprache bedingt sein (vgl. Chin \& Wigglesworth, 2007, S. 108; Myers-Scotton, 2006, S. 120). Sprache kann dementsprechend als ,Sozialdiagnostik“ fungieren, indem der Gebrauch einer bestimmten Sprache zu einer Assoziation des Sprechers mit einer bestimmten Gruppe führt (vgl. Myers-Scotton, 2006, S.110). 
Zudem gilt es die fehlende Sensibilität des monolingualen Habitus der Regelschule (vgl. Gogolin, 1994) für spracherwerbsbedingte Unterschiede in den verkehrssprachlichen sowie mehrsprachigen Kompetenzen und Voraussetzungen von bilingual aufwachsenden SuS mit MH zu kritisieren. Ein bilingualer Spracherwerb weist quantitative und qualitative Unterschiede zum monolingualen Spracherwerb auf (vgl. z. B. Schulz \& Grimm, 2012; Grimm \& Schulz, 2014, 2016; Tracy, 2008), die sich unter anderem in Kompetenzunterschieden hinsichtlich bestimmter sprachlicher Fähigkeiten der Einzelsprachen manifestieren. Andererseits sind mit Bilingualität allerdings auch bestimmte metalinguistische (vgl. z. B. Bialystok, 2013; Bien-Miller et al., 2017), diskursiv-rhetorische (vgl. z. B. Auer, 2009; Fuller, 2009; Keim, 2012; Potowski, 2009) und bildungsbezogene (vgl. z. B. Barwell, 2014; Clarkson, 2007; García \& Kano, 2014; García \& Wei, 2014; Moschkovich, 2007; Rauch et al., in Vorb.; Schüler-Meyer et al. 2017) Vorteile verbunden, die als Ressourcen für Bildungsprozesse eingesetzt werden können. Werden diese unterschiedlichen Voraussetzungen und Ressourcen bilingual aufwachsender SuS mit MH in Bildungsinstitutionen nicht adäquat und produktiv berücksichtigt und sie stattdessen anhand einer sozialen Bezugsnorm monolingual aufwachsender SuS beurteilt, kann dies zur Bildungsbenachteiligung mehrsprachig aufwachsender SuS mit $\mathrm{MH}$ beitragen. SuS mit MH, die häufig durch eigene Migrationserfahrungen oder die Migration ihrer Eltern bilingual aufwachsen, sind in Deutschland bereits früh sowie langfristig von Bildungsbenachteiligung betroffen. Dies lässt sich an einer Vielzahl empirischer Studien belegen (siehe z. B. Baumert \& Schümer, 2001; Britz, 2006; Gomolla \& Radtke, 2009; Haag et al., 2012, 2016; Kempert et al., 2016; Rauch et al., 2016; Tarelli, Schwippert \& Stubbe, 2012; Wendt \& Schwippert, 2017). Obwohl die Ursachen für die Bildungsbenachteiligung von Kindern und Jugendlichen multidimensional und komplex sind (vgl. z. B. Baumert \& Schümer, 2001; Britz, 2006), stehen sprachliche Defizite im Deutschen bis heute stets im Vordergrund des Diskurses über die Gründe für die Bildungsbenachteiligung dieser Gruppe. Allerdings kann auch eine frühzeitig einsetzende und intensive Förderung der Verkehrssprache Deutsch die Bildungsbenachteiligung dieser Schülerschaft nicht neutralisieren, was sich anhand der ernüchternden Ergebnisse flächendeckender, zum Teil verpflichtender Sprachdiagnostik und -förderung im Zuge des „PISA-Schocks“ des Jahres 2001 erkennen lässt.

Die vorliegende Studie versucht einen Beitrag zur Überwindung dieses problematischen Umgangs mit der Mehrsprachigkeit von SuS mit MH für den Regelschulkontext der Bundesrepublik Deutschland zu leisten. Hierfür wird auf Basis der Erkenntnisse der Bilingualismusforschung sowie anerkennungstheoretischer Reflexionen eine im Regelschulunterricht umsetzbare Möglichkeit zur Implementation der Mehrsprachigkeit von SuS mit MH durch ein bilinguales Interaktionsangebot innerhalb der Sozialform des Peer-Learnings 
(PL) mit Hilfe eines quasi-experimentellen Treatment-Kontrollgruppen Designs unter Verwendung von Mixed-Methods untersucht. Die Implementation der Mehrsprachigkeit von SuS mit MH in den Regelunterricht wird bereits seit einiger Zeit gefordert (vgl. z. B. Rehbein, 2011). Allerdings ist die bisherige empirische Befundlage noch nicht in der Lage, evidenzbasierte, effektive und auch umsetzbare Methoden zur Implementation und Förderung der Mehrsprachigkeit in den Regelunterricht zu empfehlen. PL, definiert als ,the acquisition of knowledge and skill through active helping and supporting among status equals or matched companions" (Topping, 2005, S. 631), scheint hierfür eine fruchtbare Methode darzustellen, da SuS mit dem gleichen Sprachprofil beim gemeinsamen Lernen bilingual interagieren können, ohne dass die Lehrkraft selbst über die nicht-verkehrssprachlichen Fähigkeiten der SuS verfügen muss.

Dementsprechend verfolgt die vorliegende Studie primär drei Ziele: 1) Zunächst soll eine adäquate Beschreibung des Phänomens Bilingualität sowie der damit verbundenen Kompetenzen und Konsequenzen erfolgen, die es für angemessene und fruchtbare Bildungsprozesse von bilingual aufwachsenden SuS mit $\mathrm{MH}$ zu berücksichtigen gilt. 2) Die Notwendigkeit der Berücksichtigung der bilingualen Disposition bei Bildungsprozessen dieser Schülerschaft wird hierbei nicht nur über die Lernwirksamkeit und positiven Effekte eines adaptiven Umgangs mit Bilingualität motiviert, sondern auch aus anerkennungstheoretischer Perspektive legitimiert (vgl. Honneth, 2003; Mecherill, 2005; Stojanov, 2006, 2011). Hierbei wird sich zeigen, dass der von dem monolingualen Habitus und der Sprachenhierarchisierung geprägte Umgang der Regelschulen der Bundesrepublik Deutschland mit der Bilingualität von SuS mit MH eine Missachtung sowohl der Anerkennungsformen des Rechts als auch der Solidarität darstellt. Diese Missachtungen hemmen ihre Entwicklung zu autonomen, selbstbestimmten Individuen, was den in der vorliegenden Studie postulierten Handlungsbedarf zu einem anderen Umgang mit Bilingualität argumentativ begründet. 3) Darauf aufbauend soll mit einem bilingualen Interaktionsangebot bei der Sozialform des PL geprüft werden, ob sich diese Methode zur Implementation der Bilingualität von bilingual aufwachsenden SuS mit MH eignet. Unter der Annahme, dass unterrichtliche Settings lediglich Angebote machen, die von den SuS unterschiedlich angenommen und umgesetzt werden können (siehe z. B. Helmke, 2017, S. 69-101; Seidel, 2014), stellt die Optionalität des bilingualen Sprachangebots eine vielversprechende Eigenschaft der PL-Intervention dar. Angesichts der fehlenden Erfahrung der SuS mit dem bildungssprachlichen Gebrauch ihrer weiteren Sprache neben dem Deutschen gilt es zu klären, ob bilinguale SuS ihre beiden Sprachen beim PL für zentrale schulische Inhalte überhaupt (förderlich) nutzen (können) oder ob sie ein solches bilinguales Sprachverhalten in Bildungsprozessen als zusätzliche Belastung empfinden.

Zur empirischen Untersuchung eines solchen bilingualen Interaktionsangebots beim PL liegt eine adäquate Datengrundlage aus der „BiPeer-Studie“ vor, 
die unter anderem das bilinguale Sprachangebot beim PL für bilingual türkisch-deutschsprachig aufwachsende SuS der dritten und vierten Klasse systematisch für die Lerninhalte Lese- und Rechenstrategien variiert. Mit Hilfe dieses quasi-experimentellen Treatment-Kontrollgruppen Designs und einem Mixed-Methods Vertiefungsdesign (Kuckartz, 2014, S. 78) als Studienanlage soll in der vorliegenden Studie die Umsetzung des bilingualen Sprachangebots durch die SuS untersucht werden. Die zentralen Erkenntnisinteressen fokussieren aus quantitativer Perspektive die Prävalenz bilingualen Sprachverhaltens, deren Abhängigkeit von individuellen Eigenschaften, von der PLGruppe, vom Lerninhalt sowie von bestimmten Phasen und Aufgaben beim PL. Hinsichtlich qualitativer Aspekte des bilingualen Interaktionsangebots beim PL werden selbstberichtete Gründe für die Annahme oder Ablehnung des Sprachangebots und die Funktionen bilingualer Gesprächsbeiträge untersucht.

Die Zielsetzungen und Argumentationsstränge der vorliegenden Studie verlangen eine interdisziplinäre Auseinandersetzung mit den zu untersuchenden Phänomenen. Die relevanten Phänomene und Teilaspekte des in der Studie umgesetzten bilingualen Interaktionsangebots beim PL werden entweder vornehmlich von einer Fachwissenschaft unter disziplineigener Perspektive erforscht oder von mehreren Fachwissenschaften mit verschiedenen Schwerpunktsetzungen untersucht. Dementsprechend werden primär diejenigen Perspektiven und Zugänge der Disziplinen Erziehungswissenschaft, Linguistik, Didaktik, Philosophie und Psychologie gewählt, die für die jeweiligen zu betrachtenden Teilaspekte am fruchtbarsten erscheinen.

Die vorliegende Studie ist folgendermaßen aufgebaut: Zunächst bedarf es einer kurzen, grundlegenden Auseinandersetzung mit dem Phänomen Sprache (Kapitel 2), um ein adäquates Verständnis des Phänomens Bilingualität erhalten zu können. Hierfür wird der Begriff „Sprache“ näher erläutert und die Bandbreite distinkter sprachlicher Formen anhand verschiedener Varietäten verdeutlicht (Abschnitt 2.1). Aufbauend auf diesem Grundverständnis von Sprache wird die Grammatik von Sprache näher betrachtet, wobei ausgehend von einem modularen Ansatz die einzelnen Teilsysteme der Grammatik sowie weitere zentrale linguistische Wissenssysteme dargestellt werden (Abschnitt 2.2). Anschließend gilt es auf Basis der in Abschnitt 2.2 erworbenen Erkenntnisse zu reflektieren, was in Anbetracht eines modularen Sprachsystems unter sprachlichem Wissen und Sprachkompetenz zu verstehen ist (Abschnitt 2.3), wobei auch die Funktion von Sprache als kognitives Werkzeug (Abschnitt 2.3.1) und metalinguistische Bewusstheit (Abschnitt 2.3.2) fokussiert werden. Abschließend werden die wichtigsten Erkenntnisse des zweiten Kapitels zusammengefasst (Abschnitt 2.4).

Im nächsten Kapitel werden zentrale Aspekte des Phänomens Bilingualität dargestellt, die für die vorliegende Studie von besonderer Relevanz sind (Kapitel 3). Zu Beginn werden der Begriff „Bilingualität“ und dessen Vielfalt 
möglicher Definitionen betrachtet, um eine Arbeitsdefinition dieses Phänomens für die vorliegende Studie festzulegen (Abschnitt 3.1). Anschließend werden verschiedene Aspekte von Bilingualität näher beleuchtet, wobei der bilinguale Spracherwerb (Abschnitt 3.2), bilinguale (Sprach-)Kompetenzen (Abschnitt 3.3), die Zusammenhänge von Identität, Kultur und Sprache bei bilingualen Sprechern (Abschnitt 3.4), bilingualer Sprachgebrauch (Abschnitt 3.5) sowie Bilingualität und Bildung (Abschnitt 3.6) fokussiert werden.

Den ersten zu vertiefenden Aspekt nach der Begriffsbestimmung von Bilingualität stellt der bilinguale Spracherwerb dar, der vom monolingualen Spracherwerb abzugrenzen ist (Abschnitt 3.2). Hierbei werden verschiedene Spracherwerbstypen präsentiert, wobei insbesondere der doppelte Erstspracherwerb (2L1a), der Zweitspracherwerb (L2a) sowie der monolinguale Spracherwerb (L1a) definitorisch voneinander abgegrenzt werden (Abschnitt 3.2.1). Anschließend werden deren Bedingungen und Entwicklungsverläufe im Kontext der Verkehrssprache Deutsch miteinander verglichen (Abschnitt 3.2.2), um potentielle Unterschiede der verschiedenen Spracherwerbstypen hinsichtlich der Sprachkompetenz im Deutschen vor allem zu Beginn und während der Grundschule angemessen einschätzen zu können. Die zentralen Erkenntnisse des Abschnitts 3.2 werden in Abschnitt 3.2.3 zusammengefasst.

Anschließend werden bilinguale (Sprach-)Kompetenzen intensiv betrachtet (Abschnitt 3.3), da diese in ihrer Bedeutung für die Wahrnehmung und Wertschätzung bilingualer Sprecher sowie für den konkreten Umgang mit bilingualen Dispositionen von hoher Bedeutung sind. Ausgehend von einem kurzen historischen Abriss der Bilingualismusforschung zu sprachlichen und kognitiven Kompetenzen von bilingualen Sprechern (Abschnitt 3.3.1) werden Theorien und empirische Befunde zu (meta-)linguistischen Kompetenzen bilingualer Sprecher (Abschnitt 3.3.2) und (meta-)kognitiven Konsequenzen von Bilingualität (Abschnitt 3.3.3) vorgestellt. In Abschnitt 3.3.2.1 wird die Besonderheit von Sprachkompetenzprofilen von bilingualen Sprechern erläutert, woraufhin theoretische Reflexionen sowie empirische Befunde zu linguistischen (Abschnitt 3.3.2.2) und metalinguistischen Kompetenzen bilingualer Sprecher präsentiert werden (Abschnitt 3.3.2.3). Angesichts der Bedeutung früher Literacyerfahrungen insbesondere zu Beginn der Grundschulzeit wird die Literacyentwicklung im Kontext der Bilingualität ebenfalls reflektiert (Abschnitt 3.3.2.4). Hinsichtlich (meta-)kognitiver Konsequenzen von Bilingualität werden sowohl der Zusammenhang von Kognition und Bilingualität (Abschnitt 3.3.3.1) als auch von exekutiven Funktionen und Bilingualität (Abschnitt 3.3.3.2) näher betrachtet. Diese beiden Aspekte sind nicht nur für die Grundlagenforschung der Kognitionspsychologie und Linguistik von hoher Relevanz, sondern können auch als potentielle, nicht auf sprachliche Fähigkeiten fokussierte Argumentationspositionen für einen förderlichen Umgang mit der bilingualen Disposition von bilingualen Sprechern fungieren. Die zentralen Erkenntnisse des Abschnitts 3.3 werden in Abschnitt 3.3.4 zusammengefasst. 
In Abschnitt 3.4 werden die Zusammenhänge von Identität, Kultur und Sprache erläutert, da diese insbesondere im Kontext der migrationsbedingten Bilingualität eine hohe Bedeutung für das psychosoziale Empfinden sowie das Selbstkonzept von bilingualen Sprechern haben. Zunächst werden sukzessiv der theoretische Zusammenhang von sowohl Identität und Sprache (Abschnitt 3.4.1) als auch von Kultur und Sprache (Abschnitt 3.4.2) betrachtet, bevor die Erkenntnisse dieser beiden Abschnitte hinsichtlich der Trias von Identität, Kultur und Sprache zusammengeführt werden (Abschnitt 3.4.3). Im Anschluss hieran werden theoretische Reflexionen und empirische Befunde zu Einstellungen zu Sprache(n) und Bilingualität präsentiert (Abschnitt 3.4.4), wobei insbesondere auch Sprachideologien und deren Bedeutung für den Umgang einer Gesellschaft mit migrationsbedingter Bilingualität diskutiert werden. Abschließend wird nochmals die identitätsstiftende Funktion von Sprache bei bilingualen Sprechern fokussiert, wobei hier stärker empirische Befunde im Kontext der migrationsbedingten Bilingualität akzentuiert werden (Abschnitt 3.4.5). Abschließend werden die zentralen Erkenntnisse des Abschnitts 3.4 in Abschnitt 3.4.6 zusammengefasst.

In Abschnitt 3.5 steht der bilinguale Sprachgebrauch im Fokus. Hierbei werden insbesondere soziolinguistisch orientierte Theorien und Modelle der Sprachwahl und des Sprachverhaltens adressiert (Abschnitt 3.5.1). Diesbezüglich wird zunächst ein Überblick über verschiedene Modelle gegeben (Abschnitt 3.5.1.1), bevor die Sprachmodi nach Grosjean (2008) näher erläutert werden (Abschnitt 3.5.1.2), da das Modell nach Grosjean (ebd.) das für die vorliegende Arbeit präferierte soziolinguistische Modell der Sprachwahl und des Sprachverhaltens bilingualer Sprecher darstellt. Nach diesen primär theoretischen Reflexionen werden empirische Befunde zur Sprachwahl sowie zum bilingualen Sprachgebrauch (Abschnitt 3.5.1.3) und zu diskursiv-rhetorischen Zwecken von Sprachwechseln und -mischungen präsentiert (Abschnitt 3.5.2). Die zentralen Erkenntnisse werden in Abschnitt 3.5.3 zusammengefasst.

Der letzte zentrale Abschnitt des dritten Kapitels widmet sich dem Zusammenhang von Bilingualität und Bildung (Abschnitt 3.6). Ausgehend von einer Begriffsbestimmung von Bildung nach Stojanov (2006) (Abschnitt 3.6.1) werden die zwei grundlegenden Positionen zum Zusammenhang von Bilingualität und Bildung vorgestellt (Abschnitt 3.6.2). Die wertschätzende Position der Bilingualität als Bildungsressource wird anhand der Threshold- (Abschnitt 3.6.3.1) und Interdependenzhypothese von Cummins (2000) näher ausgeführt (Abschnitt 3.6.3.2). Anschließend werden verschiedene Schulformen für bilinguale SuS näher betrachtet (Abschnitt 3.6.4), wobei der Submersionskontext monolingualer Bildung (Abschnitt 3.6.4.1) sowie Immersion, ,transitional bilingual education“ und ,dual language education“ bilingualer Bildung dargestellt werden (Abschnitt 3.6.4.2). Daraufhin werden bilinguale Praktiken - in der vorliegenden Studie definiert als alle vokalsprachlichen Handlungen, die 
nicht ausschließlich in der Verkehrssprache kommuniziert werden - in Bildungsprozessen beleuchtet (Abschnitt 3.6.5). In diesem Abschnitt werden zunächst sowohl das aktuell vieldiskutierte Konstrukt „Translanguaging“ (Abschnitt 3.6.5.1) als auch potentielle Motive für bilinguale Praktiken in Bildungsprozessen (Abschnitt 3.6.5.2) näher betrachtet. Darauf aufbauend wird die empirische Befundlage hinsichtlich bilingualer Praktiken in Bildungsprozessen (Abschnitt 3.6.5.3) sowie bezüglich Einstellungen von Lehrkräften zu bilingualem Sprachverhalten im Unterricht präsentiert (Abschnitt 3.6.5.4). Die zentralen Erkenntnisse des Abschnitts 3.6 werden in Abschnitt 3.6.6 zusammengefasst.

In Abschnitt 3.7 werden die wichtigsten Aspekte des dritten Kapitels anhand des soziokognitiven Interaktionsmodells der Sprachentwicklung nach Hamers und Blanc (2000) nochmals im Sinne eines Fazits zusammengeführt und miteinander verbunden. Als Ergebnis dieses Abschnittes wird die Frage aufgeworfen, ob der Umgang der deutschen Regelschule mit der Bilingualität von SuS mit MH eine Form von Bildungsbenachteiligung darstellt. Um dieses Postulat zu prüfen, wird im vierten Kapitel Bildungsgerechtigkeit unter einer anerkennungstheoretischen Perspektive näher betrachtet. Hierbei gilt es sich zunächst klar zu machen, was unter Bildungsgerechtigkeit zu verstehen ist (Abschnitt 4.1). Da die Definition, Beschreibung sowie potentielle Lösungsansätze von Bildungsgerechtigkeit von der zu Grunde gelegten Konzeption von Gerechtigkeit determiniert werden, gilt es die populärsten Ansätze zu betrachten und miteinander zu vergleichen. Zunächst wird die auf Gleichheit basierende Verteilungsgerechtigkeit nach Rawls (2014) inspiziert (Abschnitt 4.1.1). Diese Position prägt auch stark das Verständnis von Bildungsgerechtigkeit in Deutschland (Abschnitt 4.1.1.1) und dominierte auch implizit die PISA-Debatte um die Jahrtausendwende (Abschnitt 4.1.1.2). Danach wird die auf Menschenwürde basierende Verteilungsgerechtigkeit nach Nussbaums (2014) Fähigkeitenansatz dargestellt (Abschnitt 4.1.2), bevor die anerkennungstheoretische Konzeption von Bildungsgerechtigkeit nach Stojanov (2011) betrachtet wird (Abschnitt 4.1.3). Die Gemeinsamkeiten und Unterschiede der verschiedenen Konzeptionen von Bildungsgerechtigkeit werden in Abschnitt 4.1.4 adressiert, woraufhin sich der anerkennungstheoretische Ansatz als vielversprechendes Analyseinstrument zur Betrachtung von Bildungsgerechtigkeit herausstellen wird.

Dementsprechend wird das Konzept der Anerkennung näher betrachtet (Abschnitt 4.2). Zunächst werden die Bedeutungsfacetten und Dimensionen von Anerkennung inspiziert (Abschnitt 4.2.1). Anschließend wird Honneths (2003) Anerkennungstheorie erläutert (Abschnitt 4.2.2), indem sukzessive die Anerkennungsformen der Liebe (Abschnitt 4.2.2.1), des Rechts (Abschnitt 4.2.2.2) sowie der Solidarität (Abschnitt 4.2.2.3) dargestellt werden. Die primär in der Sozialphilosophie zu verortende Anerkennungstheorie Honneths 
(2003) wird anschließend hinsichtlich ihrer erziehungswissenschaftlichen/pädagogischen Rezeption ausgeführt (Abschnitt 4.2.3), wobei insbesondere Anerkennung als Strukturmoment jeder menschlichen Kommunikation (Abschnitt 4.2.3.1) sowie als Herstellung von Zuschreibungen unter Berücksichtigung von Machtstrukturen (Abschnitt 4.2.3.2) reflektiert werden. Anschließend wird die Anerkennung von SuS in Bildungsprozessen näher beleuchtet (Abschnitt 4.2.3.3), bevor die Umsetzung der Anerkennungsformen des Rechts (Abschnitt 4.2.3.4) und der Solidarität (Abschnitt 4.2.3.5) für SuS mit MH in der Bildungsinstitution Schule in Deutschland analysiert wird. Die zentralen Erkenntnisse des Abschnitts 4.2 zur Anerkennung werden in Abschnitt 4.2.4 zusammengefasst. Abschließend wird in Abschnitt 4.3 ein Fazit gezogen, welches den Handlungsbedarf hinsichtlich der Missachtung bilingual aufwachsender SuS mit MH sowie deren bilingualer Disposition durch die deutsche Regelschule auf den Anerkennungsformen des Rechts und der Solidarität als Bildungsungerechtigkeit akzentuiert und potentielle Lösungsansätze auch unter Berücksichtigung der in Kapitel 3 dargestellten Erkenntnisse der Bilingualismusforschung reflektiert. Hierbei wird sich herausstellen, dass eine Möglichkeit, die bilinguale Disposition bilingual aufwachsender SuS phasenweise in den deutschen Regelunterricht zu implementieren, in einem bilingualen Interaktionsangebot beim PL besteht.

Dementsprechend wird im fünften Kapitel die Sozialform des PL adressiert. Hierbei werden zunächst Grundlagen und Formen des PL beschrieben (Abschnitt 5.1), wobei formelles und informelles Peer-Learning nach Johnson und Johnson (1999) differenziert (Abschnitt 5.1. 1), Subtypen des Peer-Learnings am Beispiel des Peer-Tutoring dargestellt (Abschnitt 5.1.2) und die Basiselemente förderlichen PL nach Johnson \& Johnson (ebd.) ausgeführt werden (Abschnitt 5.1.3). Anschließend werden zentrale empirische Befunde zur Lernwirksamkeit von PL aus Meta-Analysen berichtet (Abschnitt 5.2), bevor die Bedeutung der Interaktion beim PL näher inspiziert wird (Abschnitt 5.3). Hierbei wird zunächst die Interaktion selbst als Determinante erfolgreichen, förderlichen PL betrachtet (Abschnitt 5.3.1), wobei insbesondere förderliche Sprachhandlungen und Dialogtypen in den Abschnitten 5.3.1.1 respektive 5.3.1.2 anhand theoretischer Reflexionen und empirischer Evidenz ausgeführt werden. Danach werden zwei weitere zentrale Phänomene für die Koordination der Gruppenaktivitäten beim PL adressiert und zwar Task-Management und metakognitive Regulation (Abschnitt 5.3.2), die ebenfalls sukzessive in den Abschnitten 5.3.2.1 respektive 5.3.2.2 reflektiert werden. Daraufhin werden die Bedeutung und Wirkmechanismen von Interaktion beim PL für Bildungs- und Lernprozesse betrachtet (Abschnitt 5.3.3), um die zuvor dargestellten Reflexionen und Befunde innerhalb adäquater lerntheoretischer Positionen zu erklären. Hierfür wird zunächst die Beziehung von Kommunikation und Lernen fokussiert (Abschnitt 5.3.3.1), bevor Piagets und Vygotskis Perspektiven auf Lernen und Entwicklung für die Sozialform des PL ausgeführt und reflektiert 
werden (Abschnitt 5.3.3.2). Zudem wird das Potential von PL zur Steigerung der Partizipation und des Outputs diskutiert (Abschnitt 5.3.3.3). Abschließend werden die zentralen Erkenntnisse zur Interaktion beim PL zusammengefasst (Abschnitt 5.3.4).

Bevor bilinguale Interaktion als Angebot beim PL theoretisch sowie hinsichtlich der aktuellen empirischen Evidenz ausgeführt wird, werden zuvor kognitive Lernstrategien näher beschrieben (Abschnitt 5.4), da diese häufig den Lerninhalt beim PL darstellen, was auch in der vorliegenden Studie der Fall ist. Zunächst werden kognitive Lernstrategien selbst näher beschrieben (Abschnitt 5.4.1), um anschließend ihren Erwerb und ihre Vermittlung (Abschnitt 5.4.2) sowie empirische Evidenz zu ihrer Lernwirksamkeit (Abschnitt 5.4.3) zu erläutern. Danach werden Lesestrategien (Abschnitt 5.4.4) sowie Halbschriftliche Rechenstrategien (Abschnitt 5.4.5) als domänenspezifische kognitive Lernstrategien näher betrachtet, die auch in der PL-Intervention der Datengrundlage der vorliegenden Studie als Lerninhalte verwendet werden. Die zentralen Erkenntnisse des Abschnitts 5.4 werden in Abschnitt 5.4.6 zusammengefasst.

Im vorletzten Abschnitt des Kapitels zu PL wird bilinguale Interaktion als Angebot beim PL reflektiert (Abschnitt 5.5). Hierbei werden primär aktuelle empirische Befunde zu bilingualer Interaktion beim Peer-Learning für die Kontexte bilingualer Bildung (5.5.1) und der Submersion (5.5.2) getrennt dargestellt, da die Voraussetzungen und der Umgang mit der bilingualen Disposition von bilingual aufwachsenden SuS sich in diesen Kontexten deutlich unterscheiden. Die Erkenntnisse dieser beiden Abschnitte werden in Abschnitt 5.5.3 zusammengefasst, wobei insbesondere die Forschungslücken und -unzulänglichkeiten der bisherigen Evidenz zu bilingualer Interaktion beim PL adressiert werden. Das Kapitel zu PL endet mit einem Fazit (Abschnitt 5.6), welches zu den übergeordneten und untergeordneten Fragestellungen der vorliegenden Studie überleitet (Kapitel 6).

Danach werden im siebten Kapitel die methodischen Aspekte der vorliegenden Mixed-Methods Studie ausgeführt, wobei zu Beginn die Motivation und Legitimation der Entscheidung für die in der vorliegenden Studie verwendete Mixed-Methods Forschungsanlage erläutert wird. Anschließend wird die Datengrundlage des Forschungsvorhabens näher beschrieben, die aus der BiPeer-Studie stammt (Abschnitt 7.1). Im Folgenden wird das Design (Abschnitt 7.1.1) sowie zentrale Bestandteile der Interventionen (Abschnitt 7.1.2) hinsichtlich der Interventionsphasen (Abschnitt 7.1.2.1), des Lese- (Abschnitt 7.1.2.2) und Rechentrainings (Abschnitt 7.1.2.3) sowie der Implementation des bilingualen Interaktionsangebots beim PL der BiPeer-Studie dargestellt (Abschnitt 7.1.2.4). Anschließend wird die Stichprobe des vorliegenden Forschungsvorhabens auf Individual- und Dyadenebene beschrieben (Abschnitt 7.2). Da bilinguale Praktiken, welche in der vorliegenden Studie sowohl in Form von monolingual türkischsprachigen als auch türkisch-deutschsprachig 
gemischten Äußerungen auftreten, das zentrale Konstrukt der vorliegenden Studie darstellen, wird deren Datengrundlage und Datenaufbereitung in Abschnitt 7.3 näher ausgeführt. Hierbei werden die Datengrundlage (Abschnitt 7.3.1), die Sequenzierung der Trainings (Abschnitt 7.3.2), die Transkription der Audioaufnahmen (Abschnitt 7.3.3), die Operationalisierung (Abschnitt 7.3.4) und Auszählung bilingualer Praktiken unter Verwendung des Programms MAXQDA (Abschnitt 7.3.5) beschrieben.

Anschließend werden die methodischen Aspekte der Analyse der quantitativen Fragestellungen in Abschnitt 7.4 sukzessive vorgestellt. Hinsichtlich der Prüfung der Abhängigkeit der Prävalenz bilingualer Praktiken von der Dyade (Abschnitt 7.4.1) werden die Stichprobe (Abschnitt 7.4.1.1), die Datengrundlage (Abschnitt 7.4.1.2) und die Analysemethode beschrieben (Abschnitt 7.4.1.3). Die Methodik der Untersuchung der Prävalenz explizit angebotener bilingualer Praktiken beim PL (Abschnitt 7.4.2) beginnt ebenfalls mit der Stichprobendarstellung (Abschnitt 7.4.2.1), bevor die verwendeten Konstrukte und Instrumente (Abschnitt 7.4.2.2), die Datengrundlage und Durchführung (Abschnitt 7.4.2.3) sowie die konkreten Analysemethoden der einzelnen Unterfragestellungen F2a bis F2c präsentiert werden (Abschnitt 7.4.2.4). Die letzte quantitative Fragestellung fokussiert potentielle Zusammenhänge zwischen sprachlichen sowie motivationalen Eigenschaften und Merkmalen mit der Prävalenz bilingualer Praktiken (Abschnitt 7.4.3). Auch hier wird zunächst die Stichprobe adressiert (Abschnitt 7.4.3.1), bevor die verwendeten Konstrukte und Instrumente (Abschnitt 7.4.3.2), die Datengrundlage und Durchführung (Abschnitt 7.4.3.3) und abschließend die konkreten Analysemethoden der einzelnen Unterfragestellungen F3a und F3b erläutert werden (Abschnitt 7.4.3.4).

Danach werden die methodischen Aspekte der qualitativen Fragestellungen in Abschnitt 7.5 sukzessive dargestellt. Zunächst wird der methodische Umgang mit den Selbstberichten zum bilingualen Sprachgebrauch im Training und dessen Begründung präsentiert (Abschnitt 7.5.1), wobei die Stichprobe (Abschnitt 7.5.1.1), die verwendeten Instrumente sowie die Durchführung der Erhebung (Abschnitt 7.5.1.2), die Analysemethode und Datenauswertung (Abschnitt 7.5.1.3) und abschließend das konkrete Kategoriensystem zur Kodierung der selbstberichteten Gründe für die Annahme oder Ablehnung des Angebots zur bilingualen Interaktion beschrieben werden (Abschnitt 7.5.1.4). Die zweite qualitative Fragestellung adressiert die Funktionen bilingualer Praktiken beim PL (Abschnitt 7.5.2). Auch hier wird zunächst die Stichprobe (Abschnitt 7.5.2.1), die Analysemethode (Abschnitt 7.5.2.2) sowie das Kategoriensystem (Abschnitt 7.5.2.3) präsentiert und abschließend das konkrete Kodiervorgehen samt Hinweisen zum Umgang mit ambivalenten Fällen und potentiellen Mehrfachkodierungen näher erläutert (Abschnitt 7.5.2.4).

In Kapitel acht werden die Ergebnisse der übergeordneten Fragestellungen sowie ihrer Unterfragestellungen sukzessive präsentiert. Die Diskussion der 
Ergebnisse im neunten Kapitel ist so strukturiert, dass zunächst die Ergebnisse der Fragestellungen einzeln reflektiert und in den bisherigen Forschungsstand eingebettet werden (Abschnitt 9.1). Anschließend werden die quantitativen und qualitativen Befunde der vorliegenden Studie in Form der Datenintegration von Mixed-Methods Untersuchungen miteinander in Beziehung gesetzt (Abschnitt 9.2). Auf Basis dieser Erkenntnisse werden praktische Implikationen abgeleitet (Abschnitt 9.3). Abschließend werden die Limitationen der Studie kritisch reflektiert sowie sich hieraus ergebende Forschungsdesiderata präsentiert (Abschnitt 9.4). Im zehnten Kapitel wird abschließend ein Fazit gezogen. 


\section{Eine kurze Einführung zum Phänomen Sprache}

In der öffentlichen Diskussion kursieren viele populäre Fehlkonzepte über Sprache sowie Mehrsprachigkeit (vgl. Kersten et al., 2011; Tracy, 2011) und deren Zusammenhang mit Bildungsprozessen. Aber auch im wissenschaftlichen Diskurs wird „Sprache“ terminologisch zum Teil als Containerbegriff gebraucht, in welchem verschiedene Varietäten, Gebrauchsweisen und Funktionen von Sprache - und somit letztlich auch sprachliche Fähigkeiten - undifferenziert betrachtet und vermischt werden. Für die vorliegende Untersuchung ist eine adäquate, wissenschaftlich fundierte Konzeption von Sprache allerdings unumgänglich, sodass zunächst eine terminologische Präzisierung des Begriffs „Sprache“ anhand der Betrachtung von sprachlichen Varietäten (Abschnitt 2.1) und die Beschreibung ihrer konstituierenden Teilsysteme (Abschnitt 2.2) vorangestellt werden. Auf Basis dieser Grundlagen werden Aspekte sprachlichen Wissens, die für die vorliegende Arbeit relevant sind, ausgeführt und Sprachkompetenz aus linguistischer Perspektive allgemein diskutiert (Abschnitt 2.3), bevor die Funktion von Sprache als kognitives Werkzeug (Abschnitt 2.3.1) und metalinguistische Bewusstheit näher beleuchtet werden und (Abschnitt 2.3.2). Abschließend werden die zentralen Inhalte des zweiten Kapitels zusammengefasst (Abschnitt 2.4).

\subsection{Begriffspräzisierung und Varietäten von Sprache}

Natürliche Sprachen werden häufig im Sinne von ,(nationalen) Einzelsprachen wie Deutsch, Schwedisch, Japanisch etc.“ (Bußmann, 2008, S. 644) verstanden und somit über politisch-geographische Kategorien (vgl. Grewendorf, Hamm $\&$ Sternefeld, 1987, S. 24) definiert. Diese sind von artifiziellen Sprachen wie z. B. Esperanto oder HTML zu unterscheiden, da natürliche Sprachen nicht explizit, zweckgebunden konstruiert werden, sondern sich im Verlauf durch deren Gebrauch entwickeln und verändern. Die politisch-geographische Reduktion des Begriffs von natürlichen Sprachen ist zu grobkörnig und im Hinblick auf dessen Bedeutung für Bildungsprozesse ungeeignet. Nichtsdestotrotz weist diese Operationalisierung von Sprache darauf hin, dass das Phänomen Sprache sowie sprachliche Fähigkeiten in der Regel mit bestimmten Einzelsprachen assoziiert werden.

Allerdings stellen Einzelsprachen keine homogenen Systeme dar, sondern zeichnen sich durch eine hohe Variabilität in ihrer Form aus. Die verschiedenen Formen der Realisierung einer Einzelsprache werden als „Varietäten“ bezeichnet. Varietäten sind ,spezifische Ausprägungen eines sprachlichen Ver- 
haltens in einem mehrdimensionalen (regional, sozial, situativ, historisch differenzierten) Varietätenraum“ (Bußmann, 2008, S. 772). Dementsprechend wird nicht eine Einzelsprache beim Sprechen produziert, sondern Varietäten der jeweiligen Einzelsprache wie z. B. Dialekte (vgl. Myers-Scotton, 2006, S. 23). Varietäten einer Einzelsprache können sich prinzipiell auf allen sprachlichen Ebenen unterscheiden, sodass sie auf der Oberfläche anhand der Aussprache, Wortwahl, syntaktischen Strukturen etc. differenziert werden können. Sie sind abhängig von verschiedenen, sich zum Teil überlagernden regionalen, sozialen, situativen und historischen Kontexten sowie Bedingungen (vgl. Bußmann, 2008, S. 772). So kann die Zugehörigkeit zu einer bestimmten sozialen Gruppe wie z. B. zu Subkulturen (z. B. Punkrocker, Surfer, Graffitisprüher etc.) oder Berufsgruppen (z. B. Juristen, Mediziner, Psychologen etc.) Einfluss auf das Sprachverhalten nehmen, insbesondere wenn Mitglieder derselben Gruppe miteinander interagieren. Diese Varietäten werden als Soziolekte bezeichnet, die primär zur Kommunikation innerhalb der Gruppe, zur Lösung gruppenspezifischer sprachlicher Anforderungen sowie in manchen Fällen auch zur Abgrenzung von anderen Gruppen verwendet wird. Kommunizieren Angehörige derselben sozialen Gruppen miteinander, die alle aus derselben Region stammen, kann die gesprochene Varietät nicht nur Merkmale des Soziolekts, sondern auch des Dialekts aufweisen. Weiterhin können auch dieselben Varietäten durch eine funktionsspezifische Nutzung in distinkte Register (vgl. Bußmann, 2008, S. 577f.) differenziert werden. So wird z. B. in einer Predigt oder Nachrichtensendung zwar die standardsprachliche Varietät genutzt, aber in einer charakteristischen, funktionsspezifischen Weise für den jeweiligen Bereich, was beispielsweise in der Sprachmelodie und der Wortwahl zum Ausdruck kommt. Dieser kurze Exkurs macht deutlich, dass nicht Einzelsprachen gesprochen werden, sondern Varietäten einer Einzelsprache, die von einer Vielzahl verschiedener Faktoren determiniert werden.

Natürliche Einzelsprachen entwickeln sich zunächst mündlich und werden häufig im weiteren Verlauf schriftsprachlich fixiert. Mündliche und schriftsprachliche Varietäten von Sprache werden als ein Kontinuum von „konzeptioneller Mündlichkeit und Schriftlichkeit" operationalisiert, welche sich in einer Vielzahl sprachlicher sowie stilistischer Merkmale voneinander unterscheiden (vgl. Koch \& Oesterreicher, 1985). So zeichnet sich die konzeptionell mündliche Varietät unter anderem durch die Aneinanderreihung von Hauptsätzen (Parataxe), einen eingeschränkten Gebrauch von Konjunktionen („und“, „weil“", „dass“ etc.), Satzabbrüche, geringere sprachliche Vielfalt etc. aus (vgl. Bußmann, 2008, S. 234f.). Dies führt dazu, dass manche sprachlichen Strukturen wie z. B. Relativsätze in der gesprochenen Sprache im Gegensatz zur geschriebenen Sprache nur äußerst selten produziert werden (vgl. Weinert, 2004). Die konzeptionelle Schriftlichkeit weist unter anderem komplexe Haupt- und Nebensatzkonstruktionen (Hypotaxe), ein präzises, spezifisches Vokabular, sprachliche Vielfalt etc. auf. Hierbei ist wichtig zu erwähnen, dass 
die sprachliche und stilistische Ausgestaltung nicht vom genutzten Medium (mündlich vs. schriftlich) abhängig ist, sondern vielmehr von den Bedingungen der Kommunikation (raumzeitliche Nähe, Kontext, Vertrautheit, Ziel etc.) beeinflusst wird (vgl. Koch \& Oesterreicher, 1985). So orientiert sich z. B. ein wissenschaftlicher Vortrag, der mündlich auf einer Konferenz präsentiert wird, sprachlich an der konzeptionellen Schriftlichkeit.

Ähnlich zur Unterscheidung von konzeptionell mündlicher und schriftlicher Varietät werden alltagsprachliche, primär zur Kommunikation genutzte und bildungsbezogene, akademische Varietäten von Sprache voneinander unterschieden (vgl. z. B. Cummins, 2008, S. 74f; Gantefort \& Roth, 2010; Heppt, et al., 2014; Schleppegrell, 2004). Die international populärste und einflussreichste Konzeption der oben angeführten alltagssprachlichen und akademischen Varietäten stellen Cummins“ 1979 erstmals postulierte „basic interpersonal communicative skills (BICS)“ und ,cognitive-academic language proficiency (CALP)“ dar (vgl. Cummins, 1979, 2008). Die Distinktion dieser Varietäten stellt die Antwort Cummins auf Ollers (1978, zit. nach Cummins, 1979, S. 198) ,global language proficiency“ dar, welche Oller in seiner starken Version - ähnlich zu Spearmans (1904) Intelligenzfaktor , g“ - als übergeordneten Faktor von Sprachfähigkeiten modellierte und in positivem Zusammenhang mit kognitiven Fähigkeiten sowie Bildungserfolg setzt (vgl. Cummins, 1979, S. 198). Dieser übergeordnete Faktor widerspricht nach Cummins (ebd.) allerdings der empirischen Evidenz, dass - mit Ausnahme von Fällen schwerer Behinderung oder autistischer Störungen - jedes Individuum basale interpersonale Kommunikationsfähigkeiten in seiner L1 unabhängig von Intelligenz und Lernerfolg erwirbt sowie ausbildet. Zudem ist Cummins (ebd.) der Ansicht, dass soziolinguistische Aspekte von Kommunikation und funktionaler Sprachgebrauch sich kaum auf einen übergeordneten Faktor reduzieren lassen. Mithilfe einer Reanalyse von Daten zur Sprachperformanz von Zweitsprachlernern in Kanada konnte Cummins (1981) die Distinktion in BICS und CALP empirisch prüfen. Sekundarstufenschüler, die nach Kanada immigriert sind, benötigen für den Erwerb von BICS der Verkehrssprache ungefähr zwei Jahre, während sie für CALP allerdings fünf bis sieben Jahre brauchen, um das Niveau ihrer monolingual aufwachsenden Peers zu erreichen. Dementsprechend lassen sich sprachliche Fähigkeiten kaum als ein kohärentes, übergeordnetes Konstrukt konzeptualisieren, sondern müssen hinsichtlich verschiedener Varietäten differenziert werden.

Cummins (2008, S. 74) unterscheidet BICS und CALP anhand der Kontextgebundenheit und des kognitiven Anspruchs. Während sich BICS durch eine hohe Kontextgebundenheit und einen niedrigen kognitiven Anspruch auszeichnet, weist die Produktion und Rezeption von CALP hingegen einen hohen kognitiven Anspruch, aber eine reduzierte Kontextgebundenheit auf. So kommunizieren z. B. Kinder beim gemeinsamen Bauen einer Sandburg im Sand- 
kasten eher in der Varietät von BICS, da die Realisation der jeweiligen Kommunikationsziele unter Einbezug des Kontexts durch Zeigegesten, Mimik oder Verwendung von Objekten die Sprachproduktion und -verarbeitung entlastet. Möchte eines dieser Kinder im Nachhinein diese Spielepisode einer anderen Person erzählen, die beim Spielen nicht anwesend war, muss sich das Kind eher der Varietät von CALP bedienen. Es muss bei der Nacherzählung die fehlenden kontextuellen Informationen mithilfe sprachlicher Mittel realisieren, was einen höheren Anspruch an die zu verwendenden sprachlichen Formen und Funktionen stellt als in der Spielsituation selbst. Hierbei gilt es allerdings zu berücksichtigen, dass Kontextgebundenheit und kognitiver Anspruch keine allgemeingültigen Größen darstellen, sondern abhängig von dem Hintergrundwissen sowie den Erfahrungen der Gesprächspartner und den in der Situation vorliegenden Informationen sind (vgl. ebd.).

Auch wenn die Dimensionen der Kontextgebundenheit und des kognitiven Anspruchs sich auch auf nicht schulische Situationen beziehen können, wird CALP stets in enger Verbindung zum schulischen Kontext konzeptualisiert. Dies zeigt sich nicht nur an der empirischen Untermauerung der Distinktion auf Basis nach Kanada eingewanderter SuS der Sekundarstufe (vgl. Cummins, 1981), sondern auch in Cummins (2000, S. 67) Definition von CALP als ,the extent to which an individual has access to and command of the oral and written academic registers of schooling". Diese Definition setzt CALP in direkten Bezug zu sprachlichen Varietäten der Schule. Für Cummins (2008, S. 79f) ist die Realisation von CALP insbesondere in geschriebenen Texten zu finden, sodass er SuS zum Erwerb sowie Verständnis der Sprachvarietät CALP empfiehlt, Texte sowohl im Leseprozess als auch in der Anschlusskommunikation kollaborativ zu bearbeiten.

Akademisch orientierte Sprachvarietäten werden im deutschsprachigen Raum auch unter dem Begriff „Bildungssprache“ (vgl. z. B. Gantefort \& Roth, 2010; Heppt, et al., 2014) untersucht. Mit dem Konstrukt Bildungssprache wird versucht, die sprachlichen Anforderungen von Schule zu identifizieren und zu operationalisieren, die in Cummins Konzept von CALP enthalten sind, von ihm selbst aber nie konkret in Form der Bestimmung zugehöriger linguistischer Formen und Funktionen spezifiziert wurden. Cummins (2008, S. 78) sieht dies auch nicht als Notwendigkeit an, da CALP „a very different set of theoretical, policy, and classroom instructional issues" adressiert und seiner Ansicht nach eher als übergeordnetes Konstrukt zur Diskussion sprachlicher Voraussetzungen für Bildung in pluralistischen Gesellschaften genutzt werden sollte.

Nach Schleppegrell (2004) tritt die Varietät „Bildungssprache“ in (schulischen) Bildungskontexten auf, um Wissen zu erwerben und zu vermitteln. Hierfür benötigt es sowohl einen ,fachspezifischen bildungssprachlichen Wortschatz, dessen Verwendung sich zumeist auf einzelne Fächer beschränkt 
(z. B. Addition, multiplizieren)“ als auch „einen allgemeinen bildungssprachlichen Wortschatz, der fächerübergreifend eingesetzt wird (z. B. eine Übersicht erstellen, vervollständigen)“ (Heppt et al., 2014, S. 140). Auf struktureller Ebene zeichnet sich Bildungssprache unter anderem durch ein erhöhtes Auftreten von komplexen Haupt- und Nebensatzkonstruktionen, Passivkonstruktionen sowie langen Nominal- und Präpositionalphrasen aus (z. B. „Die Diffusion von Molekülen eines Lösungsmittels durch eine semipermeable Membran...") (vgl. ebd.).

„Literacy“ wiederum ist zwar eng mit den Varietäten der konzeptionellen Schriftlichkeit, CALP oder Bildungssprache verbunden, wird allerdings eher als Kompetenzkomplex statt Varietät konzeptioniert. Der Begriff „Literacy“ wird von verschiedenen Autoren in unterschiedlicher Weise verwendet und hat keine genuin deutschsprachige Entsprechung (vgl. Brandenburg, 2006, S. 11). Brandenburg (ebd., S. 11ff) zählt neben dem zentralen Element der Schriftsprachkompetenz unter anderem sprachliche Abstraktionsfähigkeit, Vertrautheit mit literarischer Sprache und Gattungen sowie gesellschaftliche Kernkompetenzen wie mathematische und naturwissenschaftliche Grundkenntnisse und damit einhergehend auch den Umgang mit Schaubildern zu Literacy. Dementsprechend vereinigt das Konstrukt zunächst Kernmerkmale der Literarität, der Beherrschung von Schriftsprache als Kulturtechnik, und der Literalität, der ästhetischen Rezeptionskompetenz. Unter Hinzufügung allgemeiner sprachlicher Abstraktionsfähigkeit sowie weiteren mathematischen und naturwissenschaftlichen Grundkennnissen stellt Literacy nach Brandenburg (ebd., S. 13) das Fundament für die Befähigung zur aktiven Teilhabe an gesellschaftlichen Prozessen und die Voraussetzung zur sozialen Selbstbestimmung eines Individuums dar. Dementsprechend werden sowohl sprachspezifische als auch generelle, allgemeine Wissenskomponenten und Fähigkeiten dem Konstrukt Literacy zugeordnet.

Die bisherigen Ausführungen zeigen, dass Einzelsprachen im tatsächlichen Gebrauch eher als Varietäten realisiert werden, die abhängig von diversen Bedingungen, Kontexten und Funktionen unterschiedliche Formen aufweisen. Hierbei scheinen Varietäten, die sich eher an konzeptioneller Schriftlichkeit orientieren, für Bildungsprozesse eine besondere Relevanz zu haben. Trotz der eben dargelegten Ausführungen zu verschiedenen Varietäten von Einzelsprachen stellt sich allerdings weiterhin die Frage, welche Merkmale und Eigenschaften Sprache(n) ausmachen. Zur Identifikation zentraler Merkmale einer Einzelsprache benötigt es eine Analyse der Grammatik einer Sprache. Hiermit ist sowohl das sprachliche Wissen als auch die Beschreibung oder Theorie des jeweiligen sprachlichen Wissens gemeint (vgl. Grewendorf Hamm \& Sternefeld, 1987, S. 28). 


\subsection{Teilsysteme der Grammatik und zentrale linguistische Wissenssysteme}

Die Konzeption und Modellierung der Komponenten sprachlichen Wissens, deren Status sowie deren Strukturierung divergieren zwischen einem modularen und einem holistischen Ansatz (vgl. Schwarz 1996, S. 47-54). Der modulare Ansatz modelliert Sprache als ein angeborenes, unabhängiges System bestehend aus sprachspezifischen Prinzipien und Mechanismen, das durch Konfrontation mit sprachlichen Strukturen der Umgebung einzelsprachliche Regelungen internalisiert und grammatische Parameter setzt. Dieser Prozess ist so robust, dass Umweltbedingungen hierbei keine zentrale Rolle spielen und das sprachliche System unabhängig von anderen nicht-sprachlichen kognitiven Modulen agiert (vgl. ebd., S. 47-52). Der holistische Ansatz hingegen interpretiert Sprache als Folge von allgemeinen Kognitionsprinzipien sowie Lernmechanismen und akzentuiert Umweltbedingungen als zentrale Einflussfaktoren in einem reziproken Prozess zwischen Lerner und Umwelt. Sprache entsteht durch Interaktion innerhalb des Sozialisationsprozesses, wobei Lerner zwar über eine Prädisposition zur Konstruktion und Kategorisierung der Umwelt, aber über keine sprachspezifische Disposition verfügen (vgl. ebd., S. 52-54). Während sich die beiden Ansätze früher unvereinbar gegenüberstanden, werden diese in hybriden Modellen fruchtbar miteinander kombiniert (vgl. Kauschke, 2012, S. 147ff). Hybride Modelle akzentuieren das Zusammenspiel von Anlage und Umwelt als auch von sprachspezifischen und domänenübergreifenden Mechanismen. Zur Beschreibung der grammatischen Teilsysteme von Sprache wird im Folgenden vermehrt auf den modularen Ansatz referiert, auch wenn im Interaktionismus ebenfalls verschiedene Bereiche grammatischen Wissen postuliert werden.

Hinsichtlich des Aufbaus von Sprache werden verschiedene grammatische Teilsysteme angenommen, die sich in ein phonologisches, morphologisches, syntaktisches und semantisches Modul differenzieren lassen (vgl. Grewendorf, Hamm \& Sternefeld, 1987, S. 38ff). Natürliche Sprachen sind überwiegend Lautsprachen, in welchen Informationen über Schallereignisse vermittelt werden (vgl. Dittmann, 2006, S. 10). Die Phonologie einer Sprache umfasst zum einen das Lautinventar, bestehend aus einer endlichen Anzahl von Phonemen, den „kleinsten bedeutungsdifferenzierenden Einheiten“ einer Sprache (z. B. [p] vs. [b] in „Pein“" und „Bein“) (Grewendorf, Hamm \& Sternefeld, 1987, S. 86), zum anderen Prinzipien und Regularien, wie Phoneme zu Wörtern kombiniert werden. So können z. B. die Phoneme ,[p]“ und ,[t] “ im Deutschen nie gemeinsam am Silbenanfang, wohl aber am Silbenende stehen. Weiterhin umfasst die Phonologie prosodische Eigenschaften, die sich auf Unterschiede in der Tonhöhe, Lautstärke, Geschwindigkeit und Rhythmus beziehen (vgl. Crystal, 2008, S. 392). 
Aus der Kombination von Lauten werden über phonologische Regeln Wörter produziert. Wortbildungsprozesse sind allerdings nicht nur auf der phonologischen Ebene angesiedelt, sondern werden primär der Morphologie zugewiesen (vgl. Grewendorf, Hamm \& Sternefeld, 1987, S. 38). Wörter bestehen aus Morphemen, die als „kleinste bedeutungstragende Einheiten“ einer Sprache definiert werden (z. B. „,ver-fahr-en“, „Auto-s“, „spät-er“) (ebd., S. 254), welche wiederum diversen Regularien innerhalb der Formenlehre (Flexion) sowie verschiedener Wortbildungsprozesse (Derivation) unterliegen (vgl. ebd., S. 255 ff).

Die Syntax liefert Regeln, die die zulässigen Kombinationen von Wörtern zu Satzgliedern und Sätzen festlegen. Dementsprechend ermöglicht das syntaktische Wissen die Gliederung von Wörtern zu Wortgruppen und von Wortgruppen zu grammatisch korrekten Sätzen (vgl. ebd., S. 39). Sätze bestehen aus syntaktisch zusammengehörigen Wortgruppen, sogenannten „Phrasen“ (z. B. [[Der neue Präsident] [fliegt] [nach Peking]].), die hierarchisch nach diversen linguistischen Prinzipien strukturiert sind. Die tatsächliche Ausprägung der einzelnen Prinzipien kann sich zwischen Einzelsprachen unterscheiden. Diese Prinzipien führen dazu, dass z. B. flektierte Verben im Hauptsatz des Deutschen an der zweiten Stelle („Der neue Präsident trägt ein Toupet.“ vs. *"Der neue Präsident ein Toupet trägt."1) und im Nebensatz an der letzten Stelle stehen müssen (,Wir sehen, dass der neue Präsident ein Toupet trägt.“ vs. ,"Wir sehen, dass der neue Präsident trägt ein Toupet.“).

Die Semantik ermöglicht, die Bedeutung eines Satzes aus den Bedeutungen seiner einzelnen Wortgruppen und Wörter zu erschließen (vgl. ebd., S. 39). Alle Wörter - mit Ausnahme von Funktionswörtern (z. B. „,dass“, „,der“) tragen eine lexikalische Bedeutung. Diese lexikalischen Bedeutungen stehen allerdings nicht isoliert zueinander, sondern müssen sinnig in die Semantik der anderen lexikalischen Einheiten und in die syntaktische Struktur integriert werden. So hat z. B. der Satz „Die Konstablerwache wird zur Verhütung von Straftaten durch die Polizei videoüberwacht" zwei Lesarten. Die Phrase [durch die Polizei] kann sich entweder auf die Ausübung von Straftaten oder auf die Videoüberwachung beziehen, was zwei unterschiedliche satzsemantische Bedeutungen impliziert.

Neben diesen Teilsystemen der Grammatik existieren mit dem mentalen Lexikon und der Pragmatik zwei weitere zentrale linguistische Wissenssysteme, die aus linguistischer Perspektive allerdings keine Teilsysteme der Grammatik darstellen. Das mentale Lexikon wird als „organisierter und aktiver Speicher, in dem lexikalische Einheiten mit komplexen Beziehungen zueinander gespeichert werden“ (Rothweiler \& Kauschke, 2007, S. 42) definiert. Es wird davon ausgegangen, dass thematisch verwandte Einträge miteinander vernetzt sind (z. B. „Tier“ und „Hund“) und dass zu jedem Eintrag phonologische, 
morphologische, syntaktische, semantische, pragmatische sowie - nach vollzogenem Schriftspracherwerb - orthographische Informationen gespeichert werden (vgl. ebd., S. 42ff).

Die Pragmatik stellt die Schnittstelle zwischen sprachlichen Strukturen und deren Bedeutung in der Lebenswelt dar, denn ,language is not produced in a vacuum; it is enacted in changing dramas" (Baker, 2011, S. 4). Die Pragmatik ermöglicht es dem Sprecher, sprachliche Äußerungen adäquat unter Berücksichtigung des Kontexts zu verwenden sowie zu verstehen (vgl. Grewendorf, Hamm \& Sternefeld, 1987, S. 41). Sprecher sind in der Lage, eine Äußerung wie z. B. „da ist die Tür“ nicht nur als Information zur Lagebeschreibung des Ein- und Ausgangs eines Raumes zu verstehen, sondern unter bestimmten Umständen diese Äußerung als Aufforderung zum Verlassen des Raumes zu interpretieren.

Wie anhand dieser kurzen Einführung in die Teilsysteme der Grammatik und in zentrale linguistische Wissenssysteme deutlich wird, besteht die Grammatik einer Sprache aus einem multimodalen Wissenssystem, in welchem sich die einzelnen Module wiederum in Teilsysteme differenzieren lassen. Die verschiedenen Module sind weitestgehend unabhängig voneinander, agieren aber nichtsdestotrotz in enger Kooperation miteinander. Im Folgenden sollen zentrale, den grammatischen Teilsystemen übergeordnete Merkmale sprachlichen Wissens etwas näher betrachtet werden, um anschließend das Konstrukt der Sprachkompetenz zu reflektieren.

\subsection{Sprachliches Wissen und Sprachkompetenz}

Ein zentrales Merkmal des sprachlichen Wissens ist die Eigenschaft der „discrete infinity“ (Chomsky, 2000, S. 3ff). Das sprachliche Wissen stellt eine endliche Menge an Elementen und Regeln zur Verfügung. Aus dieser endlichen Menge kann allerdings eine unendliche Menge an Sätzen produziert werden. Es handelt sich dementsprechend um ein äußerst kreatives System, welches in der Lage ist, Äußerungen zu formulieren und zu verarbeiten, die zuvor noch nie realisiert wurden. Das sprachliche Wissen selbst ist hierbei in der Regel implizit. Grammatisch (in-)korrekte Sätze werden als solche wahrgenommen und produziert, ohne die Gründe für die (Un-)Grammatikalität explizieren zu können (vgl. Schwarz, 1996, S. 49).

Sprachliches Wissen lässt sich hinsichtlich Sprachproduktion und -verständnis differenzieren. Diese Unterscheidung erscheint zunächst trivial, sie stellt aber zwei voneinander distinkte Sprachfähigkeiten dar. Die Fähigkeit zur Sprachproduktion bestimmter Strukturen impliziert nicht zwingend das Sprachverständnis dieser und umgekehrt, was insbesondere während des kindlichen Spracherwerbs zu beobachten ist (vgl. Grimm et al., 2011). Produziert 40 
eine Person bestimmte sprachliche Strukturen nicht, so kann daraus aber auch nicht geschlossen werden, dass sie diese Strukturen nicht verstehen würde, wenn sie mit diesen konfrontiert wird.

Sprachliche Strukturen unterscheiden sich in ihrer Regelhaftigkeit. Regelhafte Strukturen zeichnen sich dadurch aus, dass sie, sofern sie einmal erworben werden, auf eine Vielzahl von Elementen angewandt werden können. So können zum Beispiel aus syntaktischer Perspektive eine unendliche Anzahl von Haupt- und Nebensätzen im Deutschen grammatisch korrekt produziert und verstanden werden, sobald der Sprecher die Verbzweitstellung respektive Verbendstellung erworben hat. Regelhafte Strukturen unterscheiden sich im Grad ihrer Eindeutigkeit der Form-Funktions-Beziehungen. So tritt z. B. das Suffix ,-er“" (nicht zu verwechseln mit dem Präfix ,er-") immer am Ende von Lexemen auf, inkorporiert allerdings eine Vielzahl von Funktionen (Nominalisierung wie bei „Lehr-er“; Pluralbildung wie bei „Häus-er“; Komparativ wie bei ,spät-er"). Im Gegensatz zu regelhaften Strukturen können sogenannte idiosynkratrische Merkmale von Wörtern nicht auf Basis genereller, zugrundeliegender Regeln vorhergesagt werden (vgl. Bußmann, 2008, S. 274f), sondern müssen einzeln erworben und im mentalen Lexikon gespeichert werden. Die Bedeutung des Verbs „berücken “" lässt sich beispielsweise nicht durch abstrakte Regeln oder durch die Bedeutungen der einzelnen drei Morpheme „be-rück-en“ vollständig ableiten, sondern Sprecher müssen diesem Wort in einem sinnvollen Kontext begegnen, um es zu erwerben. Auch grammatische Phänomene wie z. B. die Nominalflexion, mit welcher grammatische Kategorien wie Numerus, Genus und Kasus realisiert werden, zeichnen sich durch gewisse idiosynkratrische Merkmale aus (vgl. Wegener, 1995), was deren Erwerb erschwert.

Hinsichtlich der bisherigen Ausführungen stellt sich die Frage, was angesichts der Struktur und Merkmale von Sprache sowie sprachlichem Wissen unter Sprachkompetenz zu verstehen ist. Eine einheitliche oder verbindliche Definition von Sprachkompetenz gibt es nicht, sondern sie wird im Kontext der Forschungsdisziplin oder der theoretischen Fundierung erzeugt (vgl. Jude, 2008, S. 11). Aus der Perspektive der Generativen Grammatik wird der Kompetenzbegriff vom Begriff der Performanz getrennt. Die Fähigkeit, die dem Sprachgebrauch zu Grunde liegt, wird als sprachliche Kompetenz bezeichnet. Hingegen wird die Art und Weise, wie von dieser zugrunde liegenden Fähigkeit Gebrauch gemacht wird, als sprachliche Performanz definiert (vgl. Grewendorf, Hamm \& Sternefeld, 1987, S. 32). Mit Kompetenz wird vor allem das Wissen über die Sprache, über das Regelsystem der Sprache und die Fähigkeit zum Erkennen von Ungrammatikalitäten sowie Ambiguitäten bezeich-

2 Bei Fischern und Vogelfängern verwendeter Begriff, mit einem Ruck ein Fangnetz über das zu fangende Tier zu schließen. 
net. Unter Performanz wird die tatsächliche Produktions- und Verständnisleistung verstanden, die von einer Vielzahl sozialer, biologischer und psychologischer Faktoren wie z. B. Konzentration, Müdigkeit, Aufregung etc. beeinflusst wird. Dementsprechend kann von der Performanz nicht direkt auf die zugrundeliegende Kompetenz geschlossen werden.

Die Operationalisierung von Sprachkompetenz und dessen Messung ist mit diversen weiteren Schwierigkeiten verbunden. Hierzu gehören z. B. die Differenzierung rezeptiver und produktiver sowie mündlicher und schriftsprachlicher Kompetenzen, die Berücksichtigung der verschiedenen grammatischen Teilsysteme, die Auswahl geeigneter sprachlicher Phänomene und die Bestimmung von verschiedenen Niveaustufen von sprachlichen Fähigkeiten, die sich häufig adäquater als Dichotomie von ,erworben“ und ,nicht erworben“ anstatt auf Intervallskalenniveau operationalisieren lassen (vgl. z. B. Lüdtke \& Kallmeyer, 2007a; Rauch, Schastak \& Richter, 2016; Reitenbach, Schastak \& Rauch, 2018). Zudem weist der Spracherwerb hinsichtlich Registern, bestimmter Teilsysteme wie Semantik und Pragmatik sowie unter der Prämisse von Sprachwandelphänomenen kein echtes Endstadium und keine „Perfektion“ (vgl. Tracy, 2011, S. 74) auf. Dementsprechend stellt Sprachkompetenz ein äußerst dynamisches, anspruchsvolles und komplexes Konstrukt dar, dessen Eignung und Nutzen als Kategorie sowie deren Operationalisierung für das jeweilige Vorhaben intensiv reflektiert werden sollte.

Einen anderen Zugang zur Modellierung von Sprachkompetenz postulieren Hamers \& Blanc (2000) innerhalb ihres soziokognitiven Interaktionsmodells der Sprachentwicklung, welches in Abschnitt 3.7 zum Zwecke einer Zusammenfassung kurz ausgeführt wird. Anstatt Sprachkompetenz über eine Batterie ausgewählter Fähigkeiten verschiedener grammatischer Teilsysteme in Produktion und/oder Rezeption zu operationalisieren, werfen sie in Anlehnung an den Interaktionismus einen ganzheitlicheren Blick auf das Konstrukt Sprachkompetenz und verorten dieses in enger Abhängigkeit vom Sozialisationsprozess der Lerner. Hamers \& Blanc (ebd., S. 124) unterscheiden zwei zentrale Entwicklungsbereiche hinsichtlich Sprache, und zwar ,communicative linguistic competence“ und „conceptual linguistic competence“. Dementsprechend müssen sprachliche Fähigkeiten sich dahingehend entwickeln, dass sie den Sprecher zur Kommunikation mit anderen sowie zur konzeptuellen Strukturierung seiner Umwelt und Erfahrungen befähigen. Diese Entwicklungsbereiche führen wiederum zu drei grundlegenden, miteinander zusammenhängenden Funktionen bzw. Kompetenzen von Sprache: Sprache zur Kommunikation, Sprache als kognitives Werkzeug und metalinguistische Bewusstheit (vgl. ebd., S. 123). Da die Bedeutung von Sprache als Kommunikationsmittel relativ trivial erscheint und prinzipiell jeder über ein subjektives Verständnis über diese Funktion beziehungsweise Kompetenz verfügt, welches für die folgenden Ausführungen und Darstellungen zu Sprache und Bilingualität ausreicht, 
werden nur die beiden letztgenannten Funktionen beziehungsweise Kompetenzen nach Hamers \& Blanc (ebd.) ausgeführt.

\subsubsection{Sprache als kognitives Werkzeug}

Neben der Funktion zur Kommunikation akzentuieren einflussreiche Forscher der (Entwicklungs-)Psychologie wie z. B. Vygotsky (1962) oder Bruner (1975) die sich im Verlauf der (frühen) Kindheit einstellende Funktion von Sprache als kognitives Werkzeug und dessen Zusammenhang mit Kognition. Auch wenn Kommunikation bereits eine kognitive Komponente besitzt, nämlich den Austausch von konzeptueller Information, ermöglicht die Funktion von Sprache als kognitives Werkzeug primär die Organisation und Speicherung von Wissen in Form von (kombinierten) Bedeutungen (vgl. Hamers \& Blanc, 2000, S. 116f). Menschen können mithilfe von Sprache ihre Umwelt durch generelle psychologische Prozesse wie z. B. Klassifizierung, Hierarchisierung oder das Schließen von Inferenzen organisieren und strukturieren. Die Kognitionspsychologie modelliert die hierdurch entstehenden Repräsentationen wie Propositionen, Schemata, mentale Modellen etc. als zum Teil linguistisch enkodierte Elemente (vgl. Bialystok, 2013, S. 624). Propositionen werden als Wissenselemente konzeptioniert, die assoziative, semantische Prädikat-Argument-Relationen repräsentieren (z. B. [Hunde] $\rightarrow$ [bellen]). Schemata und mentale Modelle bestehen wiederum aus einer Vielzahl solcher miteinander verbundener oder in Beziehung stehender Propositionen. Mithilfe von Sprache können die jeweiligen kognitiven Schemata immer weiter ausgebaut werden, wobei aus linguistischer Perspektive durch die oben angesprochene „discrete infinity“ (Chomsky, 2000, S. 3 ff) der Ausdifferenzierung und Elaboration keine Grenzen gesetzt sind.

Der Zusammenhang von Sprache und Kognition darf hierbei allerdings nicht im Sinne von Whorfs (1956), ,linguistischer Relativität“ missverstanden werden, bei der die grammatischen Prinzipien und lexikalischen Ressourcen von Einzelsprachen die Art und Weise der Kognition der jeweiligen Sprecher beeinflussen. Gleitman \& Papafragou (2005) entkräften in ihrem Überblicksbeitrag zum Zusammenhang von Sprache und Kognition die These linguistischer Relativität. Zunächst weisen sie darauf hin, dass die linguistische Semantik kognitiv-inferenzielle Interpretationsprozesse nicht vollständig abbilden kann, sodass z. B. mentale Modelle nicht identisch mit der linguistischen Enkodierung dieser sind. Weiterhin schätzen sie Sprache als Mittel zur Kommunikation von Bedeutungen als deutlich verarmt und unterspezifiziert im Gegensatz zur Kognition ein, da die Interpretation linguistischer Stimuli, insbesondere bei mündlicher Kommunikation, abhängig von weiteren Informationsquellen sowie impliziten Prinzipien wie z. B. von Konversationsmaximen und Implikaturen (vgl. Grice, 1975) ist: „Finally, the presence of implicatures and 
other kinds of pragmatic inference ensures that - to steal a line from the Mad Hatter - although speakers generally mean what they say, they do not and could not say exactly what they mean" (Gleitman \& Papafragou, 2005, S. 637).

Nach der Inspektion relevanter empirischer Studien des Feldes hinsichtlich kognitiven Konstrukten wie der Repräsentation von Zeit, Bewegung, Orientierung sowie visuospatialer Fähigkeiten und Zahlverständnis kommen Gleitman \& Papafragou (ebd., S. 653f) zu dem folgenden Schluss: Sprache beeinflusst zwar das Denken „online“ und in vielfacher Weise, indem linguistische Markierungen z. B. bestimmte kognitive Kategorisierungen nahelegen oder den Hörer hinsichtlich einer bestimmten Interpretation „,primen“, aber distinkte grammatische Prinzipien und lexikalische Ressourcen von verschiedenen Einzelsprachen führen nicht zu unterschiedlichen kognitiven Architekturen und Prozessen beim Menschen. Anstatt potentielle kognitive Diskontinuitäten von Sprechern verschiedener Sprachen zu untersuchen, sollte sprachvergleichende Forschung eher die Gemeinsamkeiten und basalen Prinzipien zugrundeliegender linguistisch-konzeptueller Fähigkeiten von Menschen adressieren.

\subsubsection{Metalinguistische Bewusstheit}

Sprache stellt eine kognitive Aktivität dar, die aber auch gleichzeitig das Produkt der kognitiven Aktivität ist (vgl. Swain, 2000, S. 104), was die Betrachtung von Sprache als Objekt ermöglicht. Metalinguistische Bewusstheit, auch als metasprachliche Bewusstheit, explizites Sprachwissen, Sprachbewusstheit etc. bezeichnet, ist die Fähigkeit, ,sprachliche Erscheinungen losgelöst von ihren inhaltlichen Zusammenhängen zu betrachten“ oder „sprachliche Erscheinungen ins Bewusstsein zu rufen, sei es in Form einer reflektierten Kontrolle eigener sprachlicher Tätigkeiten oder als bewusstes Nachdenken über Sprache und ihre Gebrauchskontexte“ (Bien-Miller et al., 2017, S. 194f). Im Gegensatz $\mathrm{zu}$ vielen anderen sprachlichen Fähigkeiten, die eher als implizites Wissen enkodiert sind, zeichnet sich metalinguistische Bewusstheit primär durch ihren expliziten Charakter aus. Ellis (2004, S. 235ff) arbeitet die Explizitheit metalinguistischen Wissens feinkörnig heraus: Es handelt sich um bewusstes, deklaratives und somit lernbares sowie potentiell verbalisierbares Wissen, welches durch kontrollierte Verarbeitungsprozesse zugänglich ist, mit implizitem Wissen im Verarbeitungsprozess interagiert und sich in seiner Breite und Elaboration zwischen Lernern unterscheiden kann. Besonders interessant metalinguistischer Bewusstheit ist die These, dass ,,bestimmte schwierige Sprachphänomene wie die komplexe Satzbildung, der Gebrauch des Konjunktivs oder des Genitivobjektes“" sowie „späte Teilkompetenzen und der Erwerb von sprachlichen Regelungen, die durch Sprachwandel gefährdet sind, vor allem durch Maßnahmen wie Regelerarbeitung oder Gewahrwerden aufgrund von Reflexion über den Sprachgebrauch gelernt werden können“" (Eichler \& Nold, 
2008, S. 67). Dementsprechend könnten hinsichtlich bestimmter sprachlicher Strukturen metalinguistische Fähigkeiten eine notwendige Voraussetzung zum Erwerb dieser darstellen.

Zur weiteren Abgrenzung von implizitem Sprachwissen akzentuiert Ellis (2004, S. 234f) zudem, dass sich explizites Sprachwissen zu einem späteren Zeitpunkt als implizites Sprachwissen entwickelt und sich beide in ihrer neuronalen Repräsentation unterscheiden, sodass ihre Beziehung aktuell eher als Dichotomie statt als Kontinuum beschrieben werden kann. Karmiloff-Smith (1992, S. 47ff) modelliert die Entwicklung von implizitem zu explizitem Wissen über das Erreichen eines Schwellenwertes. Zunächst gilt es für den Lerner, implizites Wissen zu sammeln und zu akkumulieren, bis eine ausreichend große, kritische Menge erreicht wird. Diese Menge ermöglicht es dem Lerner durch Umstrukturierungs- und Umkodierungsprozesse, das implizite Wissens in explizite, abstrakte Wissensformen umzuwandeln sowie Verknüpfungen mit anderen Wissensbeständen zu erzeugen (vgl. Bien-Miller, 2017, S. 196).

Angesichts der Multimodalität und Komplexität von Sprache handelt es sich bei metalinguistischer Bewusstheit ebenfalls nicht um ein ,monolithisches Konzept", sondern um ein multidimensionales Konstrukt (vgl. Bialystok, 2013, S. 627). Dementsprechend unterscheidet sich auch das Spektrum an Subkomponenten und Fähigkeiten, die mit metalinguistischer Bewusstheit assoziiert werden, wobei Bezugnahmen zu den einzelnen grammatischen Teilsystemen zum Beispiel in Form von phonologischer, syntaktischer oder lexikalischer Bewusstheit naheliegen. Eine besonders breite Konzeption stellt das „Language Awareness" Konzept aus Großbritannien dar (vgl. Eichler \& Nold, 2007, S. 65-66). Hier wird metalinguistische Bewusstheit „stärker ganzheitlich, soziolinguistisch und auch politisch-gesellschaftlich" (Eichler \& Nold, 2007, S. 65) operationalisiert, sodass neben typischen metalinguistischen Fähigkeiten auch Phänomene wie z. B. Sprache und Herrschaft, Sprachgebrauch und Milieu sowie die gesellschaftliche Akzeptanz von Varietäten und Einzelsprachen betrachtet werden (vgl. Gnutzmann, 1997, S. 232-235). Sprachbewusstheit umfasst in dieser Konzeption also nicht nur spezifisch linguistische Wissenskomponenten hinsichtlich Phonologie, Lexik, Grammatik, Pragmatik etc., sondern ebenfalls eine Sensibilität für die politische, soziale und affektive Bedeutung von Sprache und Sprachgebrauch. Dementsprechend besteht die metalinguistische Bewusstheit sowohl aus sprachspezifischen und sprachenunabhängigen expliziten Wissenskomponenten und Fähigkeiten.

\subsection{Zusammenfassung}

Der Begriff der Sprache wurde hier unter einer primär sprachwissenschaftlichen und kognitionspsychologischen Perspektive präzisiert, wobei Sprache 
vor allem hinsichtlich ihrer Varietäten, grammatischen Teilsysteme, Merkmale sprachlichen Wissens und sprachlicher Kompetenzen sowie Funktionen betrachtet wurde. Sprache stellt trotz ihres Varietätenreichtums ein in einzelne Komponenten zu zerlegendes multimodales, dynamisches Wissenssystem dar, welches aus einer endlichen Anzahl regelhafter und idiosynkratrischer Elemente besteht, die eine unendliche Menge potentieller Äußerungen ermöglichen. Der Kompetenzbegriff von Sprache, welcher insbesondere hinsichtlich bildungsbezogener Phänomene eine hohe Relevanz aufweist, ist mit einer Vielzahl von theoretischen sowie methodischen Problemen und Herausforderungen behaftet. Die Funktionen von Sprache sowie die deskriptiven Merkmale der jeweiligen zu untersuchenden Varietät liefern allerdings eine erste Orientierung, die bei der Reflexion und Operationalisierung von Sprachkompetenz berücksichtigt werden sollten. Dementsprechend lassen sich sprachliche Fähigkeiten bezüglich verschiedener Varietäten 1) als Kommunikationsmittel bei der Interaktion mit anderen, 2) als kognitives Werkzeug zur Organisation und Speicherung von Wissen sowie zur Strukturierung seiner Umwelt und 3) als Objekt der Manipulation selbst durch metalinguistische Bewusstheit unterscheiden (vgl. Hamers \& Blanc, 2000, S. 123).

Die in diesem Abschnitt dargelegten grundlegenden Ausführungen gelten selbstverständlich auch für die Einzelsprachen von bilingualen Sprechern. Die Eigenschaften von Sprache müssen bei der Bezugnahme auf das Phänomen „Bilingualität" allerdings unter den Bedingungen des Erwerbs, des Vorhandenseins und der Nutzung zweier sprachlicher Systeme und bezüglich deren potentiellen Konsequenzen für das linguistische und kognitive Vermögen sowie für die konkrete Lebensrealität reflektiert werden. Hierbei kann nicht davon ausgegangen werden, dass Bilingualität eine Aggregation zweier getrennter, abgeschlossener monolingualer Systeme darstellt. Vielmehr gilt es die Verflechtung der Einzelsprachen bei bilingualen Sprechern hinsichtlich der Koordination, Kooperation und Konkurrenz der Einzelsprachen sowie deren resultierende Folgen zu berücksichtigen. 


\section{Bilingualität}

Bevor Bilingualität in seiner Heterogenität bezüglich diverser entwicklungstheoretischer, linguistischer, kognitiver, lebenspraktischer sowie bildungsbezogener Aspekte dargestellt wird, soll zunächst eine terminologische Abgrenzung erfolgen. Innerhalb der Bilingualismusforschung wird die Eigenschaft, mit mehr als einer Einzelsprache aufzuwachsen und/oder mehr als eine Sprache alltäglich zu gebrauchen, mit einer Vielzahl von Begriffen wie „Bilingualität", „Bilingualismus“, „Mehrsprachigkeit", „Multilingualismus" etc. bezeichnet. Manche Autoren nutzen hierbei unterschiedliche Begriffe in Abhängigkeit von der Quantität der vorhandenen Einzelsprachen und/oder von den jeweiligen Spracherwerbstypen, innerhalb welcher die verschiedenen Einzelsprachen erworben wurden. In der vorliegenden Studie wird eine liberale Verwendung des Begriffs „Bilingualität“ hinsichtlich des erstgenannten Aspekts verwendet: Der Begriff „Bilingualität“ wird in der vorliegenden Arbeit unabhängig von der Anzahl der tatsächlich vorhandenen Einzelsprachen gebraucht. Eine Differenzierung zwischen Zwei-, Drei- oder Viersprachigkeit erfolgt lediglich an Stellen, in denen theoretische oder empirische Distinktionen zwischen diesen Formen der Mehrsprachigkeit relevant sind. Allerdings wird hinsichtlich des zweiten Aspekts eine zentrale Einschränkung vorgenommen: Es werden nur natürlich erworbene Sprachen unter dem Begriff der Bilingualität berücksichtigt. Dementsprechend werden alle Fälle von Bilingualität, die aus einem Fremdspracherwerb resultieren, von dieser Definition ausgeschlossen. Dieser Aspekt wird näher in Abschnitt 3.2 erläutert.

Bilingualität zeichnet sich primär durch Variabilität und Heterogenität aus (vgl. z. B. Unsworth, 2016, S. 103; van Dijk et al., 2018, S. 1), was sowohl die Untersuchung des Phänomens als auch die Generalisierung zugehöriger Befunde erschwert. Sie entsteht und tritt in einer Vielzahl möglicher Arten auf (vgl. Edwards, 2013, S. 7), sodass sich bilinguale Sprecher, deren Umwelten und Sprachkombinationen immens unterscheiden können (vgl. Aronin \& Singleton, 2012, S. 118). Diese drei von Aronin und Singleton (ebd.) akzentuierten Dimensionen des Sprechers, der Umwelt und der Sprachenkombination können als übergeordneter Rahmen eine erste Orientierung hinsichtlich des Phänomens der Bilingualität liefern. So determiniert zunächst die Umwelt die zu erwerbenden Sprachen und Bedingungen, in der Sprecher ihre Bilingualität entwickeln. Die Sprecher wiederum beeinflussen ihre Umwelt durch ihr Handeln, was relevante Veränderungen der Bedingungen und auch der Sprachen selbst - z. B. durch Sprachwandelphänomene - nach sich ziehen kann. Wechselt der Sprecher zu einem Zeitpunkt seine Umwelt, finden diese reziproken Prozesse unter veränderten Bedingungen statt.

Diese abstrakte, erste Annäherung an eine strukturelle Beschreibung des Phänomens der Bilingualität weist bereits auf dessen Komplexität, Dynamik 
und Interdependenz hin. Folglich wäre ein Klassifikationssystem wünschenswert, das diese unterschiedlichen Einflussfaktoren und deren resultierende Ausprägungen von Bilingualität systematisch erfassen kann. Solche Klassifikationen oder Typologien der Bilingualität zur Strukturierung des Forschungsgegenstandes sind allerdings mit diversen Problemen und Herausforderungen behaftet: 1) Bilingualität ist keine kategoriale Variable (vgl. Bialystok, 2001, S. 8), auch wenn binäre Typologien wie ,subtractive/additive bilingualism“, „receptive/productive bilingualism“ oder „balanced/dominant bilingualism“" (vgl. Baker, 2011, S. 3-4) in der Bilingualismusforschung gebräuchlich waren und zum Teil heute noch sind. Vielmehr gilt es Bilingualität selbst als auch hinsichtlich der Einflussfaktoren kontinuierlich zu operationalisieren (vgl. z. B. Butler, 2013). 2) Die konstituierenden Merkmale von Bilingualität stehen in Abhängigkeit zueinander und beeinflussen sich gegenseitig. So determiniert z. B. die Umwelt den jeweiligen Spracherwerbstyp, welcher sich wiederum auf den Entwicklungsverlauf und auf die Sprachkompetenzen des Sprechers auswirkt. 3) Klassifikationen und Typologien können nicht der Dynamik eines sich ständig in Entwicklung befindlichen bilingualen Profils gerecht werden (vgl. ebd., S. 112-115). Angesichts dieser Probleme und Herausforderungen erscheint die Erzeugung einer allumfassenden anwendbaren Typologie sowie eines exhaustiven theoretischen Rahmens der Bilingualität illusionär (vgl. Edwards, 2013, S. 9). Trotzdem sind solche Klassifikationen und Typologien der Bilingualität hilfreich, und zwar im Sinne einer Checkliste relevanter zu berücksichtigender Variablen, die als Leitfaden für Untersuchungen und deren Datenerhebung dienen können (vgl. ebd.). Ein Beispiel für eine solche Typologie findet sich bei Butler (2013, S. 113-114), die zentrale Dimensionen benennt, die die Ausprägung der Bilingualität determinieren, und diese hinsichtlich ihrer Auswirkungen u. a. auf den (Zweit-)Spracherwerb, den Implikationen für Bildungsprozesse sowie den damit verbundenen methodologischen Herausforderungen reflektiert.

Aufgrund der reziproken Abhängigkeit der übergeordneten Einflussfaktoren auf Bilingualität, insbesondere hinsichtlich des Zusammenhangs von Nutzer und Umwelt (vgl. Aronin \& Singleton, 2012), sowie des Fokus des vorliegenden Forschungsvorhabens auf ein bilinguales Interaktionsangebot beim PL wird im Folgenden der Forschungsstand zu bilingualen Spracherwerbstypen (Abschnitt 3.2), (meta-)linguistischer Sprachkompetenz und (meta-)kognitiver Konsequenzen der Bilingualität (Abschnitt 3.3), Identität und Kultur in bilingualen Lebensrealitäten sowie zu Einstellungen zur Bilingualität (Abschnitt 3.4), zum bilingualen Sprachgebrauch (Abschnitt 3.5) und zu Bilingualität und Bildung (Abschnitt 3.6) näher betrachtet und diskutiert. Der Einflussfaktor der Sprachkombination, bei welcher vornehmlich die Anzahl und Ähnlichkeit der Sprachen fokussiert werden, wird hierbei weitestgehend ausgeklammert. Dementsprechend repräsentieren die hier selektierten Faktoren zwar nicht alle relevanten Phänomene der Bilingualität, allerdings ist diese Auswahl in der Lage, 
zentrale Bedingungen und Formen der Bilingualität der in der vorliegenden Studie untersuchten Probandengruppe türkisch-deutschsprachig aufwachsender SuS der deutschen Regelgrundschule zu erfassen. Bevor diese einzelnen Dimensionen näher betrachtet werden, soll zunächst eine Arbeitsdefinition des Begriffs „Bilingualität“ für das vorliegende Untersuchungsvorhaben festgelegt werden.

\subsection{Begriffsbestimmung Bilingualität}

Alltagssprachlich werden Sprecher als bilingual bezeichnet, wenn sie über zwei (nationale) Einzelsprachen verfügen. Das heißt, dass das Vorhandensein verschiedener Varietäten einer Einzelsprache bei einem Sprecher wie z. B. das standardsprachliche Deutsch und das Südhessische, eine Form des Hessischen Dialekts, nicht als Bilingualität kategorisiert wird. Auch im wissenschaftlichen Kontext wird Bilingualität in der Regel über das Vermögen eines Sprechers, über mehr als eine Einzelsprache zu verfügen, definiert, wobei Varietäten einer Einzelsprache ausgeschlossen werden (aber siehe Grosjean \& Li, 2013, S. 5).

Definitionen von Bilingualität nähern sich dem Begriff vor allem über die Dimension der Kompetenz (vgl. z. B. Butler, 2013, S. 111f; Edwards, 2013, S. 11f), obwohl die Intension des Begriffs der Bilingualität eine Vielzahl diverser Merkmale auf der Ebene der Sprecher, der Umwelt und der Sprache aufweist (vgl. Aronin \& Singleton, 2012), die sich potentiell ebenfalls als Definitionskriterien anbieten würden. Sprecher müssen nach kompetenzorientierten Definitionen ein festgelegtes Mindestmaß an Sprachkompetenz in beiden Sprachen vorweisen, um als bilingual bezeichnet werden zu können. Betrachtet man verschiedene Definitionen im historischen Verlauf, fällt zunächst auf, dass ältere Definitionen höhere Kompetenzgrade zu Erfüllung der Bilingualität erfordern, während spätere Definitionen liberaler in ihren Ansprüchen sind (vgl. Edwards, 2013, S. 12). So fordert z. B. Bloomfield (1933, S. 55, zit. nach. Butler, 2013, S. 111) eine muttersprachliche Kontrolle beider Sprachen, wohingegen für Haugen (1953, S. 7, zit. nach. Butler, 2013, S. 111) das bloße Produzieren bedeutungsvoller Äußerungen in einer weiteren Sprache ausreichend ist, um als bilingual zu gelten. Für Myers-Scotton (2006, S. 4) liegt Bilingualität vor, wenn man in beiden Sprachen in der Lage ist, ,a limited casual conversation" zu führen.

Wie bei den Ausführungen zum Phänomen Sprache schon dargelegt, ist die Kompetenzkategorie bei Sprache mit diversen Problemen behaftet, was selbstverständlich auch für das Phänomen der Bilingualität gilt. So bleiben Probleme wie die Kompetenz-Performanz Divergenz, die Wahl geeigneter Fähigkeitsbereiche und deren adäquate Operationalisierung oder der fehlenden Existenz von Endstadien der Sprachkompetenz bestehen. So stellt sich z. B. hinsichtlich 
der Definition von Bloomfield (1933, S. 55; zit. nach Butler, 2013, S. 111) die Frage, welche Kompetenzkriterien es für eine „muttersprachliche Kontrolle“ einer Sprache anzusetzen gilt. Auch bezüglich der Definition von Myers-Scotton (2006, S. 4) ist unklar, welche sprachlichen Fähigkeiten zum Führen einer „limited casual conversation“ (ebd.) benötigt werden? Definitionen von Bilingualität, die lediglich Kompetenzmaße zur Begriffsbestimmung heranziehen, weisen neben den theoretischen und methodischen Problemen der Sprachkompetenz als zentrales Definitionskriterium eine zu enge Intension angesichts der Heterogenität und Komplexität von Bilingualität auf. Inadäquate Intensionen von Definitionen können wiederum zu unangemessenen Denotationen führen, die bestimmte Fälle von Bilingualität ausschließen würden. Dementsprechend erscheint eine Definition von Bilingualität über die Kompetenzkategorie eher ungeeignet zu sein.

Aktuelle Begriffsbestimmungen beanspruchen zwar weiterhin zum Teil die Dimension der Kompetenz, versuchen allerdings die Bestimmung einer konkreten Mindestkompetenz in einer der oder beiden Sprachen zu umgehen und fokussieren vermehrt die Fähigkeit zur Interaktion in zwei Sprachen. So definiert z. B. Butler (2013, S. 112) die Fähigkeit zur mündlichen und/oder schriftlichen Kommunikation in mehr als einer Sprache zum Zwecke der Interaktion als das ausschlaggebende Definitionskriterium von Bilingualität, während der Kompetenzgrad in den Sprachen an keinem expliziten Kriterium festgelegt wird: „Multilanguage users are defined here as individuals or groups of people who obtain communicative competence in more than one language, with various degrees of proficiencies, in oral and/or written forms, in order to interact with speakers of one or more languages in a given society". Valdés und Figueroá (1994, S. 8) blenden in ihrer Definition ebenfalls die Bestimmung eines notwendigen Grades an Kompetenz aus, indem sie die Voraussetzungen für Bilingualität als erfüllt ansehen, wenn Sprecher ,more than one language competence" besitzen. Zwar schwächen die soeben genannten Definitionen die Relevanz der Kompetenzkategorie für die Bestimmung von Bilingualität ab, indem sie die Definition von Bilingualität nicht an ein Mindestmaß von Sprachkompetenz binden und zum Teil weitere Merkmale der Bilingualität einbinden. Nichtsdestotrotz bleibt die Kompetenzkategorie in diesen Definitionen weiterhin explizit als konstitutives Element zur Begriffsbestimmung von Bilingualität bestehen.

Im Gegensatz hierzu blenden Grosjean \& Li (2013, S. 5) die Kompetenzkategorie bei der Definition von Bilingualität komplett aus, indem sie die alltägliche Nutzung von mehr als einer Sprache (oder Varietät) als zentrales Kriterium von Bilingualität akzentuieren: ,[...], we will define bilingualism, [...], as the use of two (or more) languages (or dialects) in everyday life". Diese Definition verschiebt den Fokus von der Kompetenzkategorie hin zur Bedeutung des Gebrauchs von mehr als einer Sprache für das alltägliche Leben der Sprecher. Der Alltagsbezug des Gebrauchs der Sprachen fokussiert natürlich 
erworbene Sprachen und schließt implizit Fälle aus, in denen eine Sprache nicht im „alltäglichen Leben“ funktional ist, wie es z. B. im Fremdspracherwerb der Fall ist (siehe auch Abschnitt 3.2). Weiterhin umfasst diese Begriffsbestimmung auch Fälle von Mehrsprachigkeit, in denen Sprecher über mehr als zwei Sprachen verfügen, anstatt verschiedene Formen von Mehrsprachigkeit über die Anzahl der vorhandenen Einzelsprachen in Zwei-, Drei-, Viersprachigkeit etc. zu operationalisieren. Abschließend fällt auf, dass Grosjean \& Li (ebd.) nicht zwischen Einzelsprachen und Dialekten differenzieren, sondern für sie der Gebrauch von mehr als einem Dialekt im alltäglichen Leben ebenfalls als eine Ausprägung von Bilingualität gilt.

Für die vorliegende Studie wird die Definition von Bilingualität nach Grosjean und Li (2013, S. 5) in einer adaptierten Form favorisiert. Ihre Definition berücksichtigt die drei zentralen Elemente von Bilingualität des Nutzers, der Umwelt und der Sprachen (vgl. Aronin \& Singleton, 2012) und ist durch ihre Unbestimmtheit in ihrer Intension liberal genug, um die immense Bandbreite verschiedener Konstellationen der Bilingualität zu denotieren. Allerdings wird bezüglich dieser Definition für die vorliegende Arbeit eine zentrale Einschränkung vorgenommen: Varietäten einer Sprache wie z. B. Dialekte werden als konstitutives Merkmal von Bilingualität ausgeschlossen, sodass lediglich der Gebrauch von zwei oder mehr Einzelsprachen im alltäglichen Leben als Bilingualität definiert wird.

Um ein adäquaten Eindruck von der Vielfalt der Ausprägungen von Bilingualität zu erhalten, gilt es sich zunächst einen Überblick über potentiell divergierende Merkmale hinsichtlich der zentralen Elemente des Sprechers, der Umwelt und der Sprache sowie deren Zusammenhänge zu verschaffen. Diesbezüglich werden zunächst verschiedene Typen des bilingualen Spracherwerbs betrachtet und mit dem monolingualen Spracherwerb kontrastiert.

\subsection{Bilingualer Spracherwerb}

Der natürliche Spracherwerb kann allgemein als ein aktiver, robuster, weitestgehend von der non-verbalen kognitiven Entwicklung unabhängiger sowie ungesteuerter bzw. impliziter Prozess beschrieben werden, der durch sprachlichen Input ausgelöst und getragen wird (vgl. Schulz \& Grimm, 2012, S. 156). Sprachlicher Input bezeichnet hierbei alle sprachlichen Strukturen, die vom Sprachlerner bewusst oder unbewusst wahrgenommen werden (vgl. Unsworth, 2016). Trotz seiner Robustheit wird der Spracherwerb von diversen Faktoren wie z. B. Geschlecht, Geschwisterrang oder sozioökonomischen Status mit schwachen Effektstärken beeinflusst (vgl. z. B. Szagun, 2016, S. 180ff). Der Spracherwerb stellt ein interdisziplinär beforschtes Phänomen dar, dessen theoretische Modellierung und empirische Untersuchung sich mit zwei zentralen 
Fragen bzw. Problemen auseinandersetzen muss: 1) Wie können Kinder das komplexe System von Sprache erwerben (Lernbarkeitsproblem)? 2) Wie verändern und erweitern sich sprachliche Fähigkeiten im Verlauf des Spracherwerbs (Entwicklungsproblem) (vgl. Schulz, 2007, S. 67-69). Hinsichtlich des vorliegenden Forschungsvorhabens spielen die zugrundeliegenden Prinzipien und (Lern-)Mechanismen des Spracherwerbs allerdings nur eine untergeordnete Rolle, sodass diese nicht näher expliziert werden (für ausführliche Publikationen zum Phänomen Spracherwerb siehe z. B. Dittmann, 2006; Szagun, 2016). Vielmehr werden im Folgenden zunächst die verschiedenen bilingualen Spracherwerbstypen sowie der monolinguale Spracherwerb voneinander abgegrenzt (Abschnitt 3.2.1). Anschließend werden die Gemeinsamkeiten und Unterschiede der Entwicklungsverläufe der verschiedenen Spracherwerbstypen betrachtet (Abschnitt 3.2.2). Abschließend werden die zentralen Erkenntnisse zusammengefasst (Abschnitt 3.2.3).

\subsubsection{Spracherwerbstypen}

In der Bilingualismusforschung werden für gewöhnlich vier sich unterscheidende Formen des Spracherwerbs differenziert: Monolingualer Spracherwerb (L1a), bilingualer Erstspracherwerb bzw. doppelter Erstspracherwerb (2L1a), Zweitspracherwerb (L2a) und Fremdspracherwerb (fLa) (für einen tabellarischen Überblick siehe Reitenbach, Schastak \& Rauch, 2018, S. 105). Diese Erwerbstypen lassen sich anhand der Kombination der folgenden Bedingungen unterscheiden: 1) Der Erwerbsbeginn (Das Alter, ab dem der Lerner regelmäBig mit sprachlichem Input einer Sprache konfrontiert wird), 2) die Verkehrssprache (Die Sprache, die in der Umwelt vom Großteil der Sprecher zur Kommunikation genutzt wird) sowie 3) die Art der Unterweisung (Die Vermittlung der zu erwerbende Sprache in impliziter Form durch den sprachlichen Input und in Interaktion mit der Umwelt (ungesteuert) oder durch explizite Instruktion (gesteuert)).

Beim L1a wird der Erwerbsbeginn zur Vereinfachung der Kategorisierung auf die Geburt festgelegt, auch wenn dieser bereits pränatal ab ca. der 27 Schwangerschaftswoche beginnt, da die bis dahin entwickelte Hörfähigkeit die Wahrnehmung prosodischer Hinweisreize wie Tonhöhen und Längen erlaubt (vgl. Hennon, Hirsh-Pasek \& Golinkoff, 2000, S. 51ff). Die zu erwerbende Sprache stellt beim L1a auch die Verkehrssprache der Umwelt dar und wird ungesteuert erworben. Das bedeutet, dass monolinguale Kinder in ihrer Umwelt mit lediglich einer Sprache systematisch konfrontiert werden und der Erwerbsbeginn zu einem Zeitpunkt stattfindet, ab welchem auch alle anderen Entwicklungsbereiche ihren Ausgangspunkt nehmen. 
Das gleiche gilt auch weitestgehend für den 2L1a. Beide oder eine der beiden Sprachen stellt die Verkehrssprache dar. Der Erwerbsbeginn wird ebenfalls auf die Geburt datiert, wobei eine der beiden Sprachen auch erst sukzessive hinzukommen kann. Die kritische Grenze für die sukzessiv hinzukommende zweite Sprache, damit diese noch als weitere Erstsprache (L1) statt Zweitsprache (L2) gilt, wird je nach Autor bis vor dem Abschluss des ersten (vgl. Paradis, Genesee \& Crago, 2011) oder zweiten Lebensjahres (vgl. Schulz \& Grimm, 2012) festgelegt. Dementsprechend werden bilingual aufwachsende Kinder mit 2L1a systematisch mit zwei Sprachen konfrontiert, wobei die Quantität und die Kontexte, also welche Sprache mit welchen Personen und in welchen Kontexten wie oft gesprochen wird, sich zwischen Kindern im 2L1a deutlich unterscheiden können. So werden z. B. in Ländern mit zwei offiziellen Amtssprachen, wie dem Englischen und Französischen in Kanada, bilingual englisch- und französischsprachig aufwachsende Kinder in ihrem Alltag und in vielen (Bildungs-)Institutionen mit sprachlichem Input beider Sprachen konfrontiert. Im Gegensatz hierzu stehen türkisch-deutschsprachig aufwachsende Kinder mit 2L1a in Deutschland im familiären Kontext mit beiden Sprachen in Kontakt, aber im sonstigen Alltag und in (Bildungs-)Institutionen kommunizieren sie eher ausschließlich auf Deutsch. Auch auf der Ebene der Familie können sich die Bedingungen des 2L1a deutlich hinsichtlich des Inputs in den jeweiligen Sprachen unterscheiden. So weist z. B. das Partnerprinzip, bei dem jedem Elternteil eine bestimmte Sprache zugeordnet wird sowie eine „Familiensprache" festgelegt wird, andere Bedingungen auf als wenn beide Elternteile in beiden Sprachen mit dem Kind kommunizieren. Aber auch der 2L1a nach Partnerprinzip zeichnet sich wiederum durch eine hohe Vielfältigkeit bezüglich der Quantität und den Kontexten aus, in denen das Kind mit Input seiner jeweiligen Einzelsprachen konfrontiert wird. Zudem weisen manche Kinder durch den potentiell späteren Erwerbsbeginn einer weiteren Sprache bis zum ersten bzw. zweiten Lebensjahr einen weiter fortgeschrittenen Entwicklungsstand als bei der Geburt auf, was den 2L1a beeinflussen könnte.

Ein L2a liegt vor, wenn der Sprecher mit der Verkehrssprache in impliziter, kontinuierlicher Weise erst ab dem Alter von einem bzw. zwei Jahren regelmäßig in Kontakt kommt, sodass bis zu diesem Zeitpunkt ein monolingualer Spracherwerb einer anderen Sprache vorlag, die nicht die Verkehrssprache darstellt. Der Zweitspracherwerb wird nochmals hinsichtlich des Erwerbsbeginns in den frühen (L2 kommt im Alter zwischen 2-4 Jahren hinzu), späten (ab 6 Jahren) und erwachsenen (ab Jugendalter) L2a differenziert (vgl. Schulz \& Grimm, 2012, S. 164). Dementsprechend weisen Lerner zu Beginn des L2a bereits altersgemäße erstsprachliche und kognitive Fähigkeiten sowie Weltwissen auf (vgl. ebd.). Dieser Spracherwerbstyp ist häufig mit einer Migrationssituation verbunden, bei dem der betreffende Zweitsprachlerner migriert oder seine Eltern oder Generationen zuvor migriert sind, ihre L1 aber zumindest im familiären Kontext beibehalten. Dementsprechend unterschieden sich auch 
bei diesem Spracherwerbstyp die Kontexte, in denen die jeweiligen Sprachen auftreten und primär genutzt werden. So ist die L1 eher im familiären Kontext verhaftet, was aber nicht bedeutet, dass in diesem exklusiv in der L1 kommuniziert wird, während die L2 eher im Kontakt mit der außerfamiliären Umwelt wie z. B. in Betreuungs- und Bildungsinstitutionen, Vereinen, Ämtern, Geschäften etc. erworben und verwendet wird.

Von diesen ,natürlichen“ Spracherwerbstypen gilt es den fLa zu unterscheiden, bei welchem eine Sprache durch explizite Instruktion erworben wird, die keine Verkehrssprache im jeweiligen Erwerbskontext darstellt. Dementsprechend werden Fremdsprachlerner lediglich in bestimmten, primär unterrichtlichen Kontexten mit der Fremdsprache konfrontiert, sodass die Quantität des Inputs und die Kontextvielfältigkeit deutlich geringer als bei den anderen Spracherwerbstypen ausfallen. Der Erwerbsbeginn ist zur Abgrenzung dieses Erwerbstyps unerheblich. Fremdsprachen werden in der Regel in Bildungsinstitutionen wie Schule oder Volkshochschulkurse vermittelt, sodass der Erwerb zu einem Zeitpunkt stattfindet, an dem bereits elaborierte (schrift)sprachliche Fähigkeiten vorliegen und somit Sprach- und Schriftspracherwerb der jeweiligen Sprache häufig parallel vermittelt werden. Allerdings ist ein Fremdspracherwerb im Selbststudium unter Verwendung von Hilfsmitteln wie Lehrbücher oder -programme ebenfalls denkbar.

\subsubsection{Gemeinsamkeiten und Unterschiede der Entwicklungsverläufe der Spracherwerbstypen im Kontext des Erwerbs des Deutschen}

Die hier postulierten Altersgrenzen des Erwerbsbeginns zur Differenzierung des 2L1a vom L2a sowie hinsichtlich der drei verschiedenen Typen des L2a resultieren aus vergleichenden empirischen Untersuchungen zum Spracherwerb, die quantitative und qualitative Gemeinsamkeiten und Unterschiede zwischen den so operationalisierten Erwerbstypen feststellen. Diese Gemeinsamkeiten und Unterschiede werden häufig auf sensible Phasen (vgl. z. B. Hopp, 2007; Johnson \& Newport, 1989; Singleton \& Ryan, 2004) zurückgeführt, in welchen eine gesteigerte Sensibilität für bestimmte Umweltreize, in diesem Fall der sprachliche Input, angenommen wird. Die genauen Altersgrenzen sind allerdings noch Gegenstand linguistischer Forschung sowie eher kontinuierlich als kategorial zu betrachten und scheinen auch abhängig von dem untersuchten linguistischen Teilsystem zu sein (vgl. Grimm \& Schulz, 2016, S. 29). Zudem bewegen sich die Altersgrenzen des 2L1a (bis zwei Jahre), des frühen (zwischen 2-4 Jahren) sowie des späten L2a (ab sechs Jahre) zu Zeitpunkten, in denen mit dem Besuch institutionalisierter Betreuungs- (z. B. Krabbelstube und Kindergarten) und Bildungseinrichtungen (Schule) begonnen wird. Dem- 
entsprechend könnten die aktuellen Altersgrenzen eventuell auch durch Eigenschaften der Betreuungs- und Bildungseinrichtungen der Gesellschaft statt exklusiv durch neurologische Faktoren determiniert sein.

Hinsichtlich quantitativer und qualitativer Charakteristika der Erwerbsverläufe der natürlichen Spracherwerbstypen weisen sowohl der 2Lla als auch der frühe L2a mehr Gemeinsamkeiten als Unterschiede mit dem L1a im Deutschen auf (vgl. Grimm \& Schulz, 2016; Rothweiler, 2007; Schulz \& Grimm, 2012; Tracy, 2008). Hierbei sind die Ähnlichkeiten aber abhängig von der konkreten Erwerbsaufgabe (für einen Überblick siehe Schulz \& Grimm, 2012; Grimm \& Schulz, 2016). Hinsichtlich früh zu erwerbender linguistischer Phänomene wie z. B. Verbbedeutung, Subjekt-Verb-Kongruenz oder Präpositionen ähnelt sowohl der 2L1a als auch der frühe L2a stark dem L1a (vgl. Grimm \& Schulz, 2016, S. 37f). Allerdings weisen der 2L1a und der frühe L2a bezüglich späteren Erwerbsaufgaben wie z. B. Kasus oder Verstehen von Negation mehr Gemeinsamkeiten miteinander als mit dem Lla auf (vgl. ebd.). Der erwachsene L2a im Deutschen hingegen unterscheidet sich in seinem Erwerbsverlauf deutlich von den eben angeführten Spracherwerbstypen (vgl. Rothweiler, 2007).

Da im Gegensatz zu Nationen mit mehr als einer offiziellen Landessprache wie z. B. Indien, Südafrika, Luxemburg, Belgien etc. in Deutschland lediglich das Deutsche als offizielle Amtssprache anerkannt ist, treten natürliche bilinguale Erwerbsverläufe eher im Kontext von Migration und MH auf. Hierbei scheint die Mehrheit der bilingualen Spracherwerbsverläufe in Deutschland einem frühen L2a zu entsprechen (vgl. Schulz \& Tracy, 2011). Folglich wächst ein Großteil der Kinder mit MH in Deutschland zunächst mit einer anderen L1 als dem Deutschen im familiären Umfeld auf, bevor sie sukzessive das Deutsche als L2, in der Regel mit dem Eintritt in frühkindliche Betreuungsinstitutionen wie Kindertagesstätten (vgl. ebd.) oder in der Kommunikation mit älteren Geschwistern und/oder anderen Kindern beim Spielen (vgl. Reich, 2009, S. 64), erwerben. Auch für türkisch-deutschsprachig aufwachsende Kinder stellt das Türkische als Muttersprache der Eltern häufig auch für sie die L1 dar ${ }^{3}$, bevor sie sukzessiv Deutsch als L2 erwerben (vgl. Reich, 2009).

\subsubsection{Zusammenfassung}

Angesichts dieses kurzen Überblicks lässt sich festhalten, dass sich Spracherwerbstypen anhand der Indikatoren Erwerbsbeginn und Umgebungssprache differenzieren lassen und hinsichtlich der Erwerbsverläufe diverse Gemeinsamkeiten, aber auch Unterschiede aufweisen. Zudem unterscheiden sich bilinguale Erwerbstypen vom L1a zentral in den jeweiligen Erwerbs- und Gebrauchskontexten. Während beim L1a in fast jedem Kontext die L1 funktional

3 Für einen linguistisch fundierten Überblick zum monolingualen Erwerb des Türkischen siehe Sirim (2009). 
ist, werden bei bilingualen Erwerbstypen die jeweiligen Sprachen präferiert in bestimmten Situationen genutzt und auch erworben. Diese Bedingung der Distribution von Erwerbs- und Anwendungsmöglichkeiten einzelner Sprachen auf verschiedene Kontexte bei bilingualen Spracherwerbstypen sind allerdings abhängig von der konkreten, individuellen Lebenssituation, sodass diese auch innerhalb eines bestimmten bilingualen Erwerbstypus interindividuell deutlich divergieren können. In vielen Fällen zeichnet sich die Distribution von Erwerbs- und Anwendungsmöglichkeiten einer Sprache durch einen gewissen Anteil an Komplementarität aus. Grosjean (2010, S. 29ff) beschreibt die Kontextabhängigkeit sprachlicher Fähigkeiten sowie des Sprachgebrauchs bilingualer Sprecher als ,,complementary principle“: „Bilinguals acquire and use their languages for different purposes, in different domains of life, with different people. Different aspects of life often require different languages“. So werden z. B. bilingual türkisch-deutschsprachig aufwachsende SuS in Deutschland in institutionalisierten Bildungskontexten exklusiv mit dem Deutschen konfrontiert, während sie in familiären und/oder religiösen Kontexten häufiger von ihrer L1 umgeben sind. In diesem Beispiel würde sich die Komplementarität dahingehend auf die sprachlichen Fähigkeiten dieser SuS auswirken, dass Lesen, Schreiben sowie allgemein bildungssprachliche Varietäten primär in der L2 Deutsch erworben werden, während das mentale Lexikon zum Thema „Religion" fast ausschließlich aus türkischsprachigen Einträgen bestehen würde. Dementsprechend gilt es hinsichtlich der Bilingualität eines Individuums sowohl den zu Grunde liegenden Spracherwerbstyp als auch die individuelle Spracherwerbssituation zu berücksichtigen, da diese die Erwerbs- und Lerngelegenheiten determinieren, welche wiederum erheblichen Einfluss auf die sprachlichen Fähigkeiten in den jeweiligen Einzelsprachen haben.

\subsection{Bilinguale (Sprach-)Kompetenzen}

Die Problematik des linguistischen Kompetenzbegriffs sowie dessen fragliche Eignung zur Definition von Bilingualität wurden bereits in Abschnitt 2.3 respektive 3.1 diskutiert. Nichtsdestotrotz weisen bilinguale Sprecher charakteristische Kompetenzprofile in ihren jeweiligen Sprachen sowie relevante Kompetenzunterschiede im Vergleich zu monolingualen Sprechern auf, die es näher zu betrachten gilt. Zudem scheint Bilingualität - zumindest unter bestimmten Bedingungen - zu positiven Konsequenzen non-verbaler kognitiver Fähigkeiten führen zu können (vgl. z. B. Bialystok, 2017; Hamers \& Blanc, 2000, S. 85ff; Nicoladis, 2016; van Dijk et al., 2018). Einführend soll aber zunächst ein kurzer Blick auf die Geschichte der Bilingualismusforschung zu sprachlichen und kognitiven Kompetenzen bilingualer Sprecher geworfen werden, um die 
heterogene und teils widersprüchliche empirische Befundlage besser einordnen zu können.

\subsubsection{Historischer Abriss der Bilingualismusforschung zu sprachlichen und kognitiven Kompetenzen von bilingualen Sprechern}

Historisch vereinfacht betrachtet lässt sich die Einschätzung der Konsequenzen von Bilingualismus auf (meta-)linguistische und (meta-)kognitive Kompetenzen innerhalb der nun fast über 100 Jahre bestehenden Bilingualismusforschung folgendermaßen zusammenfassen: $\mathrm{Zu}$ Beginn wurden Bilingualismus primär negative Konsequenzen zugesprochen. Ab den 1960er Jahren bis zur Jahrtausendwende hingegen wurden vermehrt positive Konsequenzen von $\mathrm{Bi}$ lingualität in empirischen Studien identifiziert (vgl. Hamers \& Blanc, 2000, S. $85 \mathrm{ff}$ ). Aktuell zeichnet sich die Debatte durch eine gemischte, teils widersprüchliche Befundlage aus, die die Bedeutung der kontextuellen Bedingungen für die Entwicklung von Bilingualität akzentuiert.

Studien zu Beginn der Bilingualismusforschung, die Bilingualität als mentales und linguistisches Handicap diagnostizierten (für einen Überblick siehe z. B. Darcy, 1953; Peal \& Lambert, 1962), wiesen methodische Mängel wie fehlende Kontrolle des sozioökonomischen Status und Testungen in der nicht dominanten Sprache auf, die die negative Befundlage zum Teil erklären können (vgl. Hamers \& Blanc, 2000, S. 86). Erst mit der Studie von Peal \& Lambert (1962) zum Zusammenhang von Intelligenz und Bilingualität, die als Meilenstein der Bilingualismusforschung gilt, wurden die eben angeführten methodischen Mängel berücksichtigt und folglich auch andere Ergebnisse erzielt. Zwar erwarteten Peal \& Lambert (ebd.) in Übereinstimmung mit der bisherigen Empirie, dass monolinguale Sprecher bilingualen Sprechern bei verbalen Aufgaben überlegen sind und es keine Unterschiede zwischen den Gruppen bei non-verbalen Aufgaben gibt (vgl. Bialystok, 2013), allerdings wurden die Autoren von den Ergebnissen überrascht: Bilinguale Sprecher wiesen nun im Vergleich zu monolingualen Probanden bei fast allen verbalen und non-verbalen Aufgaben signifikant höhere Werte sowie eine differenziertere Intelligenzstruktur auf. Diese Studie und ihre der bisherigen Empirie widersprechenden Befunde löste ein reges wissenschaftliches Interesse an der Bilingualismusforschung auf, was die Quantität der Untersuchungen zum Bilingualismus anstiegen ließ.

Die Empirie der folgenden Dekaden wies vermehrt auf positive Folgen der Bilingualität hin, auch wenn vereinzelte Studien keine Unterschiede zwischen mono- und bilingualen Sprechern fanden und andere weiterhin negative Effekte der Bilingualität attestierten (vgl. Hamers \& Blanc, 2000, S. 92f). Hierbei 
kristallisierte sich bereits langsam heraus, dass die positiven Folgen der Bilingualität von günstigen, förderlichen Bedingungen abhängig sind, die beide Sprachen wertschätzen und diesen Möglichkeiten zur Entwicklung wie z. B. durch bilinguale Bildungsarrangements bieten. Die widersprüchliche empirische Befundlage lässt sich zu einem gewissen Teil mit der Beobachtung erklären, dass fast alle Studien mit negativen Outcomes zu den Effekten von Bilingualität in westlichen Kulturen mit Kindern von Minderheiten durchgeführt, die in der Mehrheitssprache unterrichtet wurden (vgl. ebd., S. 93). Dementsprechend liegt der Schluss nahe, dass die migrationsbedingte Bilingualität in Kontexten mit traditionell einsprachigem Selbstverständnis wie z. B. in Deutschland mit ungünstigeren Bedingungen zur Entwicklung bilingualer Kompetenzprofile assoziiert ist als in Kontexten mit bilingualem Selbstverständnis wie z. B. in Kanada. Nichtsdestotrotz scheinen in den Dekaden nach der Studie von Peal und Lambert (1962) die positiven Folgen von Bilingualität im Allgemeinen wohl etwas überschätzt worden zu sein.

Aktuell zeichnet sich die Empirie der Bilingualismusforschung hinsichtlich der Konsequenzen von Bilingualität für (meta-)linguistische und (meta-)kognitive Kompetenzen weiterhin durch eine uneinheitliche, kontroverse Befundlage aus, die abhängig von den untersuchten Fähigkeiten positive sowie negative Effekte und Nullresultate identifiziert (vgl. z. B. Altarriba \& Isurin, 2012; Bialystok, 2017; Bhatia \& Ritchie, 2013; Kempert et al., 2016; Lehtonen et al., 2018; Nicoladis, 2016). Hierbei werden auch bisher angenommene positive Effekte von Bilingualität durch aktuelle empirische Evidenz zunehmend relativiert, wofür die Debatte bezüglich des Einflusses von Bilingualität auf exekutive Funktionen ein Paradebeispiel darstellt (vgl. Bialystok, 2017; Lehtonen et al., 2018).

\subsection{2 (Meta-)Linguistische Kompetenzen Bilingualer}

Die im Abschnitt 3.2.2 erwähnten unterschiedlichen Erwerbsbedingungen und Lernkontexte des 2L1a und des L2a im Gegensatz zum L1a wirken sich auf die in der Entwicklung befindlichen Sprachkompetenzen bilingualer Sprecher aus. Die spezifische Erwerbsituation von bilingual aufwachsenden Sprechern führt nicht nur zu Unterschieden zu monolingualen Sprechern, sondern auch zu spezifischen Kompetenzprofilen hinsichtlich ihrer Einzelsprachen, welche im Folgenden näher erläutert werden.

\subsubsection{Sprachkompetenzprofile Bilingualer}

Eine prominente Kategorisierungen von bilingualen Sprechern hinsichtlich ihrer Sprachkompetenz stellt die Unterscheidungen in ,balanced/dominant bilin- 
guals" (vgl. Butler, 2013, S. 112) dar, die das Verhältnis der Kompetenzniveaus in den jeweiligen Sprachen akzentuiert. Bilinguale Sprecher, die vergleichbare Fähigkeitsniveaus in ihren Sprachen aufweisen, werden als ,balanced bilinguals" bezeichnet. Sind die Fähigkeiten in einer Sprache stärker ausgeprägt als in der anderen, wird die stärker ausgeprägte Sprache als „,dominante" Sprache des Sprechers kategorisiert oder von einer „Asymmetrie“ der Sprachkompetenz beider Sprachen gesprochen. Solche „profile effects“ werden definiert als ,differences in children's performance in distinct areas of the language they are learning or on tests measuring different aspects of knowledge" (Gathercole, 2016, S. 128). Bilinguale Sprecher mit balanciertem Kompetenzprofil stellen allerdings eher die Ausnahme als die Regel dar (vgl. Tracy, 2014, S. 19), sodass ,profile effects“ ein typisches Merkmal bilingualer Sprachkompetenz ist.

Die Unterscheidung balancierter oder asymmetrischer bilingualer Kompetenzprofile stellt durch ihre Dichotomie eine äußerst grobkörnige Kategorisierung dar. Hierbei werden die sprachlichen Fähigkeiten in den Einzelsprachen ganzheitlich betrachtet, anstatt feinkörnige Operationalisierungen hinsichtlich einzelner grammatischer Teilsysteme, sprachlicher Wissenssysteme oder Varietäten vorzunehmen. Dabei können allerdings einzelne Teilaspekte der dominanten Sprache geringer ausgeprägt sein als in der ganzheitlich als schwächer deklarierten Sprache. Die Bestimmung des Sprachkompetenzprofils ist methodisch stets auf sprachvergleichende Analysen der Kompetenzen in den jeweiligen Einzelsprachen von Bilingualen angewiesen, was mit diversen methodischen Herausforderungen verbunden ist. So können sich Sprachen z. B. auf übergeordneter Ebene hinsichtlich ihrer Typologie (z. B. fusionale Sprachen wie das Deutsche und agglutinierende Sprachen wie das Türkische), aber auch bezüglich einzelner sprachlicher Strukturen innerhalb eines Sprachtyps (z. B. Pluralflexion des Deutschen und des Englischen) deutlich unterscheiden. Aufgrund solcher distinkter Eigenschaften von Sprachen, insbesondere hinsichtlich der Transparenz und Regelhaftigkeit von Sprachen sowie der damit potentiell einhergehenden Operationalisierung der zu untersuchenden Phänomene, können sprachvergleichende Untersuchungen der Sprachkompetenzprofile bilingualer Sprecher ein äußerst schwierige Unterfangen darstellen. Aufgrund dessen werden Asymmetrien häufig über robustere, komparablere Proxys wie z. B. über den rezeptiven oder produktiven Wortschatz oder über die Selbsteinschätzung sprachlicher Fähigkeiten operationalisiert. Die Ergebnisse solcher Operationalisierungen von Sprachkompetenzen gilt es allerdings sorgfältig zu reflektieren und einzuschätzen, da z. B. aufgrund der potentiellen Komplementarität des mentalen Lexikons bezüglich bestimmter Wortfelder oder der fehlenden Objektivität bei subjektiven Einschätzungen der Sprachkompetenz die resultierenden Ergebnisse verzerrt oder nicht valide sein können.

Die asymmetrischen Kompetenzprofile können als Folge des ,,complementary principles“ (Grosjean, 2010, S. 29ff) interpretiert werden, in denen die 
jeweiligen Einzelsprachen bilingualer Sprecher in distinkten Kontexten Verwendung finden:, ,[...] they may overlap to some extent, but there are likely to be some contexts that favor one or the other language" (Gathercole, 2016, S. 128). Die Relevanz und der Gebrauch der einzelnen Sprachen in bestimmten Kontexten ist Veränderungen unterlegen (vgl. Gathercole, 2016, S. 126; Unsworth, 2016, S. 105) und somit dynamisch. Die jeweiligen Kontexte bedingen die Quantität und Qualität des Inputs, Outputs sowie der expliziten Instruktion in den Erwerbs- und Lerngelegenheiten bilingualer Sprecher in ihren jeweiligen Einzelsprachen und dementsprechend auch ihre sprachlichen Fähigkeiten.

Es wird angenommen, dass die Quantität des In- und Outputs der Einzelsprachen bei bilingualen Sprechern im Vergleich zu monolingualen Sprechern insgesamt geringer ist (vgl. Unsworth, 2016, S. 103f). Auch hinsichtlich qualitativer Aspekte des In- und Outputs sind Unterschiede zwischen bilingualen und monolingualen Sprechern zu erwarten. Die distinkten Kontexte, in denen von den einzelnen Sprachen Gebrauch gemacht wird, unterscheiden sich in ihren Themen, Interaktionsformen, Varietäten, Registern, Medien etc. und somit in ihren grammatischen, lexikalischen sowie stilistischen Anforderungen. Diese qualitativen Unterschiede der Kontexte können den Erwerb bestimmter sprachlicher Fähigkeiten determinieren, wenn z. B. ein Zweitsprachlerner lediglich in seiner L2 im schulischen Kontext mit einer dekontextualisierten, kognitiv akademischen Sprachvarietät samt expliziter Instruktion zum Schriftspracherwerb konfrontiert wird. Da sprachliches Wissen auch aus idiosynkratrischen Anteilen besteht, denen man in sinnvollen Kontexten begegnen muss, um diese zu erwerben, können sich die quantitativen und qualitativen Merkmale der Erwerbs- und Lerngelegenheiten insbesondere auf diese arbiträren, nicht regelgeleiteten Formen von sprachlichem Wissen und Fähigkeiten in den Kompetenzprofilen von Bilingualen auswirken.

Angesichts dessen erweisen sich Metaphern über bilinguale Kompetenzprofile als Summe zweier monolingualer Sprecher oder gar aus einer Defizitperspektive als „doppelt halbsprachig“ (vgl. Wiese et al., 2010) als unangemessen. Bilinguale Sprecher weisen spezifische Kompetenzprofile abhängig von ihren Lebensumständen auf, die sich vielmehr als arbeitsteilige Kooperation der Sprachen beschreiben lässt (Tracy, 2014, S. 18). Zudem können sich die Kompetenzprofile abhängig von der Lebenssituation und den damit einhergehenden Anforderungen in den jeweiligen Einzelsprachen im Verlauf verändern. Dementsprechend müssen asymmetrische Sprachkompetenzprofile beim Vergleich von bilingualen und monolingualen Sprechern hinsichtlich ihrer Sprachkompetenz berücksichtigt werden.

\subsubsection{Linguistische Kompetenzen bilingualer Sprecher}

Die Robustheit sowie die quantitative und qualitative Ähnlichkeit der Entwicklungsschritte im L1a und 2L1a sowie des frühen L2a lassen vermuten, dass 
sich hinsichtlich regelhafter, basaler, früh erworbener grammatischer Fähigkeiten wie Syntaxstrukturen, Verbalsyntax und -flexion bilinguale und monolinguale Sprecher kaum unterscheiden (vgl. Grimm \& Schulz, 2016; Rothweiler, 2007; Tracy, 2008; Schulz \& Grimm, 2012). Differenzen in diesen Fähigkeitsdomänen sind temporärer Natur, die in Abhängigkeit von dem Erwerbszeitraum stehen und sich im weiteren Erwerbsverlauf noch vor der Einschulung nivellieren.

Diverse Studien (für einen kurzen Überblick siehe Unsworth, 2016) weisen allerdings auf einen Zusammenhang von der Quantität des Inputs und komplexeren sprachlichen Strukturen auf, die sich durch zum Teil intransparente Form-Funktions-Beziehungen und einen gewissen Anteil an Idiosynkrasie auszeichnen. So gilt es z. B. im Deutschen bei der Bildung und Verarbeitung des Kasus den Valenzrahmen des Verbs als auch die korrekte Wahl und Flexion des Genus zu berücksichtigen (z. B. Donald drückt den Knopf (mask.) / die Stimmung (fem.) / das Händchen (neutr.) (Akkusativ); Horst hilft dem Populisten (mask.) / der Populistin (fem.) / dem Kaninchen (neutr.) (Dativ)), die alle keine vollkommen regelgeleiteten linguistischen Phänomene darstellen (vgl. Wegener, 1995). Dementsprechend verwundert es nicht, dass sich der 2L1a und der frühe L2a des Deutschen bezüglich des Kasuserwerbs ähneln und vom L1a bis ins Grundschulalter abweichen (vgl. Grimm \& Schulz, 2016), da beide bilinguale Spracherwerbstypen im Vergleich zum L1a eine geringere Quantität deutschsprachigen In- und Outputs aufweisen.

Der Zusammenhang von Input und Erwerb lexikalischer sowie morphosyntaktischer Strukturen scheint hierbei allerdings nicht linear zu sein, sondern abhängig von einem kritischen Schwellenwert, den es zu überschreiten gilt (vgl. Gathercole, 2016, S. 130; Unsworth, 2016, S. 111). So demonstriert z. B. Thordardottir (2015), dass ab einem Schwellenwert von ca. 50\% Input, bilinguale Sprecher sich hinsichtlich morphosyntaktischer Phänomene nicht von monolingualen Sprechern unterscheiden. Für den Erwerb besonders komplexer Phänomene wie z. B. des Pluralsystems des Walisischen scheint selbst ein Erwerbsbeginn mit der Geburt nicht zwingend ausreichend zu sein, sodass solche Phänomene eine höhere Abhängigkeit zur Quantität des Inputs aufweisen (vgl. Gathercole \& Thomas, 2009; Thomas et al., 2014) bzw. explizite, metalinguistische Reflexion benötigen könnten (vgl. Eichler \& Nold, 2008, S. 67).

Hinsichtlich lexikalischer Kompetenzen gilt als empirisch robust abgesicherter Befund, dass bilinguale verglichen mit monolingualen Sprechern über einen kleineren Wortschatz in ihren jeweiligen Sprachen verfügen (vgl. z. B. Bialystok, 2009; Kempert et al., 2016; Limbird et al., 2014; Oller \& Eilers, 2002). Aggregiert man allerdings das lexikalische Wissen der beiden Sprachen bilingualer Sprecher, verfügen diese über einen vergleichbaren Wortschatzumfang wie monolinguale Sprecher. Weiterhin weisen bilinguale Sprecher einen langsameren Zugriff auf lexikalische Einheiten im mentalen Lexikon auf, was zu einer verlangsamten Bildbenennung, höherem Auftreten von „Tip of the 
Tongue"-Erfahrungen sowie Interferenzen bei lexikalischen Entscheidungen führt (für einen Überblick siehe Bialystok, 2009, S. 55f; Kempert et al., 2016, S. 196ff). Der lexikalische Zugriff ist allerdings abhängig von der Frequenz des selektierten Wortes, sodass der Abruf von z. B. Namen oder von homonymen Übersetzungsäquivalenten (z. B. „tulpe“ (Türkisch) und „Tulpe“ (Deutsch)) bei bilingualen Sprecher ähnlich schnell wie bei monolingualen Individuen erfolgen kann (vgl. Bartolotti \& Marian, 2013, S. 21f).

Die geringere Ausprägung der lexikalischen Repertoires in den Einzelsprachen sowie der langsamere lexikalische Abruf von bilingualen im Vergleich zu monolingualen Sprechern lassen sich ebenfalls auf die reduzierte Quantität und Kontextspezifizität der Lerngelegenheiten in den jeweiligen Einzelsprachen zurückführen. Einzelsprachenspezifische Kontexte verringern die Frequenz von lexikalischen Items, da unterschiedliche linguistische Items (z. B. „Baum“ (Deutsch) und „ağaç“ (Türkisch)) zur Bezeichnung derselben Entität genutzt werden. Überzeugende Evidenz für den Einfluss der Kontextspezifizität der Lerngelegenheiten auf den Wortschatzerwerb liefern Bialystok et al. (2010), die die Items eines Wortschatztests in die lexikalischen Felder „home“ und ,school words“" separiert haben. Die Ergebnisse wiesen größere Unterschiede bei „home“ als bei „school words“ zwischen mono- und bilingualen SuS auf. Dementsprechend scheinen bilinguale Sprecher im familiären Kontext mehr Input in der (weiteren) $\mathrm{L}_{1}{ }^{4}$ zu erhalten, was sich mit anderen Ergebnissen aus Untersuchungen zur familiären Sprachwahl bilingualer Familien in Deutschland deckt (vgl. Reich, 2009). Im Gegensatz hierzu scheint aber die Quantität des Inputs in der Schule auszureichen, um einen fast vergleichbaren Wortschatzumfang wie monolinguale Sprecher in diesem semantischen Feld zu erwerben.

Solche kontextsensiblen Untersuchungen lexikalischer Fähigkeiten schränken den Allgemeinheitsanspruch des Befunds ein, dass bilinguale Sprecher über kleinere Wortschatzumfänge in ihren jeweiligen Sprachen verglichen mit monolingualen Sprechern verfügen. Parallel zum Einfluss der Quantität des Inputs auf morphosyntaktische Strukturen scheinen nicht-lineare Wachstumsmodelle mit zu übersteigendem Schwellernwert ebenfalls für lexikalisches Wissen von Bilingualen und Monolingualen zuzutreffen. So identifizierte Thordardottir (2011) einen notwendigen Anteil von 40\% und 60\% einzelsprachlichen Inputs für vergleichbare Wortschatzumfänge von mono- und bi-

4 Die Formulierung ,(weitere) L1“ wird im Folgenden verwendet, wenn in der Aussage keine Unterscheidung zwischen dem 2L1a und dem L2a gemacht wird. Die fehlende Unterscheidung kann zum einen daran liegen, dass kein Unterschied hinsichtlich des fokussierten Phänomens zwischen dem 2L1a und dem L2a besteht. Zum anderen kann die fehlende Unterscheidung aber auch durch fehlende Informationen der zitierten Studie oder Aussage resultieren. Alternativ wird auch die Formulierung ,nicht-verkehrssprachliche L1 “verwendet, um die Bezugnahme auf die weitere Sprache neben der Verkehrssprache innerhalb eines einsprachigen Kontexts zu akzentuieren. 
lingualen Sprechern hinsichtlich des rezeptiven respektive produktiven Wortschatzes. Diese Ergebnisse konnten für jüngere Kinder von Cattani et al. (2014) und Hoff et al. (2012) repliziert werden (vgl. Unsworth, 2016, S. 110).

Die Quantität des sprachlichen Inputs trägt dementsprechend entscheidend zum Wortschatzerwerb bilingualer Sprecher bei. Allerdings spielen hierbei auch qualitative Aspekte eine nicht zu unterschätzende Rolle. So gibt es Evidenz für die Förderlichkeit der Anzahl von Interaktionspartnern in einer Einzelsprache (Place \& Hoff, 2011) sowie für die Überlegenheit von muttersprachlichen gegenüber nicht-muttersprachlichen Inputs (Driessen, van der Slik \& de Bot, 2002; Place \& Hoff, 2011) hinsichtlich des Wortschatzumfangs in den Einzelsprachen (vgl. Unsworth, 2016, S. 106f). Im Bildungskontext stellt die Frequenz der Peer-Interaktion einen besseren Prädiktor für den Wortschatzerwerb in der Verkehrssprache dar als die Interaktion mit Lehrern (vgl. Palermo et al., 2014). Allerdings wirkt sich die Komplexitätsrate des Inputs operationalisiert als Anteil hypotaktischer Strukturen der Gesamtzahl der Äußerungen unter Kontrolle des Gesamtinputs - von pädagogischen Fachkräften in bilingualen Kindertagesstätten positiv auf den expressiven Wortschatz aus (vgl. Gámez \& Levine, 2013).

Aber nicht nur der Input, sondern auch der Output, also die Produktion von einzelsprachlichen Äußerungen von bilingualen Sprechern, ist relevant für den Erwerb und Ausbau linguistischer Kompetenzen, was in den letzten Jahren vermehrt in Untersuchungen fokussiert wurde (vgl. Unsworth, 2016, S. 108). So weisen z. B. Bohrnan et al. (2010) und Unsworth (2015) auf positive Effekte der Quantität des Outputs auf die Lexik und den Morphosyntaxerwerb bei bilingualen Sprechern hin. Bereits 1985 stellte Swain die Hypothese auf, dass die Produktion von Output insbesondere für den L2a von besonderer Relevanz sein könnte. Im Gegensatz zum Input führt die Produktion von Strukturen in der L2 zu einer intensiveren, tieferen sowie mental fordernden Verarbeitung. Zudem bieten Produktionen Gelegenheiten, linguistische Wissenslücken zu entdecken und die Hypothesen von Lernern über grammatische Strukturen zugänglich zu machen sowie zu testen (vgl. Swain, 2000, S. 99f). Es handelt sich hierbei folglich um Prozesse, in denen die sprachlichen Fähigkeiten bewusst betrachtet werden können. Wenn unterspezifizierte Fähigkeiten oder sprachliche Wissenslücken explizit werden, können Lerner diesen problemorientiert zum Beispiel durch Rückgriff auf Materialien (z. B. Regel- bzw. Grammatikwerke, Wörterbücher etc.), durch hilfesuchendes Verhalten (Peers, Lehrer etc. fragen) oder Üben begegnen und schließlich lösen. Dementsprechend können Sprachproduktionen zu potentiellen Lerngelegenheiten führen, wenn diese einen Anlass zur Reflexion sprachlicher Strukturen bieten, in denen Sprache losgelöst von ihrem impliziten Charakter zu einem Gegenstand expliziter Betrachtung transformiert wird.

Neben dem In- und Output beeinflusst auch der sozioökonomische Status den Spracherwerb und linguistische Kompetenzen bilingual aufwachsender 
Sprecher: „The evidence for the influence of SES factors on acquisition of vocabulary and grammar is striking" (Gathercole, 2016, S. 135). So wirkt sich ein geringer sozioökonomischer Status negativ auf linguistische sowie grammatische Fähigkeiten - operationalisiert über Satzwiederholungsaufgaben (Chiat et al., 2013) von bilingualen Kindern aus (vgl. Gathercole, 2016, S. 134). Die Bedeutung des sozioökonomischen Status für sprachliche Fähigkeiten ist selbst noch im Erwachsenenalter nachweisbar (Stadthagen-González et al., 2013) und klärt als Prädiktor mit steigendem Alter bilingualer Sprecher mehr Varianz von Sprachkompetenz auf als die zu Hause gesprochene Sprache (vgl. Gathercole, 2016, S. 134f). Der sozioökonomische Status dürfte sich hierbei nicht direkt auf den Spracherwerb und die Sprachkompetenz auswirken, sondern könnte über die Quantität und Qualität des Inputs sowie über differente Interaktionsstile den Zusammenhang mediieren (vgl. Szagun, 2016, S. 182ff). Insbesondere Aktivitäten früher Literacyförderung, die auch metalinguistische Reflexionen beinhalten, dürften als bildungsorientierte Aktivitäten vom sozioökonomischen Status abhängig zu sein. Eichler und Nold (2008, S. 67) vermuten, dass späte linguistische Teilkompetenzen sowie bestimmte komplexe, eher konzeptionell schriftsprachliche Strukturen wie zum Beispiel hypotaktische Satzstrukturen, der Konjunktiv oder der Genitiv des Deutschen nur durch ein ausreichendes Maß an metalinguistischer Bewusstheit vollkommen durchschaut und somit produktiv angewandt werden können. Dementsprechend liegt die Vermutung nahe, dass insbesondere solche linguistische Strukturen stärker vom sozioökonomischen Status beeinflusst werden als basale kommunikative Kompetenzen.

Ein Großteil dieser beschriebenen Zusammenhänge der Lebenswelt bilingual aufwachsender Kinder mit den Kompetenzen in ihren jeweiligen Sprachen lassen sich auch empirisch für türkisch-deutschsprachig aufwachsende Kinder mit MH bestätigen. Türkisch-deutschsprachig aufwachsende Kinder in Deutschland weisen im Kindergarten altersentsprechende Kompetenzen im Türkischen auf (vgl. Reich, 2009, S. 68). Die Deutschkompetenzen fallen im Vergleich zu ihren Kompetenzen im Türkischen zu Beginn des Kindergartens trotz einer immensen interindividuellen Heterogenität deutlich geringer aus (vgl. Jeuk, 2003) und unterscheiden sich auch von den Fähigkeiten monolingual deutschsprachig aufwachsender Kinder (siehe z. B. Holler-Zittlau, Dux, \& Berger, 2004; Neumann et al., 2009; Schulz \& Tracy, 2011; Schulz \& Grimm, 2012; Grimm \& Schulz, 2016). Dies ist angesichts der kurzen Erwerbsdauer und Kontaktzeit zum Deutschen im ersten Jahr des Kindergartens nicht verwunderlich. Werden Sprachstandsuntersuchungen im Kindergarten mit vierjährigen Kindern mit frühem L2a durchgeführt, dürfte ein Großteil der dieser Kinder bisher lediglich zwölf Monate regelmäßige Kontaktzeit zum Deutschen vorzuweisen haben. Gemäß typischer Verläufe des L2a kann ein Großteil der frühen Zweitsprachlerner nach zwölf Monaten Kontaktzeit mit 
der L2 Deutsch zwar bereits korrekt flektierte Hauptsätze mit Verbzweitstellung, aber keine Nebensätze produzieren, während die Nebensatzproduktion bei fast allen vierjährigen monolingual deutschsprachig aufwachsenden Kindern mit 48 Monaten Kontaktzeit erworben sein dürfte (vgl. Tracy, 2008, S. 86ff). Die Sprachkompetenzen im Deutschen von monolingualen Kindern stellen in diesem Alter keine adäquate Vergleichsbasis für Kinder mit L2a des Deutschen dar, da dies zu einer systematischen Unterschätzung von sich absolut typisch und unauffällig entwickelnden sprachlichen Fähigkeiten und somit zu Fehldiagnosen führen würde (vgl. z. B. Jeuk, 2009; Lüdtke \& Kallmeyer, 2007a; Reitenbach, Schastak \& Rauch, 2018).

Im Verlauf der Grundschule holen die Sprachkompetenzen im Deutschen von türkisch-deutschsprachig aufwachsenden bilingualen Kindern auf und übersteigen im Verlauf der Beschulung die türkischsprachigen Kompetenzen (für einen Überblick siehe Reich, 2009, S. 76). Da in der deutschen Regelschule die Kommunikations- und Unterrichtssprache exklusiv monolingual Deutsch ist (Gogolin, 1994), erfahren bilingual aufwachsende Kinder in der Schule eine immense Steigerung der Quantität und Qualität der Lerngelegenheiten für das Deutsche. Durch den Schriftspracherwerb sowie den vielfältigen Lerngelegenheiten, in denen die SuS mit einer Vielzahl unterschiedlicher Themen und Phänomenen konfrontiert werden, die zum Teil über ihre Alltagserfahrungen hinausgehen, liefert der Unterricht eine Bandbreite von Möglichkeiten zur Rezeption und Produktion deutschsprachigen Inputs. Dementsprechend verwundert es nicht, dass der Schulunterricht eine exponentielle Vergrößerung des Wortschatzumfangs anstößt (vgl. Clark, 1993; Karasu, 1995) und sich grammatische Strukturen wie Passiv- und Relativsatzkonstruktionen (weiter)entwickeln (vgl. Reich, 2009, S. 74), die eher in konzeptionell schriftsprachlichen Varietäten produziert werden (vgl. Koch \& Oesterreicher, 1985; Weinert, 2004). Zwar beansprucht die Gruppe der türkisch-deutschsprachigen bilingualen SuS im Vergleich zu anderen Gruppen intensiv den herkunftssprachlichen Unterricht (HSU) (vgl. Reich \& Hienz-De Albentiis, 1998), allerdings können sich die schriftsprachlichen Fähigkeiten in diesem Kontext nicht wie im Deutschen oder in koordinierten Alphabetisierungen entwickeln. Dementsprechend verwundert es nicht, dass türkisch-deutschsprachig aufwachsende SuS zum Ende der Grundschulzeit signifikant bessere schriftsprachliche Leistungen im Deutschen als im Türkischen erbringen (vgl. Schwippert \& Klinger, 2008).

Dementsprechend kann die Sprachkompetenz von türkisch-deutschsprachig bilingual aufwachsenden Kindern als eine sich kreuzende Entwicklung zusammengefasst werden, bei welcher eine initiale Dominanz zu Gunsten des Türkischen im weiteren Verlauf durch eine Dominanz zu Gunsten des Deutschen abgelöst wird. Diese Asymmetrie verfestigt sich in der Regel im weiteren Verlauf der schulischen, universitären und/oder beruflichen Laufbahn (vgl. Reich, 2009, S. 84f). 
Zusammengefasst sind linguistische Kompetenzen in den Einzelsprachen bilingualer Sprecher abhängig von der Quantität und Qualität des Inputs, den Möglichkeiten zur Sprachproduktion, der Anzahl sowie linguistischen Kompetenz der Interaktionspartner und dem sozioökonomischen Status, wobei dieser wohl eher über eine Vielzahl bildungsförderlicher familiärer Praktiken mediiert wird. Hierbei gilt es auf Basis des „,complementary principle“(vgl. Grosjean, 2010, S. 29ff) die unterschiedlichen Lebensrealitäten bilingual und monolingual aufwachsender Sprecher zu berücksichtigen, die zu einer geringeren Quantität einzelsprachlichen Inputs und in vielen Fällen zu komplementär verteilten Lerngelegenheiten der Einzelsprachen bilingualer Sprecher führen. Diese reduzierte Quantität des einzelsprachlichen Inputs beeinflusst vor allem die Entwicklung des mentalen Lexikons und den Erwerb komplexer, intransparenter, idiosynkratrischer grammatischer Strukturen. Allerdings eröffnen die Befunde zu potentiellen kritischen Schwellenwerten der Quantität des Inputs für vergleichbare einzelsprachliche lexikalische sowie morphosyntaktische Fähigkeiten von bilingual und monolingual aufwachsenden SuS (für einen Überblick siehe Gathercole, 2016, S. 130; Unsworth, 2016, S. 110f) diverse didaktische Perspektiven, um mit angemessenen Lernumgebungen dem einzelsprachlich reduzierten Input von bilingual aufwachsenden SuS zu begegnen.

Diese Befunde sind insbesondere für Bildungsprozesse in der Grundschule relevant, da Sprachkompetenz eine zentrale Determinante zur Teilhabe am schulischen Unterricht sowie zum Erwerb diverser schulischer Grundfähigkeiten darstellt. Gerade bildungssprachliche Varietäten zeichnen sich durch spezifisches lexikalisches Wissen aus und machen Gebrauch von grammatischen Strukturen wie z. B. Passivkonstruktionen sowie langen Nominal- und Präpositionalphrasen (Heppt et al., 2010, S. 140), welche von monolingual aufwachsenden SuS zum Teil auch schon zu Beginn der Grundschule produziert werden können. Bilingual aufwachsende SuS mit 2L1a oder L2a benötigen hierfür etwas mehr Zeit, erwerben diese grammatischen Strukturen allerdings im weiteren Verlauf der Grundschule (vgl. Schulz \& Grimm, 2012; Grimm \& Schulz, 2016). Linguistisches Wissen über Unterschiede von Spracherwerbstypen stellt eine zentrale Voraussetzung für eine adäquate Einschätzung der sprachlichen Fähigkeiten und den damit einhergehenden Leistungsbewertungen monolingual und bilingual aufwachsender SuS in der Grundschule dar. Bilingual aufwachsende SuS dürfen diesbezüglich nicht mit monolingual aufwachsenden SuS verglichen werden, da ein solcher Vergleich eventuell zu einer defizitären, devianten Einschätzung der typischen Sprachentwicklung des 2L1a oder L2a führen würde. Vielmehr gilt es diese Verzögerungen und Unterschiede als typische Folgen des 2L1a und L2a zu interpretieren und den distinkten sprachlichen Fähigkeiten adaptiv mit geeigneten didaktischen Konzepten zu begegnen. 
Neben linguistischen Kompetenzen stellen metalinguistische Kompetenzen und Literacy weitere zentrale Facetten der Sprachkompetenz dar, die insbesondere hinsichtlich Bildungsprozessen eine hohe Relevanz aufweisen. Diese Fähigkeiten sollen im Folgenden für die Population bilingual aufwachsender Sprecher näher betrachtet werden.

\subsubsection{Metalinguistische Kompetenzen bilingualer Sprecher}

Nach der Internalisierung von Sprache als Kommunikationswerkzeug kann sich Sprache in seiner Funktion als ,object of cognitive processing“ zur bewussten Analyse und Kontrolle des Sprachgebrauchs entwickeln (Hamers \& Blanc, 2000, S. 124). Während metalinguistische Kompetenzen Sprache selbst zum Gegenstand von Analyse und Reflexion machen, transformiert Literacy sprachliche Fähigkeiten zu Werkzeugen gesellschaftlicher Teilhabe und sozialer Selbstbestimmung (vgl. Brandenburg, 2006, S. 13). Sprache wird in diesem Prozess immer weiter dekontextualisiert und mehr in seiner Eigenschaft als symbolisches Repräsentationssystem internalisiert sowie realisiert. Paraverbale Informationen, die durch den Kontext, die Situation oder Erfahrung in der Kommunikation typischerweise präsent sind, treten bei diesen Facetten des Sprachgebrauchs in den Hintergrund, sodass die auszudrückenden Inhalte nur mit linguistischen Mitteln repräsentiert werden.

Der zentrale Unterschied von bilingualen und monolingualen Sprechern hinsichtlich ihrer Bedingungen zur Entwicklung metalinguistischer Fähigkeiten stellt das Aufwachsen in einer zwei- respektive einsprachigen Umwelt dar. Das Vorhandensein von zwei Sprachen in der Lebenswelt von bilingualen Sprechern und der Erwerb dieser sensibilisiert sie bereits in einem sehr frühen Alter für die Erkenntnis, dass die Form sprachlicher Elemente selten in Beziehung zu ihrer Bedeutung und Funktion stehen. So werden in der zweisprachigen Lebenswelt von Beginn an eine große Anzahl alltäglicher Entitäten durch unterschiedliche linguistische Formen repräsentiert (z. B. „Katze“ (Deutsch) und ,kedi“ (Türkisch) als Oberbegriff für lebendige, nicht menschliche, vierbeinige, miauende, Schnurhaare tragende, grazile Säugetiere), sodass die Existenz verschiedener linguistische Formen für dieselben Entitäten quasi die Standardannahme repräsentiert. Fälle von intransparenten Form-Funktions-Zusammenhängen treten ebenfalls in einsprachigen Kontexten auf. So können unter anderem Synonyme (z. B. „Karotte“ und „Möhre“), Äquivokationen (z. B. „Ball“ als kugelförmiges Spielgerät oder Tanzfestivität; „Bank“ als Sitzgelegenheit oder Geldinstitut) oder Allomorphe einer Sprache - Morpheme mit der gleichen Funktion, aber verschiedenen Formen (z. B. Pluralbildung mit „-s", „-er", ,-en“ etc.) - auch in einsprachigen Kontexten metalinguistische Reflexionen initiieren. Allerdings scheinen intransparente Form-Funktions-Beziehungen eine höhere Prävalenz und vor allem Salienz in zweisprachigen als in 
einsprachigen Lebenswelten aufzuweisen. Zudem verfügen bilingual aufwachsende Sprecher über die Möglichkeit, ihre Sprachen krosslinguistisch miteinander zu vergleichen. Während einsprachig aufwachsende Individuen hierfür lediglich auf dialektale Formen ihrer Sprache oder mit steigendem Alter auf gelernte Fremdsprachsprachen zurückgreifen können, steht bilingualen Sprechern hierfür ein weiteres sprachliches System bereits sehr früh in ihrer Entwicklung zur Verfügung. Insbesondere Sprachen unterschiedlichen Sprachtypus bieten durch ihre mannigfaltigen und auffälligen Kontraste viele Vergleichsmöglichkeiten und -anlässe im Alltag, die in einsprachigen Kontexten deutlich limitierter sind.

Bialystok (2013) fasst in ihrem Überblick die bisherige Forschung zu Unterschieden von monolingualen und bilingualen Kindern hinsichtlich der metalinguistischen Fähigkeiten Wortkonzept, syntaktischer und phonologischer Bewusstheit zusammen. Bilinguale weisen die deutlichsten metalinguistischen Vorteile in Bezug auf das Wortkonzept in fusionalen Sprachen auf. Dieses zeichnet sich vor allem durch zwei Einsichten aus, und zwar dass der kontinuierlich wahrgenommene Sprachstrom in einzelne Wörter segmentiert werden kann und dass Wörter lexikalische Arbitrarität aufweisen. So sind sich bilingual aufwachsende Kinder früher der lexikalischen Arbitrarität als monolingual aufwachsende Kinder bewusst (vgl. z. B. Bialystok, 1988; Cummins, 1978; Edwards \& Christophersen, 1988; Eviatar \& Ibrahim, 2000). Zudem scheinen sie über ein gefestigteres Verständnis lexikalischer Arbitrarität als monolinguale Kinder zu verfügen (vgl. Cummins, 1978). Dies führt auch zu einer höheren Akzeptanz von Homonymen, sobald bilinguale Sprecher über die ersten Übersetzungsäquivalente in ihren Sprachen verfügen (vgl. ByersHeinlein \& Werker, 2013), was nach Marinova-Todd (2012) zu Vorteilen beim Wortschatzerwerb führen kann (vgl. Nicoladis, 2016, S. 273f).

Bezüglich syntaktischer Bewusstheit, die häufig über grammatische Urteile wie die Beurteilung, Erklärung und Verbesserung (un-)grammatischer Sätze oder durch die Detektion von Ambiguitäten operationalisiert wird, lassen sich keine generellen, uniformen Vorteile von Bilingualität nachweisen (vgl. Bialystok, 2013, S. 630ff). Hierbei scheinen Aufgaben, die Konflikte von Form und Funktion und / oder semantische Distraktoren inkorporieren, von bilingualen besser als von monolingualen Kindern gelöst zu werden (vgl. Bialystok, 1986, 1988, Bialystok \& Majumder, 1998; Cromdal, 1999). Stärker analytisch orientierte Aufgaben zur syntaktischen Bewusstheit wie z. B. die Erklärung grammatischer Fehler oder Urteile über komplexe Phänomene scheinen hingegen eher von der Kompetenz in der jeweiligen Einzelsprache als vom Spracherwerbstyp abzuhängen (vgl. Gathercole, 1997; Gathercole \& Montes, 1997).

Phonologische Bewusstheit ermöglicht die Abstraktion von Bedeutungen bezüglich ihrer lautlichen Form und damit einhergehend einen expliziten $\mathrm{Zu}-$ griff auf phonetische sowie phonologische Strukturen und Informationen (vgl. 
Wagner \& Torgesen, 1987). Sie umfasst im engeren Sinne das Erkennen, Zählen und die Manipulation von Wörtern, Silben und Reimen und im weiteren Sinne das Erkennen und Zählen sowie die Synthese und Analyse von Phonemen (vgl. Noack \& Brändl, 2007, S. 312). Phonologische Bewusstheit besteht somit aus sprachenspezifischen und sprachenunabhängigen Wissenskomponenten. Sie kann als eine Teilkomponente der phonologischen Informationsverarbeitung interpretiert werden, welche als Nutzung von Informationen über die Lautstruktur bei der Auseinandersetzung mit gesprochener oder geschriebener Sprache definiert werden kann (vgl. Wagner \& Torgesen, 1987). Die phonologische Bewusstheit stellt aufgrund ihrer Reliabilität als Prädiktor für den Leseerwerb in Alphabetschriften eine der relevantesten metalinguistischen Fähigkeiten dar (vgl. Bialystok, 2013, S. 633). Ähnlich zur syntaktischen Bewusstheit lassen sich auch hier kaum positive Effekte von Bilingualität finden. Wenn Vorteile von Bilingualität auf die phonologische Bewusstheit nachgewiesen werden konnten, dann in der Regel nur in frühen, vorschulischen Phasen der Entwicklung bei Sprachpaaren mit einer gewissen phonologischen Ähnlichkeit und bei simplen Aufgaben (vgl. Bialystok, 2013, S. 633ff). Die Evidenz weist vielmehr darauf hin, dass sprachstrukturelle Bedingungen und Instruktion zur Literacyentwicklung einen höheren Anteil an Varianz von phonologischer Bewusstheit aufklären, sodass vorherige positive Effekte der Bilingualität neutralisiert werden (vgl. ebd.). Bilinguale Sprecher haben bezüglich der phonologischen Bewusstheit im Vergleich zu monolingulaen Sprechern allerdings den Vorteil, ihre phonologische Bewusstheit über ihre Einzelsprachen zu transferieren, selbst wenn es sich um unähnliche phonologische Systeme wie Chinesisch und Englisch handelt (vgl. Luk \& Bialystok, 2008).

Bialystok (2013, S. 642ff) fasst die gemischte Befundlage dahingehend zusammen, dass Bilingualität ,an inadequate account of the results“ darstellt, auch wenn bilingual aufwachsende Sprecher zumindest hinsichtlich des Wortkonzepts empirisch robuste Vorteile in der Entwicklung metalinguistischer Kompetenzen aufweisen. Bialystok (ebd.) akzentuiert den Einfluss weiterer Faktoren, die diese Effekte vermitteln, wobei sich die absolute Sprachkompetenz, die relative Balance zwischen den Fähigkeiten in den jeweiligen Einzelsprachen, die linguistische Ähnlichkeit der Einzelsprachen sowie die konkrete Aufgabenstellung als potentielle Mediatoren herauskristallisieren. Je höher und gleichmäßiger die Sprachkompetenzen in den Einzelsprachen ausgeprägt sind, die sich bestenfalls linguistisch strukturell ähneln, desto höher stehen die Chancen, dass die vorliegende Bilingualität ihr Potential zu positiven Effekten auf bestimmte metalinguistische Fähigkeiten entfalten kann. Eine aktuelle Studie von Bien-Miller et al. (2017) mit russisch- und türkisch-deutschsprachig aufwachsenden Grundschulkindern, die unter Nutzung eines Elizitationsverfahrens metalinguistische Äußerungen und Reflexionen hervorrufen sowie quantitativ und qualitativ auswerten, bestätigt diese Einschätzung der Befund- 
lage von Bialystok (2013). Sie weisen einen statistisch signifikanten Zusammenhang der Quantität metasprachlicher Äußerungen und Bilingualität nach, der auch unter Kontrolle von Intelligenz, Sprachkompetenz und Alter bestehen bleibt. Die Unterschiede zu der monolingualen Gruppe sind hierbei aber relativ gering, sodass weitere Faktoren, die unabhängig von Mehrsprachigkeit sind, bedeutsam für die Entwicklung metalinguistischer Fähigkeiten zu sein scheinen. Weiterhin demonstrieren sie einen Zusammenhang zwischen der Sprachkompetenz und quantitativen sowie qualitativen Ausprägungen von Sprachbewusstheit, allerdings in geringer Effektstärke und nur hinsichtlich der L2 Deutsch. Es konnte kein statistisch signifikanter Zusammenhang von metalinguistischen Fähigkeiten und den (weiteren) L1 Russisch oder Türkisch nachgewiesen werden, was die Autoren auf die Dominanz des Deutschen zurückführen. Besonders interessant an der Studienanlage von Bien-Miller et al. (2017) ist, dass bestimmte bilinguale Kinder auf Materialien wie Textvorlagen in ihrer (weiteren) L1 zurückgreifen konnten und andere nicht. Wenn bilingualen Kindern eine solche Möglichkeit vorliegt, greifen sie häufiger auf ihre (weitere) L1 für metalinguistische Reflexionen zurück, was auf die (weitere) L1 als potentielle Ressource für metalinguistische Reflexionen hinweist (vgl. ebd., S. 209).

Abhängig von der jeweiligen konkreten metalinguistischen Fähigkeit können diese auch als Teilkomponenten oder Vorläuferfertigkeiten von Literacy betrachtet werden. So sind basale Einsichten in (Schrift-)Sprache als abstraktes Symbolsystem, worunter auch metalinguistische Fähigkeiten wie z. B. das Wortkonzept oder phonologische Bewusstheit fallen, zentrale Vorläuferfertigkeiten für den Schriftspracherwerb und damit für die Entwicklung von Literacy (vgl. Brügelmann \& Brinkmann, 2001; Füssenich, 2012). Die Literacyforschung setzt allerdings andere Akzente als die Forschung zu metalinguistischer Kompetenz, sodass im Folgenden einige theoretische Reflexionen und empirische Befunde, die für die frühe Literacyentwicklung von bilingual aufwachsenden Kindern zentral sind, berichtet werden.

\subsubsection{Frühe Literacyentwicklung und Bilingualität}

Dekontextualisierte Sprache findet sich in ihrer prototypischen Form in geschriebenen Texten wider (vgl. Cummins, 2008, S. 79f, Hamers \& Blanc, 2000, S. 120), aber auch in mündlicher konzeptioneller Schriftlichkeit (vgl. Koch \& Oesterreicher, 1985) und bildungssprachlichen Varietäten (vgl. Gantefort \& Roth, 2010; Heppt et al., 2014). Insbesondere die (vor-)schulische Literacyforschung (vgl. z. B. Brandenburg, 2006; Hurrelmann, 2004; Nickel, 2004, 2007; Watson, 2003; Whitehurst \& Lonigan, 2003) weist darauf hin, dass die ersten Erfahrungen mit dekontextualisierten sprachlichen Varietäten bereits vorschulisch und primär im familiären Umfeld gemacht werden. Die Familie ist hierbei für manche Autoren nicht nur die frühste, sondern auch die 
wirksamste Instanz zur Entwicklung von Literacy (vgl. Hurrelmann, 2004). Da Kinder bis zur Einschulung in der Regel den Großteil ihres Lebens innerhalb der Familie verbringen, stellt die familiäre Sozialisation den zentralen Kontext zur Aneignung zentraler (Vorläufer-)Fertigkeiten der Literacyentwicklung dar. Nichtsdestotrotz entwickelt sich Literacy innerhalb eines komplexen Bedingungsgefüges bestehend aus reziprok interagierenden individuellen, familiären, bildungsinstitutionellen und gesellschaftlichen Faktoren, die stärker von der Komplexität der Umweltanregung als von Eigenschaften des Individuums determiniert wird (vgl. Nickel, 2004, S. 71). Hamers und Blanc (2000, S. 104) sehen im Erwerb von Literacy in beiden Sprachen eine zentrale Bedingung für die Entwicklung von Bilingualität: [...] the cognitive outcome of bilingual development relies to a large extent on the valorisation of language for literacyoriented activities, at least in Western cultures. The valorisation of literacy skills per se may have a positive effect on the child's representation of language."

Geeignete kulturspezifische prä- und paraliterale Kommunikations- bzw. Interaktionsformen im familiären Umfeld zur Förderung dieser (Vorläufer)Fertigkeiten der Literacyentwicklung stellen unter anderem das Erzählen von Geschichten, die Bilderbuchbetrachtung, das Vorlesen sowie Sprachspiele und Kinderlieder dar (vgl. Whitehurst \& Lonigan, 2003). Nach Watson (2003) lässt sich die Effektivität solcher kulturspezifischer prä- und paraliteraler Interaktionsformen in der Familie für die Literacyentwicklung durch die hierbei stattfindende Transformation von Sprache und Kognition erklären. Innerhalb dieser Interaktionsformen wird die Relevanz von metalinguistischen Fähigkeiten, Metakognition, Abstraktion und Organisation bei der Interpretation von (schrift-)sprachlichen Informationen akzentuiert. Hierbei werden konzeptionell schriftliche Merkmale von Sprache durch orale Transmission implizit vermittelt und von den Kindern im weiteren Literacyerwerb selbständig angewendet. So werden z. B. Kinder beim Geschichten erzählen mit Diskurskonventionen, die Kohäsion und Referentialität in Texten markieren und erzeugen, vertraut gemacht (vgl. Bialystok, 2013, S. 637f) oder erwerben erste basale Vorstellungen gelungener Bildbeschreibungen beim gemeinsamen Anschauen von Bilderbüchern. Diese Einsichten weisen in der Regel sprachspezifische und unabhängige Wissenskomponenten auf. So benötigt es zum einen sprachenunabhängiges Wissen über allgemeine Diskurskonventionen der Kohäsion und Referentialität, aber auch sprachspezifisches Wissen über einzelsprachliche Möglichkeiten zur Erzeugung von Kohäsion (z. B. durch Funktionswörter zur Verbindung von Propositionen) oder von Referentialität (z. B. durch Pronomen). Zudem finden solche familiären prä- und paraliteralen Kommunikationsformen idealerweise in enger, emotionaler Verbundenheit zwischen der Betreuungsperson und dem Kind statt, was eine angenehme Atmosphäre schafft und die Bindung zwischen Eltern und Kind stärken kann. 
Die förderlichen Effekte von (gelungenen) prä- und paraliteralen Interaktionsformen in der Familie auf den Literacyerwerb werden durch eine breite und robuste Empirie bestätigt (vgl. z. B. Hurrelmann, 2004; Nickel, 2007; Watson, 2003; Whitehurst \& Lonigan, 2003). Die Evidenz weist allerdings auch auf die Milieuabhängigkeit der Quantität und Qualität prä- und paraliteraler Erfahrungen hin, sodass Familien bildungsferner Milieus seltener in förderlichen Kommunikationsformen agieren und hierbei weniger fruchtbare Stile des Vorlesens sowie der Begleit- und Anschlusskommunikation pflegen als Familien aus bildungsnäheren Milieus.

Untersuchungen mit bilingualen Kindern weisen auf sprachenunabhängige, dekontextualisierte prä- und paraliterale Fähigkeiten hin, die für beide Sprachen verwendet werden können. Solche übergeordneten, mit konzeptionell schriftlichen Varietäten assoziierten Fähigkeiten, die für beide Sprachen angewandt werden können, selbst wenn diese lediglich in einsprachigen Kontexten erworben wurden, werden in der Regel auf Basis der Interdependenzhypothese nach Cummins (2000) theoretisch diskutiert. Die Interdependenzhypothese, welche in Abschnitt 3.6.3 näher erläutert wird, besagt in ihrem Kern, dass sprachenunabhängiges, deklaratives linguistisches Wissen sowie kognitiv-akademische sprachliche Fähigkeiten, die in einer Sprache enkodiert sind oder erworben wurden, auf eine andere transferiert werden können. Transfer bezeichnet hierbei die Fähigkeit, einmal Gelerntes auf neue Problemstellungen anzuwenden (Schmöe, 2016b, S. 720). Solche Transferprozesse scheinen sich bei der Entwicklung von Literacy bei bilingual aufwachsenden Sprechern zu vollziehen. So bewegen sich ihre Fähigkeiten zur Erzeugung formaler Definitionen und Bildbeschreibungen (Davidson, Kline and Snow, 1986; Snow et al., 1989) sowie ihr Verständnis von Metaphern (Johnson, 1989) in ihren beiden Sprachen auf dem gleichem Niveau, auch wenn ihr Sprachkompetenzprofil Asymmetrien aufweist (vgl. Bialystok, 2013, S. 637).

Bilinguale Sprecher können auch ihre Lesekompetenz über die verschiedenen Schriftsysteme ihrer Einzelsprachen hinweg transferieren (Bialystok, 2013, S. 641f; Cummins, 2000). Da verschiedene (Vorläufer-)Fähigkeiten in unterschiedlichen Maßen relevant für die Lesekompetenz in verschiedenen Sprachen sind (vgl. Bialystok, Luk \& Kwan, 2005), werden bei ähnlichen Sprachen in vergleichbarem Maße dieselben (Vorläufer-)Fähigkeiten zur Lesekompetenz gefördert und eingesetzt, sodass diese dann auch leichter transferiert werden können. Nach Muter und Diethelm (2001) sind die gleichen (Vorläufer-)Fähigkeiten prädiktiv für die Lesekompetenz von bilingualen und monolingualen Kindern. Die Ergebnisse von Limbird et al. (2014) widersprechen der These von Muter und Diethelm (2001) zum Teil. In ihrer Stichprobe wurde die Lesekompetenz bilingual deutsch-türkischsprachig aufwachsender Grundschulkinder stärker von ihren Wortschatzfähigkeiten beeinflusst, während die phonologische Bewusstheit sich als prädiktiver für die Lesekompetenz monolingualer Kinder herausgestellt hat. 
In der Gesamtschau akzentuiert die Literacyforschung die Bedeutung der familiären Sozialisation bezüglich des Erwerbs von (Vorläufer-)Fähigkeiten der Literacyentwicklung. Die Eltern modellieren in prä- und paraliteralen Kommunikationsformen zentrale konzeptionell schriftliche Merkmale von Sprache durch orale Transmission, welche ihre Kinder implizit verarbeiten und übernehmen. Bilingual aufwachsende Kinder können ihre Literacyfähigkeiten und deren Vorläufer auf ihre beiden Sprachen anwenden, selbst wenn diese Fähigkeiten exklusiv in einer Sprache erworben wurden und/oder ihre Sprachkompetenz deutliche Asymmetrien aufweist. Allerdings gilt trotz dieser positiven Befunde zu berücksichtigen, dass sprachspezifische Fähigkeiten hierbei eine zentrale Rolle spielen. So unterscheiden sich bilinguale und monolinguale Kinder zwar nicht hinsichtlich ihres übergeordneten, allgemeinen deklarativen und prozeduralen Wissens bezüglich Literacy, allerdings ist die konkrete einzelsprachliche Ausführung an die Fähigkeiten in der Einzelsprache gebunden.

\subsection{3 (Meta-)Kognitive Konsequenzen von Bilingualität}

Neben (meta-)linguistischer Folgen des Aufwachsens mit mehr als einer Sprache stellen (meta-)kognitive Konsequenzen der Bilingualität ein weiteres zentrales Untersuchungsphänomen der Bilingualismusforschung dar. Diese Thematik ist nicht nur aus linguistischer Perspektive interessant, sondern kann ebenfalls als natürliches Experiment zur kognitionspsychologischen Grundlagenforschung beitragen, da Sprache ein kognitives Werkzeug darstellt, mit welchem Wissen organisiert und strukturiert wird (vgl. Hamers \& Blanc, 2000, S. 117). Dementsprechend stellt der Zusammenhang von Sprache und Kognition per se ein interessantes und relevantes Forschungsphänomen dar, wobei der Vergleich von monolingual und bilingual aufwachsenden Individuen fruchtbare Einblicke hierzu liefern könnte.

Bereits in der Studie von Peal \& Lambert (1962) und den darauf folgenden Studien wurden neben potentiellen Einflüssen des bilingualen Spracherwerbs auf verbale Fähigkeiten auch eine Vielzahl weiterer non-verbaler, (meta-)kognitiver Konstrukte und Fähigkeiten untersucht (vgl. Hamers \& Blanc, 2000, S. 89-91). Es wird vermutet, dass die spezifische Erfahrungssituation des Aufwachsens und des Gebrauchs von zwei Sprachen aufgrund des engen Zusammenhangs von Sprache und Kognition sich auch auf non-verbale (meta-)kognitive Fähigkeiten auswirken könnte. Da eine extensive Auseinandersetzung mit dieser Thematik den Rahmen der vorliegenden Arbeit überschreiten würde, wird auf die Darstellung von kognitionspsychologischen Grundlagen sowie von Sprachverarbeitungsmodellen weitestgehend verzichtet und stattdessen zentrale empirische Befunde zu kognitiven Konsequenzen von Bilingualität berichtet. 


\subsubsection{Kognition und Bilingualität}

Die kognitiven Anforderungen von bilingual aufwachsenden Individuen unterscheiden sich von monolingualen Sprechern dahingehend, dass sie Wissen speichern, abrufen und verarbeiten, die in zwei unterschiedlichen Einzelsprachen repräsentiert sein können. Dementsprechend stellt sich die Frage, ob ihre kognitiven Systeme und deren Verarbeitungsmechanismen sprachspezifisch oder sprachunspezifisch strukturiert sind. Prinzipiell wären sowohl ein sprachenübergreifendes oder zwei sprachspezifische mentale Lexika und Grammatiken als auch eine parallele oder selektive Aktivierung der Einzelsprachen beim Abruf und bei der Verarbeitung von Wissen denkbar.

Die Mehrheit bilingualer Sprachverarbeitungsmodelle, unabhängig davon, ob sie visuellen und/oder auditiven sprachlichen Input fokussieren, konzeptioniert das mentale Lexikon von bilingualen Sprechern als ein sprachenübergreifendes Lexikon (vgl. Bartolotti \& Marian, 2012, S. 23-32). Die Struktur des mentalen Lexikons basiert auf Assoziationen, die durch Ko-Okkurrenzen und statistische Regularitäten sowie strukturelle Ähnlichkeiten gebildet werden (siehe z. B. BLINCS von Shook \& Marian, 2013; SOMBIP von Li \& Farkas, 2002). Dementsprechend können auch lexikalische Items aus verschiedenen Sprachen, die sich phonologisch (,hüner“ = Fertigkeit (Türkisch); „Hühner“ (Deutsch)) oder orthographisch („,biber“ = Paprika (Türkisch); „Biber“ (Deutsch)) ähneln, trotz distinkter semantischer Bedeutungen im Netzwerk miteinander assoziiert sein. Sprachenübergreifende Lexika implizieren auch eine parallele Aktivierung von Lexemen unterschiedlicher Einzelsprachen bei der Sprachverarbeitung. Die Evidenz weist hinsichtlich des Zugriffs auf das mentale Lexikon bei der Rezeption linguistischer Stimuli auf eine gemeinsame Aktivierung ähnlicher lexikalischer Items aus verschiedenen Sprachen bei bilingualen Sprechern hin (Bartolotti \& Marian, 2012, S. 17-23). So führt zum Beispiel der phonologische Input des Zielitems „,marka“ (Russisch für „Stempel“) zur Aktivierung von ,marker“ (Englisch für „Filzstift“) bei bilingualen Sprechern des Russischen und Englischen. Die parallele Aktivierung kann experimentell auch bei orthographischem Input getriggert (vgl. van Heuven et al., 2008) sowie mit elektrophysiologischen Indikatoren nachgewiesen werden (vgl. Thierry \& Wu, 2004, 2007). Dies gilt nicht nur für die Rezeption linguistischer Stimuli, sondern auch bei der Sprachproduktion, was mit einer Vielzahl von Studien zu „Tip of the Tongue“-Erfahrungen demonstriert werden konnte (vgl. Bartoletti \& Marian, 2012, S. 21-22).

Der Abruf von Wissensinhalten aus dem episodischen Gedächtnis bei Bilingualen weist ebenfalls auf einen engen Zusammenhang von Sprache und Kognition hin ist (vgl. Bartoletti \& Marian, 2012, S. 15f). Das Vorhandensein unterschiedlicher Sprachen bei Bilingualen eröffnet prinzipiell die Möglichkeit, bestimmte Erfahrungen mit einer der Sprachen bei der Konstruktion von episodischen Wissenselementen zu verknüpfen, wenn z. B. die Erfahrung in 
einem einzelsprachspezifischen Kontext gemacht wurde. Die mit der Erfahrung verknüpfte Sprache kann dann hierbei als ein zusätzlicher Cue für den Abruf dieses episodischen Wissens fungieren. Psychotherapeutische Studien unterstützen diese Annahme, da Erinnerungen häufiger, detaillierter und emotionaler abgerufen werden können, wenn dies in der Sprache erfolgt, mit welcher die abzurufende Erinnerung verknüpft ist (vgl. ebd.). Bei inkongruenten Kontexten, in welchen der Abruf einer episodischen Information, die mit Sprache A verknüpft ist, in Sprache B erfolgen soll, können spontane Übersetzungen den Abrufprozess erleichtern (vgl. ebd., S. 16).

Dementsprechend weisen bilinguale Sprecher prinzipiell einzelsprachenunspezifische Abruf- und Verarbeitungsprozesse sowie eine einzelsprachenübergreifende kognitive Architektur auf, die allerdings zumindest hinsichtlich episodischer Wissensinhalte, einzelsprachliche Verknüpfungen und Konstruktionen bilden können. Diese Befunde lassen sich aus der Perspektive der „linguistischen Relativität" (Whorf, 1956) auch bezüglich der Frage reflektieren, ob Bilingualität die Repräsentation und Funktionsweise non-verbaler kognitiver Wissenskonstruktionen beeinflusst. Prinzipiell erscheint die Annahme plausibel, dass der Erwerb oder das Lernen einer neuen Sprache die Assoziationen von Wörtern mit ihren zugehörigen Referenten sowie Denotationen beeinflusst, sodass neue Assoziationen entstehen und alte modifiziert oder adjustiert werden (vgl. Casasanto, 2008). Methodisch wird diese Annahme vor allem mit Hilfe von Beobachtungen des sprachlichen Verhaltens von bilingualen Sprechern untersucht, deren Sprachenkombination fundamentale krosslinguistische grammatische oder lexikalische Unterschiede hinsichtlich bestimmter Phänomene wie Farben, Bewegung, visuell-räumlicher Orientierung etc. aufweisen.

Die empirische Evidenz solcher Studien (für einen Überblick siehe Athanasopoulos \& Aveledo, 2013; Nicoladis, 2016) weist übergeordnet auf zwei zentrale Befunde hin: 1) Bilinguale Sprecher nähern sich dem typischen Sprachverhalten monolingualer Sprecher beim Sprechen ihrer L2 an, wobei die Sprachkompetenz und mit ihr zusammenhängende Variablen wie z. B. die Länge des Aufenthalts im zweitsprachlichen Kontext oder die Quantität des Sprachgebrauchs der Einzelsprachen die Effekte beeinflussen. 2) Allerdings weisen bilinguale Sprecher in manchen Fällen auch genuine, einzigartige Sprachverhaltensmuster auf, die von typischen Produktionen monolingualer Sprecher abweichen.

Studien zur Untersuchung bilingualer Sprecher bezüglich der Farbwahrnehmung (vgl. Athanasopoulos, 2009; Athanasopoulos et al., 2011) sowie der Nutzung von Bewegungsverben (vgl. z. B. Flecken, 2013) unterstützen die eben angeführten Befunde. So unterscheidet zum Beispiel das Griechische Hell- (,ghalazio“) und Dunkelblau (,,ble“) als zwei verschiedene Farbtöne mit eigenen Lexemen. Bilinguale Sprecher mit L1 Griechisch und L2 Englisch nä- 
hern sich bereits nach zwei Jahren Kontakt zum Englischen der Farbwahrnehmung von englischsprachigen Monolingualen an, sodass sie die Farbkontraste von Hell- und Dunkelblau als ähnlich einstufen (vgl. Athanasopoulos, 2009). Die Ergebnisse dieses Experiments wurden mit bilingualen Sprechern des Japanischen und Englischen repliziert, wobei sich hier der Sprachgebrauch als bester Prädiktor für das Verhalten bei der Farbwahrnehmung entpuppte (vgl. Athanasopoulos et al., 2011). Französisch-englischsprachig aufwachsende Kinder akzentuieren je nach Sprache unterschiedliche semantische Aspekte von Bewegungsverben, die typisch für ihre Einzelsprachen sind (vgl. Nicoladis, 2016, S. 270ff). Im Englischen verwenden sie mehr „,manner verbs“, die die Art der Bewegung semantisch inkorporieren und den Weg durch extralinguistische Elemente wie Präpositionen realisieren, während sie im Französischen häufiger auf ,path verbs“" zurückgreifen, die umgekehrt den Weg semantisch inkorporieren und die Art der Bewegung durch extralinguistische Elemente realisieren (vgl. ebd.). Im Gegensatz hierzu identifizierte Flecken (2011) bei der Untersuchung des Gebrauchs von Bewegungsverben von niederländisch-deutschsprachig aufwachsenden Kindern bei der Beschreibung von kurzen Videos einzigartige Muster in der Realisierung der Verben sowie in ihrem Blickverhalten, die von beiden monolingualen Gruppen qualitative Unterschiede aufwiesen.

Diese Effekte sollten allerdings nicht im Sinne der ,linguistischen Relativität“ (Whorf, 1956) überinterpretiert werden. Zwar weisen die Experimente auf eine einzelsprachenspezifische kognitive Verarbeitung bezüglich bestimmter Phänomene hin, allerdings könnte dies auch als Fähigkeit zu einer typischen und angemessenen Form der Sprachverwendung bilingualer Sprecher beim Gebrauch ihrer Einzelsprachen gedeutet werden (vgl. Athanasopoulos \& Aveledo, 2013, S. 249f). Die „Language as Strategy“-Hypothese (vgl. Gennari et al., 2002, S. 58) liefert eine Erklärung für solche sprachspezifischen Verarbeitungsprozesse. Bei kognitiv anspruchsvollen Aufgaben greifen Sprecher online auf Informationen des Kontextes zurück, die in den eben angeführten Studien linguistische Cues sind. Sobald allerdings die phonologische Schleife des Arbeitsgedächtnisses belegt wird, zum Beispiel durch das Wiederholen sinnloser Silben (,,verbal-shadowing"), werden die sprachspezifischen Effekte aufgehoben. Sprache scheint dementsprechend ein Hilfsmittel bei der Kommunikation und bei Sprachverarbeitungsprozessen zu sein, mit welchem Informationen im Arbeitsgedächtnis gehalten werden, um Aufgaben zu bearbeiten (vgl. Athanasopoulos \& Alvedo, 2013, S. 249). Die genuinen, einzigartigen Muster wiederum, die von der monolingualen Norm in beiden Sprachen abweichen, könnten durch den Experimentkontext beeinflusst worden sein. Die Probanden interagieren hier in der Regel mit Testleitern, die über die gleichen Sprachen wie sie selbst verfügen, sodass eine kreative, sprachenübergreifende Performanz eher als in monolingualen Kontexten akzeptiert wird kann. 
Die allgemeinen Befunde einer einzelsprachenunabhängigen kognitiven Architektur sowie einzelsprachenangemessener Sprachverarbeitungsprozesse von bilingualen Sprechern, die auch mit den Gegensätzen „Kooperation und Wettkampf" (vgl. Tracy, 2014, S. 82ff) umschrieben werden, legen die Frage nach potentiellen Transfereffekten nahe. Dies bedeutet, dass sprachliches Wissen der einen Sprache potentiell auf die andere übertragen werden kann. Die Evidenz fokussiert diesbezüglich nicht nur Transferprozesse grammatischen oder lexikalischen sprachlichen Wissens, sondern auch kognitiv akademischer sowie metalinguistischer Fähigkeiten, die in der Bilingualismusforschung häufig unter Cummins (2000) Interdependenzhypothese diskutiert werden.

Bilinguale Sprecher sind in der Lage, grammatisches Wissen der einen Sprache in ihre andere zu transferieren, auch wenn die andere Sprache die zu transferierende grammatische Kategorie nicht aufweist. So transferieren zum Beispiel französisch-englischsprachig aufwachsende Kinder das grammatische Genus des Französischen mit Maskulinum und Femininum bei einem Experiment zur Klassifizierung von Gegenständen in „Jungs“ und „Mädchen“ in das Englische, sodass „car" in Anlehnung an „la voiture“ als weiblich kategorisiert wird (vgl. Nicoladis \& Foursha-Stevenson, 2012). Aber auch geteilte grammatische Strukturen von zwei Einzelsprachen führen zu Transfereffekten. So profitieren z. B. türkischsprachige späte Zweitsprachlerner des Deutschen von der Verbendstellung im Türkischen beim Erwerb der deutschen Satzstellung (vgl. Haberzettl, 2006), da dies die Ausgangsbasis zum Erwerb der deutschen Satzstruktur im Spracherwerb darstellt (vgl. Schulz \& Grimm, 2012; Tracy, 2008). Im Gegensatz hierzu haben russischsprachige späte Zweitsprachlerner des Deutschen größere Probleme im Erwerb der Dissoziation von Verbzweit- und Verbendstellung in deutschen Haupt- respektive Nebensätzen, da das Russische stets die Verbzweitstellung realisiert (vgl. Haberzettl, 2006) ${ }^{5}$. Auch lexikalische Ähnlichkeiten z. B. in Form von Kognaten oder Internationalismen (z. B. „sympathisch“ (Deutsch), ,simpatico“ (Italienisch), „sympa(thique)“ (Französisch), „sympatisk“ (Schwedisch), „simpatıčen“ (Bulgarisch) (vgl. Schmöe, 2016a, S. 304)) können Transferprozesse initiieren und wie in diesem Fall zu einer Erleichterung des Wortschatzerwerbs führen. Trotz solcher Möglichkeiten der krosslinguistischen Beeinflussung treten Transfereffekte, die zu Fehlern in einer Einzelsprache führen, allerdings nur äußerst selten auf (vgl. Nicoladis, 2016, S. 273).

Ein relativ neuer Forschungsstrang der Bilingualismusforschung untersucht potentielle Einflüsse von Bilingualität auf Kreativität (für einen Überblick siehe van Dijk et al., 2018). Simonton (2008) definiert Kreativität als „,the act of generating new ideas or new connections between existing ideas or con-

5 Für den frühen L2a sowie den 2L1a scheint es aber keine solch ausgeprägten Unterschiede zwischen türkisch-deutsch- und russisch-deutschsprachigen Lernern zu geben (vgl. Schulz \& Tracy, 2011). 
cepts”, welcher auf Prozesse des ,divergent thinking” und „convergent thinking” zurückgreift. „Divergent thinking“" steht für die Fähigkeit, so viele Antworten wie möglich auf einen Impuls oder ein Problem zu geben, die über ein Konglomerat verschiedener Aspekte wie z. B. Gewandtheit, Flexibilität und Originalität operationalisiert wird (vgl. van Dijk et al., 2018, S. 2). Im Gegensatz hierzu fokussiert ,convergent thinking“ die Auswahl einer einzelnen, bestmöglichen Idee oder eines Ergebnisses (vgl. ebd.). Es handelt sich bei Kreativität allerdings nicht um ein stabiles Persönlichkeitsmerkmal (vgl. Jeon, Moon \& French, 2011), was auf eine Abhängigkeit der Kreativität von kontextuellen Variablen und spezifischen Testeigenschaften hinweist.

Van Dijk et al. (2018) fassen die bisherige Evidenz zum Zusammenhang von Bilingualität und Kreativität getrennt nach Untersuchungen mit bilingualen Erwachsenen und Kindern zusammen. Bilinguale Erwachsene weisen im Vergleich mit monolingualen Sprechern eine höhere Kreativität auf, wobei sie insbesondere bei den Dimensionen Gewandtheit, Flexibilität und Originalität besser als monolinguale Sprecher abschneiden (vgl. z. B. Kharkurin, 2007, 2009, 2017). Dieser Vorteil scheint allerdings nur für non-verbale Aufgabenstellungen zu gelten, da monolinguale Sprecher bei verbalen Kreativitätsaufgaben höhere Testwerte erreichen (vgl. Kharkurin, 2010). Die unterschiedlichen Erwerbstypen 2L1a und L2a scheinen einen Einfluss auf die Kreativität zu exerzieren, da Lerner mit 2L1a non-verbale Aufgaben origineller sowie flexibler als Lerner mit L2a lösen (vgl. Kostandyan \& Ledovaya, 2013). Hinsichtlich des Zusammenhangs zwischen Sprachkompetenz und Kreativität bilingualer Sprecher liegt eine gemischte Befundlage vor (vgl. van Dijk et al., 2018, S. 5).

Die Evidenz hinsichtlich der Kreativität bilingualer Kinder weist in dieselbe Richtung (vgl. ebd., S. 6). Bilingual aufwachsende Kinder erreichen höhere Testwerte hinsichtlich mathematischer Kreativität (vgl. Leikin, 2012) sowie Originalität (vgl. Leikin \& Tovli, 2014) im Vergleich zu monolingualen Kindern. Balancierte Sprachkompetenzen scheinen für Kinder allerdings zu höheren Testwerten bei Kreativitätstests zu führen (vgl. Lee \& Kim, 2010, 2011). Adi-Japha, Berberich-Artzi and Libnawi (2010) untersuchten die Strategien bei der Modifikation von bestehenden Bildern und wiesen qualitative Unterscheide zwischen bilingualen und monolingualen Kindern nach. Während bilingual aufwachsende Kinder die Bilder durch weitere Elemente oder durch die Verknüpfung von Elementen veränderten, nahmen die monolingual aufwachsenden Kinder eine Reduktion von Elementen zur Abwandlung vor.

Diese positiven Befunde zum Zusammenhang von Bilingualität und Kreativität sind allerdings mit Vorsicht zu betrachten, da die bisherige Evidenz auf relativ wenigen Studien beruht und diese zum Teil auch methodologische Unzulänglichkeiten wie z. B. kaum oder keine Kontrolle von Spracherwerbstyp, Sprachkompetenz, sozioökonomischen Status und Intelligenz aufweisen (vgl. 
van Dijk et al., 2018, S. 6). Die Autoren sehen in den häufig als besser postulierten exekutiven Funktionen bilingualer Sprecher eine potentielle (Teil-)Erklärung für die Tendenz einer höheren Kreativität von bilingualen im Vergleich zu monolingualen Sprechern: ,In order to produce an original idea or product, people have to focus and persist on the task at hand. Updating incoming information and shifting are beneficial to both divergent and convergent thinking. Furthermore, inhibiting noise and other environmental stimuli can prevent creative people from being distracted" (ebd.). Inwieweit Bilingualität tatsächlich zu besseren exekutiven Funktionen führt, wird im nächsten $\mathrm{Ab}-$ schnitt diskutiert.

\subsubsection{Exekutive Funktionen und Bilingualität}

Das Konstrukt exekutiver Funktionen beschreibt den Fähigkeitskomplex, mit welchem kognitive Prozesse während dem Ausführen komplexer kognitiver Aufgaben kontrolliert und koordiniert werden (vgl. Miyake et al., 2000, S. 50). Exekutive Funktionen werden häufig als Containerbegriff verwendet, sodass keine einheitliche operationale Definition existiert und Unklarheit über die Modellierung der Funktionsweise sowie des Zwecks von exekutiven Funktionen und angemessene Messmethoden für dieses Konstrukt besteht (vgl. Barkley, 2012). Für die Bilingualismusforschung wurde häufig auf das einflussreiche Modell exekutiver Funktionen von Miyake et al. (2000) zurückgegriffen (vgl. Bialystok, 2017, S. 248). Miyake et al. (2000) wiesen empirisch die zuvor theoretisch postulierten ,drei basalen Funktionen Hemmung (Inhibition), flexibler Aufgabenwechsel ((Set) Shifting) und Aktualisierung des Arbeitsgedächtnisses (Updating)“ als Subkomponenten exekutiver Funktionen nach (vgl. Hasselhorn \& Gold, 2017, S. 79).

Bilingualen werden im Vergleich zu monolingualen Sprechern hinsichtlich genau dieser drei von Miyake et al. (2000) empirisch nachgewiesenen exekutiven Funktionen Vorteile zugesprochen, wobei die Subkomponente der Inhibition besonders im Fokus steht (vgl. Bialystok, 2009; 2017; Bialystok, Craik \& Luk, 2012). Bei Bilingualen sind stets beide Sprachen bei der Sprachproduktion und -verarbeitung aktiviert (für einen Überblick siehe Kroll et al., 2015; Grosjean \& Li, 2013). Diese ,joint activation“ führt zu einem konstant stattfindenden Wettkampf zwischen den Einzelsprachen, bei dem bilinguale im Gegensatz zu monolingualen Sprechern in einer anderen und anspruchsvolleren Art und Weise ihre Sprachselektion und -verarbeitung kontrollieren müssen (vgl. Bialystok, 2017, S. 234): ,[...] bilinguals need to correctly select forms that meet all the linguistic criteria for form and meaning but are also part of the target language and not the competing system" (Bialystok, 2009, S. 54). Diverse Modelle haben versucht diese Sprachselektionsprozesse zu erklären, aber bisher konnte sich keines der Modelle empirisch robust durchsetzen (für 
einen kurzen Überblick siehe Bialystok, 2017, S. 235). Ein prominenter, einflussreicher Ansatz stellt das „Inhibitory Control“ Modell zur Sprachselektion von Green (1998) dar, welches Green und Abutalebi (2013) zur „Adaptive Control Hypothesis“" weiter ausbauten. Der Kern des Modells und dessen Modifikation stellt die exekutive Funktion der Inhibition in den Fokus: ,[...] a supervisory attention system was guided by top-down cues that led to the inhibition of the nontarget language so that language processing could proceed from contextually and linguistically appropriate representations. $[\ldots]$ these inhibitory processes were modified by their use in language selection and affected inhibitory control in other domains" (Bialystok, 2017, S. 235). Nach dieser Erklärung auf Basis der Ansätze von Green (1998) sowie Green und Abutalebi (2013) führt die Erfahrung mit bilingualer Sprachverarbeitung in Rezeption und Produktion zu einer allgemeinen Verbesserung der exekutiven Funktion der Inhibition, die sich auch auf non-verbale kognitive Prozesse überträgt.

Die empirisch robuste Evidenz der parallelen Aktivierung beider Einzelsprachen bei Bilingualen sowie die häufig empirisch nachgewiesenen besseren Leistungen von bilingualen Kindern bei Tests zur Messung exekutiver Funktionen - insbesondere bei Aufgaben, bei denen saliente, irreführende Information ignoriert werden müssen, aber nicht bei Aufgaben, die keine Distraktoren enthalten - unterstützen die eben ausgeführte Annahme (vgl. Bialystok, 2017, S. 248). Allerdings scheinen die Schlussfolgerungen angesichts aktueller empirischer Befunde und theoretischer Reflexionen zu früh gezogen worden zu sein (vgl. ebd., S. 249). So ist das Postulat der Inhibition als Subkomponente exekutiver Funktionen mit eigenständiger Varianzauflösung heute nicht mehr haltbar (vgl. Miyake \& Friedman, 2012). Zudem kommt es bei der Verarbeitung der Zielsprache auch zu Vereinfachungs- und Interferenzeffekten der Nicht-Zielsprache (vgl. Costa, Santesteban \& Inanova, 2006). Die Metapher eines Inhibition verlangenden Wettbewerbs zwischen den Sprachen erscheint diesbezüglich unpassend, da der Abruf von gleichen oder ähnlichen lexikalischen Items wie Namen, Kognaten und homonymen Übersetzungsäquivalenten durch die parallele Aktivierung zu einer Vereinfachung des lexikalischen Zugriffs führt. Auch die Möglichkeit der (partiellen) Deaktivierung der NichtZielsprache (vgl. z. B. Grosjean \& Li, 2013) widerspricht der Annahme der Inhibition als primären Mechanismus potentieller Vorteile bilingualer Sprecher bei exekutiven Funktionen. Zudem weisen einige aktuelle „systematic reviews" und Metaanalysen diesbezüglich gemischte Befunde und Nullergebnisse auf (für einen Überblick siehe Lehtonen et al., 2018), die die postulierten Vorteile Bilingualer hinsichtlich exekutiver Funktionen in Frage stellen. Hierbei werden insbesondere Variablen wie Erwerbsbeginn und Sprachkompetenzniveaus, die als Proxys für das Ausmaß an bilingualer Erfahrung interpretiert werden können, oder spezifische Sprachpaare als Moderatoren sowie metho- 
dische Fragen der Stichprobe, Aufgabenauswahl und Publikationsbias als potentielle Ursachen für die vermeintlichen positiven Effekte diskutiert (vgl. z. B. Kempert et al., 2016; Lehtonen et al., 2018).

Aufgrund der eben angeführten Kritik schlägt Bialystok (2017, S. 249f) vor, die potentiellen Vorteile bilingualer Sprecher hinsichtlich exekutiver Funktionen in der „executive attention“ (Engle, 2002) oder der ,working memory capacity" - ,the cognitive system in which memory and attention interact to produce complex cognition" (Shipstead, Harrison and Engle, 2015, S. 1863) - zu verorten. Aufmerksamkeit stellt den Kern der Anforderungen dar, die in Aufgaben zu exekutiven Funktionen gefragt sind, und kann somit potentiell als alternative Erklärung für die empirische Evidenz fungieren. Im Gegensatz zu der Konzeptionierung exekutiver Funktionen von Miyake et al. (2000) modellieren Shipstead, Harrison und Engle (2015) die ,,working memory capacity" sowohl als kontinuierliches Konstrukt, welches sich durch Erfahrung verändert und trainierbar ist, als auch als Ausmaß, inwieweit Ressourcen zur Kontrolle und Aufrechterhaltung von Aufmerksamkeit zur Verfügung stehen (vgl. Bialystok, 2017, S. 249). Die Anforderungen in der bilingualen Lebenswelt modifizieren das Aufmerksamkeitsverhalten durch Adaptation: ,[...] bilingualism involves an adaptation in which cognitive and brain systems used in both linguistic and nonlinguistic activities are modified as a consequence of being involved in those activities. In this sense, training through bilingual experience leads to an adaptation of cognitive and neural systems." (Bialystok, 2017, S. 250). Dementsprechend wird die Erforschung von metakognitiven Konsequenzen von Bilingualität in Zukunft andere Konstrukte wie z. B. ,working memory capacity" (Shipstead, Harrison and Engle, 2015) fokussieren und die Bedingungen der zweisprachigen Lebensrealität als Kontrollvariablen stets einbeziehen.

\subsubsection{Zusammenfassung}

Die Sprachkompetenzprofile bilingualer Sprecher werden vorschulisch primär durch ihre jeweiligen bilingualen Lebensrealitäten determiniert, deren Erwerbs- und Lerngelegenheiten zur sprachlichen Entwicklung zwischen zwei Sprachen aufgeteilt werden. Diese durch das "complementary principle“ (Grosjean, 2010, S. 29) bedingten ,profile effects“ (Gathercole, 2016, S. 128) widerlegen Beschreibungen der Sprachkompetenz bilingualer Sprecher als Summe zweier monolingualer Sprecher oder als doppelte Halbsprachigkeit, sondern akzentuieren die Distribution des kompletten Sprachvermögens bilingualer Sprecher über mehr als eine Sprache hinweg. Dementsprechend sollte die Auseinandersetzung mit der Sprachkompetenz bilingualer Sprecher stets ihre beiden Sprachen berücksichtigen. Angesichts der Abhängigkeit der 
Sprachkompetenzprofile bilingualer Sprecher von ihren konkreten Lebensrealitäten ist es nicht verwunderlich, dass sich die Bilingualismusforschung hinsichtlich (meta-)linguistischer und (meta-)kognitiver Kompetenzen durch eine uneinheitliche, kontroverse Befundlage auszeichnet.

Nichtsdestotrotz lassen sich prinzipiell keine fundamentalen, stetigen Differenzen zwischen den (meta-)linguistischen Kompetenzen von monolingual und bilingual aufwachsenden Sprechern konstatieren. Aufgrund der häufig quantitativ und qualitativ reduzierten Erwerbsmöglichkeiten verfügen bilingual aufwachsende Sprecher über einen kleineren Wortschatz in ihren einzelnen Sprachen und erwerben bestimmte grammatische Strukturen später im Vergleich zu monolingual aufwachsenden Sprechen. Allerdings stellen diese Unterschiede nur temporäre Differenzen dar, die sich im weiteren Verlauf weitestgehend nivellieren (vgl. Schulz \& Grimm, 2012; Grimm \& Schulz, 2016). Die regelmäßig postulierten Vorteile bilingualer Sprecher hinsichtlich metalinguistischer Kompetenzen müssen angesichts der empirischen Evidenz zum Teil revidiert werden. Zwar weisen bilingual aufwachsende Sprecher in ihrer Entwicklung bessere Leistungen hinsichtlich des Wortkonzepts auf und können auf Basis ihrer Einzelsprachen krosslinguistische Vergleiche bereits sehr früh vornehmen, allerdings scheinen metalinguistische Kompetenzen durch weitere, zum Teil sprachspezifische Faktoren vermittelt zu sein, sodass Bilingualität als Erklärungsansatz alleine nicht ausreicht (vgl. Bialystok, 2013, S. 642ff). Die ersten Begegnungen mit konzeptionell schriftlichen Varietäten finden in der Familie statt, sodass bei bilingual aufwachsenden Kindern diese häufig in der (weiteren) L1 stattfinden, die nicht zwingend die Verkehrs- und Unterrichtssprache darstellt. Die Erkenntnisse dieser frühen Erfahrungen mit konzeptionell schriftlichen Varietäten der L1 lassen sich prinzipiell auch auf die weitere L1 oder auf die L2 transferieren. Solche Transferprozesse benötigen allerdings auch sprachspezifisches Wissen und eventuell explizite Instruktion sowie Reflexion (vgl. Cummins, 2000), um die jeweiligen Erkenntnisse in den Einzelsprachen adäquat umzusetzen.

Die Debatten über (meta-)kognitive Unterschiede zwischen Monolingualität und Bilingualität akzentuieren ebenfalls die sich unterscheidenden sprachlichen Lebensrealitäten monolingual und bilingual aufwachsender Sprecher, die hypothetisch (meta-)kognitive Konsequenzen nach sich ziehen könnten. Zwar gibt es Hinweise auf Unterschiede zwischen monolingual und bilingual aufwachsenden Sprechern hinsichtlich bestimmter (meta-)kognitiver Eigenschaften und Fähigkeiten wie z. B. bezüglich der potentiellen Fazilitierungsund Interferenzeffekte durch sprachunabhängige mentale Lexika mit Einträgen aus mehreren Sprachen (vgl. Bartolotti \& Marian, 2012) oder den zumindest nach der momentanen empirischen Befundlage höheren Testwerten der Kreativität bilingualer im Vergleich zu monolingualen Sprechern (vgl. van Dijk, et al., 2018). Die häufig postulierten Vorteile bilingualer Individuen hinsichtlich 
exekutiver Funktionen gilt es angesichts der aktuellen widersprüchlichen empirischen Befundlage (vgl. Bialystok, 2017; Lehtonen et al., 2018) zu hinterfragen und sollten bis zu einer Klärung des Phänomens auch nicht als Interpretations- oder Erklärungsansatz für kognitive Konsequenzen der Bilingualität verwendet werden.

Die Befundlage verweist viel stärker auf die Berücksichtigung einzelsprachlicher Adaptationsleistungen sowie auf Transferprozesse bilingualer Sprecher. So nutzen bilinguale Sprecher gemäß der „Language as Strategy“Hypothese die Informationen des sprachlichen Inputs der jeweiligen Kontexte bei der kognitiven Verarbeitung, indem sie einen Output produzieren, der dem jeweiligen einzelsprachspezifischen, typischen Verhalten entspricht. Dieses Verhalten scheint wohl mit der einzelsprachlichen Sprachkompetenz zusammenzuhängen, was die Bedeutung einzelsprachlicher Fähigkeiten bei bilingualen Sprechern weiterhin akzentuiert. Zudem können bilinguale Sprecher ihr linguistisches Wissen auf andere Sprachen übertragen und zudem dieses Wissen für Aussagen über andere Bereiche anwenden, was die Studie von Nicoladis und Foursha-Stevenson (2012) mit der Anwendung des zweistelligen Genussystems des Französischen auf das einstellige Genussystem des Englischen eindrucksvoll demonstrierte.

Dementsprechend müssen insbesondere Lehrkräfte der Grundschule sensibel für diese temporären Unterschiede in den sprachlichen Kompetenzen sowie den zugrunde liegenden verschiedenen Lebensrealitäten und Entwicklungswegen von monolingual und bilingual aufwachsenden $\mathrm{SuS}$ sein. Die verzögerten linguistischen Entwicklungswege von bilingualen im Vergleich zu monolingualen Kindern sollten als spracherwerbsbedingte, typische Entwicklungsverläufe interpretiert werden, denen kein Defizit zu Grunde liegt. Zudem sollten Lehrkräfte diesen Unterschieden adaptiv und produktiv begegnen, sodass geeignete didaktische Konzepte zur Förderung der Verkehrssprache im Unterricht umgesetzt werden können. Angesichts der vorschulischen familiären Sozialisation von Literacy sowie des Potentials bilingual aufwachsender SuS zur krosslinguistischen Reflexion sprachlicher Eigenschaften stellt die Berücksichtigung und der teilweise Einbezug der (weiteren) L1 dieser SuS einen fruchtbaren Ansatz dar, um die vorschulischen Bildungserfahrungen mit dem aktuellen Lerninhalt zu verbinden. Solche didaktischen Ansätze sollten allerdings primär als Angebote gestaltet und instruiert werden, um positive Diskriminierung sowie ethnisch orientierte Segregationen zu vermeiden.

Wie bezüglich (meta-)linguistischer Kompetenzen lässt sich auch hinsichtlich (meta-)kognitiver Eigenschaften und Merkmalen bilingualer Sprecher eine doppelte Handlungsempfehlung ableiten. In Bildungsprozessen sollten Handlungsräume für bilinguale Sprecher geschaffen werden, die diese sowohl zur Nutzung und Förderung beider Sprachen als auch zur expliziten Reflexion der Gemeinsamkeiten und Unterschiede sowie den damit einhergehenden 
Transfermöglichkeiten der Einzelsprachen ermächtigen und animieren. Insbesondere die einzelsprachenunspezifische Architektur des mentalen Lexikons bei Bilingualen akzentuiert diese doppelte Handlungsempfehlung, da das semantische Netzwerk zwischen den einzelnen Einträgen verschiedener Sprachen nur durch Aktivierung beider Sprachen gestärkt werden kann.

Abschließend gilt auch wie in der kurzen Darstellung der Entwicklung der Bilingualismusforschung in Abschnitt 3.3.1 darauf hinzuweisen, dass positive Effekte der Bilingualität keine Selbstläufer sind, sondern eine sprachlich reichhaltige, förderliche und wertschätzende Umwelt zur Entwicklung benötigen. Die eben angeführten Handlungsempfehlungen fokussieren letztlich die Schaffung von Bedingungen, in der sich die Bilingualität der bilingual aufwachsenden SuS förderlich entfalten kann, sodass sich die Auftretenswahrscheinlichkeit (meta-)linguistischer und (meta-)kognitiver positiver Effekte der Bilingualität erhöht. Im Folgenden sollen dementsprechend die Zusammenhänge der Identitätsentwicklung sowie des kulturellen Selbstverständnisses mit der zweisprachigen Lebensrealität bilingualer Sprecher näher betrachtet werden, um einen Einblick in die Abhängigkeit der individuellen Selbstwahrnehmung bilingualer Sprecher von politisch-gesellschaftlichen Bedingungen zu erhalten.

\subsection{Identität, Kultur und Sprache}

Die Konstrukte Identität, Kultur und Sprache scheinen in einem engen Zusammenhang zu stehen: „In den Diskursen über Identität, über Kultur(en) sowie über Mehrsprachigkeit sind nicht nur deutliche Parallelen erkennbar, sondern auch Tendenzen der Konvergenz: Sprach(en), Kultur(en) und Identität(en) werden zusehends als unabdingbar miteinander verknüpft dargestellt" ( $\mathrm{Hu}$, 2007, S. 3). Hierbei handelt es sich allerdings nicht um drei gleichberechtigt nebeneinander zu ordnende Konstrukte. Nach Haarmann (1996, S. 219) kann in diesem Beziehungsgeflecht Identität als hierarchisch übergeordnet modelliert werden, sodass Sprache und Kultur in Abhängigkeit von dieser stehen und „ohne Identität als ihre Voraussetzung nicht denkbar" sind. Um diese Hierarchie in ihrer Systematik darstellen zu können, benötigt es zunächst eine Bestimmung und Konkretisierung der Begrifflichkeiten „Identität" und „Kultur“. Das Verständnis und der Gebrauch der Begriffe „Identität“ und „Kultur“ weisen im Alltag häufig einen essentialistischen Charakter auf (vgl. Hu, 2007, S. 7), was keine angemessene Perspektive auf diese Konstrukte eröffnet. Essentialistische Identitäts- und Kulturkonzeptionen sprechen Personen und Gruppen eine finite Menge statischer, unveränderlicher Eigenschaften und Merkmale zu, die sie in Form einer klar umrissenen Identität und Kultur charakterisieren. Unter Rekurs auf poststrukturalistisch orientierte Philosophien, kultur- 
wissenschaftlich-migrationstheoretische Reflexionen sowie sozial- und entwicklungspsychologische Erkenntnisse erweist sich eine solche Konzeption in Form klar umrissener, abgrenzbarer Entitäten als verkürzt (vgl. ebd., S. 3ff). Identität und Kultur stellen vielmehr dynamische, komplexe und hybride Konstrukte dar, die auch widersprüchliche Elemente (kohärent) in sich vereinen zu vermögen.

\subsubsection{Identität und Sprache}

Insbesondere in der Disziplin der Psychologie gibt es eine Vielzahl unterschiedlicher Konzeptionen und Modellierungen der Identität (für einen Überblick siehe Oerter \& Montada, 2002, S. 291ff). Nach Mead (1973) entwickelt sich die Identität eines Individuums durch dessen reziproken Erfahrungen und Handlungen mit der Gesellschaft und deren Mitgliedern. Identität besteht aus zwei zu differenzierenden Komponenten, dem „me“ und dem „I“. Das „I“ von Personen repräsentiert den Teil der Identität, der subjektiv handelt und sich durch Impulsivität und Spontanität auszeichnet, während das „me“ die Erfahrungen systematisiert und zu einem Selbstbild strukturiert, welches die Person objektiv reflektieren und darstellen kann (vgl. ebd.).

Eine Vielzahl von Autoren der Psychologie unterscheiden drei Perspektiven des Individuums auf sein Selbstbild, die zwar getrennt betrachtet werden können, allerdings miteinander zusammenhängen (vgl. Oerter \& Montada, 2002, S. 291f): 1) Das reale Selbst, welches am ehesten die tatsächliche Identität widerspiegelt (,,so bin ich"), 2) das ideale Selbst, welches eine idealisierte (Wunsch-)Vorstellung der Identität darstellt (,so sollte ich sein") und 3) das soziale Selbst, welches primär die Eigenschaften und Merkmale der Identität adressiert, die von anderen außenstehenden Personen wahrgenommen werden (,so sehen mich andere"). Das soziale Selbst wird von manchen Autoren als „ought-self“" operationalisiert, welches stärker ,die innere Repräsentation der Verpflichtungen und Aufgaben beinhaltet, die von Gesellschaft und Bezugspersonen herangetragen werden“ (vgl. ebd., S. 299). Diskrepanzen zwischen dem realen und sozialen Selbst beziehungsweise dem „ought-self“ können zu kognitiven Dissonanzen führen und somit zu negativen Emotionen wie unter anderem Scham, Verlegenheit, Schuld und Selbstverurteilung (vgl. ebd., S. 299f). Diese negativen Emotionen können wiederum Verhaltensänderungen initiieren, die zur Harmonisierung der verschiedenen Typen des Selbst beitragen sollen, sodass die kognitive Dissonanz aufgehoben wird.

Bei dem Prozess der Systematisierung der Erfahrungen und der Strukturierung des Selbstbildes werden nicht nur eigene, persönliche Eigenschaften herausgebildet, sondern auch Merkmale von Gruppenidentitäten inkorporiert (vgl. ebd., S. 245f). Hamers \& Blanc (2000, S. 200ff) akzentuieren genau diese Ab- 
hängigkeit der Identitätsbildung von Gruppen, die als Quellen für zu inkorporierende Merkmale und Eigenschaften der Identität dienen und als ineinander verschachtelte Fragmente zu einem Ganzen konstruiert werden. Unter Rekurs auf Tajfel $(1974,1981)$ entsteht die soziale Identität durch die Zugehörigkeit zu einer oder mehreren sozialen Gruppen einer Gesellschaft (vgl. Hamers \& Blanc, 2000, S. 201ff). Durch soziale Vergleichsprozesse des Individuums mit bestimmten Gruppen sowie unter Abgrenzung von anderen Gruppen identifiziert sich das Individuum mit allen oder einer Auswahl der entsprechenden Gruppenmerkmale und verhält sich gemäß diesen. In komplexen, multikulturellen Gesellschaften unterscheiden sich Gruppen auch hinsichtlich linguistischer, kultureller und ethnischer Merkmale, sodass diese Eigenschaften ebenfalls funktional zur Identitätskonstruktion verwendet werden können (vgl. ebd., S. 201). Die kulturelle Identität stellt einen Teil der sozialen Identität dar, die territoriale, normative und sprachliche Eigenschaften sowie bestimmte Wertüberzeugungen enthält. Die ethnische Identität wiederum ist eng mit der kulturellen verbunden, akzentuiert aber eher physiognomische Merkmale sowie die Abstammung oder Herkunft. Sofern Sprache einen relevanten Marker der kulturellen und/oder ethnischen Identität darstellt, kann Sprache eine zentrale Komponente der Identität eines Individuums werden, was Hamers \& Blanc (ebd., S. 202) als ,ethnolinguistische Identität" bezeichnen. Hierbei gilt es zu betonen, dass zur Inkorporation solcher Gruppenmerkmale bei der Identitätsbildung das Vorhandensein differenter Gruppen notwendig ist, die sich hinsichtlich kultureller, ethnischer und linguistischer Eigenschaften unterscheiden.

Sprache spielt in diesen Prozessen eine entscheidende Rolle, da sie als priorisiertes Mittel der Kommunikation die gesellschaftlichen Erfahrungs- und Tätigkeitsprozesse mitgestaltet und, wie anhand der ,ethnolinguistischen Identität" (ebd., S. 202) deutlich wird, selbst ein zentrales Merkmal von Identität darstellen kann. Auch Gogolin (1994, S. 14) akzentuiert hierbei die Bedeutung von Sprache und zählt diese, nach rechtsförmigen Merkmalen, ,zu den manifesten und zugleich stabilsten Möglichkeiten des Ausdrucks ethnischer Gemeinsamkeit bzw. zur Markierung von Verschiedenheit". Hu (2007, S. 5) weist unter Rekurs auf Chambers (1996, S. 32) auf eine weitere Funktion von Sprache hinsichtlich der Identitätsbildung hin, nämlich als ein „Werkzeug kultureller Konstruktion, mit dessen Hilfe unsere wahre Identität und unser wahrer Sinn konstruiert werden“. Dementsprechend ermöglicht Sprache in seiner Funktion als kognitives Werkzeug die Konstruktion des „me“, da unter Verwendung dieser das Selbstbild im Sinne eines mentalen Modells organisiert und kodiert werden kann. Haarmann (1996, S. 221) hebt ebenfalls diese Funktion von Sprache bei der Identitätsbildung hervor, akzentuiert allerdings noch zudem die Reziprozität dieses Prozesses: „Sprachliche Identität kann somit als eine Komponente der individuellen Identität gesehen werden. Mithilfe der 
Sprache als Medium strukturiert das Individuum die Erfahrungen des Anpassungsprozesses an die wechselnden Bedingungen der Umwelt als kollektives Ideengut und wird somit selbst in Abhängigkeit von Zeit zum Objekt der Anpassung“. Zudem kann Sprache wie im Sinne der ethnolinguistischen Identität selbstverständlich als Merkmal der Zugehörigkeit und Abgrenzung zur Konstruktion und Darstellung der eigenen Identität fungieren.

Der sprachliche Prozess, der die Konstruktion des „me“ als mentales Modell bedingt, kann als eine Form von Narration interpretiert werden: „Our own existence cannot be separated from the account we can give of ourselves. It is in telling our own stories that we give ourselves an identity. We recognize ourselves in the stories that we tell about ourselves." (Ricoeur, 1985, S. 214). Die eigene Identität wird zu einem Produkt von Sprache (vgl. Kerby, 1991, S. 4) und von Geschichten, die Personen konstruieren und sich in diesen widerfinden (vgl. Hu, 2007, S. 5). Die konkret vorliegenden politisch-gesellschaftlichen Bedingungen haben eine hohe Bedeutung für die Konstruktion der eigenen Narration, da diese die Handlungsräume und -möglichkeiten des Individuums restringieren und dementsprechend möglichst kohärent in die Narration des Individuums integriert werden müssen.

\subsubsection{Kultur und Sprache}

Wie auch das Konstrukt der Identität existiert Kultur nicht unabhängig von der Perspektive des Betrachters und scheint zumindest im wissenschaftlichen Kontext essentialistische Konzeptionen überwunden zu haben (vgl. Hu, 2007, S. 7). Allerdings ,entwickelt sich das Konzept Kultur zu einer Schlüsselkategorie, die als Basiskonzept grundlegend für jede verstehende Wissenschaft ist und gesellschaftliche Praxis als kulturelle Leistung begreift" (ebd.). Dementsprechend gilt es insbesondere bei wissenschaftlich abgeleiteten Schlussfolgerungen und Implikationen zu prüfen, ob diese nicht wieder in essentialistische Konzeptionen von Kultur verfallen oder diese in ihrer Argumentationslogik enthalten.

Nach Hu (ebd., 7ff) wird im wissenschaftlichen Diskurs von Disziplinen wie Ethnologie, Interkultureller Philosophie und Kulturwissenschaften ein diskursiv-reflexives und anthropologisches Konzept von Kultur vertreten, was sich durch drei zentrale Merkmale auszeichnet: 1) Kultur wird als Vermögen zur Bedeutungsstiftung interpretiert, welches Personen ermöglicht, ihrer Umwelt und sich selbst Bedeutung zu verleihen und somit diese zu strukturieren. Hörning \& Winter (1999, S. 9) weisen darauf hin, dass kulturelle Formen und Prozesse nicht als etwas „Sekundäres, Abgeleitetes“ zu interpretieren sind, sondern als ,dynamische, produktive Kräfte, die für die Gesellschaft selbst konstitutiv sind“". Aus dieser Perspektive sind missverständliche Formulierun- 
gen wie ,in einer Kultur leben“ hinsichtlich der aktuellen Konzeption von Kultur zu vermeiden, sondern es gilt vielmehr gesellschaftliche Praktiken als kulturelle Leistungen zu analysieren (vgl. Gutmann, 1998, S. 329). 2) Kultur und Sprache sind untrennbar miteinander verbunden, da die Konstruktion von Bedeutung in Form eines Diskurses stattfindet, der ,,sowohl unsere Handlungen als auch unsere Auffassung von uns selbst beeinflusst und organisiert" (Hall, 1994, S. 201). Dieser Diskurs wird in der Regel mittels Sprache vollzogen, in welchem kulturelle Praxis betrieben wird. Zudem kann Sprache wie bei der Identität ein inhärenter Bestandteil und Marker von Kultur in einer komplexen, diversen Gesellschaft darstellen (vgl. Hamers \& Blanc, 2000, S. 202). 3) Widerstreit und Synkretismus sind grundlegende charakteristische Eigenschaften von Kultur, sodass die früher häufig aufgegriffene Modellierung von Kultur als kollektiver Konsens aus heutiger Perspektive als „Inszenierung“ kritisiert wird: „Dagegen zeigen die Vertreter der Cultural Studies immer wieder das Aufbrechen oder das Fehlen eines Konsens in Wert- und Bedeutungsfragen und die zugrundeliegenden Konflikte auf" und können somit ,die Vorstellung einer Kultur als Trugbild entlarven“ (Hörning \& Winter, 1999, S. 9).

\subsubsection{Beziehung der Trias von Identität, Kultur und Sprache}

Nach diesen begrifflichen Klärungen kann die oben angeführte hierarchische Beziehung von Sprache und Kultur als in Abhängigkeit zur Identität stehend konkretisiert werden. Haarmann (1996, S. $219 \mathrm{ff})$ spricht der Identität unter Rekurs auf die paläoanthropolgische Forschung den Stellenwert als stärksten „Motor für die Fähigkeit des Menschen, sich an die unterschiedlichsten ökologischen Bedingungen anzupassen“, zu. In Einklang mit Watsons These (1979, S. 161), Sprache und Kultur seien ,unmittelbare und logische Folgen aus dem Besitz jener Art von Organisation [...], die jedem Individuum eine gewisse Einzigartigkeit verleiht und in seiner Form einbeschriebene Talente freisetzt", führt Haarmann (1996, S. 219ff) frühe sprachliche und kulturelle Aktivitäten wie Höhlenmalerei oder Grabbeigaben sowie die Tradierung von Erfahrung und Wissen durch Mythen und Erzählungen als Phänomene an, in welchen sich Identität ausdrückt. Hierbei kann Sprache ,,selbst in Zeit und Raum zum Objekt der Anpassung“ (ebd., S. 221) werden, z. B. wenn Jägergesellschaften wie die Eskimo in Sibirien ein komplexes, auf eine exakte räumliche Orientierung ausgerichtetes Pronominalsystem entwickeln oder Gesellschaften, die auf die Zucht bestimmter Tiere spezialisiert sind, über ein großes und ausdifferenziertes lexikalisches Netzwerk zur Beschreibung der Eigenschaften und Merkmale dieser verfügen. Solche sprachlichen Ausdifferenzierungen und Spezialisierungen finden selbstverständlich auch noch heute statt, auch wenn diese sich vornehmlich im Bereich der Lexik abspielen, was z. B. die aktuelle Digitali- 
sierung samt ihrer Folgen für Alltag und Beruf hervorragend illustriert. Dementsprechend stellen die in enger Beziehung stehende Sprache und Kultur notwendige Praktiken zur Organisation der Identität dar, die durch die Reziprozität im Verhältnis zur Identität auch Anpassungsprozesse durchlaufen. Zwar kann die Identität hierarchisch Sprache und Kultur übergeordnet werden, die gegenseitigen Abhängigkeiten und engen Verwobenheit der drei Konstrukte führen allerdings zu der Erkenntnis, dass bei der Auseinandersetzung mit einem dieser Konstrukte zwingend die anderen beiden mitreflektiert werden müssen.

Bevor die identitätsstiftende Funktion von Sprache bei hybriden Identitäten bilingualer Sprecher diskutiert werden kann, gilt es sich zuvor noch mit Einstellungen zu Sprache(n) und Bilingualität auseinanderzusetzen, da die Konstruktion von Identität sowie Kultur eng mit der Ausbildung und Inkorporation von Einstellungen verbunden sind. Es handelt sich hierbei um ein reziprokes Verhältnis, da Einstellungen die Konstruktion der Identität bedingen, aber auch bestimmte Identitätsmerkmale wie die Gruppenzugehörigkeit die Einstellung zu einzelnen Themen und Phänomenen mitbestimmen.

\subsubsection{Einstellungen zu Sprache(n) und Bilingualität}

Einstellungen werden innerhalb der Psychologie als relativ stabile Dispositionen definiert, die Individuen in einer beständigen Weise auf eine Person, Gruppe oder Situation reagieren lassen (vgl. z. B. Fischer, Jander \& Krüger, 2018, S. 95f). Sie weisen drei übergeordnete, häufig miteinander verbundene Komponenten auf: 1) Eine kognitive Komponente, bestehend aus Überzeugungen und Vorstellungen, 2) eine affektive bzw. emotionale Komponente, die mit der kognitiven Komponente verknüpft sein kann und 3) eine Handlungskomponente, die in Abhängigkeit der kognitiven und affektiven Komponente entscheidet, wann es hinsichtlich seiner Einstellungen zu handeln gilt (vgl. ebd., S. 96). Einstellungen entstehen durch individuelle Erfahrungen, die allerdings nicht zwingend direkt selbst erlebt werden müssen, um einen Einfluss auf die Einstellungsbildung vorzunehmen. So können z. B. auch die Einstellungen signifikanter Anderer oder gesellschaftliche Werteüberzeugungen auf die Einstellungsentwicklung von Individuen wirken. Dementsprechend sind Einstellungen trotz ihrer relativen Stabilität als Disposition auch dynamisch und veränderbar (vgl. Kessler \& Fritsche, 2018, S. 54).

Diese übergeordneten Eigenschaften von Einstellungen gelten selbstverständlich auch bezüglich Spracheinstellungen (vgl. Chin \& Wigglesworth, 2007, S. 108f), sodass Spracheinstellungen auch eher dauerhafter Natur sind, auf Erfahrungen beruhen und sich auf Handlungen auswirken können. Eine Besonderheit weisen Spracheinstellung im Gegensatz zu anderen Einstellun- 
gen allerdings auf, und zwar scheinen Einstellungen zu einer Sprache oder einer Varietät in besonderer Weise von der Wahrnehmung der Sprechergruppe der jeweiligen Sprache oder Varietät abhängig zu sein (vgl. Chin \& Wigglesworth, 2007, S. 108; Myers-Scotton, 2006, S. 120). Sprache kann hierbei als „Sozialdiagnostik“ fungieren: „[...] its use leads others to associate the speaker with a particular social group or set of activities" (Myers-Scotton, 2006, S.110). Dieser Aspekt erscheint einleuchtend, da insbesondere Sprache eine äußerst saliente Eigenschaft von Gruppen ist (vgl. ebd., S. 111). Wenn z. B. eine bestimmte Sprache oder Varietät vornehmlich von ungebildeten Menschen mit MH gesprochen wird, kann diese Sprache oder Varietät als Etikettierung fungieren, die allen Sprechern dieser Sprache oder Varietät die oben angeführten Eigenschaften zuspricht (vgl. ebd.). Dementsprechend stellen individuelle Spracheinstellungen nicht nur subjektive Evaluationen von Sprachen oder Varietäten dar, sondern inkludieren auch die Wahrnehmung ihrer Sprecher (vgl. ebd., S. 120).

Spracheinstellungen sind abzugrenzen von Sprachideologien (vgl. ebd., S. 109f). Ideologien lassen sich als „Aussagekomplexe ohne Wahrheitsbezug bezeichnen, die bestimmte Funktionsweisen von gesellschaftlichen Institutionen und Interessen von Gruppen nachträglich zu legitimieren versuchen" (Stojanov, 2011, S. 155) oder als ,patterns of belief and practice, which make some existing arrangements appear natural and others not" (Myers-Scotton, 2006, S. 135) definieren. Sie zeichnen sich vor allem durch fehlende Dialogizität, welche keine Offenlegung und Problematisierung der eigenen Prämissen inkorporiert, Naturalisierungen, nachträgliche Rechtfertigungen von vordiskursiven Meinungen und Positionen sowie der Verschleierung des Zusammenhangs der Funktionen und Gruppeninteressen mit den nachträglichen Rechtfertigungen aus (vgl. Stojanov, 2011, S. 158-160). Für dominante Mehrheiten erscheinen durch Ideologien geprägte Bedingungen als natürlich gegeben und nicht $\mathrm{zu}$ hinterfragen, da sie diese Bedingungen selbst bewusst oder unbewusst herbeigeführt haben (vgl. Myers-Scotton, 2006, S. 135). So kann z. B. die Prämisse, dass das Aufwachsen mit einer anderen L1 als dem Deutschen in einer Familie mit $\mathrm{MH}$ in der deutschen Regelschule zu Nachteilen im Bildungsverlauf führt, als eine Form von Sprachideologie interpretiert werden (vgl. Stojanov, 2011, S. 161). Dementsprechend können Ideologien und Einstellungen hinsichtlich Sprache dahingehend differenziert werden, dass erstgenannte eher interessenund zielorientierte Perspektiven und letztgenannte eher subjektive sowie stärker unbewusste Bewertungen von Varietäten und deren Sprechern darstellen (vgl. ebd., S. 136).

Anschließend an die Differenzierung von Spracheinstellungen und -ideologien stellen sich die Fragen, wie Einstellungen zu Sprachen sowie Bilingualität entstehen und von welchen Faktoren diese beeinflusst werden. Innerhalb der Bilingualismusforschung werden hierbei insbesondere das Prestige von 
Sprachen, welches in engem Zusammenhang mit politischen Sprachregelungen und sozioökonomischen Kräften steht, sowie die Sprachcommunity als zentrale Einflussgrößen diskutiert (vgl. z. B. Chin \& Wigglesworth, 2007; Hélot, 2006; Myers-Scotton, 2006). Aus linguistischer Perspektive sind alle natürlichen Sprachen in ihrem Wert gleich, da sie letztlich alle auf denselben Teilsystemen sowie Strukturen beruhen und sich lediglich in ihren spezifischen Ausprägungen und Parametern unterscheiden. Präferenzen und Hierarchisierungen von Sprachen nach abstrakten Kategorien wie „Wohlklang“, „Eleganz“ oder "Schönheit" stellen subjektive Bewertungen dar, die linguistisch nicht haltbar sind. Eine solch egalitäre Position zu Sprache Perspektive lässt sich auf die politisch-gesellschaftliche Perspektive nicht übertragen. Abhängig vom jeweiligen (nationalen) Kontext erfahren bestimmte Sprachen durch ihre oben bereits erwähnten Assoziationen mit bestimmten Gruppen eine hohe Wertschätzung, während andere Sprachen wiederum negativ konnotiert sind, da sich die mit ihnen assoziierten Gruppen z. B. im Besitz von attraktiven sozialen sowie materiellen Ressourcen und schließlich hinsichtlich ihres Prestiges unterscheiden (vgl. Hélot, 2006, S. 49). In diesem Sinne kann Sprache unter Rekurs auf Bourdieu (1982) als kulturelles oder symbolisches Kapital auf einem „Sprachmarkt" und somit als Quelle von Macht interpretiert werden (vgl. Hélot, 2006, S. 50; Myers-Scotton, 2006, S. 114f). Bereits 1974 wies Lambert auf den Zusammenhang von soziokulturellem Kontext und der Entwicklung von Bilingualität hin, welchen er in seiner Soziokulturellen Interdependenzhypothese theoretisch als Dichotomie von zwei Entwicklungswegen der Bilingualität abhängig von der Internalisierung soziokultureller Werte und Sprachstatus beschreibt (vgl. Hamers \& Blanc, 2000, S. 99f). Als ,,additive Bilingualität“ bezeichnet Lambert (1974) die Entwicklung in zwei Sprachen und mit mehr als einem kulturellen Orientierungssystem innerhalb eines beide Sprachen und Kulturen wertschätzenden soziokulturellen Kontexts. Hingegen entwickelt sich ,subtraktive Bilingualität" in Kontexten, in denen die zwei Sprachen und kulturellen Zugehörigkeiten bilingualer Sprecher innerhalb der Gesellschaft nicht gleichermaßen wertgeschätzt werden, sodass diese Sprachen und Zugehörigkeiten nicht vereinbar erscheinen und in einem Wettbewerb stehen. In solchen Kontexten können sich ethnolinguistische Minderheiten entweder an die Mehrheitsgesellschaft assimilieren oder sich von dieser abgrenzen. Im Falle der additiven Bilingualität ist die Wahrscheinlichkeit erhöht, balancierte Kompetenzprofile mit hoher Sprachkompetenz in beiden Sprachen sowie ein der Bilingualität positiv eingestelltes Selbstkonzept zu entwickeln, während im Kontext der subtraktiven Bilingualität asymmetrische Kompetenzprofile und eher negative Einstellungen zur Bilingualität und zu einer der beiden Sprachen auftreten.

Statusunterschiede von Sprachen werden stark durch politische Sprachregelungen beeinflusst, die unter anderem bestimmen, welche Amtssprachen zulässig sind oder welche Sprachen im Bildungssystem berücksichtigt werden. 
Politische Sprachregelungen basieren wiederum auf Sprachideologien, die sich an der ökonomisch, demographisch und politisch dominanten Mehrheit orientieren (vgl. Bourhis, 2001, S. 9f). Bourhis (ebd., S. 9-16) identifiziert vier Typen von Sprachideologien innerhalb eines Kontinuums, wobei die von einer Sprachideologie beeinflussten jeweiligen offiziellen politischen Sprachregelungen sich nicht zwingend in der tatsächlichen alltäglichen Praxis widerspiegeln müssen: 1) Die ,pluralist ideology“ (vgl. ebd., S. 10-11) lässt soziale Minderheiten frei über private Werte und kulturelle Praktiken wie z. B. Spracherwerb, -wahl und -gebrauch entscheiden und erlaubt ihnen diese auszuüben, solange sie zivile Rechte und gesellschaftlich vereinbarte Werte anerkennen und nicht gegen sie verstoßen. Die Mehrheit der Gesellschaft akzeptiert hierbei, dass bestimmte nationale (z. B. Bildungssystem, Rechtssystem, Verwaltung etc.) und private Institutionen Änderungen zum Zwecke der Akkulturation durchlaufen wie beispielsweise in Form von weiteren Amtssprachen, der Verankerung bestimmter Sprachen im schulischen Curriculum oder bilingualem Unterricht. Dementsprechend erkennt der Staat die Existenz und Praktiken seiner Minderheiten an und fördert Akkulturation durch finanzielle Unterstützung sowie soziale Wertschätzung. Die Akkulturation dürfte sich aber auf lange Sicht eher zu einer Aufnahme der Werte der Mehrheitsgesellschaft durch die Minderheiten entwickeln als umgekehrt. Ein Beispiel für eine solche Sprachideologie ist Kanada mit ihrem „Official Language Act“, der dem Englischen und Französischen einen gleichberechtigten Status als Amts- und Verkehrssprache garantiert und somit Bilingualität als offizielle Sprachregelung festschreibt, und ihrem „Multiculturalism Act“, der unter anderem die Multikulturalität als definierendes Merkmal Kanadas anerkennt und kulturellen Minderheiten die gleichen Rechte wie der Mehrheitsgesellschaft zuspricht und ihre kulturellen Praktiken wertschätzt und fördert (vgl. ebd., S. 11f). 2) Die „,civic ideology“ (ebd., S. 11-13) spricht ebenfalls Minderheiten Freiheit im privaten Bereich hinsichtlich ihrer Werte und Praktiken zu, aber bietet aktiv keine finanzielle oder soziale Unterstützung zum Erhalt oder zur Förderung der Kultur und Werte von Minderheiten an, sondern akzeptiert Initiativen und Gruppen, die mit eigenen Mitteln bestimmte kulturelle Praktiken und Werte unterstützen. Dafür fördert der Staat Mehrheitswerte als „,neutrale“ Positionen und regelt den Sprachgebrauch als ,freien Sprachmarkt“, der letztlich zu einer Akzentuierung der Mehrheitssprache und zum Sprachverlust von Minderheitensprachen führt. Als Beispiel für die „civic ideology“ kann Großbritannien genannt werden, die lange Zeit ihre kulturellen Minderheiten lediglich toleriert haben und erst in den letzten Jahrzehnten dazu übergegangen sind, pluralistischer in ihrem Umgang mit diesen zu werden (vgl. ebd., S. 12). 3) Auch die ,assimilation ideology“ (ebd., S. 13-14) fordert Minderheiten auf, ziviles Recht und gesellschaftliche Werte anzuerkennen und greift in der Regel nicht in die Privatsphäre von Minderheiten ein. Allerdings erwartet der Staat, dass 
sich gesellschaftliche Minderhei-ten im Lauf der Zeit freiwillig an die Mehrheitsgesellschaft anpassen und somit deren Werte, kulturelle Praktiken und Sprache überneh-men und ausüben. Dieser Assimilationsdruck wird von manchen Staaten forciert, indem sie Gesetze und Regelungen zur Assimilation durchsetzen. Die Vereinigten Staaten von Amerika können diesbe-züglich als Beispiel angeführt werden, da die nachkommenden euro-päischen Siedler ihre originären Werte und kulturellen Praktiken zu Gunsten eines „Amerikanismus" aufgeben oder zumindest adaptieren sollten (vgl. ebd., S. 13).

4) Die „ethnist ideology“ (ebd., S. 14-15) beansprucht für sich das Recht, in die Privatsphäre von Minderheiten einzugreifen, auch wenn deren Werte und Praktiken nicht gegen Gesetze der Mehrheitsgesellschaft verstoßen, sodass z. B. Minderheiten ihre Sprachen aufgeben und in der Mehrheitssprache kommunizieren müssen. Aber selbst bei Assi-milationsbestrebungen von Minderheiten erschweren oder verwei-gern gar manche Staaten mit „ethnist ideology“ die Akzeptanz und Anerkennung ihrer Minderheiten. Dies kann so weit gehen, dass Minderheiten aufgrund zugesprochener unüberwindbarer Unterschie-de hinsichtlich Werten und Praktiken hierbei zu einer Bedrohung der Mehrheitsgesellschaft stilisiert werden, was zur Exklusion, Apartheid oder gar Genozid führen kann (vgl. Baker, 2011, S. 391).

Sprachideologien können über ihren Einfluss auf Sprachregelungen auf die Einstellungen von Gesellschaftsmitgliedern gegenüber bestimmten Sprachen und den damit assoziierten Gruppen wirken (vgl. Chin \& Wigglesworth, 2007, S. 127). Die Einstellung zu Bilingualität ergibt sich schließlich aus den sprachideologisch geprägten Sprachregelungen eines Staates und den individuellen Spracheinstellungen der Gesellschaftsmitglieder. Positive Einstellungen zur Bilingualität entstehen zum Beispiel in Staaten wie Kanada, die Bilingualität als ein definierendes Merkmal des Selbstverständnisses des kanadischen Staates beanspruchen, welches es zu erhalten und zu fördern gilt. In anderen Staaten wie zum Beispiel in Deutschland, das zwischen den Polen der „civic“ und ,assimilation ideology“ diffundiert, führt die Ambivalenz zwischen sprachpolitischer Zugeständnisse - wie z. B. dem Herkunftssprachlichen Unterricht oder Türkisch als Abiturfach in einzelnen Bundesländern (Schröder \& Küppers, 2016) - und Assimilationsdruck - wie z. B. durch die Festsetzung einer Amtssprache, des stetig wiederkehrenden Diskurses einer „Deutschpflicht auf dem Schulhof" oder der faktisch falschen Behauptung „Deutschland ist kein Einwanderungsland“ - zu einer ambivalenten Einstellung gegenüber Bilingualität. Als Mitglied der EU ist Deutschland eigentlich verpflichtet, Mehrsprachigkeit anzustreben, die Erstsprachen seiner Einwohner zu fördern und die Gesellschaft zu einer positiven, wertschätzenden Einstellung gegenüber Bilingualität und sprachlicher Vielfalt zu bewegen (vgl. Yağmur, 2016).

Trotz des Zusammenhangs von Sprachideologien und -regelungen auf Spracheinstellungen gilt es zu betonen, dass bestimmte Individuen mit einer spezifischen gesellschaftlichen Position wie z. B. pädagogische Fachkräfte, 
Lehrer, Ärzte oder Wissenschaftler hinsichtlich der individuellen Spracheinstellungen der Gesellschaftsmitglieder zu Bilingualität einen besonderen Einfluss nehmen, da sie zum einen aufgrund ihrer Profession ein gewisses gesellschaftliches Prestige genießen und zum anderen durch professionelle Praktiken und Beratung den Umgang mit und die Einstellung zu Bilingualität mitbestimmen (vgl. Chin \& Wigglesworth, 2007, S. 123-127). Tracy (2014, S. 20) unterstellt einem gewissen Anteil dieser Berufsgruppen hinsichtlich ihrer Einstellungen zu Bilingualität gar eine „Doppelmoral“, da der „,frühe Erwerb von Sprachen, die innerhalb des Bildungssystems einen Wert haben, weil sie Teil des fremdsprachlichen Kanons sind, von Kinderärztinnen und Pädagoginnen deutlich positiver gesehen und für sinnvoller erachtet als der Erwerb von Sprachen, die in der Schule keine Existenzberechtigung haben $[\ldots]^{\prime \prime}$. Diese Kritik wird für Bildungskontexte im Abschnitt 3.6 näher ausgeführt.

\subsubsection{Die identitätsstiftende Funktion von Sprache bei bilingualen Sprechern}

Hinsichtlich des Zusammenhangs von Identität mit Sprache und Kultur weisen bilinguale Sprecher - insbesondere im Kontext der migrationsbedingten Bilingualität - einige zu berücksichtigende Besonderheiten und spezifische Bedingungen im Gegensatz zu monolingual aufwachsenden Sprechern auf. Bilinguale Sprecher mit MH verfügen über jeweils zwei Sprachen und ein durch die eigene Migration oder die der Eltern beeinflusstes Vermögen zur Bedeutungsstiftung. Die ,subjektiven Erfahrungen unter den Bedingungen der Mehrsprachigkeit“ führen zu einem „ständigen Prozess der Verortung“, sodass „,Zwischen Assimilation und Akkommodation [...] das Selbst immer wieder neu positioniert wird" (Hu, 2007, S. 2). Die in diesem Prozess entstehenden Identitäten werden als „transnationale“ (vgl. Krumm, 2009; Mecherill, 2003) oder „hybride Identitäten“ (vgl. z. B. Hall, 1994; Hu, 2007; Stojanov, 2006; Tracy, 2011) beschrieben, die von den Individuen sowohl mithilfe beider Sprachen als auch dem ihnen zur Verfügung stehenden kulturellen Praktiken und Wissen konstruiert werden.

Hybride Identitäten sind hierbei nicht als Produkt oder Summe zweier zu unterscheidender Identitäten und/oder Kulturen zu verstehen, sondern als Identitäten, „die im Übergang zwischen verschiedenen Positionen schweben, die zur gleichen Zeit auf verschiedene kulturelle Traditionen zurückgreifen und die das Resultat komplizierter Kreuzungen und kultureller Verbindungen sind, die in wachsendem Maße in einer globalisierten Welt üblich werden" (Hall, 1994, S. 218). Das Fusionsmodell von Akkulturation, in welchem Akkulturation als reziproker Wandel von Kultur durch das dauerhafte Aufeinandertreffen unterschiedlicher kultureller Praktiken zu verstehen ist (vgl. Arends-Tóth \& van de Vijver, 2006), akzentuiert ähnliche Prozesse und Praktiken. Hierbei 
integriert ein Individuum unterschiedliche kulturelle und sprachliche Ressourcen bei der Identitätsbildung zu einem neuen Vermögen der Bedeutungsstiftung, welches entweder als Mischung konzipiert ist oder gar in eigene, neue Positionen münden, die atypisch für die ursprünglichen kulturellen Quellen sind (vgl. ebd., S. 38). Dementsprechend kann die Hybridität als ein „,dritter Raum" (vgl. Bhabha, 1990, S. 211) interpretiert werden, in dem auch losgelöst von den originären kulturellen Praktiken, neue, kreativ gebildete Identitäten entstehen können. Hierbei können sich die so entstandenen hybriden Identitäten von Individuum zu Individuum deutlich unterscheiden.

Ein weiteres Bild zur Illustration hybrider Identitäten stellt die Metapher der „Übersetzung“ (vgl. Hall, 1994, S. 218) dar. So charakterisiert z. B. Stojanov (2006, S. 180) die Übersetzung als ,einen Vorgang der Transformation der primär einsozialisierten kulturellen Narrative“, bei der „eine Überschreitung der kontextuellen Grenzen der Wirklichkeitsdeutungsmuster der entsprechenden kulturellen Gruppe“" erfolgt. Hierbei gilt es darauf hinzuweisen, dass hybride Identitäten sich nicht immer zwingend durch Mischungen oder Transformationen sprachlicher und kultureller Ressourcen innerhalb eines dritten Raumes auszeichnen, sondern auch Akzentuierungen vornehmen können, die von einer Toleranz bis zur Abwertung und Missachtung bestimmter Quellen kultureller Praktiken reichen können (vgl. z. B. Bohnsack \& Nohl, 2001; Krumm, 2009).

Hybride Identitäten lassen sich empirisch mit unterschiedlichen methodischen Zugängen nachzeichnen. Verschiedene (biographische) Interviewstudien entdecken unterschiedliche Formen hybrider Identitäten, wobei auch Identitäten des dritten Raumes als unikale, übersetzte Identitäten vorzufinden sind (vgl. Stojanov, 2006, S. 184ff). So identifizieren z. B. Bohnsack \& Nohl (2001) bei Jugendlichen mit türkischem Migrationshintergrund unter Verwendung der Dokumentarischen Methode vier Typen des Umgangs mit einer erlebten „Sphärendifferenz“ zwischen der ,inneren“ Sphäre der Familie und ethnischen Community und der ,äußeren“ Sphäre des gesellschaftlichen öffentlichen Zusammenlebens in z. B. Schule, Freizeit und Beruf. Zwei Typen („Exklusivität der inneren Sphäre“ und „Primordialität der inneren Sphäre“) weisen eine Präferenz und Akzentuierung der inneren Sphäre auf, die sich lediglich hinsichtlich der Wertung der Normen und Praktiken der äußeren Sphäre unterscheiden. Der dritte Typus („Sphären(dif)fusion“) zeichnet sich durch eine „Orientierung an einer Kongruenz bzw. Fusion von innerer und äußerer Sphäre bei gleichzeitiger Diffusion in der Handlungspraxis" aus. Der vierte Typus („Die Suche nach einer dritten Sphäre“) distanziert sich von „,beiden Sphären, ihrer Moral und ihren lebenszyklischen Erwartungen und Ablaufmustern" und konstituiert eine dritte, neue Sphäre (Bohnsack \& Nohl, 2001, S. 24). Stojanov (2006, S. 189) sieht im vierten Typus eine Bewältigungsstrategie, die aus den Erfahrungen im dritten Typus resultiert, was auf die Dynamik von hybriden Identitäten hinweist. 
Auch Keim (2007, S. 14) identifiziert hybride Identitäten in ihrer ethnographisch-soziostilistischen Fallstudie einer Gruppe junger deutsch-türkischsprachig aufwachsender Mädchen in Mannheim, ,, die noch in der Migrantenpopulation verwurzelt ist, sich aber auf dem Weg nach „draußen“ befindet und an der Herausbildung eines eigenständigen Selbstbildes arbeitet“. Diese Gruppe durchläuft eine Entwicklung von einer „Phase der trotzigen ethnischen Selbsterhöhung, die in Reaktion auf die erlebte Ausgrenzung durch die deutsche Schule erfolgte“ (ebd., S. 204) hin zu einem Selbstkonzept, „das partielle Zugehörigkeit zu beiden Bezugsgesellschaften und die Aufnahme entsprechender Erfahrungen erlaubt, mit der Möglichkeit, daraus etwas Eigenständiges, Neues und Selbstbestimmtes zu entwickeln.“ (ebd., S. 202-203). Keims (2007, S. 186) ,türkische Powergirls“ sehen in ihrem Sprachgebrauch einen Marker ihrer Identitätskonzeption. Sowohl in der Phase der Erhöhung ihrer ethnischen Identität als auch in der Phase des Erarbeitens des oben angeführten neuen hybriden Selbstbildes, führen die Probandinnen die Mischung von deutschund türkischsprachigen Varietäten beim Sprachgebrauch als konstituierenden Teil ihrer Identität an. Zudem wird ihr sprachlicher Stil auch als Unterscheidungsmerkmal zu in der Türkei aufwachsenden Jugendlichen genutzt, von denen sie sich abgrenzen (vgl. ebd., S. 188).

Für eine ganzheitliche, subjektive Perspektive auf das Verhältnis von Identität und Sprache eignen sich insbesondere für jüngere Kinder sogenannte Sprachportraits (vgl. z. B. Gogolin \& Neumann, 1991; Krumm, 2001, 2009). Mehrsprachig aufwachsende Kinder und Jugendliche verorten hierbei ihre Sprachen in einer prototypischen männlichen oder weiblichen Silhouette, indem sie ihre Sprachen in diesen Silhouetten kreativ malerisch umsetzen und anschließend auf Nachfrage ihr Sprachenportrait kommentieren. Vor allem die funktionale Verortung der Sprachen zu bestimmten Körperteilen sowie die quantitativ eingenommene Fläche der jeweiligen Sprache unter Einbezug kurzer, sich anschließender Selbstberichte der Probanden ermöglichen fruchtbare Einblicke in die subjektive Bedeutung der Sprachen für die Probanden und deren Erfahrungen mit diesen.

Der Großteil der mit mehr als einer Sprache aufwachsenden Kinder und Jugendliche visualisiert hierbei ihre Identität multilingual, unabhängig von ihren vorhandenen sprachlichen Kompetenzen in der (weiteren) L1 und/oder L2. Diese multilingualen Identitäten können als hybride Identitäten kategorisiert werden (vgl. Krumm, 2009, S. 239-242). Allerdings weisen ungefähr ein Viertel der Sprachenporträts eine „Konflikt-Zweisprachigkeit“" auf, bei der entweder die nicht-verkehrssprachliche L1 oder die als L2 erworbene Verkehrssprache abgewertet wird (ebd., S. 239-241). Insgesamt identifiziert Krumm (ebd., S. 243) in seiner langjährigen Erfahrung mit der Methode der Sprachenportraits verschiedensprachige Eltern/Großeltern, ein vielsprachiges Lebensumfeld, emotionale Bezugnahmen zum sprachlichen Profil sowie die bedeutende 
Rolle des herkunftssprachlichen, Fremd- und Deutschunterrichts als wiederkehrende Konstanten, die in den jeweiligen visuellen Umsetzungen und Selbstberichten adressiert werden. Angesichts der Befunde dieses empirischen $\mathrm{Zu}$ gangs lässt sich der Zusammenhang von Sprache und Identität im Kontext des MH folgendermaßen charakterisieren (ebd., S. 245): „Migrantinnen und Migranten selbst, Erwachsene wie Kinder, sehen, verstehen und gebrauchen ihre Sprachen nicht primär unter funktionalen Aspekten, sondern vor allem im Rahmen ihrer Identitätsbewusstwerdung und ihrer Familienbindung - Kindergarten und Schule sollen diese Entwicklung stärken, nicht schwächen“.

\subsubsection{Zusammenfassung}

Zusammenfassend lässt sich schließen, dass die Konstrukte Identität, Kultur und Sprache in engen, reziproken Zusammenhängen und Wechselbeziehungen stehen, die im Kontext der migrationsbedingten Mehrsprachigkeit in einer besonderen Qualität besteht, da die sprachliche und kulturelle Vielfältigkeit der Lebenswelt narrativ in verschiedene Konstruktionen hybrider Identitäten münden können (vgl. Hu, 2007). Die identitätsstiftende Funktion der (weiteren) L1 (vgl. z. B. Keim, 2007; Krumm, 2009), die bei vielen, aber nicht allen bilingualen Sprechern mit MH vorhanden ist, sowie die kulturellen Übersetzungsleistungen bilingual aufwachsender Individuen (vgl. Stojanov, 2006) weisen auf die Relevanz einer möglichst positiven politisch-gesellschaftlichen Einstellung zu und eines förderlichen, wertschätzenden Umgangs mit von der Verkehrssprache abweichenden Erstsprachen hin. Da Einstellungen zu Sprachen eng mit der Wahrnehmung der mit ihnen assoziierten gesellschaftlichen Gruppe verbunden sind (vgl. Chin \& Wigglesworth, 2007, S. 108; Myers-Scotton, 2006, S. 120), können die Einstellungen zu den jeweiligen Gruppen ebenfalls Einfluss auf den Umgang mit der ihr verbunden Sprache ausüben.

Wenn das subjektive Selbst bilingual aufwachsender Individuen mit MH der hybriden Identität entspricht, die von der nationalen Sprachideologie beeinflussten politisch-gesellschaftlichen Rahmenbedingungen allerdings ein durch Assimilationsdruck eher monokulturell und monolingual geprägtes soziales Selbst favorisieren und propagieren, kann das Individuum diese Kontradiktion als kognitive Dissonanz wahrnehmen, was zu negativen Emotionen wie z. B. Scham, Schuld und Selbstverurteilung führen kann. Um die kognitive Dissonanz aufzulösen, kann das Individuum bestimmte Einstellungen und Perspektiven adaptieren oder gar aufgeben. So könnte z. B. das ideale und subjektive Selbst an das soziale Selbst oder das soziale Selbst abgewertet werden, was durch eine Abgrenzung zu den von der Mehrheitsgesellschaft nahegelegten Einstellungen erreicht werden kann. Solche kognitiven Umdeutungen zur Harmonisierung der verschiedenen Typen des Selbst könnten als zugrunde lie- 
gende Prozesse zur Erklärung der von Krumm (2009, S. 239-241) identifizierten „Konflikt-Zweisprachigkeit“ sowie der von Bohnsack und Nohl (2001) konstruierten Identitätstypen der „Exklusivität der inneren Sphäre“ und der „Primordialität der inneren Sphäre“ beitragen. So wird von einem Teil der Individuen mit MH die nicht-verkehrssprachliche L1 in ihrer Bedeutung erhöht und die L2 wiederum abgewertet, was als eine Form der Abgrenzung von der Mehrheitsgesellschaft gedeutet werden könnte. Andererseits könnte die ebenfalls empirisch identifizierte Abwertung der nicht-verkehrssprachlichen L1 als Folge einer Internalisierung von ideologisch geprägten Spracheinstellungen der Aufnahmegesellschaft, die die Erstsprachen von Kindern und Jugendlichen mit MH nicht wertschätzen, interpretiert werden.

Im Gegensatz hierzu steht die ,pluralist ideology“ (Bourhis, 2001, S. 1011) im Einklang mit hybriden Identitäten, da diese reziproke Akkulturationsprozesse gesetzlich legitimiert und durch finanzielle Unterstützung sowie soziale Wertschätzung fördert. Ein solcher politisch-gesellschaftlicher Rahmen ermöglicht eine Harmonisierung der verschiedenen Typen des Selbst bei hybriden Identitäten, sodass kognitive Dissonanzen gar nicht erst entstehen können. Prinzipiell stellt eine solche Sprachideologie auch keine Gefährdung des subjektiven Selbst monolingualer und monokultureller Mehrheiten dar, da jegliche Identitätsformen, sofern sie ziviles Recht und gesellschaftlich vereinbarte Werte anerkennen, in solchen Gesellschaften als gleichberechtigte Gesellschaftsmitglieder anerkannt werden.

Im Verlauf der Aufbereitung theoretischer Perspektiven und empirischer Evidenz zentraler mit Bilingualität assoziierter Phänomene kristallisiert sich in der vorliegenden Arbeit immer deutlicher heraus, dass die konkrete zweisprachige Lebenswelt samt ihrer politisch-gesellschaftlich restringierten Bedingungen bei der Untersuchung der Bilingualität von Individuen und Gruppen zu berücksichtigen ist: ,[...] the bilingual's development and behaviour cannot be considered independently from society, its structure and its cultural dimension“ (Hamers \& Blanc, 2000, S. 198). Das Aufwachsen in sprachideologisch pluralistisch geprägten Gesellschaften weist andere Entwicklungsmöglichkeiten und Herausforderungen der Bilingualität auf als in Assimilationsdruck ausübenden Gesellschaften. Diese unterschiedlichen politisch-gesellschaftlichen Rahmenbedingungen restringieren nicht nur die potentiellen (meta-)linguistischen Kompetenzen und (meta-)kognitiven Konsequenzen der Bilingualität, sondern beeinflussen auch die Identitätsbildung und das Vermögen zur Bedeutungsstiftung bilingual aufwachsender Individuen. Die Zusammenhänge von Identität, Kultur und Sprache sowie deren Abhängigkeit von der vorliegenden Sprachideologie sind selbstverständlich auch für den Kontext Schule als eine zentrale Instanz der Subjektbildung relevant, sodass auch „Lernmotivation, Lern-, Verstehens- und Interpretationsprozesse allgemein [...] nicht vom Phänomen der Mehrsprachigkeit und Mehrkulturalität abkoppelbar" (Hu, 2007, S. 2) sind. Sofern „Lernerorientierung als pädagogisches Grundprinzip“ (ebd.) in 
Bildungsinstitutionen ernst genommen werden soll, gilt es die Verwobenheit von Identität, Kultur und Sprache insbesondere im Kontext der migrationsbedingten Bilingualität durch einen möglichst förderlichen, wertschätzenden Umgang mit hybriden Identitäten und bilingualem Sprachvermögen zu berücksichtigen.

\subsection{Bilingualer Sprachgebrauch}

Die Beziehung und Verwendung der Einzelsprachen von bilingualen Sprechern kann als eine in Konkurrenz befindliche „Kooperation“ und „Arbeitsteilung" beschrieben werden (vgl. Tracy, 2011, S. 82). Beide Sprachen eines Sprechers konkurrieren zwar um Input und Anwendungsgelegenheiten, allerdings wird abhängig vom Kontext schließlich diejenige sprachliche Form verwendet, die die höchste Wahrscheinlichkeit zur Erreichung der Kommunikationsziele birgt. In zweisprachigen Kontexten kann es zu Formen von Sprachwechseln und -mischungen kommen, bei denen der Sprecher durch die Verwendung von Lexemen und Strukturen aus zwei unterschiedlichen grammatischen Systemen versucht, seine Kommunikationsabsichten zu realisieren. So kommunizieren z. B. bilingual aufwachsende Kinder und Jugendliche in Deutschland im familiären Umfeld (vgl. Reich, 2009) und in ihrer bilingualen Peer-Group (vgl. Keim, 2012) in ihren beiden Sprachen. Die in der zweisprachigen Kommunikation auftretenden Sprachwechsel und -mischungen bilingualer Sprecher stellen systematische, kreative Varietäten ihres stilistischen Repertoires dar, welche zielorientiert eingesetzt werden und nicht als defizitäre Beherrschung der Einzelsprachen interpretiert werden dürfen (vgl. z. B. Kersten et al., 2011, S. 90; Tracy, 2011, S. 82ff).

Innerhalb der englischsprachigen Literatur werden die Phänomene des Sprachwechsels und der -mischung häufig unter dem übergeordneten Terminus „Code-Switching“ zusammengefasst (vgl. Gardner-Chloros, 2009, S. 4; Özdil, 2010, S. 7, 41). Sprachwechsel und -mischungen stellen facettenreiche Phänomene dar (vgl. Gardner-Chloros, 2009, S. 7), die in verschiedensten Kontexten, diversen Formen sowie zu unterschiedlichen Zwecken auftreten und von diversen Disziplinen mit unterschiedlichen Methoden untersucht werden. Dementsprechend werden zur Beschreibung von Code-Switching möglichst breite, unspezifische Definitionen verwendet. So wird Code-Switching im Sinne eines Überbegriffs für Sprachwechsel- und mischungsphänomene z. B. als ,a speaker's employment of linguistic tools taken from two or more languages in order to interactively realize a plan of action" (Özdil, 2010, S. 7) oder als ,use of several languages or dialects in the same conversation or sentence by bilingual people" (Gardner-Chloros, 2009, S. 4) definiert. 
Eine weit verbreitete terminologische Abgrenzung stellt die Differenzierung von Sprachwechseln als „Code-Switching“ und Sprachmischungen als „Code-Mixing“ dar, die abhängig von satzstrukturellen Eigenschaften differenziert werden: ,the use of various linguistic units (words, phrases, clauses, and sentences) primarily from two participating grammatical systems across sentence boundaries within a speech event" wird als Code-Switching bezeichnet, während ,the mixing of various linguistic units (morphemes, words, modifiers, phrases, clauses, and sentences) primarily from two participating grammatical systems within a sentence" (Ritchie \& Bhatia, 2013, S. 376) mit dem Begriff Code-Mixing umschrieben wird. Hierbei wird also der Wechsel zwischen den Sprachen über Satzgrenzen hinaus (Code-Switching) und das Mischen von Sprachen innerhalb eines Satzes (Code-Mixing) voneinander abgegrenzt. Eine spezifische Form des Code-Mixings stellt das sogenannte „Borrowing“ dar, bei dem einzelne, lexikalische Entlehnungen eines Morphems, Wortes oder eines kurzen Ausdrucks von einer Sprache in die Produktion der anderen Sprache inkorporiert wird (vgl. Grosjean, 2008, S. 44).

Trotz dieser möglichen Differenzierung der Phänomene Code-Switching, Code-Mixing und Borrowing anhand syntaktischer und lexikalischer Indikatoren erscheint eine absolut trennscharfe linguistische Abgrenzung in der Praxis nicht möglich: „Efforts to distinguish code-switching, code-mixing and borrowing are doomed" (Eastman, 1992, S. 1). Insbesondere die mündliche Kommunikation zeichnet sich durch Satzabbrüche, Auslassungen, Wiederholungen, Reparaturen etc. aus, sodass in manchen Fällen Satzgrenzen oder syntaktische Strukturen nur schwer zu identifizieren sind. Zudem weisen bestimmte Sprachpaare Äquivokationen oder dieselben Lexeme und Morpheme auf, was die eindeutige Identifikation der konkret vorliegenden bilingualen Praktik erschwert.

Es stellt sich die grundlegende Frage, ob ,die beobachteten bilingualen Phänomene entweder als ein Prozess der Entlehnung und somit des Sprachwandels zu interpretieren sind oder als solitäre Erscheinungsform zu Tage treten, die jeweils sozial motivierte Gründe aufweisen" (Özdil, 2010, S. 42f). Die Bilingualismusforschung diffundiert diesbezüglich zwischen einem primär morphosyntaktischen und einem soziolinguistischen Zugang (vgl. ebd., S. 46). Morphosyntaktische Arbeiten wie z. B. Poplacks (1980) „Variationist Model“ oder Myers-Scotton (1993) „Matrix Language-Frame Model“ postulieren grammatische Restriktionen von Sprachwechsel- und -mischungsphänomenen, die von den grammatischen Eigenschaften der zu mischenden Sprachen determiniert werden (vgl. ebd., S. 47). Ohne hierauf näher einzugehen, gilt es allerdings diesbezüglich darauf hinzuweisen, dass Sprachwechsel- und -mischungsphänomene aus morphosyntaktischer Perspektive keine arbiträren, idiosynkratrischen Formen annehmen, sondern aufgrund der Restriktionen der zu mischenden Sprachen in systematischer, regelhafter Gestalt auftreten (vgl. ebd., S. 46-62). Mithilfe soziolinguistisch orientierter Arbeiten, „die v. a. mit 
der ethno-methodologischen Konversationsanalyse den sozial und kulturell motivierten Sinn eines Codewechsels in der Kommunikation untersuchen, wird analysiert, warum ein Interaktant in welcher Situation sich für eine Sprache oder einen Sprachmodus entscheidet" (ebd., S. 62). Diesbezüglich kann in Anlehnung an McClure (1981, S. 73) zwischen „situational Code-Switching” als die sozial motivierte Wahl einer Sprache und „stylistic Code-Switching” als diskursiv-rhetorisch begründete Sprachwechsel und -mischungen unterschieden werden (vgl. Özdil, 2010, S. 62f). Zusammengefasst fokussiert die morphosyntaktische Perspektive die strukturellen grammatischen Bedingungen, während der soziolinguistische Zugang die individuellen und kontextuellen Ursachen sowie diskursiven Funktionen von Sprachwechsel- und -mischungsphänomenen akzentuiert.

In der vorliegenden Arbeit spielen strukturelle Aspekte des Code-Switchings kaum eine Rolle, sodass für eine vertiefte Auseinandersetzung mit dieser Thematik auf die Ausführungen von Gardner-Chloros (2009, S. 91-116) und MacSwan (2013) verwiesen wird. Im Gegensatz hierzu sind allerdings für das Forschungsdesiderat individuelle und kontextuelle Ursachen sowie diskursiv-rhetorische Funktionen von Sprachwechsel- und -mischungsphänomenen von zentraler Bedeutung, die im Folgenden näher ausgeführt werden. Zunächst wird ein kurzer Überblick über soziolinguistisch orientierte Theorien und Modelle zu Sprachwechsel und -mischungen gegeben, wobei die Sprachmodi nach Grosjean (2008; Grosjean \& Li, 2013) näher ausgeführt werden. Anschließend werden die individuellen sowie kontextuellen Ursachen sowie diskursiv-rhetorische Funktionen von Sprachwechseln und -mischungen betrachtet.

\subsubsection{Soziolinguistisch orientierte Theorien und Modelle der Sprachwahl und des Sprachverhaltens}

Diverse soziolinguistisch motivierte Theorien und Modelle versuchen, die sprecherbezogenen, kontextuellen und konversationellen Ursachen für Sprachwechsel und -mischungen zu erklären. Diese Theorien und Modelle wurden nicht exklusiv für bilinguale Kommunikation konzeptioniert, sondern können auch hinsichtlich verschiedener Varietäten einer Einzelsprache angewandt werden. Im Folgenden sollen kurz die „Communication Accomodation Theory“ (vgl. Sachdev, Giles \& Pauwel, 2013), das „Markedness Model“" von Myers-Scotton (2006, S. 158ff) sowie die „Conversational Analyses“"von Auer (1988) betrachtet werden, um die Vielfalt der Ansätze zu illustrieren. 


\subsubsection{Erklärungsmodelle zur Sprachwahl und zum Sprachverhalten bilingualer Sprecher}

Die „Communication Accomodation Theory“, welche von Giles und Kollegen ab den 1970er Jahren aufgestellt und über die Jahre modifiziert wurde, fokussiert sozialpsychologische Strategien und Prozesse der Kommunikation (vgl. Sachdev, Giles \& Pauwel, 2013). Nach diesem Ansatz passen Sprecher ihr kommunikatives Verhalten bezüglich einer Reihe (para-)linguistischer und nonverbaler Indikatoren dahingehend an, dass sie entweder auf ihrem eigenen Stil beharren („Maintenance") oder sie sich ihrem Gegenüber sprachlich ähnlich (Konvergenz) oder unähnlich (Divergenz) verhalten (vgl. ebd., S. 394f). Sprecher, die ihr Kommunikationsverhalten an das des Gegenübers anpassen, versuchen durch Effekte der ,similarity attraction“ dem Gegenüber sympathischer zu erscheinen, um Anerkennung sowie Zugehörigkeit und damit verbunden auch Zugang zu dessen sozialen Netz zu erhalten (vgl. ebd., S. 395). Sprecher, die in ihrem Kommunikationsverhalten deutlich vom Gegenüber abweichen, schaffen Divergenz zwischen den Interaktionspartnern und somit folglich eine Distanz, welche in der Regel eine Markierung der unterschiedlichen Gruppenzugehörigkeiten der Gesprächspartner intendiert. Dementsprechend können bilinguale Sprecher, die über die gleichen Einzelsprachen verfügen, unter Verwendung von Sprachwechseln und -mischungen (partielle) Konvergenz oder Divergenz markieren.

Das „Markedness Model“ von Myers-Scotton (2006, S. 158ff) fußt auf der Prämisse, dass sich Sprecher gemäß der normativen Erwartungen in Form von „rights and obligations“, also abstrakten, implizit vereinbarten sozialen Umgangsformen während der Interaktion, situationsspezifisch verhalten. Dementsprechend sollten bilinguale Sprecher abhängig vom Kontext in der normativ angemessenen Sprache oder Varietät kommunizieren, sodass z. B. im Kontext eines monolingualen Arbeitsfeldes die mit allen Kollegen geteilte Verkehrssprache genutzt werden sollte. Kommt es in solchen Situationen zu einem Sprachwechsel oder zu -mischungen, eröffnet dieser Bruch mit den normativen Erwartungen einen neuen Kontext, in welchem andere, neue Rechte und Verpflichtungen der Interaktion firmieren (sollen). Myers-Scotton (ebd., S. 161) kategorisiert solche Brüche als bewusste, intendierte Prozesse, da die jeweilige Sprachwahl eine zielorientierte Entscheidung darstellt.

Die Konversationsanalyse nach Auer (1988) fokussiert die vorliegende Konversation selbst als zentralen Einflussfaktor auf die gewählte Sprache und/oder auf Sprachwechsel und -mischungen, sodass externe Kategorien als Ursachen innerhalb dieses Ansatzes vermieden werden. Dementsprechend existiert eine Ebene innerhalb von Konversationen, die unabhängig von äußeren Einflüssen die Praktiken der Sprecher bedingt. Um diese Ebene oder Indikatoren dieser zu identifizieren, benötigt es sowohl eine feinkörnige, sukzes- 
sive Analyse der einzelnen Redebeiträge in ihrer Dynamik als auch die Berücksichtigung größerer Organisationsstrukturen der Interaktion. Dementsprechend können bilinguale Sprecher in der Interaktion auch durch die jeweiligen sprachlichen Handlungen während einer Konversation selbst zu Sprachwechseln und -mischungen motiviert werden.

Während die „Communication Accomodation Theory“ (vgl. Sachdev, Giles \& Pauwel, 2013) und das „Markedness Model“ (Myers-Scotton, 2006, S. 158ff) eher deduktive Ansätze darstellen und Sprechern bewusste Motive und Ziele für ihr Kommunikationsverhalten zusprechen, stellt die Konversationsanalyse ein eher induktives Vorgehen dar, bei der die Interaktion selbst als zentrale Determinante für das jeweilige bilinguale Sprachverhalten fokussiert wird (vgl. Myers-Scotton, 2006, S. 173). Diese drei Ansätze schließen sich nicht gegenseitig aus, sondern akzentuieren lediglich unterschiedliche Ursachen der Sprachwahl sowie potentieller Sprachwechsel und -mischungen. So können neben sozialpsychologischen Strategien der Konvergenz und Divergenz, um Gemeinsamkeiten respektive Unterschiede zu markieren, auch normative Erwartungen sowie intendierte Brüche mit diesen und das Interaktionsgeschehen selbst die Sprachwahl und Sprachwechsel sowie -mischungen während der Kommunikation beeinflussen. Weiterhin ist zu vermuten, dass die Motivation für Sprachwahl und Sprachwechsel sowie -mischungen aus einer Kombination der oben angeführten möglichen Ursachen entsteht und sich auch innerhalb der Kommunikation dynamisch verändern kann. Ein Modell, welches durch den Verzicht auf die Akzentuierung einer der verschiedenen potentiellen Einflussquellen offen genug ist, um die verschiedenen Faktoren der Sprachwahl und Sprachwechsel sowie -mischungen zu inkorporieren und zudem exklusiv für die bilinguale Kommunikation von Sprechern konzeptioniert wurde, stellen die Sprachmodi nach Grosjean (2008; Grosjean \& Li, 2013) dar.

\subsubsection{Sprachmodi nach Grosjean (2008)}

Grosjean (2008, S. 39) definiert den Begriff „Sprachmodus” als „the state of activation of the bilingual's languages and language processing mechanisms at a given point in time". Wie in Abschnitt 3.3.3 bereits diskutiert, sind bei der Sprachverarbeitung bilingualer Sprecher immer beide Sprachen aktiviert, aber der Aktivierungsgrad der Einzelsprachen kann sich unterscheiden (für einen Überblick siehe Kroll et al., 2015). Trotz dieser parallelen Aktivierung postuliert Grosjean (2008, S. 40f) eine „Basissprache“, die im jeweiligen Moment primär für die Verarbeitung zuständig ist. Beide Einzelsprachen können unabhängig von ihrem Aktivierungsgrad potentiell als Basissprache fungieren und ein Wechsel der Einzelsprachen als Basissprache kann auch während der Interaktion vorkommen. 
Auf Basis dieser psycholinguistischen Prämissen modelliert Grosjean (ebd., S. 40) ein Kontinuum verschiedener Sprachmodi von bilingualen Sprechern, auf welchem er drei verschiedene Sprachmodi positioniert: Bilingualer Sprachmodus, gemischtsprachiger Sprachmodus und monolingualer Sprachmodus. Beim bilingualen Sprachmodus kommunizieren bilinguale Sprecher miteinander, die über die gleichen Sprachen verfügen. Beide Einzelsprachen der Sprecher weisen hierbei eine hohe Aktivierung auf und es kommt zu intensiven Formen des Code-Switchings und -Mixings. Auch beim gemischtsprachigen Sprachmodus interagieren zwei bilinguale Sprecher miteinander, die die gleichen Sprachen teilen. Dieser Sprachmodus zeichnet sich ebenfalls durch Phänomene des Sprachwechsels und des Mischens von Sprachen aus, allerdings treten diese seltener als im bilingualen Sprachmodus auf, da eine der beiden Einzelsprachen eine geringere Aktivierung aufweist. Beim monolingualen Sprachmodus kommunizieren zwei bilinguale Sprecher fast ausschließlich in einer der Einzelsprachen, wobei die andere Einzelsprache nur in leichtem Maße aktiviert ist und es nur äußerst selten oder gar nicht zu Formen des Code-Switchings und -Mixings kommt. Ein bilingualer Sprecher befindet sich auch in einem monolingualen Sprachmodus, wenn er mit seinem Gesprächspartner nur eine gemeinsame Sprache teilt oder dieser monolingual ist (vgl. ebd.). Während der Kommunikation können die Sprecher dynamisch zwischen den einzelnen Sprachmodi abhängig von sich ändernden Bedingungen wechseln. Sowohl die Initialposition auf dem Kontinuum als auch das Wechseln zwischen den Sprachmodi erfolgt hierbei nicht zwingend auf Basis einer bewusst getroffenen, zielorientierten Entscheidung der Sprecher, sondern kann implizit von Statten gehen. Zudem müssen die Interaktanten sich nicht im selben Sprachmodus befinden (vgl. ebd., S. 45f).

Grosjean (ebd., S. 42ff) benennt fünf übergeordnete Faktoren, die die Positionierung der Sprecher auf dem Kontinuum der Sprachmodi beeinflussen: 1) Teilnehmer der Kommunikation, 2) Situation, 3) Form und Inhalt der Kommunikation, 4) Funktion des Sprechaktes und 5) spezifische Forschungsfaktoren. Hinsichtlich der Teilnehmer der Kommunikation stellen neben weiteren individuellen Merkmalen dieser insbesondere die Sprachkompetenz, die Einstellungen zu ihren Sprachen, die typische Sprachpraxis im Allgemeinen als auch zwischen den Gesprächspartnern sowie deren Beziehung zentrale Einflussquellen auf das Sprachverhalten dar. Interaktanten, die über eine hohe Kompetenz in beiden Sprachen verfügen, positive Einstellungen zu ihrer Bilingualität besitzen und in einer engen Beziehung, wie z. B. einem Verwandtschaftsverhältnis oder einer Freundschaft stehen, dürften mit einer höheren Wahrscheinlichkeit im bilingualen Sprachmodus miteinander kommunizieren als in einem anderen.

Die konkrete Situation, in der die Kommunikation stattfindet, wirkt sich ebenfalls auf die Sprachmodi aus, sodass z. B. der Ort, die Rahmenbedingungen der Interaktion oder der Grad der Formalität die Sprachmodi bedingen 
können. Diesbezüglich können auch die durch den Kontext implizierten normativen Erwartungen, die im „Markedness Model“ (Myers-Scotton, 2006, S. $158 \mathrm{ff}$ ) akzentuiert wurden, eine zentrale Rolle spielen. Innerhalb eines professionellen Arbeitskontextes, in welchem lediglich die Verkehrssprache genutzt wird und der sich ansonsten durch einen hohen Grad an Formalität auszeichnet, werden selbst vertraute bilinguale Interaktanten, wie im Beispiel oben dargestellt, sehr wahrscheinlich im monolingualen Modus miteinander kommunizieren. So nutzen z. B. auch bilinguale SuS die Sprache zur Bearbeitung von Lerninhalten, die sie aus dem Unterricht gewohnt sind (vgl. Clarkson, 2007, S. 194).

Formale und inhaltliche Aspekte der zu kommunizierenden Information stellen einen weiteren Faktor zur Beeinflussung des Sprachmodus dar. So können z. B. die zu verwendende Varietät oder das Thema der Kommunikation samt der hierfür benötigten Lexik und grammatischen Strukturen bestimmte sprachliche Anforderungen an die Kommunikation stellen, die eventuell nur in einer der beiden Einzelsprachen oder durch Zuhilfenahme von Code-Switching und -Mixing von den Gesprächspartnern bewältigt werden können.

Die intendierte Funktion des jeweiligen Sprechaktes kann ebenfalls die Wahl des Sprachmodus beeinflussen. So kann z. B., wie bereits bezüglich der "Communication Accommodation Theory“" (vgl. Sachdev, Giles \& Pauwel, 2013) dargestellt, ein Sprachwechsel oder ein distinktes sprachliches Verhalten als der Gesprächspartner Distanz schaffen, Abgrenzung markieren oder gar jemanden ausschließen. Unter Verwendung von Code-Switching und -Mixing können auch eine Reihe weiterer rhetorisch-diskursiver Zwecke realisiert werden wie z. B. die Betonung einer Information, die Markierung eines Widerspruchs oder des Einverständnisses mit dem zuvor Gesagten, welche in Abschnitt 3.5.2 näher ausgeführt werden.

Abschließend gilt es spezifische Forschungsfaktoren zu berücksichtigen, die sich auf den Sprachmodus von bilingualen Sprechern während einer wissenschaftlichen Untersuchung auswirken können. So können z. B. die jeweiligen Ziele der Studie sowie das implizite oder explizite Wissen der Probanden über die Studie, die eingesetzten Stimuli und der Untersuchungskontext den Sprachmodus von bilingualen Sprechern beeinflussen. Dementsprechend sollten bei Studien der Bilingualismusforschung die potentiell durch den Untersuchungsaufbau implizierten Sprachmodi weitestgehend kontrolliert werden, um mögliche Konfundierungen zu vermeiden (vgl. Grosjean, 2008, S. 56). Grosjean (ebd., S. 57ff) schlägt vor, abhängig vom Forschungsinteresse eher einen monolingualen oder bilingualen statt eines gemischtsprachigen Sprachmodus zu etablieren, um die einzelsprachlichen oder bilingualen Kompetenzen, Einstellungen, Sprachpraktiken etc. trennscharf zu erfassen und Interferenzen zu vermeiden. Hierfür sollen je nach Sprachmodus mono- oder bilinguale Testleitungen sowie ein- oder zweisprachige Materialien eingesetzt werden. So sollte z. B. eine einzelsprachliche Sprachdiagnostik von einem monolingualen 
Sprecher mit dem bilingualen Probanden durchgeführt werden, damit der bilinguale Proband nicht in einem gemischtsprachigen oder bilingualen Sprachmodus interagiert, welche zu Sprachwechseln und -mischungen führen und somit (Teil-)Antworten in der nicht zu testenden Sprache elizitieren könnten (vgl. Kroffke \& Rothweiler, 2004).

Weitere Autoren stellen ähnliche Taxonomien potentieller Faktoren auf, die den Sprachgebrauch bilingualer Sprecher beeinflussen. So postuliert Gardner-Chloros (2009, S. 42-43) sprecherabhängige (z. B. Kompetenz, soziales Netzwerk, Einstellungen, Selbst- und Fremdwahrnehmung etc.) und sprecherunabhängige Faktoren (ökonomische Marktkräfte, Prestige, Machtrelationen, Assoziationen von Varietäten und Lebensstil etc.) sowie konversationelle Ursachen im Sinne diskursiv-rhetorischer Funktionen als einflussnehmende Faktoren, wobei diese Sets von Faktoren diverse Überschneidungen aufweisen können und sich zum Teil gegenseitig bedingen. Ritchie \& Bhatia (2013, S. 386) führen zusammenfassend die Sprecher, situationelle und sozialpsychologische Faktoren (z. B. Dominanzverhältnisse, Gruppenzugehörigkeit) sowie pragmatische, diskursiv-rhetorische Funktionen als mögliche Motivationen für Sprachwechsel und -mischungen an: „Based on a number of factors such as with whom (participants: their backgrounds and relationships), about what (topic, content), when and where a speech act occurs, bilinguals make their language choice" (ebd., S. 378). Dementsprechend herrscht zwischen den verschiedenen Ansätzen weitestgehend Einigkeit hinsichtlich der übergeordneten Faktoren Sprecher, Kontext und Konversationsmerkmale als zentrale Einflussfaktoren auf den Sprachgebrauch bilingualer Sprecher.

Die Vielzahl der potentiellen Einflussfaktoren auf das Sprachverhalten bilingualer Sprecher, deren impliziter Charakter und die zum Teil engen Zusammenhänge dieser erschweren eine klare, trennscharfe Identifikation der in einer Interaktion momentan wirkenden Determinanten des bilingualen Sprachgebrauchs. Kommunikation an sich und einige der oben angeführten Einflussfaktoren wie kontextuelle Faktoren oder das Gesprächsthema stellen dynamische Konstrukte und Entitäten dar, die sich im Verlauf einer Interaktion verändern können. Die Gesprächspartner sind sich den konkreten Ursachen ihres Sprachgebrauchs nicht zwingend bewusst und können in der impliziten Wahrnehmung dieser und ihren jeweiligen (übergeordneten) Kommunikationszielen deutlich divergieren, sodass sie auch in unterschiedlichen Sprachmodi miteinander kommunizieren können (vgl. Grosjean, 2008, S. 45f). 


\subsubsection{Empirische Evidenz zur Sprachwahl und zum Sprachgebrauch bilingualer Sprecher}

Empirische Untersuchungen im Feld (vgl. z. B. Poplack, 1981; Treffers-Dallers, 1998) als auch in experimentellen Settings (vgl. z. B. Grosjean, 1997) bestätigen die Postulate der Sprachmodi nach Grosjean (2008) sowie deren Einflussfaktoren. So liefert z. B. Poplack (1981) Evidenz für den Einfluss der Gesprächsteilnehmer und des Kontextes auf den Sprachmodus bei bilingualen Sprechern. Die aus Puerto Rico stammenden und in New York lebenden bilingual spanisch-englischsprachigen Probanden wiesen eine vier- bis zu fünffach höhere Prävalenz von Sprachwechsel- und -mischungsphänomenen in informellen und umgangssprachlichen Gesprächen mit bilingualen Sprechern der gleichen Gemeinschaft auf als in formellen Interaktionen mit diesen und in informellen Unterhaltungen mit bilingualen Personen, die nicht zur gleichen Gemeinschaft gehören. Grosjean (1997) ließ bilinguale Probanden ein Telefonat mit ihnen unbekannten Personen führen. Vor dem Telefonat erhielten die Probanden biographische Skizzen zu ihren Gesprächsteilnehmern, was ausreichte, um die verschiedenen Sprachmodi in erwartungsgemäßer Weise zu etablieren.

Die Form und Funktion von Sprachwechseln und -mischungen scheint sich im Verlauf des bilingualen Spracherwerbs zu entwickeln: ,[...] some studies suggest that children's codeswitching patterns change over time, with early codeswitching consisting mainly of lexical items and later codeswitching consisting of longer constituents" (Potowski, 2009, S. 89). Potowskis (ebd., S. 8992) Überblick zur bisherigen Evidenz weist auf alters- und kompetenzabhängige Unterschiede hinsichtlich der Form von Sprachwechsel und -mischungsphänomenen von Kindern hin, was wiederum eine zu Grunde liegende Entwicklungslogik vermuten lässt. So weisen z. B. zehnjährige bilinguale Kinder eine höhere Prävalenz sowie eine größere Breite an diversen Funktionen von Code-Switching und -Mixing im Gegensatz zu Siebenjährigen auf (Reyes, 2004). Andere Autoren wie z. B. Cantone (2007) hingegen argumentieren, dass Sprachwechsel- und -mischungsphänomene von Kindern wie die von Erwachsenen analysiert werden können, da die Quantität dieser auf eigenen Entscheidungen und nicht auf weiteren Faktoren wie Sprachentwicklung und Asymmetrien beruht.

Obwohl Sprachwechsel und -mischungsphänomene bilingualer Sprecher aus linguistischer Perspektive eine systematische, regelgeleitete kreative Facette ihres linguistisch-stilistischen Repertoires darstellen und auch als ein konstituierendes Merkmal der (Gruppen-)Identität von bilingualen Sprechern fungieren kann (vgl. z. B. Keim, 2007, 2012; Krumm, 2009; Stojanov, 2006) vertreten der Großteil bilingualer Sprecher negative Einstellungen zu CodeSwitching und -Mixing (vgl. Gardner-Chloros, 2009, S. 81f; Ritchie \& Bhatia, 2013, S. 389). Sie interpretieren Sprachwechsel und -mischungen als Zeichen 
von Faulheit, als ungewollte sowie „unreine“ Produktionen und als „linguistische Dekadenz", welche ihre sprachliche Performanz abwerten (vgl. Ritchie \& Bhatia, 2013, S. 389). Diese Einstellungen scheinen erlernt zu sein (vgl. Gardner-Chloros, 2009, S. 81f), da z. B. für die Gruppe griechisch-zypriotischer Einwanderer in London die negativen Einstellungen zu Sprachwechsel und mischungsphänomenen mit steigender Aufenthaltsdauer zunehmen (vgl. Gardner-Chloros, McEntee-Atalianis \& Finnis 2005). Zudem konnten GardnerChloros McEntee-Atalianis und Finnis (ebd.) für diese Gruppe noch einen negativen Zusammenhang von Bildungsgrad mit Einstellungen zu Code-Switching und -Mixing identifizieren, was auf assimilatorische Tendenzen hinweist.

\subsubsection{Diskursiv-rhetorische Zwecke von Code-Switching und - Mixing}

Hinsichtlich der Kommunikation mit anderen bilingualen Sprechern, die die gleichen Sprachen teilen, ermöglichen Sprachwechsel und -mischungen die Realisierung einer Vielzahl konversationeller Funktionen und rhetorischer Sprachpraktiken (vgl. z. B. Auer, 2009; Fuller, 2009, S. 120-129; GardnerChloros, 2009, S. 67ff; Keim, 2012, S. 157ff; McClure, 1981; Potowski, 2009; Reyes, 2004; Ritchie \& Bhatia, 2013, S. 384ff; Zentella, 1997). Code-Switching und -Mixing können als eine diskursive Strategie eingesetzt werden, die die „Einstellung des Sprechers zum Gesagten kontextualisieren kann und so dessen Bedeutung mit bestimmt [sic]“ (Auer, 2009, S. 102). Der Einsatz solcher diskursiver Elemente kann sowohl bei Kindern und Jugendlichen (vgl. z. B. Auer, 2009; Keim, 2012, S. 157ff; Potowski, 2009; Reyes, 2004) als auch bei Erwachsenen (vgl. z. B. Gardner-Chloros, 2009, S. 67ff; Ritchie \& Bhatia, 2013, S. 384ff) identifiziert werden. Die durch Sprachwechsel und -mischungen potentiell zu realisierenden konversationellen Funktionen operieren auf verschiedenen Diskurstypen wie z. B. narrative, dialogische, informationsvermittelnde Diskurse etc. sowie unterschiedlichen Diskurselementen wie z. B. Adressierungen, Reparaturen, Strukturierungen etc. (vgl. Keim, 2012, S. 157ff). Da das Erkenntnisinteresse der vorliegenden Arbeit nicht auf konversationellen Funktionen von Code-Switching und -Mixing fokussiert ist, erscheint eine exhaustive Darstellung aller potentiell zu realisierender Funktionen nicht zielgerichtet, sodass im Folgenden lediglich einige typische Zwecke dargestellt werden.

Sprachwechsel und -mischungen stellen eine Möglichkeit zur Überwindung von Verständnis- sowie Formulierungsproblemen und zur Markierung von Reparaturen dar (vgl. Keim, 2012, S. 157). So können z. B. lexikalische Lücken durch Entlehnungen aus der anderen Sprache gefüllt werden (vgl. 
Potowski, 2009, S. 95), um das Kommunikationsziel zu erreichen. Äußerungsabbrüche, Korrekturen, Reformulierungen können durch Sprachwechsel markiert werden, sodass der Rezipient diese eventuell besser verarbeiten kann. Reformulierungen sowie Übersetzungen können hierbei z. B. den zu illustrierenden Sachverhalt oder die zu kommunizierende Information in einer abgewandelten Weise darstellen, um das Verständnis zu erhöhen (vgl. Moschkovich, 2007).

Sprachwechsel eignen sich insbesondere in dialogischen und narrativen Diskursen zur Organisation und Strukturierung dieser (vgl. Auer, 2009, S. 98; Keim, 2012, S. 158f; Reyes, 2004, 84f). So können Sprachwechsel in dialogischen Diskursen z. B. Sprecher-, Situations-, Themenwechsel und Sprechakte wie Fragen oder Befehle markieren (vgl. Reyes, 2004, S. 84f) sowie Handlungen oder Handlungsstränge (vgl. Keim, 2012, S. 159) voneinander abgrenzen. Bei der Aushandlung von Streitigkeiten kann durch das Beibehalten der Sprache des vorausgegangenen Redebeitrags des Gesprächspartners Zustimmung signalisiert werden, während durch einen Sprachwechsel Widerspruch markiert werden kann (vgl. Keim, 2012, S. 167ff). Zudem können mit Sprachwechseln Adressierungen vorgenommen werden (vgl. Keim, 2012, S. 158f; Reyes, 2004, S. 84f), um Adressaten zu wechseln, auszuschließen oder bestimmten Adressaten exklusive Inhalte zu vermitteln. In narrativen Diskursen lassen sich indirekte und direkte Redewiedergaben sowie Zitate und Imitationen (vgl. Auer, 2009, Keim, 2012, S. $161 \mathrm{ff}$; Reyes, 2004, S. 84f) durch Sprachwechsel und -mischungen markieren, was auch zur Steigerung der Authentizität der Erzählung beitragen kann.

Weiterhin können Äußerungen Nachdruck verliehen werden, indem diese in der anderen Sprache wiederholt werden (vgl. Auer, 2009, S. 97; Potowski, 2009, S. 94). Die oben bereits angesprochenen Übersetzungen und Reformulierungen von Äußerungen in einer anderen Sprache können ebenfalls zum Zwecke der Erläuterung oder Erklärung eines Sachverhalts eingesetzt werden, um die Wahrscheinlichkeit des Verständnisses dieser zu erhöhen (vgl. Kern, 1994; Moschkovich, 2007). Eventuell weisen bestimmte Lexeme der einen Sprache eine geeignetere Semantik und Konnotation als die in der anderen auf, was zu einer semantischen Präzisierung sowie einer Erhöhung der Realisierung der Äußerungsintention führen kann.

Die oben angeführten konversationellen Funktionen von Sprachwechsel und -mischungen können prinzipiell auch von monolingualen Sprechern mit anderen para- und nonverbalen Mitteln realisiert werden (vgl. Gardner-Chloros, 2009, S. 77; Fuller, 2009, S. 116). Hierfür eignen sich zum Beispiel prosodische Markierungen wie Betonungen oder Stimmhöhenwechsel sowie Zeigegesten oder Blickkontakt. Über diese para- und nonverbalen Mittel verfügen selbstverständlich auch bilinguale Sprecher, sodass durch die Kombination 
von Sprachwechseln und typischen prosodischen und nonverbalen Markierungen, die Salienz der konversationellen Funktion noch weiter erhöht werden kann.

\subsubsection{Zusammenfassung}

Die Sprachwahl und der Sprachgebrauch bilingualer Sprecher hängen primär von den Gesprächsteilnehmern, dem Kontext und dem Inhalt der Konversation ab. Dementsprechend ist es nicht verwunderlich, dass bilingual aufwachsende Kinder und Jugendliche in Deutschland im familiären Umfeld (vgl. Reich, 2009) und in ihrer bilingualen Peer-Group (vgl. Keim, 2012) in ihren beiden Sprachen interagieren. Die Identifikation der im konkreten Moment wirkenden beeinflussenden Faktoren, die den Sprachmodus letztlich bestimmen, stellt ein schwieriges Unterfangen dar, da die einzelnen Faktoren sich gegenseitig bedingen, den Sprechern in der Regel nicht bewusst sind und nicht stabil sein müssen, sodass sich die Gesprächsteilnehmer innerhalb eines Gesprächs dynamisch auf dem Kontinuum der Sprachmodi bewegen können (vgl. Grosjean, 2008). Insbesondere hervorzuheben ist die Instabilität der Faktoren während einer Konversation, da z. B. eine sich verändernde Konstellation der Gesprächsteilnehmer, ein Orts- oder Themenwechsel bereits zu Änderungen des Sprachmodus führen können.

Sprachwechsel- und -mischungsphänomene treten sowohl in Form als auch Funktion in vielfältiger Weise auf. Die Formen von Code-Switching und -Mixing scheinen gewisse Entwicklungstendenzen aufzuweisen (vgl. Potowski, 2009) und unterliegen grammatischen Beschränkungen, sodass folglich keine arbiträren, sondern interindividuell ähnliche Sprachmischungen über die gleichen Sprachpaare produziert werden. Sprachwechsel- und -mischungsphänomene stellen angesichts des „complementary principle“ (vgl. Grosjean, 2010, S. 29ff) bei fehlendem einzelsprachlichen Wissen oder Fähigkeiten eine kreative und legitime Kompetenz zur Überwindung von Ausdrucks- und Verständnisproblemen dar. Erst durch die Nutzung beider Sprachen in Formen des Code-Switchings und -Mixings, was in vielen Fällen die typische Sprachsozialisation bilingual aufwachsender Sprecher darstellt, können bilinguale Sprecher auf ihr komplettes Vermögen sprachlicher Ressourcen zurückgreifen. Defizitperspektiven auf Sprachwechsel und -mischungen sind angesichts der Erkenntnisse der Bilingualismusforschung nicht haltbar. Diesbezüglich gilt es auch bilinguale Sprecher selbst zu überzeugen, die negative Einstellungen zu Sprachmischungen im Kontext der Migration mit steigender Aufenthaltsdauer entwickeln (vgl. Gardner-Chloros, McEntee-Atalianis \& Finnis, 2005). Dementsprechend scheinen politisch-gesellschaftliche Kontexte, die sprachideologisch einen gewissen Assimilationsdruck ausüben, sich nicht nur auf die Einstellungen zu Einzelsprachen selbst auszuwirken, sondern ebenfalls negative 
Einstellungen bei bilingualen Sprechern zu ihrem lebensweltlich bedingten, typischen Sprachverhalten hervorrufen zu können.

Kontexte, die bilinguale Praktiken verbieten und $\mathrm{zu}$ monolingualem sprachlichen Handeln zwingen, schränken nicht nur das Kommunikationspotential bilingualer Sprecher ein, sondern verwehren ihnen auch einen vollumfänglichen Rückgriff auf ihre sprachlichen Ressourcen als kognitive Werkzeuge. Zudem ist die Einhaltung konsequenter Sprachverbote schwierig umzusetzen, da die Wahl des Sprachmodus nicht zwingend der bewussten Kontrolle unterliegt. Diese beiden Aspekte werden im folgenden Abschnitt zu Bilingualität und Bildung näher betrachtet und ausgeführt.

\subsection{Bilingualität und Bildung}

Die Entwicklung der bilingualen Sprachkompetenz ist von den jeweiligen Erwerbs- und Lerngelegenheiten abhängig, die die Lebensrealität bilingualer Individuen zur Entwicklung ihrer Fähigkeiten in ihren Einzelsprachen bietet. Diese Gelegenheiten werden nicht nur von dem unmittelbaren Umfeld und der familiären Sozialisation bilingualer Sprecher determiniert, sondern werden ebenfalls von der vorherrschenden politisch-gesellschaftlichen Sprachideologie beeinflusst. Die Institution Schule stellt die zentrale Bildungsinstanz für Kinder und Jugendliche dar, in denen sie grundlegendes Wissen und Fähigkeiten erwerben, die sie zur Teilhabe an und Gestaltung des gesellschaftlichen Lebens befähigen. Die konkrete Ausgestaltung der in der Schule stattfindenden Lehr- und Lernprozesse determiniert die Erwerbs- und Lerngelegenheiten der $\mathrm{SuS}$ - insbesondere hinsichtlich bildungssprachlicher Varietäten - welche zum Teil auch durch die jeweilige vorherrschende Sprachideologie bedingt sind. Dementsprechend sollen im Folgenden grundlegende Positionen zu Bilingualität und Bildung dargestellt werden und monolinguale und bilinguale Schultypen hinsichtlich ihrer sprachlichen Handlungsräume und Eignung für eine bilinguale Schülerschaft näher betrachtet werden. Darauf aufbauend werden bilinguale Praktiken hinsichtlich ihres Bildungspotentials und ihrem konkreten Auftreten in Bildungsprozessen reflektiert. Hierbei werden auch die Einstellungen der zentralen Akteure der Schule - SuS sowie Lehrkräfte - zu bilingualen Praktiken in Bildungsprozessen dargestellt und diskutiert. Bevor auf diese Aspekte näher eingegangen werden kann, gilt es allerdings zunächst den Begriff der Bildung zu präzisieren. 


\subsubsection{Begriffsbestimmung Bildung}

Eine allgemeingültige Definition von „Bildung“ für den erziehungswissenschaftlichen Diskurs ist zum Scheitern verurteilt, da so viele verschiedene Bedeutungen von Bildung existieren, ,wie es Varianten von Erziehungswissenschaft gibt" (Tenorth, 2007, S. 94). Im Folgenden wird der Bildungsbegriff nach Stojanov (2006, S. 19-67) präferiert, da dieser an die grundlegenden sowie bedeutsamen Vorarbeiten zu Bildung von Dewey (1916) und Humboldt (1905) anknüpft und unter Rekurs auf McDowell (1996) einen für die Gegenwart adäquaten Bildungsbegriff erzeugt. Zudem unterzieht Stojanov (ebd., S. 54-66) „Bildung“ einer intersubjektivitätstheoretischen Rekonstruktion, die in einer hohen Anschlussfähigkeit an anerkennungstheoretische Reflexionen (vgl. Balzer \& Ricken, 2010; Honneth, 2003; Mecherill, 2005; Stojanov, 2011) mündet, hervorragend mit den von Hamers und Blanc (2000) postulierten übergeordneten Funktionen beziehungsweise Kompetenzen von Sprache vereinbar ist und zentralen Thesen sozial-konstruktivistischer Ansätze entspricht (vgl. Hogan \& Tudge, 2009).

Unter Verwendung der begriffsanalytischen Methode (vgl. Stojanov, 2006, S. 21-25) arbeitet Stojanov (ebd., S. 25-27) zunächst zwei widersprüchliche Bedeutungen des alltagssprachlichen Bildungsbegriffes heraus. Dieser wird „einerseits als Selbstbestimmung und andererseits als pädagogische Unterweisung"verwendet (ebd., S. 26). Verstanden als Selbstbestimmung verweist der Bildungsbegriff auf die Entwicklung eines Individuums zu einem autonomen Subjekt, wobei Autonomie zum einen das Ziel dieser Entwicklung ist, zum anderen aber auch den Vorgang selbst beschreibt, ,der sich durch seine EigenLogik auszeichnet und sich von außen nicht vorbestimmen sowie nur begrenzt steuern lässt" (ebd., S. 25). Bildung als pädagogische Unterweisung in einem „Bildungswesen“ verkehrt das eben angeführte Verständnis dahingehend um, dass Bildung vor allem Wissensvermittlung in institutionalisierten, asymmetrischen Interaktionen bedeutet, in der die jüngere von der älteren Generation „,zu einem bestimmten Reifungsstand geführt und in die bestehende Gesellschaft eingegliedert werden sollen" (ebd.).

Diese semantische Differenz des alltäglichen Bildungsbegriffs stellt keine deutschsprachige Besonderheit dar, sondern lässt sich auch in anderen Sprachen wie z. B. dem Russischen finden (vgl. ebd., S. 28). Zur Zusammenführung des eben dargestellten Widerspruchs der zentralen Bedeutungskomponenten des alltäglichen Bildungsbegriffs schlägt Stojanov (ebd. S. 26) vor, „dass die Formung und Entfaltung eines autonomen Individuums“ als „,interaktiv vermittelt" im Sinne einer „Initiierung und Ermöglichung der individuellen Entwicklung" zu verstehen ist. Hierbei greift Stojanov (ebd., S. 28-33) sowohl auf Deweys (1916) Begriff der „Education“, welcher die eben erwähnte Annäherung von autonomer Eigenentwicklung und externer Wissensvermittlung in Interaktionen in sich vereint, als auch auf Humboldts (1905) 
Differenzierung von „Welt“ und „Umwelt", in der die Umwelt als wahrzunehmende Entität zu verstehen ist, welche es auf abstrakte Allgemeinheiten der Welt zu transzendieren gilt, zurück. Weiterhin inkorporiert Stojanov (ebd.) das kritische, hinterfragende Moment von Bildung nach McDowell (1996) in seinen wissenschaftlichen Bildungsbegriff. Nach McDowell (1996) sind Individuen ,ansozialisierten und habituell überlieferten Selbst- und Wirklichkeitswahrnehmungsmustern“ ausgeliefert, welche sich „weitgehend seiner Reflexion und seiner willentlichen Entscheidungsmacht" entziehen. Nichtsdestotrotz sind sie in der Lage, ,die intelligiblen, ,welthaften“ Realien wie eben Normen und Gründe" wahrzunehmen, zu reflektieren und sie hinsichtlich ihrer Existenzberechtigung, Funktionalität sowie Gültigkeit kritisch zu prüfen (Stojanov, 2006, S. 32). Der zuletzt genannte Prozess des kritischen Reflektierens und Prüfens ,findet [...] in den faktisch-sozialen Verflechtungen des Einzelnen, in den konkreten, ihm vorgegebenen Umwelten seines Aufwachsens statt“ und stellt letztlich das dar, was als Bildung zu bezeichnen ist (ebd.).

Ausgehend von diesen Rekonstruktionen der Bedeutungsfacetten des alltagssprachlichen und wissenschaftlichen Bildungsbegriffs umfasst nach Stojanov (ebd., S. 33-35) der aktuelle Bildungsbegriff unter ,posttraditionellen Bedingungen“ (vgl. ebd., S. 38-41) drei zentrale Komponenten: 1) Der Vorgang der Selbst-Entwicklung als „Entwicklung eines eigenständigen Selbst“ und als „eigenlogischer Prozess der Selbstentwicklung (ebd., S. 33). 2) Dieser Vorgang verschränkt sich mit der Welt-Erschließung, wobei „Welt“ vor allem als ,konzeptuelle Inhalte, die auf Gründe und Normen verweisen und eine universelle bzw. transkontextuelle Gültigkeit beanspruchen“ (ebd., S. 34), zu verstehen ist. 3) Diese beiden parallel stattfinden Prozesse ereignen sich in den ,alltäglichen pluralen und interaktiv strukturierten sozialen Erfahrungen des Einzelnen“" (ebd., S. 35):

„Der gegenwärtige Bildungsbegriff bezeichnet in seinem Kern den parallelen Vorgang der Selbst-Entwicklung und der Welt-Erschließung des Einzelnen. Unter posttraditionellen Bedingungen lässt sich dieser Vorgang nicht mehr als eine Hinführung in den objektiven Geist bzw. als eine Aneignung eines Kanons hochkultureller Objektivationen angemessen auffassen. Vielmehr muss der Bildungsprozess als sich in alltäglich-sozialen Interaktionserfahrungen ereignend konzeptualisiert werden." (ebd. S. 66)

Dementsprechend behält der Bildungsbegriff nach Stojanov (ebd.) seine zentrale und prominente Bedeutungskomponente einer "Selbstentwicklung zur Autonomie", wobei die hierzu scheinbar widersprüchliche Bedeutungskomponente einer pädagogischen Unterweisung durch soziale Interaktionserfahrungen versöhnlich integriert wird. Die in diesen Prozessen stattfindende WeltErschließung fokussiert als Erwerbsgegenstand auf Normen und Werte verweisende, verbindliche Universalien, die allerdings hinsichtlich ihrer Gültigkeit der Reflexion des Einzelnen offen stehen. Ein solcher Bildungsbegriff ist 
anwendbar auf das volle Spektrum von informellen, spontanen und eigenverantwortlichen bis formellen, geplanten und institutionalisierten Bildungsprozessen und akzentuiert die Intersubjektivität des Subjekt-Objekt-Dualismus der Selbstentwicklung und Welt-Erschließung.

\subsubsection{Grundlegende Positionen zum Zusammenhang von Bilingualität und Bildung}

Die herausragende Bedeutung von Sprache und sprachlichen Fähigkeiten für Bildung ist in der öffentlichen und wissenschaftlichen Diskussion unumstritten (vgl. z. B. Cummins, 2000; Gogolin, 2013; Kempert et al., 2016; Schleppegrell, 2004). Die drei zentralen Komponenten der „Selbst-Entwicklung“ und „Welt-Erschließung“ durch „alltäglich-soziale Interaktionserfahrungen“ des Bildungsbegriffs nach Stojanov (2006, S. 66) sind auf Sprache und sprachliche Fähigkeiten angewiesen. Die von Hamers \& Blanc (2000, S. 123) postulierten übergeordneten Funktionen oder Kompetenzen von Sprache zur Kommunikation, als kognitives Werkzeug und als Reflexionsgegenstand selbst weisen auf die Relevanz von Sprache zum Erwerb von Wissen und Fähigkeiten hin. Sprache wirkt durch Kommunikation als Medium zum Erlernen von Wissen sowie Fähigkeiten und konstruiert durch Informationsverarbeitung sowie mentale Modellbildung die Repräsentation des Erlernten. Zum einen stellen sprachliche Rezeptionsfähigkeiten die "Grundlage für das Instruktionsverständnis“ und somit eine wichtige „Determinante des Schulerfolgs“ (Schrader, 2006, S. 571) dar. Zum anderen sind produktive sprachliche Fähigkeiten zentral für typische Praktiken der Zertifizierung von Bildungsprozessen durch schriftsprachliche oder mündliche Prüfungsformen, insbesondere da deren Bewertung nicht nur von der inhaltlichen Korrektheit abhängt, sondern zu einem gewissen Anteil stets auch von der sprachlichen Form abhängt.

Hierbei scheinen bildungsbezogene Sprachvarietäten wie CALP oder Bildungssprache von größerer Bedeutung als basale Kommunikationsfähigkeiten zu sein. Eine Vielzahl empirischer Studien weist anhand diverser Indikatoren wie z. B. der Einschulungsvoraussetzungen, der Leistungsentwicklung auch in Sachfächern wie Mathematik oder des Übergangs von Primar- zu Sekundarstufe I auf die Bedeutung sprachlicher Fähigkeiten in der Unterrichtssprache als zentrale Fähigkeit für Bildungserfolg hin (für einen Überblick siehe Kempert et al., 2016, S. 167-180). Für in Deutschland aufwachsende Kinder und Jugendliche mit MH, die häufig Deutsch als L2 erwerben (vgl. Schulz \& Tracy, 2011), „lässt sich festhalten, dass über die gesamte Alterspanne von der Kindergartenzeit bis hin zum Ende der Sekundarstufe I deutliche Disparitäten in der Verkehrssprache Deutsch“ im Vergleich mit monolingual aufwachsenden SuS nachgewiesen wurden (Kempert et al., 2016, S. 167). Zwar kann ein Großteil dieser Disparitäten für die meisten Herkunftsgruppen durch die Kontrolle 
von sozioökonomischen Merkmalen aufgeklärt werden, aber ein gewisser Anteil unaufgeklärter Varianz bleibt z. B. für die Gruppe der Kinder und Jugendlichen mit türkischem Migrationshintergrund bestehen (vgl. ebd., S. 167). Dementsprechend kann die Bildungsbenachteiligung von Kindern und Jugendlichen mit Migrationshintergrund in Deutschland (vgl. Haag, 2012; Haag et al., 2016; Rauch et al., 2016; Tarelli, Schwippert \& Stubbe, 2012; Wendt \& Schwippert, 2017) neben soziökonomischen Gründen zumindest zu einem gewissen Teil auch auf sprachliche Fähigkeiten zurückgeführt werden.

Die Organisation und Ausgestaltung von (bilingualen) Bildungssystemen basiert nicht nur auf normativen, pädagogischen, psychologischen und didaktischen Prinzipien, sondern inkorporiert auch immer soziokulturelle, politische und ökonomische Aspekte (vgl. Baker, 2011, S. 208). Der Wert, der der Bilingualität für institutionalisierte Bildungsprozesse sowie für die Gesellschaft zugesprochen wird, determiniert den Umgang mit der natürlich erworbenen Mehrsprachigkeit von SuS sowie den Erwerb von Fremdsprachen im schulischen Kontext. Für das deutsche Bildungssystem kann konstatiert werden, dass dem Erwerb bestimmter Fremdsprachen wie z. B. Englisch, Französisch oder Latein eine hohe Bedeutung beigemessen wird, da diese Sprachen bereits früh und langfristig im Curriculum verankert sind. Allerdings wird der Nutzen sowie Umgang mit der natürlich erworbenen Mehrsprachigkeit von SuS, die keine der Sprachen des Fremdsprachenkanons, sondern die Sprachen ihrer eingewanderten Eltern oder Großeltern wie z. B. Türkisch, Italienisch, Polnisch etc. natürlich erworben haben, im wissenschaftlichen Diskurs kontrovers diskutiert (für einen Überblick siehe z. B. Gogolin \& Neumann, 2009). Hierbei stehen sich primär zwei divergierende Überzeugungen gegenüber, die auf unterschiedlichen theoretischen Annahmen beruhen.

Die Vertreter eines nicht wertschätzenden Umgangs samt weniger Angebote zur Förderung bilingualer Fähigkeiten sehen in der (weiteren) L1 Bilingualer, die nicht die Verkehrs- oder Unterrichtssprache darstellt, keine Ressource für Bildungsprozesse (vgl. z. B. Esser, 2009). Diese Vertreter berufen sich häufig auf die „Time-on-Task“" Hypothese, die ursprünglich von Carroll (1963) aufgestellt wurde und in ihrem Kern schulisches Lernen als Funktion von aufgewandter und benötigter Lernzeit definiert. Die benötigte Lernzeit ergibt sich aus der individuellen Begabung und dem Vorwissen des Lerners sowie der Qualität der Instruktion. Die aufgewandte Lernzeit wiederum besteht zum einen aus der zur Verfügung gestellten Lernzeit zum Erwerb des Lerngegenstandes und der von den SuS tatsächlich aufgebrachten Zeit, in der sie sich mit dem Lerngegenstand auseinandersetzen. Die Abhängigkeit des Lernerfolgs von der Funktion aufgewandter und benötigter Lernzeit wird als Argument eingesetzt, Bildungsprozesse von mehrsprachigen Kindern in der Bundesrepublik Deutschland nur einsprachig in der Instruktionssprache stattfinden zu lassen, um die Gelegenheiten zum Erwerb (bildungs-)sprachlicher Fähigkeiten in der 
Instruktionssprache zu maximieren, auf die es hinsichtlich Bildungserfolg und Chancen auf dem Arbeitsmarkt ankommt (Esser, 2009).

Angesichts der Inputabhängigkeit des Spracherwerbs und den durch das „complementary principle“ (vgl. Grosjean, 2010, S. 29ff) quantitativ sowie zum Teil auch qualitativ reduzierten Lerngelegenheiten zum Erwerb der Einzelsprachen bei bilingual aufwachsenden Sprechern erscheint die „Time-onTask" Hypothese (vgl. Carroll, 1963) für den Erwerb sprachlicher Fähigkeiten intuitiv einleuchtend. Es gibt zwar empirische Evidenz aus dem Wortschatzund Morphosyntaxerwerb des L2a und 2L1a, dass es lediglich einen kritischen Schwellenwert an Input zu überschreiten gilt, um vergleichbare (konzeptionell) mündliche morphosyntaktische und lexikalische Kompetenzen wie monolingual aufwachsende Sprecher zu erlangen (vgl. Gathercole, 2016, S. 130; Unsworth, 2016, S. 111). Aber nichtsdestotrotz benötigt es einzelsprachliches Wissen und dementsprechend Input sowie Output - insbesondere hinsichtlich idiosynkratrischer linguistischer Elemente und Strukturen einer Einzelsprache - um eine Sprache zu erwerben. Ebenfalls sind allgemeine Erfahrungen, Einsichten sowie (meta-)linguistische Erkenntnisse zur Form und Funktion (konzeptionell) schriftsprachlicher Varietäten für die Sprachkompetenz relevant, um Sprache als kognitives Werkzeug verwenden zu können (vgl. Hamers \& Blanc, 2000, S. 116-120). Auch bei diesen Varietäten benötigt es neben allgemeinen Erkenntnissen zum dekontextualisierten, akademisch anspruchsvollen Sprachgebrauch auch Wissen darüber, mit welchen sprachlichen Mitteln diese Varietät in den jeweiligen Einzelsprachen umzusetzen sind (Heppt et al., 2014, S. 140). Dementsprechend erweist sich die Auslegung der „Time-on-Task“ Hypothese nach Carroll (1963) als äußerst anschlussfähig für die Sprachentwicklung.

Die Befürworter eines die Mehrsprachigkeit fördernden und wertschätzenden Umgangs mit der Bilingualität von SuS berufen sich auf diverse Argumente, die zum Großteil in der vorliegenden Studie bereits diskutiert oder zumindest angeschnitten wurden. So werden unter anderem die spracherwerbsbedingten Unterschiede zwischen mono- und bilingual aufwachsenden Kindern und folglich die Notwendigkeit der Berücksichtigung dieser differenten Voraussetzungen in der Schule durch didaktisch angemessene, adaptive Konzepte akzentuiert. Weiterhin stellen das Anknüpfen an vorschulische Bildungsprozesse in der familiären Sozialisation und der Einbezug des Potentials metalinguistischer Kompetenzen bilingual aufwachsender SuS potentielle Ressourcen für institutionalisierte Bildungsprozesse dar. Zudem wird auf die möglichen positiven (meta-)kognitiven Konsequenzen von Bilingualität sowie auf die Bedeutung von Sprache für die Identität verwiesen. Auch bilinguale Praktiken wie Sprachwechsel und -mischungen akzentuieren die Tatsache, dass das sprachliche Vermögen Bilingualer zur Kommunikation und als kognitives Werkzeug an mehr als eine Einzelsprache gebunden ist, sodass monolinguale Zwänge auch mit einer Reduktion dieses Vermögens einhergehen. Im Zentrum 
der Argumentation der Vertreter eines die Mehrsprachigkeit fördernden und wertschätzenden Umgangs mit der natürlich erworbenen Bilingualität von SuS steht allerdings häufig der Zusammenhang von (konzeptionell) schriftsprachlichen, kognitiv-akademischen sprachlichen Fähigkeiten der beiden Sprachen von bilingualen SuS. So kann diese Varietät in der verkehrssprachlichen L1 oder L2 gefördert werden, indem diese Varietät in der nicht-verkehrssprachlichen L1 ebenfalls ausgebaut wird. Einen theoretischen Überbau für diese Annahme liefert Cummins“ (2000) „Threshold-“ und „Interdependenzhypothese“, die im Folgenden näher ausgeführt werden.

\subsubsection{Die Threshold- und Interdependenzhypothese nach Cummins (2000)}

Cummins (2000, S. 174) formulierte die „Thresholdhypothese“, im Deutschen auch als „Schwellenwerthypothese“ bezeichnet, und die „Interdependenzhypothese" aufgrund der zwei folgenden Beobachtungen: 1) Viele bilingual aufwachsende SuS scheitern im Bildungssystem und weisen eine geringe Literacy in ihren beiden Sprachen auf, wenn sie Schulen besuchen, in denen nur exklusiv in der Verkehrssprache unterrichtet wird, die für viele dieser Kinder die L2 darstellt. Allerdings scheinen im Gegensatz hierzu bilingual aufwachsende SuS, die im schulischen Kontext in ihren beiden Sprachen unterrichtet werden, positive Effekte auf ihre kognitiven und akademischen Fähigkeiten zu erfahren. 2) Im Gegensatz zu den Vorhersagen der ,Time-on-Task“ Hypothese (vgl. Carroll, 1963) wirkt sich Unterricht in der nicht-verkehrssprachlichen L1 bilingualer SuS im Kontext diverser bilingualer Schulprogramme nicht negativ auf ihre akademische Entwicklung in der Verkehrssprache aus. Dies gilt für alle SuS unabhängig davon, ob sie mit ihrer L1 zur Minderheit oder Mehrheit der Gesellschaft gehören. Diese zwei Beobachtungen sowohl eines potentiellen Zusammenhangs der akademisch-sprachlichen Fähigkeiten der beiden Einzelsprachen als auch die Einschränkung der Auslegung der „Time-on-Task“ Hypothese (vgl. ebd.) für die akademische Entwicklung in der Verkehrssprache führen dementsprechend zur Notwendigkeit einer Reflexion der Bedeutung sprachlicher Fähigkeiten der nicht-verkehrssprachlichen L1 für Bildungsprozesse.

\subsubsection{Die Thresholdhypothese nach Cummins (2000)}

Die Thresholdhypothese fokussiert den Zusammenhang von kognitiv-akademischen sprachlichen Fähigkeiten der beiden Sprachen bilingualer Sprecher. Wenn sich akademisch-sprachliche Fähigkeiten in beiden Sprachen in schulischen Bildungsprozessen entwickeln können, dann ergeben sich nach Cummins (2000, S. 175) positive Effekte auf (meta-)linguistischer, akademischer 
und möglicherweise (meta-)kognitiver Ebene: „[...] the continued development of bilingual children's two languages during schooling is associated with positive educational and linguistic consequences. [...] bilingualism is not just a societal resource, it is also an individual resource that potentially can enhance aspects of bilingual children's academic, cognitive and linguistic functioning". Dementsprechend scheinen bilingual aufwachsende Kinder und Jugendliche von der schulischen Förderung kognitiv-akademischer sprachlicher Fähigkeiten ihrer beiden Sprachen stark zu profitieren, während eine exklusive Fokussierung auf die Verkehrssprache diese Effekte nicht hervorrufen kann.

Studien, die bilingual aufwachsende Kinder und Jugendliche hinsichtlich ihrer Sprachkompetenzprofile gruppieren und bezüglich diverser metalinguistischer, akademischer und non-verbaler kognitiver Fähigkeiten vergleichen, liefern Evidenz zur Unterstützung der Thresholdhypothese (vgl. ebd., S. 178182). So weisen z. B. die Ergebnisse von Ricciardelli $(1992,1993)$ darauf hin, dass bilinguale Kinder, die in ihren beiden Sprachen (Englisch und Italienisch) kompetent sind, gegenüber monolingualen Kindern mit hoher Sprachkompetenz im Englischen und bilingualen Kindern, die eine Asymmetrie zu Gunsten sprachlicher Fähigkeiten im Englischen aufweisen, signifikant bessere Leistungen bei metalinguistischen, verbalen und non-verbalen Aufgaben erbringen. Lasagabaster (1998) untersuchte den Zusammenhang der Sprachkompetenz in den Einzelsprachen und metalinguistischer Kompetenz bei Kindern und Jugendlichen, die in der Schule Baskisch, Spanisch und Englisch nutzen. Die Gruppe mit hoher einzelsprachlicher Kompetenz in allen Sprachen wies unter Kontrolle von non-verbalen Fähigkeiten, sozioökonomischen oder soziokulturellen Status signifikant höhere metalinguistische Fähigkeiten auf als alle anderen Gruppen.

Die Thresholdhypothese wurde im wissenschaftlichen Diskurs als auch in der pädagogischen Praxis häufig inkorrekt rezipiert, was auch zu unzulässigen praktischen Schlussfolgerungen führte (vgl. Cummins, 2000, S. 176f). So rezipiert z. B. Esser (2009, S. 78) als Beispiel für den deutschsprachigen Raum die Thresholdhypothese von Cummins (2000) folgendermaßen falsch: „Nur im Rahmen muttersprachiger Fertigkeiten ließe sich eine Zweitsprache erlernen, wie es die sog. Cummins-Hypothese besagt". Sowohl im deutschsprachigen Eintrag zu Jim Cummins (Wikipedia, 2018a) als auch im englischsprachigen Beitrag zur Hypothese selbst (Wikipedia, 2018b) in der freien Online-Enzyklopädie „Wikipedia“ stellt die Thresholdhypothese nicht korrekt dar. Allerdings gibt Cummins (2000, S. 175) selbst zu, dass die erste Postulierung und Ausführung der Thresholdhypothese in vielerlei Hinsicht zu vage war, was die häufigen Fehlinterpretationen zum Teil erklären könnte. Insbesondere die Postulierung von Schwellenwerten, die es zu erreichen gilt, um die oben angeführten positiven Effekte auf (meta-)linguistische, akademische und kognitive Fähigkeiten zu erhalten, führte zu diversen Fehlinterpretationen und 
falschen Schlussfolgerungen. Eine dieser Fehlinterpretationen ist die Annahme, dass ein bestimmter Schwellenwert sprachlicher Fähigkeiten der L1 zu erreichen sein muss, damit sich sprachliche Fähigkeiten in einer L2 entwickeln können (vgl. ebd., S. 176), was der oben angeführten Fehlinterpretation der „Cummins-Hypothese“ von Esser (2009, S. 78) nahe kommt. Eine solche These widerspricht natürlich der in Abschnitt 3.2 ausgeführten empirischen Evidenz zu bilingualen Spracherwerbstypen, die sich grob mit der Formel ,the younger, the better" (vgl. Singleton \& Ryan, 2004) zusammenfassen lässt. Je früher der Erwerbsbeginn einer weiteren L1 oder einer L2 beginnt, desto eher gleicht der Erwerb den Erwerbsverläufen monolingualer Lerner. Insbesondere Lerner, die eine L2 im Erwachsenenalter beginnen zu erwerben, weichen fundamental in quantitativen als auch qualitativen Aspekten vom monolingualen Spracherwerb und frühen bilingualen Spracherwerbstypen ab (vgl. Rothweiler, 2007).

Cummins (1991, S. 86) schätzt die Bedeutsamkeit der Identifikation der Schwellenwerte als weniger relevant ein als die praktischen Implikationen der Thresholdhypothese:

„[...] whether or not there are specific "thresholds" associated with these metalinguistic and cognitive outcomes is unclear. The issue may be only of academic interest since the practical implication of the data is the same: schools should attempt to encourage minority students to develop their L1 abilities to as great an extent as possible both to stimulate transfer to L2 and to reap the significant personal and more subtle educational benefits of additive bilingualism $[\ldots]^{\text {“". }}$.

Dementsprechend sollte nicht die Operationalisierung von sprachlichen Mindestkompetenzen als Schwellenwerte fokussiert, sondern didaktische Möglichkeiten und Angebote zur Förderung der nicht-verkehrssprachlichen L1 akzentuiert werden.

\subsubsection{Die Interdependenzhypothese nach Cummins (2000)}

Das soeben angeführte Zitat weist auch auf einen der möglichen Mechanismen hin, die die positiven Effekte durch balancierte Fähigkeiten der L1 und L2 bzw. der beiden L1 bedingen, und zwar auf Transfereffekte, die zentral für die Interdependenzhypothese sind. Die Interdependenzhypothese ist nach Cummins (2000, S. 175) relevant für das Verständnis der akademischen Ergebnisse bilingual aufwachsender SuS und für die Planung sowie Durchführung von angemessenen Bildungsprogrammen für diese Schülerschaft. Sie postuliert Transferprozesse von deklarativem sprachlichen Wissen sowie kognitiv-akademischen sprachlichen Fähigkeiten zwischen der L1 und L2 bzw. weiteren L1. „Transfer“ bezeichnet - wie in Abschnitt 3.3.2.4 bereits definiert - allgemein die Fähigkeit, „einmal Gelerntes auf neue Problemstellungen anzuwen- 
den“ (Schmöe, 2016b, S. 720). Im Kontext der Interdependenzhypothese können damit Prozesse beschrieben werden, bei denen einzelsprachenunabhängiges, deklaratives linguistisches Wissen sowie kognitiv-akademische sprachliche Fähigkeiten, die in einer Sprache enkodiert sind oder erworben wurden, auf die andere Sprache bilingualer Sprecher ,transferiert“ werden können. So können deklaratives linguistisches Wissen und kognitiv-akademische sprachliche Fähigkeiten wie z. B. phonologische Bewusstheit, kognitive Lernstrategien, Erkenntnisse zur Rechtschreibung oder Textkomposition zwischen Sprachen transferiert werden. Aber auch basale in der L1 erlangte Erkenntnisse im Kontext früher Literacyerfahrungen, wie z. B. dass Kohäsion und Referentialität erzeugende Diskurskonventionen beim Erzählen von Geschichten durch bestimmte lexikalische Mittel und syntaktische Strukturen realisiert werden, können auch auf das Erzählen von Geschichten in der L2 oder weiteren L1 transferiert werden. Hierbei müssen allerdings die für die L2 oder weiteren L1 spezifischen lexikalischen und syntaktischen Marker sowie Strukturen der Diskurskonventionen erworben werden, um diese beim Erzählen von Geschichten in der L2 oder weiteren L1 auch umsetzen zu können.

Die ursprüngliche Modellierung des Systems, welches Transferprozesse möglich macht, konzipierte Cummins (2000, S. 191) als ,common underlying proficiency“. In diesem System sind die kognitiv-akademischen Konzepte und Fähigkeiten sowie das deklarative linguistische Wissen enthalten, das durch eine oder beide Sprachen generiert wurde und welches auf beide Sprachen angewandt werden kann. Diese Modellierung würde Cummins (ebd.) mittlerweile eher als dynamisches, zentrales Verarbeitungssystem konzeptualisieren, durch welches die Interdependenz von L1 und L2 bzw. weiterer L1 aus drei potentiellen Quellen entstehen kann: 1) Durch die Verwendung derselben kognitiven und linguistischen Fähigkeiten für die Entwicklung der Literacy. 2) Durch den Transfer von allgemeinem konzeptuellen Wissen über die Einzelsprachen hinweg, wobei die L1 als Basis oder Schemata gilt, auf der der Erwerb in der L2 oder (weiteren) L1 aufbaut. 3) Durch den Transfer von linguistischem Wissen und Fähigkeiten, die sich in beiden Sprachen gleichen oder zumindest ähneln. Dementsprechend kann Transfer reziprok von Statten gehen und gelingt umso besser, je ähnlicher sich die L1 und L2 bzw. weitere L1 sind (ebd., S. 190). Diese Transferprozesse dürfen allerdings nicht zwingend als implizite, automatisch ablaufende Prozesse interpretiert werden, sondern bedürfen unter Umständen auch expliziter, formaler Unterweisung in beiden Einzelsprachen (vgl. ebd., S. 194).

Dementsprechend kann die Interdependenzhypothese als Gegentheorie zur „Time-on Task“ Hypothese bezüglich des Erwerbs kognitiv-akademischer sprachlicher Fähigkeiten in der L2 oder weiteren L1 betrachtet werden, insbesondere wenn die Quantität der Lerngelegenheiten als relevantester Prädiktor für den Erwerb kognitiv-akademischer sprachlicher Fähigkeiten in der der L2 oder weiteren L1 interpretiert wird (vgl. ebd., S. 174). Aus der Perspektive der 
Interdependenzhypothese kann die L1 von bilingual aufwachsenden Kindern eine Ressource zum Erwerb kognitiv-akademischer sprachlicher Fähigkeiten in der Instruktionssprache und für Lernprozesse im Allgemeinen darstellen (vgl. ebd., S. 173), sodass ein Teil der formalen Instruktionszeit in die Förderung ausgewählter erstsprachlicher Fähigkeiten investiert werden sollte. Im Gegensatz hierzu würde im Sinne der „Time-on-Task“ Hypothese nach Carroll (1963) die Ausbildung erstsprachlicher Fähigkeiten zur Förderung verkehrssprachlicher Fähigkeiten nicht zielführend erscheinen, da es zu einem Verlust potentieller Instruktionszeit zum Erwerb verkehrssprachlicher Fähigkeiten kommen würde.

Es existiert „overwhelming evidence in support of the interdependence hypothesis" (ebd., S. 174-175). Zur Überprüfung der Interdependenzhypothese sind zwei verschiedene empirische Zugänge zentral, die Cummins (ebd., S. 182-190) überblicksartig zusammenfasst: 1) Die Untersuchung von Zusammenhängen zwischen kognitiv-akademischer sprachlicher Fähigkeiten in L1 und L2 bzw. weiterer L1 sowie 2) Untersuchungen zum Transfer kognitiv-akademischen sprachlichen Wissens zwischen L1 und L2 bzw. weiterer L1 in verschiedenen mono- und bilingualen Schulformen (ebd., S. 182). Die Korrelationsstudien zur Überprüfung der Interdependenzhypothese vergleichen die Höhe des Zusammenhangs verschiedener sprachlicher Fähigkeiten über beide Einzelsprachen bei bilingualen Sprechern. So weist die Literacy in der L2 oder (weiteren) L1 einen höheren Zusammenhang mit Literacy in der L1 als mit mündlichen Fähigkeiten in der L2 oder (weiteren) L1 auf (vgl. California State Departement of Education, 1985). Auch Vorläuferfertigkeiten für den Leseerwerb wie die phonologische Bewusstheit scheinen über Einzelsprachen hinweg zusammenzuhängen. So erklärt die phonologische Bewusstheit im Spanischen $47 \%$ der phonologischen Bewusstheit im Englischen und phonologische Bewusstheit und Buchstabenerkennung im Spanischen klären insgesamt 84\% der Varianz der Buchstabierfähigkeit im Englischen bei spanisch-englischsprachig aufwachsenden Erstklässlern in bilingualen Schulprogrammen auf (vgl. Durgunoğlu, 1998). González $(1986,1989)$ diagnostiziert bei SuS desselben bilingualen Schulprogramms, dass spanisch-englischsprachige SuS, die vor ihrer Migration in die Vereinigten Staaten von Amerika in Mexiko für mindestens zwei Jahre beschult wurden, bessere Lesefähigkeiten in der L1 und L2, aber schlechtere mündliche sprachliche Fähigkeiten in der L2 im Vergleich zu bilingual spanisch-englischsprachig aufwachsenden SuS haben, deren Beschulung in den Vereinigten Staaten von Amerika begann. Zudem stehen auch Schreibfähigkeiten der L1 und L2 in Interdependenz zueinander, selbst wenn sich die Sprachen oder Schriftsysteme (z. B. Englisch und Japanisch) deutlich unterscheiden (vgl. Canale, Frenette \& Bélanger, 1987; Cumming, 1989). Die Korrelationen von sich ähnlichen Sprachpaaren fallen allerdings höher als bei unähnlicheren Sprachpaaren aus (vgl. Genesee, 1979). 
Diesen Studien entsprechend stellen die kognitiv-akademisch sprachlichen Fähigkeiten in der L1 aufgrund ihres Zusammenhangs mit diesen Fähigkeiten in der L2 oder weiteren L1 eine Ressource für die kognitiv-akademische Entwicklung der L2 oder weiteren L1 dar. Hierbei scheint die Dauer des Sprachkontakts zur Verkehrssprache für mündliche sprachliche Fähigkeiten äußerst relevant zu sein, während für kognitiv-akademische sprachliche Fähigkeiten in der Verkehrssprache der Dauer eine geringere Bedeutsamkeit zugesprochen wird (vgl. Cummins, 2000, S. 184). Diese Befunde widersprechen dem alleinigen Erklärungsanspruch der „Time-on-Task“ Hypothese für die Entwicklung (konzeptionell) schriftsprachlicher, kognitiv-akademisch sprachlicher Fähigkeiten in der Verkehrssprache.

Der Transfer zwischen L1 und L2 bzw. der weiteren L1 könnte zu einem kompensatorischen Effekt führen, der die reduzierte Instruktionszeit ausgleicht (vgl. ebd., S. 186). So untersuchten z. B. Ramírez (1992) und Ramírez, Yuen \& Ramey (1991) in einer Längsschnittuntersuchung über 2300 bilingual spanisch-englischsprachige aufwachsende Kinder in den Vereinigten Staaten von Amerika vom Kindergarten bis zur sechsten Klasse, die „english-only“ Schulen oder Schulen mit „transitional bilingual education“ besuchen. Beim letztgenannten Schultyp werden bilingual aufwachsende SuS zunächst unter Verwendung ihrer L1 unterrichtet bis sie früher oder später in ,english-only“ Klassen wechseln, in denen nur monolingual Englisch kommuniziert und gelernt wird (vgl. Baker, 2011, S. 215ff). Die Ergebnisse widersprechen den Implikationen der „Time-on-Task“ Hypothese für den Erwerb verkehrssprachlicher Fähigkeiten, da die SuS, die früh von einem monolingualen Unterricht in der L1 (Spanisch) zu einem monolingualen Unterricht in der L2 oder der weiteren L1 (Englisch) wechseln, trotz der deutlich reduzierten Instruktionszeit ähnliche sprachliche Fähigkeiten im Englischen wie exklusiv monolingual Englisch unterrichtete bilingual spanisch-englischsprachig aufwachsende SuS aufweisen. Zudem holen SuS, die erst spät von einem monolingual spanischsprachigen $\mathrm{zu}$ einem monolingual englischsprachigen Unterricht wechseln, im Vergleich zu der monolingual beschulten Schülerpopulation hinsichtlich ihrer akademischen Leistungen auf, obwohl sie lediglich ca. 40\% der Instruktionszeit im Englischen hatten.

Zusammenfassend lässt sich schließen, dass die empirische Evidenz zur Threshold- und Interdependenzhypothese (vgl. Cummins, 2000) das Potential der Förderung kognitiv-akademischer sprachlicher Fähigkeiten in der L1 für den Erwerb kognitiv-akademischer sprachlicher Fähigkeiten in der L2 bzw. weiteren L1 und somit für Bildungsprozesse im Allgemeinen akzentuieren. Die Bedeutung früher Literacyerfahrungen sowie metalinguistischer Bewusstheit samt sprachvergleichender Reflexionen für zentrale Vorläuferfähigkeiten des Schriftspracherwerbs und zum Erwerb kognitiv-akademischer sprachlicher Fähigkeiten können als anschlussfähige Grundlagen und Prozesse zur Initiation von Transferprozessen genutzt werden, die es angemessen didaktisch zu 
instruieren und umzusetzen gilt. Die Institution Schule, insbesondere die Grundschule, als zentraler Ort zum Erwerb und zur Förderung basaler kognitiv-akademischer sprachlicher Kompetenzen und Fähigkeiten stellt hierfür geeignete professionelle Strukturen und Personal zur Verfügung, die diese Prozesse unterstützen können. Allerdings unterscheiden sich Schultypen interund intraindividuell in ihrem Umgang mit und ihren Einstellungen zur Bilingualität ihrer SuS, sodass die Nutzung der L1 von bilingual aufwachsenden SuS zur Förderung ihrer Kompetenzen in der L2 oder weiteren L1 sowie deren Bildungsprozesse im Allgemeinen in nicht zu unterschätzender Weise von den konkreten sprachlichen Bedingungen in der jeweiligen Bildungsinstitution determiniert werden. Dieser Aspekt wird im Folgenden in Form einer Inspektion der Bedingungen bilingualer Bildung in mono- und bilingualen Schulformen näher ausgeführt.

\subsubsection{Mono- und bilinguale Schulformen für bilinguale Schülerinnen und Schüler}

Die Bandbreite an verschiedenen Schulformen und -programmen für bilingual aufwachsende SuS ist immens. Im Folgenden sollen vier populäre Schultypen näher beschrieben werden, die sich in ihren sprachlichen, sozialen und gesellschaftlichen Zielsetzungen sowie hinsichtlich ihres Umgangs mit der (natürlich erworbenen) Bilingualität ihrer SuS zum Teil deutlich unterscheiden (vgl. Baker, 2011, S. 209): Submersion, Immersion, ,transitional bilingual education" und „dual language education“. Während der erste Schultyp monolinguale Schulformen repräsentiert, weisen die drei zuletzt genannten Schultypen die Gemeinsamkeit auf, beide Sprachen ihrer SuS zumindest temporär in Bildungsprozesse zu integrieren.

\subsubsection{Monolinguale Bildung: Submersion}

Baker (2011, S. 211) beschreibt die Bedingungen des Schultyps der Submersion für bilinguale SuS in metaphorischer Anlehnung an die Bedeutung des englischen Wortes „submersion“ im Sinne von „untertauchen“ folgendermaßen:

„Submersion contains the idea of a language minority student thrown into the deep end and expected to learn to swim as quickly as possible without the help of floats or special swimming lessons. The language of the pool will be the majority language $[\ldots]$ and not the home language of the child $[\ldots]$. The language minority student will be taught all day in the majority language, often alongside fluent speakers of the majority language. Both teachers and students will be expected to use only the majority language in the classroom, not the home language. Students will sink, struggle or swim." 
Dementsprechend werden mit Submersion Schulformen bezeichnet, in denen eine Minderheit bilingual aufwachsender SuS mit einer Mehrheit monolingual aufwachsender SuS gemeinsam sowie gleichermaßen nur in der Verkehrssprache der Mehrheitsgesellschaft beschult werden -mit Ausnahme des Fremdsprachenunterrichts - und in der Regel keine adaptiven sprachlichen Hilfen oder spezieller Unterricht zur Förderung ihrer L2 oder weiteren L1 vorgesehen sind. Wenn doch sprachliche Unterstützungsmaßnahmen im Kontext der Submersion angeboten werden, treten diese in der Regel in Form vom Regelunterricht abgetrennter, exklusiv an bilingual aufwachsende Kinder gerichteter zusätzlicher Fördermaßnahmen auf. Ein Beispiel hierfür stellen die in vielen deutschen Regelschulen selbstentwickelten und -organisierten Förderangebote für „Deutsch als Zweitsprache“ dar. Dabei ist die Effektivität von segregierender präventiver und kompensatorischer Sprachförderung der Verkehrssprache als ernüchternd einzustufen (vgl. Kempert et al., 2016, S. 205-206; Thomas \& Collier, 2002). Die Förderung der nicht verkehrssprachlichen L1 wird, sofern überhaupt von den einzelnen Schulen angeboten, ebenfalls aus dem Regelunterricht exkludiert und in den freiwilligen, in der Regel nicht versetzungsrelevanten HSU (vgl. Schröder \& Küppers, 2016) verlegt, welcher in Kapitel 4 näher beschrieben wird. Die Submersion zielt letztlich auf eine hohe Sprachkompetenz in der Verkehrssprache sowie auf eine Assimilation der bilingual aufwachsenden SuS an die Mehrheitsgesellschaft ab.

Der Umgang mit der sprachlichen Heterogenität im Submersionskontext der deutschen Regelschule wird von Gogolin (1994) in ihrer Monographie „der monolinguale Habitus der multilingualen Schule“ historisch rekonstruiert und anhand von Unterrichtsbeobachtungen und Lehrerbefragungen empirisch geprüft. Gogolins (ebd., S. 3) These zum Umgang mit der sprachlichen Heterogenität im Submersionskontext der deutschen Regelschule expliziert sie folgendermaßen:

„Die zentrale These meiner Untersuchung lautet, daß das nationalstaatlich verfaßte deutsche Bildungswesen im Zuge der Entwicklung im 19. Jahrhundert ein monolinguales Selbstverständnis herausbildete. Dieses trägt bis heute [...]. Unter Umständen zunehmender Pluralisierung der Schülerschaft aber, die sich als eine Konsequenz der Migration für Bildung und Erziehung einstellt, erweist sich dieses Selbstverständnis mehr und mehr als dysfunktional: Es begrenzt die Kompetenzen, deren es zur Bewältigung der Komplexität schulischer Arbeit unter den Umständen sprachlicher Vielfalt bedarf.“

Gogolin (ebd.) wirft dem deutschen Schulsystem vor, auf seinem monolingualen Selbstverständnis zu beharren, obwohl sich die Schülerschaft durch Migrationsbewegungen hinsichtlich ihrer sprachlichen Voraussetzungen und Eigenschaften pluralisiert hat. Die monolinguale Orientierung der Schule, die sich vor allem im Handeln und im Umgang der Lehrkräfte mit der sprachlichen Vielfalt im Unterricht widerspiegelt, beschreibt Gogolin (1994) als „monolin- 
gualen Habitus“. Sie greift hierfür auf das Konzept des Habitus als „Erzeugungsmodus von Praxisformen" nach Bourdieu (1979, S. 164; zit. nach ebd., S. 30) zurück. Habitus fungieren für Individuen als „Wahrnehmungs-, Handlungs- und Denkmatrix“ und werden unter „objektiven Strukturen - wie etwa den eine Klasse charakterisierenden materiellen Existenzbedingungen - erworben" (Gogolin, 1994, S. 31). Der Habitus ermöglicht Individuen in Form einer Interpretationsschablone, auf neue, unvorhergesehene Situationen spontan im Sinne des Habitus zu reagieren (vgl. ebd.). Berufstypische Haltungen, Handlungsweisen und Produkte stellen einen „fachspezifischen Habitus“ dar, der innerhalb eines Wechselspiels von Strukturen der jeweiligen relevanten Institutionen und deren beteiligten Akteuren entsteht. Es handelt sich um ein selbsterhaltendes, sich verändernden Bedingungen trotzendes System, da der Habitus das Handeln der Individuen leitet und diese Handlungen wiederum die Strukturen des Habitus erfüllen sowie bestärken (vgl. ebd.).

Für die Profession der Lehrkräfte bedeutet dies, dass der monolinguale Habitus der Institution Schule ihre Wahrnehmungen, Einstellungen sowie Handlungen hinsichtlich sprachlicher Vielfalt im Schulkontext beeinflusst. Grundlegend für den monolingualen Habitus ist die weit verbreitete, falsche Annahme, dass Menschen, Gesellschaften und Nationen in der Regel einsprachig aufwachsen und sind (vgl. z. B. Gogolin, 2015, S. 293; Tracy, 2011, S. 72). Aus dieser Überzeugung wiederum speisen sich ihre Einstellungen und Bezugsnormorientierungen zu sprachlicher Vielfalt in der Schule und somit letztlich auch ihre Erwartungen an ihre SuS. Selbst wenn die Einstellungen der Lehrkräfte zur sprachlichen Vielfalt positiv ausfallen, kann der monolinguale Habitus auf einer unbewussten Ebene intervenieren (vgl. Gogolin, 1994, S. 3235) oder sich in allgemeinen Schulpraktiken niederschlagen, wie z. B. in Form der Exklusion anderer Sprachen zum Lernen und kommunizieren als der Verkehrssprache Deutsch (mit Ausnahme von Fremdsprachen des Fremdsprachenkanons). Dies kann so weit gehen, dass es zur öffentlichen Diskussion einer "Deutschpflicht auf dem Schulhof" in der Gesellschaft kommt (vgl. Seibel, 2016) oder die Empfehlung für Eltern mit Migrationshintergrund ausgesprochen wird, mit ihren Kindern in Deutsch statt in ihrer L1 zu kommunizieren (vgl. Reimann, 2014; Kersten et al., 2011). Solche Diskurse und Empfehlungen beruhen auf keiner wissenschaftlichen, evidenzbasierten Grundlage und tragen mindestens Merkmale einer ,assimilation ideology“ (vgl. Bourhis, 2001). Die Exklusion der (weiteren) L1 führt selbstverständlich auch zu einer Negation des Bildungspotentials erstsprachlicher Fähigkeiten. Noch bedeutsamer ist allerdings, dass der monolinguale Habitus zu einer Orientierung der Lehrkräfte an den monolingualen Standard führt. Die sprachlichen Fähigkeiten der monolingual aufwachsenden SuS werden als soziale Bezugsnorm zur Beurteilung herangezogen, obwohl sich die Ausgangsvoraussetzungen und Lerngelegenheiten von Kindern mit Deutsch als L2 oder weiteren L1 von diesen 
fundamental unterscheiden, was in Abschnitt 3.2 und 3.3 bereits theoretisch sowie empirisch untermauert wurde.

Wie die Ausführungen zum monolingualen Habitus im multilingualen Schulkontext (vgl. Gogolin, 1994) bereits vermuten lassen, gilt es die Schulform der Submersion hinsichtlich der Lernbedingungen und Lernergebnisse für bilingual aufwachsende SuS kritisch zu betrachten (vgl. Baker, 2011, S. 212f). Einige bilingual aufwachsende SuS weisen insbesondere am Schulbeginn sprachliche Verständnisprobleme auf, was sowohl die Partizipation am Unterricht als auch den Erwerb der zu lernenden Inhalte beeinträchtigt. Diese sprachlichen Verständnisprobleme werden zum Teil von den SuS aktiv verdeckt, indem sie z. B. sich an dem Verhalten anderer Klassenkameraden orientieren, Routinen durchschauen und sich an diese anpassen sowie zur Kaschierung grammatischer Fehler schnell und/oder undeutlich Sprechen (vgl. Knapp, 1999). Aber auch wenn Lehrkräfte sprachliche Verständnisprobleme erkennen, fehlt vielen die Expertise im Umgang mit einer solchen sprachlichen Heterogenität (vgl. Baker, 2011, S. 212), sodass potentielle Unterstützungshilfen wie sprachliche Unterstützungsangebote oder adaptive Formulierungen der Instruktionen nicht umgesetzt werden können. Potentielle sprachliche Verständnisprobleme zu Beginn der Beschulung können auch fortwähren, da die Orientierung an der monolingualen Norm im Submersionskontext sprachliche Fähigkeiten voraussetzt, die nicht zwingend von allen bilingual aufwachsenden SuS erfüllt werden (vgl. z. B. Grimm \& Schulz, 2016; Schulz \& Grimm, 2012; Tracy, 2008). So benötigen nach Collier (1992) Zweitsprachlerner im Submersionskontext, die keine Beschulung in ihrer L1 erhalten haben, sieben bis zehn Jahre Unterricht in der L2, um die Sprachkompetenz ihrer monolingualen Peers zu erreichen. Dieses Resultat bleibt auch nach Kontrolle der L1, Ethnie und dem sozioökonomischen Status bestehen. Dies wirkt sich zwangsläufig auch negativ auf die Entwicklung ihrer (konzeptionell) schriftsprachlichen Fähigkeiten in der L2 aus. So weisen z. B. SuS in New York, die Englisch als L2 erworben haben und in der Grundschule sieben Jahre in Submersion beschult wurden, noch beim Eintritt in die High School geringere Literacyfähigkeiten als ihre monolingual aufwachsenden Peers auf (vgl. Menken, 2009; Menken \& Kleyn, 2010). Die Bildungsbenachteiligung bilingual aufwachsender SuS mit MH in Deutschland (siehe z. B. Haag et al., 2012, 2016; Kempert et al., 2016; Rauch et al., 2016; Tarelli, Schwippert \& Stubbe, 2012; Wendt \& Schwippert, 2017), welche in Kapitel 4 näher ausgeführt wird, könnte dementsprechend zum Teil auf dem monolingualen Habitus in Kombination mit der Heterogenität der sprachlichen Fähigkeiten im Klassenverbund beruhen. Die geringeren sprachlichen Kompetenzen in der Verkehrssprache schränken die Möglichkeiten zur Partizipation am Unterricht ein und die soziale Bezugsnormorientierung am monolingualen Durchschnitt führt zu schlechteren Bewertungen ihrer Lernleistungen, die in der Regel am mündlichen und/oder schriftsprachlichen Output der Lerninhalte gemessen werden. Die negativen Folgen 
der Submersion für bilingual aufwachsende SuS beschränken sich nicht nur auf ihre Sprachkompetenz und ihren Bildungserfolg, sondern können sich auch negativ auf ihr Selbstkonzept auswirken. Die in der Schulform der Submersion eingeschriebene Exklusion der (weiteren) L1 bilingual aufwachsender SuS, welche inhärent Assimilationstendenzen sowie eine implizite Defizitorientierung enthält, können zu sozialen und emotionalen Problemen führen, da dieser Umgang als eine Form von Diskriminierung empfunden werden kann:

„It is not just the child's home language that is deprecated. The identity of the child, the parents, grandparents, the home, community, religion and culture appear to be deprecated, discredited and disparaged. It is not only the students' language that is denied. It also denies or denounces what they hold most sacred: self-esteem, identity, relationships, roots, religion and sometimes race." (Baker, 2011, S. 213)

Die SuS nehmen diesen missachtenden bis diskriminierenden Umgang mit ihnen bewusst war und können ihre Erfahrungen diesbezüglich konkret artikulieren (vgl. z. B. Krumm, 2009; Keim, 2007). Hierbei sprechen einige bilingual aufwachsende Individuen ihren Lehrkräften indirekt die Schuld für die Exklusion und Negation ihrer (weiteren) L1 sowie die daraus resultierenden negativen Konsequenzen für ihre Bildungswege zu:

„We are treated like garbage. I kept getting suspended because when I spoke Spanish with my homeboys, the teachers thought I was disrespecting them. They kept telling me to speak in English because I was in America. I wasn't going to take that. [...] So I left and never went back. Some of those teachers don't want us. That hurts, that really hurts." (Ribadeneira, 1992, S. 7; zit. nach Auerbach, 1993, S. 9).

Die in der Submersion vertretene monolinguale Sprachpolitik spiegelt die asymmetrisch verteilten Machtverhältnisse zwischen der monolingualen Mehrheit und bilingualen Minderheiten bestimmter Gesellschaften wider (vgl. Auerbach, 1993, S. 16). Die Exklusion der (weiteren) L1 von bilingual aufwachsenden SuS darf hinsichtlich ihrer negativen Konsequenzen für die Identität und das Selbstkonzept dieser Schülerschaft nicht unterschätzt werden, da sie ,the child's most intense existential experience” (Phillipson, 1992, S. 189) negiert: , $[\ldots]$ their sense of powerlessness is reinforced either because they are de facto excluded from the classroom or because their life experiences and language resources are excluded" (Auerbach, 1993, S. 18).

Letztlich stellen die Bedingungen des Submersionskontexts auch für Lehrkräfte eine Herausforderung dar (vgl. Baker, 2011, S. 213). Die Heterogenität der Sprachkompetenz der einzelnen SuS im Klassenverbund der Submersion stellt hohe Anforderungen an Lehrkräfte hinsichtlich der Umsetzung der Basisdimensionen qualitativ hochwertigen Unterrichts (vgl. z. B. Klieme, Schümer \& Knoll, 2001; Klieme, Pauli \& Reusser, 2009). Qualitativ hochwertiger Unterricht zeichnet sich durch gute Klassenführung, kognitive Aktivierung und soziale Eingebundenheit aus (vgl. ebd.). In Klassen mit hoher Heterogeni- 
tät stellt die kognitive Aktivierung aller SuS im Klassenverbund ein anspruchsvolles Ziel dar, da der Unterricht binnendifferenziert, adaptiv an die verschiedenen Fähigkeitsniveaus angepasst werden muss. Ein Misslingen der kognitiven Aktivierung aller SuS im Klassenverbund kann sich negativ auf die Klassenführung auswirken, da sowohl Über- als auch Unterforderung der SuS zu Unruhe bis Renitenz im Klassenverbund führen können. Werden die in der (weiteren) L1 gemachten Lebenserfahrungen und sprachlichen Ressourcen (vgl. Auerbach, 1993, S. 18) durch den monolingualen Habitus ausgeschlossen und nicht wertgeschätzt, kann sich dies auch negativ auf die subjektiv wahrgenommene soziale Eingebundenheit im Klassenverbund der bilingual aufwachsenden SuS auswirken. Insgesamt erweist sich die von Gogolin (1994, S. 3) angeprangerte Dysfunktionalität des monolingualen Habitus der multilingualen Schule, der die Kompetenzen begrenzt, ,deren es zur Bewältigung der Komplexität schulischer Arbeit unter den Umständen sprachlicher Vielfalt bedarf", als zentrale Schwachstelle der Submersion sowohl im Hinblick auf die bilingual aufwachsende Schülerschaft als auch deren Lehrkräfte.

\subsubsection{Bilinguale Bildung: Immersion, Transitional Bilingual Education und Dual Language Education}

Baker (2011, S. 210) unterscheidet zwischen schwachen und starken Formen bilingualer Bildung. $\mathrm{Zu}$ den starken Formen zählen die Immersion und ,dual language education", die auf hohe Kompetenzen in zwei Sprachen ohne Vernachlässigung der schulischen Lerninhalte abzielen, während schwache Formen wie „transitional bilingual education“ die L1 nur zeitweise zu Beginn der Beschulung einbeziehen und im weiteren Verlauf in die Submersion übergehen.

Der Schultyp der Immersion stellt eine Form bilingualer Bildung dar, die auf eine Elterninitiative in Kanada der 1960er Jahre mit den Zielen zurückgeht (vgl. ebd., S. 239). Englischsprachig aufwachsenden Kindern sollte es ermöglicht werden, sich zu bilingualen, biliteralen und bikulturellen Sprechern des Englischen und Französischen zu bilden, ohne den Erwerb schulischer Lerninhalte zu vernachlässigen (vgl. ebd). Diese etwas schwammige Beschreibung ohne eindeutige Definition ist der Vielfalt und Variabilität von Immersionsprogrammen geschuldet, sodass einige Autoren Immersionsprogramme eher anhand von Kernmerkmalen und variierenden Eigenschaften definieren (vgl. ebd., S. 242f). Zwei zentrale Kernmerkmale sind die folgenden: 1) Die Sprache der Immersion stellt in diesen Kontexten keinen expliziten Lerngegenstand dar, sondern wird primär als Medium des Unterrichts verstanden. 2) Mit der Immersionssprache wird das gleiche schulische Curriculum wie in der jeweiligen L1 erworben (vgl. McMillan \& Turnbull, 2009, S. 18). Die mit Immersionsprogrammen intendierte Form eines inzidenten Sprachenlernens durch die Auseinandersetzung mit Lerninhalten des Curriculums in einer zu lernenden 
Sprache erinnert an das Konzept des „Content and Language Integrated Learning" (CLIL), welches eine vor allem europäische und US-amerikanische Adaptation kanadischer Immersionsprogramme darstellt (vgl. Baker, 2011, S. 245f). Die SuS sind bei Immersionsprogrammen und CLIL mit einer bildungssprachlichen Varietät der zu lernenden Sprache konfrontiert. Es wird angenommen, dass ein solcher inzidenter Erwerb der zu lernenden Sprache schneller von Statten geht als ein lediglich auf Sprache fokussierter Fremdsprachenerwerb, da die Einbettung des Sprachenlernens in den Erwerb von Wissen und Fertigkeiten des Curriculums authentische, bedeutungsvolle Lerngelegenheiten schafft, in denen Sprache als kognitives Werkzeug fungiert (vgl. Davison $\&$ Williams, 2001). Weitere Kernmerkmale dieses Schultyps sind unter anderem die Optionalität des Besuchs dieser Schulform, die schulische Förderung beider Sprachen, bilinguale Lehrkräfte sowie eher homogene Sprachkompetenzen der SuS eines Klassenverbunds, da die Lerngelegenheiten zum Erwerb der Sprache der Immersion zu einem Großteil auf den Unterrichtskontext beschränkt sind (vgl. Baker, 2011, S. 242).

Die zentralen variablen Merkmale der Immersion sind das Alter bei Eintritt (z. B. ab Kindergarten, Grundschule, Sekundarstufe etc.) und der Anteil von Unterricht in der Immersions- und Erstsprache (z. B. partielle Immersion mit $50 \%$ des Unterrichts in der Immersionssprache) (vgl. McMillan \& Turnbull, 2009, S. 17). Am häufigsten werden frühe Immersionsprogramme (mit Eintritt in den Kindergarten oder in die Grundschule) mit einer zunächst exklusiven Beschulung in der Immersionssprache gewählt (vgl. Baker, 2011, S. 239f). Der Anteil der Immersionssprache nimmt für gewöhnlich über die Schulkarriere $\mathrm{ab}$, sodass in der Sekundarstufe nur noch vereinzelte Fächer in der Immersionssprache gewählt werden (vgl. McMillan \& Turnbull, 2009, S. 187). Weitere variable Merkmale sind unter anderem die Kontinuität der Immersion über die verschiedenen Bildungsstufen hinweg, die Menge an bereitgestellter sprachlicher Unterstützung für die SuS (vgl. Baker, 2011, S. 242f) und die Rolle der L1 im Unterricht in der Immersionssprache (für einen Überblick siehe Turnbull \& Dailey-O'Cain, 2009). Die Nutzung der L1 in Immersionsprogrammen stellt eine ähnlich kontrovers geführte Debatte (vgl. McMillan \& Turnbull, 2009, S. 15) wie die Diskussion über den Nutzen sowie den Umgang mit natürlich erworbenen Sprachen, die nicht dem Fremdsprachenkanon des jeweiligen Bildungssystems entsprechen (vgl. Gogolin \& Neumann, 2009), dar. Der exklusive Gebrauch der Immersionssprache für SuS als auch für Lehrkräfte in Unterrichtseinheiten, die in der Immersionssprache vollzogen werden sollen, galt als eines der Kernprinzipien kanadischer Immersionsprogramme. Diese Praxis wird allerdings seit Beginn der 2000er Jahre vermehrt dahingehend hinterfragt, ob ein limitierter, situationsspezifischer und angemessener Gebrauch der Erstsprache in solchen Kontexten nicht doch sinnvoll wäre (vgl. McMillan \& Turnbull, 2009, S. 15). Nichtsdestotrotz wird in dieser Schulform der 
Sprachgebrauch organisatorisch separiert, sodass Phänomene wie Code-Switching und -Mixing keine legitimen Sprachpraktiken darstellen und zu vermeiden sind.

Dementsprechend kann Immersion, in Anlehnung an Bakers (2011, S. 211) Metapher des „Untertauchens“ bezüglich des Schultyps der Submersion, als ein optionales sowie temporär limitiertes „Eintauchen“ in das Sprachbad der Immersionssprache beschrieben werden. Die SuS erhalten „Schwimmhilfen“ wie z. B. adaptive Instruktionen sowie partieller Gebrauch der L1, um sie vor dem „Untergehen“ zu bewahren. Die eher als homogen zu bezeichnenden Sprachkompetenzen in der Immersionssprache erleichtern eine adaptive kognitive Aktivierung im Klassenverbund. Der Großteil der SuS werden nicht sinken, sondern zunächst unter Mühen versuchen den „Kopf über Wasser zu halten“ und letztlich sich zu „Schwimmern“" entwickeln, auch wenn sie in der Regel keine erstsprachliche Sprachkompetenz in der Immersionssprache entwickeln (vgl. ebd., S. 241).

"Transitional bilingual education“ ist eine vor allem in den Vereinigten Staaten von Amerika verbreitete Schulform für SuS von Sprachminderheiten, die sich im Kern durch eine temporäre, teilweise Beschulung in ihrer L1 auszeichnet, die im Verlauf sukzessive reduziert und durch Lernen und Lehren in der Verkehrssprache ersetzt wird (vgl. ebd., S. 215ff). Den bilingualen Lehrkräften sowie den SuS ist es dementsprechend zu einem gewissen Grad erlaubt, die L1 der SuS beim Lehren, Lernen und Interagieren zu verwenden. Das Ziel hierbei ist nicht die Förderung beider Sprachen zur Entwicklung balancierter bilingualer und biliteraler Sprachfähigkeiten durch die Bearbeitung des schulischen Curriculums unter Verwendung von zwei Sprachen, sondern die Assimilation dieser SuS an die Mehrheitsgesellschaft durch einen schrittweisen Übergang zur Submersion (vgl. ebd.). Die Beschulung in der L1 wird so gesehen zu einem gewissen Grad geduldet, aber nur bis die SuS eine Sprachkompetenz in der Verkehrssprache erreichen, die die exklusive Beschulung in dieser ermöglicht. Dieser Schultyp differenziert sich in zwei verschiedene Unterkategorien, die sich durch die Dauer der Beschulung in der L1 unterscheiden. Bei ,early exit"--Programmen wird der Rückgriff auf die L1 zur Beschulung auf maximal die ersten zwei Schuljahre beschränkt, während bei „late exit"Programmen die Inklusion der Erstsprache bis zur sechsten Klasse fortgeführt werden kann, wobei die Erstsprache aber nicht mehr als 40\% der Zeit genutzt werden sollte (vgl. ebd., S. 216).

Im Gegensatz zur „transitional bilingual education“ zielt die „,dual language education“" zum einen auf die Förderung erstsprachlicher Fähigkeiten zum Erhalt oder zur Weiterentwicklung dieser sowie der damit assoziierten Kultur und Identität und zum anderen auf die Entwicklung der Sprachkompetenz in beiden Sprachen ohne Vernachlässigung des Curriculums ab (vgl. ebd., S. 207). Diese Programme lassen sich zum Teil nur schwerlich von Immersions- 
programmen differenzieren, da die beiden Schulformen mehr Gemeinsamkeiten als Unterschiede aufweisen. Als „dual language education“ können nach Baker (ebd., S. 222) Schulen bezeichnet werden, bei denen ungefähr die gleiche Anzahl an SuS der Sprachminderheit und -mehrheit gemeinsam unter Verwendung beider Sprachen unterrichtet werden. Es werden dabei die gleichen Ziele wie in der Immersion verfolgt, nämlich eine hohe mündliche und schriftliche Sprachkompetenz in zwei Sprachen sowie die Ausbildung von positiven interkulturellen Einstellungen bei vergleichbaren inhaltlichen Lernerfolgen wie SuS in monolingualen Schulen (vgl. ebd., S. 224). Zudem sollen durch die Anerkennung der L1 als offizieller Schulsprache ebenfalls die im Submersionskontext für bilingual aufwachsende SuS potentiell zu beobachtenden negativen Effekte auf die Identität und das Selbstbewusstsein verhindert werden. Die sprachliche Adaptation der Instruktion sowie des Unterrichts leistet ebenfalls einen Beitrag zu mehr Bildungsgerechtigkeit (vgl. ebd., S. 225).

Trotz des kennzeichnenden Merkmals von „dual language education“, beide Sprachen für schulische Bildungsprozesse im Unterricht zu verwenden, wird die Nutzung der einzelnen Sprachen nach organisatorischen Richtlinien wie sprachspezifische Tage, Fächer, Unterrichtseinheiten etc. separiert, sodass bilinguale Phänomene wie Code-Switching und -Mixing in der Klasseninteraktion nicht intendiert sind (vgl. ebd., S. 223). Zwar kommt es hin und wieder zu bilingualer Interaktion, wie in allen Schulformen mit mehrsprachigen Schülern üblich (vgl. z. B. Baker, 2011; Barwell, 2009, 2014; Cook, 2002; García \& Li Wei, 2014; Turnbull \& Dailey-O'Cain, 2009), allerdings handelt es sich hierbei nicht um einen geplanten, strukturierten und systematischen parallelen Gebrauch beider Sprachen, sondern vielmehr um das Gegenteil: „A central idea in dual language bilingual schools is language separation and compartmentalization" (Baker, 2011, S. 226). Diese Trennung gilt es sensibel und reflexiv zu planen, sodass keine der beiden Sprachen benachteiligt wird, wie z. B. wenn die Verkehrssprache häufiger oder primär für prestigeträchtigere Fächer wie Naturwissenschaften verwendet wird. Die Sprachseparation eröffnet allerdings auch Möglichkeiten, die Heterogenität in den sprachlichen Kompetenzen der Schülerschaft produktiv zu verwenden, da somit unterschiedliche Kinder je nach aktuell zu nutzender Sprache als „Experten“ agieren können (vgl. ebd., S. 228).

Die Zusammensetzung der Schülerschaft und die sprachliche Orientierung dieser prägen die schulischen Sprachpraktiken bilingualer Schulen mit. In der Regel stellen SuS, deren L1 nicht die Verkehrssprache ist, die Mehrheit der Schülerschaft von Schulen mit „dual language education“ dar (vgl. ebd., S. 224), da bilinguale Bildung für Eltern von bilingual aufwachsenden Kindern äußerst attraktiv ist. Für monolinguale Eltern hingegen müssen solche Schulen sich neben dem bilingualen Sprachangebot durch weitere Vorteile wie z. B. eine gute Reputation oder Effektivität auszeichnen, um diese zur Einschreibung ihrer Kinder in solche Schulen zu überzeugen. Trotz des größeren Anteils 
an SuS, deren L1 nicht die Verkehrssprache ist, lässt sich bei diesen durchaus eine Präferenz der Mehrheits- bzw. Verkehrssprache identifizieren, was auch zur Herausbildung von Sprachasymmetrien führt (vgl. ebd., S. 223).

Die Messung der Effektivität von bilingualen im Vergleich zu monolingualen Schulformen ist mit diversen methodischen Herausforderungen verbunden (vgl. ebd., S. 254ff). So hängen typische Leistungsindikatoren von Schuleffektivität wie z. B. Sprachkompetenz(en), akademische Leistungen in verschiedenen Schulfächern sowie motivationale, soziale und emotionale Eigenschaften und Fähigkeiten von einer Vielzahl verschiedener, in Interaktion stehender Faktoren auf Individual- (z. B. Vorwissen, Intelligenz, Arbeitsgedächtnis, Motivation, Volition etc.), Familien- (z. B. soziökonomischer Status, Bildungsaspiration, kulturelles Kapital etc.) Unterrichts- (z. B. fachliche, didaktische und pädagogische Kompetenz der Lehrkräfte, Unterrichtsqualität, Klassenzusammensetzung etc.), Schul- (z. B. Schulklima, Ausstattung etc.) und politisch-gesellschaftlicher Ebene (z. B. Bildungsgerechtigkeit, (sprachbezogene) Bildungsideologien, Selektionsprozesse etc.) ab (vgl. z. B. Bos, Klieme \& Köller, 2010; Creemers \& Kyriakides, 2008; Hattie, 2008; Helmke, 2017, S. 69-101; Seidel, 2014). Die Inklusion weiterer Sprachen neben der Verkehrssprache in bilingualen Schulformen ist also nur eine von vielen, in Zusammenhang stehenden Einflussfaktoren auf die Effektivität von Schulen. Zudem verringert die inter- und intraindividuelle Heterogenität der verschiedenen bilingualen Schultypen die Generalisierbarkeit der empirischen Evidenz.

In schulvergleichenden Studien schneiden bilinguale Schulformen in der Regel vergleichbar oder besser als monolinguale Schulformen ab (für einen Überblick siehe Baker, 2011, S. 258ff), wobei die zentralen Annahmen der Threshold- und Interdependenzhypothese von Cummins (2000) bestätigt werden. So attestiert zum Beispiel die Meta-Studie von Rolstad, Mahoney und Glass (2005) „dual language education“-Programmen eine bessere Effektivität hinsichtlich der Förderung von Lesekompetenz und mathematischen Fähigkeiten. Die Meta-Studien von Willig (1985) und Greene (1998) liefern Evidenz für die Bedeutung erstsprachlicher Fähigkeiten für den Bildungserfolg und sprechen somit implizit für ,transitional bilingual education“ und ,dual language education", da durch die Inklusion der L1 bessere akademische Leistungen erzielt werden respektive der Erwerb der Verkehrssprache unterstützt wird. Thomas \& Collier (2002) schließen auf Basis ihrer Ergebnisse zur Effektivität von Schulen, dass „dual language education“ ab der Grundschule die optimale Schulform für langfristigen akademischen Erfolg für bilinguale SuS von Sprachminderheiten darstellen. Auch Slavin \& Cheung (2005) empfehlen auf Basis ihrer Synthese zu effektiver Leseinstruktion für bilingual aufwachsende Lerner des Englischen trotz der quantitativ geringen empirischen Befundlage bilinguale Bildungsprogramme für bilinguale SuS.

Die Effektivität von Immersionsprogrammen lässt sich nicht nur aufgrund der internationalen Verbreitung dieser Schulform, die mittlerweile auch in 
Deutschland zu beobachten ist (vgl. Möller et al., 2017), legitimieren, sondern auch auf Basis der empirischen Evidenz zur Effektivität - insbesondere hinsichtlich akademischer Fähigkeiten - dieses Schultyps (für einen Überblick siehe Baker, 2011, S. 265-271; Johnstone, 2002). Dies erscheint zunächst überraschend, da SuS mit MH in der Submersion zumindest hinsichtlich des Kernmerkmals der Immersion - Lernen der Inhalte des Curriculums durch eine Sprache, die nicht die L1 darstellt - prinzipiell unter ähnlichen Bedingungen wie SuS in der Immersion lernen. Andererseits gibt es auch zentrale Unterschiede, die die distinkten Bildungserfolge der Schulformen der Immersion und Submersion zum Teil determinieren könnten (vgl. Baker, 2011, S. 240f): Durch die anvisierte Biliteralität und die Nutzung bildungssprachlicher Varietäten in den beiden Sprachen können positive Effekte auf (meta-)linguistischer und möglicherweise (meta-)kognitiver Ebene sowie Transferprozesse im Sinne von Cummins' (2000) Threshold- respektive Interdependenzhypothese ausgelöst werden, die wiederum zu positiven akademischen Ergebnissen beitragen. Die homogenen Ausgangsbedingungen der Sprachkompetenzen in der Immersion erleichtern einen adaptiven Unterricht und führen zu einer angemessenen sozialen Bezugsnormorientierung, die in der Submersion zu Gunsten monolingual aufwachsender Kinder verzerrt auftritt. Häufig werden in Immersionsprogrammen international prestigeträchtige Sprachen -z. B. Englisch und Französisch in Kanada - oder zumindest regional angesehene Sprachen z. B. Spanisch und Katalanisch in Spanien - inkorporiert, was die Einstellung der Schülerschaft zu diesen Sprachen positiv beeinflussen und ihre Lernmotivation steigern könnte. Immersionsschulen vertreten dementsprechend auch eher pluralistische Sprachideologien, die die verschiedenen Sprachen wertschätzen, und stellen einen optionalen Schultypus dar, für den sich Eltern und/oder SuS bewusst entscheiden, was sich beides ebenfalls positiv auf das Engagement und die Motivation auswirken könnte.

Studien zur Analyse der Effektivität verschiedener Schultypen auf Bildungsindikatoren von bilingual aufwachsenden SuS liefern aufgrund der vielfältigen potentiellen Störvariablen allerdings nur sehr grobe, konfundierende Einblicke auf die Bedeutung der Integration beider Sprachen für die Bildungsprozesse der bilingualen Schülerschaft. Eine feinkörnigere, validere Perspektive auf die Relevanz der Berücksichtigung der Bilingualität von bilingualen $\mathrm{SuS}$ in Bildungsprozessen versprechen die Untersuchung bilingualer Praktiken sowie die diesem Sprachverhalten zugrunde liegenden Motive in konkreten Lehr-Lernprozessen in Bildungsinstitutionen. Diesbezüglich werden im Folgenden kurz das relativ neue Konzept des „Translanguaging“ vorgestellt und anschließend potentielle Motive sowie empirische Evidenz zu bilingualen Praktiken in Lehr-Lernprozessen ausgeführt. 


\subsubsection{Bilinguale Praktiken in Bildungsprozessen}

Bevor zentrale empirische Befunde zu bilingualem Sprachverhalten in Bildungsprozessen (Abschnitt 3.6.5.3) sowie zu Einstellungen von Lehrkräften und pädagogischen Fachkräften zu diesem (Abschnitt 3.6.5.4) präsentiert werden, gilt es zunächst den Begriff des sowie das Konzept von „Translanguaging“ zu erläutern (Abschnitt 3.6.5.1). Translanguaging ist ein relativ neues, aus dem Bildungskontext entstandenes Konzept von Bilingualität und bilingualem Sprachgebrauch. Dieses Konzept wird innerhalb der Bilingualismusforschung kontrovers diskutiert, was sicherlich auch zu dessen Popularität beigetragen hat. Hieran anschließend werden potentielle Gründe und Motive für bilinguale Praktiken von SuS und Lehrkräften in Lehr-Lernprozessen aus theoretischer Perspektive reflektiert, wobei insbesondere kognitionspsychologische Aspekte von bilingualem Sprachverhalten bei Bildungsprozessen betrachtet werden (Abschnitt 3.6.5.2).

\subsubsection{Translanguaging}

Das Phänomen „Translanguaging“ hat im wissenschaftlichen Diskurs der Linguistik und Bilingualismusforschung in den letzten Jahren eine enorme Popularität erlangt, was neben der Fruchtbarkeit dieses Konzepts auch an der Weiterentwicklung dessen durch eine Vielzahl von Forschern liegt (vgl. García \& Kano, 2014, S. 260). Translanguaging wurde in diesem Prozess von einigen Autoren zu einer eigenständigen linguistischen Position erhoben, die von Forschern wie z. B. Ofelia García, Li Wei, Ricardo Otheguy und Wallis Reid vertreten wird. Diese primär soziolinguistisch fundierte Theorie hegt transformative Ambitionen auf politischer und gesellschaftlicher Ebene und löste kontroverse Diskussionen in der Bilingualismusforschung aus, da sie etablierte linguistische Modelle sowie robuste empirische Befunde der Bilingualismusforschung in Frage stellt.

Ursprünglich wurde der Begriff „Translanguaging“ von Cen Williams (1994, 1996) eingeführt, um den geplanten und systematischen Gebrauch von zwei Sprachen innerhalb des schulischen Unterrichts zu beschreiben (vgl. Baker, 2011, S. 288). Hierunter fallen pädagogische und didaktische Praktiken von Lehrkräften und SuS zum Gebrauch von zwei Sprachen in Bildungsprozessen. Baker (ebd.) definiert „Translanguaging“ als ,,process of making meaning, shaping experiences, gaining understanding and knowledge through the use of two languages“. Lewis, Jones \& Baker (2012, S. 644) führen diese Definition weiter aus und konkretisieren sie folgendermaßen:

„The process of translanguaging uses various cognitive processing skills in listening and reading, the assimilation and accommodation of information, choosing and selecting from the brain storage to communicate in speaking and 
writing. Thus, translanguaging requires a deeper understanding than just translating as it moves from finding parallel words to processing and relaying meaning and understanding."

So können zum Translanguagingbegriff von Baker (2011, S. 288) und von Lewis, Jones \& Baker (2012, S. 644) auf der Seite der Lehrkräfte explizite Instruktionen, Angebote und Aufgaben zum Gebrauch beider Sprachen in Lernprozessen wie z. B. bilinguale Unterrichtsmaterialien, Initiation sprachvergleichender, metalinguistischer Reflexionen oder Lesen eines Textes in Sprache X und die Widergabe dessen in Sprache Y etc. gezählt werden. Hinsichtlich bilingualer SuS umfasst dieses Konzept bilinguale Praktiken in Gestalt von Sprachwechseln sowie -mischungen und Übersetzungen, aber auch allgemeine mentale Prozesse, bei welchen die SuS in Denkprozessen auf beide Sprachen zurückgreifen. Prinzipiell werden solche bildungsbezogenen mehrsprachigen Prozesse nicht nur in (vor-)schulischen Institutionen von bilingual aufwachsenden SuS verwendet, sondern in einer Vielzahl von (Bildungs-)Institutionen und Bildungsprozessen sowie von verschiedenen Akteuren genutzt. So gebrauchen z. B. Ärzte, die ihre Approbation im Ausland erhalten haben und sich für eine Anerkennung dieser in Deutschland auf die sprachliche und medizinische Prüfung vorbereiten, ihre L1 und das Deutsche in der Vorbereitung, um ihr in der L1 enkodiertes medizinisches Wissen auch in der L2 Deutsch zugänglich zu machen und widergeben zu können. Ein weiteres Beispiel stellen Studierende der Romanistik an deutschen Universitäten dar, die sich auf spanischsprachige Prüfungen vorbereiten, indem sie z. B. die englischsprachig publizierte Fachliteratur zu syntaktischen Strukturen des Portugiesischen lesen, zentrale Theorien, Konzepte und Befunde beim Aufbereiten der Studien in Deutsch zusammenfassen und elaborieren, um abschließend das im Lernprozess erlangte Wissen in der Prüfung im Spanischen widerzugeben.

Das Konzept „Translanguaging“ wurde im weiteren Verlauf von diversen Autoren aus der exklusiven Verankerung im Bildungskontext gelöst und als Überbegriff für bilinguales Sprachhandeln eingesetzt: , , ... ] to refer to both the complex language practices of plurilingual individuals and communities, as well as the pedagogical approaches that use those complex practices" (García \& Li Wei, 2014, S. 20). Die Fokussierung auf Sprache als Aktivität ist in dem Begriff „Languaging“ inhärent verankert, der von Li Wei (2011, S. 1124) als „process of using language to gain knowledge, to make sense, to articulate one"s thougt and to communicate about using language“ definiert wird. „Languaging" verschiebt dementsprechend die Perspektive von Sprachen als Strukturen zu Sprachen als Aktivität, Repertoire zur Kommunikation und kognitives Werkzeug. Diese Perspektivenverschiebung wird auch mit Verweis auf die These von Makoni \& Pennycook (2007, S. 2) legitimiert, die Sprachen als Erfindungen sozialer, kultureller und politscher Bewegungen interpretieren: „Languages do not exist as real entities in the world and neither do they emerge 
from our represent real environments; they are, by contrast, the inventions of social, cultural and politic movements".

Der Brückenschlag zum Kontext der Bilingualität wird durch das Präfix „trans-“vollzogen. García und Li Wei (2014, S. 21) interpretieren das Präfix „trans “" von Translanguaging in Anlehnung an den Begriff „transculturación“" des Anthropologen Fernando Ortiz und folgern hieraus, dass das Sprachhandeln bi- und multilingualer Sprecher neue Sprachpraktiken generiert, die keine hybriden Mischungen oder Synthesen ihrer Sprachen darstellen, sondern die als genuin neue, einzigartige Sprachpraktiken zu verstehen sind. Diese Konzeption erinnert auch an die in Abschnitt 3.4 diskutierten hybriden Identitäten und an Bhabhas (1990) Kulturverständnis bilingual- und bikulturell aufwachsender Menschen als „Dritter Raum“, in dem neue kulturelle Praktiken entstehen. Translanguaging eröffnet demnach einen „translanguaging space“, der nicht nur neue Sprachpraktiken erzeugen kann, sondern über transformative Kräfte zur Generierung neuer Identitäten, Praktiken und Werte durch Kreativität und Kritikfähigkeit verfügt. Diese Prämissen führen García und Li Wei (2014, S. 14) zu der theoretischen Annahme, dass bilinguale Sprecher ,(a) single array of disaggregated features that is always activated" besitzen, anstatt zwei separate, autonome Einzelsprachen in sich zu vereinen: ,[...] language practices of bilinguals are complex and interrelated; they do not emerge in a linear way or function separately since there is only one linguistic system". Oder anders ausgedrückt: ,bilinguals have one linguistic repertoire from which they select features strategically to communicate effectively" (ebd.). Der Kern dieser Konzeption sprachlichen Wissens und Fähigkeiten bilingualer Sprecher akzentuiert das Repertoire linguistischer Funktionen und Praktiken statt Grammatiken von Einzelsprachen. Das Repertoire besteht aus einem komplexen Netzwerk semiotischer Zeichen, aus welchem einzelne „language features“ ausgewählt und zu sprachlichen Praktiken zusammengeführt werden, die adaptiv an die Situation sowie die Kommunikationsbedürfnisse und -ziele des Sprechers angepasst werden (vgl. García \& Kano, 2014, S. 260f).

Diese starke, selbstbewusst vertretene Position der Anhänger des Translanguaging Konzepts nach García und Li Wei (2014) widerspricht diversen bisherigen linguistischen Theorien und robusten empirischen Befunde. Hinsichtlich dieser Position gibt es einige Kritikpunkte zu äußern, von denen eine Auswahl allerdings nur kurz angerissen werden soll. Zunächst fällt auf, dass bereits wissenschaftlich etablierte Phänomene und Konstrukte der Linguistik und Bilingualismusforschung von den Autoren zum Teil adaptiert und schließlich in ihren eigenen übergeordneten, transformativen Framework des Translanguaging integriert werden. Dementsprechend handelt es sich bei diesem Konzept nicht um ein neues Paradigma, sondern es vereint primär robuste (sozio-)linguistische Befunde der Bilingualismusforschung, welche sich allerdings auch in anderen Theorien wie dem dynamischen Modell der Bilingualität (Herdina 
\& Jessner, 2002) oder dem holistischen Bilingualismus (Grosjean, 1982) wiederfinden lassen. Der Hauptkritikpunkt ist allerdings die Annahme eines linguistischen Systems bilingualer Sprecher unter Negation der psychologischen Realität der Existenz von Einzelsprachen als autonome, getrennte Systeme. Zwar geht der Großteil der Bilingualismusforschung von einem sprachenübergreifendes Lexikon bilingualer Sprecher aus (vgl. Bartolotti \& Marian, 2012, S. 23-32), ansonsten steht das Translanguaging-Konzept nach García und Li Wei (2014) mit einigen robusten Befunden der Spracherwerbsforschung (siehe Abschnitt 3.2) und Psycholinguistik, insbesondere hinsichtlich Bilingualismus und Kognition (siehe Abschnitt 3.3.3) sowie bilingualem Sprachgebrauch (siehe Abschnitt 3.5), in Kontradiktion. Eine ausführliche Widerlegung dieser Annahme anhand der Unterscheidung von Repertoires und Grammatiken und empirisch robusten Befunden zu Code-Switching, bilingualem Spracherwerb und psycholinguistischer Forschung kann bei MacSwan (2017) nachgelesen werden. Nichtsdestotrotz sieht auch MacSwan (ebd., S. 191) in dem Konzept von Translanguaging einen fruchtbaren Ansatz zur Betrachtung von Bilingualität, da dieser konventionelle Perspektiven auf Sprachpraktiken von bilingual aufwachsenden Kindern im Bildungskontext hinterfragt und die potentielle Förderlichkeit von bilingualen Sprachpraktiken in Bildungskontexten akzentuiert. Dementsprechend wird Translanguaging in den folgenden Ausführungen weitestgehend wie in der ursprünglich intendierten Definition und Konzeption von Baker (2011, S. 288) verstanden und verwendet.

\subsubsection{Potentielle Motive für bilinguale Praktiken in Bildungsprozessen}

Wie anhand der bisherigen Darstellung von Bilingualität und (institutionalisierter) Bildung vermutet werden kann, wird die Förderlichkeit von bilingualen Praktiken in Bildungsprozessen eher kritisch eingeschätzt. Im Kontext der Submersion werden bilinguale Praktiken in der Regel aus einer Defizitperspektive betrachtet, deren Auftreten vermeintlich durch eine geringe Sprachkompetenz in der Verkehrssprache bedingt ist. Aber selbst bei den Schultypen der Immersion und „dual language education“ ist bilinguales Sprachhandeln häufig unerwünscht, da der Unterricht in diesen Schulformen sich primär durch Sprachenseparation auszeichnet (vgl. Baker, 2011, S. 226; García \& Kano, 2014, S. 261). So konnten zum Beispiel Worthy et al. (2013, S. 314) trotz intensiver Literaturrecherche für den Grundschulkontext keine Studien identifizieren, in denen Schulen beschrieben oder beforscht werden, die auf Schulebene Praktiken des Translanguaging programmatisch in ihr Konzept inkorporieren und explizit fordern sowie fördern. Im Falle der Immersion gilt der Rückgriff auf die L1 sowie Sprachwechsel und -mischungen für einige Akteure bis heute noch als kontraproduktiv zum Erwerbsziel einer elaborierten Sprachkompetenz in der L2 (vgl. MacMillan \& Turnbull, 2009). Ausgehend 
von der Interdependenzhypothese (Cummins, 2000) kann bilinguales Sprachverhalten hinsichtlich diverser Phänomene wie z. B. Vorwissensaktivierung, Konzepterwerb, Textverständnis, Wortschatzerwerb, Schriftspracherwerb etc. als eine förderliche Praxis zur Initiation impliziter und/oder expliziter Transferprozesse sein. Transfer kann schließlich nur gelingen, wenn auf die entsprechenden Ressourcen zurückgegriffen werden kann und diese anschließend übertragen werden, was unter anderem reziproke Prozesse des Vergleichs, der Elaboration, Modifikation und Adaptation erfordert. Zudem befinden sich bilinguale Sprecher, unter bestimmten Bedingungen, in einem bilingualen Sprachmodus, bei dem beide Sprachen aktiviert sind, sodass bilinguale Praktiken und sprachliche Interferenzen kaum zu vermeiden sind. Dementsprechend ist es verwunderlich, dass selbst in bilingualen Schulformen weitestgehend an der Sprachenseparation festgehalten wird.

Für den Bildungskontext lässt sich allerdings eine robust auftretende bilinguale Sprachpraxis bilingualer SuS in allen Schulformen und zum Teil auch für Lehrkräfte in bilingualen Schulen identifizieren (vgl. Culligan, 2015, S. 3), die sich folgendermaßen pointiert umschreiben lässt: ,[...], the L1 creeps back in, however many times you throw it out with a pitch-fork" (Cook, 2001, S. 405). Diesbezüglich stellt sich zum einen die Frage, welche Motive dem renitenten Verhalten bilingualer SuS trotz impliziten oder expliziten Verboten des Gebrauchs der (weiteren) L1 zu Grunde liegen und zum anderen, wieso auch Lehrkräfte unter bestimmten Bedingungen den Rückgriff auf die (weitere) L1 oder bilingualen Sprachgebrauch erlauben? Für bilingual aufwachsende SuS gibt es hierzu einige potentielle, nicht direkt bildungsbezogene Erklärungen. So stellen für bilingual aufwachsende Kinder z. B. der bilinguale Sprachmodus und somit auch bilinguale Praktiken die typische Kommunikationsform innerhalb der Familie (vgl. z. B. Reich, 2009) und der bilingualen Peer-Group (vgl. z. B. Keim, 2012) dar. Anscheinend gelingt es im Kontext der Schule nicht immer, in einem monolingualen Sprachmodus zu verbleiben. Zudem könnte das Auftreten bilingualer Praktiken in der Schule auch durch das Bedürfnis der Markierung von Identität oder Gruppenzugehörigkeit initiiert werden. Für Lehrkräfte lassen sich kaum nicht direkt bildungsbezogene Erklärungen identifizieren. So könnten sie bilinguale Praktiken zu bestimmten Zeitpunkten dulden oder gar explizit erlauben, um z. B. ihre Wertschätzung gegenüber der Bilingualität, Kultur oder Identität ihrer bilingualen SuS auszudrücken.

Die überzeugendsten potentiellen Erklärungen für die zugrundeliegenden Motive zur Verwendung bilingualer Praktiken im Unterricht stellen allerdings direkt bildungsbezogene Gründe dar. Nach Baker (2011, S. 289f) können bilingualen Praktiken im Unterricht vier zentrale Vorteile für Lern- und Bildungsprozesse zugesprochen werden: 1) Sie können zur Förderung der mündlichen sowie bildungssprachlichen Varietäten in der schwächer ausgeprägten Sprache beitragen. Diese These wurde bereits bei der Darstellung der Schwel- 
lenwert- und Interdependenzhypothese nach Cummins (2000) indirekt diskutiert. Zudem lassen sich die positiven Effekte auf den Erwerb der L2 durch eine Beschulung unter Einbezug der Erstsprache zum Beispiel anhand der berichteten Meta-Studien von Willig (1985) und Greene (1998) oder Längsschnittstudien von Ramírez (1992) beziehungsweise Ramírez, Yuen und Ramey (1991) untermauern. 2) Sie können Eltern mit geringen verkehrssprachlichen Fähigkeiten ermöglichen, ihre Kinder beim Erwerb schulischer Lerninhalte zu unterstützen. Die im Unterricht in der Verkehrssprache vermittelten zu erwerbenden Lerninhalte können so zuhause mit den Eltern in einer anderen Sprache besprochen werden, was die Teilhabe der Eltern an schulischen Lernprozessen ihrer Kinder steigert. 3) Sie können aber auch die Partizipation und Integration von Zweitsprachlernern mit verschiedenen zweitsprachlichen Fähigkeitsniveaus im Unterricht erhöhen. Hinzu kommt, dass bilinguales Sprachverhalten auch die Interaktion zwischen SuS vereinfachen und verbessern kann. Diese These wird in Abschnitt 5.4 aufgegriffen sowie anhand Evidenz zum zweisprachigen Peer-Learning illustriert und dementsprechend in den folgenden Darstellungen zunächst ausgespart. 4) Der relevanteste und überzeugendste Vorteil von bilingualen Praktiken ist allerdings, dass sie im Bildungskontext $\mathrm{zu}$ einem tieferen, umfassenderen Verständnis des Lerninhalts führen können (vgl. Baker, 2011, S. 289f). Diese These kann auch dahingehend erweitert werden, dass Translanguaging in manchen Fällen das Verstehen von Lerninhalten erleichtert oder gar erst ermöglicht. García und Kano (2014, S. 265) differenzieren hierbei zwischen „dependent" und ,independent translanguaging“ in Abhängigkeit der Sprachkompetenzniveaus der SuS. SuS, die über geringere Sprachfähigkeiten in der Unterrichtssprache verfügen, sind bei der Erarbeitung von Lerninhalten in dieser Sprache abhängig von Praktiken des Translanguaging zur Verarbeitung des Lerninhalts. SuS mit hohen Sprachkompetenzniveaus in beiden Sprachen sind nicht zwingend auf Praktiken des Translanguaging angewiesen, sie können diese aber flexibel und strategisch in förderlicher Weise einsetzen. So verwendet die erstgenannte Schülerschaft bilinguale Praktiken primär als Unterstützung, die Lern- oder Verstehensprozesse ermöglicht, während die letztgenannte Schülerschaft diese Praktiken zur Verbesserung oder Steigerung ihrer Lern- und Verstehensprozesse nutzen können.

Praktiken des Translanguaging lassen sich als spezifische Formen von kognitiven Elaborations- und/oder Organisationsstrategien (vgl. Hasselhorn \& Gold, 2017; Mandl \& Friedrich, 2006) betrachten. Zum einen greifen Lernprozesse häufig auf Vorwissen zurück, welches bei bilingualen Sprechern auch in der (weiteren) L1 enkodiert sowie in ihrem multikulturellen Horizont eingebettet sein könnte, zum anderen gilt es Wissen zu konstruieren und zu artikulieren, was in der Regel unter Verwendung sprachlicher Mittel mündlich oder schriftlich erfolgt (vgl. Baker, 2011, S. 289). Wenn der Rückgriff und die Nutzung der Ressource der (weiteren) L1 als kognitives Werkzeug für Lernprozesse unterbunden wird, sodass die SuS nur in der schwächer ausgeprägten 
Sprache lernen dürfen, kann dies einerseits zu einer Reduktion ihrer „thinking power" führen oder gar mit ,asking the child to fail" gleichgesetzt werden, insbesondere wenn es sich um kognitiv anspruchsvolle Aufgaben handelt (ebd.). Andererseits sind Praktiken des Translanguaging bei der Integration oder dem Aufbau von Wissen in geringerem Maße abhängig von der hierfür verwendeten Informationsgrundlage. Bei der Wissenskonstruktion in der Sprache der vorliegenden Informationsgrundlage können Bedeutungen, Formulierungen und ganze Schemata prinzipiell kopiert oder adaptiert werden, was kein echtes Verständnis des zu prozessierenden Lerninhalts benötigt (vgl. ebd.). Bei Prozessen des Translanguaging hingegen müssen die Konzepte in einer anderen Sprache als die der Informationsgrundlage mit eigenen Worten der anderen Sprache konstruiert und artikuliert werden, was ein höheres Verständnis der Lerninhalte voraussetzt.

Der eben angeführte Unterschied zwischen der Verarbeitung von Lerninhalten in derselben oder einer anderen Sprache als die der Informationsgrundlage lassen sich anhand der Reflexion der hierfür benötigten (mentalen) Übersetzungsprozesse illustrieren. Übersetzungen dürfen nicht nur als Zugang zur Bedeutung einzelner Wörter, Phrasen oder Sätzen missverstanden werden. Kern (1994, S. 442) definiert Übersetzungen im Kontext des L2a als „,mental reprocessing“ und bezieht sich dabei explizit auf die Ausführungen von $\mathrm{O}$ “ Malley et al. (1985, S. 33), welche Übersetzungen als ,using the first language as a base for understanding and / or producing the second language" verstehen, und auf Chamot \& Kupper (1989, S. 16), die Übersetzungen als „rendering ideas from one language to another in a relatively verbatim manner" beschreiben. Angesichts dieser Definitionen stellen Übersetzungen im Bildungskontext vielmehr eine Transformation von Informationen in eine zugängliche, nutzbare mentale Repräsentation dar und können zur Erlangung von Kontrolle über propositionale Inhalte eingesetzt werden. Dementsprechend können Übersetzungsprozesse eher als übergeordnete metakognitive Strategie des Monitorings des eigenen Lernprozesses interpretiert werden (vgl. Kern, 1994, S. 453).

Im Kontext des Fremdsprachenunterrichts mit Universitätsstudierenden identifiziert Kern (ebd., S. 447ff) unter Verwendung von ,reading task interviews" mit ,think aloud“" potentielle Vorteile und strategische Einsatzmöglichkeiten von Übersetzungen. So vereinfachen Übersetzungen die semantische Verarbeitung von Inhalten, da diese den Zugriff auf elaboriertere Repräsentationen und Konzepte in der L1 erlauben. Dies wirkt sich positiv auf die Arbeitsgedächtniskapazität aus, da das Arbeitsgedächtnis entlastet wird, wenn bereits bekannte statt neue Repräsentationen und Konzepte in diesem gehalten werden. Weiterhin können die Informationen bei Übersetzungen auch in stärker komprimierte und somit weniger belastende Informationseinheiten transformiert werden, was wiederum zu frei werdenden Arbeitsgedächtniskapazitäten führt. Zudem reduzieren Übersetzungsprozesse affektive Barrieren, die sich negativ auf die Motivation und das Selbstkonzept auswirken, wenn durch 
Übersetzungen die Inhalte für die Leser verständlich werden, was das Selbstbewusstsein erhöht. Die Studierenden verfolgen diverse Ziele, die mit Hilfe des strategischen Gebrauchs von Übersetzungen erreicht werden sollen (vgl. ebd., S. 452f). So werden Übersetzungen primär zur Konsolidation von Bedeutung, Sicherung von kontextuellen Informationen, Klärung syntaktischer Rollen, Verifizierung von grammatischen Zeitformen und Überprüfung des eigenen Verständnisses eingesetzt. Zusammengefasst können dementsprechend bilinguale Praktiken durch deren Verwendung als Elaborations- und Organisationsstrategien, zur metakognitiven Selbstregulation und als motivationale Mechanismen Lern- und Verstehensprozesse unterstützen. Im Folgenden soll die zuletzt genannte These von Baker (2011, S. 289f), dass bilinguale Praktiken zu einem tieferen, umfassenderen Verständnis des Lerninhalts führen können, anhand diverser empirischer Studien untermauert werden.

\subsubsection{Empirische Evidenz zu bilingualen Praktiken in Bildungsprozessen}

Die Evidenz zu bilingualem Sprachgebrauch in Bildungsprozessen gilt es differenziert nach verschiedenen Schulformen und Erhebungskontexten zu betrachten. Wie oben dargestellt, unterscheiden sich die mono- und bilingualen Schultypen hinsichtlich ihrer Schüler- und Lehrerschaft, dem Umgang mit der Bilingualität ihrer SuS, ihren Bildungszielen, den in der jeweiligen Nation zu Grunde liegenden Sprachideologien etc., was zu distinkten Bedingungen für den Gebrauch bilingualer Praktiken führt. Diese kontextuellen Bedingungen determinieren den sprachlichen Handlungsraum in Bildungsprozessen der bilingual aufwachsenden SuS und dementsprechend ebenfalls die Entfaltungssowie Entwicklungsmöglichkeiten ihrer bilingualen Disposition.

Im Kontext von „transitional bilingual education“ und „dual language education“ kann bilingualer Sprachgebrauch unter optimierten Bedingungen für bilingual aufwachsende SuS und hinsichtlich einer Vielzahl von Lerninhalten untersucht werden. Dies gilt in ähnlicher Weise auch für den Schultyp der Immersion, wobei sich die bilingualen Schulprogramme durchaus bezüglich einiger zentraler Konditionen wie z. B. der Homo- und Heterogenität der Sprachkompetenzprofile ihrer SuS unterscheiden. Im Gegensatz zu den bilingualen Schultypen zeichnen sich die Bedingungen für bilingualen Sprachgebrauch in Bildungsprozessen in Submersionskontexten primär durch die Akzentuierung der Verkehrssprache unter Negation der natürlich erworbenen (weiteren) L1 (mit Ausnahme der Kanonsprachen des Fremdsprachenunterrichts) aus, sodass weder explizite Instruktion noch regelmäßiger Kontakt mit bildungssprachlichen Varietäten der (weiteren) L1 der bilingual aufwachsenden SuS (mit Ausnahme des HSU) zu erwarten sind. Zudem assoziiert die Submersion eher eine defizitorientierte Perspektive mit bilingualem Sprachgebrauch und verbietet diesen zum Teil explizit. Dementsprechend lässt sich nicht von vergleichbaren 
Bedingungen zum bilingualen Sprachhandeln in Bildungsprozessen zwischen bilingualen und monolingualen Schultypen sprechen.

Dementsprechend werden im Folgenden empirische Befunde zum Gebrauch bilingualer Praktiken in Bildungsprozessen und $\mathrm{zu}$ den Einstellungen sowie Überzeugungen signifikanter Akteure in Bildungsinstitutionen unter kurzer Einordnung des Erhebungskontextes berichtet. Hierbei wird die Evidenz zu bilingualen Praktiken in Gruppenarbeiten von SuS noch nicht ausgeführt, da diese in Abschnitt 5.5 nach grundlegender Darstellung der Sozialform des „Peer-Learnings“ präsentiert werden.

Bilinguale Sprecher nutzen ihre (weitere) L1 zum Teil für nicht unterrichtsbezogene Gespräche untereinander, aber vor allem „on-task“ für den Erwerb von Lerninhalten (vgl. z. B. Barwell, 2014; Clarkson, 2007; Culligan, 2015; Dirim, 1998; García, 2011; García \& Kano, 2014; Worthy et al., 2013). So beobachtet Barwell (2014) im Mathematikunterricht einer kanadischen Grundschulklasse im Submersionskontext mit SuS der Minderheit „Cree“ unter Verwendung eines ethnographischen Zugangs, dass die SuS ihre (weitere) L1 Cree zum gegenseitigen Erklären von mathematischen Inhalten verwenden. Hierbei merken die SuS explizit in den Interviews an, dass sie die Erklärungen untereinander in der L1 als hilfreich erachten (ebd., S. 918).

Culligan (2015) interviewt SuS der elften Klasse im Kontext kanadischer Immersionsprogramme zu ihren sprachlichen Erfahrungen im Mathematikunterricht. Einige SuS berichten (vgl. ebd., S. 8ff), dass sie mit ihren Peers eher in der L1 (Englisch) kommunizieren, da sie Unbehagen empfinden, mit Freunden in der L2 (Französisch) zu interagieren. Sie empfinden keinen großen Unterschied zwischen der Instruktion mathematischer Inhalte in der L1 oder L2, aber manche SuS merken an, dass sie bei Instruktionen in der L2 aufmerksamer und konzentrierter sind. Wenn die zu lernenden Konzepte allerdings eine Herausforderung darstellen, greifen sie auf ihre L1 zurück. Zudem wird der Erwerb konzeptueller mathematischer Inhalte in der L2 von einer Schülerin als zusätzliche Belastung empfunden, was auf eine erhöhte kognitive Belastung des Prozessierens von Inhalten in einer L2 schließen lässt (vgl. ebd.).

García et al. (2011) untersuchen Praktiken des Translanguaging bei 5-6 Jahre alten spanisch-englischsprachig aufwachsenden Kindern in einem New Yorker Kindergarten, der ein balanciertes, sprachenseparierendes Konzept der „dual language education“ verfolgt. Trotz der offiziellen Sprachenseparation kreieren die Kinder unter Verwendung von bilingualen Praktiken ohne Bedenken oder Scheu einen „dritten Raum“ sprachlicher Praktiken (vgl. Li Wei, 2011), in welchem sie Translanguaging für sechs übergeordnete Funktionen verwenden: 1) Vermittlung von Sachverhalten untereinander, 2) Ko-Konstruktion von Bedeutungen, 3) Konstruktion von Bedeutungen für sich selbst, 4) Inklusion anderer, 5) Exklusion anderer und 6) Darstellung von Wissen. Hierbei ist es einigen Kindern in manchen Fällen nur mit Hilfe dieser bilingualen 
Praktiken möglich, ihre Kommunikationsabsichten zu vollziehen, dem Geschehen zu folgen und Lernprozesse anzuregen. Die Ko-Konstruktion von Bedeutungen und die Konstruktion von Bedeutungen für sich selbst treten hierbei am häufigsten auf und eröffnen stets Möglichkeiten sowohl zum Erwerb des Englischen als auch des Spanischen. Insbesondere die Funktion der Darstellung von Wissen unter Verwendung von Translanguaging kann nicht nur die Partizipation und Möglichkeiten zum Wissenserwerb erhöhen, sondern auch zu einer positiv konnotierten Identität als selbstbewusster, kompetenter bilingualer Sprecher führen (vgl. García et al., 2011, S. 45ff).

Worthy et al. (2013) untersuchen bilinguale Praktiken bei einer fünften Klasse mit „dual language education“ mit bilingual spanisch-englischsprachig aufwachsenden Kindern und ihrer bilingualen Lehrkraft, die ihren Unterricht bewusst nach Prinzipien des Konzepts von Translanguaging führt. Bei der Diskussion im Klassenverbund einer von der Lehrerin vorgelesenen Geschichte, die von den Migrationserfahrungen eines mexikanischen Mädchens und ihrer Familie in die Vereinigten Staaten von Amerika handelt, untersuchen die Forscher das bilinguale Sprachhandeln und replizieren einige der identifizierten Funktionen bilingualer Praktiken von García et al. (2011). So werden bilinguale Praktiken zur Verständnissicherung und Ko-Konstruktion von Bedeutungen primär in Form von Übersetzungen, dem Rückgriff auf sprachliches Wissen des Spanischen und durch auf Spanisch artikulierte Bezüge zu ihren eigenen Erfahrungen eingesetzt (vgl. Worthy et al., 2013, S. 317ff).

Äußerst fruchtbare Einblicke in die (strategische) Nutzung sowie Zwecke bilingualer Praktiken im Bildungskontext können durch Selbstberichte von bilingualen SuS gewonnen werden, die ihr sprachliches Handeln bei der Aufgabenbearbeitung online oder durch retrospektive Betrachtung kommentieren und erläutern. Hierzu sollen zwei Studien von García \& Kano (2014) und Clarkson (2007) etwas detaillierter ausgeführt werden.

García \& Kano (2014) untersuchen im Kontext der den Vereinigten Staaten von Amerika zehn japanisch-englischsprachig aufwachsende Kinder zwischen 12-16 Jahren mit MH und unterschiedlichen sprachlichen Kompetenzniveaus, die einen privaten Kurs zur Produktion englischsprachiger Texte zur Vorbereitung des Studierfähigkeitstests ,scholastic achievement test“ besuchen. Der Kurs wird von einer bilingual japanisch-englischsprachigen Kursleiterin unterrichtet, die explizit Praktiken des Translanguaging in den Kurs miteinbezieht. So werden zweisprachige Textmaterialien in Form von gleichen Texten durch Übersetzung oder verschiedene Texte zum gleichen Thema angeboten, wobei die Diskussion der Textinhalte primär in Japanisch vollzogen wird, während die zu verfassenden Essays im Englischen produziert werden. Die zentralen Förderziele des Kurses fokussieren die Textorganisation sowie die inhaltliche Qualität der im Text produzierten Konzepte, Ideen und Darstellungen. Das erstgenannte Ziel wurde gewählt, da sich das Englische und Japanische in der 
Textorganisation und in ihren schriftsprachlichen Diskurskulturen dahingehend unterscheiden, dass englischsprachige Texte einen deduktiven und japanischsprachige Texte einen induktiven Ansatz verfolgen (vgl. ebd., S. 262f). Die Förderung der inhaltlichen Qualität der im Text produzierten Konzepte, Ideen und Darstellungen liegt in den kulturellen Unterschieden der Staaten Japan und USA begründet, da diese unterschiedliche Ideologien und Kulturen vorweisen, die differente Konzepte akzentuieren. So sind den bilingual aufwachsenden SuS mit japanischem MH Konzepte wie Harmonie und Kooperation vertrauter als Unabhängigkeit, Demokratie und Individualismus (vgl. ebd.). Dementsprechend fokussiert der Kurs nicht primär sprachliche Fähigkeiten im Englischen, sondern allgemeine bildungssprachliche Fähigkeiten der Textproduktion sowie konzeptuelles Wissen unter (schrift-)kulturell unterschiedlich geprägten Perspektiven. García und Kano (2014) interviewen die SuS des Kurses unter Verwendung von „stimulated recall“, bei welcher die SuS einzelne Videoaufnahmen von sich bei Lernprozessen im Kurs kommentieren und erläutern.

García und Kano (ebd.) identifizieren Praktiken des Translanguaging als Unterstützung, Erweiterung sowie Verbesserung der Aufgabenbearbeitung in Abhängigkeit der Kompetenzniveaus der untersuchten SuS. Die Autoren differenzieren Praktiken des Translanguaging von SuS mit geringeren Fähigkeitsniveaus als ,dependent" und von SuS mit einer hohen bilingualen Sprachkompetenz als ,independent translanguaging“. Weiterhin wird deutlich, dass sich die Praktiken durch eine große Heterogenität auszeichnen, sich Formen metakognitiver Selbstregulation in bestimmten Praktiken zeigen und diese auch metalinguistische Bewusstheit fördern sowie (er-)fordern.

Alle untersuchten SuS verwenden Praktiken des Translanguaging im bilingualen Förderkurs. Die SuS mit geringeren Sprachkompetenzniveaus weisen interindividuell unterschiedliche bilinguale Textrezeptions- und -produktionsstrategien auf. Eine Schülerin liest den zu bearbeitenden Text auf Englisch und greift bei Verständnisproblemen auf die korrespondierenden Paragraphen im japanischen Text zurück. Ein anderer Schüler liest zunächst den Text auf Japanisch, um die Thematik in seiner Vollständigkeit zu erfassen und zu verstehen, denkt aber bei der Produktion des englischsprachigen Textes auf Englisch. Eine andere Probandin wiederum denkt im Japanischen, wenn sie den englischsprachgien Text schreibt. Unabhängig von der letztlich konkret gewählten Strategie wird bei diesen SuS deutlich, dass sie Praktiken des Translanguaging produktiv nutzen, um die Textrezeptions- und -produktionsaufgaben zu bearbeiten.

Andere Probanden sind nicht mehr zwingend auf die japanischsprachigen Textäquivalente angewiesen und verwenden Translanguaging eher zur Erweiterung ihres Textverständnisses und zur Vorbereitung auf die Textproduktion. So enthalten die ersten Essayentwürfe einer Probandin auch japanischsprachige Ausführungen zu Textstellen und Konzepten, die sie noch nicht komplett 
begreift, um ihr Verständnis zu erweitern, ihre Textproduktion zu beschleunigen und um kognitive Ressourcen sowie Zeit zu sparen (vgl. García \& Kano, 2014, S. 269). Eine andere Probandin berichtet von unbewussten erstsprachlichen Interferenzen bei der Suche nach Synonymen im Englischen (ebd., S. 269f):

,[...] I couldn't think of synonyms in English because Japanese words kept shooting through my mind one after another. So, I depended on my Japanese. [For example], we had 'use up' among the list. When I understood the word in Japanese (" 消費'), an English word “consume" came through my mind. This way, I used the Japanese language for the task."

Aus diesem Selbstbericht werden zwei zentrale Phänomene des Translanguaging evident: Zum einen treten Interferenzen zwischen den Sprachen auf, die keiner bewussten Kontrolle unterliegen, sondern impliziten Aktivierungsprozessen des sprachlichen Wissens folgen, welche die Probandin bildlich als Wörter, die ihr „durch den Kopf schießen“, beschreibt. Zum anderen kann dieser Vorgang im Sinne der Interdependenzhypothese interpretiert werden, da der implizite Rückgriff auf erstsprachliche Fähigkeiten zur Aktivierung des passenden, bildungssprachlichen Prädikats „to consume“ des Englischen führt, was als eine Form von Transfer interpretiert werden kann.

Der Einsatz von Translanguaging von SuS mit hoher bilingualer Kompetenz verfolgt primär den Zweck einer Verbesserung der Aufgabenbearbeitung. So greift ein Proband bei der Recherche zu Vor- und Nachteilen von Mobiltelefonen auf englisch- und japanischsprachige Texte zurück, um seinen Text auf einer vielfältigen, interkulturellen Informationsgrundlage zu begründen. Derselbe Proband berichtet auch von bewussten kognitiven Übersetzungsprozessen bei der Rezeption des Englischen. Durch diese Praktik können SuS selbstreguliert ihr lexikalisches Wissen nicht nur in der Zielsprache sondern auch in ihrer L1 fördern. Eine andere Schülerin, die ihrer Meinung nach über einen höheren Wortschatz im Englischen als Japanischen verfügt, nutzt ihr aus der Rezeption des englischsprachigen Textes entstandenes mentales Textmodell zur Bearbeitung des japanischsprachigen Äquivalents, um die Bedeutung unbekannter japanischer Lexeme zu klären. Translanguaging stellt dementsprechend keine unidirektionale Unterstützung, Erweiterung oder Verbesserung sprachlicher Fähigkeiten in der L2 durch den Rückgriff auf die L1 dar, sondern ist bidirektional auch als Förderung erstsprachlicher Fähigkeiten zu interpretieren.

Eine ähnliche Studie wurde von Clarkson (2007) mit 85 vietnamesischenglischsprachig aufwachsenden Kindern der vierten Klasse in Australien durchgeführt. Die SuS bearbeiteten Sprachkompetenztests in Vietnamesisch und Englisch, drei verschiedene Mathematiktests, unter anderem mit Rechen, Text- und offenen Problemlöseaufgaben, sowie Fragebögen zu ihren Hintergrunddaten und ihren kognitiven Prozessen bei der Aufgabenbearbeitung. Auf 
Grundlage dieser Daten wurden anschließend vier Probanden ausgewählt, die über eine hohe mathematische und bilinguale Kompetenz verfügen. Mit diesen $\mathrm{SuS}$ wurden Interviews durchgeführt, bei welchen sie unter Videographie mathematische Textaufgaben bearbeiten und nach der Aufgabenbearbeitung ihre kognitiven Prozesse während der Betrachtung der Videos retrospektiv erläutern. Einige der Probanden, die in den Fragebögen angaben, bei der Aufgabenbearbeitung auf Vietnamesisch zurückzugreifen, wurden in einer Follow-upUntersuchung zwei Jahre nach der Untersuchungsanlage erneut interviewt.

Zwischen 40-50\% aller Probanden gaben an, bei mindestens einer Aufgabe der drei Mathematiktests auf ihre (weitere) L1 Vietnamesisch zurückgegriffen zu haben. Der Rückgriff auf die (weitere) L1 steht in einem signifikant positiven Zusammenhang von $\mathrm{r}=.4$ mit den Leistungen der offenen Problemlöseaufgaben. Zudem zeigt sich ein signifikanter Zusammenhang in der Höhe von $\mathrm{r}=.7$ zwischen der Itemschwierigkeit der Textaufgaben und den Angaben zur Nutzung der (weiteren) L1 bei der Aufgabenbearbeitung. Dementsprechend scheint die mentale Nutzung der (weiteren) L1 sich bei bestimmten Aufgabentypen positiv auf die Lösungswahrscheinlichkeit auszuwirken und bei anderen Aufgabentypen eine Ressource darzustellen, von der bei ansteigender Schwierigkeit Gebrauch gemacht wird. Die SuS wurden zudem abhängig von ihren Kompetenzen in ihren Einzelsprachen kategorisiert. Hierbei zeigte sich unter Kontrolle der kognitiven Entwicklung, dass SuS, die in ihren beiden Sprachen geringe Kompetenzen aufweisen, signifikant schlechtere Leistungen bei den Textaufgaben und den offenen Problemstellungen erbringen als bilinguale Sprecher mit hohen sprachlichen Fähigkeiten in beiden Sprachen oder asymmetrischen Fähigkeitsprofilen. Dieses Ergebnis kann als weitere Unterstützung der Schwellenwerthypothese von Cummins (2000) interpretiert werden, da hohe sprachliche Kompetenzen in beiden Sprachen mit akademischem Erfolg zusammenhängen.

Drei der vier interviewten Probanden gaben im Interview an, von Vietnamesisch bei schulischen Lernprozessen Gebrauch zu machen. Zwei dieser SuS berichten, dass sie über Aufgaben im Vietnamesischen nachdenken, wenn die Aufgaben für sie schwierig zu lösen sind. Bei den Textaufgaben besteht die Schwierigkeit allerdings nicht im Rechnen selbst, sondern eher in dem Verständnis des sprachlich formulierten mathematischen Sachverhalts der Textaufgabe. Einer dieser Probanden weist dabei eine gewisse Kontextabhängigkeit vom Ort auf, da er eher bei der Bearbeitung von Hausaufgaben statt in der Schule auf seine beiden Sprachen zurückgreift. Im Follow-up-Interview zwei Jahre nach der ersten Untersuchungsanlage gibt dieser Schüler allerdings an, dass er nicht mehr zwischen den Sprachen bei der Aufgabenbearbeitung wechselt. Die dritte Probandin, die zweisprachiges Handeln bei der Aufgabenbearbeitung berichtet, macht von Vietnamesisch nur bei bestimmten Aufgabentypen Gebrauch. So sind für sie Bruchrechnungen, die sie von ihrer Mutter und 
Schwester beigebracht bekommen hat, in Vietnamesisch einfacher zu lösen als im Englischen.

Die Ergebnisse der Interviews lassen sich folgendermaßen zusammenfassen (vgl. Clarkson, 2007, S. 209ff): Die Wechsel vom Englischen in das Vietnamesische scheinen keine kurzfristige Lösungsstrategie partiell auftretender Probleme darzustellen, sondern eher eine übergeordnete Strategie zu sein, auch wenn diese wohl weitestgehend unbewusst und ungeplant eingesetzt wird. Diese übergeordnete Strategie kann aber mit steigenden Sprachfähigkeiten der Unterrichtssprache verworfen werden. Zudem kann der Ort der Aufgabenbearbeitung (z. B. Schule oder zu Hause) das zweisprachige Handeln beim Lösen mathematischer Aufgaben beeinflussen. Besonders spannend hierbei ist die anekdotische Evidenz, dass selbst den bilingualen Lehrkräften der SuS nicht bewusst war, dass diese bei der Bearbeitung mathematischer Aufgaben zwischen ihren Sprachen wechseln (vgl. ebd., S. 212).

Abschließend soll die Studie von Dirim (1998) in ihren Grundzügen dargestellt werden, da diese eindrucksvoll zeigt, dass Angebote bilingualer Praktiken im Submersionskontext implementiert werden können und hierfür nicht zwingend stets eine bilinguale Lehrkraft zur Verfügung stehen muss. Dirim (ebd.) untersucht unter Verwendung eines ethnographischen Zugangs den Sachunterricht einer Grundschulklasse, die zum Großteil aus deutsch- und türkisch-deutschsprachigen SuS besteht. In der untersuchten Klasse erhält das Türkische neben dem Deutschen den Status als offizielle Unterrichtssprache, die nicht nur exklusiv unter den türkisch-deutschsprachigen SuS Verwendung findet, sondern auch im vollen Klassenverbund genutzt wird. Die Klasse wird im Team-Teaching von zwei Lehrerinnen unterrichtet, die kein Türkisch sprechen. Die türkisch-deutschsprachigen SuS erhalten eine koordinierte Alphabetisierung unter Mitwirkung des Lehrers des HSU. Dieser Untersuchungsklasse wird eine weitere Klasse gegenübergestellt, die quasi als Kontrollgruppe fungiert. Die Beobachtungen zeigen, dass Deutsch die dominante Sprache im Unterricht darstellt und dies auch von allen SuS akzeptiert wird. Die deutsch-türkischsprachigen SuS verwenden das Türkische in Situationen, in denen sie es als legitim betrachten (vgl. ebd., S. 147). Sie verwenden bilinguale Praktiken primär zum Task-Management, aber auch zur Aufgabenbearbeitung. Die hierbei auftretenden Übersetzungsprozesse lösen weiterführende Denk- und Reflexionsprozesse aus, insbesondere hinsichtlich metalinguistischer Bewusstheit. Selbst im Gespräch im Klassenverband greifen sie auch auf Entlehnungen aus dem Türkischen zurück. Zentrale inhaltliche Begriffe werden allerdings stets im Deutschen produziert. Die „Kontrollfunktion“ der Lehrkraft wird dahingehend berücksichtigt, dass in Anwesenheit der Lehrkraft in der Regel nur deutschsprachige Äußerungen produziert werden. Nichtsdestotrotz hat das Türkische auch bei der Interaktion unter den deutsch-türkischsprachigen SuS einen geringeren Stellenwert als das Deutsche (vgl. ebd., S. 144). Ein weiterer 
interessanter Befund ist, dass das Türkische in dieser Klasse ein gruppenkonstituierendes Merkmal darstellt. In dieser Klasse gehen die deutsch-türkischsprachigen SuS engere Peerbeziehungen ein als in der Beobachtungsklasse, in der das Türkische keinen offiziellen Status als Unterrichtssprache hat. Dementsprechend scheint Sprache in Bildungskontexten, in denen die Sprache funktional genutzt und anerkannt wird, als ein gruppenkonstituierendes Merkmal zu fungieren.

Die eben angeführten potentiellen Vorteile von Translanguaging im Bildungskontext sowie deren theoretische und empirische Untermauerung stellen die Angemessenheit monolingualer Praktiken der Submersion sowie die zum Teil strikte Sprachenseparation in bilingualen Bildungsprogrammen als Beschulungsformen von bilingual aufwachsenden Kindern in Frage. Eine gelingende Implementation von bilingualen Praktiken in den Unterricht muss allerdings die Einstellungen und Überzeugungen von Lehrkräften berücksichtigen, da diese die zentralen Akteure der Umsetzung von Unterrichtsangeboten und praktiken darstellen. Dementsprechend sollen im Folgenden zentrale Befunde zu Einstellungen und Überzeugungen von Lehrkräften zu bilingualen Praktiken im Unterricht betrachtet werden, da es diese bei der Entwicklung von Methoden zur Integration bilingualer Praktiken in den Unterricht ernst zu nehmen und zu berücksichtigen gilt.

\subsubsection{Empirische Evidenz zu Einstellungen und Überzeugungen von Lehrkräften und pädagogischen Fachkräften zu bilingualen Praktiken in Bildungsprozessen}

Hinsichtlich der Untersuchung von Einstellungen wird im Kontext von Lehrkräften und pädagogischen Fachkräften eher der Begriff „Überzeugungen“ gebraucht. Nach Trautmann (2005, S. 39) umfassen Überzeugungen „Einstellungen, Werte, Meinungen oder Handlungsstrategien“. Die Bewusstheit und Beeinflussbarkeit von Überzeugungen sowie deren Abgrenzung von konkretem Wissen wird kontrovers diskutiert (vgl. Hammer, Fischer \& Koch-Priewe, 2016, S. 149). Sofern Wissen und Überzeugungen miteinander verbunden sind, entweder in reziprokem Zusammenhang oder in Form von Überzeugungen als einem Teil von Wissen, können Überzeugungen durch Wissen beeinflusst und somit verändert werden (vgl. ebd., S, 149). Dementsprechend können Überzeugungen als ,subjektiv geprägte und als legitim angesehene Thesen“ definiert werden, ,die die Analysen von und Reaktionen auf Sachverhalte, Situationen und Personen beeinflussen" (vgl. ebd., S. 150).

Die interviewte Lehrkraft in der Studie von Barwell (2014) im Kontext der Submersion ist bezüglich des Gebrauchs der L1 zwiegespalten. Einerseits verbietet die Lehrkraft explizit den Gebrauch der (weiteren) L1, was auch mit dem Schriftzug „Remember to speak in English“ an einer Wand des Klassenraums 
dauerhaft visuell präsent ist, da seine SuS neben Mathematik auch die L2 Englisch erwerben sollen. Bei Zuwiderhandlungen dessen weist die Lehrkraft seine SuS darauf hin, in Englisch zu kommunizieren, selbst wenn es sich um Erklärungen handelt. Andererseits sieht er aber auch positive Effekte im Rückgriff auf die (weitere) L1, insbesondere wenn einige SuS die Instruktionen der Lehrkraft im Englischen nicht verstehen und diese in ihrer (weiteren) L1 von Peers erklärt bekommen und wenn sie sich untereinander mathematische Konzepte erklären (ebd., S. 917). Die Lehrkraft würde gerne diese Erklärungen untereinander verstehen, um Einblick in das (fehlende) Verständnis der Lerninhalte seiner $\mathrm{SuS}$ zu erhalten und diese mit der ganzen Klasse zu teilen.

Wlossek und Rost-Roth (2016) interviewten 17 Lehrende von Mittelschulen unter Verwendung von „stimulated recalls“ mit Videoszenen aus dem eigenen Unterricht, wobei acht Lehrkräfte Regelklassen (RK) unterrichten und die restlichen neun in Übergangsklassen (ÜK) tätig sind, die aus kürzlich nach Deutschland zugewanderten SuS bestehen. Das Erkenntnisinteresse der Studie fokussiert die Lehrerüberzeugungen zum Aufwachsen in mehrsprachigen Kontexten, zu Erfahrungen mit der (weiteren) L1 der SuS im Unterricht und zur Einschätzung von Konzepten der Mehrsprachigkeitsdidaktik.

Der Großteil der Lehrkräfte der ÜK sowie vereinzelt der RK teilen eine positive Einschätzung zum Aufwachsen in einem mehrsprachigen und multikulturellen Umfeld. Hierbei wird die (weitere) L1 als „Schatz“ bezeichnet, die eine Ressource für das berufliche Weiterkommen darstellt, und ein potentieller Sprachverlust als bedauerlich betrachtet. Viele der Lehrenden in RK glauben, dass ihre SuS ihrer Mehrsprachigkeit selbst keine hohe Bedeutung zusprechen, da diese über eher schwach ausgeprägte Fähigkeiten in ihrer (weiteren) L1 verfügen. Allgemein berichtet der Großteil der Lehrkräfte beider Klassenformen von ihrer Ambivalenz hinsichtlich der Wertschätzung der (weiteren) L1 einerseits und der Förderung des L2a, welche in einem vermeintlichen Konkurrenzverhältnis stehen. Typische Mythen der Mehrsprachigkeit sind ebenfalls in den Überzeugungen der interviewten Lehrkräfte zu identifizieren, da Code-Switching und -Mixing mit einer ,,doppelten Halbsprachigkeit“" und einer Verlangsamung des L2a in Verbindung gebracht werden.

Der Gebrauch der (weiteren) L1 im Unterricht stellt für den Großteil der Lehrkräfte der RK kein typisches Phänomen dar, da die SuS nur vereinzelt auf ihre (weitere) L1 zurückgreifen und diese nur selten thematisiert oder in den Unterricht integriert wird. Im Gegensatz hierzu berichten die Lehrkräfte der ÜK, dass eine Einbindung der L1 der SuS nicht zu vermeiden ist, sondern den Normalfall darstellt. Die L1 wird hierbei vor allem bei der Wortschatzarbeit, für Sprachvergleiche und in Form von Übersetzungen als Hilfestellungen für andere Peers verwendet. Die Integration der L1 verläuft aber mit Ausnahme von zwei Lehrkräften spontan und ungeplant, sodass beim Großteil dieser Lehrkräfte nicht von einem expliziten, systematischen Ansatz des Translanguaging gesprochen werden kann. Interessant ist, dass einige Lehrkräfte der ÜK 
der Einbindung der L1 einen positiven Beitrag auf die Identitätskonstruktion ihrer SuS zusprechen.

Hinsichtlich der Implementation von Konzepten der Mehrsprachigkeitsdidaktik in den Regelunterricht äußert der Großteil der Lehrkräfte Zweifel an deren Umsetzbarkeit. Das Curriculum selbst beansprucht schon genügend Instruktionszeit und der Erwerb bestimmter Lerninhalte unter Verwendung der Einbindung der (weiteren) L1 würde angesichts der schwach ausgeprägten Sprachkompetenzen noch mehr Zeit beanspruchen. Lediglich zwei Lehrkräfte sprechen sich für die Einbindung der (weiteren) L1 vor allem in RK aus, da sie dies als notwendig erachten, damit ihre SuS ihre eigene Mehrsprachigkeit entdecken und eine positive Identität ausbilden können. Für jüngere SuS könnte die (weitere) L1 spielerisch eingebunden werden und für ältere SuS sollte sie primär sprachvergleichend eingesetzt werden. Bei der Umsetzung mehrsprachigkeitsdidaktischer Konzepte gilt es nach Ansicht der Lehrkräfte darauf zu achten, dass diese in Verbindung mit dem Curriculum stehen. Zudem sollte die Klassenzusammensetzung keine Gruppendominanz einer bestimmten sprachlichen Minderheit aufweisen.

Zusammengefasst vertritt der Großteil der Lehrkräfte in der Studie von Wlossek \& Rost-Roth (ebd.) kritische Positionen gegenüber (migrationsbedingter) Mehrsprachigkeit im Bildungskontext. Sie postulieren ein vermeintliches Konkurrenzverhältnis zwischen (weiterer) Erst- und Verkehrssprache im Sinne der Time-on-Task Hypothese und in der sehen in der Einbindung der (weiteren) L1 in RK einen zusätzlichen Zeitverlust auf Kosten des Curriculums. Mehrsprachigkeit wird prinzipiell nicht als Ressource für Lernprozesse und Bildung gesehen, sondern als Vorteil auf dem Arbeitsmarkt und im Falle der Lehrkräfte der ÜK als vorübergehende, notwendige Strategie für Bildungsprozesse interpretiert, was an das Konzept von „dependent translanguaging“ von García und Kano (2014) erinnert. Zudem reproduzieren diverse Lehrkräfte populäre Mythen der Mehrsprachigkeit in Form einer Defizitperspektive auf Code-Switching und -Mixing. Allerdings weisen einige der Lehrkräfte der ÜK zum Teil positive und im Einklang mit aktuellen Forschungsbefunden stehende Überzeugungen hinsichtlich Mehrsprachigkeit und Bildung auf, da sie z. B. die Bedeutung der (weiteren) L1 für die Identitätsbildung akzentuieren.

Für den Kontext der Immersion identifiziert Culligan (2015) ähnliche Überzeugungen der Immersionslehrkräfte wie die Lehrkräfte der ÜK von Wlossek und Rost-Roth (2016). Die Lehrkräfte weisen darauf hin, dass der Rückgriff auf die L1 in bestimmten Fällen notwendig ist. Einige Lehrkräfte sehen im Lernen der Inhalte in der L2 Französisch eine zusätzliche Belastung für manche SuS. Hierbei akzentuieren sie die Herausforderung, die die Balance zwischen der Vermittlung der mathematischen Lerninhalte, also des Curriculums, und der Förderung des L2a erzeugt. Beide Ziele sind nicht immer parallel 
miteinander zu vereinen, sodass manchmal auf das ,Werkzeug“ Englisch zurückgegriffen werden muss, wie z. B. in Form von Übersetzungen bei lexikalischen Verständnisproblemen (vgl. Culligan, 2015, S. 11ff).

Auch im Kontext des Fremdsprachenunterrichts existiert ein vermeintliches Konkurrenzverhältnis zwischen der Nutzung der L1 und dem Erwerb der Fremdsprache (vgl. Carless, 2008). Die Lehrkräfte fühlen sich „uncomfortable or somewhat guilty", wenn SuS ihre L1 verwenden, insbesondere da sie bei der Erstsprachennutzung der SuS untereinander in Gruppenarbeiten nicht unterrichtsbezogene Interaktionen vermuten (ebd., S. 333). Nach den Beobachtungen der Lehrkräfte tritt der Rückgriff auf die L1 in Abhängigkeit der Aufgabenschwierigkeit und -komplexität auf. Dementsprechend rufen insbesondere offene Aufgaben, die sich in der Regel durch Komplexität und damit einhergehend durch einen gewissen Anspruch auszeichnen, Interaktionen in der L1 hervor (vgl. ebd., S. 337f).

Die eben berichteten Studien untersuchten praktizierende Lehrkräfte mit Berufserfahrung. Es stellt sich die Frage, ob die kommende Generation von Lehrkräften ähnliche Überzeugungen hinsichtlich Mehrsprachigkeit aufweist. Hammer, Fischer und Koch-Priewe (2016) untersuchen Zusammenhänge von Überzeugungen hinsichtlich Mehrsprachigkeit und dem Wissen zu Deutsch als L2 bei insgesamt 427 Lehramtsstudierenden verschiedener Fächer. Hierfür validieren sie einen Fragebogen zu Überzeugungen hinsichtlich Mehrsprachigkeit, der aus den Dimensionen „Sprachsensibilität im Fachunterricht“, „Zuständigkeit für Sprachförderung " und „Wertschätzung von Mehrsprachigkeit“ besteht. Zudem erheben sie mit Hilfe eines Tests Wissen zu Deutsch als L2 und mit einem weiteren Fragebogen soziodemographische Informationen der Probanden. Hammer, Fischer und Koch-Priewe (ebd.) weisen signifikante positive Zusammenhänge zwischen dem Wissen über Deutsch als L2 als auch der Semesteranzahl und positiven Überzeugungen zu Mehrsprachigkeit insgesamt und auf allen drei Dimensionen nach. Angehende Lehrkräfte des Fachs Mathematik zeichnen sich durch eher negative Überzeugungen hinsichtlich Mehrsprachigkeit aus, insbesondere hinsichtlich der Sprachsensibilität im Fachunterricht. Unter Verwendung der verschiedenen Datenquellen identifizieren Hammer, Fischer und Koch-Priewe (ebd., S. 159ff) drei verschiedene Typen von Lehrkräften bezüglich Überzeugungen zu Mehrsprachigkeit. Mehr als die Hälfte $(56,9 \%)$ der angehenden Lehrkräfte können dem Typus ,bewusster Unterstützer" zugeordnet werden, der sich durch eine hohe Wertschätzung der Mehrsprachigkeit der SuS, die Übernahme von Verantwortung für Sprachförderung sowie Praxiserfahrung auszeichnet. 28,3\% der Lehramtsstudierenden werden als „,naive Beschützer“ kategorisiert, da sie positive Absichten verfolgen, aber mehrsprachige SuS vor sprachlicher Komplexität schützen wollen und sie somit in ihren Kompetenzen unterschätzen. Der Typus „erfahrungsarme Fachorientierte", der sich durch die Priorisierung der zu vermittelnden 
Lerninhalte statt Sprachbildung sowie durch einen Mangel an Praxiserfahrung auszeichnet, stellt mit 14,8\% den am seltensten zugeordneten Typus dar.

Die hohe Prävalenz des Typus „bewusster Unterstützer“ lässt vermuten, dass die Phänomene Mehrsprachigkeit und Deutsch als L2 in der Lehramtsausbildung in Deutschland eine gewisse Rolle spielen. Zudem zeigen die Ergebnisse, dass positive Überzeugungen bezüglich Mehrsprachigkeit mit dem Wissen über Deutsch als L2 zusammenhängen, was die Bedeutsamkeit der Thematisierung dieser Phänomene in der Lehramtsausbildung weiterhin unterstreicht. Neben dem Wissen scheinen aber auch Erfahrungen in der Praxis sich positiv auf die Überzeugungen von angehenden Lehrkräften hinsichtlich Mehrsprachigkeit auszuwirken. Insbesondere die Unterscheidung der „erfahrungsarmen Fachorientierten“ und „bewussten Unterstützer" anhand der Quantität der in der Praxis gesammelten Erfahrung könnte darauf hinweisen, dass die Erkenntnis der Relevanz sprachlicher Fähigkeiten sowie die Notwendigkeit sprachlicher Bildung für das Lernen in allen Fächern primär durch Praxiserfahrung beim Vermitteln der Lerninhalte gewonnen wird.

Die Überzeugungen hinsichtlich und der Umgang mit der Bilingualität von Kindern ist bereits in vorschulischen pädagogischen Institutionen relevant, da bilingual aufwachsende Kinder bereits in Kindertagesstätten mit der vorherrschenden gesellschaftlichen Sprachideologie konfrontiert werden. Obwohl nahezu alle Bildungspläne der Bundesländer explizit die Wertschätzung und Förderung von Zwei- und Mehrsprachigkeit als Ziele frühkindlicher Bildung benennen (vgl. Viernickel \& Schwarz, 2009), ist eine konkrete, praktische Umsetzung dieser Ziele kaum vorzufinden (vgl. Kratzmann et al., 2017, S. 238ff). Angesichts der Heterogenität der (weiteren) L1 der Kinder gilt es umsetzbare Möglichkeiten zu identifizieren, die die natürlich erworbene Mehrsprachigkeit der Kinder wertschätzen und fördern. Konzepte zur Implementation von Mehrsprachigkeit ,nennen daher als übergreifende Ziele die Förderung von Respekt vor anderen Kulturen, den Aufbau einer sprachlichen Brücke zur Familie und den weiteren Kompetenzerwerb in der Erstsprache der Kinder" (ebd., S. 239).

Kratzmann et al. (2017) haben die Einstellungen zu und den Umgang mit Mehrsprachigkeit bei 119 pädagogischen Fachkräften in 19 Kindertagesstätten hinsichtlich der eben genannten übergeordneten Ziele der Integration von Mehrsprachigkeit in Kindertagesstätten untersucht. Hierfür nutzten sie eine Vielzahl von Datenquellen wie Fragebögen zur Erhebung von Einstellungen und Hintergrundvariablen, einen Wissenstest $\mathrm{zu}$ Mehrsprachigkeit und Deutsch als L2, Ratingverfahren zur mehrsprachigen Ausstattung (z. B. Medien wie Bücher, Zeitschriften, Informationsmaterialien, Spiele etc.) und Raumgestaltung (z. B. Poster, Schilder etc.) sowie ein Instrument zur Untersuchung mehrsprachiger Interaktion. Die Stichprobe bestand nur aus pädagogischen Fachkräften, die eine Gruppe (stellvertretend) leiten. Die verschiedenen Datenquellen wurden unter Verwendung von Mehrebenen- und Pfadmodellen in Bezug zueinander gesetzt. 
Die von Kratzmann et al. (ebd.) untersuchten pädagogischen Fachkräfte vertreten signifikant stärker kompensatorische Überzeugungen im Sinne eines Ausgleichs der Sprachkompetenzen mono- und bilingual aufwachsender Kinder sowie multilingual-pädagogische Einstellungen, die Mehrsprachigkeit als Kompetenz und Normalfall, von denen auch monolinguale Kinder profitieren können, akzentuieren. Assimilatorische Einstellungen stehen hierbei in einem negativen Zusammenhang mit der Berufsausbildung, allerdings in einem positiven Zusammenhang mit der Kenntnis einer anderen Sprache. Dementsprechend erscheinen Quereinsteiger als auch überraschenderweise mehrsprachige pädagogische Fachkräfte als weniger geeignet für die Umsetzung der übergeordneten Ziele des Umgangs mit Mehrsprachigkeit. Die pädagogischen Fachkräfte erreichen im Mittel einen Wert von 7,70 samt einer Standardabweichung von 2,60 bei maximal 14 zu erreichenden Punkten im Wissenstest. Im Gegensatz zu der Untersuchung von Hammer, Fischer und Koch-Priewe (2016) lassen sich allerdings keine signifikanten Zusammenhänge zwischen dem Wissen über Mehrsprachigkeit und den Einstellungen identifizieren.

Die untersuchten Kindertagesstätten weisen eine geringe Anzahl von mehrsprachigen Angeboten durch Medien (Mittelwert von 4,76 Medien pro Kindertagesstätte) sowie eine geringe Sichtbarkeit von Mehrsprachigkeit (Mittelwert des Ratings von 1,47 und Maximum von 3 bei fünfstufiger Likertskala) auf. Die mehrsprachige Ausstattung und Raumgestaltung hängt in zu erwartender Weise mit den eben berichteten Einstellungen zusammen, sodass zum Beispiel assimilatorische Einstellungen auf der Ebene der Kindertagesstätte negativ mit dem Vorhandensein mehrsprachiger Materialien und Raumgestaltung korreliert. Mehrsprachige Interaktionen treten in den untersuchten Kindertagesstätten im Mittel zu 16\% auf. Allerdings umfasst die Operationalisierung auch englischsprachige Interaktionen, sodass der Anteil an Interaktionen unter Verwendung der natürlich erworbenen, migrationsbedingten Sprachen deutlich geringer ausfallen dürfte.

Die Ergebnisse von Kratzmann et al. (ebd.) lassen den Schluss zu, dass die Umsetzung der übergeordneten Ziele der Integration der Mehrsprachigkeit in Kindertagesstätten bis heute kaum vollzogen sind. Zwar weisen die pädagogischen Fachkräfte eher als positiv zu beurteilende Einstellungen zur Mehrsprachigkeit auf, aber von einer Wertschätzung und Förderung der sprachlichen Dispositionen bilingual aufwachsender Kinder durch mehrsprachige Medien, Raumgestaltung und Interaktionen kann angesichts der Ergebnisse nicht gesprochen werden.

\subsubsection{Zusammenfassung}

Die Bedeutung von Sprache und sprachlicher Fähigkeiten für Bildungsprozesse ist nicht zu hinterfragen. Sprache ist eng mit der Identität (vgl. Hu, 2007) 
und dementsprechend auch mit der Subjekt-Entwicklung verbunden. Die Welt-Erschließung erfolgt zu einem Großteil in alltäglich-sozialen Interaktionserfahrungen (vgl. Stojanov, 2006), wobei sich insbesondere der Erwerb von konzeptuellen Inhalten in Form von Wissen und Fähigkeiten primär durch sprachliche Kommunikation und Sprache als kognitives Werkzeug (vgl. Hamers \& Blanc, 2000) vollzieht. Der enge Zusammenhang von Sprache und Bildung wird auch von den verschiedenen monolingualen und bilingualen Schultypen akzentuiert, wobei sich diese allerdings inter- sowie intraindividuell im Umgang mit den erstsprachlichen Fähigkeiten sowie den bilingualen Praktiken ihrer bilingual aufwachsenden Schülerschaft unterscheiden (vgl. Baker, 2011). Während „dual language education“ und Immersionsprogramme auf hohe sprachliche Kompetenzen in beiden Sprachen zielen, wird die nicht verkehrssprachliche L1 in „transitional bilingual education“ temporär zum Zwecke der Assimilation und in der Submersion - mit Ausnahme des HSU - nicht gefördert. Nichtsdestotrotz orientieren sich alle Schultypen in unterschiedlicher Intensität an dem Postulat der Time-on-Task-Hypothese hinsichtlich des sprachlichen Lernens, sodass eine Erhöhung der Quantität der Lerngelegenheiten mit der Entwicklung sprachlicher Fähigkeiten in den Einzelsprachen gleichgesetzt wird. Selbst der Großteil bilingualer Schultypen wie „dual language education" und Immersionsprogramme zeichnen sich durch eine systematisch organisierte Separation von Sprachen nach Zeit, Fächern, Tagen etc. aus, auch wenn sie durch ihr Ziel der hohen Sprachkompetenz in beiden Sprachen prinzipiell die grundlegenden Prämissen der Schwellenwert- und Interdependenzhypothese von Cummins (2000) verfolgen. Dabei weist die empirische Evidenz zu bilingualen Praktiken in Bildungsprozessen auf eine Vielzahl förderlicher Zwecke und Verwendungsweisen von bilingualen Praktiken in Bildungsprozessen hin (vgl. z. B. Barwell, 2014; Clarkson, 2007; Culligan, 2015; Dirim, 1998; García, 2011; García \& Kano, 2014; Worthy et al., 2013), die zum Teil implizit und automatisiert ablaufen. Abhängig von dem vorliegenden Sprachkompetenzprofil sowie der konkreten Aufgabe treten bilinguale Praktiken zumindest temporär in bildungsbezogenen Denkprozessen bilingual aufwachsender SuS auf. Diese bilingualen Praktiken sind für manche SuS zum Teil notwendig, für andere hingegen dienen diese als Erweiterung zur Verbesserung ihrer Lernprozesse (vgl. García \& Kano, 2014). Wie anhand der theoretischen Inspektion von Übersetzungsprozessen als Transformation von Informationen in zugänglichere, nutzbare mentale Repräsentation, die zur Erlangung von Kontrolle über propositionale Inhalte eingesetzt werden können (vgl. Kern, 1994), lässt sich in Anlehnung an Baker (2011, S. 289f) konstatieren, dass bilinguale Praktiken zu einem tieferen, umfassenderen Verständnis des Lerninhalts führen können. Hiervon gilt es Lehrkräfte und pädagogische Fachkräfte zu überzeugen, da diese zwar generell positive Einstellungen zu Mehrsprachigkeit haben, bilinguale Praktiken in Bildungsprozessen allerdings eher aus einer Defizitperspektive betrachten (vgl. Kratzmann et al., 2017; Wlossek 
\& Rost-Roth, 2016). Zudem vermuten sie ein Konkurrenzverhältnis im schulischen Kontext zwischen der Entwicklung der Einzelsprachen bilingualer Sprecher, bei dem die Entwicklung der einen Sprache auf Kosten der Entwicklung der anderen verläuft (vgl. Wlossek \& Rost-Roth, 2016), was letztlich zu einer Reduktion der Quantität der Lerngelegenheiten führen soll. Dementsprechend sollte die Implementation von mehrsprachigen Unterrichtskonzepten der Mehrsprachigkeit in den Regelunterricht mit zentralen Inhalten des Curriculums vereinbar sein, da die zusätzliche Förderung der (weiteren) L1 der SuS zusätzliche zeitliche Ressourcen benötigen würde.

\subsection{Fazit}

Bilingualität, definiert als Gebrauch von zwei oder mehr Einzelsprachen im alltäglichen Leben (vgl. Grosjean \& Li, 2013, S. 5), entwickelt sich innerhalb eines natürlichen Erwerbs in den Spracherwerbstypen 2L1a oder L2a (vgl. Grimm \& Schulz, 2016; Schulz \& Grimm, 2012; Tracy, 2008). Diese Spracherwerbstypen determinieren die Sprachentwicklung der bilingual aufwachsenden Individuen, wobei die konkrete individuelle Spracherwerbssituation und die damit verbundenen Lern- und Erwerbsgelegenheiten sich zwischen, aber auch innerhalb desselben Spracherwerbstyps unterscheiden können. Für bilinguale Spracherwerbstypen lässt sich aber eine zentrale Gemeinsamkeit konstatieren, die für den Großteil der bilingual aufwachsenden Sprecher gilt, und zwar die (partielle) komplementäre Verteilung des Gebrauchs der beiden Einzelsprachen abhängig von verschiedenen Kontexten und Bereichen ihrer zweisprachigen Lebensrealität (vgl. Grosjean, 2010, S. 29ff). Nichtsdestotrotz lassen sich primär temporäre Unterschiede zwischen dem L1a und dem 2L1a sowie frühen L2a hinsichtlich basaler sprachlicher Kompetenzen zur Kommunikation identifizieren (vgl. Grimm \& Schulz, 2016; Schulz \& Grimm, 2012; Tracy, 2008). Die potentiellen kognitiven Unterschiede zwischen monolingual und bilingual aufwachsenden Sprechern, insbesondere hinsichtlich Metakognition und exekutiver Funktionen, scheinen angesichts der kontroversen Befundlage überschätzt worden zu sein(vgl. Bialystok, 2017; Lehtonen et al., 2018). Diesbezüglich dürften weitere potentielle Einflussfaktoren die positiven Effekte auf exekutive Funktionen erzeugen, die zwar mit Bilingualität assoziiert sind, aber nicht über Bilingualität selbst hervorgerufen werden. Die im jeweiligen Kontext vorherrschende Sprachideologie (vgl. Bourhis, 2001), die die Einstellungen $\mathrm{zu}$ Bilingualität, $\mathrm{zu}$ bestimmten Einzelsprachen bilingualer Sprecher und über diese Form von Wertschätzung ebenfalls die strukturellen und organisatorischen Fördermöglichkeiten bilingualer Sprachkompetenz restringieren, scheint eine zentrale Größe für die bilinguale Sprachentwicklung sowohl auf (meta-)linguistischer als auch (meta-)kognitiver Ebene darzustellen. 
Die positiven Effekte von Bilingualität auf exekutive Funktionen wurden vornehmlich bei Stichproben identifiziert, die im Kontext einer pluralistischen Sprachideologie leben und eher hohe, balancierte Sprachkompetenzen in beiden Sprachen vorweisen (vgl. Bialystok, 2017). Bereits 1974 wies Lambert auf den Zusammenhang des soziokulturellen Kontextes und der Entwicklung der Bilingualität sowie den damit assoziierten sprachlichen Fähigkeiten hin, welcher sich über die folgenden Dekaden anhand einer Vielzahl von empirischen Befunden weiter erhärtet.

Die temporären Unterschiede zwischen den BICS monolingual und bilingual aufwachsender SuS vollziehen sich allerdings noch über den Altersbereich der Grundschule, sodass die zeitlich begrenzten sprachlichen Entwicklungsunterschiede sich dennoch negativ auf ihre Bildungsprozesse auswirken können. Wenn Lehrkräfte durch den monolingualen Habitus (vgl. Gogolin, 1994) monolingual aufwachsende SuS als soziale Bezugsnorm definieren und die mehrsprachige Disposition ihrer bilingualen SuS aus einer Defizitperspektive betrachten, wirkt sich dies negativ auf die Bildungsprozesse sowie die die Ausbildung von CALP von bilingual aufwachsenden SuS aus. Hierbei gilt es darauf hinzuweisen, dass erste Erfahrungen mit konzeptionell schriftsprachlichen Varietäten sowie allgemein mit Literacy von bilingual aufwachsenden $\mathrm{SuS}$ in manchen Fällen durchaus exklusiv - mit Ausnahme von frühen Literacyerfahrungen in Kindertageseinrichtungen - in der (weiteren) L1 innerhalb der familiären Sozialisation gemacht werden. Sofern die Grundschule an diese Erfahrungen nicht explizit anknüpft, nutzt sie nicht das Potential zur Initiation von Transferprozessen bilingual aufwachsender SuS, sodass diese hinsichtlich des Transfers auf sich alleine gestellt sind. Dementsprechend lässt sich zumindest für (meta-)linguistische Kompetenzen bilingual aufwachsender SuS schließen, dass zwar sprachliche Differenzen zwischen den BICS monolingual und bilingual aufwachsender SuS zu Beginn und noch während der Grundschule bestehen, ein adaptiver Unterricht samt Einbezug der erstsprachlichen Literacyerfahrungen sowie der metalinguistischen Fähigkeiten zum Sprachvergleich von bilingual aufwachsenden SuS diesen Unterschieden allerdings fruchtbar begegnen könnten.

Der Zusammenhang von Identität, Kultur und Sprache (vgl. Hu, 2007) akzentuiert ebenfalls die Bedeutung der Sprachideologie auf bilinguale Sprachentwicklung und allgemein auf Bildungsprozesse von bilingual aufwachsenden SuS. Da sowohl sprachliche als auch kulturelle Merkmale von Individuen in ihre Identitätsentwicklung einbezogen werden, bilden sich im Kontext der migrationsbedingten Mehrsprachigkeit auch hybride Identitäten (vgl. Keim, 2007, 2012; Krumm, 2009; Mecherill, 2005; Stojanov, 2006) aus. Hybride Identitäten können nach Stojanov (2006, S. 180) als Übersetzung interpretiert werden, die ,einen Vorgang der Transformation der primär einsozialisierten kulturellen Narrative“ hervorruft, bei dem ,eine Überschreitung der kontextu- 
ellen Grenzen der Wirklichkeitsdeutungsmuster der entsprechenden kulturellen Gruppe" erfolgt. Dementsprechend weisen hybride Identitäten im Kontext der Migration kulturelle und sprachliche Merkmale sowohl der Mehrheitsgesellschaft als auch der jeweiligen zugehörigen Minderheitsgesellschaft und neue, originäre Aspekte auf. Sprachideologien, die assimilatorische Tendenzen vertreten, können kognitive Dissonanzen des subjektiven Selbst mit dem idealisierten und/oder sozialen Selbst hervorrufen, die implizit zentrale Identitätsmerkmale in Frage stellen und sich negativ auf das Selbstbewusstsein und schließlich auch auf Bildungsprozesse auswirken können. In solchen Kontexten werden bilinguale Sprachpraktiken häufig aus einer Defizitperspektive betrachtet, obwohl sie typische Phänomene bilingualen Sprachverhaltens darstellen, die durch morphosyntaktische Bedingungen restringiert sind und sich auf grammatischer Ebene allgemein durch Systematik und Regelhaftigkeit auszeichnen (vgl. z. B. Gardner-Chloros, 2009; Potowski, 2009; Özdil, 2010). Phänomene des Code-Switchings und -Mixings sind als durch das „complementary principle" (vgl. Grosjean, 2010, S. 29ff) determinierte, kreative und angemessene sprachliche Praktiken zu interpretieren, durch welche das komplette Sprachvermögen bilingual aufwachsender SuS produktiv werden kann. Die negativen Einflüsse assimilatorischer Sprachideologien können so weit gehen, dass bilinguale Sprecher selbst negative Einstellungen zu ihrer Bilingualität entwickeln (vgl. Gardner-Chloros, 2009, S. 81f; Ritchie \& Bhatia, 2013, S. 389) oder sich von der Mehrheitsgesellschaft abgrenzen, indem sie ihre kulturellen und sprachlichen Dispositionen ihrer gesellschaftlichen Minderheit erhöhen (vgl. Bohnsack \& Nohl, 2001).

Hamers und Blanc (2000, S. 124) akzentuieren in ihrem soziokognitiven Modell der Sprachentwicklung die lebensweltlichen Bedingungen als Rahmen der Sprachentwicklung, was sie auch innerhalb des Schaubildes ihres Modells illustrativ umsetzen (siehe Abbildung 1). Im Folgenden soll dieses Modell kurz bezüglich (bilingualer) Sprachentwicklung umrissen werden, um die engen Zusammenhänge und Abhängigkeiten der konkreten sozialen Lebensrealität mit der individuellen (bilingualen) Sprachentwicklung zu verdeutlichen und die im Kapitel 2 und 3 zugehörigen zentralen Erkenntnisse nochmal zusammenzufassen. Eine detaillierte, erschöpfliche Darstellung des Modells kann bei Hamers und Blanc (ebd., S. 110-134) nachgelesen werden. 
Abbildung 1

Soziokognitives Modell der Sprachentwicklung nach Hamers und Blanc (2000, S. 124)

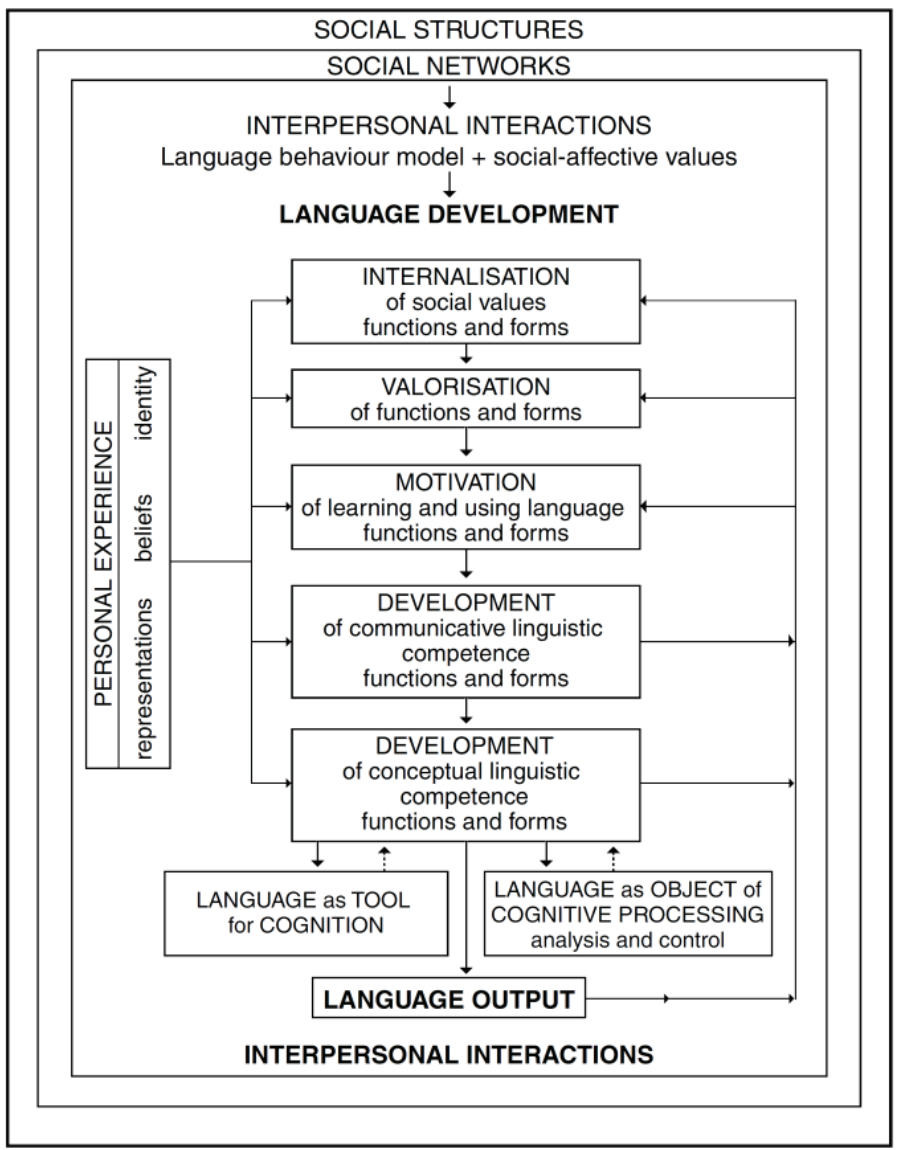

Die sozialen Strukturen repräsentieren die politisch-gesellschaftlichen Konditionen der Makroebene, die die gesetzlichen, rechtlichen und soziökonomischen Bedingungen einer Gesellschaft restringieren. Hierzu zählen auch die Werte und Normen einer Gesellschaft, die hinsichtlich Bilingualität durch Sprachideologien geprägt werden. Die sozial-strukturellen Bedingungen sowie Werte und Normen werden vornehmlich in zentralen gesellschaftlichen Institutionen wie z. B. der Schule umgesetzt sowie vermittelt. 
Das soziale Netzwerk des Individuums hingegen stellt die primäre und zentrale Sozialisationsinstanz für die Funktionalität und die Wertschätzung sprachlicher Varietäten dar, wobei im Kontext des Aufwachsens mit mehr als einer Sprache im sozialen Netzwerk die Bedeutung der Bilingualität selbst als auch der jeweiligen Einzelsprachen für das Individuum vermittelt sowie konstruiert werden. Das soziale Netzwerk bedingt die interpersonalen Interaktionen des Individuums, wobei innerhalb dieser Interaktionen die Gesprächspartner als Sprachmodelle sowohl für den Gebrauch als auch für die mit den jeweiligen Sprachen und deren Varietäten assoziierten sozial-affektiven Wertschätzungen fungieren. Die interpersonalen Interaktionen determinieren die persönlichen Erfahrungen von Individuen, die wiederum ihre jeweiligen Repräsentationen, Überzeugungen und letztlich auch ihre Identität mitformen, und sich folglich auch auf die individuelle Sprachentwicklung auswirken. Das Kind wird in und durch Sprache abhängig vom konkreten Sprachgebrauch in seinem sozialen Netzwerk sozialisiert und nimmt hierbei den Status seiner Gruppe sowie der Sprache der Gruppe innerhalb der Gesamtgesellschaft und den sozialen Strukturen samt der ihnen zugesprochenen Werte und Attributionen wahr (vgl. ebd., S. 111f). Sprachliche Sozialisation kann innerhalb dieses Modells als ,the interface between a particular social network, which is part of a larger social structure but which has its own pattern of language use, and the individual's social representations, which shape the child's relation to language and languages" (vgl. ebd., S. 112) verstanden werden.

Die interpersonalen Interaktionen und die damit assoziierten persönlichen Erfahrungen bedingen die Internalisierung der Werte und Normen, die Wertschätzung sprachlicher Funktionen und Formen, die Motivation zum Erlernen und Gebrauch dieser und dementsprechend letztlich auch die Entwicklung von Sprache zur Kommunikation und als konzeptuelle Kompetenz aus. Diese Prozesse beeinflussen sich in der eben genannten Reihenfolge, sodass die Internalisierung der Werte und Normen die Wertschätzung sprachlicher Funktionen und Formen bedingt, die wiederum auf die Motivation zum Erwerb und den Gebrauch bestimmter sprachlicher Formen und Funktionen wirkt und so weiter. Allerdings wirken sich auch Entwicklungen hinsichtlich der kommunikativen und konzeptuellen Kompetenz wiederum auf die Prozesse der Internalisierung, Wertschätzung sowie der Motivation aus. Hinsichtlich Sprache als konzeptuelle Kompetenz unterscheidet das Modell den Gebrauch von Sprache als kognitives Werkzeug und als metalinguistisches Objekt selbst. Hamers und Blanc (ebd., S. 124) akzentuieren ebenfalls die Bedeutung der Nutzung von Sprache als konzeptuelle Kompetenz und von sprachlichem Output für die Sprachentwicklung, da die erstgenannten Faktoren durch ihren Gebrauch ihre Entwicklung vorantreiben und der Output wiederum auf die Prozesse der Internalisierung, Wertschätzung sowie der Motivation wirkt. Angesichts der Output-Hypothese von Swain (2000) könnte das Modell um direkte Pfeile vom 
Output zu den Entwicklungsbereichen Kommunikation und konzeptueller Gebrauch von Sprache ergänzt werden, statt exklusiv über die Prozesse der Internalisierung, Wertschätzung und Motivation zu operieren.

Der eben dargestellte durch die sozialen Strukturen und das soziale Netzwerk gerahmte Teil des Modells von Hamers \& Blanc (2000, S. 124) akzentuiert hervorragend die Abhängigkeit der Sprachentwicklung von psychosozialen Prozessen der Internalisierung und Wertschätzung sowie der hieraus entstehenden Motivation zum Erwerb und zum Gebrauch bestimmter sprachlicher Formen und Funktionen. Sprache darf nicht auf grammatische, strukturelle Eigenschaften reduziert werden, sondern muss hinsichtlich des konkreten Gebrauchs zur Kommunikation sowie zur Strukturierung der Umwelt in der mehrsprachigen Lebensrealität bilingual aufwachsender Individuen als soziales Phänomen interpretiert werden. Diesbezüglich kann wiederholt auf Bakers (2011, S. 4) pointierte, treffende Beschreibung des Zusammenhangs von Sprache und der Lebensrealität rekurriert werden: „Language is not produced in a vacuum; it is enacted in changing dramas."

Für die bilinguale im Gegensatz zur monolingualen Sprachentwicklung gilt es innerhalb dieses Modells auf fünf zentrale Einflussfaktoren der Sprachentwicklung beider Sprachen hinzuweisen (vgl. Hamers \& Blanc, 2000, S. 125127), die im Modell selbst nicht illustriert sind, da es sich um ein vom Spracherwerbstyp unabhängiges Modell handelt: 1) Zunächst gilt es die Beziehung der Einzelsprachen zueinander auf individueller und sozialer Ebene zu berücksichtigen. Wenn sich der Status, die Wertschätzung sowie die Funktionalität beider Sprachen auf sozialer und/oder individueller Ebene unterscheiden, werden die beiden Einzelsprachen nicht als äquivalent betrachtet. Dies führt zu Relevanz- und Bedeutungsunterschieden der Einzelsprachen für das Individuum, sodass sich mit hoher Wahrscheinlichkeit asymmetrische Kompetenzprofile sowie distinkte Gebrauchskontexte und Verwendungsweisen der Einzelsprachen bilingualer Sprecher ausbilden. 2) Zudem gilt es den Grad der Überlappung der Zuordnung bestimmter Sprachen für bestimmte Funktionen zu berücksichtigen. Angesichts des „complementary principle“ (vgl. Grosjean, 2010, S. 29ff) können sich die Funktionen der beiden Einzelsprachen komplementär unterscheiden oder zum Teil überschneiden (vgl. ebd., S. 126). Wenn eine Sprache nur in bestimmten Kontexten verwendet wird, limitiert dies selbstverständlich die Möglichkeiten, einzelsprachliche Formen und Funktionen über diese Kontexte hinaus zu erwerben. Lediglich in den eher selten auftretenden Ausnahmefällen der balancierten Bilingualität wird mit beiden Sprachen in denselben Kontexten operiert. 3) Hamers und Blanc (ebd., S. 127) differenzieren bezüglich des ,,complementary principle“ zwischen dem 2L1a und dem L2a, wobei sie den Einzelsprachen im 2L1a eher komplementäre Funktionen zusprechen, während im L2a die gleichen Funktionen der L1 sukzessiv in der L2 ebenfalls erworben werden. Diese These kann durchaus für den er- 
wachsenen L2a zutreffen. Hinsichtlich des frühen L2a erscheint die These unangebracht, da sich im frühen L2a im Submersionskontext bestimmte sprachliche Formen und Funktionen wie z. B. die konzeptionell schriftsprachliche Varietät erst entwickeln. Abschließend wirkt sich 4) der Grad der Internalisierung der Wertschätzung der beiden Sprachen auf die Motivation zum Spracherwerb und -gebrauch der jeweiligen Sprachen aus und 5) der Beitrag, den die einzelnen Sprachen zur Entwicklung kommunikativer und konzeptueller Kompetenz leisten, determiniert wiederum deren Entwicklung (vgl. ebd., S. 127).

Angesichts der bisherigen Ausführungen des Fazits, welche an dem Modell von Hamers und Blanc (2000, S. 124) nochmals kurz ausgeführt und illustriert wurden, agieren die in Deutschland herrschenden sozialen Strukturen sowie sozialen Netzwerke in staatlichen Bildungsinstitutionen wie der Schule kontraproduktiv zur Sprachentwicklung bilingual aufwachsender SuS. Der Submersionskontext samt des monolingualen Habitus innerhalb einer eher sich durch Assimilation auszeichnenden Sprachideologie bietet weder Wertschätzung noch systematische, adäquat didaktisch aufbereitete Angebote und Möglichkeiten zur Entwicklung der nicht-verkehrssprachlichen L1. Dies wirkt sich nicht nur negativ auf die Ausbildung von CALP in der nicht-verkehrssprachlichen L1, sondern auch auf die Entwicklung von CALP in der L2 sowie allgemein auf die Bildungsprozesse dieser Schülerschaft aus, da wie in Kapitel 3 verdeutlicht wurde, sie nicht auf ihr komplettes Sprachvermögen bei Bildungsprozessen zurückgreifen können und ihre Identität sowie ihr bilingualer Sprachgebrauch aus einer Defizitperspektive betrachtet und behandelt werden. Dies widerspricht der in Abschnitt 3.6 dargelegten Evidenz zur Schwellenwerthypothese von Cummins (2000) sowie zu bilingualen Praktiken in Bildungsprozessen, die vielfältige förderliche Funktionen für und positive Effekte auf die Bildungsprozesse bilingual aufwachsender SuS identifizieren (vgl. z. B. Barwell, 2014; Clarkson, 2007; Culligan, 2015; Dirim, 1998; García, 2011; García \& Kano, 2014; Worthy et al., 2013).

Dementsprechend soll im folgenden Kapitel geprüft werden, ob ein solcher Umgang des Submersionskontextes mit der mehrsprachigen Disposition der bilingual aufwachsenden SuS als eine Form der Bildungsbenachteiligung definiert werden kann. Hierbei wird verstärkt auf das Konzept der Anerkennung rekurriert, da sowohl die Anerkennungstheorie Honneths (2003) als auch die erziehungswissenschaftliche oder pädagogische Rezeption und Adaptation dieses Konzepts sich als Analyseinstrumente zur Identifikation von Bildungsungerechtigkeit auf der Ebene des Rechts und der sozialen Wertschätzung bilingual aufwachsender SuS und ihrer sprachlichen Disposition eignen. 


\section{Bildungsgerechtigkeit und Anerkennung}

Wie in Kapitel 3 - insbesondere in den Abschnitten 3.4 sowie 3.6 - deutlich wurde, stellen Einstellungen zu und der Umgang mit Bilingualität in der Gesellschaft und der Institution Schule zentrale Einflussfaktoren auf die Bildung von bilingual aufwachsenden SuS mit MH dar. Im Folgenden sollen die Einstellungen und der Umgang mit der Bilingualität von SuS mit MH in der Institution Schule hinsichtlich Bildungsgerechtigkeit näher betrachtet werden. Hierbei werden anerkennungstheoretische Zugänge zu dieser Thematik akzentuiert, wobei insbesondere auf die Anerkennungstheorie von Honneth (2003) sowie pädagogischen und erziehungswissenschaftlichen Rezeptionen und Adaptation dieser durch Autoren wie Balzer und Ricken (2010), Stojanov (2006, 2011), Mecherill (2005) beziehungsweise Do Mar Castro Varela und Mecherill (2010) sowie Schäfer und Thompson (2010) zurückgegriffen werden. Die anerkennungstheoretische Perspektive wurde neben diversen Vorteilen dieses Blickwinkels primär auf Basis von zwei ihrer zentralen Eigenschaften ausgewählt: Zum einen verschiebt die Anerkennungstheorie als Gerechtigkeitstheorie den Fokus der Betrachtung von Bildungsgerechtigkeit als ein Problem der gerechten Verteilung von „Bildungsgütern“ hin zu der Frage, wie Mitgliedschaften und Interaktionsverhältnisse in der Institution Schule gestaltet werden sollten, um allen SuS die Entwicklung zu einem autonomen Subjekt zu ermöglichen. Zwar sind Fragen der gerechten Verteilung von „Bildungsgütern", welche z. B. in den prominenten Ansätzen der auf Gleichheit basierenden Verteilungsgerechtigkeit nach Rawls (1971) oder der auf Menschenwürde basierenden Verteilungsgerechtigkeit nach Nussbaum (2010) reflektiert werden können, ein zentraler und relevanter Bestandteil von Bildungsgerechtigkeit. Allerdings sind diese nicht in der Lage, die Bildungsbenachteiligung von $\mathrm{SuS}$ mit MH in der Bundesrepublik Deutschland adäquat zu beschreiben. Zum anderen eignet sich die Perspektive der Anerkennungstheorie als Analyseinstrument, um spezifische bildungsbenachteiligende Praktiken zu identifizieren, und liefert hierbei auf Basis ihres zu Grunde liegenden theoretischen Rahmens Denkanstöße für eine Ersetzung dieser durch anerkennendes, bildungsgerechtes Handeln.

Zunächst wird der Begriff der Bildungsgerechtigkeit näher erläutert (Abschnitt 4.1). Anschließend werden drei prominente Gerechtigkeitstheorien „Gerechtigkeit als Fairness“ (Rawls, 1971) (Abschnitt 4.1.1), ,auf Menschwürde basierende Gerechtigkeit“" (Nussbaum, 2010) (Abschnitt 4.1.2) und ,,auf Anerkennung basierende Gerechtigkeit" (Stojanov, 2006) (Abschnitt 4.1.3) kurz ausgeführt und hinsichtlich ihrer Anwendbarkeit und Fruchtbarkeit für das Phänomen Bildung verglichen und reflektiert (Abschnitt 4.1.4). Da die aktuelle Konzeption von Bildungsgerechtigkeit in Deutschland am ehesten der 
auf Gleichheit basierenden Verteilungsgerechtigkeit entspricht, wird diese Perspektive für die Bundesrepublik Deutschland in Abschnitt 4.1.1.1 näher betrachtet, wobei insbesondere die Deutung und der Umgang mit der in PISA 2000 (vgl. Baumert et al., 2001) identifizierten Bildungsbenachteiligung von Kindern und Jugendlichen mit MH dargelegt wird (Abschnitt 4.1.1.2). Danach wird das Konzept von Anerkennung in Abschnitt 4.2 fokussiert, indem zunächst einige Bedeutungsfacetten und Dimensionen der Anerkennung in seiner alltäglichen und wissenschaftlichen Verwendung unter Berücksichtigung des historischen Wandels der Konzeption sozialer Wertschätzung diskutiert werden (Abschnitt 4.2.1), bevor die Anerkennungstheorie nach Honneth (2003) erläutert wird (Abschnitt 4.2.2). Auf Grundlage von Honneths (ebd.) Anerkennungstheorie werden einige für das vorliegende Erkenntnisinteresse relevante pädagogische oder erziehungswissenschaftliche Rezeptionen, Reflexionen sowie Modifikationen der Anerkennungstheorie ausgeführt (Abschnitt 4.2.3), wobei insbesondere Anerkennung als Strukturmoment jeder menschlichen Kommunikation und Praktik (Abschnitt 4.2.3.1), Anerkennung als Herstellung von Zuschreibungen unter Berücksichtigung von Machtstrukturen (Abschnitt 4.2.3.2) und Anerkennung in Bildungsprozessen (Abschnitt 4.2.3.3) akzentuiert werden. Der zuletzt genannte Abschnitt wird weiter vertieft, indem die Anerkennung von Kindern und Jugendlichen mit $\mathrm{MH}$ und deren migrationsbedingte Mehrsprachigkeit in Bildungsprozessen hinsichtlich der Anerkennungsform des Rechts (Abschnitt 4.2.3.4) und der Anerkennungsform der Solidarität (4.2.3.5) eingehend reflektiert werden. Abschließend werden die zentralen Erkenntnisse zu Anerkennung in Abschnitt 4.2.4 zusammengefasst, bevor ein Fazit für Kapitel 4 gezogen wird (Abschnitt 4.3).

\subsection{Bildungsgerechtigkeit}

Diskurse über Bildungsgerechtigkeit, terminologisch auch mit Begriffen wie "Chancengleichheit", „Chancengerechtigkeit", „Bildungschancen“ etc. bezeichnet (vgl. Wigger, 2015, S. 73), werden nicht erst seit dem „PISA Schock“ Anfang des 21. Jahrhunderts geführt, sondern waren bereits in den 1970er Jahren in Deutschland ein „dominierendes Thema im Gerechtigkeitsdiskurs im Erziehungssystem“(Lenzen, 2015, S. 123). Nichtsdestotrotz wurde der Begriff „kaum einer stringenten begriffsanalytischen Reflexion unterzogen“ und sein „Bedeutungsgehalt kaum zum Gegenstand systematischer Rekonstruktionsversuche gemacht" (Stojanov, 2011, S. 11). Dies ist äußerst problematisch, da sowohl der grammatische Kopf „Gerechtigkeit“ als auch der semantisch spezifizierende Kern „Bildung“ des Kompositums „Bildungsgerechtigkeit“ historisch gewachsene, mehrdeutige und somit unterschiedlich interpretierbare Abstrakta sind (vgl. Wigger, 2015, S. 73). Dementsprechend erscheinen eine 
nähere Begriffsbestimmung der Einzelbestandteile sowie deren Komposition als Ausgangspunkt der Reflexion über ein gerechtes Bildungswesen umso dringlicher und notwendiger zu sein.

Etymologisch beinhaltet der Gerechtigkeitsbegriff im Deutschen und zum Teil in anderen europäischen Sprachen eine Doppeldeutung von ,gerade“ und „rechtmäßig“ (vgl. Lenzen, 2015, S. 112), was semantisch eine allgemein nachvollziehbare, stringente Perspektive auf gerechtigkeitsbezogene Entscheidungsprozesse impliziert. Allerdings weisen Gerechtigkeitskonzepte diverse, sich teils widersprechende Orientierungsgrundlagen auf, sodass in vielen Fällen unklar ist, was als „,gerecht“ zu bezeichnen ist (vgl. Horster, 2015). Die Gerechtigkeitskultur moderner demokratischer Staaten weist einen hohen Grad an Komplexität auf, in welchem es sowohl Vergangenes als auch $\mathrm{Zu}$ künftiges und verschiedene Prinzipien hinsichtlich Gerechtigkeitsfragen gegeneinander abzuwiegen gilt (vgl. Krebs, 2000, S. 26f). Die unterschiedlichen normativen Perspektiven, aus denen Gerechtigkeit in Bezug auf die jeweiligen Phänomene reflektiert werden, weisen verschiedene grundlegende Perspektiven auf (z. B. gütertheoretische Verteilungsgerechtigkeit oder Gestaltung sozialer Verhältnisse) und akzentuieren distinkte Zielkonstrukte der Gerechtigkeit (z. B. Gleichheit, Menschenwürde, Anerkennung). Dementsprechend kommt es bei der Betrachtung von Gerechtigkeit hinsichtlich eines Phänomens wie z. B. „Bildung“ aufgrund dieser unterschiedlichen Perspektiven und Zielkonstrukte zu diversen Beschreibungen, Problemdefinition und Lösungsvorschlägen, die sich nicht immer vereinen lassen. Hierbei kann bereits dem Diskurs über Gerechtigkeit eine hohe Relevanz zugesprochen werden, da in diesem die Deskription und Deutung des Gerechtigkeitsproblems und somit die Perspektive festlegt wird, die den ganzen Diskurs und womöglich auch die hieraus zu ziehenden praktischen Schlussfolgerungen beeinflussen.

Historisch betrachtet münden die „Hauptlinien des Gerechtigkeitsdiskurs“ in eine aktuelle Charakterisierung von Gerechtigkeit als Gleichheit, welches als verallgemeinertes Prinzip unter staatlicher Obhut verbindlich ist, zu potentiellen Freiheitseinschränkungen Einzelner führen kann sowie paradoxerweise Ungleichheiten nicht nur enthält, sondern auch legitimiert (vgl. Lenzen, 2015, S. 119). Neben diesem auf Gleichheit fokussierten Konzept von Gerechtigkeit lassen sich im aktuellen Diskurs die Anzahl von Gerechtigkeitskonzepten in zwei weitere Ansätze grob unterteilen, nämlich in auf Menschenwürde oder auf Anerkennung basierende Gerechtigkeit (vgl. Horster, 2015, S. 43). 


\subsubsection{Auf Gleichheit basierende Verteilungsgerechtigkeit}

Die gleichheitsbasierte Gerechtigkeit - auch als Egalitarismus bezeichnet - definiert die Gleichheit von Lebensaussichten als das „Ziel“" von Gerechtigkeit, welches einen ,moralischen Selbstzweck oder Eigenwert" darstellt und durch die gleiche Verteilung von zu definierenden Grundgütern ,relational oder komparativ" in Bezug auf andere zu konzeptionieren ist (vgl. Krebs, 2000, S. 10). Ungleichheit ist innerhalb dieser Konzeption nur moralisch vertretbar, wenn die Ungleichheit eine Folge selbst zu verantwortender Handlungen darstellt. Entsteht Ungleichheit für Betroffene durch Eigenschaften oder Vorkommnisse, die außerhalb ihrer Verantwortung wie z. B. durch genetische Anlagen, Erbe, Geschenke etc. liegen, dann gilt es diese Ungleichheit (in der Regel durch Wohlfahrt) zu egalisieren (vgl. ebd., S. 11). Dementsprechend stellt das Prinzip der Meritokratie (vgl. Solga, 2008), in welchem gesellschaftliche Ordnungen durch „Leistung“ und „Verdienst“ strukturiert werden, ein zentrales Prinzip auf Gleichheit basierender Verteilungsgerechtigkeitskonzepte dar. Meritokratische Gesellschaften zeichnen sich nach Goldthorpe (1996, S. 255f) durch die folgenden Prinzipien aus: 1) verantwortungsvolle Positionen werden auf Basis demonstrierter Kompetenz zugeteilt, 2) Bildungsmöglichkeiten sind an das ,natürliche Fähigkeitspotential“" zu binden und 3) Leistung ist die Basis und Legitimation von sozialen Ungleichheiten.

Die prominenteste Version einer auf Gleichheit basierenden Verteilungsgerechtigkeit stellt Rawls (1971) „Gerechtigkeit als Fairness“ dar. Mit lediglich zwei hierarchisch strukturierten Prinzipien, die methodisch mit dem Gedankenspiel des „Schleier des Nichtwissens“ (ebd., S. 139f) abgeleitet werden, lässt sich hiernach eine faire, auf Gleichheit abzielende Gerechtigkeit erzeugen. Das übergeordnete Prinzip besagt, dass jede Person ,den gleichen unabdingbaren Anspruch auf ein völlig adäquates System gleicher Grundfreiheiten, das mit demselben System von Freiheiten für alle vereinbar ist" (ebd., S. 78) hat, was gleiche Grundfreiheiten gewährleistet sowie einen gerechten Staat etabliert. Das untergeordnete Prinzip legitimiert soziale und ökonomische Ungleichheiten unter den folgenden Bedingungen: „erstens müssen sie mit Ämtern und Positionen verbunden sein, die unter Bedingungen fairer Chancengleichheit allen offenstehen; und zweitens müssen sie den am wenigsten begünstigten Angehörigen der Gesellschaft den größten Vorteil bringen“" (ebd., S. 78) ${ }^{6}$. Dementsprechend kombiniert der Egalitarismus ,ein Gleichheitsprinzip bezüglich unverdienter Lebensaussichten mit einem Wohlfahrtsprinzip“,

6 Dementsprechend wäre z. B. eine exorbitante Entlohnung von Managementposten in der Finanzwirtschaft legitim, wenn sich theoretisch alle Gesellschaftsmitglieder diese Stelle erarbeiten könnten und sich durch den Entgeltanreiz diejenigen Kandidaten angesprochen fühlen, die die Aufgabe gewinnmaximierend erfüllen, sodass diese Gewinne in Form erhöhter Steuerabgaben des Unternehmens in die Gesellschaft zurückgeführt werden. Dies erinnert an die 
dass ,gewisse Abstriche an Gleichheit um einer größeren Wohlfahrt willen hin" (Krebs, 2000, S. 14) akzeptiert.

Folglich operationalisiert eine auf Gleichheit basierende Verteilungsgerechtigkeit Bildung als ein zu verteilendes Gut, zu welchem jedem der gleiche Zugang zu verschaffen ist und Bildungszertifikate fair nach dem Prinzip der Meritokratie zu verteilen sind. Dementsprechend gilt es nach dieser Theorie Bildungsungleichheiten aufgrund nicht selbstverschuldeter Ursachen wie z. B. durch „ungünstige genetische Anlagen“ von staatlicher und institutioneller Seite zu kompensieren, während selbst zu verantwortende Bildungsungleichheiten wie z. B. als Folge mangelhafter Anstrengungsbereitschaft aus dieser Perspektive legitim sind. Diese Perspektive fokussiert vor allem die Allokationsfunktion von Schule (vgl. Wigger, 2015, S. 89), die nach meritokratischen Prinzipien selektiert und gesellschaftliche Positionen mit Bildungszertifikaten verbindet. Solga (2008) beschreibt die meritokratische Leitfigur sozialer Ungleichheit in Bezugnahme auf die Institutionalisierung ungleicher Bildungschancen anhand fünf kennzeichnender Merkmale: 1) „natürliche“ Fundierung sozialer Ungleichheit in unterschiedlichen Fähigkeitspotentialen, 2) Ungleichheit als gesellschaftliches Funktionserfordernis der gesellschaftlichen Arbeitsteilung, 3) Notwendigkeit organisierter Bildungsprozesse zur Erzeugung von vergleichbaren Qualifikations- und Kompetenznachweisen in Form von Bildungszertifikaten, 4) individuelle anstatt kategoriale Ungleichheitsdefinitionen zur Umdeutung struktureller Risiken und sozioökonomischer Herkunftsabhängigkeit des Bildungserfolges in individuell zu verantwortende Entscheidungen und 5) Entpersonifizierung der Definition von Leistung durch Bildungsinstitutionen als „Gatekeeper“ mit Definitionsmacht über Leistungskriterien und der Verantwortlichkeit zur Einhaltung dieser. Dementsprechend präsentiert sich institutionalisierte Bildung nach diesen meritokratischen Merkmalen als Chance, die im Sinne eines „Survival of the Fittest“ zur Legitimation von sozialer Ungleichheit strukturell gezwungen ist und die Verantwortung für Bildungserfolg an die SuS und ihre Erziehungsberechtigten abgibt. Bildungsmisserfolg wird bezüglich dieser Bedingungen für die ,Verlierer" des Bildungssystem handlungsrelevant, da das eben dargelegte Narrativ Bildungsmisserfolg als selbst zu verantwortend deutet (vgl. ebd., S. 31).

Ein Beispiel für eine Konzeption von Bildung als eine auf Gleichheit basierende Verteilungsgerechtigkeit stellt das Jahresgutachten 2007 des Aktionsrats Bildung (vbw, 2007) zum Thema Bildungsgerechtigkeit dar. Der Aktionsrat spricht von Bildungsungerechtigkeit, wenn ,Menschen trotz gleicher kognitiver Ausgangsvoraussetzungen nicht die gleichen Chancen besitzen, ein entsprechend hohes Kompetenzniveau zu erreichen“ und „wenn ein Bildungssystem Unterschiede in den Ausgangsvoraussetzungen nicht so weit wie möglich

„Trickle-down-Theorie“, welche ein „Durchsickern“ von Wohlstand in die Allgemeinheit durch unregulierte Märkte postuliert, aber als falsifiziert gilt (vgl. Dabla-Norris et al., 2015). 166 
ausgleicht“ (ebd., S. 136). In diesem kurzen Zitat sind die zwei zentralen Prämissen von Bildungsgerechtigkeit als „Ausgleich primär-sozialisatorischer Ungleichheiten auf der einen Seite und als formell-gleiche Behandlung aller Schüler/innen nach ihren Leistungen auf der anderen" (Stojanov, 2011, S. 28) enthalten. Wigger (2015, S. 78) folgert, dass für den Aktionsrat Bildung Bildungsgerechtigkeit vor allem ,herkunftsunabhängige Chancengleichheit“ bedeutet, also ,die Gleichheit der Chancen, Kompetenzniveaus und letztlich Bildungszeugnisse zu erreichen, die den jeweiligen ,kognitiven Ausgangsvoraussetzungen" entsprechen.

Angesichts des Aspekts, dass sich kognitive Ausgangsvoraussetzungen zu einem Großteil erst im Bildungsverlauf entwickeln und somit primär sozial konstruiert sind (vgl. Solga, 2008, S. 24-26), erscheint eine solche Konzeption von kognitiven Ausgangsvoraussetzungen als gegebene Determinante für den Bildungserfolg erstaunlich bis paradox. Nach Wigger (2015, S. 79) wird Bildungsgerechtigkeit vom Aktionsrat Bildung vor allem aus einer ökonomischen Perspektive betrachtet, in welcher Bildung ,als eine Ressource für die Wettbewerbsfähigkeit der Wirtschaft und Zukunftsfähigkeit des Standortes Deutschland" gilt und die Erzeugung von Bildungsgerechtigkeit durch finanzielle Investitionen auf staatlicher Seite Kosten verursacht. Hierbei führen Maßnahmen zur Etablierung von Bildungsgerechtigkeit auch zu Pflichten, die denjenigen aufoktroyiert werden, die von bildungsgerechtigkeitsfördernden Maßnahmen betroffen sind. Hierbei gilt allerdings zu erwähnen, dass der Aktionsrat Bildung von der Vereinigung der Bayerischen Wirtschaft finanziert wird ${ }^{7}$, die unter anderem von Fördermitgliedern wie der Philip Morris GmbH, Pöschl Tabak GmbH \& Co. KG, EADS Deutschland GmbH oder ProSiebenSat1. Media AG unterstützt werden (vgl. vbw, 2018), sodass eine ökonomische Perspektive auf Bildung zu erwarten ist.

Solche primär meritokratischen Konzeptionen von Bildungsgerechtigkeit werden von einer Vielzahl von Erziehungswissenschaftlern kritisiert (vgl. z. B. Giesinger, 2008, 2015; Gomolla \& Radtke, 2009; Solga, 2008; Stojanov, 2011; Wigger, 2015). Bildung wird hiernach vor allem aus einer gesellschaftlich funktionalen, ökonomischen Perspektive betrachtet, die Kindern und Jugendlichen sowie ihren Eltern eine hohe Selbstverantwortung für den Bildungsprozess zuspricht, obwohl sich Kinder und Jugendliche innerhalb der frühkindlichen und schulischen Sozialisation erst auf dem Weg zu autonomen Subjekten befinden (vgl. Stojanov, 2011). Diese Perspektive akzentuiert die Eigenschaften und Fähigkeiten der Kinder und Jugendlichen als Determinanten für den Bildungserfolg, sodass eine adaptive Verteilung individueller Förderung und Sonderbehandlung als Lösung zur Nivellierung ungleicher (Ausgangs-)Vo-

7 Für die Bertelsmann Stiftung als ein weiteres Beispiel eines nicht demokratisch legitimierten Akteurs, der aus wirtschaftlichen Interessen politischen Einfluss auch auf das Bildungs- und Wissenschaftssystems ausübt, siehe Lohmann (2007) oder Schuler (2010). 
raussetzungen gesehen wird. Die auf Gleichheit basierende Verteilungsgerechtigkeit übersieht hierbei, dass Bildungsinstitutionen selbst soziale Bildungsungleichheiten erzeugen (vgl. Gomolla \& Radtke, 2009). Da die auf Gleichheit basierende Bildungsgerechtigkeit auch für die Bildungslandschaft in Deutschland die zentrale Perspektive zur Diskussion und Reflexion von Bildungsgerechtigkeit darstellt, werden im Folgenden einige zentrale Befunde zu den Praktiken und Auswirkungen des auf Gleichheit basierenden Bildungssystems in Deutschland berichtet. Hierbei wird am Beispiel von SuS mit MH deutlich, dass die vermeintliche Lösung einer intensivierten präventiven oder kompensatorischen Förderung und Sonderbehandlung nicht in der Lage ist, die Bildungsungleichheit in Deutschland aufzuheben.

\subsubsection{Auf Gleichheit basierende Verteilungsgerechtigkeit in Deutschland}

Wie bereits angesprochen, stellt Bildungsgerechtigkeit in Deutschland seit den 1970er Jahren ein „dominierendes Thema im Gerechtigkeitsdiskurs im Erziehungssystem“ (Lenzen, 2015, S. 123) dar. Anstatt eines holistischen Blicks auf Bildungsgerechtigkeit sowie auf die Strukturen und Praktiken des Bildungssystems, die zur Bildungsgerechtigkeit beitragen, wurden in Debatten über Bildungsgerechtigkeit primär bestimmte bildungsbenachteiligte Gruppen fokussiert. Während in den 1970er Jahren das „katholische Arbeitermädchen vom Lande" im Diskurs über Bildungsgerechtigkeit im Fokus stand, wird seit den 1980er Jahren vermehrt die Bildungsbenachteiligung von „Migrantenkindern“ diskutiert (vgl. Gomolla \& Radtke, 2009, S. 21f). Die zuletzt genannte Gruppe steht auch noch heute unter anderer Etikettierung als „Kinder und Jugendliche

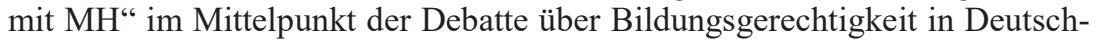
land, was sicherlich zum Teil auch durch die Interpretation potentieller Ursachen des schlechten Abschneidens Deutschlands bei der PISA-Studie des Jahres 2000 bedingt ist. In allen drei Fällen werden eine Reihe von Kollektivmerkmalen zur Konstruktion und Operationalisierung von bildungsbenachteiligten Gruppen kombiniert. Während das „,katholische Arbeitermädchen vom Lande“ anhand der Merkmale „Konfession, soziale Schicht, Geschlecht und Wohnort“ (ebd., S. 21) definiert wird, werden bei „Migrantenkindern“ oder Kindern mit MH primär ethnische, kulturelle und sprachliche Eigenschaften als konstituierende Eigenschaften akzentuiert. Zudem wird mit dieser Gruppe häufig auch die Zugehörigkeit zu niedrigeren sozioökonomischen Schichten verbunden. Mit diesem Vorgehen der Konstruktion von Gruppen anhand nicht direkt bildungsbezogener Eigenschaften können ,die Unterschiede in der Bildungsbeteiligung, die zwischen verschiedenen Schülergruppen, differenziert etwa nach Sozialstatus oder Migrationshintergrund der Eltern, gemessen werden, ursächlich auf deren Wahlverhalten“ (ebd., S. 10) zurückgeführt werden. Diese Praxis stellt eine Perspektive auf Bildungsgerechtigkeit dar, bei der Eigenschaften 
und Merkmale von zuvor konstruierten Gruppen ursächlich für deren Bildungsbenachteiligung interpretiert werden, die es im Sinne einer gerechten Verteilung von Bildungschancen durch zusätzliche Förderung oder eine Sonderbehandlung zu kompensieren gilt. Ein zutreffendes Beispiel für diese Handlungslogik stellen die bildungspolitischen Reaktionen der Bundesrepublik Deutschland auf den „PISA-Schock“ zu Beginn des 21. Jahrhunderts dar, welches im Folgenden kurz ausgeführt werden soll.

4.1.1.2 Die Reaktionen auf den „PISA-Schock“ in Deutschland als Beispiel für eine auf Gleichheit basierende Verteilungsgerechtigkeitsperspektive

Der „PISA-Schock“ für Deutschland fußt primär auf zwei Ergebnissen der PISA-Studie aus dem Jahre 2000: 1) Deutschland weist für die untersuchten Fähigkeiten der Lesekompetenz, der mathematischen und naturwissenschaftlichen Grundbildung signifikant geringere Leistungen als der OECD-Durchschnitt auf und 2) in Deutschland ist der Zusammenhang des sozioökonomischen Status mit schulischen Kompetenzen im Vergleich zu allen anderen untersuchten Ländern am höchsten (vgl. Baumert et al., 2001). Zudem schnitt die Gruppe von Jugendlichen mit MH in ihren Leistungen schlechter als Jugendliche ohne MH ab, selbst wenn die Sozialschichtzugehörigkeit der Herkunftsfamilie, die Verweildauer der Jugendlichen und die Sprache in der Familie kontrolliert wurden (vgl. Baumert \& Schümer, 2001, S. 379). Neben dem Kompetenzniveau gibt es eine Vielzahl weiterer Indikatoren wie z. B. Rückstellung von der Einschulung, Verteilung auf Förder-, Haupt-, Realschule und Gymnasium, Klassenwiederholungen, Abgang ohne Schulabschluss, Finden von Lehrstellen etc. (vgl. Britz, 2006), die auf die Bildungsbenachteiligung von Kindern und Jugendlichen mit MH hinweisen, die aber nicht zwingend in internationalen Schulleistungsuntersuchungen wie PISA, TIMSS oder IGLU berücksichtigt werden.

Anstatt aufgrund dieser Befunde, insbesondere hinsichtlich des hohen $\mathrm{Zu}-$ sammenhangs der Kompetenzniveaus mit dem sozioökonomischen Status der Eltern in Deutschland, das Bildungssystem und dessen Strukturen selbst bezüglich strukturellen, institutionalisierten Mechanismen der Bildungsbenachteiligung bestimmter Gruppen zu reflektieren, rückte die Gruppe der Kinder und Jugendlichen mit MH sowie deren vermeintliche Sprachdefizite in den Fokus der Bildungsdebatte in Deutschland. So behauptete z. B. der damalige Ministerpräsident Bayerns und Kanzlerkandidat Edmund Stoiber, dass die sprachlichen Defizite von Jugendlichen mit türkischem Migrationshintergrund ein Grund für das schlechte Abschneiden Deutschlands bei PISA seien (vgl. Brinck, 2003). Auch die für Bildung zuständigen Minister der Länder forderten im Anschluss an die Kultusministerkonferenz 2002 „Maßnahmen zur Verbesserung der Sprachkompetenz bereits im vorschulischen Bereich“" (KMK, 2002, 
S. 6) und „Maßnahmen zur wirksamen Förderung bildungsbenachteiligter Kinder, insbesondere auch der Kinder und Jugendlichen mit Migrationshintergrund" (ebd., S. 7). Obwohl die Ursachen für die Bildungsbenachteiligung von Kindern und Jugendlichen multidimensional und komplex sind (vgl. z. B. Baumert \& Schümer, 2001; Britz, 2006), standen sprachliche Defizite im Deutschen stets im Vordergrund des Diskurses über die Gründe für die Bildungsbenachteiligung dieser Gruppe. Die naheliegende Lösung wurde anschließend in präventiver und kompensatorischer Sprachförderung im Deutschen gesehen (siehe z. B. Kany \& Schöler, 2007; Lüdtke \& Kallmeyer, 2007b; Reitenbach, Schastak \& Rauch, 2018; Stojanov, 2006, 2011), was auch im Koalitionsvertrag zwischen CDU, CSU und FDP (2009, S. 59f.) in Form verbindlicher, bundesweit vergleichbarer Sprachtests sowie (vor-)schulischer Sprachförderung festgeschrieben wurde (siehe z. B. Kany \& Schöler, 2007; Lüdtke \& Kallmeyer, 2007b). Auch der Aktionsrat Bildung (vbw, 2007, S. 146) fordert „die unerlässliche Unterrichtung in der Verkehrssprache Deutsch und deren Praktizierung im öffentlichen wie im privaten Raum“ zum Zwecke einer „konsequenten Akkulturation" (vgl. Stojanov, 2011, S. 146).

Das Ergebnis der Problemidentifikation sowie des darauf folgenden Lösungsansatzes der Bildungsbenachteiligung von Kindern und Jugendlichen mit MH kann in Anbetracht empirischer Befunde als ernüchternd bezeichnet werden. Zwar nimmt die Bildungsbenachteiligung von Kindern und Jugendlichen mit MH etwas ab, allerdings besteht bis heute eine persistente Bildungsbenachteiligung hinsichtlich dieser Gruppe (siehe z. B. Haag et al., 2012, 2016; Kempert et al., 2016; Rauch et al., 2016; Tarelli, Schwippert \& Stubbe, 2012; Wendt \& Schwippert, 2017).

Auf Basis dessen wird deutlich, dass die zu Grunde liegende Handlungslogik für den Diskurs über die Bildungsbenachteiligung von Kindern und Jugendlichen mit MH und der institutionelle Umgang mit ihnen kritisch zu betrachten ist. Primär wird eine Defizitperspektive eingenommen, bei der die sprachlichen Fähigkeiten von Kindern und Jugendlichen mit MH vom vermeintlichen monolingualen Standard dahingehend abweichen, dass sie für die Beschulung in der Regelschule häufig nicht genügend entwickelt seien. Mit dieser Defizitperspektive sind sekundär implizite, subjektive Theorien zur Erklärung der sprachlichen Differenzen zwischen Kindern und Jugendlichen mit und ohne MH verbunden, die die Unterschiede nicht auf die distinkten vorliegenden Spracherwerbstypen zurückführen, sondern kulturelle und zum Teil ethnische Differenzen anführen. Letztlich wird mit dieser Perspektive das „Wahlverhalten“ der Eltern und deren Kindern mit MH als Ursache für die Bildungsbenachteiligung bestimmt (vgl. Gomolla \& Radtke, 2009, S. 10). Dementsprechend verwundert es nicht, dass Forderungen nach deutschsprachiger Kommunikation innerhalb von Familien mit MH (vgl. Kersten et al., 2011; Reimann, 2014) oder einer Deutschpflicht auf dem Schulhof (vgl. Sei- 
bel, 2016) medienwirksam diskutiert werden. Nach Bourhis (2001) Kategorisierung von Sprachideologien entsprechen solche Forderungen einer Assimilationsideologie, wobei die Forderung nach deutschsprachiger Kommunikation innerhalb von Familien mit MH (vgl. Kersten et al., 2011; Reimann, 2014) gar Züge einer „ethnist ideology“ aufgrund des potentiellen Eingriffs in die Privatsphäre trägt.

Diese gesellschaftlichen und bildungspolitischen Reaktionen auf die Bildungsbenachteiligung von Kindern und Jugendlichen mit MH unterscheiden sich fundamental von der Handhabung der Bildungsbenachteiligung der „katholischen Arbeitermädchen vom Lande" in den 1970er Jahren. Bei der letztgenannten Gruppe wurden keine Zuschreibungen von devianten Merkmalsausprägungen vorgenommen, die sich vermeintlich negativ auf ihre Bildung auswirken und dementsprechend eine Sonderbehandlung in Form von zusätzlicher Förderung verlangen. Stattdessen führte die Auseinandersetzung mit der Bildungsbenachteiligung dieser Gruppe zu einer „Politisierung der Diskussion über Ungleichheit und Ungleichbehandlung, in deren Folge es zu einer Reorganisation der Struktur des Bildungsangebots für Mädchen, einer Änderung der Selektionspraktiken in den Schulen und einer Delegitimation von Begründungshaushalten kam, die bis dahin die Entscheidungen gültig machten" (Gomolla \& Radtke, 2009, S. 22). Diese Lösungsansätze waren erfolgreich, sodass die institutionalisierte Ungleichbehandlung dieser Gruppe durch das Bildungssystem aufgehoben wurde.

Dementsprechend wurde bei der Gruppe der „katholischen Arbeitermädchen vom Lande" eine vollkommen andere Perspektive auf die Bildungsbenachteiligung als bei Kindern und Jugendlichen mit MH verfolgt, bei der die Institution Schule als Akteur der Produktion von Bildungsbenachteiligung akzentuiert wurde, und zwar in Form einer ,soziologischen, auf die Prozeduren der Organisation gerichteten, die Selektion betonenden Beschreibungsweise der Schule" (ebd., S. 26). Eine solche Perspektive legt andere praktische Handlungsmöglichkeiten nahe als eine Defizitperspektive auf vermeintlich zu Bildungsbenachteiligung führender, devianter Merkmalsausprägungen. Die Beteiligung der deutschen Regelschule als Produzent der Bildungsbenachteiligung von Kindern und Jugendlichen mit MH durch ,institutionelle Diskriminierung" (ebd.) wird näher in Abschnitt 4.2.3 in Anlehnung an pädagogische und erziehungswissenschaftliche Rezeptionen der Anerkennungstheorie ausgeführt. Im Folgenden sollen allerdings zunächst zwei weitere Gerechtigkeitskonzepte reflektiert werden, deren Anwendung auf das Bildungssystem denkbar sind. 


\subsubsection{Auf Menschenwürde basierende Verteilungsgerechtigkeit}

Auf Menschenwürde basierende Gerechtigkeitskonzepte fokussieren ebenfalls eine gerechte Verteilung von Gütern, allerdings aus der Perspektive des Humanismus und nach Prinzipien, „die der Würde des Menschen entsprechen“ (Horster, 2015, S. 44), um ein „gutes Leben in modernen Gesellschaften“ (Wigger, 2015, S. 83) zu ermöglichen. Solche Konzepte operieren mit Schwellenwerten im Sinne von Mindestmaßen, die es für ein menschenwürdiges Leben zu erreichen und bereitzustellen gilt, auch um die Teilhabe am gesellschaftlich-politischen Leben zu ermöglichen (vgl. Stojanov, 2011, S. 39). Oberhalb der einzelnen Schwellenwerte können durchaus Ungleichheiten bestehen, die nicht als ungerecht zu interpretieren sind (vgl. Horster, 2015, S. 45).

Eine häufig rezitierte Ausführung des hier angeführten Gerechtigkeitskonzepts stellt der Fähigkeitenansatz von Martha Nussbaum (2010) dar. Nussbaum (ebd., S. 104) akzentuiert in ihrem Konzept die ,philosophischen Grundlagen einer Theorie grundlegender menschlicher Ansprüche, die von allen Regierungen als von der Menschenwürde gefordertes absolutes Minimum geachtet und umgesetzt werden sollten“ und „dass diese Idee eines basalen sozialen Minimums am besten von einem Ansatz umgesetzt werden kann, der menschliche Fähigkeiten [...] in den Mittelpunkt stellt". Menschliche Fähigkeiten sind hierbei als Zwecke an sich und nicht als Mittel zu Zwecken anderer zu verstehen sowie hinsichtlich Schwellenwerten zu messen, die die Teilhabe an gesellschaftlichen Prozessen ermöglichen (vgl. ebd., S. 105). Nussbaum (ebd., S. 112-114) postuliert zehn zentrale menschliche Fähigkeiten wie unter anderem die Fähigkeit zum Leben, zur körperlichen Gesundheit und Integrität, zur praktischen Vernunft, aber auch die Fähigkeit zur Anteilnahme an anderen Spezies und der Natur, die Fähigkeit zum Spiel und zur Kontrolle der eigenen Umwelt. Diese zentralen Fähigkeitskategorien gilt es unter Achtung des Pluralismus für jede Gesellschaft gemäß ihres kulturellen Selbstverständnis zu modifizieren und auszuhandeln (vgl. ebd., S. 115). So dürfte zum Beispiel die Fähigkeit zur Anteilnahme an anderen Spezies und der Natur in Teilen Indiens, die den Vegetarismus fest in ihrer Kultur verankert haben, anders ausgelegt werden als in Deutschland. Manche Fähigkeiten müssten allerdings stark umgedeutet werden, da zum Beispiel in buddhistischen Kulturen eher eine Verschmelzung mit der Umwelt statt einer Kontrolle dieser angestrebt wird (vgl. Stojanov, 2011, S. 39).

Trotz der Fokussierung auf menschenwürdige Prinzipien des Humanismus unterscheidet sich diese Konzeptualisierung von Bildungsgerechtigkeit als Verteilungs- oder Teilhabegerechtigkeit nur durch abgeschwächte Formen der zentralen Prämissen der zuvor angeführten Gerechtigkeitsperspektive. Der Ausgleich herkunftsbedingter Bildungsbenachteiligungen und die Garantie von Chancengleichheit im Sinne fairer Bedingungen bleiben existent, aber sind 
nur noch relevant hinsichtlich des Erreichens der zu antizipierenden Schwellenwerte (vgl. ebd., S. 39f). Dieser Ansatz akzentuiert primär die Qualifikations-, Sozialisations- und Integrationsfunktion der Schule (vgl. Wigger, 2015, S. 89-90), lässt allerdings offen, wie nach dem Erreichen der Schwellenwerte die Bildungsgüter zu verteilen sind (vgl. ebd., S. 83). Die auf Menschenwürde basierende Verteilungsgerechtigkeit lässt zudem zwei weitere zentrale Fragen innerhalb ihres Theorierahmens offen: Zum einen stellt sich die Frage, wie ein „gutes Leben“ zu definieren ist (vgl. ebd.) und zum anderen bleibt unklar, wer die Definitionsmacht über die Auswahl der bildungsrelevanten Fähigkeiten und die Operationalisierung der einzelnen Schwellenwerte besitzt.

\subsubsection{Bildungsgerechtigkeit als Anerkennungsgerechtigkeit}

Das Konzept der Bildungsgerechtigkeit als Anerkennungsgerechtigkeit wurde im deutschsprachigen Raum primär von Krassimir Stojanov ab 2006 in der Monographie „Bildung und Anerkennung“ ausgearbeitet und 2011 von ihm in der Monographie „Bildungsgerechtigkeit. Rekonstruktion eines umkämpften Begriffs" detailliert expliziert. Hierbei kritisiert und distanziert sich Stojanov (2011) nicht nur von auf Gleichheit basierenden Verteilungsgerechtigkeitsansätzen unter meritokratischen Bedingungen, sondern analysiert auch die Konstruktion des MH als bildungsrelevantes Merkmal (ebd., S. 139-148) und versucht sich an einer Neufassung des Konzepts der institutionellen Diskriminierung von Gomolla \& Radtke (2009) (ebd., S. 151-162). Im Gegensatz zu den oben angeführten Gerechtigkeitskonzepten bezieht sich Stojanov (2011) explizit auf den Bereich der Bildung und Bildungsgerechtigkeit. Die folgenden Ausführungen zu Anerkennung allgemein sowie zu den jeweiligen Anerkennungsformen bleiben in den unmittelbar folgenden Ausführungen etwas unterspezifiziert, werden aber in Abschnitt 4.2 näher erläutert.

Unter Bildungsgerechtigkeit versteht Stojanov (2011, S. 57f) zum einen die Anerkennung des Potentials eines jeden Einzelnen zur Entwicklung eines autonomiefähigen Subjekts im Sinne seines in Abschnitt 3.6 erläuterten Bildungsbegriffs und zum anderen eine auf den von Honneth (2003) postulierten Anerkennungsformen der Liebe, des Rechts und der Solidarität basierende Qualität von sozialen Beziehungen, die Individuen ermöglicht, Erfahrungen der Empathie, des moralischen Respekts und der sozialen Wertschätzung kontinuierlich im Verlauf ihrer Subjektentwicklung zu machen (vgl. Wigger, 2015, S. 85f). Im Gegensatz zu den auf Gleichheit basierenden Verteilungsgerechtigkeitskonzeptionen ist für Stojanov (2011) Bildung kein quantitativ zu verteilendes Gut, dass abhängig von vermeintlich natürlich determinierten, unterschiedlichen Fähigkeitspotentialen zu verteilen ist. Dies ist auch nicht möglich, da sich die Fähigkeitspotentiale und das kognitive Leistungsvermögen erst im Verlauf des Bildungsprozesses entwickeln (vgl. ebd., S. 49ff) und zwar 
zu einem beträchtlichen Anteil in der Institution Schule selbst. Die Entwicklung der Bildung eines Individuums ist folglich vielmehr ,abhängig von der konkreten Beschaffenheit seiner sozialen Erfahrungen“" (vgl. ebd., S. 57). Dementsprechend gilt es die Anerkennungsformen der Empathie, des moralischen Respekts und der sozialen Wertschätzung zu institutionalisieren und als Maßstäbe für pädagogisches Handeln zu operationalisieren (vgl. ebd., S. 65ff). Um die Anerkennungsformen für die Ausgangsvoraussetzungen eines jeden Individuums umsetzen zu können, sind ungleiche Bedingungen und Praktiken legitim. Dementsprechend gilt es Strukturen zu schaffen, ,, in denen die Einzelnen Bedingungen der Möglichkeit zum Handeln vorfinden, die ihren basalen Handlungsdispositionen, aktuale oder qua kultureller Zugehörigkeit antizipierten Dispositionen entsprechen und „antworten“““ (Do Mar Castro Varela \& Mecherill, 2010, S. 94).

Diese Forderungen hätten nicht nur immense Folgen auf mikrosoziologischer Ebene hinsichtlich der Struktur und Organisation von Unterricht, sondern beträfen zentrale Funktionslogiken der Institution Schule selbst wie z. B. die Selektion zur Erfüllung der Allokationsfunktion von Schule. Die Selektion im Bildungssystem wird von Stojanov (2011, S. 59ff) scharf kritisiert, da unter anderem gravierende herkunftsabhängige Unterschiede in Lernleistungen erst durch diese in der Sekundarstufe I entstehen und Selektion eine Missachtung des moralischen Respekts vor der nicht abschließbaren Bildsamkeit von Individuen darstellt.

Stojanovs (2011) Konzeption der Bildungsgerechtigkeit als Anerkennungsgerechtigkeit stellt in seiner Radikalität hohe Hürden an die Machbarkeit und Umsetzung seiner Ideen. So kritisiert Wigger (2015, S. 86), dass die theoretische Ausschließung der finanziellen Machbarkeit und der staatlich gewollten sozialen Stratifizierung ,weder die Kriterien der Verteilung noch [...] eine gerechte Verteilung" klärt oder begründet. Zudem ist die Abschaffung von Selektionsprozessen in der Schule im Sinne eines Schonraums des Bildungsprozesses bis zum Übergang in das Berufsleben mit dem Problem verbunden, dass keine Erfahrungen mit Selektionsprozessen gemacht werden können, welche aber außerhalb eines solchen Schonraums Schule immanent sind (vgl. ebd., S. 87).

\subsubsection{Zusammenfassung}

Die drei eben aufgeführten Gerechtigkeitskonzeptionen der Gleichheit, Menschenwürde und Anerkennung verfolgen distinkte Zielkonstrukte, fokussieren verschiedene Funktionen von Schule und unterscheiden sich in ihren Mitteln und Wegen zur Zielerreichung. Die auf Gleichheit und auf Menschenwürde bezogenen Gerechtigkeitskonzeptionen akzentuieren eine gerechte Verteilung von Gütern wie z. B. Bildung. Während der erste Ansatz diese als relationale, 
meritokratische Verteilung unter (scheinbar) gleichen Ausgangsbedingungen operationalisiert, definiert der zweitgenannte die Verteilung hinsichtlich eines menschenwürdigen Minimalmaßes und eines zu erreichenden Schwellenwertes. Eine ungleiche Verteilung von Gütern wird bei gleichheitsorientierten Gerechtigkeitskonzeptionen durch das meritokratische Prinzip legitimiert und bei auf Menschenwürde basierenden Gerechtigkeitskonzeptionen akzeptiert, sofern diese Ungleichheiten oberhalb des definierten Minimalanspruchs liegen. Während gleichheitsorientierte Gerechtigkeitskonzeptionen primär die Selektions- und Allokationsfunktion von Schule bedienen, fokussieren auf Menschenwürde basierende Gerechtigkeitskonzeptionen stärker die Qualifikations-, Sozialisations- und Integrationsfunktion von Schule (vgl. Wigger, 2015, S. 89). Das anerkennungstheoretisch orientierte Konzept der Bildungsgerechtigkeit nach Stojanov (2011) operationalisiert im Gegensatz hierzu Gerechtigkeit über die Gestaltung sozialer Verhältnisse (vgl. Horster, 2015, S. 43). Bildungsgerechtigkeit besteht dann, wenn im Bildungssystem Empathie, moralischer Respekt und soziale Wertschätzung kontinuierlich im Bildungsprozess von allen Teilnehmenden erfahren werden. Die Missachtung sowie der Ausschluss bestimmter Personen(-gruppen) von mindestens einer dieser Formen der Anerkennung erzeugt Bildungsungerechtigkeit. Da Stojanovs (2011) Bildungsgerechtigkeit explizit keine distributive Operationalisierung zu Grunde liegt, stellen sich Fragen einer ,gleichen“ Verteilung nicht. Vielmehr wird die auf Hegel (1974; zit. nach Horster, 2015, S. 45) zurückzuführende These der „Gleichheit aller Menschen in ihrer Verschiedenheit" als grundlegende Prämisse erachtet. Durch die Fokussierung der Entwicklung zu autonomiefähigen Subjekten bedient Stojanovs (2011) Konzept primär die Personalisationsfunktion von Schule (vgl. Wigger, 2015, S. 89).

In der Gesamtschau lässt sich folgendes schließen: 1) Obwohl die drei dargestellten Gerechtigkeitskonzeptionen aufgrund ihrer unterschiedlichen Orientierungen und Ziele nur schwerlich vereinbar erscheinen, identifiziert Horster (2005, S. 43) „eine unbegründete Ungleichbehandlung“ als verbindendes Element dieser Ansätze. Unbegründete Ungleichbehandlungen verletzen das Prinzip der Gleichheit, der Menschenwürde und anerkennender Sozialbeziehungen, sodass unbegründete Ungleichbehandlungen im Bildungssystem im Verbund aller drei Gerechtigkeitskonzeptionen beschrieben und kritisiert werden können. Aus diesen drei Perspektiven kann anschließend ein breiteres Spektrum potentieller Lösungsansätze abgeleitet werden, die abhängig vom konkreten Fall einen, mehrere oder alle drei Perspektiven in der Diskussion und Umsetzung berücksichtigen. 2) Aus den Ausführungen wird aber auch deutlich, dass Gleichheit ein Abstraktum und eine Illusion darstellt, die konkret in der Realität nie umsetzbar sein wird (vgl. auch Krebs, 2000, S. 29f) und dementsprechend kein „Ziel“ von Bildungsgerechtigkeit darstellen sollte. Innerhalb der distributiv orientierten Ansätze sind Ungleichheiten in ihren jeweiligen 
Funktionslogiken inhärent eingeschrieben und vertretbar. Der anerkennungstheoretisch orientierte Ansatz setzt Gleichheit in Form der Gleichheit aller Menschen in ihrer Verschiedenheit (vgl. Horster, 2015, S. 45) normativ voraus und legitimiert somit ebenfalls ungleiche Bedingungen, wenn hierdurch alle Individuen sich zu autonomiefähigen Subjekten entwickeln können. Fragen der "Gleichheit" gilt es dementsprechend im Diskurs der Bildungsgerechtigkeit mitzudenken, allerdings eher hinsichtlich ihrer Ursachen sowie RechtmäBigkeit für eine Ungleichbehandlung. 3) Jedes der drei Konzepte allein kann Bildungsungerechtigkeit nicht verhindern (vgl. Wigger, 2015, S. 89). Allerdings erweisen die jeweiligen Perspektiven der verschiedenen Ansätze fruchtbare Einblicke in die Vielschichtigkeit der Problematik und könnten unter $\mathrm{Zu}$ sammenführung dieser einen analytisch feinkörnigeren Blick auf Bildungsgerechtigkeit liefern. So lassen sich die zentralen Prämissen der beiden gütertheoretisch orientierten Gerechtigkeitskonzepte prinzipiell in eine anerkennungstheoretisch fundierte Bildungsgerechtigkeit implementieren. Die Gleichheit aller Menschen in ihrer Verschiedenheit (Horster, 2015, S. 45) sowie der Anerkennung des Potentials eines jeden Einzelnen zur Entwicklung zu einem autonomiefähigen Subjekt (Stojanov, 2011, S. 57f) könnten als normative Grundlage der Prämisse, Ämter und Positionen sollten unter Bedingungen fairer Chancengleichheit allen offenstehen (vgl. Rawls, 1971), interpretiert werden. Die Ermöglichung der Erfahrung von Empathie, moralischen Respekts und sozialer Wertschätzung muss zwangsläufig aufgrund der Verschiedenheit der Menschen für jedes Individuum unterschiedlich ausfallen, sodass Ungleichbehandlungen nicht per se zu vermeiden sind. Vielmehr stellt die Ungleichbehandlung eine Voraussetzung zur Entwicklung eines autonomiefähigen Subjekts dar, sodass Rawls (ebd.) weiteres Postulat, Ungleichbehandlungen sollten den am wenigsten begünstigten Angehörigen der Gesellschaft den größten Vorteil bringen, ebenfalls inkorporiert werden könnte. Schließlich kann die Entwicklung zu einem autonomiefähigen Subjekt als Überbegriff für die von Nussbaum (2010) postulierten notwendigen Fähigkeiten verstanden werden, die die Menschenwürde betreffen und letztlich die Teilhabe an der Gesellschaft ermöglichen.

Unabhängig von den teils radikalen Forderungen von Stojanovs (2011) Konzept der Bildungsgerechtigkeit sowie den Kritikpunkten daran erscheinen anerkennungstheoretische Überlegungen zur Analyse schulischer Bildungsprozesse zum Zwecke der Reduktion von Bildungsungerechtigkeit äußerst fruchtbar. Dementsprechend wird im Folgenden die Anerkennung hinsichtlich ihrer theoretischen Grundlagen ausgeführt sowie ihrer Rezeption im erziehungswissenschaftlichen Diskurs bezüglich der Bildungsgerechtigkeit von mehrsprachig aufwachsenden SuS mit MH ausgeführt. 


\subsection{Anerkennung}

Seit über zwei Dekaden erfährt Anerkennung als Forschungsobjekt innerhalb einer Vielzahl verschiedener kultur- und sozialwissenschaftlicher Disziplinen eine hohe Aufmerksamkeit (vgl. Balzer \& Ricken, 2010, S. 36). Anerkennung kann als ein anthropologisches Grundbedürfnis (vgl. Micus-Loos, 2012, S. 303) interpretiert werden, dass in Abhängigkeit von den jeweilig vorherrschenden Gesellschaftsstrukturen von Individuen und Gruppen antizipiert oder „erkämpft“ (Honneth, 2003) wird. Aufgrund gesellschaftlicher Wandlungsprozesse, die die soziale Wertschätzung nicht mehr an vertraute gesellschaftliche Positionen und Hierarchien bindet (vgl. Balzer \& Ricken, 2010, S. 45ff), wird Anerkennung zu einem „Schlüsselbegriff der Gegenwart, da Anerkennung in der Gegenwart zu einem Problem geworden ist" (Do Mar Castro Varela \& Mecherill, 2010, S. 90). Anerkennung selbst stellt ein äußerst komplexes und zum Teil paradoxes mikro- und makrosoziales Phänomen dar, welches nicht nur als ethisch-moralische Kategorie fungiert, sondern ebenfalls als kulturell-politische (Macht-)Problematik zu verstehen ist (vgl. Balzer \& Ricken, 2010).

Angesichts der Relevanz, Multidimensionalität und Wirkungsmacht der Anerkennung gilt es zunächst sich einen kurzen Überblick über dessen Bedeutungsfacetten und Dimensionen zu verschaffen (Abschnitt 4.2.1). Anschließend wird Honneths Anerkennungstheorie (2003) erläutert (Abschnitt 4.2.2) und dessen Rezeption im pädagogischen und erziehungswissenschaftlichen Diskurs (Abschnitt 4.2.3) vor allem hinsichtlich Anerkennung als Praktik der Adressierung und der Etikettierung ausgeführt. Abschließend soll die Anerkennung von SuS mit MH in der Institution Schule insbesondere bezüglich der Einstellungen zu und dem Umgang mit ihrer migrationsbedingten Mehrsprachigkeit reflektiert werden (Abschnitt 4.2.3.3 - Abschnitt 4.2.3.5).

\subsubsection{Bedeutungsfacetten und Dimensionen von Anerkennung}

Um zu verstehen, warum Anerkennung einen „Schlüsselbegriff der Gegenwart“" (Do Mar Castro Varela \& Mecherill, 2010, S. 90) darstellt, erscheint die Betrachtung der Wandlung dieses Begriffs sowie der Strukturen westlich individualisierter Gesellschaften hilfreich. Aus gesellschaftlich-kontextueller Perspektive benennen Balzer \& Ricken (ebd., S. 45ff) vier zentrale Entwicklungen, die der aktuellen Relevanz der Anerkennung auf mikro- und makrosoziologischer Ebene den Weg geebnet haben: 1) Im Übergang von traditionellen gesellschaftlichen Hierarchien zu modernen Gesellschaften hängt die soziale Wertschätzung nicht mehr allein vom sozialen Status ab, der primär durch Herkunft und der beruflichen Rolle determiniert wurde. Anerkennung muss nun 
erworben bzw. „erkämpft“ (Honneth, 2003) werden und zwar nach meritokratischen Prinzipien. 2) Hiermit steigen auch die Anforderungen an Identitätskonstruktionen, da weitere gesellschaftliche Bereiche neben den Indikatoren „Herkunft“ und „Beruf“ hinzukommen, in denen Anerkennung erworben werden kann und muss. Es gilt nun innerhalb verschiedener gesellschaftlicher Bereiche aus diversen vorgegebenen Kategorien, die durchaus in ambivalenten Spannungsverhältnissen stehen können, originelle und authentische Identitäten auszubilden. 3) Diese neuen Formen von Identitäten müssen wiederum artikuliert sowie in die Gesellschaft eingebracht und von dieser akzeptiert sowie wertgeschätzt werden, damit ein gesellschaftliches Zusammenleben dieser neuen Identitätsformen durch reziproke Anerkennung funktioniert. 4) Die drei eben angeführten Entwicklungen bedingen schließlich eine Neuinterpretation des Subjektverständnisses, sodass Anerkennung als zentrale Voraussetzung zur Subjektwerdung wird und diese ohne einen bestimmten Grad an Sozialität nicht erreichbar ist. Dementsprechend stehen heutzutage Individuen und Gruppen vor anderen Herausforderungen der Sozialisation und Subjektwerdung als in traditionellen gesellschaftlichen Hierarchien, die fruchtbar mit dem Konzept der Anerkennung erfasst werden können.

Begriffsgeschichtlich lässt sich ebenfalls ein Wandel in der Semantik von Anerkennung konstatieren. Der früher vorherrschende Ehrbegriff, welcher den Status von Personen(-gruppen) determinierte, wird in „Würde - als allgemeiner Kennzeichnung des Menschlichen - und „Identität“ - als individueller Kennzeichnung von Verdiensten etc.“ differenziert (Balzer \& Ricken, 2010, S. 43f). Der Anerkennungsbegriff wird anschließend im weiteren Verlauf positiv konnotiert und von Rousseau als ein zentrales menschliches Bedürfnis deklariert (vgl. ebd.). Darauf aufbauend arbeitet Hegel (1807; zit. nach ebd., S. 44) in seiner „Phänomenologie des Geistes“ den paradoxen Charakter der Anerkennung, ,in der eigenen Unabhängigkeit von anderen abhängig zu sein“ aus, auf dessen Vorarbeiten auch Honneth (2003) rekurriert. Dementsprechend enthält der Anerkennungsbegriff bereits seit dem 18. Jahrhundert auf begrifflicher Ebene die Komponente sozialer Wertschätzung der spezifischen Eigenschaften und Fähigkeiten von Individuen (vgl. Honneth, 2003, S. 196ff) sowie den intersubjektiven Charakter der Autonomie in Anerkennungsprozessen.

Aktuell wird alltagssprachlich Anerkennung als ein bedeutungsheterogener und mehrdimensionaler Begriff gebraucht, mit welchem Personen(-gruppen) oder Entitäten (Y) von jemandem (X) als etwas (Z) wahrgenommen, bestätigt, bekräftigt, ein-, ab- oder wertgeschätzt sowie berechtigt oder legitimiert werden (vgl. Ricken \& Balzer, 2010, S. 39f). In Anerkennungsprozessen handelt es sich dementsprechend um eine „dreistellige Relation“, in welcher X Y als Z anerkennt (vgl. ebd.). Hierbei wird Y von X nicht nur „passiv“ als Existenz (mit oder ohne positivem oder negativem Werturteil) bestätigt, sondern mit der Kategorie $Z$ versehen, welche z. B. Ansprüche von X an Y legitimieren könnte. Dieser Prozess der Anerkennung kann somit auch als ein ,aktiver“ Vorgang 
beschrieben werden (vgl. ebd.). Durch die Designation von $\mathrm{Y}$ als $\mathrm{Z}$ wird $\mathrm{Y}$ einer bestimmten Kategorie zugeordnet, also $\mathrm{Y}$ als $\mathrm{Z}$ konstituiert, wodurch der Handlungsspielraum von $\mathrm{Y}$ als zur Kategorie $\mathrm{Z}$ zugehörig beeinflusst wird. Diese beiden Dimensionen von Anerkennung, die passiv wahrnehmende und bewertende sowie die aktiv produktive und herstellende, sind nicht getrennt $\mathrm{zu}$ betrachten, sondern stellen vielmehr inhärente und parallel auftretende Facetten von Anerkennung in jedem Anerkennungsakt dar (vgl. ebd., S. 41).

Trotz dieses zunächst wertneutralen „Doppelcharakters“ (ebd.) der dreistellig relationalen Anerkennungsstruktur wird Anerkennung im alltäglichen Diskurs häufig als „Würdigung“, „Akzeptanz“ oder „Respekt" gegenüber Personen verstanden, die den Subjektstatus von Individuen bestätigt. Anerkennung wird somit alltagssprachlich primär als positives Werturteil zur Bestätigung einer Person genutzt. Diese dominante Verwendungsweise des Anerkennungsbegriffs konstatieren Balzer \& Ricken (ebd., S. 42) auch für den wissenschaftlichen Diskurs. Zudem weisen sie darauf hin, dass hinter dieser primären Verwendungsweise sowohl im alltagssprachlichen Gebrauch als auch im wissenschaftlichen Diskurs ein implizit normativ motivierter Appell zu anerkennenden Handlungen steht, der eine ,,analytische Wendung“ des Anerkennungsbegriffs erschwert (ebd., S. 43). Im Gegensatz zum Alltagsverständnis von Anerkennung trägt Honneths (2003) theoretische Modellierung von Anerkennung essentiell zu einem analytischen Verständnis von Anerkennungsprozessen bei, die zudem eine hohe Anschlussfähigkeit an erziehungswissenschaftliche und pädagogische Reflexionen von Anerkennung aufweist. Dementsprechend soll Honneths (ebd.) Anerkennungstheorie im Folgenden näher expliziert werden.

\subsubsection{Anerkennungstheorie nach Honneth}

Die prominenteste Ausführung einer auf Anerkennung basierenden Theorie stellt Axel Honneths 1990 vorgelegte Habilitation „Kampf um Anerkennung: zur moralischen Grammatik sozialer Konflikte" dar. Nach Honneth (2003, S. 304) erfüllt seine Anerkennungstheorie drei Funktionen: 1) Als Gesellschaftstheorie kann sie real geschichtliche Veränderungsprozesse erklären, 2) als Gerechtigkeitskonzept ermöglicht sie die Bewertung sozialer Veränderungsprozesse und 3) als Moralpsychologie gibt sie der Perspektive der Betroffenen eine Stimme. Honneth wählt hierbei das Konzept der menschlichen Autonomie als Ausgangspunkt seiner Theorie (vgl. Halbig \& Kohl, 2004, S. 67). Autonomie, definiert im Kant'schen Verständnis der Neuzeit als „Freiheit, seinen Willen der Vernunft zu unterstellen" (Schäfer \& Thompson, 2010, S. 8), spielt in westlich individualisierten Gesellschaften eine zentrale Rolle. Autonomie akzentuiert die Verantwortlichkeit des Einzelnen, welche als strukturgebendes Merkmal gesellschaftlichen Zusammenlebens interpretiert werden kann: „Die Zurechnung einer individuellen Verantwortlichkeit strukturiert die sozialen 
Beziehungen“, sodass jegliche sozialen Phänomene auf individueller Verantwortlichkeit und somit auf Autonomie zurückgeführt werden können (vgl. ebd., S. 8f).

Anerkennung stellt für Honneth (2003) einen reziproken, intersubjektiven Prozess dar, der durch „Kämpfe“ errungen wird. Hinsichtlich dieser eben angeführten Prämissen adaptiert Honneth das Stufenmodell der Liebe-RechtSittlichkeit von Hegel (1986) und greift auf die Persönlichkeitsidentität und konstitution von Mead (1973) für sein intersubjektivitätstheoretisches Personenkonzept zurück (vgl. Marfels, 2011, S. 210-212). Das aktuelle Anerkennungskonzept Honneths wurzelt im Deutschen Idealismus von Fichte und Hegel (vgl. Horster, 2015, S. 45; Micus-Loos, 2012, S. 304). Nach Hegel (1974; zit. nach Horster, 2015, S. 45) ist ,die wechselseitige Anerkennung eine Notwendigkeit jeder zwischenmenschlichen Existenz in der individualisierten staatlichen Gemeinschaft“, wobei ,die Gleichheit aller Menschen in ihrer Verschiedenheit" anerkannt wird. Um den von Hegel (1807; zit. nach Balzer \& Ricken, 2010, S. 44) postulierten paradoxen Charakter der Anerkennung, ,in der eigenen Unabhängigkeit von anderen abhängig zu sein“, sozialpsychologisch und -philosophisch zu begründen, bedient sich Honneth der von Mead (1973) dargelegten intersubjektiven Konstitution von Identität. Identität kann ausgebildet werden, wenn Individuen in der Lage sind, ,die Perspektiven der anderen auf die Situation und auf sich einzunehmen“, um „, für sich Objekt zu sein, sich selbst anzuschauen und darin zu erfassen" (Schäfer \& Thompson, 2010, S. 13). Dementsprechend benötigt die eigene Autonomie ein soziales Gegenüber, sodass sie sich in der reziproken Anerkennung „zwischen“ den Individuen, also intersubjektiv, entwickeln kann. Wie bereits in Abschnitt 3.4 dargelegt, führt die Übernahme der Perspektive auf sich selbst zur Ausbildung des „me“, in welchem gesellschaftliche Kategorien und Konstrukte adaptiert werden. Das „I“ stellt die „Reaktion auf das gesellschaftlich vermittelte und übernommene ,me“" dar, welches sich durch Unvorhersagbarkeit, Freiheit sowie durch eine gewisse Spontanität auszeichnet (vgl. Oerter \& Montada, 2002, S. 214). In der Identitätsentwicklung und im gesellschaftlichen Handeln treten stets diese beiden Identitätskonstruktionen dynamisch auf, sodass sich „Sozialität und Subjektivität in einer produktiven Wechselseitigkeit zeigen“" (Schäfer \& Thompson, 2010, S. 14). Dementsprechend kann bereits aus den Ausführungen von Hegel und Mead geschlossen werden, dass Anerkennungsprozesse subjektiv sowie inhärent sozial abhängig sind und die Autonomiebildung in mikro- sowie makrosoziologischen Kontexten formt.

Unter dem oben kurz angerissenen Rückgriff auf Hegel und Mead postuliert Honneth (2003) drei Anerkennungsformen als ,subjektphilosophisches Stufenmodell“" (Marfels, 2011, S. 214), und zwar die Anerkennungsform der Liebe, des Rechts und der Solidarität. Auf jeder dieser sukzessiv zu absolvierenden Stufen gilt es reziprok Anerkennung im intersubjektiven Austausch mit 
anderen zu erfahren sowie zu zollen, um durch die jeweiligen zu entwickelnden praktischen Selbstbeziehungen zu einem autonomen Subjekt zu werden. Jede dieser Anerkennungsformen zeichnet sich durch unterschiedliche Anerkennungsweisen, betroffene Persönlichkeitsdimensionen, Entwicklungspotentiale, praktische Selbstbeziehungen sowie Missachtungsformen samt bedrohter Persönlichkeitskonzepte aus (für einen tabellarischen Überblick siehe Honneth, 2003, S. 211). Honneths (vgl. ebd., S. 150ff) methodisches Vorgehen zur Validierung seiner Theorie beinhaltet 1) die Konstruktion einer phänomenologisch angelegten Typologie der drei Anerkennungsformen, 2) eine sich mit der Empirie deckenden Beschreibung dieser, die die Operationalisierung prüfbar machen soll, und 3) die Konzeption negativer Äquivalente der Missachtung wie Misshandlung und Vergewaltigung, Entrechtung und Ausschließung sowie Entwürdigung und Beleidigung.

\subsubsection{Die Anerkennungsform der Liebe}

Die Anerkennungsform der Liebe ist nach Honneth (ebd., S. 153) erfahrbar in Primärbeziehungen, z. B. als erotische Zweierbeziehungen, Freundschaften sowie Eltern-Kind-Beziehungen, und zwar in Form von emotionaler Zuwendung, die die Bedürfnis- und Affektnatur von Individuen betrifft. Diese ist folglich von einem Partikularismus, einer begrenzten Anzahl ,geliebter“ Menschen geprägt (ebd., S. 174). Innerhalb der Primärbeziehungen führt die reziproke Erfahrung emotionaler Zuwendung zur Erkenntnis, dass Individuen in ihrer Bedürftigkeit von anderen abhängig sind. Das Interaktionsverhältnis kann als ,prekäre Balance zwischen Selbständigkeit und Bindung“ (ebd.) beschrieben werden, in welchem sich Individuen reziprok einander anvertrauen, aber dennoch sich als selbständige Individuen temporär aus der Bindung lösen können. Innerhalb dieses Prozesses kommt es zur Ausbildung von Selbstvertrauen, der auszubildenden praktischen Selbstbeziehung innerhalb der Anerkennungsform der Liebe, welche eine notwendige Voraussetzung zur autonomen Teilhabe an der Gesellschaft darstellt (vgl. ebd., S. 154).

Honneth (ebd., S. 155ff) greift auf die Forschungstradition der Objektbeziehungstheorie zur empirischen Prüfung seiner Deskription der Anerkennungsform der Liebe zurück. In Bezugnahme auf frühkindliche entwicklungspsychologische Studien von vor allem Winnicott $(1984,1989)$ zeichnet Honneth (2003, S. 174) die ,prekäre Balance zwischen Selbständigkeit und Bindung " in der von Winnicott (ebd.) postulierten Phase der relativen Abhängigkeit nach. In dieser Phase erkennt der Säugling seine Mutter als eigenständiges Wesen durch Erfahrungen der „Zerstörung“" (z. B. in Form von Beißen der Mutter) und mit Hilfe von „Übergangsphänomenen“ (z. B. Aufbau einer emotional aufgeladenen Beziehung zu Objekten wie Kuscheldecken, Stofftieren, Spielzeugen etc. als Vermittlungsglieder zwischen Symbiose und Trennung) 
an. Durch diese Erfahrungen entwickelt der Säugling die Fähigkeit zum Alleinsein. In der vorher stattfindenden Phase der absoluten Abhängigkeit ist der Säugling noch in einem solch hohen Maße von der Mutter abhängig, dass er in einer Symbiose mit seiner Mutter lebt, in welcher eine undifferenzierte Intersubjektivität vorliegt. Das heißt, der Säugling erkennt weder sich noch seine Mutter als eigene, voneinander verschiedene Wesen an, sondern sieht sich und seine Mutter als eine zusammengehörige Entität.

Honneth (ebd., S. 214f) postuliert Misshandlung und Vergewaltigung als zeitlich sowie kulturell invariable negative Äquivalente der Anerkennungsform der Liebe. Hierbei erfährt das Individuum einen Autonomieentzug über den eigenen Körper, in welchem das Individuum ,dem Willen eines anderen Subjekts schutzlos bis zum sinnlichen Entzug der Wirklichkeit ausgesetzt" (ebd., S. 214) ist. Dies führt mit hoher Wahrscheinlichkeit zu einem Zusammenbruch des Vertrauens in die Zuverlässigkeit der sozialen Welt und damit der eigenen Selbstsicherheit (vgl. ebd., S. 215).

\subsubsection{Die Anerkennungsform des Rechts}

Die Anerkennungsform des Rechts hat sich im Gegensatz zur Anerkennungsform der Liebe erst im historischen Verlauf entwickeln können (ebd. S. 175ff). Innerhalb traditionsgebundener Gesellschaften war das Recht des Einzelnen an seine soziale Rolle gebunden, sodass unterschiedliche Rechtsansprüche für Individuen abhängig ihres Status innerhalb einer Gesellschaft bestanden. Hierbei waren der rechtliche Status und die soziale Wertschätzung eines Individuums unter dem Etikett der „Achtung“ miteinander verbunden (vgl. ebd., S. 180ff). In modernen westlichen Gesellschaften löst sich diese Bindung von Recht und sozialer Rolle auf. Geltende Rechte benötigen nun eine transparente und nachvollziehbare Begründung und rechtliche Ausnahmen und Privilegien werden weitestgehend abgeschafft, da Rechte der Erfüllung gesellschaftlicher Interessen dienen sollen. Das Recht wird hierbei zu einer universellen, allgemein gültigen Eigenschaft von Individuen, während sich die Bedeutung sozialer Wertschätzung zu einer besonderen Eigenschaft im Sinne eines distinkten Merkmals zwischen Individuen verschiebt (vgl. ebd.).

Im Laufe der Geschichte westlicher Gesellschaften kommt es zu einer kumulativen Erweiterung individueller Rechtsansprüche als Prozess von Kämpfen um Anerkennung zur Teilnahme an der gesellschaftlichen Willensbildung (vgl. ebd. S. 185ff). In der Rechtswissenschaft lassen sich liberale Freiheitsrechte, politische Teilnahmerechte und soziale Wohlfahrtsrechte unterscheiden, deren Durchsetzung nach Marshall (1963; zit. nach Honneth, 2003, S. $185 \mathrm{ff}$ ) in dieser Reihenfolge sukzessive auf das 18., 19. und 20. Jahrhundert datiert werden können. Nach Honneth (2003, S. 185ff) weist die Erlangung dieser Rechtsansprüche die Struktur von „Kämpfen“ auf, bei welchen Gruppen 
die entsprechende Rechtslosigkeit vor allem durch Proteste öffentlich anprangerten und die Rechte letztlich argumentativ erzwangen.

Die Anerkennungsform des Rechts vollzieht sich als reziproke kognitive Achtung, die die moralische Zurechnungsfähigkeit von Individuen betrifft. Die Selbsterkenntnis als Träger von Rechten kann nur unter dem Wissen erfahren werden, welche normativen Pflichten es anderen gegenüber einzuhalten gilt und zwar in Form kognitiver Perspektivübernahme eines ,generalisierten Anderen“ (vgl. ebd., S. 174). Die Erkenntnis, dass Individuen die normativen Pflichten, die sie anderen gegenüber einzuhalten haben, auch für sich selbst in Anspruch nehmen können, stellt die reziproke, intersubjektive Erfahrung der Anerkennungsform des Rechts dar. Um diese kognitive Operation einer wechselseitigen Achtung von Individuen als Rechtssubjekte vollführen zu können, müssen Individuen in der Lage sein, in ,individueller Autonomie über moralische Fragen vernünftig zu entscheiden“"(ebd., S. 185). Dementsprechend sieht Honneth (ebd.) nur diejenigen Individuen als Rechtsmitglieder an, die über moralische Zurechnungsfähigkeit verfügen. In diesem Sinne werden z. B. Kindern und Jugendlichen in vielen modernen Rechtsstaaten keine vollständige moralische Zurechnungsfähigkeit zugesprochen, da bestimmte Rechte wie z. B. Ehemündigkeit, Fahrerlaubnis, Wahlrecht, Konsum bestimmter Rauschmittel etc. erst ab einem gewissen Alter beansprucht werden dürfen. Die intersubjektive Erfahrung rechtlicher Anerkennung ermöglicht den Erwerb von Selbstachtung, ,weil individuelle Rechte zu besitzen bedeutet, sozial akzeptierte Ansprüche stellen zu können“, und somit „statten sie das einzelne Subjekt mit der Chance zu einer legitimen Aktivität aus, anhand derer es sich selber vor Augen führen kann, dass es die Achtung aller anderen genießt" (ebd., S. 194).

Als negative Äquivalente im Sinne der Missachtung der Anerkennungsform des Rechts definiert Honneth (ebd., S. 215) die Entrechtung und Ausschließung. Unterprivilegierte Gruppen, denen gleiche Rechtsansprüche versagt wurden wie z. B. das Wahlrecht der schwarzen Afroamerikaner zu Zeiten der Rassentrennung in den Vereinigten Staaten von Amerika bis zum 06. 08. 1965 oder der Frauen in der Schweiz bis zum 16. 03. 1971, war es dementsprechend nicht möglich, Selbstachtung reziprok und intersubjektiv auszubilden. Diese Entrechtung führte auch zum Ausschluss an der politischen Partizipation. Das Wahlrecht konnte in beiden Fällen durch „Kämpfe“ erlangt werden. Durch die Bürgerrechtsbewegung schwarzer afroamerikanischer Bürger in den 1950er und 1960er Jahren wurde das Wahlrecht durch gewaltfreien Widerstand, zivilen Ungehorsam und (Massen-)Proteste erkämpft. Auch der Frauenbewegung in der Schweiz gingen über Jahrzehnte diverse Aktivitäten in Form von Vorstößen und Protesten voraus, aus denen schließlich eine Volksabstimmung über den Anspruch auf das Wahlrecht von Frauen in der Schweiz resultierte, bei der allerdings nur Männer stimmberechtigt waren. In beiden Fällen artikulierten die benachteiligten Gruppen ihre Forderungen und standen für ihre zu erlangenden Rechte trotz hieraus potentiell resultierender Gefahren ein, 
sodass die eventuell zu Beginn etwas „extrem“ anmutende Metapher des „Kampfes um Anerkennung“ zumindest für diese beiden Beispiele legitim und nachvollziehbar erscheint.

\subsubsection{Die Anerkennungsform der Solidarität}

Die Anerkennungsform der Solidarität ist nach Honneth (ebd., S. 196ff) erfahrbar als soziale Wertschätzung der spezifischen Eigenschaften und Fähigkeiten von Individuen, ,durch die Menschen in ihren persönlichen Unterschieden charakterisiert sind" (ebd., S. 197). Durch soziale Wertschätzung werden Individuen als wertvolle Mitglieder der Gesellschaft angesehen. Das kulturelle Selbstverständnis einer Gesellschaft, worunter Honneth (vgl. ebd., 198) die ethischen Werte und Ziele einer Gesellschaft versteht, gibt diejenigen Kriterien vor, was als sozial wertzuschätzen gilt und was nicht. Hierbei stellt sich die Frage, welche Kriterien es gesellschaftlich als potentielle Begründungen für die $\mathrm{Zu}$ - oder Absprache sozialer Wertschätzung anzulegen gilt. Zunächst kann festgehalten werden, dass die Kriterien geschichtlichem Wandel unterliegen (vgl. ebd., 198). In Bezugnahme auf die Gegenwart sind diese so zu erzeugen, dass die wertzuschätzenden Eigenschaften und Fähigkeiten einen Beitrag zur Verwirklichung gesellschaftlicher Zielvorgaben leisten (vgl. ebd., S. 204). Sowohl die gesellschaftlichen Zielvorgaben als auch die hierfür zu leistenden Beiträge sind keine bereits vorliegenden, durch Kompromisse erzeugten Richtlinien, sondern benötigen eine ,sekundäre Deutungspraxis“ zur Aushandlung dieser (ebd., S. 205). Honneth (ebd., S. 205ff) interpretiert diese Deutungspraxis als einen kulturellen Dauerkonflikt sozialer Gruppen in einem Kampf um 1) die Deutungshoheit, welche Eigenschaften wertzuschätzen sind, 2) die Darstellung der eigenen Gruppe als besonders wertzuschätzend und 3) ökonomische Ressourcen. Die Lösung des eben angeführten Konflikts sieht Honneth (vgl. ebd., S. 207ff) in der Solidarität, in welcher Individuen und Gruppen „wechselseitig an ihren unterschiedlichen Lebenswegen Anteil nehmen, weil sie sich untereinander auf symmetrische Weise wertschätzen" (vgl. ebd., S. 208). Hierbei gilt es allerdings zu betonen, dass der Solidaritätsanspruch Individuen oder Gruppen nicht von der Notwendigkeit entbindet, den gesellschaftlichen Wert der von ihnen repräsentierten Eigenschaften und Fähigkeiten überzeugend zu artikulieren und einzubringen, um sozial wertgeschätzt zu werden.

Wenn ein Individuum in der Lage ist, die erfahrene soziale Wertschätzung auf sich als Individuum zu beziehen, kommt es zur Ausbildung von Selbstschätzung (ebd., S. 209). Als negative Äquivalente der Anerkennungsform der Solidarität definiert Honneth (ebd., S. 217) Entwürdigung und Beleidigung. Beide Missachtungsformen können die soziale Wertschätzung eines Individuums negieren, sodass dieses seine Eigenschaften und Fähigkeiten nicht als beitragsleistenden Teil der Gesellschaft wahrnehmen kann. 
Nach dem Erlangen von Selbstvertrauen und der darauf folgenden Ausbildung von Selbstachtung stellt das Erreichen von Selbstschätzung die letzte Etappe der Entwicklung eines Individuums zu einem autonomen Subjekt dar. Das so entstandene autonome Subjekt hat Anerkennung in Form von Liebe, Recht und Solidarität reziprok erfahren und seinen „Kampf um Anerkennung“ erfolgreich bestritten.

Honneths (2003) Anerkennungstheorie weist diverse zu kritisierende Punkte auf (für einen Überblick siehe Stojanov, 2006, S. 170-181). So kann der Anerkennungstheorie im Allgemeinen z. B. vorgeworfen werden, dass sie nicht direkt an der sozial-empirischen Realität ansetzt, sondern indirekt über psychologische Mechanismen von Subjekten und Subjektgruppen operiert, was den Anerkennungsbegriff verschwimmen lässt (vgl. ebd., S. 170f). Aber auch bestimmte Postulate auf den einzelnen Anerkennungsformen weisen konzeptuelle Schwachpunkte auf. So stellen sich z. B. die Fragen, ob der Ausbau von Selbstvertrauen ohne erfahrene Liebe wirklich unmöglich ist (vgl. Marfels, 2011, S. 220) oder ob Personen ohne autonome moralische Zurechnungsfähigkeit tatsächlich keine Selbstachtung ausbilden können (vgl. ebd., S. 228ff). Zudem erscheint das normative Prinzip der Anerkennung für alle Subjekte auf Basis der Solidarität im Widerspruch zur Notwendigkeit des Leistens eines Beitrags zur Verwirklichung gesellschaftlicher Zielvorgaben zu stehen (vgl. Ikaheimo, Laitinen \& Quante, 2004, S. 82f). Die diversen Kritikpunkte an Honneths (2003) Anerkennungstheorie können allerdings für die Zwecke der vorliegenden Untersuchung vernachlässigt werden. Viel zentraler hierfür sind die Fragen, wie das Anerkennungskonzept Honneths (ebd.) sich fruchtbar auf erziehungswissenschaftliche und pädagogische Phänomene wie z. B. Bildungsprozesse in der Bildungsinstitution Schule anwenden lässt und welche neuen analytischen Perspektiven diese auf konkrete Praktiken und Handlungen im Umgang mit migrationsbedingter Mehrsprachigkeit in Bildungsinstitutionen eröffnet. Diesen Fragen soll im Folgenden Abschnitt näher betrachtet werden.

\subsubsection{Rezeption der Anerkennungstheorie im erziehungswissenschaftlichen/pädagogischen Diskurs}

Die bisherigen Ausführungen zu Anerkennung weisen darauf hin, dass Anerkennungsprozesse selbstverständlich eine hohe Relevanz in erziehungswissenschaftlichen/pädagogischen ${ }^{8}$ Kontexten haben und dementsprechend auch aus

8 Im Folgenden werden stets beide Bezeichnungen als Verweise auf die wissenschaftliche Disziplin, die sich im Kern mit Fragen der Erziehung und Bildung auseinandersetzt, verwendet, da sowohl eine Verortung des Phänomens der Anerkennung zu einer der beiden Bezeichnun- 
einer spezifisch erziehungswissenschaftlichen/pädagogischen Perspektive beleuchtet werden sollten. Im Folgenden soll zunächst Anerkennung aus einem bildungsbezogenen, erziehungswissenschaftlichen/pädagogischen Blickwinkel hinsichtlich seiner aktiven, produktiven Seite unter Berücksichtigung von Machtstrukturen sowie der Frage, in welchen Handlungen und Praktiken Anerkennung auftritt, diskutiert werden. Abschließend soll Anerkennung für Bildungsprozesse und -gerechtigkeit hinsichtlich des Umgangs der Institution Schule mit SuS mit MH sowie mit ihrer migrationsbedingten Mehrsprachigkeit reflektiert werden.

\subsubsection{Anerkennung als Strukturmoment jeder menschlichen Kommunikation}

Während Honneth (2003) Anerkennung primär aus makrosoziologischer Perspektive betrachtet, fokussieren Balzer \& Ricken (2010, S. 72ff) unter anderem auch die Frage, welche Handlungen und Praktiken tatsächlich Anerkennung beinhalten. Balzer \& Ricken (ebd.) verweisen unter Rückbezug auf den passiven Charakter von Anerkennung im Sinne der Wahrnehmung oder Wertung auf Todorovs (1998; zit. nach ebd.) Lesart der Anerkennung als Adressierung, bei der „Anerkennung ebenso wie Nichtanerkennung sich voneinander mindestens strukturell nicht trennen lassen, weil beide - aufgrund der rezeptiven wie responsiven Existenzverfasstheit - als bedeutsame Adressierungen verstanden werden [...] müssen“ (Balzer \& Ricken, 2010, S. 73-74). Unabhängig davon, ob mit einer Handlung eines Sprechers ein Lob oder eine Missbilligung intendiert ist, muss der Empfänger die Handlung zunächst für sich deuten. Die Deutung an sich ist hinsichtlich des Anerkennungsgeschehens selbst gleichgültig, da durch die Interaktion sowohl Sprecher als auch Empfänger sich zumindest im passiven Charakter der Wahrnehmung oder Wertung anerkennen. Balzer \& Ricken (ebd., S. 75) schlagen vor, „Adressierungen als Anerkennungsakte zu lesen“, da „,alles, was passiert, [...] sich als ein adressierender und attribuierender Akt [...] verstehen und rekonstruieren" lässt. Dementsprechend definieren Balzer \& Ricken (ebd., S. 73) Anerkennung ,als ein spezifisches Strukturmoment einer jeden menschlichen Kommunikation und Praktik“ beziehungsweise ,als ein spezifisches Moment an (Sprech-)Handlungen selbst $[\ldots]$ und als die jeweilige Form, Funktion und auch inhaltliche Gestaltung von Adressierungen und deren impliziter Normativität".

Ein solches Verständnis von Anerkennung als Adressierung verdeutlicht, dass eine Analyse von pädagogischen Kontexten hinsichtlich der Möglichkei-

gen als auch eine wissenschaftstheoretische Reflexion der Distinktion einer ,erziehungswissenschaftlichen“ und einer ,pädagogischen“ Disziplin keinen Mehrwert für das vorliegende Erkenntnisinteresse birgt. 
ten für anerkennendes Handeln und einer Quantifizierung auftretender Anerkennungspraktiken zu kurz greift. Vielmehr gilt es die von den Akteuren eingenommenen Rollen samt ihrer konstitutiven und konstituierenden Zuschreibungen in pädagogischen Interaktionen sowie deren zu Grunde liegenden Strukturen, Ordnungen, Prinzipien und Normen zu analysieren (vgl. ebd., S. 76f). Diese Perspektive ist in der Lage, Bildungsungerechtigkeit hinsichtlich hierarchisierender, benachteiligender oder gar diskriminierender Formen sozialer Beziehungen in pädagogischen Kontexten zu untersuchen sowie Wege und Möglichkeiten aufzuzeigen, solche Strukturen und Praktiken aufzuheben.

\subsubsection{Anerkennung als Herstellung von Zuschreibungen unter Berücksichtigung von Machtstrukturen}

Die erste explizit pädagogische/erziehungswissenschaftliche Anerkennungstheorie wurde von Annedore Prengel 1993 mit ihrem Konzept einer „Pädagogik der Vielfalt" vorgelegt (vgl. Micus-Loos, 2012, S. 310; Stojanov, 2006, S. 166). Prengel (1993) bezieht sich explizit auf Honneths Anerkennungstheorie und operationalisiert die drei Anerkennungsformen als normative Prinzipien pädagogischen Handelns, wobei sie die Anerkennungsform der sozialen Wertschätzung in ihrer Konzeption für den erziehungswissenschaftlichen/pädagogischen Diskurs besonders hervorhebt. Sie interpretiert diese als „,egalitäre Differenz“ in Form einer „Anerkennung zwischen soziokulturell unterschiedlich individuierten Personen“ (Prengel, 1993, S. 61) und zwar als „Anerkennung der Zugehörigkeit zu (sub)kulturellen Gemeinschaften“ (ebd. S. 184). In diesen Ausführungen von Prengel (1993) wird nicht nur die dominante Verwendungsweise von Anerkennung als positives Werturteil zur Bestätigung einer Person verwendet, sondern ebenfalls der konstitutive Charakter von Anerkennung als aktiv herstellende Praxis impliziert.

Anerkennungsansätze produzieren Kategorien der Differenz und der Distinktion von Personen(-gruppen) anhand von Merkmalen wie Herkunft, Kultur, Beruf, Bildung, familiärem Status etc. (vgl. z. B. Balzer \& Ricken, 2010; Do Mar Castro Varela \& Mecherill, 2010; Mecherill, 2005; Schäfer \& Thompson, 2010, Stojanov, 2006) und bestätigen somit dominante Unterscheidungen und hegemoniale Identifizierungen (vgl. Mecherill, 2005, S. 323f). So verweist z. B. die Kategorie „Migrant“ bzw. „Migrationshintergrund“ nicht nur auf die „Anerkennung der Zugehörigkeit zu (sub)kulturellen Gemeinschaften“ (Prengel, 1993, S. 184) hinsichtlich der oben angeführten Kategorien Herkunft und Kultur, sondern etikettiert auch Personen(-gruppen) als „Migrant“" bzw. „Mensch(en) mit Migrationshintergrund“ in Form einer Kategorie des „Anderen“ (vgl. Do Mar Castro Varela \& Mecherill, 2010, S. 102). Zwar werden somit Personen(-gruppen) hinsichtlich eines Teils ihrer individuellen Eigenschaften anerkannt, erhalten aber auch gleichzeitig definierende Zuschreibun- 
gen auf Basis einiger nicht selbst ausgewählter Merkmale. Diese paradoxe pädagogische Anerkennungspraktik hinsichtlich Migration und MH fasst Mecherill (2005, S. 325) folgendermaßen hervorragend zusammen: „Das heißt, dass es unter einer migrationspädagogischen Perspektive sowohl darum geht, sich für Strukturen einzusetzen, in denen Andere und Nicht-Andere als Handlungssubjekte anerkannt werden; zugleich geht es aber auch um einen dekonstruktiven Bezug auf identitätsbestätigende Praxen der Anerkennung. "Wie bereits in Abschnitt 3.4 dargelegt, können Menschen mit MH kulturell hybride Identitäten und Handlungspraktiken aufweisen (vgl. Hu, 2007; Keim, 2007, 2012; Krumm, 2009; Stojanov, 2006), die in einem kreativen Prozess ihre multikulturell sozialisierten „Selbst- und Wirklichkeitsdeutungsmuster interperspektivisch transformierend [...] artikulieren, oder sie mit „fremden" Horizonten von Selbst- und Weltwahrnehmung [...] synthetisieren" (Stojanov, 2006, S. 199). Eine Differenz erzeugende Kategorisierung in „Fremde“ oder „Andere“ kreiert nicht nur ein inadäquates Bild der Identität und Kultur der betroffenen Menschen, sondern übergeht auch die Komplexität sowie die Übersetzungsleistungen dieser Gruppe hinsichtlich der Identitätsbildung und kulturellen Praktiken. Diese Fehlzuschreibung kann nach Stojanov (ebd.) als Missachtung auf der Ebene der Solidarität interpretiert werden, da ,die Nicht-Wahrnehmung und die Nicht-Beachtung der erwähnten partikular-ansozialisierten Selbst- und Wirklichkeitsdeutungen des Einzelnen“" sowie „seine oder ihre Fixierung auf diese Deutungen [...] durch die Brille der kulturellen Zugehörigkeit geschieht".

An dieser Stelle soll auf eine Besonderheit von Anerkennungsverhältnissen in pädagogischen Kontexten hingewiesen werden. Honneth (2003) hebt die Reziprozität und Intersubjektivität von Anerkennungsprozessen hervor und impliziert mit dieser Beschreibung ein symmetrisches Verhältnis der Akteure. Professionelle Beziehungen in pädagogischen Kontexten zwischen Pädagogen und Edukanden zeichnen sich aber inhärent durch eine Asymmetrie aus (vgl. Stojanov, 2011, S. 72), in welcher der Pädagoge über mehr „Macht“ in dieser Beziehung verfügt als der Edukand. Der Pädagoge erzieht und bildet den Edukanden zur Autonomie, wobei auf Mittel des Zwangs zurückgegriffen werden kann, sofern dies notwendig erscheint. Dies stellt für Stojanov (ebd., S. 72f) keinen Widerspruch zur Prämisse reziproker, intersubjektiver Anerkennungsverhältnisse dar. Edukanden verfügen über das Potential zur Entwicklung zu autonomen Subjekten. Dementsprechend besteht auch innerhalb asymmetrischer pädagogischer Kontexte ein Verhältnis zwischen Pädagogen und Edukanden, in welchem sie sich, ,wechselseitig in ihrer reellen oder potentiellen Subjektivität anerkennen“ und der ,intersubjektiv-reziproke Aspekt“ im ,asymmetrischen Moment der Zwangsausübung seitens des Pädagogen übergeordnet" (Stojanov, 2011, S. 72f) ist. Auch Ricken und Balzer (2010, S. 53ff) weisen darauf hin, dass auch negierende, sanktionierende Handlungen in der 
pädagogischen Praxis als anerkennende Akte zu interpretieren sowie zu legitimieren sind. Gerade der Verzicht auf Zwang und Sanktion in pädagogischen Situationen, in denen diese angebracht oder gar notwendig wären, verneint implizit das Vertrauen in die Bildsamkeit des Edukanden aus seinen Fehlern zu lernen, was letztlich als eine Missachtung des Potentials zur Autonomiewerdung verstanden werden kann.

Es stellt sich letztlich die Frage, wer über die Entscheidungsgewalt verfügt, wem welche Kategorien unter welchen Bedingungen zugeschrieben werden. Die Dominanzgesellschaft fungiert diesbezüglich als Machtordnung, sodass Anerkennungspraktiken „als Ausdruck von natio-ethno-kulturellen Machtund Dominanzverhältnissen“ (Do Mar Castro Varela \& Mecherill, 2010, S. 101) zu verstehen sind. Sie besitzt nicht nur die Definitionshoheit über die zu vergebenden Kategorien, sondern bestimmt auch in Anlehnung an Honneth (2003) diejenigen wertzuschätzenden Fähigkeiten und Eigenschaften unter Verwendung einer ,sekundären Deutungspraxis“ (ebd., S. 205), die einen Beitrag zur Verwirklichung gesellschaftlicher Zielvorgaben leisten (ebd., S. 204). Hierdurch werden bestimmte Lebensweisen, Eigenschaften oder Fähigkeiten präferiert und andere erschwert oder gar verboten (vgl. Mecherill, 2005, S. 323f). Wird dieser Gedanke weitergedacht, so wird deutlich, dass Anerkennung auch mit Praktiken der Unterwerfung und Unterordnung verknüpft ist, was Balzer und Ricken (2010, S. 67f) unter Zitation Butlers (2001, S. 24f) eindrucksvoll darlegen:

„Ein Subjekt, so Butler, ist ,genötigt, nach Anerkennung seiner eigenen Existenz in Kategorien, Begriffen und Namen zu trachten, die es selbst nicht hervorgebracht hat" (Butler, 2001, S. 25), denn es wird nicht anerkannt, ohne sich den ,gesellschaftlichen Kategorien“" einer anerkennungsfähige[n] [...] sozialen Existenz (ebd., S. 24) zu beugen bzw. zu unterwerfen. Unterordnung und Unterwerfung (unter historisch kontingente Normen der Anerkennbarkeit) und die „Annahme von Machtbedingungen“" sind daher der „Preis der Existenz“ (ebd. S. 25) und „die nüchterne Grundlage der Subjektwerdung“ (ebd.)“.

Dementsprechend gibt es aus anerkennungstheoretischer Perspektive, im Gegensatz zu Prengels (1993, S. 184) normativem Anspruchs ,egalitärer Differenz" einer „Anerkennung der Zugehörigkeit zu (sub)kulturellen Gemeinschaften“, eigentlich ,keinen normativen Anspruch auf die Anerkennung der eigenen kulturellen Zugehörigkeit“ (Stojanov, 2006, S. 168). Um „kulturellbiographische Anerkennung" (Stojanov, 2006, S. 199) in Form sozialer Wertschätzung zu erhalten, gilt es ,seine ,ansozialisierten“ kulturspezifischen Horizonte und Wertvorstellungen als wertvoll für die gesamte Gesellschaft" (ebd., S. 168) in Form eines fruchtbaren Beitrags zu gesellschaftlichen Zielvorgaben oder einer Bereicherung für diese zu artikulieren. Mecherill (2005, S. 314) weist diesbezüglich darauf hin, dass die Artikulation des Anspruchs auf Anerkennung von den jeweiligen Betroffenen ausgehen sollte, aber Diskurse über die Anerkennung von Minderheiten häufig von Mehrheitsangehörigen geführt 
werden. Solche Praktiken der Vertretung der Betroffenen durch nicht zu dieser Gruppe gehörenden Unterstützer schwächen den Anspruch auf und Kampf um symmetrische, reziprok intersubjektive Anerkennungsverhältnisse sowie letztlich der Etablierung von Solidarität. Nichtsdestotrotz gilt hier natürlich einzuwenden, dass der Verzicht auf oder das Fehlen von Unterstützung von Individuen aus der Mehrheitsgesellschaft den Kampf um Anerkennung von Minoritäten deutlich erschwert oder gar nicht erst stattfinden kann. So sind z. B. aktuell die Bedingungen für die Gruppe geflüchteter Menschen bei der Migration in andere Länder angesichts diverser Nachteile wie z. B. ihrem reduzierten Rechtsstatus und geringer ökonomischer Mittel suboptimal, um rechtliche und solidarische Anerkennung reziprok mit der Mehrheitsgesellschaft zu erfahren.

\subsubsection{Anerkennung von Schülerinnen und Schülern in Bildungsprozessen}

Da ein bedeutender Anteil von Bildung innerhalb von Institutionen stattfindet, sollten Anerkennungsprozesse insbesondere in solchen Kontexten untersucht werden. Die Institution Schule trägt hierbei eine herausragende Rolle, da deren Besuch in der Bundesrepublik Deutschland gesetzlich verpflichtend ist und in dieser relevante Bildungsprozesse sowie -zertifizierung erfolgen, die sich auf die zukünftige gesellschaftliche Teilhabe auswirken. Zudem weist die Bildungsinstitution Schule professionelle Strukturen und Personal auf, sodass problematische oder missachtende Anerkennungspraktiken hier eher modifiziert werden können als in ,natürlichen“ Sozialisationsinstanzen wie der Familie oder des Wohnsitzes.

Hinsichtlich bildungstheoretischer Reflexionen der Anerkennung konstatiert Stojanov (2006, S. 168f), dass ,,der Selbstverwirklichungsprozess der Einzelnen“ der Gegenstand der Anerkennung ist und „Anerkennungsverhältnisse nicht das Ziel von Bildung, zu dem diese hinführen muss“, sondern „vielmehr Voraussetzung und Triebwerk für Bildung sind“. Die Qualität der sozialen Beziehungen in der Institution Schule determinieren zu einem nicht zu unterschätzenden Anteil die Möglichkeiten der Selbst-Entwicklung und Welt-Erschließung, sodass Schule und Unterricht die reziproken, intersubjektiven Interaktionsverhältnisse der Anerkennungsformen der Empathie, des Rechts und der Solidarität in ihren Praktiken berücksichtigen sollten. Hierbei kann die Entwicklung von Selbstvertrauen, -achtung und -schätzung (vgl. Honneth, 2003) ebenfalls als Ziel von Bildung definiert werden, da die Entwicklung zu einem autonomen Subjekt einen Teil des Bildungsprozesses darstellt, welcher diese praktischen Selbstbeziehungen benötigt. Allerdings sollten hinsichtlich der Institution Schule die beiden letztgenannten praktischen Selbstbeziehungen und deren zugehörige Anerkennungsformen akzentuiert werden, da die Ausbildung von Selbstvertrauen nach Honneth (2003, S. 153ff) primär im familiären Umfeld sowie in der frühen Kindheit verortet ist, auch wenn selbstverständlich 
Empathie in institutionalisierten professionellen, pädagogischen Anerkennungsverhältnissen eine relevante Rolle spielt. Dementsprechend gilt es insbesondere die Rahmenbedingungen, unter welchen nicht nur personelle und finanzielle Ressourcen, sondern auch zentrale Funktionslogiken und Praktiken von Schule zu verstehen sind, sowie die institutionalisierten Interaktionsverhältnisse der Schule hinsichtlich der Anerkennungsform des Rechts und der Solidarität für bilingual aufwachsende $\mathrm{SuS}$ zu untersuchen, da diese aus anerkennungstheoretischer Perspektive zentrale Voraussetzungen für Bildung sowie relevante Indikatoren für Bildungsgerechtigkeit darstellen.

\subsubsection{Die Anerkennungsform des Rechts von Schülerinnen und Schülern mit Migrationshintergrund in der Bildungsinstitution Schule}

Die Rahmenbedingungen und Interaktionsverhältnisse in der Institution Schule sollten hinsichtlich der Anerkennungsform des Rechts so strukturiert sein, dass erstens alle SuS prinzipiell über die gleichen Rechte verfügen und zweitens die jeweiligen vorliegenden Handlungsdispositionen aller SuS berücksichtigt werden, sodass diese sich bewähren und entwickeln können. Während der erste Aspekt die Gleichheit der SuS akzentuiert, macht der zweite Aspekt auf die unterschiedlichen Voraussetzungen und Eigenschaften der SuS aufmerksam, was zunächst widersprüchlich erscheint. Diesbezüglich kann auf Hegels (1974; zit. nach Horster, 2015, S. 45) Prämisse der „Gleichheit aller Menschen in ihrer Verschiedenheit" zurückgegriffen werden. Zwar sollten alle SuS über dieselben Grundrechte und -pflichten verfügen, aber die zweite Voraussetzung der Berücksichtigung der jeweiligen vorliegenden Handlungsdispositionen legitimiert potentielle Unterschiede in den jeweiligen Anerkennungsverhältnissen. Somit sollte die Institution Schule es allen SuS unabhängig ihrer Eigenschaften, Fähigkeiten und Merkmale ermöglichen, ,ihr sozial vorstrukturiertes Vermögen wirksam werden zu lassen und sich in affirmative und transformative Welt- und Selbstverhältnisse zu begeben" (Mecherill, 2005, S. 321). Die Schaffung solcher Rahmenbedingungen und Interaktionsverhältnisse könnte als ein universelles Recht der SuS interpretiert werden, dessen konkrete Umsetzung im Einzelfall die besonderen Eigenschaften und Fähigkeiten des Subjekts berücksichtigen sollte.

Die Rechte und Pflichten von SuS in Deutschland bezüglich ihrer Bildung in der Institution Schule finden sich in den gesetzlichen Schulregelungen der Bundesländer wieder. Im Folgenden werden relevante gesetzliche Regelungen für die Gestaltung von Anerkennungsverhältnissen in der Institution Schule am Beispiel des Hessischen Schulgesetzes (HSchG) in der Fassung vom 30. Juni 2017 dargelegt und diskutiert. Prinzipiell haben alle SuS die gleichen Rechte und Pflichten. So ist zum Beispiel unter $§ 1$ HSchG das Recht aller Kinder auf schulische Bildung unabhängig von „Geschlecht, Behinderung, Herkunftsland 
oder Religionsbekenntnis [...] wirtschaftliche[r] oder gesellschaftliche[r] Stellung der Eltern" festgehalten. Zudem gilt es nach den Erziehungs- und Bildungsaufträgen der Schule unter §2 Abs. 4 HSchG Kinder zu befähigen, „Beziehungen zu anderen Menschen nach den Grundsätzen der Achtung und Toleranz, der Gerechtigkeit und der Solidarität zu gestalten“. Systematische Benachteiligungen oder Bevorzugungen aufgrund differierender Eigenschaften und Merkmale werden in $\S 3$ Abs. 3 HSchG unterbunden: „Die Schule darf keine Schülerin und keinen Schüler wegen des Geschlechts, der Abstammung, der Rasse, der Sprache, der Heimat und Herkunft, einer Behinderung, des Glaubens und der religiösen oder politischen Anschauungen benachteiligen oder bevorzugen“. Allerdings ist gleichzeitig ein potentiell distinkter Umgang mit verschiedenen SuS gesetzlich verankert, da die Berücksichtigung der jeweiligen Handlungsdispositionen der SuS zur Gewährleistung der Verwirklichung des Erziehungs- und Bildungsauftrags rechtlich in $\$ 3$ Abs. 6 HSchG legitimiert ist: , ,...] und jede Schülerin und jeder Schüler unter Berücksichtigung der individuellen Ausgangslage in der körperlichen, sozialen und emotionalen sowie kognitiven Entwicklung angemessen gefördert wird. Es ist Aufgabe der Schule, drohendem Leistungsversagen und anderen Beeinträchtigungen des Lernens, der Sprache sowie der körperlichen, sozialen und emotionalen Entwicklung mit vorbeugenden Maßnahmen entgegenzuwirken“. Hinsichtlich der sprachlichen Fähigkeiten von SuS, deren „Sprache nicht Deutsch ist", werden jedoch in $\S 3$ Abs. 14 HSchG konkrete Handlungsanweisungen zu den Rechten und Pflichten der Institution Schule sowie der SuS formuliert: „Schülerinnen und Schüler, deren Sprache nicht Deutsch ist, sollen unabhängig von der eigenen Pflicht, sich um den Erwerb hinreichender Sprachkenntnisse zu bemühen, durch besondere Angebote so gefördert werden, dass sie ihrer Eignung entsprechend zusammen mit Schülerinnen und Schülern deutscher Sprache unterrichtet und zu den gleichen Abschlüssen geführt werden können.“ Die besonderen Angebote werden in $§ 8 \mathrm{a}$ Abs. $1 \mathrm{HSchG}$ als „,besondere Unterrichtsangebote zum Erwerb der deutschen Sprache oder zur Verbesserung der deutschen Sprachkenntnisse" konkretisiert.

Die soeben beispielhaft am HSchG ausgeführten gesetzlichen Bestimmungen illustrieren, dass sowohl die Schule als Institution als auch SuS selbst zur Achtung der Anerkennungsformen des Rechts und der Solidarität verpflichtet sind. Diskriminierung durch Benachteiligung oder Bevorzugung von SuS aufgrund differierender Eigenschaften und Merkmale ist folglich gesetzlich untersagt. Allerdings sind Ungleichbehandlungen zu vertreten und manchmal notwendig, wenn hiermit auf unterschiedliche Ausgangslagen von SuS reagiert und/oder „drohendem Leistungsversagen und anderen Beeinträchtigungen des Lernens" ( $§ 3$ Abs. 6 HSchG) begegnet wird. Diese Bestimmung, einerseits auf die Eingangsvoraussetzungen in nicht bevorteilender oder benachteiligender Weise einzugehen und andererseits mit vorbeugenden Maßnahmen Leistungsversagen und Beeinträchtigungen des Lernens entgegenzuwirken, lässt einen 
gewissen Interpretations- und Handlungsspielraum offen, der flexibel abhängig vom konkreten Fall ausgelegt werden kann. Für den Bereich sprachlicher Förderung von SuS, deren Sprache nicht Deutsch ist, wird der Handlungsspielraum allerdings deutlich eingeschränkt. Sprachliche Fähigkeiten im Deutschen, die für die Beschulung in der Verkehrs- und Unterrichtssprache Deutsch als nicht ausreichend betrachtet werden, gilt es verpflichtend durch eigenes Bemühen und zusätzliche Förderangebote zu verbessern (vgl. §3 Abs. 14 HSchG).

Die Terminologie „SuS, deren Sprache nicht Deutsch ist“ ist relativ breit formuliert. Die Formulierung lässt sich im Kontext der Submersion wahrscheinlich dahingehend interpretieren, dass hiermit alle Spracherwerbstypen außer dem L1a und 2L1a gemeint sind. Dementsprechend werden hiermit primär Zweitsprachlerner des Deutschen adressiert. Angesichts des monolingualen Habitus der deutschen Regelschule ist es nicht verwunderlich, dass sprachliche Defizite im Sinne der Time-on-Task Hypothese durch eigenes Bemühen sowie durch zusätzliche Förderangebote im Deutschen behoben werden sollen, sodass dies gar als Pflicht der SuS deklariert wird. Es finden sich weder Ausführungen zu einer (temporären) Beschulung unter Verwendung der L1 der betroffenen SuS wie es in (transitionalen) bilingualen Bildungsprogrammen üblich ist noch zu Unterrichtspraktiken, die die L1 im Sinne der Interdependenzhypothese nach Cummins (2000) involvieren. Zusätzliches professionelles pädagogisches Personal, welches die L1 von bilingual aufwachsenden SuS teilt und diese in ihren Lernprozessen, zumindest in einer ersten Übergangsphase, unterstützt, ist ebenfalls nicht vorgesehen. Der monolinguale Habitus der deutschen Regelschule zeichnet sich allerdings nicht nur durch konkrete gesetzliche Handlungsrichtlinien aus, sondern lässt sich primär als „Wahrnehmungs-, Handlungs- und Denkmatrix" (Gogolin, 1994, S. 31) verstehen, die konkrete Schul- und Unterrichtspraktiken determinieren. Der oben angeführte Handlungsspielraum hinsichtlich der Berücksichtigung der Ausgangsvoraussetzungen der SuS sowie der Praktiken, um Leistungsversagen und Lernbeeinträchtigungen entgegenzuwirken, wird dementsprechend auch durch den monolingualen Habitus als Orientierungsrahmen weiter eingeschränkt. Die in Abschnitt 3.6.4.1 dargelegte Evidenz zum Umgang der Institution Schule mit der L1 von SuS mit MH sowie hinsichtlich der Einstellungen und Überzeugungen von Lehrkräften zum Gebrauch der L1 von SuS mit MH im Submersionskontext (Abschnitt 3.6.4.2) lassen schließen, dass Schul- und Unterrichtspraktiken in Form einer systematischen Berücksichtigung und Einbindung der L1 von SuS mit MH zur Förderung ihrer Bildungsprozesse in der Regelschule nicht anerkannt und implementiert werden. Solche Praktiken der Institution Schule können aus anerkennungstheoretischer Perspektive als Missachtung der Anerkennungsform des Rechts und somit auch als eine Form von Bildungsbenachteiligung interpretiert werden. 
Weitere Evidenz für bildungsbenachteiligende Praktiken der Institution Schule hinsichtlich SuS mit MH, die über die Kategorien Herkunft, Kultur und Sprache operieren, liefern Gomolla und Radtke (2009). Gomolla und Radtke (ebd., S. 20f) vertreten die These, dass ein ,nicht unbedeutender Teil der Ungleichheit in der Bildungsbeteiligung von deutschen im Vergleich mit nichtdeutschen Schülern [...] nicht auf die Eigenschaften der Kinder und ihre[r] migrationsbedingten Startnachteile“ zurückzuführen ist, sondern ,,in der Organisation Schule selbst erzeugt" wird. Zur Unterstützung ihrer These zeichnen Gomolla und Radtke (2009) auf Basis von Interviews die Mechanik direkter und indirekter Diskriminierung von SuS mit MH durch die Institution Schule z. B. anhand der Selektionsprozesse bei der Einschulung und dem Übergang zur Sekundarstufe I nach.

Ein zentraler Befund der Autoren betrifft die Eigenschaft institutioneller Diskriminierung, kein stabiles, fortwährendes Phänomen in Schulen darzustellen, sondern diese eher als eine flexible, anforderungsbezogene Praktik der Selektions- und Allokationsfunktion der Schule zu verstehen (vgl. ebd.). Gesellschaftliche Ordnungsmuster und Orientierungen, die diskriminierende Einstellungen und Überzeugungen gegenüber SuS mit MH in Form von „kulturalisierenden (Stereo-)Typisierungen“" (ebd., S. 274) enthalten, werden weder passiv „von äußeren Ordnungsmustern“ in die Institution getragen noch aktiv von dem pädagogischen Personal etabliert (ebd., S. 268). Stattdessen eignet sich die Institution Schule ,solche institutionellen Muster im Prozeß des Organisierens an“ und diese werden ,in die eigenen Operationen dann eingearbeitet, wenn sie gebraucht werden - aber auch nur dann" (ebd.). Hierbei akzentuieren die Autoren die Bedeutung der Voraussetzungen und der Erwartungen an die Mitgliedschaft in der Institution Schule zur Steuerung des Prozesses der Organisation von Selektion und Allokation (vgl. ebd., S. 274). Das Bildungssystem verfügt über eine begrenzte Anzahl an materiellen und personellen Ressourcen, die die Aufnahme und Verteilung von SuS auf Schulzweige und Schulen determinieren. Hierbei gilt es, die vorhanden Mitgliedschaften der Schulzweige und Schulen so auf die SuS zu verteilen, dass die jeweiligen Schulzweige und Schulen gefüllt werden. Zudem müssen SuS bestimmte Erwartungen bei der Einschulung und dem Übergang in die Sekundarstufe I erfüllen, die als notwendige Voraussetzungen zur Realisierung des Erziehungs- und Bildungsauftrags in den jeweiligen Schulen vorhanden sein müssen. Bei SuS mit $\mathrm{MH}$ werden neben den für die Beschulung allgemeingültigen Eigenschaften wie unter anderem den kognitiven, sozialen oder emotionalen Entwicklungsstand Kriterien wie „Deutschkenntnisse“, „Sprachkompetenz im Deutschen“, „kultureller Hintergrund“ und „häuslicher Hintergrund“ bei Bedarf hinzugezogen (vgl. ebd., S. 271). Die Kriterien sind in allen Fällen ,unscharf genug, um flexibel angewendet werden zu können“" (ebd., S. 269). Kommt es nun zu einer fehlenden Passung zwischen den vorhandenen materiellen und personellen Ressourcen der Schulzweige und Schulen und den auf Basis der Fähigkeiten 
der SuS zu verteilenden Mitgliedschaften, dominiert letztlich „die Eigenrationalität der Organisation, die ihre Mitgliedschaftsbedingungen behauptet" und schließlich flexibel an die Erfordernisse der Organisation der Selektion und Allokation adjustiert (ebd., S. 270). Die Institution Schule ist dementsprechend an der Hervorbringung und Legitimation gesellschaftlicher Hierarchien auf Basis „ethnischer Differenzierung“ beteiligt, ,indem sie Unterschiede macht und diese mit der geläufigen Semantik über Migranten begründet" (ebd., S. 276).

Zur Illustration dieser Praktik eignet sich ein Gedankenexperiment: Wenn theoretisch in einem Schuljahr ein immenser Anteil der SuS der Grundschulklassen in Hessen am Ende der vierten Klasse hervorragende Leistungen in zentralen schulischen Kompetenzen wie Lesen, Schreiben und Rechnen vorweisen würden, die sie für den Übergang in das Gymnasium qualifizieren würden, müssten trotzdem die vorhanden Mitgliedschaften der Förder-/Sonder-, Haupt- und Realschulen auf diese SuS verteilt werden. Um eine unangemessene Verteilung zu legitimieren, gilt es Begründungslogiken anzuführen, die die Erfüllung des Erziehungs- und Bildungsauftrags der Schule trotz der hervorragenden Ausgangsvoraussetzungen der SuS gefährden könnte. Hierbei können sich die jeweiligen Institutionen auf die bereits oben angeführten, zusätzlichen Kriterien der Selektion und Allokation von SuS mit MH berufen. Der Ausschluss aus der Mitgliedschaft lässt sich dann im Falle des Beispiels des Gedankenexperiments mit einer abweichenden kulturellen oder häuslichen Sozialisation legitimieren.

Gomolla und Radtke (ebd., S. 275) definieren dementsprechend Mechanismen der institutionellen Diskriminierung bei SuS mit MH folgendermaßen:

„Von Mechanismen der institutionellen Diskriminierung von Migrantenkindern in der Schule soll gesprochen werden, - wenn regelmäßig von der Organisation Schule vorgenommene (Selektions-)Entscheidungen, die in ihrer eigenen Logik und Pragmatik getroffen werden, ungleiche Wirkungen auf die Schüler haben, und - wenn diese in der Organisation selbst hergestellten Unterschiede durch Merkmale/Eigenschaften, die der benachteiligten Gruppe zugeschrieben werden, mit Sinn ausgestattet werden, und - wenn es sich dabei um das Kollektivmerkmal der „nationalen Herkunft“/ „Kultur“" handelt.“

Hierbei differenzieren Gomolla und Radtke (ebd., S. 277ff) zwischen Mechanismen direkter und indirekter institutioneller Diskriminierung. Hinsichtlich Mechanismen direkter institutioneller Diskriminierung kann zum einen zwischen ,positiver Diskriminierung“, die durch den Rückgriff auf formell-rechtliche Bestimmungen SuS mit MH aus einer Förderperspektive Angebote und Maßnahmen der Sonderbehandlung auferlegt, und zum anderen dem ,strategische[n] Umgehen schulrechtlicher Regelungen, die gezielt eingeführt wurden, um einer verfrühten und unangemessenen Selektion von Kindern aufgrund von Sprache und kultureller Herkunft entgegenzuwirken“"(ebd., S. 277) unterschie- 
den werden. Mechanismen indirekter Diskriminierung bestehen aus der fehlenden Berücksichtigung der individuellen Eingangsvoraussetzungen und Handlungsdispositionen in Form einer durch die Mitgliedschaft in der Institution Schule legitimierten Gleichbehandlung von bilingual aufwachsenden SuS mit MH und monolingual aufwachsenden SuS (vgl. ebd., S. 281).

Die Quintessenz der Befunde von Gomolla und Radtke (2009) hat Stojanov (2011, S. 153) folgendermaßen hervorragend zusammengefasst, wobei ein Hinweis auf die Flexibilität der Praktiken der institutionellen Diskriminierung noch hinzuzufügen wäre: „Bildungsinstitutionen vermeiden die Komplexitätserhöhung für Unterricht und pädagogischen Umgang mit Kindern und Jugendlichen, die sich durch eine Abweichung vom Postulat des in einer Mittelschichtfamilie monolingual und im Einklang mit der „Leitkultur" sozialisierten „Normalschülers“ ergeben würde, indem sie „Argumentationshaushalte“ produzieren, welche ,kulturelle“ Ursachen für Bildungsmisserfolge [...] behaupten." Stojanov (ebd., S. 154f) kritisiert den Ansatz von Gomolla und Radtke (2009) als eklektizistisch, da sie mit einem normativen Gerechtigkeitsbegriff arbeiten, obwohl eine zentrale systemimmanente Funktion der Institution Schule die Produktion von Ungleichheit als gesellschaftliches Funktionserfordernis der gesellschaftlichen Arbeitsteilung ist. Stojanov (2011, S. 155) präferiert hierbei einen ideologiekritischen Ansatz der Konstruktion sprachlich-kultureller Differenz, der seiner Ansicht nach besser für die „doppelte Aufgabe der Rekonstruktion der soziopolitischen Funktionen diskursiver Zuschreibungen und der Überprüfung ihres Wahrheitsbezugs" geeignet ist. Die Mechanismen der institutionellen Diskriminierung und die zugrundeliegenden Begründungslogiken lassen sich dementsprechend folgendermaßen besser beschreiben:

„So wird bei der - ideologischen - Behauptung, dass Bildungsdefizite von Kindern und Jugendlichen ,,mit Migrationshintergrund“ auf einen „Identitätskonflikt“, oder auf einen schwierigen „Spagat zwischen den Kulturen“ zurückzuführen seien (insbesondere dann, wenn sich ihre Familien nicht assimiliert haben), von der stillschweigenden Prämisse ausgegangen, dass der Mensch grundsätzlich ein monokulturelles Wesen sei, oder zumindest, dass die Identitätsbildung des Menschen eine monokulturelle Zugehörigkeit voraussetze. Diese Prämisse wird von den Proponenten der „Identitätskonflikt-These“ als eine Selbstverständlichkeit behandelt, die keiner weiteren Erläuterungen und Begründungen bedarf, und die daher eine Berücksichtigung von Gegenpositionen und -argumenten nicht erfordert.“ (ebd., S. 158).

Im Gegensatz zu Gomolla und Radtke (2009) infiltrieren nach Stojanov (2011) differenzschaffende Ideologien beziehungsweise ,äußere Ordnungsmuster“ (Gomolla \& Radtke, 2009, S. 268) die Institution Schule und werden als Prämissen für die Selektions- und Allokationsfunktion angewandt, ohne diese zu hinterfragen. Unabhängig davon, welches Erklärungsmodell besser geeignet zur Beschreibung der Mechanismen institutioneller Diskriminierung erscheint, 
weisen beide Ansätze auf differenzierende, Unterschiede herstellende Praktiken der Institution Schule bezüglich der Gruppe von bilingual aufwachsenden SuS mit MH im Vergleich zu monolingual aufwachsenden „Normalschülern“ hin, die als Bildungsungerechtigkeit zu deklarieren sind und zu Bildungsbenachteiligung führen.

In der Gesamtschau lässt sich die differenzschaffende Auslegung der schulgesetzlichen Bestimmungen sowie der durch den monolingualen Habitus und gesellschaftlichen Ideologien geprägten Schul- und Unterrichtspraktiken zur Erfüllung der Selektions- und Allokationsfunktion als auch des Erziehungsund Bildungsauftrags der Institution Schule hinsichtlich bilingual aufwachsender SuS mit MH als Formen flexibel eingesetzter institutioneller Diskriminierung rekonstruieren. Dies affirmiert die Interpretation einer Missachtung der Anerkennungsform des Rechts im Sinne Honneths (2003) als Entrechtung und Ausschließung für die Gruppe von bilingual aufwachsenden SuS mit MH. Die persistente Bildungsbenachteiligung von SuS mit MH, die sich nicht allein auf Basis typischer Variablen mit hoher Varianzaufklärung für Bildungserfolg wie Vorwissen, Kompetenz, sozioökonomischer Status etc. erklären lässt, erhält durch die Mechanismen der institutionellen Diskriminierung auf Basis ideologischer, vermeintlich sprachlich-kultureller Differenz beruhender Begründungslogiken die Legitimation für diverse bildungsbenachteiligende Praktiken. Zudem ignoriert das Bildungssystem mit seiner Akzentuierung verkehrssprachlicher Fähigkeiten als zentrale sprachliche Variable für Bildungserfolg die Evidenz zur Effektivität bilingualer Bildungsprogramme für akademischen Erfolg und L2a sowie die Schwellenwert- und Interdependenzhypothese (Cummins, 2000) unterstützende empirische Evidenz. Die Handlungsdispositionen von SuS mit MH, die häufig Deutsch als L2 erwerben (vgl. Schulz \& Tracy, 2011), werden durch die fehlende oder positiv diskriminierenden Berücksichtigung ihrer zweisprachigen Lebensrealität und der Missachtung ihrer L1 als Ressource für Bildungsprozesse in ihren Bildungsmöglichkeiten eingeschränkt. Insbesondere die selektive Frühförderung von SuS mit MH wie z. B. die abhängig vom jeweiligen Bundesland optionalen oder obligatorischen Vorlaufkurse zur Förderung des Deutschen beinhaltet Einflussnahmen oder gar „massive Einschränkungen der Freiheit der Eltern bei der Erziehung ihrer Kinder, bei der kulturellen Selbstbestimmung der Familien sowie bei der Wahl ihrer Sprache(n), in der Familienangehörige miteinander kommunizieren“ (Stojanov, 2011, S. 145). Bilingual aufwachsende SuS mit MH werden auf Basis der Kategorien Herkunft, Kultur und Sprache als andersartig im Vergleich zur Mehrheitsgesellschaft etikettiert, die in Unterstellungen z. B. von „Identitätskonflikten“, einer „Zerrissenheit" oder eines „Spagats zwischen Kulturen“, „Integrationsunwilligkeit der Familien“, „Sprachdefiziten“ oder gar „doppelte Halbsprachigkeit“ münden. Diese Prämissen diskreditieren nicht nur das bilinguale Sprachvermögen von bilingual aufwachsenden SuS mit MH, 
sondern missachten auch ihre potentiellen transformativen Übersetzungsleistungen zur Konstruktion von hybriden Identitäten und eines unikalen, originellen kulturellen Vermögens.

Angesichts einer holistischen Perspektive auf die Lebensrealität und Handlungsdispositionen von bilingual aufwachsenden SuS mit MH kann der gegenwärtige Umgang der Institution Schule mit diesen folglich als Missachtung der Anerkennungsform des Rechts in Form einer Entrechtung und Ausschließung interpretiert werden. SuS mit MH werden von der Institution Schule unter Assimilationsdruck gesetzt anstatt in Form einer pluralistischen Ideologie reziproke Akkulturation in Schul- und Unterrichtspraktiken zu implementieren. Diesbezüglich können in einer kulturell und sprachlich pluralistischen Gesellschaft die eben beschriebenen ,nationalkulturelle[n]“" Einengungen durch „mononationale, monokulturelle und monolinguale Verharrung“ (Mecherill, 2005, S. 322) als Missachtung der Anerkennungsformen des Rechts affirmiert werden. Werden die potentiellen hybriden Identitäten und kulturellen Vermögen sowie die bilingualen Handlungsdispositionen und -praxen bei bilingual aufwachsenden SuS mit MH von der Institution Schule systematisch ignoriert oder flexibel als defizitär ausgelegt, können diese „Missachtungen [...] die Entwicklung von Handlungsfähigkeit und Subjektstatus verhindern oder zumindest erschweren“" (ebd.), was zu Bildungsbenachteiligung führt. Dementsprechend kann in Zweifel gestellt werden, ob die Institution Schule schulrechtliche Bestimmungen für bilingual aufwachsende $\mathrm{SuS}$ mit $\mathrm{MH}$, wie sie zum Beispiel in $§ 3$, Abs. 9 HSchG formuliert sind, gewährleisten können: „Die Schule ist zur Wohlfahrt der Schülerinnen und Schüler und zum Schutz ihrer seelischen und körperlichen Unversehrtheit, geistigen Freiheit und Entfaltungsmöglichkeit verpflichtet. Darauf ist bei der Gestaltung des Schul- und Unterrichtswesens Rücksicht zu nehmen“.

\subsubsection{Soziale Wertschätzung von Schülerinnen und Schülern mit Migrationshintergrund in der Bildungsinstitution Schule}

Die Anerkennungsform der Solidarität kann, wie in Abschnitt 4.2.2.3 bereits dargelegt, als soziale Wertschätzung der spezifischen Eigenschaften und Fähigkeiten von Individuen, ,durch die Menschen in ihren persönlichen Unterschieden charakterisiert sind“" (Honneth, 2003, S. 197) definiert werden. Bilingual aufwachsende SuS mit MH zeichnen sich - wie alle anderen Kinder und Jugendliche - primär durch ihre individuelle Einzigartigkeit sowie durch Heterogenität hinsichtlich ihrer schulrelevanten Eigenschaften und Fähigkeiten aus. Im Gegensatz zu monolingual aufwachsenden SuS lässt sich ihre Sozialisation und Lebensrealität allerdings als bilingual und -kulturell bezeichnen. Wie in Abschnitt 3.2 und 3.4 dargestellt, können sich allerdings bilinguale Spracherwerbstypen respektive die individuelle Konstruktion beziehungsweise Übersetzung von Kultur und Identität bei bilingual aufwachsenden SuS 
mit MH interindividuell unterscheiden, sodass auch bezüglich dieser Eigenschaften Heterogenität herrscht. Nichtsdestotrotz können die migrationsbedingte Bilingualität und das Potential, ,die ,,enkulturierten“ Selbst- und Wirklichkeitsdeutungsmuster interperspektivisch transformierend zu artikulieren oder sie mit „,fremden“" Horizonten von Selbst- und Weltwahrnehmung zu synthetisieren" (Stojanov, 2006, S. 199), als zwei Merkmale interpretiert werden, die für die Anerkennungsform der Solidarität als sozial wertzuschätzende Eigenschaften für diese Gruppe angeführt werden können.

Stojanov (2006) hat in seiner Monographie „Bildung und Anerkennung“ bereits die „kulturell-biographische Anerkennung“, auf welche in den vorangegangen Kapiteln dieser Arbeit wiederholt rekurriert wurde, ,als eine bildungstheoretisch immens wichtige Unterform von sozialer Wertschätzung rekonstruiert" (Stojanov, 2006, S. 199). Stojanov (2006) identifiziert eine Missachtung dieser Unterform der sozialen Wertschätzung durch die Institution Schule, da das Bildungssystem die transformativen Übersetzungsleistungen und das Potential hierzu nicht wahrnimmt oder nicht beachtet und stattdessen diese als „Spagat zwischen den Kulturen“ und „Identitätskonflikte“ ideologisch deutet. Dies wurde auch bei der Analyse der Anerkennungsform des Rechts diskutiert, allerdings als Legitimation der flexibel angewandten Begründungslogik zur Entrechtung sowie zum Ausschluss in Selektions- und Allokationsprozessen der Institution Schule. Dementsprechend soll in diesem Abschnitt insbesondere die soziale Wertschätzung der Bilingualität in der Institution Schule diskutiert werden.

Das linguistische Selbstverständnis Deutschlands liegt in der Monolingualität (vgl. Gogolin, 1994). Der L1a wird als Norm angesehen (vgl. Kersten et al., 2011), zentrale Bildungsinstitutionen wie die Schule zeichnen sich durch einen monolingualen Habitus aus (vgl. Gogolin, 1994) und die Sprachkompetenz in der Verkehrssprache ist ein zentrales Kriterium für Bildungserfolg und gesellschaftliche Teilhabe (vgl. z. B. Cummins, 2000; Esser, 2009; Kempert et al., 2016). Gleichzeitig erfährt Mehrsprachigkeit selbst eine hohe soziale Wertschätzung, da diese Eigenschaft in einer globalisierten Welt eine zentrale Fähigkeit im 21. Jahrhundert darstellt, die auch im Curriculum in Form des Fremdsprachenunterrichts in der Institution Schule seit langem fest verankert ist. Das Motto des EU-Parlaments hinsichtlich der Multilingualität ihrer EUBürger kann mit „communication in mother tongue plus two"9 (Yağmur, 2016, S. 98) beschrieben werden, alle frühkindlichen Bildungspläne der Länder benennen explizit die Wertschätzung und Förderung von Zwei- und Mehrsprachigkeit als Ziele frühkindlicher Bildung (vgl. Viernickel \& Schwarz, 2009) und auch in schulischen Bildungsplänen stellt Mehrsprachigkeit ein explizites Bildungsziel dar.

9 Als zusätzliche Sprachen zur Muttersprache wünscht sich das EU-Parlament eine Fremdsprache (typischerweise Englisch) und die Sprache eines angrenzenden Landes der europäischen Union (vgl. Yağmur, 2016, S. 98). 
Die Sprachideologie der Bundesrepublik Deutschland zeichnet sich allerdings durch eine implizite Hierarchie zwischen verschiedenen Sprachen aus, die das Deutsche sowie Sprachen des Fremdsprachenkanons wie Englisch, Französisch und Latein (vgl. Tracy, 2014, S. 20) präferieren und priorisieren. Das Englische genießt den Status als „Weltsprache“ und Latein ist für einige Berufe wie z. B. im Bereich der (Tier-)Medizin oder Pharmazie eine Voraussetzung, was das Prestige dieser Sprachen erklären könnte. Andere, natürlich erworbene Sprachen, die wie zum Beispiel das Türkische, Polnische, Arabische oder Italienische, die häufig von bilingual aufwachsenden Kindern in Deutschland als Erstsprache oder als eine Erstsprache erworben werden, haben ein geringeres Prestige als das Deutsche und die Sprachen des Fremdsprachenkanons (vgl. ebd.). Diese Sprachenhierarchie könnte neben der in Deutschland herrschenden Sprachideologie der Assimilation zum Teil auch auf dem Befund zum Zusammenhang von Einstellungen zu bestimmten Sprachen und der Wahrnehmung der Sprecher der Sprache (vgl. Chin \& Wigglesworth, 2007, S. 108; Myers-Scotton, 2006, S. 120) beruhen, da diese Sprachen primär von Sprechergruppen mit $\mathrm{MH}$ gesprochen werden, die sich häufig durch einen geringeren sozioökonomischen Status auszeichnen (vgl. z. B. Rauch et al., 2016; Wendt \& Schwippert, 2017) und von Teilen der Mehrheitsgesellschaft eventuell bis heute nicht anerkannt werden.

Eine ähnliche Tendenz der Sprachenhierarchisierung lässt sich auch für die Institution Schule feststellen. Auf der Ebene des Systems Schule und hinsichtlich des Curriculums spielen die Sprachen von Gruppen mit MH in der deutschen Regelschule kaum eine Rolle. Der monolinguale Habitus der Regelschule samt seiner Orientierung an der Time-on-Task Hypothese sieht in den natürlich erworbenen Erstsprachen von bilingual aufwachsenden SuS mit MH keine Ressource für Lern- und Bildungsprozesse. Dies wird auch deutlich an den schulischen Angeboten zum Unterricht in den Erstsprachen von bilingual aufwachsenden SuS mit MH. Dieser findet fast ausschließlich im HSU statt. Die Struktur und Organisation des HSU unterscheidet sich fundamental von anderen Schulfächern (vgl. z. B. Schröder \& Küppers, 2016). Wie die Benennung vermuten lässt, akzeptiert der HSU nur SuS, die die Sprache des HSUs als (eine) Erstsprache erwerben, sodass von einem exklusiven Angebot gesprochen werden kann (vgl. ebd., S. 194f). Der HSU stellt ein freiwilliges Zusatzangebot dar, welches eher Grundschulen anbieten und der nur zustande kommt, wenn auch ausreichend SuS der jeweiligen Sprache in der Schule vorhanden sind sowie auch daran teilnehmen möchten. Der HSU wird in der Regel nicht von der Schule allein angeboten, sondern häufig in Zusammenarbeit mit Konsulaten, Vereinen oder engagierten Eltern organisiert. Dementsprechend sind nicht alle Lehrkräfte des HSU im Sprachunterricht ausgebildet. Zentral ist, dass der HSU kein versetzungsrelevantes Fach ist, in dem nur selten Noten vergeben werden und der inhaltlich nicht mit anderen Schulfächern verknüpft ist, wie z. B. in Form einer koordinierten Alphabetisierung. 
Im Gegensatz hierzu wird Unterricht in Sprachen von Gruppen mit $\mathrm{MH}$ selten in einer inklusiven Form angeboten, an dem auch SuS teilnehmen können, die die jeweilige Sprache nicht als (eine) L1 erwerben (vgl. ebd., S. 195f). Die Formen dieses Unterrichts sind ebenfalls sehr heterogen. So gibt es z. B. in einigen Schulen in Nordrheinwestfalen, Hamburg und Bremen Türkischunterricht als dritte Fremdsprache, der auch von SuS ohne Türkisch als (eine) L1 gewählt werden kann. Dieser Unterricht findet wie regulärer Fremdsprachenunterricht statt und ist dementsprechend auch versetzungsrelevant. Manche Gymnasien bieten diesen gar als Leistungskurs der gymnasialen Oberstufe an (vgl. ebd.). Vereinzelt werden auch bilinguale Bildungsprogramme angeboten, die prinzipiell nach den Grundsätzen von (transitionalen), ,dual language education" Programmen mit Sprachenseparation aufgebaut sind (für Beispiele türkisch-deutschsprachiger bilingualer Programme siehe ebd., S. 196f). Zusammengefasst stellt der Großteil der schulischen Angebote zu Sprachen von Gruppen mit MH keine systematisch in das Curriculum der deutschen Regelschule eingebetteten, zertifizierten Schulfächer dar. Mit Ausnahme der positiven Beispiele der Integration von Sprachen von Gruppen mit $\mathrm{MH}$ als versetzungsrelevante Schulfächer in den Bundesländern Nordrheinwestfalen, Hamburg und Bremen, spielt die Förderung erstsprachlicher Fähigkeiten selbst oder als Ressource für Bildungsprozesse in der deutschen Regelschule keine Rolle. Die in Abschnitt 3.6.5.4 berichtete Evidenz zu Einstellungen von (angehenden) Lehrkräften zu Mehrsprachigkeit lassen sich ebenfalls als Indikatoren der sozialen Wertschätzung migrationsbedingter Bilingualität interpretieren. Die Befunde von Wlossek und Rost-Roth (2016) sowie Hammer, Fischer und KochPriewe (2016) weisen für einen Großteil der befragten Probanden positive Einstellungen zur migrationsbedingten Mehrsprachigkeit auf, was von einer gewissen Wertschätzung der Mehrsprachigkeit zeugt. Trotzdem steht nach der Meinung eines Großteils der Lehrkräfte die (weitere) L1 in einem vermeintlichen Konkurrenzverhältnis zum Erwerb des Deutschen sowie der Bearbeitung des Curriculums (vgl. Wlossek \& Rost-Roth, 2016). Zudem werden bilinguale Praktiken in der Regel aus einer Defizitperspektive als Zeichen mangelnder Sprachkompetenz betrachtet, statt als typisches bilinguales Kommunikationsverhalten (vgl. ebd.). Die L1 wird von den Lehrkräften in dieser Studie mit Ausnahme von zwei Probandinnen auch nicht als Ressource für Lern- und Bildungsprozesse wertgeschätzt. Für die frühkindliche Bildung in Institutionen wie Kindertagesstätten ergibt sich nach den Befunden von Kratzmann et al. (2017) ein ähnliches Bild. Zwar werden vermehrt positive Einstellungen zur Mehrsprachigkeit von den pädagogischen Fachkräften berichtet, die Umsetzung der Implementation von Konzepten der Mehrsprachigkeit in Form der „Förderung von Respekt vor anderen Kulturen“, dem „Aufbau einer sprachlichen Brücke zur Familie“ und des weiteren „Kompetenzerwerb[s] in der Erstsprache der Kinder" (ebd., S. 239) kann angesichts der empirischen Ergebnisse dieser Studie als gescheitert interpretiert werden. 
Es stellt sich die Frage, ob diese implizite Sprachhierarchie selbst eine Form von Missachtung darstellt. Das Deutsche hat als Verkehrssprache hinsichtlich Teilhabe an der Gesellschaft und persönlicher Zukunftsperspektiven Priorität, was auch von Menschen mit MH als Meinung geteilt wird (vgl. Gogolin, 2015, S. 294). Hinsichtlich des Kanons der Fremdsprachen gibt es plausible Gründe, die die Relevanz dieser im Schulkontext legitimieren. Eine Missachtung von natürlich erworbenen Sprachen im Schulkontext, die nicht die Verkehrssprache darstellen oder dem Fremdsprachkanon angehören, müssen nach Honneth (2003) hinsichtlich der Anerkennungsform der Solidarität den Tatbestand einer Entwürdigung oder Beleidigung aufweisen. Die fast flächendeckende Exklusion natürlich erworbener Erstsprachen von SuS mit MH aus dem prüfungs- und versetzungsrelevanten Curriculum manifestiert aber per se keine Missachtung in Form einer Entwürdigung oder Beleidigung. Diese Praxis könnte theoretisch auch mit organisatorischen, strukturellen und ressourcenbedingten Gründen legitimiert werden. Eine Missachtung tritt erst auf, wenn diese Sprachen explizit als Hemmnisse für den Deutscherwerb und/oder das schulische Lernen artikuliert und problematisiert würden. Erst diese Deutung kann als Entwürdigung oder Beleidigung verstanden werden, da hiermit eine Fähigkeit, die objektiv und wertneutral betrachtet lediglich die Anzahl an potentiellen Kommunikationspartnern sowie den Handlungsspielraum zur Kommunikation selbst erhöht, durch die produktive Seite von Anerkennungspraktiken ein negatives, kontraproduktives Werturteil erhalten würde und folglich als keinen für die Gesellschaft bietenden Mehrwert interpretiert werden könnte. Die wiederkehrenden, medial verbreiteten und stets in Zusammenhang mit Bildung stehenden Mythen über Mehrsprachigkeit (vgl. Kersten et al., 2011), die Mehrsprachigkeit aus einer ideologisch geprägten Defizitperspektive betrachten, stellen Missachtungen der Anerkennungsform der Solidarität dar. Wenn behauptet wird, dass mehrsprachiges Aufwachsen zu Identitätskonflikten führt, typische bilinguale Sprachpraktiken als defizitär oder gar als „doppelte Halbsprachigkeit“ interpretiert werden und Familien mit MH nahe gelegt wird, zuhause Deutsch zu sprechen (vgl. Reimann, 2014), da sich dies angeblich positiv auf die Bildung ihrer Kinder auswirkt, liegt die Interpretation einer Entwürdigung oder Beleidigung nahe. Der Kern der Entwürdigung und Beleidigung dieser Narration liegt allerdings nicht in den Mythen über Mehrsprachigkeit selbst, die die in Kapitel 3 dargestellten wissenschaftlich validierten Phänomene, Eigenschaften, Prozesse und Potentiale der Bilingualität ignorieren oder gar widersprechen. Die Missachtung der Anerkennungsform der Solidarität hinsichtlich migrationsbedingter Bilingualität liegt in dem Umstand, dass diese Mythen nicht für alle Sprachen gelten. So sind z. B. Sprachen des Fremdsprachenkanons von diesen Prämissen ausgeschlossen. SuS, die z. B. eine bilinguale englisch-deutschsprachige Schule besuchen, stellen keine Adressaten dieser sprachideologisch geprägten Vorwürfe dar. Die implizite Sprachenhierarchie, die abhängig von der jeweiligen Sprache unterschiedliche 
Narrative der sozialen Wertschätzung der jeweils vorliegenden Form der Bilingualität enthält, ist das zentrale Moment der Missachtung der Anerkennungsform der Solidarität. Das Absprechen der sozialen Wertschätzung migrationsbedingter bilingualer Fähigkeiten, die nicht dem Fremdsprachenkanon entsprechen, ist aus sprachwissenschaftlicher Perspektive unangemessen, auf gesellschaftlich-funktionaler Ebene allerdings eine Ideologie im gesellschaftlichen „Kampf“ um Anerkennung in Deutschland. Angesichts dessen erhärtet sich die oben bereits geäußerte Vermutung, dass die soziale Wertschätzung von Sprachen von Gruppen mit MH wie z. B. das Türkische, das Polnische oder das Italienische nicht exklusiv durch die Funktionalität und Bedeutung begründet sind, sondern zu einem gewissen Teil auch an der Assoziation von Sprachen mit ihren Sprechern liegt. Da Sprache - wie in Abschnitt 3.4. bereits erörtert - eng mit der Identität verbunden sind (vgl. z. B. Hu, 2007; Keim, 2007, 2012; Krumm, 2009; Stojanov, 2006), transzendiert die fehlende soziale Wertschätzung der sprachlichen Sozialisation und bilingualen Fähigkeiten von bilingual aufwachsenden SuS mit MH auch auf die Ausbildung von Selbstschätzung. Dementsprechend verwundert es nicht, dass manche SuS im Zuge der Sozialisation in Institutionen frühkindlicher Betreuung oder der Schule selbst negative Einstellungen zu ihren Erstsprachen entwickeln, sodass sie diese vor anderen verheimlichen (vgl. Krumm, 2009) und dass bilinguale Praktiken für viele bilinguale Sprecher selbst ebenfalls negativ konnotiert sind (vgl. Gardner-Chloros, 2009, S. 81f; Ritchie \& Bhatia, 2013, S. 389).

Die Implementation von Sprachen von bilingual aufwachsenden SuS mit MH in inklusive, versetzungsrelevante Fremdsprachenangebote oder gar in bilinguale Bildungsprogramme (vgl. Schröder \& Küppers, 2016) können als eine Möglichkeit angesehen werden, einen Wandel hinsichtlich der sozialen Wertschätzung dieser Sprachen und der migrationsbedingten Bilingualität anzustoßen. Durch die Integration dieser Sprachen in das Curriculum würden sie einen offiziellen, legitimen Status erhalten, der die bisher bestehende Sprachenhierarchie aufweicht. Zur Erlangung von sozialer Wertschätzung muss die migrationsbedingte Mehrsprachigkeit 1) argumentativ als Bereicherung oder Beitrag zur Erreichung gesellschaftlicher Zielvorgaben und 2) die Gruppe der Sprecher als besonders wertzuschätzend dargestellt werden. Beide Punkte sollten in Anlehnung an Mecherill $(2005,314)$ von den Betroffenen selbst im Diskurs artikuliert und begründet werden, da ,ein Symptom und Motor von Gerechtigkeit darin besteht, dass gesellschaftliche Gruppen [...] sich selbst vertreten und darstellen“. Die Evidenz der Bilingualismusforschung liefert eine breite Basis hinsichtlich diverser Phänomene und Bereiche, die die migrationsbedingte Bilingualität als Bereicherung für die Gesellschaft darstellen könnten. Hierfür könnten sich angesichts des international führenden Modells des Wirtschaftskapitalismus und -liberalismus insbesondere Argumentationsstrukturen anbieten, die die migrationsbedingte Bilingualität als Ressource für Lern- und Bildungsprozesse sowie für den internationalen Handel wertschätzen, was kombiniert 
als Stärkung des Wirtschaftsstandorts Deutschlands einen Beitrag zur Erreichung gesellschaftlicher Zielvorgaben leisten könnte.

\subsubsection{Zusammenfassung}

Anerkennung stellt ein Grundbedürfnis von Menschen dar (vgl. Micus-Loos, 2012, S. 303), das Individuen sowohl passiv wahrnimmt sowie bewertet als auch diese aktiv mit Zuschreibungen konstruiert (vgl. Balzer \& Ricken, 2010). Honneths (2003) Anerkennungstheorie - insbesondere die konkrete Ausformulierung der drei verschiedenen Anerkennungsformen - stellt eine vielseitig einsetzbare Gesellschafts- und Gerechtigkeitstheorie, die sich vor allem zur Untersuchung der konkreten Anerkennungspraktiken bezüglich gesellschaftlicher Minderheiten eignet. Erziehungswissenschaftlich/Pädagogisch geschärfte anerkennungstheoretische Perspektiven können hierbei fruchtbar auf das Phänomen Bildungsgerechtigkeit sowie auf die konkreten Praktiken und Handlungen in der Bildungsinstitution Schule angewandt werden. Dies liegt zum einen an Honneths (ebd.) Modellierung von Anerkennung als ,subjektphilosophisches Stufenmodell“" (Marfels, 2011, S. 214) mit dem Ziel der Autonomie durch die Ausbildung bestimmter praktischer Selbstbeziehungen in reziproken, intersubjektiven Anerkennungsverhältnissen mit anderen Individuen, die sich hervorragend mit dem von Stojanov (2006, S. 33-66) rekonstruierten Bildungsbegriff verbinden lässt. Zum anderen eröffnen anerkennungstheoretische Reflexionen neue Perspektiven auf Bildungsgerechtigkeit, da nicht eine gerechte Verteilung von Bildungsgütern unter den Prämissen von Gleichheit und Meritokratie fokussiert wird, sondern die Qualität von sozialen Beziehungen sowie deren determinierende Rahmenbedingungen und Praktiken im Mittelpunkt stehen (vgl. Stojanov, 2011). Diese Perspektive ist nicht unidirektional von der Institution Schule auf ihre Schülerschaft zu reflektieren, sondern bidirektional als reziprokes, intersubjektives Anerkennungsverhältnis. Sowohl die Ursachenzuschreibung der Bildungsbenachteiligung von bestimmten Gruppen anhand der Eigenschaften und Merkmale der Betroffenen selbst als auch bildungsbenachteiligende Praktiken der Institution Schule (vgl. Gomolla \& Radtke, 2009) lassen sich unter Verwendung anerkennungstheoretischer Reflexionen analysieren.

Aus anerkennungstheoretischer Perspektive kann der Umgang der Institution Schule mit SuS mit MH sowie hinsichtlich ihrer migrationsbedingten Bilingualität als Missachtung der Anerkennungsformen des Rechts und der Solidarität interpretiert werden. Hinsichtlich der Anerkennungsform des Rechts besteht die Missachtung von SuS mit MH primär in der von Gomolla und Radtke (2009) beschriebenen flexibel eingesetzten institutionellen Diskriminierung auf Basis ideologischer, vermeintlich auf sprachlich-kultureller Diffe- 
renz beruhender Begründungslogiken und der fehlenden oder positiv diskriminierenden Berücksichtigung der zweisprachigen Lebensrealität sowie der Negation der (weiteren) L1 als Ressource für Bildungsprozesse bei bilingual aufwachsenden SuS mit MH. Die erste Missachtung kann dementsprechend in der Terminologie Honneths (2003) als Ausschließung bezeichnet werden, während die zweite Missachtung eher einer Entrechtung entspricht. Die Missachtung der Anerkennungsform der Solidarität besteht primär in der impliziten Sprachenhierarchie der deutschen Regelschule, die durch den MH vorhandene, natürlich erworbene Sprachen ihrer Schülerschaft samt ihrer typischen bilingualen Praktiken aus einer Defizitperspektive problematisiert, während dieses Narrativ für bestimmte andere (weitere) Erstsprachen und/oder Personengruppen nicht gilt. Im Rahmen der Anerkennungstheorie Honneths (ebd.) könnte diese Missachtung als eine Entwürdigung interpretiert werden.

\subsection{Fazit}

SuS mit MH sind im Vergleich zu SuS ohne MH in Deutschland systematisch in zweifacher Weise bildungsbenachteiligt. So weisen sie zum einen statistisch schlechtere sozioökonomische Ausgangsbedingungen auf (vgl. Rauch et al., 2016; Wendt \& Schwippert, 2017), die eng mit dem Bildungserfolg in Deutschland assoziiert sind (vgl. Baumert et al., 2001), und werden innerhalb des Bildungssystems institutionell in direkter und indirekter Weise diskriminiert (vgl. Gomolla \& Radtke, 2009). Diese Bildungsbenachteiligung lässt sich ebenfalls aus anerkennungstheoretischer Perspektive auf den Anerkennungsformen des Rechts und der Solidarität für die Bildungsinstitution Schule in Deutschland nachzeichnen, da auf Basis nationaler, ethnischer, kultureller und/oder sprachlicher Merkmale SuS mit MH missachtet werden.

Um die Missachtung der Bilingualität von zweisprachig aufwachsenden $\mathrm{SuS}$ mit MH durch die Institution Schule in Form von Ausschließung, Entrechtung und Entwürdigung aufzuheben, müssten die (weiteren) Erstsprachen von bilingual aufwachsenden SuS mit MH in der Schule und im Unterricht anerkannt werden. Die in Kapitel 3 und Kapitel 4 dargelegten theoretischen Modelle und empirischen Befunde zu Bilingualität respektive Bildungsgerechtigkeit und Anerkennung stellen eine robuste, evidenzbasierte Basis an Argumenten zur Anerkennung der migrationsbedingten Mehrsprachigkeit von SuS mit MH in der Bildungsinstitution Schule in Deutschland zur Verfügung. Dementsprechend stellt sich vielmehr die Frage, wie die Anerkennung von migrationsbedingter Mehrsprachigkeit von SuS mit MH konkret in der Regelschule umgesetzt werden könnte. Hierbei gilt es auch die strukturellen, personellen und materiellen Ressourcen zu berücksichtigen, damit die Anerkennung migrationsbedingter Bilingualität in der Regelschule realisiert werden kann. Dies 
schließt umfangreiche, kostenintensive Reformen wie z. B. die flächendeckende Etablierung bilingualer Bildungsprogramme oder die Verpflichtung zusätzlicher bilingualer Lehrkräfte zur Unterstützung der erstsprachlichen Fähigkeiten von SuS mit MH aus. Solche „top down“ administrierten Lösungsvorschläge scheiterten bisher z. B. auch im Kontext der frühkindlichen Bildung, bei dem Empfehlungen und Vorschläge zur Umsetzung der Implementation von Mehrsprachigkeit in den Kindertagesstätten selbst kaum umgesetzt wurden (vgl. Kratzmann et al., 2017). Zudem widersprechen sie auch dem Postulat, dass Anerkennungsansprüche durch die Betroffenen selbst artikuliert und erkämpft werden sollten.

Der bisherige Forschungsstand zu bilingualen Praktiken sowie die empirischen Befunde zur Einstellung von Lehrkräften hinsichtlich Mehrsprachigkeit, welche im Abschnitt 3.6 dargelegt wurden, liefern diverse Hinweise auf zu berücksichtigende Faktoren, um die Anerkennung von migrationsbedingter Bilingualität von SuS mit MH in Schule und Unterricht zu realisieren. Zunächst einmal ist zu konstatieren, dass bilingual aufwachsende SuS in ihren Bildungsprozessen mental auf ihre (weitere) L1 zurückgreifen (vgl. z. B. Clarkson, 2007; García \& Kano, 2014) sowie bilingual im Unterricht kommunizieren, obwohl ihnen letzteres explizit verboten wurde (vgl. z. B. Barwell, 2014; Culligan, 2015). An dieser Stelle soll wiederholt auf die Unausweichlichkeit mental oder vokalsprachlich exerzierter bilingualer Praktiken in Bildungsprozessen von bilingual aufwachsenden SuS mit Cooks (2001, S. 405) durchaus illustrer Metapher erinnert werden: ,[...] the L1 creeps back in, however many times you throw it out with a pitchfork". Das renitente Verhalten von SuS, mit anderen SuS, die ihre bilinguale Disposition teilen, bilingual zu kommunizieren, könnte als eine Form des Kampfes um Anerkennung im Sinne einer Ablehnung monolingualer Beschränkungen interpretiert werden. Diese regelverletzenden Kommunikationspraktiken zwischen bilingualen SuS könnten produktiv als anerkannte Praxis des Lernens umgedeutet werden, wenn im Unterricht diesen bilingualen SuS das Angebot unterbreitet wird, bei der Zusammenarbeit mit anderen bilingualen SuS desselben Sprachprofils zweisprachig kommunizieren zu dürfen. Wichtig ist, dass diese Unterrichtspraktik als optionales Angebot formuliert wird, um mit der aktiven, produktiven Seite von Anerkennung keine positiv diskriminierenden, segregierenden Zuschreibungen zu vollziehen (vgl. Mecherill, 2005). Ein solches Angebot benötigt auch keine bilingualen Lehrkräfte, da sich Gruppenarbeiten von SuS durch die Übernahme von Verantwortung und Koordination des Lernprozesses durch sie selbst auszeichnen (vgl. Topping, 2005). Auch die Studie von Dirim (1998) weist darauf hin, dass zur Legitimation weiterer Sprachen als offizielle Unterrichtssprachen nicht zwingend bilinguale Lehrkräfte vorhanden sein müssen. In den von Wlossek und Rost-Roth (2016) geführten Interviews mit Lehrkräften wurde konstatiert, dass die Implementation von Konzepten zur Einbindung der migrationsbedingten Mehrsprachigkeit sich auch zur Bearbeitung des Curriculums 
eignen sollte. Hinsichtlich Gruppenarbeiten von SuS gibt es eine breite, robuste empirische Befundlage, die die Effektivität von kollaborativen Lernformen auch für zentrale schulische Lerninhalte wie Lesen (vgl. z. B. Rohrbeck et al., 2003; Rosenshine \& Meister, 1994) oder Mathematik (vgl. Robinson et al., 2005; Rohrbeck et al., 2003) meta-analytisch nachgewiesen haben. Dementsprechend scheint ein optionales Angebot zur bilingualen Interaktion für bilingual aufwachsende SuS mit MH eine Möglichkeit darzustellen, um ihre migrationsbedingte Mehrsprachigkeit als Ressource für Bildungsprozesse in die Unterrichtspraxis zu integrieren und letztlich somit anzuerkennen. Diese Möglichkeit soll im folgenden Kapitel im Kontext der Methode des „PeerLearnings" reflektiert werden. 


\section{Peer-Learning}

Im Folgenden wird die Sozialform des PL als Methode für selbstverantwortliche Bildungsprozesse von gleichgestellten Individuen in Gruppen näher betrachtet. Hierbei gilt es zu reflektieren, ob PL eine fruchtbare und umsetzbare Möglichkeit darstellt, das bilinguale Sprachvermögen bilingual aufwachsender $\mathrm{SuS}$ zumindest temporär in den Regelunterricht zu implementieren. Diesbezüglich wird zunächst die Methode des PL selbst vorgestellt (Abschnitt 5.1), wobei die Unterscheidung von informellem und formellem PL (Abschnitt 5.1.1), die Vielfältigkeit von Subtypen des PL (Abschnitt 5.1.2) und förderliche Basiselemente für erfolgreiches PL (Abschnitt 5.1.3) näher beschrieben werden. Anschließend werden meta-analytische Befunde zur Lernwirksamkeit von PL präsentiert (Abschnitt 5.2). Danach wird die zentrale Bedeutung der Interaktion beim PL näher erörtert (Abschnitt 5.3), wobei förderliche Sprachhandlungen und Diskurskulturen (Abschnitt 5.3.1), Task-Management und metakognitive Regulation (5.3.2) sowie die Wirkmechanismen der Förderlichkeit einer bildungsförderlichen Kommunikation beim PL auf Basis verschiedener theoretischer Perspektiven des Konstruktivismus (Abschnitt 5.3.3) näher beleuchtet werden. Die zentralen Erkenntnisse des Abschnitts 5.3 werden in Abschnitt 5.3.4 zusammengefasst. Weiterhin werden kognitive Lernstrategien allgemein dargestellt sowie als Lerngegenstand beim PL betrachtet (Abschnitt 5.4) und abschließend werden empirische Befunde zu bilingualen Praktiken beim PL vorgestellt (Abschnitt 5.5). Die zentralen Erkenntnisse der beiden letztgenannten Abschnitte werden in Abschnitt 5.4.6 respektive 5.5.3 zusammengefasst.

\subsection{Grundlagen und Formen des Peer-Learnings}

PL kann zunächst auf Basis der Bedeutungen der Wortteile „Peer“ und „Learning“ als gemeinsames Lernen von „Gleichgestellten“ oder „Ebenbürtigen“ (vgl. Kessels \& Hannover, 2009) beschrieben werden. Eine elaboriertere Definition, die in der vorliegenden Studie präferiert wird, stellt die Bestimmung von PL nach Topping (2005, S. 631) als ,,acquisition of knowledge and skill through active helping and supporting among status equals or matched companions" dar. Dementsprechend ermöglicht PL sozial interaktive Lernprozesse (vgl. Thurston et al., 2007), bei denen die Rolle der Lehrkraft in den Hintergrund tritt. Die Lehrkraft übernimmt eine Moderationsfunktion, die zwar das PL-Format plant, strukturiert und modelliert, in die PL-Prozesse aber möglichst nicht eingreifen sollte. 
Die Definition von Topping (ebd.) erfasst die zentralen, grundlegenden Komponenten von PL als prinzipiell kollaborative Lernform in Dyaden oder Gruppen von Personen, die innerhalb des Kontextes des PL weitestgehend gleichgestellt sind. Solche unspezifisch anmutenden Definitionen sind für eine Annäherung an PL notwendig, da sich diese Methode in einer Vielzahl distinkter Subtypen umsetzen lässt, welche sich wiederum primär in organisationalen Variablen unterscheiden (vgl. Topping, 2005). Bevor auf einzelne Subtypen und zentrale organisatorische Variablen des PL eingegangen wird, soll zunächst auf eine in der Praxis zu beobachtende und relevante Unterscheidung eingegangen werden, und zwar auf die Differenzierung von formellem und informellem PL nach Johnson und Johnson (1999).

\subsubsection{Formelles und informelles Peer-Learning nach Johnson und Johnson (1999)}

Johnson und Johnson (ebd., S. 69) differenzieren zwischen formellen und informellen Formen des PL. Während beim formellen PL die Implementation der Methode vorher geplant wird, sodass Unterstützungs- und Überwachungsprozesse strukturell integriert werden, zeichnet sich das informelle PL durch Spontanität und fehlende Strukturierung aus. Die zuletzt genannte Form kann auch als ,pseudo learning group“ (ebd., S. 68) bezeichnet werden und trifft wohl auf den Großteil von Gruppenarbeiten in der Praxis zu (vgl. Adl-Amini, 2018, S. 26; Littleton, 2010, S. 152). Zwar arbeiten die SuS hierbei zusammen, eine echte Kollaboration erfolgt allerdings aufgrund der fehlenden Struktur und Planung nicht. Kollaboration, definiert als ,co-ordinated, continuing attempt to solve a problem or in some other way construct common knowledge" (Littleton \& Mercer, 2010, S. 273), ist auf diverse Kooperationsbedingungen und -praktiken angewiesen. Hierzu gehört primär die Etablierung von „Intersubjektivität", welche nicht im anerkennungstheoretischen Sinne zu verstehen ist, sondern beim PL den mentalen Zustand der Peers beschreibt, bei dem alle über das selbe Verständnis der Zielsetzung, der Aufgabe oder des Problems verfügen (vgl. ebd.). Weiterhin stellen neben einem gemeinschaftlich geteilten Verständnis der Ziele und Aufgaben die gegenseitige Anteilnahme sowie kontinuierliche Aushandlungsprozesse zentrale Komponenten von Kollaboration dar (vgl. ebd.). Diese Kooperationsbedingungen und -praktiken lassen sich ad-hoc ohne Struktur sowie Instruktion nur schwerlich umsetzen. Nichtsdestotrotz scheinen viele Lehrkräfte zu glauben, dass sie formelles PL bereits durch die Administration von Gruppenarbeit implementieren, obwohl ,all they are really doing is putting children together and hoping for the best" (Topping, 2005, S. 632). Informelle Formen des PL sind dementsprechend eher als ineffizient zu bezeichnen, sodass in solchen Fällen individuelles Lernen zu präferieren ist (vgl. Johnson \& Johnson, 1999, S. 68). Innerhalb des formellen PL 
gibt es eine Vielzahl verschiedener Subtypen, die durch unterschiedliche Ausprägungen zentraler organisatorischer Variablen des PL determiniert sind, welche im Folgenden näher betrachtet werden.

\subsubsection{Subtypen des Peer-Learnings am Beispiel „Peer-Tutoring“}

Formelle Varianten des PL unterschieden sich in einer Vielzahl von organisatorischen Strukturierungsmöglichkeiten. Topping (2005, S. 633-634) benennt insgesamt zwölf organisatorische Variablen, in denen sich verschiedene Formen des PL unterscheiden können. Hierzu zählen unter anderem der Lerninhalt, die Gruppengröße, Alter, Vorwissen oder Kompetenz der Gruppenmitglieder, Vorhandensein von Verstärkern und Rollen. Je nachdem, wie diese Variablen im jeweiligen PL umgesetzt werden, können sich unterschiedliche Varianten des PL ergeben, die sich wiederum innerhalb ein und desselben Formates durchaus unterscheiden können. So kann zum Beispiel PL als „kooperatives Lernen“ (vgl. ebd., S. 632) gestaltet werden, bei der eine Gruppe von sechs leistungsheterogenen SuS eine mathematische Textaufgabe mit mehreren potentiellen Lösungswegen ohne spezifische Rollenzuteilung mit dem Ziel bearbeiten, ihre Lösung als Gruppe argumentativ im Klassenverbund zu präsentieren. Da die Interaktion keine wirkliche Strukturierung durch Rollen oder Interaktionsskripte vorweist, dürfte hierfür eine gewisse Trainingsphase voranzustellen sein, um allen eine gleichberechtigte Teilhabe an der Bearbeitung der Aufgabe zu ermöglichen und um Synergieeffekte zu erzeugen (vgl. ebd., S. 632). Diese Variante von „kooperativem Lernen“ könnte auch mit einer Gruppengröße von vier leistungshomogenen SuS vollzogen werden, die Abschnitte eines Textes zusammenfassen sollen und hierbei jeder Peer für ein bestimmtes Qualitätsmerkmal von Zusammenfassungen verantwortlich ist, bevor das Gruppenergebnis im Klassenverbund vorgestellt und von diesem evaluiert wird. Hierbei könnten die Verantwortlichkeiten so verteilt sein, dass ein Peer für den ersten Vorschlag einer Zusammenfassung, ein weiterer für die Verwendung von eigenen Worten, ein anderer zur Prüfung überflüssiger Details und der übrig gebliebene für die Kürze der Zusammenfassung zuständig ist, um der Aufgabenbearbeitung innerhalb der Gruppe Struktur zu verleihen. Angesichts dessen wird deutlich, dass sich selbst Subtypen des PL in einer Vielzahl organisatorischer und interaktionsstrukturierender Aspekte unterscheiden können. Neben dem „kooperativen Lernen“ stellt das „Peer-Tutoring“ die am häufigsten angewandte, etablierteste und am intensivsten untersuchte Variante des PL dar (vgl. ebd., S. 639). Dieses Format des PL entspricht am ehesten der PLIntervention, welche die Datengrundlage der vorliegenden Studie bildet. Dementsprechend sollen im Folgenden die organisatorischen und strukturellen Merkmale dieser Form des PL näher erläutert werden. 
Nach Adl-Amini (2018, S. 28) kann Peer-Tutoring als eine Unterrichtsmethode definiert werden, bei der ,sich zwei heterogene Lernpartner nach einem strukturierten Vorgehen in wechselnden Rollen als Tutor und Tutand in Bezug auf ein gemeinsames Lernziel" gegenseitig unterrichten. Peer-Tutoring findet aufgrund dieser Rollenverteilung in Tutor und Tutand in der Regel in Dyaden statt, wobei die jeweiligen Rollen entweder fest den jeweiligen Peers anhand ihres Vorwissens und/oder Kompetenzen zugeordnet oder reziprok abwechselnd von den beiden Peers bedient werden. Das gegenseitige Unterrichten kann mit Hilfe von Skripten strukturiert werden, die die jeweiligen Verantwortlichkeiten sowie Handlungsmöglichkeiten der Rollen Tutor und Tutand definieren, um einen Rahmen für einen kollaborativen Dialog zu etablieren. Hierbei werden häufig die fünf folgenden Interaktionsschritte verwendet (vgl. ebd., S. 29): 1) Der Tutor fordert den Tutanden auf, eine bestimmte Aufgabe zu bearbeiten oder stellt ihm eine Frage. 2) Der Tutand bearbeitet die Aufgabe, löst diese oder liefert eine Antwort auf die vom Tutor gestellte Frage. 3) Der Tutor kommentiert die Lösung oder die Antwort des Tutanden indem er Feedback gibt. 4) Anschließend wird zusammen oder mit Hilfe der Lehrkraft die Qualität der Lösung oder Antwort näher geprüft und unter Umständen gemeinschaftlich verbessert. 5) Abschließend wird auf Basis des zuvor vollzogenen Schrittes das Verständnis des Lerninhalts evaluiert, was wiederum als Grundlage für den nächsten Turnus der eben geschilderten Abfolge verwendet werden kann. Je nach Lerninhalt und Lernziel können diese Schritte modifiziert werden, um diesen möglichst gerecht zu werden. So ist z. B. auch denkbar, dass der Tutand im zweiten Schritt zunächst keine eigenen Bearbeitungsprozesse vornimmt, sondern Lösungswege oder Antworten von einer (fiktiven) dritten Person kommentiert und evaluiert. Der dritte Schritt wäre dann so anzupassen, dass der Tutor die Einschätzung des Tutanden der Güte des Lösungsweges der (fiktiven) dritten Person kommentiert, bevor sich eine gemeinsame Verbesserung des Lösungsweges anschließt. Wie für das „kooperative Lernen“ gilt auch für das „Peer-Tutoring“ als Variante von PL, dass ,eine Vielzahl von Praxiskonzepten zur Umsetzung von tutoriellem Lernen im Unterricht verschiedener Fächer" (ebd.) denkbar ist und existiert. Dementsprechend zeichnet sich Peer-Tutoring durch den Erwerb oder das Einüben von Wissen und Fähigkeiten innerhalb klarer Prozeduren zur Interaktionsstrukturierung durch asymmetrische Rollen wie Tutor und Tutand aus, die kontinuierlich festgelegt sind oder abwechselnd reziprok von zwei leistungsheterogenen Peers in einer Dyade belegt werden können (vgl. Adl-Amini, 2018, S. 29; Topping, 2005, S. 632).

Das Potential, PL anhand diverser organisatorischer Variablen so zu modifizieren, sodass dieses den Eigenschaften der SuS sowie den vorhandenen Ressourcen entspricht, weist für die Praxis eine vielversprechende Möglichkeit für binnendifferenzierenden, adaptiven Unterricht auf. Für die PL-Forschung hingegen stellt die Vielfalt diverser Formen des PL eine Herausforderung dar, da 
hierdurch allgemeine Aussagen zur Lernwirksamkeit und den Wirkmechanismen von PL erschwert werden. Dementsprechend gilt es aus wissenschaftlicher Perspektive diejenigen Elemente von PL zu identifizieren, die die Effektivität dieser Methode primär begründen.

\subsubsection{Förderliche Basiselemente des Peer-Learnings nach Johnson \& Johnson (1999)}

Angesichts der Vielzahl potentieller Formen und Varianten des PL stellt sich die Frage, wie PL zu organisieren ist und welche Fähigkeiten die Peers benötigen, um eine möglichst effektive Kollaboration beim PL zu ermöglichen. Hierfür wird üblicherweise auf die von Johnson und Johnson (1999) postulierten fünf Basiselemente von PL in der PL-Forschung referiert (vgl. Adl-Amini, 2018, S. 21). Johnson und Johnson (1999, S. 70-71) identifizieren und beschreiben fünf basale Elemente von PL, die zum einen als definitorische Merkmale zur Abgrenzung von formellen und informellen Formen des PL verwendet werden können (vgl. Adl-Amini, 2018, S. 21) und zum anderen als förderliche Komponenten für effektives, lernwirksames PL gelten: 1) positive Interdependenz, 2) individuelle Verantwortung, 3) förderliche Interaktion, 4) soziale Kompetenz und 5) Gruppenevaluation.

Positive Interdependenz in einer Gruppe besteht, wenn der Eindruck für jedes Gruppenmitglied entsteht, dass man nur erfolgreich sein kann, wenn die Gruppe erfolgreich ist. Eine positiv strukturierte gegenseitige Abhängigkeit der Gruppenmitglieder kann z. B. durch zusätzliche Belohnung für gute Gruppenleistungen, verschiedene Ressourcenzuteilungen (z. B. exklusive Distribution von Informationen und Materialen an einzelne Gruppenmitglieder), komplementäre Rollen etc. gefördert werden.

Individuelle Verantwortung kann beim PL etabliert werden, indem jedes Gruppenmitglied einen Teil zum Ergebnis oder dem Produkt der Gruppe beiträgt, der nicht von einem anderen Gruppenmitglied kompensiert werden kann. Zur Sicherstellung dessen kann z. B. die individuelle Testung eines jeden einzelnen Gruppenmitglieds, die zufällige Wahl des Produkts eines Gruppenmitglieds zur Repräsentation der Gruppenleistung sowie das Sichtbarmachen oder gar Bewerten der Einzelleistungen der Gruppenmitglieder eingesetzt werden. Sowohl die positive Interdependenz als auch die individuelle Verantwortung fokussieren organisationale Variablen der Strukturierung der Bearbeitung des Inhalts des PL, die Motivationsverlusten durch leistungsmindernde sozialpsychologische Effekte in Gruppen wie dem ,Trittbrettfahren“ oder dem ,sozialen Faulenzen" (vgl. Kessler \& Fritsche, 2018, S. 126) entgegen wirken sollen. Diese beiden Merkmale können auch Koordinationsverluste verringern, wenn z. B. einzelne, parallel zu bearbeitende Aufgabenschritte bestimmten Gruppenmitgliedern zugeordnet oder diesen differente Rollen zugewiesen werden. 
Hierbei gilt allerdings noch zu erwähnen, dass die Gruppenleistung bei PLFormaten mit positiver Interdependenz und individueller Verantwortung auch abhängig von der Aufgabe ist (vgl. ebd., S. 126f). So zeichnen sich z. B. disjunktive Aufgaben wie Mathematikaufgaben dadurch aus, dass die Leistung des besten Gruppenmitglieds die Gruppenleistung bestimmt. Bei konjunktiven Aufgaben wie der Bewältigung eines Hindernisparcours als Gruppe im Sportunterricht, bei dem das Gruppenergebnis durch das Absolvieren des Parcours aller Gruppenmitglieder gemessen wird, bestimmt die Leistung des schlechtesten Gruppenmitglieds das Gruppenergebnis. Dementsprechend müssen sich weder positive Interdependenz und individuelle Verantwortung für alle Aufgabenformate beim PL noch umgekehrt sich alle Aufgabenformate für interdependentes PL samt individueller Verantwortung zwingend empfehlen.

Während die ersten beiden Merkmale organisationale Variablen fokussieren, die primär auf die Etablierung einer förderlichen Gruppenstruktur zielen, in der sich die Gruppenmitglieder als Teil der Gruppe verstehen, zu der jeder einen Beitrag zu leisten hat, unterstreichen die Punkte förderliche Interaktion und soziale Kompetenz die Relevanz sprachlicher, kommunikativer und interpersoneller Fähigkeiten für PL. PL erfolgt zu einem Großteil in Form von Interaktion über das Medium Sprache. Mithilfe von Sprache verständigen sich die Gruppenmitglieder über die zu bearbeitende Aufgabe, indem sie ihre Meinungen, Ideen und ihr Wissen kommunizieren, gegenseitig aufeinander referieren und im besten Falle Lösungen ko-konstruieren. Solche Interaktionen verlangen diverse diskursförderliche sprachliche Handlungen wie unter anderem Externalisierungen mentaler Modelle und Propositionen (vgl. z. B. Howe et al., 2007), Argumentationen (vgl. z. B. Asterhan \& Schwarz, 2009; Chinn \& Clark, 2013) und Erklärungen (vgl. z. B. Webb et al., 2009). Zudem gilt es mit den durch diese sprachlichen Handlungen transportierten Propositionen in kooperativer, produktiver Weise als Gruppe umzugehen, was Fähigkeiten wie Entscheidungsfindung, Konfliktmanagement, Vertrauensbildung oder Führung beansprucht (vgl. Johnson \& Johnson, 1999, S. 71). Solche Formen der Interaktion sind keine Selbstverständlichkeit, sondern treten eher selten auf und müssen dementsprechend durch Instruktion und Übung beim PL etabliert werden (vgl. z. B. Asterhan \& Schwarz, 2009, S. 375; Littleton, 2010, S. 152; Littleton \& Mercer, 2010, S. 281). Die Voraussetzungen und Variablen zur Etablierung eines kooperativen, produktiven Dialogs und dessen Bedeutung für die Effektivität von PL wird durch eine Vielzahl empirischer Studien belegt (für einen Überblick siehe z. B. Howe, 2010; Littleton \& Mercer, 2010) und stellt neben der Bedeutung von Metakognition und Regulation für erfolgreiches PL einen aktuellen Fokus der PL-Forschung dar (vgl. Littleton, 2010, S. 152). Diese Phänomene werden aufgrund ihrer Relevanz für die Effektivität von PL, aber auch wegen der Passung zum vorliegenden Erkenntnisinteresse in Abschnitt 5.3 näher ausgeführt. 
Die Evaluation der Zusammenarbeit sowie der Ergebnisse der Gruppe stellen ein weiteres förderliches Element von effektivem PL dar. Hierbei ist die Gruppe aufgefordert, zwischen produktivem und unproduktivem Gruppenverhalten zu differenzieren und die Ergebnisse der Evaluation in zukünftigen Gruppenprozessen zu berücksichtigen. Metakognitive Fähigkeiten, definiert als Wissen und Regulation der eigenen kognitiven Aktivitäten und Lernprozesse (vgl. Veenman, van Hout-Wolters \& Afflerbach, 2006, S. 3), werden bei der Reflexion der Gruppenprozesse zur Bewertung sowie Einschätzung der Qualität der Zusammenarbeit und der Zielerreichung benötigt. Die Umsetzung der Ergebnisse dieser Reflexionsprozesse in den folgenden PL Arbeitsphasen beansprucht wiederum regulative Prozesse des Selbst, der anderen sowie der ganzen Gruppe (vgl. z. B. de Backer et al., 2016; Iiskala, Vauras \& Lehtinen, 2004; Whitebread et al., 2009) wie z. B. in Form von Monitoring und Kontrolle. Hierbei werden metakognitive und regulative Prozesse nicht nur zur Koordination und Effizienzsteigerung des PL eingesetzt, sondern auch zur Adressierung und Regulierung emotionaler und motivationaler Zustände der Gruppenmitglieder (vgl. Whitebread et al., 2009). Metakognitive Fähigkeiten und regulative Prozesse treten in der aktuellen PL-Forschung vermehrt in den Vordergrund und werden nicht nur in evaluativen Prozessen betrachtet, sondern primär bei der Interaktion der Peers im PL selbst (vgl. z. B. de Backer et al., 2016; Iiskala, Vauras \& Lehtinen, 2004; Whitebread et al., 2009).

Diese fünf Elemente förderlichen PL gilt es vor der praktischen Einführung dieser Methode angemessen hinsichtlich der Eigenschaften der Peers, des Lerninhalts, des Ziels des PL sowie der vorhandenen Ressourcen und organisatorischen Bedingungen zu reflektieren und möglichst effektiv an die vorliegenden Gegebenheiten anzupassen. Dies scheint aber gerade in der Praxis nicht zu geschehen. Es herrscht zwischen der PL-Forschung und der Umsetzung der empirischen Befunde in die Praxis durch Lehrkräfte eine Asymmetrie, da Lehrkräfte zwar angeben, kooperative Lernformen einzusetzen, allerdings werden diese von den Lehrkräften nicht nach den in der Forschung identifizierten förderlichen Basisdimensionen strukturiert und implementiert (vgl. Adl-Amini, 2018, S. 26; Littleton, 2010, S. 152). Dementsprechend sind die in der Forschung identifizierten positiven Effekte von PL nicht zwingend auch in der Praxis ohne wissenschaftliche Begleitung zu erwarten. Im Folgenden soll zunächst die Lernwirksamkeit und Effektivität von PL hinsichtlich diverser Phänomene anhand von Meta-Analysen betrachtet werden, bevor näher auf die Bedeutung der Interaktion sowie metakognitiver und regulativer Prozesse beim PL eingegangen wird. 


\subsection{Befunde von Meta-Analysen zur Effektivität von Peer- Learning}

In ihren Anfängen fokussierte die PL-Forschung zunächst die Fragestellung, ob kollaboratives Lernen effektiver als individuelles Lernen ist (vgl. Littleton, 2010, S. 152). Diesbezüglich lässt sich eine breite, robuste empirische Basis identifizieren, die die Überlegenheit von kooperativen Lernformen gegenüber individuellem Lernen demonstriert (vgl. Hattie, 2014, S. 250-253). Anschließend wurden vermehrt verschiedene schulische Kompetenzen sowie soziale und motivationale Outcomes in den Blick genommen, indem Variablen wie Gruppengröße, Zusammensetzung der Gruppen nach Geschlecht und Vorwissen oder Kompetenz und verschiedene Aufgabentypen hinsichtlich ihres Einflusses auf die Effektivität von PL untersucht wurden (vgl. ebd.). Hierzu wurden auch diverse meta-analytische Studien durchgeführt (vgl. z. B. GinsburgBlock, Rohrbeck \& Fantuzzo, 2006; Robinson, Schofield \& Steers-Wentzell, 2005; Rohrbeck et al., 2003; Rosenshine \& Meister, 1994), deren zentrale Ergebnisse im Folgenden kurz ausgeführt werden sollen. Auf einzelne Studien wird hierbei nur referiert, wenn bestimmte Variablen der Lernwirksamkeit in den Meta-Analysen nicht adressiert werden. Zudem gilt es hierbei zu berücksichtigen, dass in manchen Meta-Analysen eine Vielzahl verschiedener Formate des PL einbezogen (vgl. z. B. Rohrbeck et al., 2003), während in anderen bestimmte Varianten des PL wie z. B. das Peer-Tutoring fokussiert werden (vgl. Robinson, Schofield \& Steers-Wentzell, 2005). Für einen umfangreichen Überblick zur Empirie beim PL im Allgemeinen sowie insbesondere für das PL-Format des Peer-Tutorings kann auf die Ausführungen von Adl-Amini (2018) verwiesen werden.

Diverse Meta-Analysen weisen positive Effekte von Formen des PL für zentrale schulische Kompetenzen wie Lesen (vgl. Adesope et al., 2011; Rohrbeck et al., 2003; Rosenshine \& Meister, 1994) und Rechnen (vgl. Robinson, Schofield \& Steers-Wentzell, 2005; Rohrbeck et al., 2003) nach. Auch in Hatties (2014) „Meta-Meta-Analysen“ erzielen populäre Formen des PL wie PeerTutoring und Reziprokes Lehren mittlere bis hohe Effekte auf Lernergebnisse. Die Effektgrößen unterscheiden sich allerdings nach den eingesetzten Testverfahren. Mit standardisierten Verfahren werden deutlich geringere Effektgrößen identifiziert als mit trainingsnahen, von den jeweiligen Forschern erstellten Testinstrumenten (vgl. Rosenshine \& Meister, 1994, S. 498). In rollenspezifizierten PL-Formaten wie dem Peer-Tutoring profitieren sowohl der Tutand als auch der Tutor vom PL (vgl. Hattie, 2014, S. 221; Robinson, Schofield \& Steers-Wentzell, 2005, S. 334-335). Bezüglich des Lerninhalts Lesen fällt auf, dass insbesondere die Leseflüssigkeit und/oder Lesestrategien fokussiert werden (vgl. z. B. Brown \& Palincsar, 1982; Fuchs et al. 1997; Munser-Kiefer \& 
Kirschhock, 2012; Nix, 2011; Palincsar \& Brown, 1984; Philipp \& Schilcher, 2012; Spörer, Brunstein \& Kieschke, 2009).

Mithilfe von PL können allerdings nicht nur positive kognitive Effekte hervorgerufen werden, sondern PL kann sich ebenfalls günstig auf soziale (kooperative Fähigkeiten, Konfliktlösung, Einstellungen zu Peers, Steigerung des Zugehörigkeitsgefühls etc.) und motivationale Eigenschaften (akademisches Selbstkonzept, Selbstkonzept bezüglich diverser zentraler schulischer Fähigkeiten, Selbstbewusstsein, Selbstwert, Autonomie- und Kompetenzerleben etc.) sowie auf das Verhalten von SuS im Unterricht (Arbeitsverhalten, Teilnahme, Anstrengungsbereitschaft, Frustrationstoleranz etc.) auswirken (vgl. Ginsburg-Block, Rohrbeck \& Fantuzzo, 2006; Robinson, Schofield \& SteersWentzell, 2005; Rohrbeck et al., 2003). Hierbei scheinen insbesondere PLFormate effektiv zu sein, die den Peers mehr Autonomie und Kontrolle über ihre Gruppenprozesse bezüglich der Zielsetzung, des Monitorings der Aufgabenbearbeitung, der Evaluation der Performanz oder der Auswahl von Verstärkern einräumen, anstatt diese Aufgaben primär den Lehrkräften zu überlassen (vgl. Rohrbeck et al., 2003, S. 251).

Diese positiven kognitiven, sozialen, motivationalen und behavioralen Effekte des PL lassen sich bei allen Alters- und Klassenstufen beobachten (vgl. Ginsburg-Block, Rohrbeck \& Fantuzzo, 2006; Rohrbeck et al., 2003). Hinsichtlich SuS der Grundschule scheinen allerdings jüngere (Jahrgangsstufe 13) als ältere SuS der Grundschule (Jahrgangsstufe 4-6) sowie SuS mit niedrigem sozioökonomischen Status oder der Zugehörigkeit zu einer Minderheit besonders stark vom PL zu profitieren (Rohrbeck et al., 2003, S. 250). Die Effektivität von PL ist zudem nicht abhängig von der Dauer der Intervention (Robinson, Schofield \& Steers-Wentzell, 2005, S. 341; Rohrbeck et al., 2003, S. 251; Rosenshine \& Meister, 1994, S. 500), dem vorhandenen Vorwissen oder der Kompetenz der SuS in dem jeweiligen Lerninhalt (Robinson, Schofield \& Steers-Wentzell, 2005, S. 345). Zusätzliche signifikante positive Effekte lassen sich durch den Einsatz von Verstärkern erzielen, insbesondere wenn die jeweiligen Gruppen autonom über ihre Belohnungen entscheiden dürfen, wie z. B. durch die Wahl zwischen verschiedenen Verstärkern und deren Menge (vgl. Robinson, Schofield \& Steers-Wentzell, 2005, S. 344; Rohrbeck et al., 2003, S. 252).

Robinson, Schofield \& Steers-Wentzell (2005, S. 347-349) sind der Ansicht, dass eine Vielzahl der positiven sozialen, motivationalen und behavioralen Effekte vor allem beim Reziproken Lehren, aber auch beim PL im Allgemeinen, mit den Erfahrungen der SuS zusammenhängen, die sie in den Rollen als Tutor oder Tutand machen. Sie (ebd.) berufen sich diesbezüglich auf Rollentheorien, die postulieren, dass bestimmte Einstellungen und Handlungen mit sozialen Rollen verbunden sind. Bei der Ausübung von sozialen Rollen übernimmt man die mit der Rolle assoziierten Einstellungen, Überzeugungen sowie Handlungen und versucht sie in einer konsistenten Weise auszuüben. 
Beim Reziproken Lehren übernehmen die SuS (abwechselnd) die Rollen als Tutor und Tutand, die den Rollen von Lehrer und Schüler ähneln. Robinson, Schofield \& Steers-Wentzell (ebd.) sind der Ansicht, dass insbesondere die Rolle als Tutor SuS erkennen lässt, welche Verhaltensweisen von SuS förderlich für das Lernen sind, sodass diese im PL von den SuS gefordert und eingesetzt werden. Diese Prozesse sind nicht nur in PL-Formaten mit definierten Rollen zu erwarten, sondern auch in kooperativen Lernformen ohne spezifische Rollenverteilung. In solchen kollaborativen Lernformen regulieren sich die Peers gegenseitig (vgl. z. B. de Backer et al., 2016; Iiskala, Vauras \& Lehtinen, 2004; Whitebread et al., 2009) und es treten Praktiken des Argumentierens, Erklärens sowie der Ko-Konstruktion auf(vgl. z. B. Asterhan \& Schwarz, 2009; Chinn \& Clarke, 2013; Littleton \& Mercer, 2010; Howe, 2010; Webb, 2009). Die letztgenannten Aushandlungsprozesse können zum Teil in asymmetrischer Weise erfolgen, sodass z. B. Gruppenmitglieder mit höherem Vorwissen Gruppenmitglieder mit niedrigerem Vorwissen innerhalb der Kollaboration vermehrt anleiten und unterstützen. Die Ausübung solcher Praktiken könnte dementsprechend auch ohne spezifische Rollenverteilung zu Einsichten führen, was eine gute Schülerin und Schüler sowie Lehrerin und Lehrer ausmacht. Unabhängig davon, ob diese Erkenntnisse in rollenstrukturierten oder freien PL-Formaten erfolgen, können diese wiederum in das Unterrichtsgeschehen im Klassenverbund transferiert werden, sodass die SuS vermehrt förderliche Verhaltensweisen in ihrer Rolle als SuS ausüben.

Ein weiterer Forschungsstrang bezüglich der Effektivität von PL untersucht die Gruppenzusammensetzung. Bisherige Untersuchungen fokussierten primär die Gruppenzusammensetzung bezüglich des Leistungsstands (vgl. Fuchs et al., 1997; McMaster, Fuchs \& Fuchs, 2006) und des Geschlechts (vgl. Robinson, Schofield \& Steers-Wentzell, 2005; Rohrbeck et al., 2003). AdlAmini (2018, S. 43, 49) fasst für das PL-Format des „Peer-Tutoring“ die Evidenz dahingehend zusammen, dass leistungsheterogene Gruppen eine höhere Lernwirksamkeit beim Peer-Tutoring aufweisen als leistungshomogene Gruppen (vgl. z. B. Fuchs et al., 1997; McMaster, Fuchs \& Fuchs, 2006). Angesichts der Bedeutung eines produktiven Dialogs für die Effektivität von PL durch diskursförderliche sprachliche Handlungen wie unter anderem Externalisierungen mentaler Modelle und Behauptungen (vgl. z. B. Howe, 2010), Argumentationen (vgl. z. B. Asterhan \& Schwarz, 2009; Chinn \& Clark, 2013) und Erklärungen (vgl. z. B. Webb et al., 2009) erscheinen heterogene Gruppenzusammensetzungen auch hinsichtlich anderer PL-Formate als empfehlenswert, da Leistungsheterogenität eher zur Erzeugung kognitiver Konflikte führen sollte, die zur Lösung die oben angeführten sprachlichen Handlungen benötigen.

Hinsichtlich der Gruppenzusammensetzung nach dem Geschlecht führen gleichgeschlechtliche Gruppenzusammensetzungen zu einer höheren Lernwirksamkeit als gemischtgeschlechtliche Gruppen (vgl. Robinson, Schofield 
\& Steers-Wentzell, 2005, S. 346f; Rohrbeck et al., 2003, S. 251). Allerdings gibt es Hinweise darauf, dass dies hinsichtlich bestimmter Rollenstrukturen in Abhängigkeit des Geschlechts nicht in jedem Fall zwingend zutreffen muss. So demonstrieren z. B. Topping \& Whiteley (1993), dass weibliche Tutoren beim Peer-Tutoring einen größeren Lernzuwachs mit männlichen als mit weiblichen Tutanden erfahren.

Sprachliche Variablen wie Sprachhintergrund, Sprachkompetenz, Diskursfähigkeit etc. als individuelle Eingangsvoraussetzungen zur Gruppenzusammensetzung wurden bisher weitestgehend in der PL-Forschung ignoriert (aber siehe Rauch et al., in Vorb.). Dabei sind sprachliche Fähigkeiten für erfolgreiches PL aus theoretischer Perspektive des Konstruktivismus (siehe z. B. De Lisi \& Golbeck, 1999; Hogan \& Tudge, 1999), den förderlichen Basisdimensionen von PL nach Johnson und Johnson (1999) sowie der bisherigen empirischen Befundlage zu förderlichen Sprachhandlungen beim PL (siehe z. B. Asterhan \& Schwarz, 2009; Chinn \& Clark, 2013; Howe, 2010; Littleton \& Mercer, 2010; Webb et al., 2009) höchst relevant. Dementsprechend sollen im folgenden Abschnitt 5.3 die Interaktion beim Peer-Learning sowie die potentiellen zu Grunde liegenden Wirkmechanismen von förderlichen Dialogformen im PL aus empirischer und theoretischer Perspektive näher betrachtet werden.

\subsection{Interaktion beim Peer-Learning}

Wie in Abschnitt 5.1.3 bereits erwähnt, liegt ein aktueller Schwerpunkt der PLForschung auf den Voraussetzungen, Bedingungen und Prozessen, die Formen eines kooperativen Dialogs beim PL ermöglichen. Zu einem förderlichen Dia$\log$ beim PL gehören aber auch Task-Management sowie die eigene, gegenseitige und gemeinschaftliche metakognitive Regulation der Peers. Diese Phänomene des PL werden im Folgenden näher betrachtet.

\subsubsection{Förderliche Sprachhandlungen und Diskurskulturen beim Peer-Learning}

Angesichts der Bedeutung von Interaktion für Bildungs- und Lernprozesse beim PL stellt sich die Frage, welche Form(en) von Diskurs für PL lernwirksam sind. Diesbezüglich können prinzipiell zwei distinkte empirische Zugänge unterschieden werden (vgl. Littleton \& Mercer, 2010, S. 274): 1) Die Identifikation und Operationalisierung förderlicher Sprachhandlungen oder 2) förderlicher Dialogtypen für PL. Der erste Ansatz versucht aus einer interaktionsana- 
lytischen Perspektive einzelne Sprachhandlungen wie z. B. Propositionen/Assertionen, Fragen, Argumentationen oder Erklärungen zu bestimmen, deren Prävalenz und/oder Qualität mit den individuellen Lernzuwächsen zusammenhängen (vgl. z. B. Chi et al., 2001; Howe, 2010; Schwarz \& Linchevski, 2007; Webb et al., 2009). Der zweite Ansatz nimmt eher eine holistische Perspektive ein, aus welcher entweder der Dialog hinsichtlich seiner charakteristischen Eigenschaften in mehr oder weniger lernförderliche Dialogtypen kategorisiert (für einen Überblick siehe Littleton \& Mercer, 2010) oder die Quantität und/oder Qualität spezifischer Interaktionen wie z. B. kontrastierende Meinungen, Ko-Konstruktionen oder Elaborationen etc. (Asterhan \& Schwarz, 2009; Howe, 2010) fokussiert wird, welche sich auf Lernprozesse beim PL auswirken sollten. Auch hier werden präferiert korrelative Methoden zur Überprüfung des Zusammenhangs dieser Dialogtypen mit Lernzuwächsen verwendet.

Letztlich unterscheiden sich diese beiden übergeordneten Perspektiven primär in ihren methodischen Zugängen, Operationalisierungen und Auswertungen als in den untersuchten Konstrukten. Die untersuchten Dialogtypen und Interaktionen des zweiten Ansatzes werden über die Quantität und/oder Qualität von Sprachhandlungen respektive über Zusammenhänge und Abfolgen von verschiedenen Sprachhandlungen durch Ratings und Kategorisierungen operationalisiert. Dementsprechend können diese eher holistischen Zugänge als Typologien, Aggregationen, Cluster und Abfolgen von den im ersten $\mathrm{Zu}-$ gang untersuchten Sprachhandlungen interpretiert werden. Im Folgenden sollen zentrale empirische Ergebnisse und theoretische Konzeptionen der beiden Zugänge ausgeführt werden.

\subsubsection{Förderliche Sprachhandlungen beim Peer-Learning}

Beim PL können sich bereits bloße Externalisierungen von Proposition positiv auf das Lernen auswirken. Unter Externalisierung von Propositionen können unter anderem Aussagen, Ideen, Vermutungen und Beobachtungen verstanden werden, die SuS beim PL äußern und ihre aktuellen mentalen Modelle zu einem Thema repräsentieren. So identifizieren Howe et al. (2007) bei SuS der Grundschule signifikante positive Korrelationen von .26 und .27 zwischen der Prävalenz der Äußerung von Propositionen und Lernzuwächsen bei physikalischen (Kraft und Bewegung) respektive chemischen Themen (Kondensierung und Vaporisierung). Diese Zusammenhänge konnten nur beim PL beobachtet werden, während für den Unterricht im Klassenverbund diese signifikanten Zusammenhänge nicht auftraten. Die Autoren vermuten (vgl. ebd., S. 560), dass die Externalisierung von Propositionen selbst nicht zwingend lernwirksam ist, sondern der Umgang mit diesen in der Interaktion. Während Äußerungen von Propositionen im Klassenverbund eher Feedback oder Korrekturen der Lehrkraft evozieren, dürften diese beim PL eher einen gegenseitigen Austausch über die Lerninhalte initiieren und eine Koordination der verschiedenen 
Propositionen der Gruppenmitglieder hervorrufen, was wiederrum zu weiteren förderlichen Interaktionen wie Elaborationen, Ko-Konstruktionen oder argumentativ geführten Aushandlungsprozessen führen könnte. Diese Interpretation lässt sich für eine Vielzahl von Lerninhalten durch den robusten Befund unterstützen, dass insbesondere die Externalisierung von kontrastierenden Meinungen beim PL sich positiv auf die Lernwirksamkeit auswirken (für einen Überblick siehe Howe, 2010, S. 32ff). In manchen PL-Untersuchungen stellt diese Interaktionsform den stärksten Prädiktor für die Ergebnisse im Post-Test und Follow-up-Test dar (vgl. ebd.). Hierbei müssen die Widersprüche nicht zwingend innerhalb der Gruppe gelöst werden, da sich positive Effekte zum Teil erst in den Follow-up-Tests ergeben (vgl. ebd., S. 36) und die Lösung von Widersprüchen auch eher selten bei Gruppen mit SuS im mittleren Kindesalter in PL-Prozessen auftritt. Wenn Widersprüche doch innerhalb der Gruppe gelöst werden, dann allerdings nur selten durch argumentative Aushandlungsprozesse, sondern eher durch unbegründete Einigung (vgl. ebd., S. 35). Howe (2010) formuliert auf Basis dieser Beobachtung die Hypothese, dass ungeklärte Widersprüche während des Diskurses beim PL individuelle „Post-Gruppen-Prozesse" hervorrufen, bei denen das Individuum nach dem PL die bestehenden Widersprüche für sich löst und Effekte somit erst in Follow-up-Untersuchungen evident werden. Dieses Phänomen ist eventuell auch durch Einschränkungen des aktuellen Entwicklungsstandes bedingt (vgl. ebd., S. 41).

Die Resolution von Widersprüchen und kontrastierenden Meinungen beim PL stellt hohe Ansprüche an die kollaborative Klärung dieser. So gilt es zunächst den Widerspruch zu erkennen sowie zu evaluieren, bevor die kontrastierenden Meinungen reflektiert sowie elaboriert werden können und die Gruppe sich schließlich auf eine (neue) Lösung einigt (vgl. ebd., S. 35f). Der konstruktive, produktive Umgang mit kontrastierenden Meinungen beim PL benötigt diskurstheoretisch eine argumentative Auseinandersetzung über die sich widersprechenden Positionen, bei denen die Gruppe primär durch Argumente und Erklärungen die beste Lösung auf Basis der geäußerten Propositionen (ko-)konstruiert.

Argumentation gilt es von Erklärungen zu differenzieren, da beide Sprachhandlungen ,quite different epistemic actions“ darstellen, die unterschiedliche (sozio-)kognitive Prozesse widerspiegeln und sich wohl für „,different task designs, different domains, and different types of concepts" unterschiedlich gut eignen (Asterhan \& Schwarz, 2009, S. 376). Argumentationen sind Sprachhandlungen, mit welchen Gesprächspartner die Akzeptanz einer oder mehrerer Ideen durch Begründungen, Schlussfolgerungen oder Erörterungen steigern oder verringern (vgl. ebd., S. 375f). Erklärungen sind von Argumentationen nicht einfach zu differenzieren, da sie über ähnliche syntaktische und formale Strukturen verfügen (vgl. ebd., S. 376f): Beide Sprachhandlungen bestehen aus mindestens zwei aufeinander bezogene Propositionen, können über die glei- 
chen Operatoren eingeleitet werden (z. B. „,weil“, „da“, „wegen“, „,dementsprechend", ,aufgrund von“, „,infolge dessen“ etc.) und sind transaktionaler Natur, bei dem ein Sprecher einen Rezipienten durch nachvollziehbare und angemessene Begründungen, Schlussfolgerungen oder Erläuterungen zu überzeugen versucht. Allerdings unterscheiden sich Argumentationen und Erklärungen in ihrem Zweck: „An explanation has a clarifying function within a dialog, in the sense that the recipient should come to understand something better as a result of the explanation. In argumentation [...] the proponent proposes reasons for the recipient to come to accept or refute a certain thesis" (ebd., S. 377). Dementsprechend sollen Argumentationen des Sprechers zur Überzeugung des Gegenübers führen, während Erklärungen das Verständnis des Rezipienten erweitern, sodass die Unterscheidung zwischen diesen Sprachhandlungen nach Asterhan und Schwarz (ebd.) auf Basis des Kontexts und des Ziels der Sprachhandlung getroffen werden sollte. Allerdings stellt auch diese Differenzierung keine trennscharfe Operationalisierung dar, da bei Dialogen, in welchen die jeweiligen Intentionen der Gesprächspartner nicht transparent gemacht werden, Argumente zur Überzeugung des Rezipienten wie Äußerungen zur Klärung von Propositionen oder zur Erweiterung des Verständnisses vermittelt werden können und umgekehrt. Dementsprechend stellt die parallele Operationalisierung und Erhebung von Argumentationen und Erklärungen beim PL in einer Studie eine methodische Herausforderung dar, die eher vermieden wird, sodass der Großteil der Studien eines dieser Phänomene fokussiert (vgl. z. B. Chinn \& Clark, 2013; Webb et al., 2009) oder diese undifferenziert zusammenfasst (vgl. z. B. Howe et al., 2007).

Unabhängig davon, welche der jeweiligen Sprachhandlungen beim PL untersucht wurden, führen sowohl Argumentationen (für einen Überblick siehe Chinn \& Clark, 2013) als auch Erklärungen (für einen Überblick siehe Webb et al., 2009) beim PL zu positiven Effekten. Positive Effekte ergeben sich diesbezüglich nicht nur für die Rezipienten von Argumentationen und Erklärungen, sondern auch für die Sprecher, da bei diesen Sprachhandlungen das eigene mentale Modell durch die Externalisierung, Begründung und Vermittlung an andere auf Unklarheiten, Inkonsistenzen, Widersprüche und Lücken geprüft werden kann. Ähnlich zu dem Befund, dass nicht davon ausgegangen werden kann, dass SuS beim PL spontan förderlich miteinander einen kooperativen Dialog führen (vgl. Littleton, 2010, S. 152), kann ebenso nicht zwingend erwartet werden, dass SuS beim PL konstruktiv argumentieren oder erklären. SuS können ,argumentieren“ durchaus mit „streiten“ (vgl. Chinn \& Clark, 2013, S. 316) oder „erklären“ mit „Lösung vorgeben“ verwechseln. Dementsprechend empfiehlt es sich bei PL-Formaten auch Formen eines konstruktiven, produktiven kooperativen Dialogs zu modellieren und Sprachhandlungen wie Meinungen externalisieren, Argumentieren und Erklären explizit zu besprechen (vgl. Chinn \& Clark, 2013; Littleton \& Mercer, 2010; Webb et al., 2009) sowie explizit strukturell im PL zu verankern. Hierfür gibt es diverse 
Vorschläge (vgl. z. B. Chinn \& Clark., 2013, S. 324ff) sowie ganze Förderprogramme (siehe Littleton \& Mercer, 2010, S. 283), die sich sowohl positiv auf die Quantität von förderlichen Sprachhandlungen als auch auf die Lernergebnisse auf Individual- und Gruppenebene auswirken und letztlich zu förderlicheren Dialogtypen führen (vgl. ebd.). Allerdings gilt es darauf hinzuweisen, dass selbst nach der Förderung konstruktiver, produktiver Sprachhandlungen und Dialogformen sich die Gruppendiskurse durch einen gewissen Anteil ungeeigneter Sprachhandlungen und -praktiken auszeichnen (vgl. Chinn \& Clark, 2013, S. 326). In diesen Fällen sollte überprüft werden, ob das jeweilige PLFormat die organisatorischen und strukturellen Bedingungen der fünf Basiselemente für förderliches PL nach Johnson \& Johnson (1999) erfüllen, ob zu wenig Vorwissen der SuS zur Bearbeitung des jeweiligen Lerninhalts vorhanden ist oder ob sich der Lerninhalt überhaupt für PL eignet (vgl. Chinn \& Clark, 2013, S. 327ff).

\subsubsection{Förderliche Dialogtypen und Interaktionen beim Peer-Learning}

Förderliche Dialogtypen und Interaktionen beim PL zeichnen sich durch lerninhaltsbezogene, begründende sowie schlussfolgernde Sprachhandlungen., konstruktiv kollaborative Einstellungen und Praktiken der Individuen und intersubjektive, gruppenorientierte Prozesse der gemeinschaftlichen Organisation und Aufgabenbearbeitung aus. Zur Differenzierung und Erfassung förderlicher und hinderlicher Formen von PL-Dialogen erzeugen diverse Forscher Kategoriensysteme und Typologien, mit denen die übergeordnete Diskurskultur in den jeweiligen PL-Gruppen geratet werden können, anstatt produktive Sprachhandlungen und einzelne Interaktionen aus einer Mikroperspektive qualitativ zu bewerten und quantitativ auszuzählen. So unterscheiden z. B. Littleton \& Mercer (2010, S. 276ff) drei Formen von PL-Dialogen, die sich allgemein hinsichtlich der Orientierungen sowie den Praktiken innerhalb der Gruppe und der Qualität sowie Quantität förderlicher Sprachhandlungen differenzieren: 1) Unter ,disputational talk“ fassen die Littleton und Mercer (vgl. ebd.) PL-Dialoge, die sich durch individuelle Orientierungen, fehlende Intersubjektivität sowie Konsens auszeichnen und deren Interaktionen primär über kurze Assertionen und geschlossene Fragen erfolgen. Entscheidungen werden weder kollaborativ in der Gruppe getroffen noch argumentativ begründet oder erläutert. 2) Im Gegensatz hierzu partizipieren (fast) alle Gruppenmitglieder am sogenannten ,cumulative talk“, bei dem die geäußerten Ideen in der Gruppe wahrgenommen werden, allerdings in einer unkritischen Weise. Hierbei kommt es zwar zum Teil zu Elaborationen, wenn z. B. eine der Propositionen eine andere (zufällig) ergänzt, primär treten allerdings isolierte Assertionen auf, wobei Wiederholungen zu erwarten sind. Die Propositionen werden innerhalb der Gruppe kumulativ angehäuft, aber nicht innerhalb der Gruppe evaluiert, begründet und schließlich integriert, sodass diese Form des Dialogs 
echte gegenseitige Bezugnahmen vermissen lässt. 3) PL-Dialoge der Form „explorative talk“ zeichnen sich durch ein hohes Maß an Intersubjektivität, Engagement, Offenheit sowie kritisch-konstruktiver Diskussion aus. Die Ideen der Gruppenmitglieder werden nicht nur sukzessive angehäuft, sondern kritisch reflektiert und problematisiert, was argumentative Aushandlungsprozesse und die Erklärung der eigenen sowie der anderen Propositionen verlangt. Hierdurch erhöht sich die Wahrscheinlichkeit für von der Gruppe wahrgenommene Widersprüche der externalisierten Propositionen sowie deren Lösung durch förderliche Interaktionen wie z. B. kollaborative Argumentation, bei der die Widersprüche der argumentativ dargelegten Externalisierungen näher exploriert werden (vgl. Chinn \& Clark, 2013, S. 315). Dementsprechend werden Entscheidungen bei Gruppen mit ,explorative talk“ innerhalb der Gruppe begründet und gemeinsam getroffen.

PL-Dialoge der Form ,explorative talk“ weisen eine hohe Lernwirksamkeit auf Gruppen- und Individualebene hinsichtlich der Lerninhalte als auch förderlicher Interaktionen auf (vgl. Littleton \& Mercer, 2010, S. 281). Der Großteil der Dialoge beim PL entspricht allerdings den Formen „disputational“ und „cumulative talk“ (vgl. ebd.). Lehrkräfte scheinen für diese Problematik bei der Implementation von PL noch keine ausreichende Sensitivität entwickelt zu haben, auch weil förderliche Diskursformen eher selten explizit besprochen werden, sodass die SuS sich nicht zwingend darüber im Klaren sind, was mit Sprachhandlungen wie „diskutieren“, „erklären“ oder „,begründen“ beim PL gemeint ist. Angesichts des förderlichen Typus des ,explorative talks“ sollten auch eher Praktiken wie Tipps geben, Primen, Unterstützen und Helfen anstatt Evaluieren und Korrigieren stärker beim Einüben eines förderlichen PL-Dia$\operatorname{logs}$ in den Fokus rücken (vgl. Howe, 2010, S. 43f). Formen des ,explorative talks" lassen sich vermitteln und trainieren (vgl. Littleton \& Mercer, 2010, S. 283f), sodass vor der Implementation von PL eine explizite Diskussion sowie Förderung produktiver, kollaborativer Dialogformen und Gruppenprozesse vorangestellt werden sollte.

Angesichts der hohen kognitiven und interaktionalen Anforderungen des „explorative talks“, der liberale, offene und gleichberechtigte Diskurse verlangt, stellt sich die Frage, ob PL-Formate mit Dyaden und hierarchisierten Rollen, wie z. B. es häufig beim Peer-Tutoring der Fall ist, organisatorisch und strukturell geeignet sind, um eine solche Form der Interaktion zu etablieren. Die Hierarchisierung in Tutor und Tutand impliziert ein asymmetrisches Machtverhältnis über den Prozess sowie die Bewertung der Güte der Aufgabenbearbeitung, welche insbesondere in den Schritten drei und vier des gegenseitigen Unterrichtens (vgl. Adl-Amini, 2018 S. 29) sichtbar wird und dem Tutor ein gewisses Vetorecht zuspricht. Der Tutor hat die Aufgabe, den Tutanden so anzuleiten und zu unterstützen, dass dieser in die Lage versetzt wird, die Aufgabe zu erfüllen, was eher Praktiken wie Tipps geben, Primen, Unterstützen und Helfen (vgl. Howe, 2010, S. 43f) präferiert, damit der Tutand sein 
mentales Modell sukzessive elaboriert. Hierbei ist es angesichts der verspäteten Effekte von PL nach nicht aufgelösten Widersprüchen nicht zwingend notwendig, zu einer kohärenten Lösung innerhalb der Gruppe zu kommen, da der Tutand diese womöglich post-dialogisch für sich selber erzeugt (vgl. ebd.). Die Gruppengröße dyadischen Peer-Tutorings lässt auch nicht viel Spielraum für eine Vielzahl alternativer Ideen zur Aufgabenbearbeitung und -lösung sowie deren Begründung. Hierbei ist auch zu hinterfragen, ob bei PL-Formaten, die primär das Erwerben und Einüben von Wissen und Fähigkeiten fokussieren, Interaktionen in Form des ,explorative talks" notwendig oder angemessen erscheinen. So könnten Formen des „cumulative talks“ mit dem Fokus auf die Elaboration des mentalen Modells des Tutanden ausreichen, bei dem der Tutor sich stärker auf die Praktiken des Evaluierens und eines korrektiven, Reflexionen anregenden Feedbacks konzentriert. Hierbei könnten auch kognitive Ressourcen beim Tutor frei werden, die für die Etablierung eines effizienten TaskManagements sowie zur metakognitiven Regulation des Tutanden verwendet werden könnten. Auf diese beiden zentralen Prozesse des Task-Managements sowie der metakognitiven Regulation für lernzielgerichtete Interaktionen beim PL wird im Folgenden eingegangen.

\subsubsection{Task-Management und metakognitive Regulation beim Peer- Learning}

Neben einem lerninhalts- und zielorientierten Dialog zum Erwerb von Wissen und Fähigkeiten ist die gemeinsame Organisation, Koordination und Regulation der Gruppenprozesse eine Voraussetzung für effektives PL (vgl. z. B. de Backer et al., 2016; Iiskala, Vauras \& Lehtinen, 2004; Johnson \& Johnson, 1999). Hierfür sind insbesondere Prozesse des „Task-Managements“ als auch der metakognitiven Regulation relevant, die in den nächsten Abschnitten näher ausgeführt werden.

\subsubsection{Task-Management beim Peer-Learning}

Die Organisation und Koordination der inhaltlichen Bearbeitung der Lerninhalte, Aufgaben sowie der Zusammenarbeit wird im Folgenden mit dem Begriff „Task-Management“ bezeichnet. Hierzu gehören z. B. die Zuteilung von Rollen und Aufgaben, die Initiation sowie Terminierung von Arbeitsschritten und -phasen oder die Beschaffung von benötigten Materialen. Intersubjektivität stellt hierbei eine zentrale Bedingung dar, da ohne ein von den Gruppenmitgliedern geteiltes Verständnis der Zusammenarbeit die Koordination des Handelns der Individuen nur schwerlich gelingen kann. Task-Management erfolgt zum Großteil über sprachliche Interaktion, wobei auch nicht-sprachliche 
Praktiken wie Zeigegesten oder die Handhabung von Materialien zur Organisation und Koordination der Gruppenprozesse eingesetzt werden können.

Task-Management berührt primär die von Johnson und Johnson (1999, S. 71) als förderlich für PL postulierten sozialen Kompetenzen wie z. B. „leadership, decision-making, trust-building, communication, and conflict-management skills“. Insbesondere Fähigkeiten wie Führung, Kommunikation und Entscheidungsfindung gilt es in Gruppenprozesse so einzubringen, dass gemeinschaftlich die Aufgabe des Task-Managements bewältigt werden kann. Aber auch strukturelle Bedingungen des PL-Formats wie z. B. individuelle Verantwortlichkeiten, Rollenzuteilungen samt ihrer zugehörigen Aufgaben und Handlungen oder unterstützende Materialien wie Interaktionsskripte, explizite Abfolgen in Form von Teilschritten der Aufgabenbearbeitung oder Checklisten beeinflussen das Task-Management. Dementsprechend sollte bei der Konzeption von PL-Formaten ebenfalls berücksichtigt werden, welche Strukturen und Hilfen implementiert werden, um Notwendigkeiten des TaskManagements möglichst zu minimieren und die durch Task-Management erzeugte kognitive Ladung z. B. durch zusätzliche Materialien zu reduzieren.

\subsubsection{Metakognitive Regulation beim Peer-Learning}

Sowohl für das Task-Management als auch für die gemeinschaftliche Aufgabenbearbeitung beim PL sind Prozesse der metakognitiven (Selbst-)Regulation zentral. Regulatorische Prozesse beim Lernen werden in der Psychologie auf der Ebene der Metakognition verortet. Unter Metakognition wird weitestgehend das Wissen über und die Regulation der eigenen kognitiven Aktivitäten und Lernprozesse verstanden (vgl. Veenman, Hout-Wolters \& Afflerbach, 2006, S. 4). Dieses Konstrukt hat seit seiner Postulierung Ende der 1970er Jahre hohe Aufmerksamkeit erhalten und sich in einer Vielzahl von Studien robust als relevante, trainierbare Eigenschaft von Individuen für Lernprozesse sowie als Prädiktor für Lernwirksamkeit herausgestellt (für einen Überblick siehe z. B. Hacker, Dunlosky, Graesser, 2009; Hasselhorn \& Gold, 2017, S. 88-100; Whitebread et al., 2009, S. 67). Trotz dessen zeichnet sich Metakognition bis heute durch Heterogenität und fehlende Kohärenz hinsichtlich ihrer Definition und Konzeption aus (vgl. Veenman, Hout-Wolters \& Afflerbach, 2006, S. 4f). So ist z. B. unklar, ob es sich bei Metakognition um bewusste oder automatische Prozesse sowie um ein generelles oder domänenspezifisches Konstrukt handelt (vgl. ebd., S. 6f). Eine weitere zentrale Herausforderung stellt die Differenzierung von Kognition und Metakognition dar. Eine Vielzahl der Konzeptionen von Metakognition modelliert diese als Teil von Kognition, der kognitive Prozesse überwacht, was allerdings dem „Homonkulus-Problem" entspricht:

„Most conceptualizations of metacognition have in common that they take the perspective of "higher-order cognition about cognition". There is a higher-order agent 
overlooking and governing the cognitive system, while simultaneously being part of it. This is the classical homunculus problem or Comte's paradox: One cannot split one's self in two, of whom one thinks whilst the other observes him thinking." (ebd., S. 5).

Diese zirkuläre Beziehung von Kognition und Metakognition erschwert die methodische Trennung beider Konstrukte. Nichtsdestotrotz kann Metakognition nicht mit intellektuellen Fähigkeiten gleichgesetzt werden, was im Umkehrschluss allerdings auch bedeutet, dass metakognitive Fähigkeiten auch kognitive Limitierungen kompensieren können (vgl. ebd., S. 6).

Metakognition umfasst nach der oben von Veenman, Hout-Wolters und Afflerbach (ebd. S. 4) vorgeschlagenen Definition sowohl deklaratives Wissen als auch prozedurale Fähigkeiten. Diese Komponenten werden häufig in metakognitives Wissen und metakognitive Regulation differenziert, wobei Ersteres als ,the individual's knowledge about personal, task and strategy variables affecting their cognitive performance” und Letztere als ,the cognitive processes taking place during ongoing activities i.e. planning, monitoring, control and evaluation“ (Whitebread et al., 2009, S. 72) beziehungsweise als ,a set of self-regulatory skills and strategies used by students to actively control and coordinate their learning" (de Backer et al., 2016, S. 188) beschrieben werden können. Zudem werden regulatorische Prozesse nicht nur auf die Kognition begrenzt, sondern auch auf Emotion und Motivation bei Lernprozessen als „,the learner's ongoing monitoring and control of emotions and motivational states during learning tasks" (vgl. Whitebread et al., 2009, S. 72) angewendet.

De Backer et al. (2016) akzentuieren hinsichtlich metakognitiver Regulation die Prozesse der Planung, der Kontrolle/Monitoring sowie der Evaluation. Die Autoren (vgl. ebd., S. 189) verstehen unter metakognitiven Regulationsprozessen der Planung unter anderem die Selektion und Sequenzierung des Problems, die Beschaffung von Ressourcen sowie die Planung des Bearbeitungsvorgehen. Metakognitiven Regulationsprozessen der Evaluation ordnen sie unter anderem die Bewertung von Arbeitsprozessen und Ergebnissen zu. Sie fassen zudem die übrig gebliebenen Prozesse des Monitorings und der Kontrolle als Überwachung und Überprüfung der Bearbeitungs- und Verstehensprozesse zusammen.

Angesichts der relativ geringen empirischen Grundlage ist die Rolle von Metakognition beim PL noch ungeklärt (für einen Überblick siehe de Backer, van Keer \& Valcke, 2015, S. 65f). Prinzipiell scheint Metakognition allerdings für PL bedeutsam zu sein, da die SuS nicht nur klären müssen, was gelernt wird, sondern wie etwas gelernt wird (vgl. de Backer et al., 2016, S. 190). Die Forschung zu Metakognition und PL verfolgt bisher vor allem die folgenden zwei Perspektiven: 1) PL als Methode zur Förderung von metakognitiver Regulation und 2) metakognitive Regulation als eine von der Gruppe geteilte Aktivität von Peers beim PL (vgl. de Backer, van Keer \& Valcke, 2015, S. 65). 
Die erste Perspektive betont das Potential von PL, eine Vielzahl von Lerngelegenheiten für metakognitive Regulation zu ermöglichen, da die Peers als Gruppe die Verantwortung zur Organisation und Koordination des Task-Managements sowie der Aufgabenbearbeitung übernehmen, sodass sie selbst zum Großteil für die Planung, Überwachung, Kontrolle und Evaluation ihrer Prozesse zuständig sind. Diese Notwendigkeit zur metakognitiven (Selbst-)Regulation der Gruppe ruft metakognitive Regulationsprozesse hervor und führt zu positiven Effekten auf die individuellen Fähigkeiten zu metakognitiver Regulation (vgl. Iiskala, Vauras \& Lehtinen, 2004, S. 148; de Backer, van Keer \& Valcke, 2015, S. 66; de Backer et al., 2016, S. 190). Manche Rollen beim PL zeichnen sich auch primär durch Aufgaben der metakognitiven Regulation der Gruppenprozesse aus. So ist z. B. die Rolle des Tutors beim Peer-Tutoring eng mit Aufgaben wie der Führung, Überwachung, Kontrolle und Evaluation des Tutanden und seiner Arbeitsprozesse assoziiert. Diese Aufgaben könnten auf zwei Weisen sich positiv auf metakognitive Regulationsfähigkeiten auswirken (vgl. de Backer, van Keer \& Valcke, 2015, S. 66): Die Ausübung von metakognitiver Regulation durch den Tutor kann zum einen als Modell für den Tutanden fungieren, an welcher er sich für die Ausübung seiner metakognitiven Selbstregulation orientieren kann. Zum anderen ist auch denkbar, dass bei reziproker Rollenverteilung die metakognitiven Regulationspraktiken in der Tutorenrolle womöglich auch in die Tutandenrolle transferiert werden könnten. Dies gilt prinzipiell auch für andere PL-Formate, in denen mindestens eine Rolle mit Aufgaben der metakognitiven Regulation ausgestattet ist.

Der zweite Forschungsstrang akzentuiert die Interindividualität von Regulationsprozessen beim PL. Metakognitive Regulation wurde bisher selbst innerhalb des Kontextes von PL primär aus einer individuellen Perspektive betrachtet (vgl. Iiskala, Vauras \& Lehtinen, 2004, S. 148), obwohl beim PL die Organisation und Koordination des Task-Managements und der Aufgabenbearbeitung eine in der Gruppe zu erfüllende Aufgabe darstellt, welche gemeinschaftliche als auch gegenseitige Regulationsprozesse verlangt. Hierbei werden drei potentielle Arten metakognitiver Regulation beim PL unterschieden (vgl. de Backer, van Keer \& Valcke, 2015, S. 65; Iiskala, Vauras, Lehtinen, 2004, S. 150; Whitebread et al., 2009; S. 73): 1) Selbst-Regulation, 2) KoRegulation und 3) sozial geteilte Regulation. Als Selbst-Regulation werden diejenigen metakognitiven Prozesse kategorisiert, die die eigenen Prozesse regulieren ohne einen Einfluss auf die anderen Gruppenmitglieder auszuüben. Ko-regulative metakognitive Prozesse zielen auf die Einflussnahme eines Individuums auf ein anderes Gruppenmitglied ab, was ein asymmetrisches Verhältnis zwischen dem Regulierenden und dem Regulierten impliziert (vgl. de Backer, van Keer \& Valcke, 2015, S. 65). Diese Form der metakognitiven Regulation ist nicht exklusiv, aber vermehrt beim Peer-Tutoring in Dyaden zu erwarten, da die Rollen Tutor und Tutand die eben angeführte Asymmetrie 
widerspiegeln (vgl. Whitebread et al., 2009, S. 73). Als sozial geteilte Regulation definieren de Backer, van Keer und Valcke (2015, S. 65) ,shared metacognitive awareness and egalitarian, interdependent adoption of regulation skills towards joint learning objectives in groups operating as genuine social entities". Diese Formen der Regulation treten eher in Gruppenkonstellationen auf, die sich durch Symmetrie, Balance sowie eine gleichberechtigte Diskurskultur auszeichnen (vgl. Iiskala, Vauras \& Lethinen, 2004, S. 150), und adressieren alle Gruppenmitglieder (vgl. Whitebread et al., 2009, S. 73). Dementsprechend zeichnet sich sozial geteilte metakognitive Regulation primär durch die egalitäre Verteilung der Verantwortung für die metakognitive Regulation der Gruppe an alle Gruppenmitglieder aus, wobei die Ausübung von metakognitiver Regulation sich an alle Gruppenmitglieder richtet. Sozial geteilte metakognitive Regulation stellt hohe Ansprüche an die regulativen Fähigkeiten der Gruppenmitglieder und benötigt Zeit, sich in Gruppen zu entwickeln (vgl. de Backer, van Keer \& Valcke, 2015, S. 65).

Diese drei verschiedenen Formen von metakognitiver Regulation können auch sukzessiv oder parallel auftreten. So können metakognitive Regulationen weitere regulative Akte elizitieren (vgl. ebd., S. 65), sodass z. B. eine SelbstRegulation von Peer X als Modell für Peer Y fungiert, der wiederum in Folge Peer Z ko-reguliert und anschließend alle drei Gruppenmitglieder intersubjektiv in sozial geteilter metakognitiver Regulation agieren. Weiterhin dürfte ein gewisser Grad an metakognitiven selbst- und ko-regulativen Fähigkeiten eine Voraussetzung für sozial geteilte metakognitive Regulation sein (vgl. Iiskala, Vauras \& Lehtinen, 2004, S. 174). Es gibt erste empirische Hinweise darauf, dass sozial geteilte metakognitive Regulation beim PL sich positiv auf die Gruppenleistung, individuelle regulative Fähigkeiten sowie die Lernwirksamkeit auswirkt und zu einer Steigerung der Reflexion der mentalen Modelle sowie der Problemlösestrategien innerhalb der Gruppe beiträgt (für einen Überblick siehe de Backer, van Keer \& Valcke, 2015, S. 65). Weiterhin scheinen Prozesse sozial geteilter metakognitiver Regulation abhängig von der Aufgabenschwierigkeit zu sein, da bei simplen Aufgaben diese Form der Regulation beim PL kaum, jedoch bei als schwierig empfundenen Aufgaben vermehrt auftritt (vgl. Iiskala, Vauras \& Lehtinen, 2004, S. 171). Zudem gilt es darauf hinzuweisen, dass Praktiken der metakognitiven Ko-Regulation von sozial geteilten metakognitiven Regulationen nicht immer klar zu differenzieren sind und dass beide Formen der metakognitiven Regulation nicht zwingend nur durch vokalsprachliche Äußerungen vermittelt werden, sondern auch mit nonverbalen Praktiken wie z. B. Mimik, Blickkontakt, Zeigegesten oder Berührungen erfolgen können (vgl. ebd., S. 173). 


\subsubsection{Bedeutung und Wirkmechanismen von Interaktion beim Peer-Learning für Bildungs- und Lernprozesse}

Insbesondere in den Abschnitten 2.3.2, 3.6.2 sowie indirekt in 3.6.5 wurde die zentrale Bedeutung von Sprache als kognitives Werkzeug respektive von sprachlichen Fähigkeiten für Bildungsprozesse erläutert und anhand empirischer Evidenz dargestellt. Dementsprechend ist es nicht verwunderlich, dass der Interaktion eine Schlüsselfunktion für effektives PL zugesprochen wird. Hierbei gilt es allerdings näher zu bestimmen, welche Wirkmechanismen der Lernwirksamkeit von förderlichen Dialogen beim PL zu Grunde liegen und welche theoretischen Perspektiven sich zur Erklärung dieser eignen. Bevor hierauf näher eingegangen wird, soll kurz die theoretische Modellierung von PL im Allgemeinen kommentiert werden.

Hinsichtlich theoretischer Reflexionen von PL werden typischerweise motivationale und soziale Ansätze sowie Elaborations- und Entwicklungsperspektiven gewählt (vgl. Adl-Amini, 2018, S. 24). Diese Perspektiven werden in theoretische Modelle von PL inkorporiert, die sich unter anderem in der Schwerpunktsetzung der jeweiligen Komponenten, der Modellierung der $\mathrm{Zu}-$ sammenhänge verschiedener Prozesse beim PL sowie der Wirkrichtungen unterscheiden (vgl. Topping et al., 2005). Die Auswahl der eben angeführten theoretischen Perspektiven zur Rahmung von PL sowie die theoretische Modellierung von PL kann hinsichtlich diverser Aspekte kritisch betrachtet werden (vgl. Adl-Amini, 2018, S. 24f): 1) Die Auswahl an verwendeten theoretischen Zugängen für PL erscheint etwas willkürlich, da auch andere theoretische Perspektiven, die zur Aufklärung der Ursachen und Wirkmechanismen der Effektivität von PL beitragen könnten, kaum Beachtung finden. So könnte z. B. die Self-Determination Theory nach Ryan und Deci (2018) oder rollentheoretische Reflexionen (vgl. Robinson, Schofield \& Steers-Wentzell, 2005) zur Erläuterung motivationaler Effekte des PL zu Grunde gelegt werden anstatt extrinsische Motivation durch Verstärker beim PL zu akzentuieren. Auch theoretische Perspektiven und Modelle aus der empirischen Bildungs- und Unterrichtsforschung wie Unterricht als Angebot (vgl. Helmke, 2017, S. 69-101; Seidel, 2014) oder Basisdimensionen guten Unterrichts (vgl. Klieme, Schümer \& Knoll, 2001; Klieme, Pauli \& Reusser, 2009) könnten sich als theoretische Perspektiven für PL angesichts der meta-analytischen Befunde zu positiven Effekten von PL auf Arbeitsverhalten, Time-on-Task, Zugehörigkeitsgefühl etc. (vgl. Rohrbeck et al., 2003) eignen. 2) Die vier typischerweise angeführten Perspektiven stellen keine trennscharfen Theoriekomplexe dar, sondern überschneiden sich zum Teil und ergänzen sich gegenseitig. Zudem sind diese Perspektiven keine einheitlichen, geschlossenen Theoriemodelle, sondern beinhalten wiederum diverse Theorien und zum Teil gar verschiedene Paradigmen, sodass auch innerhalb der Perspektiven es zu klären gilt, welche jeweiligen 
Ansätze zur Erklärung der Wirksamkeit von PL gewählt werden. 3) Die theoretischen Modelle zum PL selbst sind äußerst komplex und widersprechen somit dem Prinzip der Sparsamkeit der Hypothesen- und Theoriebildung (vgl. Sarris \& Reiß, 2005, S. 50). Anstatt solcher multidimensionaler Theoriekomplexe könnte eine theoretische Betrachtung von PL aus der Perspektive der fünf Basiselemente für effektives PL nach Johnson und Johnson (1999) unter Umständen eine fruchtbarere, praktischere Alternative darstellen, die hinsichtlich der Implementation und Adaptation von PL für die individuell vorliegenden Bedingungen anwendungsfreundlicher erscheint.

Eine ausführliche Diskussion theoretischer Perspektiven und Modellierungen von PL ist kein zentrales Erkenntnisinteresse der vorliegenden Arbeit. Stattdessen sollen im Folgenden ausgehend von der These ,thinking is an individualized form of interpersonal communication" (Sfard, 2015, S. 249) ausgewählte Aspekte der kognitiven Elaborations- und Entwicklungsperspektive sowie einer Erweiterung der „Output Hypothesis“ (Swain, 2000) kurz ausgeführt werden, da sich diese zur Erklärung der Wirkmechanismen eines förderlichen, kollaborativen Dialogs eignen.

\subsubsection{Die Beziehung von Kommunikation und Lernen}

Nach Stojanov (2006, S. 66) vollziehen sich Bildungsprozesse der „Selbst-Entwicklung“ und der „Welt-Erschließung“ in ,,alltäglich-sozialen Interaktionserfahrungen", welche für SuS zu einem großen Anteil in der Bildungsinstitution Schule erfolgen. Auch konstruktivistische Entwicklungsperspektiven auf Prozesse des Lernens nach Piaget (vgl. de Lisi \& Golbeck, 2009) oder nach Vygotski (vgl. Hogan \& Tudge, 2009) akzentuieren die Bedeutung von Kommunikation für Lernen in verschiedener Weise. So werden durch Interaktionen z. B. Lerngelegenheiten erzeugt und Lernprozesse ausgelöst (vgl. de Lisi \& Golbeck, 2009) sowie innerhalb von Interaktionen intramentales Wissen externalisiert und Sprache als intermentales Werkzeug zur Erzeugung neuer Erkenntnisse eingesetzt (vgl. Hogan \& Tudge, 2009). Die in solchen sozialen, konstruktivistischen Prozessen externalisierten oder gemeinschaftlich erzeugten Propositionen können wiederum aus der Perspektive kognitiver Elaboration zur Modifikation der individuellen mentalen Modelle beitragen. Dementsprechend erscheint die Erkenntnis, dass Kommunikation und Lernen eng zusammenhängen und sich gegenseitig bedingen, angesichts der eben erwähnten kognitiven Lerntheorien und der in Abschnitt 5.3.1 ausgeführten empirischen Evidenz zur Lernwirksamkeit kollaborativer Dialoge beim PL fast trivial. Nichtsdestotrotz gilt es die konkrete Beschaffenheit des Zusammenhangs von Kommunikation und Lernen zu klären, um die Effektivität von förderlichen Interaktionen beim PL theoretisch fundieren zu können.

Anna Sfard (2015) reflektiert die Beziehung von Kommunikation und Lernen anlässlich einer 2011 von der American Educational Research Association 
(AERA) initiierten Konferenz zu sozialer Interaktion und Lernen in der Schule. Hierfür rezitiert Sfard (ebd., S. 248) einige zentrale Annahmen, die innerhalb der eben erwähnten Konferenz von führenden Forschern auf diesem Gebiet geäußert wurden: „We are using [and changing] language to learn something“; ,[A properly shaped] discourse is the most promising path to development of thinking”; "The learning of mathematics has been improved thanks to a change in the forms of classroom talk”; „Dialogical inquiry [...] enhances academic skills". Diese Assertionen weisen alle dieselbe Argumentationsstruktur auf, da in allen Fällen eine Veränderung in A eine Veränderung in B verursacht. Somit implizieren letztlich alle diese Annahmen einen kausalen Ursache-WirkungsZusammenhang (vgl. ebd.). Hierbei stellen die Variablen A und B, die mit verschiedenen Konstrukten wie Diskurs, Sprache, Gespräch und Dialog respektive Entwicklung von Denken, Lernen (von Mathematik) und akademische Fähigkeiten in den jeweiligen Annahmen besetzt werden, unterschiedliche Facetten derselben Konstrukte dar: Kommunikation (A) und Wissen sowie Fähigkeiten von Lernern (B). Sfard (ebd., S. 249) ist der Meinung, dass angesichts der zugrunde liegenden Konstrukte von A und B letztlich kein kausaler Ursache-Wirkungs-Zusammenhang zwischen diesen beiden besteht, sondern beide Variablen eher ,two sides of the same coin“ repräsentieren. Hierbei konzeptioniert Sfard (ebd.) Denkprozesse, welche sowohl dem Wissen als auch den Fähigkeiten eines Lerners zu Grunde liegen oder diese erzeugen, als ,individualized form of interpersonal communication". Hieraus schließt sie, dass Kommunikation im Mittelpunkt von Lernprozessen steht anstatt diesem Konstrukt lediglich den Status eines Mittels zum Zweck zuzusprechen: „Communication, rather than playing a secondary role as the means for learning, is in fact the centerpiece of the story - the very object of learning" (ebd.). Die Förderung von bildungsbezogener Kommunikation ist also in direkter Weise mit der Förderung des Lernens verbunden, da beide vergleichbaren Prozessen unterliegen. In der Interaktion bezüglich eines schulischen Lerngegenstandes müssen z. B. SuS beim PL ihr Wissen in Propositionen externalisieren, die für die Gruppenmitglieder zu verarbeiten sind und deren Anschlussfähigkeit oder Inkompatibilität mit ihren jeweiligen mentalen Modellen möglichst argumentativ sowie evidenzbasiert innerhalb der Gruppe ausgehandelt werden. Bei der individuellen Aufgabenbearbeitung müssen SuS zentrale Propositionen identifizieren und in ihr aktuelles mentales Modell integrieren oder das mentale Modell so modifizieren, dass die neuen Propositionen an dieses anschlussfähig werden. Die hierfür zu vollziehenden Denkprozesse können in ähnlicher Weise wie die Aushandlungsprozesse in der Gruppe modelliert werden, da das Individuum ein kohärentes mentales Modell konstruieren muss, bei welchem die Zusammenhänge der zu Grunde liegenden Propositionen in einer begründeten Weise argumentativ zu rechtfertigen sind. Der zentrale Unterschied besteht lediglich darin, dass beim PL Teile der Modellbildung in der Gruppe externali- 
siert und gemeinschaftlich diskutiert und (eventuell) konstruiert werden, während bei individuellen Denkprozessen die Konstruktion internalisiert in einer Form von Selbstgespräch verläuft. Eine förderliche Veränderung in der Interaktion zwischen SuS geht dementsprechend einher mit einer positiven Veränderung der individuellen Denkprozesse und folglich des Lernens von einzelnen SuS.

Nach Sfard (ebd., S. 250f) gibt es zwei zu differenzierende Ebenen von Veränderungen beim Lernen: „object-level change“ und „meta-level change“. Die erstgenannte Ebene bezieht sich auf Veränderungen, die den Diskurs als Ganzes transformiert, während die zweitgenannte Ebene die Regeln des Diskurses modifiziert. Bei „object-level“ Veränderungen bauen die Lernprozesse auf einem gewissen Vorwissen hinsichtlich eines in Teilen bekannten Gegenstands auf, welcher in der Interaktion mit anderen weiter bearbeitet wird, sodass neue Erkenntnisse hinsichtlich dieses Lerngegenstands innerhalb der Kommunikation gewonnen werden können. So können z. B. SuS, die positive Kardinalzahlen samt basaler Grundrechenarten erworben haben, ihr Wissen in diesem Bereich durch Kommunikation mit anderen festigen, erweitern und zur Problemlösung anwendungsbezogener Aufgaben aus anderen Bereichen einsetzen. Im Gegensatz hierzu führen „,meta-level“ Veränderungen neue, vorher unbekannte Konstrukte ein, die in ihren Grundlagen zunächst durchschaut werden müssen, um mit diesen in einem nächsten Schritt individuell oder gemeinschaftlich operieren zu können. Ein Beispiel hierfür wäre die Einführung negativer Zahlen, deren Charakteristika und mathematische Zusammenhänge selten in der alltäglichen Lebenswelt zum Tragen kommen und folglich ein eher in der Schule behandeltes Phänomen darstellen. Um mit einem solchen Gegenstand wie negativen Zahlen produktiv umgehen zu können, müssen deren Grundlagen zumindest in basaler Weise verstanden werden. Angesichts dieser beiden Ebenen sind verschiedene Sozialformen des Lernens abhängig vom jeweiligen Gegenstand und dem vorhandenen Vorwissen der SuS zu präferieren. So eignet sich PL für die Initiation von „object-level“ Veränderungen, während Frontalunterricht, bei dem die Lehrkraft einen Gegenstand didaktisch aufbereitet den SuS näher bringt, eher bei der Einführung neuer Konzepte und somit für „meta-level“ Veränderungen zu empfehlen ist (vgl. ebd. S. 251).

Die Hypothese von Sfard (2015), Kommunikation ins Zentrum von Lernprozessen zu rücken, da Denkprozesse individualisierte Formen interpersonaler Kommunikation darstellen, ist anschlussfähig an Howes (2010) Hypothese zur Erklärung verzögerter Effekte nicht aufgelöster Widersprüche beim PL. Howe (ebd.) vermutet, dass nicht aufgelöste Widersprüche beim PL zu einem späteren Zeitpunkt von den Gruppenmitgliedern in individueller Weise selbst gelöst werden, was die verspätet auftretenden Effekte von PL erklären könnte. Eventuell wirkt sich PL hierbei nachträglich auf die Fähigkeit aus, widersprüchliche Propositionen von Lerninhalten argumentativ in einem mentalen Selbstgespräch zu klären, da die kommunikativen Praktiken beim PL eventuell 
auch bei der individuellen Reflexion von Gegenständen übernommen werden können.

Zusammenfassend lässt sich aus der These von Anna Sfard (2015) schließen, dass die Förderung von produktiven bildungsbezogenen Dialogen beim PL sich ebenfalls positiv auf die individuellen Denk- und Reflexionsprozesse auswirken könnte. Die konstruktivistischen Lerntheorien von Piaget und Vygotski leisten einen weiteren Beitrag zum Verständnis der Wirkmechanismen von (förderlicher) Interaktion beim PL. Diese werden im Folgenden kurz ausgeführt, wobei lediglich die für die Kommunikation beim PL relevanten Aspekte ihrer Theoriekomplexe erläutert werden.

\subsubsection{Piagets und Vygotskis Perspektiven auf Lernen und Entwicklung beim Peer-Learning}

Piagets (vgl. de Lisi \& Golbeck, 2009) und Vygotskis Perspektiven auf PL (Hogan \& Tudge, 2009) weisen einige Gemeinsamkeiten, allerdings auch konträre Positionen auf. Beide Ansätze sind gut mit der Perspektive der kognitiven Elaboration vereinbar, da ihr Konstruktivismus sowohl die Entwicklung von Subjekten als Verbesserung oder Anpassung ihres kognitiven Systems über die Zeit als auch die Anwendung des kognitiven Systems zur Herstellung von Bedeutung in der Umwelt umfasst (vgl. de Lisi \& Golbeck, 2009, S. 5). Wenn SuS beim PL ihre mentalen Modelle externalisieren und auf diese gegenseitig reagieren, kann dies zur Modifikationen ihrer mentalen Modelle führen und dementsprechend Entwicklungsprozesse initiieren.

Piaget fokussiert primär kognitive Aspekte des Konstruktivismus (vgl. de Lisi \& Golbeck, 2009). In Piagets Theoriekomplex bezeichnet Äquilibrium den „desired state for intellectual functioning“ (vgl. ebd., S. 12), der eine Form adäquat konzipierter mentaler Modelle beschreibt, die sich durch eine Balance zwischen der subjektiven, mentalen Konzeption einer Entität und deren Eigenschaften und Merkmalen in der Umwelt auszeichnet (vgl. ebd., S. 8). Sofern kein Äquilibrium hinsichtlich einer Entität besteht, gibt es zwei distinkte Prozesse, mit denen ein Äquilibrium zumindest temporär erzeugt werden kann: Assimilation und Akkommodation. Assimilation stellt einen inkorporierenden Prozess dar, bei welchem ein Objekt oder eine Proposition so in das kognitive System integriert wird, dass der mentalen Konzeption eine der Umwelt entsprechende Bedeutung verliehen wird und dieses folglich in der Auseinandersetzung mit seiner Umwelt angewandt werden kann (vgl. ebd.). Im Gegensatz hierzu bezeichnet Akkommodation einen Prozess, bei welchem ein Objekt oder eine Proposition dem aktuellen kognitiven System in einer Weise widerspricht, sodass eine Integration dessen in das mentale Modell nicht möglich ist. Folglich wird das kognitive System dahingehend modifiziert, dass die vorher inkompatible Proposition schließlich anschlussfähig wird und einen Bestandteil des adaptierten mentalen Modells darstellt (vgl. ebd.). Beiden Prozessen 
geht in der Regel eine Erfahrung voraus, die Probleme oder Lücken des aktuellen mentalen Modells offenbart und dementsprechend als Störung (Pertubation) wahrgenommen wird, welche schließlich zur Aufhebung des Äquilibriums führt. Prozesse der Assimilation und/oder Akkommodation regulieren und kompensieren diese Störung letztlich, sodass der Zustand eines (temporären) Äquilibriums erzeugt werden kann (vgl. ebd., S. 12).

Nach Piaget kann die Interaktion beim PL als Lerngelegenheit interpretiert werden, die durch von den Gruppenmitgliedern initiierten Pertubationen zu Restrukturierungen kognitiver Systeme in Form von Assimilation und Akkommodation führen kann (vgl. ebd., S. 18ff). So kann z. B. die Externalisierung eines mentalen Modells von Peer X eine Pertubation bei Peer Y erzeugen, wenn dieses mit seinem mentalen Modell nicht übereinstimmt, sodass die sich widersprechenden Propositionen der mentalen Modelle im Folgenden zwischen Peer X und Peer Y ausgehandelt werden müssen. Andererseits können hierbei auch Peer X Inkonsequenzen und Inkonsistenzen auffallen, die ihm vor der Erläuterung seines mentalen Modells in der individuellen Reflexion selber nicht bewusst waren (vgl. ebd., S. 18). Weiterhin ermöglicht nach Piaget die soziale Interaktion im PL Rollenübernahmen und Perspektivwechsel, die bei asymmetrischen Verhältnissen wie z. B. zwischen Erwachsenen und Kindern aufgrund der Hierarchie nicht so einfach zu vollziehen sind (vgl. ebd., S. 22).

Allgemein postuliert Piaget qualitative Unterschiede in der Interaktion zwischen symmetrischen Peer- und asymmetrischen Erwachsenen-Kinder-Beziehungen. So sind Kinder in Peer-Interaktionen aktiver (vgl. ebd., S. 27) und orientieren sich stärker an ihrem Gegenüber als bei Interaktionen zwischen Erwachsenen und Kindern (vgl. ebd., S. 24). Während Piaget Erwachsenen-Kinder-Interaktionen eher Prozesse der Akkommodation durch z. B. hierarchisierte regulative Prozesse sowie Modellierungen zuspricht, zeichnen sich kooperative Prozesse beim PL durch eine erhöhte Wahrscheinlichkeit zur Erzeugung eines Äquilibriums auf Basis gegenseitigen Respekts aus (vgl. ebd., S. 10).

Vygotski (1997, S. 18; zit. nach Hogan \& Tudge, 2009, S. 41) akzentuiert stärker die Bedeutung von Kultur und damit einhergehend von Historizität für die Entwicklungs- und Lernprozesse von Menschen:

„Culture creates special forms of behavior, it modifies the activity of mental functions, it constructs new superstructures in the developing system of human behavior [...]. In the process of historical development, social man changes the methods and devices of his behavior, transforms natural instincts and functions, and develops and creates new forms of behavior - specifically cultural."

Anstatt lediglich zeitlich invariable, genetisch determinierte Entwicklungsprozesse von Menschen zu postulieren, weist Vygotski auf die Abhängigkeit der menschlichen Sozialisations- und Lernprozesse von den jeweiligen Gegebenheiten hin, wobei dem Konzept Kultur in diesen Prozessen eine zentrale Rolle 
zugesprochen wird. Kultur als Vermögen zur Bedeutungsstiftung ermöglicht nämlich Personen, ihrer Umwelt und sich selbst Bedeutung zu verleihen und somit diese zu strukturieren. Nach Vygotski gilt es das Zusammenspiel von sich entwickelnden Kindern und Kultur zu berücksichtigen, da die Umwelt des Kindes kulturell geprägtes Verhalten und Wissen liefert, welches das Kind erwirbt, internalisiert und wiederum auf seine Umwelt anwendet (vgl. ebd., S. 42).

In Vygotskis Konzeption von Lernen spielt die von ihm postulierte „Zone der proximalen Entwicklung“ (ZPD) eine herausragende Rolle. Die ZPD beschreibt, was ein Lerner auf Basis seines Vorwissens und Hilfestellungen durch ein weiteres, kompetenteres Individuum als nächstes lernen kann (vgl. ebd., S. 43). Eine häufig in der Literatur anzutreffende Misskonzeption der ZPD ist ihre Operationalisierung über ein klar definiertes Kriterium, welches unabhängig von der gemeinsamen Aktivität mit einer weiteren Person existiert (vgl. ebd.). Vielmehr entsteht die ZPD erst durch die Kollaboration mit anderen, die das Überschreiten der Grenzen des Vorwissens des Individuums erst ermöglicht. Die spezifischen Mechanismen, die zu Entwicklung führen, stellen „Internalisation“ und „Externalisation“ dar (vgl. ebd., S. 43f). Bei der Internalisation übernehmen Kinder auf Basis ihrer individuellen Eigenschaften, Erfahrungen und Vorwissen den Umgang von kompetenteren Individuen mit der Umwelt. Entwicklung kann hierbei als Prozess der Reorganisation von mentalen Strukturen in Relation zu anderen Menschen in der Umwelt interpretiert werden. Die Externalisation bezeichnet die Produktion eines mentalen Modells für einen Adressaten, welches diesem in der Regel durch Sprachhandlungen erläutert und erklärt wird. Sowohl bei der Internalisation als auch bei der Externalisation werden soziale und kognitive Prozesse stets gemeinsam betrachtet, da intrapsychologische Prozesse durch interpersonale Erfahrungen initiiert werden. Sozio-kulturell betrachtet bilden sich psychologische Prozesse dementsprechend zunächst in kollektivem Verhalten aus und werden erst in einem weiteren Schritt internalisiert, wobei diese Mechanismen von semiotischen Werkzeugen, insbesondere von Sprache und Interaktion, mediiert werden (vgl. Swain, 2000, S. 103).

Im Gegensatz zu Piaget favorisiert Vygotskis Theoriekomplex eher asymmetrische soziale Beziehungen in Lernprozessen, da sich diese besser zur Erzeugung der ZPD als homogene, gleichberechtigte Interaktionspartner eignen (vgl. ebd., S. 50). Hinsichtlich der Asymmetrie steht hierbei allerdings die Kompetenz hinsichtlich des jeweiligen Lerngegenstands als zu Grunde liegende Argumentation im Vordergrund. Leistungsheterogenen Individuen wird im Vergleich zu leistungshomogeneren Partnern eher die Fähigkeit zugesprochen, adaptiv an das Vorwissen des Gesprächspartners anzuknüpfen und dieses mit adäquaten Hilfsmitteln an die ZPD heranzuführen, sodass es zu Prozessen der Internalisation kommen kann (vgl. ebd., S. 55). Dementsprechend kann 
sich auch PL als effektive Sozialform aus der Perspektive des sozial-kulturellen Konstruktivismus des Lernens erweisen, sofern die jeweiligen Gruppenmitglieder sich in ihrer Kompetenz bezüglich des Lerngegenstands unterscheiden und der kompetentere Peer auch tatsächlich zur Erzeugung der ZPD fähig ist.

Unabhängig von der jeweiligen theoretischen Perspektive als auch der sich in ihrer Konzeption ähnelnden kognitiven Mechanismen kann die Interaktion beim PL Lernprozesse initiieren, die primär durch Unterschiede zwischen der Kompetenz oder den mentalen Modellen der Peers ermöglicht werden. Folglich sprechen sich diese beiden theoretischen Perspektiven für heterogene Gruppenmitglieder beim PL aus, wobei noch zu klären ist, ob tatsächlich Kompetenzunterschiede zwischen den Peers herrschen müssen oder ob organisatorische Variablen wie asymmetrische Rollen und Hilfsmaterialien ebenfalls Pertubationen und/oder Internalisations- und Externalisationsprozesse hervorrufen können, die in der Interaktion ausgehandelt werden.

Abschließend soll auf einen weiteren potentiell fruchtbaren Wirkmechanismus von PL eingegangen werden, dessen Kern in einer Steigerung der Partizipation und Beteiligung der SuS durch die Reduktion potentieller Teilnehmer an Aushandlungsprozessen in der Kleingruppe liegt.

\subsubsection{Peer-Learning zur Steigerung der Partizipation und des Outputs}

Im Gegensatz zum Unterricht im Klassenverbund stellt PL eine äußerst intime, teilnehmerreduzierte Sozialform des Lernens dar. Hierdurch wird eine höhere aktive Beteiligung aller SuS ermöglicht (vgl. Adl-Amini, 2018, S. 33) oder gar notwendig, da die Aufgabenbearbeitung in der Verantwortung von ca. zwei bis sechs SuS liegt, anstatt wie im Klassenverbund über mehr als 20 SuS distribuiert wird. Mit dieser erhöhten Beteiligung geht auch eine Steigerung der Wahrscheinlichkeit einher, dass alle SuS die Chance erhalten, ihr Wissen zu externalisieren, Lösungsvorschläge zu äußern, Externalisierungen anderer SuS zu kommentieren und weitere lernförderliche kommunikative Aktivitäten zu vollziehen. Die meta-analytischen Befunde von Robinson, Schofield und SteersWentzell (2005) weisen auf positive Effekte von PL auf lernförderliches Verhalten von SuS hin. Während die Autoren (ebd., S. 349ff) diese Effekte vor allem unter Verwendung von Rollentheorien versuchen zu erklären, könnten diese aus einer Angebots-Nutzungs-Perspektive des Unterrichts (vgl. Helmke, 2017, S. 69-101; Seidel, 2014) als eine erhöhte Nutzungsfrequenz von Lernangeboten gedeutet werden. Dementsprechend würde PL nicht nur mehr Lerngelegenheiten aufgrund der reduzierten Teilnehmerzahl in den Kleingruppen erzeugen, sondern die positive Interdependenz und individuelle Verantwortung zwingt quasi die SuS das im PL eingebettete Lerngebot vermehrt zu nutzen, und zwar in einer aktiven, produktiven Weise. 
Diese Annahme soll anhand der Output-Hypothese von Swain (2000), welche bereits in Abschnitt 3.3.2.2 kurz im Kontext des Erwerbs linguistischer Kompetenzen durch In- und Output angerissen wurde, erweitert werden. Swain (ebd.) reflektiert und stützt evidenzbasiert eine Erweiterung ihrer Output-Hypothese für den L2a durch kollaborative Dialoge. Swain (ebd., S. 99) spricht Output, verstanden als externalisierte, wahrnehmbare Sprachproduktion, bei der Sprache als Objekt zugänglich wird, eine besondere Bedeutung für den L2a $\mathrm{zu}$, da Sprachproduktionen Lerner zu einer intensiveren, tieferen und anstrengenderen Verarbeitung von Sprache als Sprachverstehensprozesse zwingen. Hierbei können zwei Prozesse sich lernförderlich auf den L2a auswirken, nämlich „,noticing“ und „hypotheses testing“ (vgl. ebd., S. 99f). „Noticing“ beschreibt Erkenntnisse von Sprachlernern bei der Sprachproduktion, bei welchen ihnen Lücken in ihrem linguistischen Wissen und Können ,at the very moment of attempting to produce it" (vgl. ebd., S. 100) auffallen. Lerner sind sich ihrer sprachlichen Möglichkeiten und Grenzen nicht zwingend bewusst, sodass unter Umständen erst bei der Produktion bestimmter Strukturen den Lernern klar wird, was sie bereits können und was nicht. „Hypotheses testing“ bezeichnet die implizite Externalisierung von Hypothesen über sprachliche Formen und Funktionen, die bei der Sprachproduktion erkennbar werden (vgl. ebd.). Werden Fehler in der Sprachproduktion erkannt, können auf Basis dieser die zu Grunde liegenden Hypothesen über die ungrammatisch produzierten Strukturen auf ihre Adäquatheit hin getestet werden. Sowohl beim „noticing“ als auch beim ,hypotheses testing“ ermöglicht die Sprachproduktion, Sprache als Objekt metalinguistisch hinsichtlich seiner Wohlgeformtheit und Angemessenheit zu betrachten. Prinzipiell können beide Prozesse nicht nur für das Konstrukt Sprache angewandt werden, sondern auch auf andere Lerninhalte und Konzepte angewandt werden, da bei der Externalisierung von mentalen Modellen ebenfalls Lücken und inadäquate zu Grunde liegende Hypothesen evident werden können.

Diese Erweiterung von Swains (2000) Output-Hypothese für mentale Modelle spricht der Sozialform PL nicht nur eine erhöhte Beteiligung der SuS samt einer erhöhten Wahrscheinlichkeit für Externalisierungen sowie der Nutzung von Lernangeboten zu, sondern akzentuiert die Möglichkeit zum Erkennen von Inkonsistenzen, Inkohärenzen und falschen zu Grunde liegenden Annahmen ihrer mentalen Modelle. Hierbei sind die Lerner nicht auf sich allein gestellt, sondern werden von ihren Peers unterstützt, die aus einer metakognitiven Perspektive als weitere Kontrollinstanz zur Prüfung der Korrektheit der Externalisierungen fungieren. PL-Formate eignen sich dementsprechend nicht nur zum Lernen und Üben von schulischen Lerninhalten, sondern auch zum Erwerb von (Zweit-)Sprachen in der Interaktion mit anderen. 


\subsubsection{Zusammenfassung}

Eine förderliche, kooperative Interaktion ist ein absolut zentraler Aspekt der Lernwirksamkeit von PL (vgl. Howe, 2010; Johnson \& Johnson, 1999; Littleton \& Mercer, 2010). Hierbei scheinen Sprachhandlungen wie Propositionen oder Assertionen äußern sowie Widersprüche, Argumentationen und Erklärungen lernwirksam zu sein, da deren Prävalenz und/oder Qualität mit individuellen Lernzuwächsen zusammenhängen (vgl. z. B. Chi et al., 2001; Howe, 2010; Schwarz \& Linchevski, 2007; Webb et al., 2009). Insbesondere Interaktionen beim PL des Typs „,explorative talk“ stellen förderliche Dialogformen für lernwirksames PL dar (vgl. Littleton \& Mercer, 2010, S. 277), die sich durch Intersubjektivität, Engagement, Offenheit sowie einer kritisch-konstruktiven Diskussionskultur auszeichnen, bei welcher die Beiträge der Gruppenmitglieder kritisch reflektiert und problematisiert werden, was argumentative Aushandlungsprozesse und die Erklärung der eigenen Propositionen verlangt. Die Kommunikation beim PL ist aber nicht nur für das Verständnis und den Erwerb des Lerninhalts relevant, sondern auch zur Koordination der Gruppenarbeitsprozesse. Hierzu gehören auch metakognitive Regulationsprozesse, die beim PL nicht nur aus individueller Perspektive betrachtet werden dürfen, sondern auch Ko-Regulationen sowie sozial geteilte Regulationen umfassen (vgl. de Backer et al., 2016; Iiskala, Vauras \& Lehtinen, 2004; Whitebread et al., 2009). Da nicht von förderlichen Dialogkulturen bei SuS ohne explizite Thematisierung kooperativer Interaktionsformen ausgegangen werden kann (vgl. Littleton \& Mercer, 2010, S. 281), sich solche Interaktionstypen allerdings trainieren lassen (vgl. ebd., S. 283ff), sind Instruktions- und Übungsphasen zu förderlicher Kommunikation beim PL vor der Umsetzung von PL-Formaten zu empfehlen.

Wenn Denken als ,individualized form of interpersonal communication“ interpretiert werden kann, verschiebt sich die Rolle von Kommunikation als Vermittler von Wissen und Fähigkeiten zu ,the very object of learning“ (vgl. Sfard, 2015, S. 249), sodass die Kommunikation beim PL selbst einen zentralen Teil des Lernprozesses darstellt. Die innerhalb der Kommunikation stattfindenden Assertions-, Argumentations- und Erklärungsprozesse eines Diskurses können dementsprechend als epistemologische Handlungen verstanden werden, die im weiteren Verlauf als Vorlagen für Reflexionsprozesse zum Erwerb von Wissen und Fähigkeiten verwendet werden können (vgl. Howe, 2010). Die argumentativ zu vollführende Überzeugung von Gesprächspartnern von einer Assertion und die Erklärung von Inhalten zur Aufklärung über oder zur Ermöglichung des Verständnisses eines Sachverhalts in einem Diskurs ähnelt der zur Konstruktion eines mentalen Modells notwendigen Orchestrierung und Kohärenzherstellung der dem Modell zu Grunde liegenden Propositionen. Eine Position vor sich und anderen in der Interaktion zu rechtfertigen oder ver- 
ständlich zu machen gleicht den intramentalen Reflexionsprozessen der Konstruktion mentaler Modelle, sodass nicht nur mentale Modelle innerhalb von Kommunikation externalisiert werden, sondern ebenfalls erzeugt werden.

Bei der Kommunikation im PL können sowohl aus Piagets (vgl. de Lisi \& Golbeck, 2009) als auch Vygotskis Perspektive des Konstruktivismus (vgl. Hogan \& Tudge, 2009) die Peers auf Basis ihrer jeweiligen mentalen Modelle, Wissen und Kompetenzen verschiedene lernförderliche Prozesse wie Pertubation, Assimilation, Akkommodation, Internalisierung sowie Externalisierung initiieren und fruchtbare Handlungsräume wie eine ZPD schaffen. Hierbei ist von einem erhöhten Auftreten dieser Prozesse als im Klassenverbund auszugehen, da die Gruppengröße beim PL die Partizipation jedes Individuums fordert und fördert, sodass die SuS mehr Möglichkeiten erhalten, Lücken und/oder falsche Annahmen innerhalb ihres mentalen Modells zu identifizieren (vgl. Swain, 2000) und mit Hilfe der Gruppe zu korrigieren.

PL eignet sich aufgrund seiner Mehrperspektivität und der Möglichkeit zur gegenseitigen und gemeinschaftlichen Regulation für „object-level change“ Lernprozesse, in welchen neue Erkenntnisse durch den Rückgriff auf sowie der gemeinschaftlichen Anwendung von bereits bestehendem Wissen und Fähigkeiten erworben werden. Der Erwerb kognitiver Lernstrategien sowie deren Anwendung zur Erreichung eines bestimmten Lernziels stellen fruchtbare Lernaktivitäten für „object-level“ Veränderungen beim PL dar. Zwar gilt es bestimmte deklarative, prozedurale und konditionale Wissensaspekte sowie Voraussetzungen von kognitiven Lernstrategien zunächst durch explizite Instruktion und Modellierung einzuführen, was eher „,meta-level“ Veränderungen entspricht. Allerdings eignet sich PL als Sozialform für die sich an die Instruktion und Modellierung anschließenden übungs-, unterstützungs- und reflexionsintensiven Prozesse des Erwerbs kognitiver Lernstrategien oder für die Anwendung kognitiver Lernstrategien zum Erwerb eines anderen Lerninhalts. Im Folgenden werden kognitive Lernstrategien allgemein und insbesondere Lesestrategien sowie Halbschriftliche Rechenstrategien näher betrachtet, da sie die Lerninhalte der dieser Studie zu Grunde liegenden Datengrundlage darstellen.

\subsection{Kognitive Lernstrategien beim Peer-Learning}

Bevor empirische Studien zu bilingualen Praktiken beim PL vorgestellt werden, sollen kognitive Lernstrategien beim PL betrachtet werden. Kognitive Lernstrategien -insbesondere Lesestrategien - werden häufig als Lerninhalt für PL-Formate implementiert (vgl. z. B. Fuchs et al., 1997; Munser-Kiefer \& Kirschhock, 2014; Philipp \& Schilcher, 2012; Spörer et al., 2016). Dement- 
sprechend soll im Folgenden zunächst geklärt werden, was kognitive Lernstrategien sind und wie diese möglichst effizient erworben und vermittelt werden können. Anschließend werden zentrale Befunde zur Lernwirksamkeit kognitiver Lernstrategien berichtet und Lese- sowie Halbschriftliche Rechenstrategien der Addition und Subtraktion näher betrachtet.

\subsubsection{Kognitive Lernstrategien}

Obwohl schon in den 1960er Jahren die Relevanz strategischer Handlungen beim Lernen nachgewiesen wurde (vgl. Hasselhorn \& Gold, 2017, S. 88), hielten kognitive Lernstrategien erst im Zuge der Mitte der 1990er Jahre geführten Debatten über die Aufgaben, Struktur, Funktionen und Effektivität von Schule in Deutschland Einzug in die Lehrpläne (vgl. Friedrich \& Mandl, 2006, S. 12). Friedrich und Mandl (ebd., S. 1) definieren kognitive Lernstrategien allgemein als ,jene Verhaltensweisen und Gedanken, die Lernende aktivieren, um ihre Motivation und den Prozess des Wissenserwerbs zu beeinflussen und zu steuern“. Die zwei zentralsten Eigenschaften von kognitiven Lernstrategien sehen Hasselhorn und Gold (2017, S. 88) in den für kognitive Lernstrategien invariablen, charakteristischen Merkmalen der Zielgerichtetheit sowie der notwendigen zusätzlichen Tätigkeiten, die über die inhärenten obligatorischen Verarbeitungsprozesse der jeweiligen Lernaktivität hinausgehen. Allerdings zeichnen sich kognitive Lernstrategien potentiell durch sechs weitere, ambivalente und zum Teil widersprüchliche Merkmale aus, da sie „(1) absichtlich, (2) bewusst und (3) spontan eingesetzt werden“ können, ,vom Lernenden (4) ausgewählt und (5) kontrolliert werden und dass der Strategieeinsatz (6) Anteile der begrenzten Kapazität des Arbeitsgedächtnisses verbraucht" (vgl. ebd.). Diese Ambivalenzen und zum Teil widersprüchlich anmutenden Eigenschaften sind allerdings notwendig, um die Bandbreite und Vielfalt kognitiver Lernstrategien zu erfassen. Hierbei ist von der Prämisse auszugehen, dass nicht alle diese Merkmale beim Lernstrategieeinsatz umgesetzt werden müssen. Die zuvor angeführten invariablen und eben dargelegten flexiblen Aspekte berücksichtigen Hasselhorn und Gold (ebd., S. 89) in ihrer Definition von kognitiven Lernstrategien: „Unter Lernstrategien versteht man Prozesse bzw. Aktivitäten, die auf ein Lern- oder Behaltensziel ausgerichtet sind und die über die obligatorischen Vorgänge bei der Bearbeitung einer Lernanforderung hinausgehen. Lernstrategien weisen wenigstens eine zusätzliche Eigenschaft auf, indem sie entweder intentional, bewusst, spontan, selektiv, kontrolliert und/oder kapazitätsbelastend sind bzw. eingesetzt werden."

Für einen effektiven Einsatz strategischer Lernhandlungen gilt es über deklaratives, prozedurales und konditionales Wissen über kognitive Lernstrategien zu verfügen (vgl. Bräuer, 2010, S. 99ff; Philipp, 2015, S. 49). Lerner benötigen zunächst deklaratives Wissen darüber, welche Lernstrategien es gibt. 
Darüber hinaus müssen sie über prozedurales Wissen einzelner Lernstrategien verfügen, damit sie diese auch korrekt umsetzen können. Zudem sollten sie über konditionales Wissen verfügen, mit welchem sie beurteilen können, wann sich eine Strategie abhängig vom Ziel der Lernaktivität eignet. Hierbei gilt es darauf hinzuweisen, dass - abhängig vom jeweiligen Lerngegenstand - kognitive Lernstrategien in ihrer Wirksamkeit nützliche bis gar notwendige Werkzeuge für Lernaktivitäten darstellen.

Aufgrund der Vielgestaltigkeit und der schieren Menge an potentiellen kognitiven Lernstrategien wird versucht, diese in Form von Taxonomien zu organisieren. Allerdings dürfen diese Taxonomien nicht als absolute, trennscharfe Kategorisierungen interpretiert werden, da einzelne Strategien sich durch Multifunktionalität auszeichnen und somit für verschiedene Zwecke eingesetzt werden können (vgl. Friedrich \& Mandl, 2006, S. 2; Philipp, 2015, S. 47). Eine der prominentesten Taxonomien von kognitiven Lernstrategien stellt die Differenzierung nach ,kognitiven Strategien, metakognitiven Strategien und Stützstrategien des externen Ressourcenmanagements" (Hasselhorn \& Gold, 2017, S. 89) dar.

Innerhalb der kognitiven Lernstrategien können Wiederholungs-, Organisations- und Elaborationsstrategien unterschieden werden, die die konkrete Bearbeitung des Lernstoffes betreffen und funktional nach ihrem Zweck differenziert werden können (vgl. ebd., S. 90ff). Wiederholungsstrategien fokussieren das Halten von Informationen im Arbeitsgedächtnis zur Transferierung in das Langzeitgedächtnis und somit zur dauerhaften Speicherung, indem z. B. bestimmte Fakten auswendig gelernt werden. Organisationsstrategien zielen hingegen auf eine Reduktion und Verknüpfung der zentralen Inhalte des neu zu lernenden Stoffes ab und werden dementsprechend primär für die Organisation und Strukturierung von Inhalten eingesetzt. Hierbei werden unter anderem die relevanten Informationen identifiziert, sinnvoll zueinander in hierarchische, zeitliche und/oder funktionale Beziehungen gesetzt und zu größeren Sinneinheiten gebündelt, was die Anzahl an im Arbeitsgedächtnis zu haltenden Einheiten verringert und somit dieses entlastet. Geeignete kognitive Organisationsstrategien zur Strukturierung des zu lernenden Materials stellen z. B. Mindmaps, Zusammenfassungen oder Flussdiagramme dar. Elaborationsstrategien dienen dem Verständnis des zu lernenden Stoffes, indem die relevanten Inhalte näher ausgearbeitet werden, um diese mit dem Vorwissen sinnvoll zu verknüpfen. Diese Strategien werden auch als generative Strategien bezeichnet, da die Verknüpfung mit dem Vorwissen zur Konstruktion oder zumindest zur Modifikation von mentalen Modellen führen kann. Hierfür eignen sich z. B. Analogiebildungen, Generierung von Beispielen, Verknüpfungen mit Alltagsphänomenen herstellen oder weiterführende Fragen zur Thematik erzeugen.

Metakognitive Strategien, deren Grundlagen bereits im Abschnitt 5.3.2.2 beschrieben wurden, fokussieren im Gegensatz zu kognitiven Lernstrategien nicht den Lerninhalt, sondern die beim Lernen stattfindenden Lernprozesse der 
Lerner. Diese Strategien werden auch als „Selbstkontroll- und Selbstregulationsstrategien“ bezeichnet, die die ,situations- und aufgabenangemessene Steuerung des Lernprozesses samt Planung, Überwachung, Bewertung und Regulation“ (Friedrich \& Mandl, 2006, S. 5) fokussieren. Die Lerner planen, überwachen und kontrollieren während des Lernens ihre Lernaktivitäten und regulieren diese entsprechend der Anforderungen der Aufgabe und des Lernziels. So gilt es z. B. sich zunächst für das konkrete Vorgehen der Bearbeitung des Lernstoffes zu entscheiden, die Bearbeitung im Prozess zu überwachen und nach der Beendigung zu kontrollieren, ob das anvisierte Ziel tatsächlich erreicht wurde. Metakognitive Strategien können sich hierbei sowohl auf das Lernen selbst als auch auf die konkrete, kritisch reflektierte Anwendung von kognitiven Lernstrategien beziehen (vgl. Hasselhorn \& Gold, 2017, S. 92).

Die Stützstrategien des externen Ressourcenmanagements betreffen die Lernumwelt des Lerners, welche möglichst angemessen zu strukturieren ist (vgl. ebd., S. 88f). Hierzu gehört unter anderem die Gestaltung des Arbeitsplatzes, die Nutzung von lernförderlichen Angeboten wie z. B. Bibliotheken, Sprachlaboren, Datenbanken, Computerräumen, aber auch die Bildung von Arbeitsgruppen zum kooperativen Lernen (vgl. ebd.)

Neben diesen drei übergeordneten Strukturierungsdimensionen nach Hasselhorn und Gold (ebd., S. S. 90ff) stellt die Spezifizität von kognitiven Lernstrategien (vgl. Philipp, 2015, S. 44f) eine weitere fruchtbare Dimension zur Kategorisierung dieser dar. Kognitive Lernstrategien unterscheiden sich anhand der Vielfältigkeit ihrer Anwendungsmöglichkeiten, wobei Philipp (ebd.) zwischen allgemeinen, domänenspezifischen und aufgabenspezifischen Strategien differenziert. Allgemeine Strategien zeichnen sich durch ihre prinzipiell universellen Einsatzmöglichkeiten aus. So kann z. B. die Organisationsstrategie „Zusammenfassen“, bei welcher die zentralen Inhalte eines Gegenstandes identifiziert und in komprimierter Form paraphrasiert werden, auf eine Vielzahl verschiedener Lerninhalte angewandt werden. Domänenspezifische Strategien sind in ihrer Anwendung auf eine bestimmte Domäne beschränkt. So können z. B. Lesestrategien zwar für jeden beliebigen Text eingesetzt werden, allerdings sind sie nicht ohne weiteres auf andere Medien wie Bilder, Musik, Filme etc. anwendbar. Ähnliches gilt auch für aufgabenspezifische Strategien, deren Spezifizität allerdings noch höher und deren Anwendungsmöglichkeiten dementsprechend weiter eingeschränkt sind. Hierzu zählt z. B. die halbschriftliche Rechenstrategie „Ergänzen“, die nur bei Subtraktionsaufgaben appliziert werden kann. Auch wenn domänen- und aufgabenspezifische kognitive Strategien auf allgemeinen kognitiven Strategien basieren, stellen diese Modifikationen für einen bestimmten Zweck dar und benötigen domänen- sowie aufgabenspezifisches Wissen und Fertigkeiten.

Zusammenfassend lässt sich schließen, dass Lernstrategien einen ,im Kern modularen Charakter" (ebd., S. 46) haben, sodass diese sowohl einzeln als auch im Verbund durch Verknüpfung und/oder Sequenzierung angewandt 
werden können. Sie zeichnen sich allgemein durch Zielgerichtetheit sowie durch zusätzlich zum eigentlichen Lerninhalt zu vollziehende Arbeitsprozesse und im Speziellen durch weitere, je nach Lernstrategie divergierende variable Eigenschaften aus (vgl. Hasselhorn \& Gold, 2017, S. 89). Zum einen können verschiedene Lernstrategien auf dasselbe Ziel hinarbeiten (vgl. Philipp, 2015, S. 46) wie z. B. die Erstellung von Mindmaps, Zusammenfassungen und Schaubildern, die alle als Organisationsstrategien umgesetzt werden können. Zum anderen können allerdings differente Zwecke mit den gleichen Strategien verfolgt werden (vgl. ebd., S. 47). So kann z. B. die Strategie der Textmarkierung als Organisationsstrategie zur Reduktion der relevanten Informationen genutzt werden, aber auch als metakognitive Überwachungsstrategie, mit der unbekannte Wörter markiert werden, deren Bedeutung es in einem späteren Schritt zu klären gilt. Im Folgenden wird der Erwerb und die Vermittlung kognitiver Lernstrategien näher betrachtet.

\subsubsection{Erwerb und Vermittlung kognitiver Lernstrategien}

Angesichts der benötigten deklarativen, prozeduralen und konditionalen Wissenskomponenten für einen effektiven Einsatz kognitiver Lernstrategien ist es nicht verwunderlich, dass der Erwerb von kognitiven Lernstrategien selbst eine anspruchsvolle, zeitintensive Lernaktivität darstellt. Die „Conceptual Change Theorie“ (vgl. Hasselhorn \& Mähler, 1998) kombiniert mit dem „Cognitive Apprenticeship Approach" nach Collins, Brown und Newman (1987) und dem graduellen Transfer der Verantwortung für Lernprozesse nach Pearson und Gallagher (1983) stellen einen fruchtbaren Rahmen dar, um kognitive Lernstrategien individuell oder gemeinschaftlich im PL zu erwerben und zu vermitteln. In einer ersten Phase werden die kognitiven Lernstrategien unter vorheriger Aktivierung des Vorwissens der Lerner zunächst von einer Lehrkraft explizit erklärt und modelliert. Hierbei erwerben die Lerner primär deklaratives und konditionales Wissen. Danach beginnen die Lerner in der zweiten Phase (gemeinsam) die kognitiven Strategien auszuprobieren und anzuwenden, wobei die Unterstützung der Lehrkraft graduell abnimmt, sodass die Verantwortlichkeit der Lerner für die Anwendung der kognitiven Lernstrategien sukzessiv ansteigt. Die Lerner üben (gemeinsam) eigenständig weiter, um den Einsatz der Strategien langfristig zu automatisieren. Bei Bedarf können sie hierbei die Hilfe der Lehrkraft oder anderer Peers in Anspruch nehmen, welche sie durch Prozesse des „Coachings“ oder „Scaffoldings“ unterstützen. In dieser zweiten Phase entwickeln die Lerner allmählich das benötigte prozedurale Wissen der $\mathrm{zu}$ erwerbenden kognitiven Lernstrategien. In einer dritten Phase explorieren sowie transferieren die Lerner (gemeinsam) die zu erwerbenden kognitiven Strategien und artikulieren sowie reflektieren ihre bisherigen Erfahrungen. Die 
damit einhergehenden Elaborationsprozesse vertiefen ihr deklaratives, prozedurales und konditionales Wissen zu kognitiven Lernstrategien.

In der zweiten Phase des Erwerbs kommt es in der Regel zu sogenannten Produktionsdefiziten (vgl. Hasselhorn \& Gold, 2017, S. 96). In diesem Stadium verfügen die Lerner zwar über die grundlegenden Handlungsschritte von kognitiven Lernstrategien, sie setzen diese allerdings nicht spontan sowie selbstinitiiert ein und sind ebenfalls noch nicht von der Fruchtbarkeit des Einsatzes von Lernstrategien überzeugt (ebd.). Aber selbst wenn Lerner im weiteren Verlauf spontan kognitive Lernstrategien einsetzen, müssen sich kognitive Lernstrategien noch nicht zwingend positiv auf die Lernaktivität auswirken, da vor dem effizienten Einsatz dieser zum Teil Nutzungsdefizite auftreten (vgl. ebd., S. 96f). Die kognitiven Lernstrategien werden zwar selbstinitiiert eingesetzt, wirken sich aber noch nicht lernwirksam aus. Hierbei gilt es darauf hinzuweisen, dass die Anwendung von kognitiven Lernstrategien zu Beginn zusätzlichen „Cognitive Load“ erzeugt. Die Leistungen können zunächst sogar abnehmen, da das Arbeitsgedächtnis zusätzlich belastet wird, was auf Kosten der eigentlichen, übergeordneten Lernaktivität gehen kann (vgl. ebd.). Dementsprechend könnte dem Nutzungsdefizit eine fehlende Automatisierung sowie unzureichendes konditionales Wissen zu Grunde liegen, aber auch durch Motivationsdefizite in Folge der zusätzlichen Arbeitsgedächtnisbelastung sowie dem ausbleibenden Lernerfolg verursacht werden (vgl. ebd., S. 97).

\subsubsection{Empirische Evidenz zur Lernwirksamkeit von kognitiven Lernstrategien}

Kognitiven Lernstrategien wird insgesamt eine hohe Lernwirksamkeit zugesprochen (für einen Überblick siehe z. B. Mandl \& Friedrich, 2006; Martin \& Nicolaisen, 2015, S. 43-48). Während Martin und Nicolaisen (ebd.) einige ausgewählte, groß angelegte Studien zur Wirksamkeit von Lernstrategien hinsichtlich kognitiver und motivationaler Outcomes berichten, werden diverse einzelne (meta-)kognitive, motivationale sowie emotionale Strategien, Stützstrategien und übergreifende Aspekte zu Lernstrategien und deren Lernwirksamkeit in den einzelnen Beiträgen der Herausgeberschaft von Mandl und Friedrich (2006) thematisiert. Allerdings sind die Effektgrößen abhängig von der Operationalisierung kognitiver Lernstrategien sowie der damit verbundenen abhängigen Variable (vgl. ebd., S. 12f). So erzeugen kognitive Lernstrategien, die über retrospektive Angaben zur Nutzung dieser in Fragebogenskalen operationalisiert wurden, eher schwache Effekte auf Lernleistungen. Zudem wirken sich kognitive Lernstrategien nur geringfügig positiv auf Lerninhalte aus, die nicht zwingend mit kognitiven Lernstrategien bearbeitet werden müssen wie z. B. das Auswendiglernen von Fakten. Die abhängige Variable muss sich dementsprechend auch für kognitive Lernstrategien eignen, um wirksame 
Effekte von kognitiven Lernstrategien hervorzurufen (vgl. ebd., S. 13). Im Gegensatz hierzu weisen Studien, die kognitive „Lernstrategien prozessnah bzw. handlungsnah erfassen" sowie für angemessene Lernaufgaben wie z. B. Verstehensleistungen bei Problemlöseaufgaben einsetzen, hohe und robuste Effekte von kognitiven Lernstrategien auf den Lernerfolg auf (ebd., S. 12f).

Kognitive Lernstrategien rufen positive Effekte bei Kindern (vgl. z. B. Whitebread et al., 2009), Jugendlichen (vgl. z. B. Artelt et al., 2002; Martin, 2014) sowie Studierenden (für einen Überblick siehe Streblow \& Schiefele, 2006) hinsichtlich ihrer Lernleistungen hervor. Gemäß Hatties (2014, S. 224ff) „Meta-Meta-Studie“ der empirischen Unterrichtsforschung sind kognitive Lernstrategien äußerst lernwirksam und rangieren auf dem vierten Platz der lernwirksamsten individuellen Faktoren nach der Selbsteinschätzung des eigenen Leistungsniveaus, dem Vorwissen und der Intelligenz (vgl. Martin \& Nicolaisen, 2015, S. 47). Hinsichtlich der Leseleistung stellt das Wissen über Lernstrategien gemeinsam mit der Lesegeschwindigkeit den zweitbesten Prädiktor nach kognitiven Grundfähigkeiten dar (vgl. Artelt et al., 2002). Erfolgreiche und nicht erfolgreiche Lerner unterschieden sich in der Studie von Martin (2014), ,in erster Linie aufgrund ihrer Lernstrategie-Kompetenz und -Erfahrung“, wobei diejenigen Lerner am besten abschneiden, „die ein breites Lernstrategie-Repertoire haben und dieses auch gezielt einsetzen" sowie häufig darauf zurückgreifen (vgl. Martin \& Nicolaisen, 2015, S. 45). Lernstrategien wirken nicht nur positiv auf kognitive Outcomes, sondern weisen signifikante, hohe positive Zusammenhänge mit lernrelevanten motivationalen Variablen wie z. B. Lernfreude, Selbstwirksamkeit, Schulisches Selbstkonzept und Selbstständigkeit auf (vgl. Martin 2014). Diese Zusammenhänge gelten für kognitive, metakognitive sowie motivationale Lernstrategien und für Stützstrategien, wobei die gemittelte Aggregation dieser vier Strategietypen für fast alle motivationalen abhängigen Variablen die höchsten Zusammenhänge birgt (vgl. Martin \& Nicolaisen, 2015, S. 48).

Allerdings existiert widersprüchliche Evidenz hinsichtlich der konkreten Wirkmechanismen und Zusammenhänge von verschiedenen kognitiven Lernstrategietypen untereinander sowie hinsichtlich bestimmter abhängiger Variablen. So gibt es z. B. zum einen empirische Befunde dafür, dass kognitive Lernstrategien vermittelt über metakognitive Lernstrategien positiv auf den Lernerfolg wirken, zum anderen allerdings auch Evidenz, die eher kognitiven Strategien den Status als Mediator zuspricht (vgl. Friedrich \& Mandl, 2006, S. 14f). Ähnliche widersprüchliche Ergebnisse zu den konkreten Wirkmechanismen von kognitiven Lernstrategien zeigen sich auch hinsichtlich motivationaler Outcomes sowie der Beziehung von Lernerfolg, aufgewandter Lernzeit und kognitiven Lernstrategien (vgl. ebd., S.15). 


\subsubsection{Lesestrategien}

Lesestrategien sind domänenspezifische Lernstrategien, deren übergeordnetes Ziel es ist, den Leser bei der Konstruktion eines adäquaten, kohärenten Textmodells zu unterstützen (vgl. Philipp, 2015). Lesestrategien stellen besonders relevante domänenspezifischen Lernstrategien dar, da sie prinzipiell auf jeden Text angewandt werden können (vgl. ebd., S. 42). Insbesondere für schulische Lernprozesse und -aktivitäten stellt das Lesen einen zentralen Modus zur Wissensaneignung dar. Abhängig von der Textschwierigkeit sowie der Komplexität des im Text vermittelten Inhalts können Lesestrategien zum Teil gar als eine notwendige Bedingung zur Konstruktion eines adäquaten Textmodells sowie des mentalen Modells des im Text dargestellten Inhalts interpretiert werden.

Im Allgemeinen können die oben angeführten Merkmale, Eigenschaften, Wirksamkeiten, Erwerbs- sowie Vermittlungsbedingungen und -herausforderungen von kognitiven Lernstrategien auch auf Lesestrategien übertragen werden. So gilt z. B. auch für Lesestrategien, dass unterschiedliche Lesestrategien spezifisches deklaratives, prozedurales und konditionales Wissen benötigen, distinkte sowie dieselben Ziele fokussieren können, einzeln, in Kombination sowie in Sequenzen angewandt werden können, ihre Nutzung abhängig von der Intention des Lesers ist etc. (vgl. Philipp, 2015, S. 42-47; Rosebrock \& Nix 2008, S. 59ff). Zudem sind die positiven Effekte von Lesestrategien auf Lesekompetenz ebenfalls vielfältig und robust belegt (vgl. z. B. Artelt et al., 2002). Dementsprechend soll im Folgenden lediglich ein näherer Blick sowohl auf die Lesestrategien „Wortbedeutungen klären“, „Zusammenfassen“ und „Vorhersagen", welche auch in der Intervention der vorliegenden Dissertation vermittelt werden, als auch auf Lesestrategien beim Peer-Learning geworfen werden.

Die Strategie „Wortbedeutungen klären“ fokussiert für den Leser unverständliche Wörter, deren Semantik sich aufgrund ihres Fehlens im mentalen Lexikon nicht ergeben und/oder im satzsemantischen Kontext nicht inferiert werden können. Das Ziel der Strategie ist es, die Semantik dieser Wörter zu klären und in den satzsemantischen Kontext sinnvoll zu integrieren. Dekodierprozesse auf der Wortebene erfolgen nach dem „Dual Route Model“ (vgl. Coltheart et al., 2001) durch einen holistischen, direkten Zugriff auf das Wort, sofern dieses im sogenannten Sichtwortschatz bereits gespeichert vorliegt, oder durch sukzessiv synthetisierendes „Erlesen“ des Wortes. Angesichts des „Simple View of Reading“ (Hoover \& Gough, 1990) reichen Dekodierprozesse zum Leseverständnis alleine allerdings nicht aus, sondern benötigen ebenso linguistische Verstehensprozesse. Hinsichtlich Wortbedeutungen können linguistische Verstehensprozesse als Zugriff auf die Wortbedeutung im mentalen Lexikon oder als das Ziehen von Inferenzen auf Basis der im Text repräsentierten Informationen interpretiert werden. Das flüssige Dekodieren 
von Wörtern sowie deren Verständnis und Integration in die Satzsemantik zur Konstruktion der Satzproposition stellen zentrale Anforderungen der hierarchieniedrigen Prozessebene im Lesen dar (vgl. Rosebrock \& Nix 2008, S. 17ff). Diese Ebene darf in ihrer Bedeutung nicht unterschätzt werden, da aufgrund der Limitierung der Arbeitsgedächtniskapazität automatisierte Dekodierprozesse sowie linguistische Verstehensprozesse hierarchiehöhere Textverständnisprozesse wie z. B. die globale Kohärenzbildung erst ermöglichen (vgl. ebd.).

Die Strategie „Wortbedeutungen klären“ kann zunächst den metakognitiven Lernstrategien zugeordnet werden, da bei der Überwachung des Leseprozesses oder bei der Kontrolle des Verständnisses des Textes unverständliche Wörter und Proposition identifiziert werden. Allerdings weist diese Strategie eine weitere zentrale Komponente neben der metakognitiven Identifikation von Verständnisschwierigkeiten auf, nämlich die Lösung dieser durch die Klärung der Wortbedeutung. Hierfür gilt es sich die Bedeutung des Wortes durch den Rückgriff auf weitere Informationen wie z. B. den Text selbst oder Lexika zugänglich zu machen oder sich Hilfe in Form einer Erklärung der Wortbedeutung von einer anderen Person einzuholen. Diese zweite Komponente entspricht eher den Stützstrategien des externen Ressourcenmanagements (vgl. Hasselhorn \& Gold, 2017, S. 88f).

Die Strategie „Zusammenfassen“ zielt auf die Reduktion der im Text repräsentierten Informationen ab, indem die relevantesten Propositionen identifiziert, paraphrasiert und sinnvoll miteinander verknüpft werden. Hierfür müssen Leser sowohl lokale als auch globale Kohärenzen bilden, indem sie zum einen die zentralen Propositionen von einzelnen Abschnitten, zum anderen aber auch des ganzen Textes erfassen und sinnig miteinander in Beziehung setzen (vgl. Rosebrock \& Nix 2008, S. 17ff). Hierfür sind, wie oben bereits angedeutet, automatisierte hierarchieniedrige Prozesse notwendig, damit genügend Arbeitsgedächtniskapazität vorhanden ist, um die Kohärenzbildung zu vollziehen. Allerdings dürfen die lokale und globale Kohärenzbildung nicht bereits als Formen der Lesestrategie "Zusammenfassen“ missverstanden werden. Diese bilden eher die Grundlage für das Zusammenfassen, mit welchen der Leser abhängig von seinem Vorwissen, Lesezielen und -intentionen die für ihn zentralen Propositionen in komprimierter Form paraphrasiert und verbindet. Auf inhaltlicher Ebene zeichnen sich Zusammenfassungen primär durch die Identifikation der zentralen Protagonisten oder Themen sowie der relevanten Propositionen über diese aus. Auf formaler Ebene sollten die komprimierten Paraphrasierungen der zentralen Propositionen in möglichst eigenen, sich von der Textgrundlage unterscheidenden Formulierungen artikuliert werden, wobei es insbesondere auf Details zu verzichten gilt (vgl. z. B. Fuchs et al., 1997). 
Dementsprechend stellt die Lesestrategie „Zusammenfassen“ primär eine prototypische Organisationsstrategie dar, die auf die Reduktion von Informationen durch die Identifikation und Verknüpfung der Relevantesten abzielt. Prinzipiell kann diese Strategie aber auch als metakognitive Strategie zur Kontrolle des Textverständnisses verwendet werden. Wenn die zentralen Propositionen eines Textes in komprimierter Form paraphrasiert werden können, dann kann dies als mindestens rudimentäres Textverständnis gewertet werden.

Bei der Strategie „Vorhersagen“ werden auf Basis des bisherigen Textmodells anhand von Teilen des Textes Prognosen über die folgenden Inhalte oder Propositionen aufgestellt. „Vorhersagen“ zielt dementsprechend auf eine Antizipation zukünftiger Textinhalte $a b$, ohne über weitere Informationen mit Ausnahme der bisher gelesenen Textteile sowie des individuellen Vorwissens zu verfügen. Für diese Strategie sind neben lokalen und globalen Kohärenzen vor allem die hierarchiehöheren Prozesse des Erkennens von Superstrukturen, also der internen Organisation des Textes, und der Identifikation von Darstellungsstrategien, worunter im allgemeinen die übergeordnete Zielsetzung des Textes zu verstehen ist (vgl. Rosebrock \& Nix 2008, S. 17ff), relevant. Sowohl das Erkennen von Superstrukturen als auch die Identifikation von Darstellungsstrategien basieren neben dem mentalen Textmodell der Leser zum einen auf dem Vorwissen der Leser zu dem im Text behandelten Thema, zum anderen aber auch von ihrem Wissen über Textsorten. So unterscheiden sich literarische Texte von Sach- und Informationstexten deutlich in ihrer internen Strukturlogik und deren übergeordneter Zielsetzung (vgl. Rosebrock, 2007).

Angesichts dessen kann die Lesestrategie „Vorhersagen“ primär als eine Elaborationsstrategie beschrieben werden, bei der durch die Verbindung des aktuellen mentalen Textmodells und des Vorwissens zukünftige Textinformationen antizipiert werden. Allerdings enthält diese Strategie auch eine metakognitive Komponente, da hiermit das Verständnis des aktuellen Textmodells dahingehend geprüft werden kann, ob dieses für das Vorhersagen zukünftiger Textinhalte ausreicht.

Die drei Strategien „Wortbedeutungen klären“, „Zusammenfassen“ und „Vorhersagen“ lassen sich hervorragend sequentiell anwenden. Mit dem Klären unbekannter Wortbedeutungen erzeugt der Leser die Grundlage, sich die Inhalte eines Textes oder Textabschnitts auf der Oberfläche zugänglich zu machen. Daran anschließend kann mit der Organisationsstrategie des Zusammenfassens eine dem Leseziel entsprechende Identifikation der zentralen Inhalte vorgenommen werden, die zu Reduktion der Textinformationen führt. Die hieraus entstandene Paraphrase des relevanten Inhalts des Textes oder Abschnitts sowie das Vorwissen des Lesers zum Text selbst als auch zu der Thematik des Textes kann daraufhin als Basis für die Antizipation der folgenden Inhalte genutzt werden. Hieraus entsteht eine Erwartungshaltung, die als Orientierung die Aufmerksamkeit im sich anschließenden Leseprozess leiten kann. Durch 
diese Sequenz werden alle zentralen Aspekte der Prozessebene von Lesekompetenz (vgl. Rosebrock \& Nix, 2008) explizit berücksichtigt und durch bewusste, strategische Handlungen reflektiert, was sich förderlich auf die Konstruktion des Textmodells auswirken sollte.

Die Sozialform des PL zum Erwerb sowie zur Einübung von Lesestrategien eignet sich nicht nur hinsichtlich der Förderung der Prozessebene der Lesekompetenz, sondern adressiert ebenfalls sowohl die Subjektebene als auch die soziale Ebene von Lesekompetenz (vgl. Rosebrock \& Nix, 2008, S. 21ff). Die Subjektebene der Lesekompetenz repräsentiert das Wissen, die Beteiligung, die Motivation sowie die Einstellung des Lesers, welche der Leser in den Leseprozess einbringt und die im Leseprozess vom Text beansprucht sowie beeinflusst werden (vgl. ebd.). PL kann diesbezüglich die Partizipation sowie Motivation mit der Auseinandersetzung des Textes erhöhen und sich somit positiv auf lernrelevante motivationale Variablen wie z. B. das Selbstkonzept auswirken (vgl. z. B. Martin, 2014; Robinson, Schofield \& Steers-Wentzell, 2005). Die soziale Ebene der Lesekompetenz akzentuiert die Anschlusskommunikation als Bestandteil des Lesens, welcher nicht nur als isolierter, individueller Prozess zu interpretieren ist, sondern Gesprächsanlässe erzeugt, die kommunikativ im Anschluss an die Lektüre ausgehandelt werden können (vgl. Rosebrock \& Nix, 2008, S. 23ff). Angesichts der kooperativen Grundstruktur samt positiver Interdependenz, individueller Verantwortlichkeit und möglichst förderlicher Interaktion (vgl. Johnson \& Johnson, 1999) fordert die Sozialform des PLs die Gruppenmitglieder auf, ihr jeweiliges Wissen und ihre Erkenntnisse über den Text sowie dessen Thema in den kollaborativen Dialog einzubringen, zu erläutern, begründen und zu erklären (vgl. z. B. Howe, 2010; Littleton \& Mercer, 2010).

In der Gesamtschau lässt sich konstatieren, dass sich PL aufgrund seiner Kongruenz zur Prozess-, Subjekt- und sozialen Ebene der Lesekompetenz nach Rosebrock und Nix (2008) für die Vermittlung und den Erwerb von Lesestrategien eignet. Die Lesestrategien selbst operieren zwar hinsichtlich ihrer Zielsetzung primär auf der Prozessebene der Lesekompetenz, können allerdings in ihrer Anwendung in der Sozialform des PLs auf der subjektiven und sozialen Ebene zusätzlich wirken. Dementsprechend werden beim PL zu Lesestrategien alle Ebenen der Lesekompetenz adressiert und aktiviert. Zudem stellen die Lesestrategien Wörter klären, Zusammenfassen und Vorhersagen relativ ergebnisoffene Lerntätigkeiten dar, bei welchen die Mehrperspektivität des PL fruchtbar die Aushandlungsprozesse sowie Lösungen bereichern kann. 


\subsubsection{Halbschriftliche Rechenstrategien bei Addition und Subtraktion}

Halbschriftliche Rechenverfahren lassen sich mathematikdidaktisch zwischen dem Kopfrechnen und dem schriftlichen Rechnen einordnen (vgl. Padberg, 2011). Beim Kopfrechnen werden Rechenprozesse mental ohne äußere Hilfsmittel gelöst und beim Schriftlichen Rechnen werden arithmetische Aufgaben mit einem invariablen, gleichförmigen, notationsgestützten Rechenverfahren gelöst, wobei für jeden mathematischen Operator ein eigenes Verfahren vorliegt. Halbschriftliche Rechenverfahren zeichnen sich ähnlich wie schriftliche Rechenverfahren durch schrittweise, notationsgestützte Rechenprozesse aus. Das zu lösende arithmetische Problem wird hierbei aber entweder so manipuliert, dass es in einem Rechenschritt zu lösen ist oder sukzessive in einzelne Rechenoperationen zerlegt wird, wobei mit den resultierenden Zwischenergebnissen dieser Einzelschritte weiter gerechnet wird, bis die Aufgabe gelöst ist. So wird z. B. beim „Schrittweise Rechnen“, welches ein in der Schule vermitteltes und von SuS häufig eingesetztes Globalverfahren des Halbschriftlichen Rechens darstellt, der zweite Summand der Aufgabe $657+248$ in seine Stellen zerlegt und sukzessive mit dem ersten Summand verrechnet, wobei die Zwischenergebnisse ab dem ersten Rechenschritt zum neuen ersten Summanden werden. Dementsprechend wird zunächst 657 mit 200 addiert, anschließend 857 mit 40 und abschließend 897 mit 8 verrechnet, sodass die Lösung 905 in insgesamt drei Schritten erzeugt wird.

Im Gegensatz zu den Schriftlichen Rechenverfahren sind Halbschriftliche Rechenverfahren allerdings weder an eine verbindliche Notationsform gebunden noch auf starre, immer gleiche Bearbeitungsprozesse, die Algorithmen ähneln, festgelegt. Halbschriftliche Rechenverfahren zeichnen sich vielmehr durch ihre Vielfältigkeit, Flexibilität und Adaptivität in der Anwendung aus, sodass sie häufig mit strategischen Rechenprozessen verbunden werden (vgl. Selter, 2009). Für manche Autoren stellt diese strategische Komponente gar ein definitorisches Element von Halbschriftlichen Rechenverfahren dar. So definiert z. B. Bauer (1998, S. 180) Halbschriftliches Rechnen als ,ein flexibles, je auf die Besonderheit der vorliegenden Aufgaben und des Zahlenmaterials bezogenes Rechnen unter Verwendung geeigneter Strategien“, bei dem „Zwischenschritte, Zwischenrechnungen, Zwischenergebnisse fixiert bzw. Rechenwege verdeutlicht sowie Rechengesetze und Rechenvorteile ausgenutzt" werden. Neben der explizit notierten schrittweisen Bearbeitung von Rechenaufgaben akzentuiert diese Definition ein an der konkreten Aufgabenstellung orientiertes Vorgehen, welches flexibel spezifische Eigenschaften der Rechenaufgabe berücksichtigt und produktiv zur Lösung dieser nutzt. Dementsprechend vereinfachen Halbschriftliche Rechenverfahren den Rechenprozess durch eine schriftlich fixierte und somit notationsgestützte Manipulation des originären 
mathematischen Problems unter Ausnutzung dessen spezifischer Eigenschaften.

Die Mathematikdidaktik fokussiert seit mittlerweile fast drei Dekaden stärker flexible, adaptive Fähigkeiten von SuS zur Lösung mathematischer Probleme statt einer unreflektierten Bearbeitung mathematischer Aufgaben mit hoher Lösungswahrscheinlichkeit im Bereich der Arithmetik: „The emphasis in teaching arithmetic has changed from preparation of disciplined human calculators to developing children's abilities as flexible problem solvers" (Anghileri, 2001, S. 79). SuS sollen heutzutage im Mathematikunterricht eher ,adaptive expertise" entwickeln, die sich durch einen effizienten, kreativen und flexiblen Einsatz eines Repertoires an erworbenen Rechenstrategien auszeichnet (vgl. Torbeyns et al., 2009, S. 581). Dieser Forderung entsprechend nimmt seit den 1990er Jahren auch die Bedeutung des Halbschriftlichen Rechnens in der Didaktik der Grundschulmathematik in Deutschland zu und wurde kurz darauf fest in den Lehrplänen der einzelnen Bundesländer verankert (vgl. Padberg, 2011, S. 172f; Ratgheb-Schnierer, 2014, S. 28). Halbschriftliche Verfahren werden hierbei vor allem im Tausenderraum (Zahlenraum von 0-1000), prinzipiell aber auch im Hunderterraum (Zahlenraum von 0-100) in der Grundschulmathematik verwendet. Mathematische Aufgaben in diesen Zahlenräumen überfordern die Arbeitsgedächtniskapazitäten der SuS beim Kopfrechnen im Verlauf der ersten Grundschuljahre, sodass halbschriftliche Verfahren mit expliziter Notation von Zwischenschritten hier kognitiv entlastend wirken (vgl. Padberg, 2011, S. 169f).

Rechenstrategien bei der Addition und Subtraktion mit halbschriftlichen Rechenverfahren stellen die zentralen Inhalte der Rechenstrategieintervention der vorliegenden Studie dar und werden dementsprechend im Folgenden fokussiert, wobei die verschiedenen Rechenstrategien erst im Abschnitt 7.1.2.2 konkret benannt und erläutert werden. Die Halbschriftlichen Rechenstrategien der Addition und Subtraktion unterscheiden sich deutlich von den bisher angeführten Lern- und Lesestrategien. Im Kern wird bei diesen Rechenstrategien eine Manipulation der originären Aufgabe vorgenommen, bei der die Aufgabenstellung und/oder die Rechenprozesse so modifiziert werden, dass es entweder zu einer Reduktion der Anzahl an Rechenschritten oder zu einer Vereinfachung der zu vollführenden Rechenoperationen kommt. So wird z. B. mit Hilfe der Strategie „Hilfsaufgabe“ (vgl. ebd., S. 182) ein mathematisches Problem wie 375 - 298 zunächst zum Term 375 - 300 transformiert, der angesichts des glatten Hunderters des Subtrahenden einfach zu lösen ist. Die Differenz dieses manipulierten Terms muss abschließend mit dem Wert zwei addiert werden, da dies die Modifikation der originären Aufgabe wieder aufhebt und zur korrekten Lösung führt. In Anbetracht der Definition von kognitiven Lernstrategien von Hasselhorn und Gold (2017, S. 89) erscheint eine Kategorisierung von halbschriftlichen Rechenstrategien als kognitive Lernstrategien zu- 
nächst schwierig. Die zwei zentralen Merkmale von Lernstrategien - die Fokussierung auf ein „Lern- oder Behaltensziel“" sowie zusätzliche, ,über die obligatorischen Vorgänge bei der Bearbeitung einer Lernanforderung“ hinausgehende Handlungen (ebd.) - scheinen zunächst nicht auf Halbschriftliche Rechenstrategien zuzutreffen. Wenn allerdings das Lösen halbschriftlicher mathematischer Aufgaben als Lernziel und die Manipulationen der originären Aufgabenstellung als zusätzliche Handlungsschritte interpretiert werden, dann können Halbschriftliche Rechenstrategien durchaus als kognitive Lernstrategien interpretiert werden und am ehesten den aufgabenspezifischen kognitiven Lernstrategien zugeordnet werden.

Theoretisch lassen sich zwei Perspektiven - der Strategiewahlansatz und der Emergenzansatz - zur Modellierung arithmetischer Rechenstrategien unterscheiden. Der Strategiewahlansatz (siehe z. B. Torbeyns et al. 2009) konzeptioniert arithmetische Rechenstrategien als ein Repertoire an Rechenstrategien, aus welchem adäquate Rechenverfahren in Abhängigkeit zu der jeweiligen zu lösenden Aufgabe flexibel ausgewählt werden. Im Gegensatz hierzu besagt der Emergenzansatz (vgl. Threlfall, 2009), dass Rechenstrategien nicht einfach invariabel und algorithmisch angewandt werden, sondern im Rechenprozess in Abhängigkeit vom individuellen Wissen und der konkreten Aufgabe entstehen. Dementsprechend beschreibt der Emergenzansatz die Anwendung von Rechenstrategien als ein situationsabhängiges, individuelles Reagieren auf spezifische Aufgabenmerkmale, welches zur Konstruktion von Rechenwegen während des Lösungsprozesses führt. Während beim ersten Ansatz Rechenstrategien vermittelt und als standardisierte Verfahren eingesetzt werden können, ist dies beim Emergenzansatz nicht möglich, da die Rechenstrategien erst im Prozess kreativ entstehen. Eine aktuelle quasiexperimentelle Interventionsstudie zum Vergleich beider Ansätze mit SuS der dritten Klasse, bei dem die didaktischen Konsequenzen der Ansätze identifiziert und umgesetzt wurden, kann keine Unterschiede in der Lernwirksamkeit zwischen den Ansätzen konstatieren (Schwabe et al., 2014). Unabhängig vom Niveau und den Fähigkeiten der SuS sind beide Ansätze effektiv, sodass der eine oder andere Ansatz oder eine Kombination beider zur Instruktion von arithmetischen Rechenstrategien verwendet werden kann.

Beide Ansätze akzentuieren allerdings die Merkmale Flexibilität, Kreativität und Adaptivität als zentrale Eigenschaften von arithmetischen Rechenstrategien. Während Flexibilität sich durch die Fähigkeit auszeichnet, zwischen verschiedenen Rechenstrategien zu wechseln, beschreibt Kreativität die Fähigkeit, neue Rechenstrategien zu erzeugen oder bereits erworbene Strategien zu modifizieren (vgl. Selter, 2009, S. 620). Im Gegensatz hierzu ermöglicht Adaptivität, Rechenstrategien anhand der spezifischen Merkmale der zu lösenden Aufgabe angemessen auszuwählen oder zu erschaffen (vgl. ebd.). Alle drei Konzepte lassen sich aber nur schwer voneinander trennen, da Flexibilität und Kreativität als Voraussetzung für Adaptivität interpretiert werden können (vgl. 
ebd.). Dies spiegelt sich auch in der Terminologie wider, da sowohl mit den Begriffen ,adaptive expertise“ (vgl. z. B. Torbeyns et al., 2009) als auch „flexibles Rechnen" (vgl. z. B. Ratgheb-Schnierer, 2014) arithmetische Rechenstrategien bezeichnet werden.

Flexibles Rechnen erfordert ein fundiertes Wissen über Zahlen und Rechenoperationen, die Automatisierung von mathematischen Basisfakten wie z. B. das „Einspluseins“, das Beherrschen strategischer Werkzeuge und das Erkennen von Aufgabenmerkmalen und Zahlbeziehungen (vgl. Ratgheb-Schnierer, 2014, S. 31). SuS verwenden zur Bearbeitung von Additions- und Subtraktionsaufgaben mit Halbschriftlichen Verfahren in der Regel aber nur eine Hauptstrategie zur Bearbeitung aller Aufgaben (vgl. Padberg, 2011, 196f). Bei der Addition nutzen SuS präferiert das Stellenweise Rechnen, während sie bei der Subtraktion das Schrittweise Rechnen bevorzugen (vgl. ebd., S. 193-194). Diese beiden Verfahren stellen streng genommen keine echten Rechenstrategien dar, da sie als Universalverfahren für alle arithmetischen Aufgaben angewandt werden können und keine spezifischen Aufgabenmerkmale zur Vereinfachung des Rechenprozesses ausnutzen. Dementsprechend gilt es Halbschriftliche Rechenstrategien explizit mit dem Fokus auf eine flexible, kreative und adaptive Anwendung zu instruieren (vgl. ebd., S. 196).

Zusammengefasst scheint sich die Sozialform des PLs ebenfalls für Halbschriftliche Rechenstrategien der Addition und Subtraktion zu eignen. Die Wahl eines adaptiven Lösungswegs sowie das flexible Anwenden von Strategien zeichnen sich durch eine gewisse Offenheit und Komplexität aus, die durch die Mehrperspektivität sowie durch die gegenseitige oder geteilte Regulation des PL unterstützt werden können. Die Peers können sich hierbei gegenseitig oder geteilt dahingehend überwachen, nicht nur lediglich eine Strategie einzusetzen, sondern verschiedene Strategien auszuprobieren. Insbesondere innerhalb der zweiten und dritten Phase des „Cognitive Apprenticeship Approach" (vgl. Collins, Brown \& Newman, 1987), in welchen das intensive Üben, Explorieren, Transferieren und Reflektieren der Lerninhalte fokussiert wird, kann die Mehrperspektivität des PL zur Schulung des Erkennens von Aufgabenmerkmalen und Zahlbeziehungen beitragen, indem die Vor- und Nachteile der Strategien in Abhängigkeit der Aufgabenmerkmale gemeinsam erprobt, diskutiert und schließlich ergründet werden können.

\subsubsection{Zusammenfassung}

Kognitive Lernstrategien können abhängig vom jeweiligen Lernziel als förderliche bis gar notwendige Lernaktivitäten betrachtet werden, die mittlerweile einen festen Bestandteil des schulischen Curriculums darstellen (vgl. Friedrich \& Mandl, 2006, S. 12). Sie stellen als zielgerichtete, das eigentliche Lernziel 
überschreitende Lernaktivitäten (vgl. Hasselhorn \& Gold, 2017, S. 88) aufgrund ihrer benötigten deklarativen, prozeduralen und konditionalen Wissensaspekte (vgl. Bräuer, 2010, S. 99ff; Philipp, 2015, S. 49) einen anspruchsvollen, eigenständigen Lerninhalt dar, deren Anwendung und Ausführung nicht zwangsläufig einem starren Handlungsmuster folgen und nicht stets in einer unikalen, allgemeingültigen Lösung münden müssen. Unter Verwendung der Sozialform des PL kann der Offenheit sowie der Komplexität kognitiver Lernstrategien durch die Mehrperspektivität respektive die gegenseitige oder geteilte Regulation der Peers beim PL fruchtbar begegnet werden, indem die verschiedenen Ideen der Gruppenmitglieder zur Lösung beitragen und die Gruppenmitglieder ihre Arbeitsprozesse gemeinschaftlich koordinieren sowie überwachen können.

PL eignet sich dementsprechend hervorragend als Methode und Sozialform für solche übungs-, unterstützungs- und reflexionsintensiven Erwerbs- und Vermittlungsprozesse zum Erwerb kognitiver Lernstrategien. Die Peers können phasenweise alleine und gemeinsam die Anwendung der Lernstrategien üben, hierbei als Modelle fungieren, sich gegenseitig helfen, überwachen sowie kontrollieren und gemeinsam die Chancen und Herausforderungen bei der Strategieanwendung diskutieren. Insbesondere in den Phasen des Produktionsund/oder Nutzungsdefizits beim Erwerb von kognitiven Lernstrategien (vgl. Hasselhorn \& Gold, 2017, S. 96f) könnte die gegenseitige Unterstützung und Regulation der Peers den kontinuierlichen, konsequenten Einsatz der Strategien initiieren sowie potentielle Motivationsverluste aufgrund fehlender Automatisierung, erhöhtem Arbeitsaufwand und zunächst ausleibendem Lernerfolg kompensieren.

Da beim PL als Lerngegenstand komplexere Lerninhalte und Probleme zu präferieren sind, die im besten Falle verschiedene, argumentativ zu begründende Perspektiven zulassen oder gar benötigen (vgl. Chinn \& Clark, 2013, S. 326), stellen kognitive Lernstrategien selbst oder kognitive Lernstrategien zur Lösung eines Problems oder für den Erwerb von Inhalten einen geeigneten Lerninhalt oder eine angemessene Lernaktivität für PL dar. Dementsprechend können kognitive Lernstrategien aufgrund ihrer Offenheit und Komplexität Diskurse sowie (ko-)konstruktive Aushandlungsprozesse innerhalb der Gruppen initiieren, die sich förderlich auf den Erwerb des im PL fokussierten Lernziels auswirken können. Diese Passung des Lerninhalts kognitiver Lernstrategien und der Sozialform PL spiegelt sich auch in der Vielzahl an publizierten PL-Förderprogrammen zur Vermittlung von Lernstrategien (vgl. Artelt, 2006, S. 346f) - insbesondere von Lesestrategien (vgl. Philipp \& Schilcher, 2012) wider. 


\subsection{Bilinguale Interaktion als Angebot beim Peer-Learning}

Der Abschnitt 5.3 hat gezeigt, dass der Interaktion beim PL eine zentrale Bedeutung zukommt. Die Interaktion ist hierbei nicht nur hinsichtlich der Bearbeitung der Lerninhalte und dem Wissenserwerb relevant, sondern auch für die Organisation, Koordination und gegenseitige sowie geteilte metakognitive Regulation der Zusammenarbeit in einer möglichst produktiven Weise zu vollziehen. Hinsichtlich des PL von bilingualen SuS gelten prinzipiell die gleichen Aspekte und Wirkmechanismen für konstruktive kooperative Interaktionen wie für monolinguale SuS mit einer Ausnahme: Bilingualen SuS stehen prinzipiell zwei Sprachen zur Interaktion beim PL zur Verfügung. Abhängig vom jeweiligen Schulkontext bestehen unterschiedliche Bedingungen, Voraussetzungen, Einstellungen und Perspektiven sowohl auf Schul- als auch Schülerseite hinsichtlich bilingualer Praktiken beim PL, welche bezüglich der Bewertung und Einordnung empirischer Studien zu bilingualen Praktiken beim PL berücksichtigt werden sollten. Dementsprechend gilt es den Forschungsstand zu bilingualen Praktiken beim PL differenziert nach den jeweiligen Erhebungskontexten einzuschätzen.

Untersuchungen im Kontext von bilingualen Bildungsprogrammen weisen auf Potentiale und Grenzen von bilingualen Praktiken beim PL unter scheinbar optimalen Bedingungen für bilinguale Interaktion beim PL hin. Die Schülerschaft bilingualer Bildungsprogramme verfügt über Erfahrung im bildungssprachlichen Umgang mit ihren beiden Sprachen hinsichtlich verschiedener schulischer Fächer sowie auch über schriftsprachliche Fähigkeiten in beiden Sprachen. Ihre Lehrkräfte sind zum Großteil ebenfalls bilingual, sodass sie beim PL auftretende bilinguale Praktiken in der Interaktion ihrer SuS verstehen und potentiell in der Lage sind, ihnen interaktions- und lernförderliche bilinguale Kommunikationsformen und -praktiken zu vermitteln. So können sie hierbei ihre SuS z. B. dahingehend sensibilisieren, dass lerninhaltsbezogene Propositionen mit verschiedenen Sprachen alternativ sprachlich konstruiert und durchdrungen werden können (vgl. z. B. Schüler-Meyer et al., 2017), Assertionen, Argumente und Erklärungen mit Sprachwechseln zu unterschiedlichen Darstellungen und Elaborationen dieser führen (vgl. z. B. Klingner \& Vaughn, 2000; Moschkovich, 2007) oder sprachvergleichende Betrachtungen metalinguistische Reflexionen über Wort- und Morphembedeutungen, Ambiguitäten und Textkonventionen elizitieren (vgl. Hopewell, 2011). Zudem vertreten bilinguale Bildungsprogramme liberale und pluralistische Sprachideologien (vgl. Baker, 2011, S. 208), welche ein förderliches, wertschätzendes Schulklima für bilinguale Interaktion als Bildungsressource impliziert. Allerdings verfügen bilinguale Bildungsprogramme nur scheinbar über optimale Bedingungen für bilinguales PL, da die häufig implementierte strikte Spra- 
chenseparation, einzelne Sprachen zu bestimmten Zeiten oder Fächern möglichst exklusiv zu verwenden, dem parallelen Gebrauch zweier Sprachen beim Lernen und der Interaktion widerspricht. Dementsprechend ist bei bilingualen Bildungsprogrammen mit strikter Sprachenseparation eine Unterbindung bilingualer Praktiken beim PL durch die Lehrkraft oder durch die SuS selbst zu erwarten. Lediglich in Fällen von bilingualer Bildung, die explizit Praktiken des Translanguaging implementieren, sowie in transitionalen Phasen bilingualer Bildungsprogramme, bei welchen der Rückgriff auf die (weitere) L1 temporär erlaubt sowie gefördert und unterstützt wird, dürften bilinguale Praktiken beim PL willkommen sein und von den förderlichen Rahmenbedingungen bilingualer Bildungsprogramme profitieren.

Im Kontext der Submersion können die Bedingungen, Voraussetzungen, Einstellungen und Perspektiven hinsichtlich bilingualer Praktiken beim PL als suboptimal bezeichnet werden. Bilinguale SuS mit MH verfügen in der Regel kaum über Erfahrung im bildungssprachlichen sowie schriftsprachlichen Umgang mit ihrer (weiteren) L1, wobei ihr bilinguales Profil sich bereits in der Grundschule in Richtung einer Dominanz in der Verkehrssprache entwickelt (vgl. Reich, 2009). Zudem bilden manche bilinguale SuS mit MH bereits während der Grundschule negative Einstellungen zu ihrer nicht-verkehrssprachlichen L1 aus (vgl. Krumm, 2009, S. 239ff), was die Annahme von Angeboten zur bilingualen Interaktion beim PL zusätzlich erschwert. Die Lehrkräfte teilen in diesem Kontext nicht die weiteren Sprachen aller ihrer SuS, sodass sie bei der Wahrnehmung bilingualer Praktiken beim PL ihre Moderationsrolle gefährdet sehen könnten, da sie keinen Einblick in die Verstehensprozesse der SuS erhalten (vgl. Barwell, 2014, S. 917) und eventuell nicht lerninhaltsbezogene Interaktionen vermuten würden (vgl. Carless, 2008, S. 333). Beim Auftreten bilingualer Praktiken dürfte diesbezüglich ein striktes Durchgreifen zur Unterbindung bilingualer Interaktion zu erwarten sein. Andererseits können in der Submersion bilinguale Praktiken beim PL auch nur dann auftreten, wenn sich überhaupt mindestens zwei SuS in einer Gruppe befinden, die über dieselben Sprachen verfügen und diese auch gebrauchen (wollen). Weiterhin sind Bildungsinstitutionen im Kontext der Submersion der deutschen Regelschule durch einen „monolingualen Habitus“ (vgl. Gogolin, 1994) geprägt, sodass die zugrunde liegende Sprachideologie häufig zwischen ,assimilation“ oder gar „ethnist ideology“ (vgl. Bourhis, 2001) diffundiert und - zumindest in Deutschland - Bildungsungerechtigkeiten hinsichtlich Kindern und Jugendlichen mit MH durch die Bildungsinstitution Schule selbst erzeugt werden (vgl. Gomolla \& Radtke, 2009). Zudem identifizieren die in Abschnitt 4.2.3 ausgeführten pädagogisch/erziehungswissenschaftlich orientierten anerkennungstheoretischen Analysen des Umgangs der deutschen Regelschule mit bilingual aufwachsenden SuS mit MH und deren Mehrsprachigkeit diverse Praktiken der Missachtung der Anerkennungsform des Rechts sowie der Solidarität, die die bilingualen Handlungsdispositionen dieser SuS entrechtet und entwürdigt. 
Im Folgenden wird ein Überblick über die bisherige empirische Evidenz zu bilingualen Praktiken beim PL getrennt nach den Kontexten bilingualer Bildung und der Submersion diskutiert, welche im Abschnitt 3.6 ausgespart wurden. Hierbei werden nur Studien berücksichtigt, die (in-)formelle PL-Formate mit einem expliziten Angebot oder dem Verzicht auf ein explizites Verbot sowie Sanktionen zur bilingualen Interaktion untersuchen. Da die bisherige Empirie zu dieser Thematik relativ überschaubar ist, werden auch Studien im Kontext der Hochschulbildung (vgl. Storch \& Wigglesworth, 2003) integriert, sofern ihre Ergebnisse einen fruchtbaren Beitrag zum Stand der Evidenz liefern. Diese Studien werden dem Submersionskontext zugeordnet. Die jeweiligen Kontexte der Studien werden bei der Darstellung dieser kurz ausgeführt, um eine Einordnung der Evidenz abhängig von den jeweiligen vorliegenden $\mathrm{Be}-$ dingungen $\mathrm{zu}$ ermöglichen, die selbst innerhalb bilingualer Bildungsprogramme und der Submersion äußerst heterogen ausfallen können.

\subsubsection{Evidenz zu bilingualer Interaktion beim Peer-Learning im Kontext bilingualer Bildung}

Hopewell (2011) untersucht im Kontext der „dual language education” 49 spanisch-englischsprachig aufwachsende und literalisierte SuS im „English as a second language“-Unterricht innerhalb eines „,within group repeated measures design" unter Verwendung von Mixed-Methods. Die SuS erhalten in einer Sitzung das Angebot, sowohl das Spanische als auch das Englische bei der Bearbeitung der Aufgaben zu verwenden, während in der anderen Sitzung die Bearbeitung auf das Englische beschränkt ist. Die SuS bearbeiten in Gruppen zwei Texte, wobei ein Text einen höheren Lebensweltbezug aufweist (Mathematik im Garten) und ein anderer eher typischen Texten in der Schule entspricht (Erfindungen). Hierbei wird die Quantität sowie die Nutzungsweisen der bilingualen Praktiken und die Leistung der SuS in ,written recalls“, bei welcher zentrale Inhalte der Texte in Einzelarbeit schriftlich widergegeben werden sollen, in beiden Konditionen analysiert. Zunächst lesen die SuS die Texte einzeln im Englischen und anschließend schreiben sie ihre „written recalls“. Innerhalb der monolingualen Kondition dürfen die ,written recalls“ nur im Englischen produziert werden, in der bilingualen Kondition im Englischen oder Spanischen oder in beiden Sprachen gemischt. Anschließend diskutieren die SuS den Text in Kleingruppen, wobei sie Lesestrategien wie das Klären von Wörtern, Fragen generieren und beantworten und die Nennung von einer zentralen Proposition, die sie aus dem Text gelernt haben, anwenden. Hinsichtlich des PL-Formats dürfte es sich um informelles PL handeln, da Hopewell (ebd.) keine strukturellen und organisatorischen Eigenschaften des PL expliziert. In der bilingualen Kondition dürfen die SuS bilingual kommunizieren, 
während in der monolingualen Kondition nur auf Englisch interagiert werden darf.

Hinsichtlich der ,written recalls“ gaben die SuS in der bilingualen Kondition deskriptiv im Mittel mehr Inhalte des Textes wider als in der monolingualen Kondition. Dieser Unterschied konnte aber inferenzstatistisch nicht abgesichert werden, da der p-Wert des Gruppenvergleichs mit .055 knapp an der Signifikanzgrenze von .05 vorbeischrammt. Allerdings identifiziert Hopewell (ebd. S. 615) einen Interaktionseffekt zwischen der Textgrundlage und der bilingualen Kondition. Während in der bilingualen Kondition der Text mit Lebensweltbezug zu einem signifikanten Anstieg der Quantität der ,written recalls“ führt, sinkt diese bei dem Text zu Erfindungen leicht ab.

Interessanterweise treten bilinguale Praktiken in beiden Konditionen auf (vgl. ebd., S 612), was die Unvermeidlichkeit bilingualer Interaktion selbst bei explizitem Verbot unterstreicht (vgl. Cook, 2001, S. 405). Hopewell (ebd., S. $612 \mathrm{f}$ ) identifiziert innerhalb der bilingualen Kondition eine Vielzahl verschiedener förderlicher Nutzungszwecke bilingualer Praktiken beim PL. So wird das Spanische zur Erkennung von Kognaten und zur Lösung von Ambiguitäten, für metalinguistische Analogien auf Wort- und Morphemebene, Übersetzungen, alternative Erklärungen sowie zum expliziten Vergleich von Textkonventionen eingesetzt (vgl. ebd., S. 617). Dementsprechend nutzten ihre Probanden ihre bilinguale Disposition vor allem für metalinguistische Zwecke und zur Förderung des Textverständnisses.

Insgesamt interpretiert Hopewell (ebd., S. 616) ihre Ergebnisse dahingehend, dass sprachliche Restriktionen wie Sprachenseparation die Lern- und Lehrmöglichkeiten von SuS respektive Lehrkräften einschränken sowie zentrale Lernvoraussetzungen und -praktiken der SuS nicht berücksichtigen: „Restricting students to only one language denies them access to their augmented resources, ignores the fact that knowledge and vocabulary are distributed differently across languages, overlooks the intertwined and reciprocal nature of linguistic-based learning, and limits opportunities to learn by imposing unnecessary constraints and obstacles". Zudem akzentuiert sie den Befund der Interaktion zwischen den Textinhalten und dem (Nicht-)Angebot der bilingualen schriftlichen Widergabe, da zumindest bei Themen mit Lebensweltbezug das Textverständnis durch den Rückgriff auf die (weitere) L1 erhöht werden kann (vgl. ebd., S. 617).

Swain und Lapkin (2000) untersuchen englisch-französischsprachig aufwachsende SuS der achten Klasse, die seit dem Kindergarten im Immersionskontext Französisch erwerben. Diese SuS wurden bis zur dritten Klasse exklusiv im Französischen unterrichtet, wobei die Instruktionszeit im Englischen und Französischen ab der fünften Klasse als weitestgehend gleichberechtigt bezeichnet werden kann. Die insgesamt 44 Probanden arbeiten in Dyaden ohne Rollenverteilung sowie ohne nähere Instruktionen zur gemeinsamen Aufgabenbearbeitung zusammen, sodass diese Form als informelles PL zu bewerten 
ist. Zwölf Dyaden schreiben gemeinsam eine Nacherzählung zu einem zweimal auditiv präsentierten Text (,dictogloss task“). Innerhalb der restlichen zehn Dyaden erhält jedes Gruppenmitglied vier Bilder der Geschichte, die bei der ,dictogloss task“ auditiv präsentiert wurde, mit der Aufgabe, gemeinsam in der Dyade die Bilder in die richtige Reihenfolge zu bringen und auf Basis dessen eine gemeinsame Nacherzählung zu schreiben (,jigsaw task"). Swain und Lapkin (ebd.) analysieren die Prävalenz von englischsprachigen Äußerungen auf der Ebene des Turns im Zusammenhang mit der Aufgabe (,dictogloss/jigsaw task") sowie mit der Qualität der Nacherzählung und erfassen die Nutzungszwecke des Rückgriffs auf die L1 Englisch anhand eines Kategoriensystems.

Die Prävalenz englischsprachiger Turns liegt bei der, ,jigsaw task“ bei $29 \%$ und bei der ,dictogloss task" bei $21 \%$, wobei die Standardabweichungen mit $21 \%$ respektive $18 \%$ relativ groß ausfallen und sich die Prävalenzen nicht signifikant unterscheiden. Am häufigsten wird auf die L1 sowohl bei der, ,jigsaw task" als auch der „dictogloss task“ zum Task-Management (43\% respektive $35 \%$ ) zurückgegriffen, während zum Finden angemessenen Vokabulars im Französischen (27\% respektive $14 \%$ ) sowie zum Verständnis von Textinformationen und der Geschichte selbst deutlich seltener auf die L1 zurückgegriffen wird (10\% respektive 22\%). Das Englische wird in beiden Gruppen zu gleichen Anteilen zur Diskussion nicht lernbezogener Inhalte (11\% respektive $12 \%$ ) und der Grammatikalität der Nacherzählung (8\% in beiden Gruppen/Aufgaben) verwendet. Swain und Lapkin (ebd.) identifizieren für beide Aufgaben einen negativen Zusammenhang zwischen der Prävalenz englischsprachiger Turns und der inhaltlichen und sprachlichen Bewertung der Nacherzählungen, wobei aber nur die Korrelation bei der ,jigsaw task“ mit -.66 respektive -.64 signifikant ist. Bei der „dictogloss task“ werden Zusammenhänge in den Größen von -.14 und -.16 identifiziert.

Anhand einer Fallanalyse von zwei Dyaden der ,jigsaw task", die über eine ähnlich hohe Prävalenz von englischsprachigen Turns verfügen, sich aber deutlich in der sprachlichen und inhaltlichen Qualität unterscheiden, lassen sich deskriptiv differente Nutzungshäufigkeiten des Englischen für verschiedene Zwecke identifizieren. Während die Dyade mit der guten Leistung auf das Englische primär zum Zwecke des Task-Managements (35\%) und dem Verständnis (22\%) zurückgreift, zeichnet sich die Nutzung des Englischen des Tandems mit schlechter Leistung durch das Auffinden passender französischsprachiger Vokabeln aus (45\%), während sie das Englische nicht für das Textverständnis verwenden $(0 \%)$.

Swain und Lapkin (ebd., S. 268) interpretieren ihre Ergebnisse aus soziokultureller Perspektive und sehen im Gebrauch der L1 ein Werkzeug, dass den SuS auf verschiedene Weise beim PL behilflich ist: „to understand and make sense of the requirements and content of the task; to focus attention on language form, vocabulary use, and overall organization; and to establish the tone 
and nature of their collaboration". Verbote, auf seine L1 bei linguistisch und kognitiv als komplex sowie anspruchsvoll einzuschätzenden Aufgaben zurückzugreifen, kritisieren die Swain und Lapkin (ebd.) scharf, da somit den $\mathrm{SuS}$ ein wichtiges kognitives Werkzeug entzogen wird, mit welchem sowohl der Erwerb akademisch anspruchsvoller Inhalte als auch der L2 unterstützt werden kann.

Rubinstein-Ávila (2003) untersucht in einer Fallstudie im Kontext von „dual language education“ zwei bilingual englisch-portugiesischsprachige Grundschülerinnen, die im Peer-Tutoring mit reziproken Rollen Lesestrategien anwenden. Die Autorin betrachtet die Dyade vor allem hinsichtlich der Ausübung von Macht. Hierbei konstatiert Rubinstein-Ávila (ebd., S. 95), dass die Bestimmung der Interaktionssprache stärker an die Tutorenrolle gebunden ist, da je nach Sprachpräferenz des Tutors, entweder das Englische oder Portugiesische prominenter ist. Dementsprechend scheinen Machtstrukturen auch durch die Sprachnutzung beim bilingualen Peer-Tutoring markiert werden zu können.

\subsubsection{Evidenz zu bilingualer Interaktion beim Peer-Learning im Kontext der Submersion}

Planas und Setati (2009) untersuchen die Prävalenz und Funktion der Sprachwahl beim Mathematikunterricht im Klassenverbund sowie in Kleingruppen in einer Klasse mit 24 ca. zwölf Jahre alten SuS in Barcelona. Die Unterrichtssprache in der Schule ist normalerweise das Katalonische, allerdings befinden sich in der untersuchten Klasse einige SuS mit MH, deren Eltern aus Lateinamerika nach Spanien eingewandert sind und deren L1 Spanisch ist. Die Probanden setzten sich über fünf Sitzungen von jeweils 50 Minuten mit geometrischen Transformationen wie z. B. Rotation oder Symmetrie in ,open-ended“ Aufgaben auseinander, wobei sie auch im Klassenverbund, aber die meiste Zeit in Kleingruppen agierten. Die Kleingruppen wurden abhängig von der jeweiligen Sprachdominanz gebildet, sodass auch Gruppen entstanden, die primär aus spanisch-dominanten SuS bestanden, wobei eine dieser spanisch-dominanten Gruppen im Fokus der Untersuchung steht. Die bilinguale Lehrkraft erlaubt ihren SuS explizit bilingual oder im Spanischen miteinander in den Kleingruppen, aber auch im Klassenverbund zu interagieren. Beide Sozialformen wurden mit Kameras, Mikrophonen und Audiogeräten aufgenommen.

Abhängig von der jeweiligen Sozialform unterscheiden sich die Prävalenzen des Spanischen und des Katalanischen in den spanisch-dominanten Kleingruppen (vgl. ebd., S. 45). In der Interaktion im Klassenverbund wird von diesen Gruppen das Katalanische präferiert, während in den Kleingruppen vornehmlich das Spanische verwendet wird. Abhängig vom jeweiligen Kontext 
produzieren fast alle SuS der spanisch-dominanten Gruppen im Klassenverbund mindestens mehr als 85\% ihrer Äußerungen im Katalanischen, während sich dieses Verhältnis in den Kleingruppen umkehrt, sodass in diesem Kontext das Spanische zu mindestens $85 \%$ produziert wird. Innerhalb der Kleingruppen wiederum unterscheidet sich die Sprachwahl abhängig von den Phasen der Aufgabenbearbeitung und den damit verbundenen Handlungen (vgl. ebd., S. 46, 52). Sie verwenden primär das Katalanische, um sich mit der Aufgabenstellung vertraut zu machen, neu eingeführte mathematische Fachbegriffe sowie deren Bedeutung für die Aufgabe zu eruieren und die ersten Arbeitsschritte zu planen. Dementsprechend interagieren sie zu Beginn der Kleingruppenarbeit vornehmlich im Katalanischen. Anschließend wechseln sie ins Spanische, um sich gemeinschaftlich mit den mathematischen Inhalten auseinandersetzen, wobei sie Argumente einbringen sowie Erklärungen und Lösungsansätze diskutieren (vgl. ebd.). Diese Sprachhandlungen werden selten auch im Katalanischen vollzogen, sobald es allerdings zu Verständnisproblemen kommt, wechseln die spanisch-dominanten Gruppenmitglieder wieder in das Spanische. Am Ende der Sitzungen diskutieren die Gruppen ihre Ergebnisse im Klassenverbund. Hierbei führt das bilinguale Sprachangebot allerdings nicht zu einer erhöhten Partizipation der spanisch-dominanten Gruppen im Klassenverbund, obwohl sie innerhalb der Kleingruppe in manchen Fällen die beste Lösung erarbeitet haben.

Planas und Setati (ebd., S. 55ff) interpretieren die Sprachwahl der spanischdominanten SuS in der Kleingruppe im Rahmen von Grosjeans (2010, S. 29ff) „,complementary principle“, da diese SuS ihre Sprachwahl abhängig von den jeweiligen sozialen Normen und mathematischen Anforderungen anpassen. Sie greifen auf ihre L1 bei der Erarbeitung und Aushandlung der mathematischen Inhalte zurück, insbesondere bei anspruchsvollen, problembeladenen Inhalten, folgen allerdings der Norm des Katalanischen im Klassenverbund. Die ausbleibende Partizipation der erfolgreichen spanisch-dominanten Kleingruppe, deren Mitglieder im Klassenverbund sich eher in der Identität als „,Zuhörer" präsentieren, verbinden die Autoren mit den Erfahrungen dieser SuS mit $\mathrm{MH}$ als Minderheit in der Interaktion mit anderen: „These experiences may explain in part the reasons for the different uses of the two languages and the difficulties in certain types of social participation structures“" (ebd., S. 56f).

Während Planas und Setati (2009) die Sozialformen des Klassenverbundes und des PL hinsichtlich bilingualer Praktiken untersuchen, fokussieren Klingner und Vaughn (2000) die Arbeit in Kleingruppen mit einer ähnlichen Altersgruppe. Klingner und Vaughn (ebd.) untersuchen das Hilfe- und Unterstützungsverhalten von $37 \mathrm{SuS}$ einer heterogenen fünften Grundschulklasse beim PL zu Lesestrategien zu einem biologischen Thema (der menschliche Körper) im Submersionskontext der Vereinigten Staaten von Amerika. Mit Ausnahme von zwei SuS haben alles SuS Spanisch als L1 und Englisch als weitere L1 
oder als L2 erworben. Bevor die SuS in Kleingruppen die Lesestrategien anwenden und üben, erläutert und modelliert die Lehrkraft über fünf Sitzungen, wie die verschiedenen Lesestrategien kollaborativ zu implementieren sind. Die PL-Gruppen wurden so gebildet, dass zwei SuS mit hoher Sprachkompetenz in Spanisch und Englisch mit mindestens zwei SuS mit eher geringen sprachlichen Fähigkeiten zusammenarbeiten, wobei die SuS verschiedene Rollen (Teamleitung, Motivator, Zeitmanager etc.) in einem Rotationssystem einnehmen und mit Hilfe von Unterstützungsmaterialien die jeweiligen rollenbezogenen Aufgaben ausführen. Die Interaktionen innerhalb der Gruppen wurden in Spanisch und Englisch geführt und mit einem Audiogerät aufgenommen, allerdings wird in der Studie nicht näher erläutert, ob die bilinguale Interaktion als Angebot explizit formuliert wurde oder ob dies eine typische Praktik innerhalb der Klasse darstellt, die unabhängig von der Lernmethode und dem -inhalt auftritt, obwohl es sich bei der Schule nicht um ein bilinguales Bildungsprogramm handelt. Zudem wurden als schwierig eingeschätzte Wörter der Textgrundlage von den Autoren identifiziert, zu welchen die SuS eine Definition im Prä- und im Posttest verfassten. Die Interaktion der SuS wurde hinsichtlich der angewandten Lesestrategien, ihres Hilfeverhaltens und des Diskurses über die Lerninhalte mit Hilfe von Kategoriensystemen analysiert.

Klingner und Vaughn (ebd.) beobachteten beim PL der Gruppen viele Fälle von Code-Switching zur Erklärung von Wortbedeutungen und Konzepten über den menschlichen Körper. Hierbei mussten die SuS, die ein Wort oder ein Konzept erklären wollen, zunächst einschätzen, ob ihre Gruppenmitglieder lediglich ein spezifisches Wort oder das zugrunde liegende Konzept nicht verstehen und ob dieses für den Text zentral ist, was wiederum die Ausführlichkeit der Erklärung bedingt. Dementsprechend wurden sowohl kurze Übersetzungen von Wörtern des Englischen ins Spanische gegeben als auch elaborierte Erklärungen zu zentralen Konzepten wie „Gefäße“ oder „Knorpel“ im Spanischen ausgeführt (vgl. ebd., S. 88f). Angesichts des Wortschatztests identifizieren Klingner und Vaughn (ebd., S. 90f) höchstsignifikante Zuwächse, wobei alle SuS profitierten, allerdings die ,high“ und ,average achieving“ SuS die größten Zuwächse verzeichneten.

Die bilingualen Praktiken zur Erklärung von Wortbedeutungen und Konzepten bezeichnen die Autoren in Anlehnung an Moll (1992; zit. nach Klingner \& Vaughn, 2000, S. 92) als „language brokering“. Diese sind nicht nur als Übersetzungsprozesse zu verstehen, sondern ermöglichen und vereinfachen die sprachlichen Verstehensprozesse ihrer „low achieving“ Gruppenmitglieder, indem sie die entsprechenden Bedeutungen und Konzepte in ihrer L1 verarbeiten und anschließend transferieren, sodass sie diese Bedeutungen und Konzepte auch in ihrer L2 verstehen und erläutern können (vgl. ebd., S. 70f). Dementsprechend kann die L1 von SuS durch die bilinguale Interaktion beim PL eine Vermittlungsfunktion einnehmen, mit welcher der Transfer von Wis- 
sen in Formen der Verkehrssprache erfolgen kann. Hierbei gilt zudem anzumerken, dass die SuS nicht spezifisch in förderlichen Interaktionsformen und Hilfeverhalten beim PL instruiert wurden: ,[...] whether students scaffold may be a question not of whether students are capable of doing so but of whether they know how to provide assistance, whether they are motivated to do so, and whether the environment is structured to provide an optimal level of support" (ebd., S. 92). Der Rückgriff auf die L1 in Form von „language brokering" scheint eine intuitive Strategie bilingualer SuS darzustellen, die sie innerhalb eines förderlichen organisatorischen Rahmens des PLs spontan und effektiv einsetzen können.

Während Klingner und Vaughn (2000) förderliche bilinguale Praktiken des „language brokering“ von sprachkompetenteren Peers für Gruppenmitglieder mit geringeren sprachlichen Fähigkeiten beim PL beschreibt, die nach García und Kano (2014, S. 265) eher der Kategorie ,dependent translanguaging“ zuzuordnen sind, weist Moschkovich (2007) anhand einer Fallstudie auf Formen des ,independent translanguaging“ hin, obwohl die untersuchten Schülerinnen über keine ausgeprägten bildungssprachlichen Fähigkeiten in ihrer L1 verfügen. Moschkovich (2007) untersucht zwei spanisch-englischsprachige Schülerinnen der neunten Klasse beim informellen PL im Submersionskontext der Vereinigten Staaten von Amerika, die Aufgaben zur Steigung von Geraden gemeinsam bearbeiten. Eine der beiden Schülerinnen hat etwas Erfahrung mit Mathematik im Spanischen, die andere keine.

Innerhalb der Dyade kommt es bei der Diskussion und Bearbeitung der Aufgabe zu Sprachwechseln vom Englischen ins Spanische. An einer Interaktion illustriert Moschkovich (ebd., S. 135), dass Sprachwechsel als eine „resource for elaborating ideas while expanding, repeating or adding information for another speaker" (vgl. ebd., S. 137) bei bildungsbezogenen Diskursen fungieren können. Die Schülerin G. stellt eine Vermutung zur Steigung einer Geraden im Graphen im Englischen auf, welche von ihrer Partnerin M. als Unsicherheit hinsichtlich des visuellen Vergleichs der Steigung der verschiedenen Geraden interpretiert wird. M. wechselt daraufhin ins Spanische und weist G. auf die Nähe der Gerade zur X-Achse als einen Indikator für eine geringe Steigung hin. Anschließend wechselt sie ins Englische, um die Aufmerksamkeit von G. auf eine Gerade im Graphen zum Vergleich zu lenken und beschreibt deren Position mit einer Assertion im Spanischen. Nachdem G. mit einem Nicken ihre Zustimmung bzw. ihr Verständnis signalisiert, weist M. G. wiederholt auf die Nähe zur x-Achse als Indikator für Steigung hin. Unter Verwendung von Code-Switching konnte M. ihre Assertion im Spanischen und Englischen ausdrücken, ohne die Proposition mit den gleichen Wörtern zu wiederholen. In ihren spanischsprachigen Gesprächsbeiträgen verwendete sie hierbei eine weniger fachsprachliche Varietät als im Englischen. Diese elaborierende Wiederholung einer Assertion in Form unterschiedlicher Varietäten in ver- 
schiedenen Sprachen durch Code-Switching weist darauf hin, dass unter Verwendung von Code-Switching dieselbe Proposition in abgeänderter Weise formuliert werden kann, was wiederum den Verstehensprozess fördern kann.

Storch und Wigglesworth (2003) boten sechs von zwölf Lernpaaren Universitätsstudierender in einer Fallstudie bilinguale Praktiken bei Aufgaben zur Textrekonstruktion und zum Textverfassen auf Englisch an und interviewten diese im Anschluss. Die Dyaden, die das Angebot zur bilingualen Interaktion erhalten haben, bestanden aus entweder zwei Studierenden mit Indonesisch oder Mandarin als L1, die in Australien studieren. Storch und Wigglesworth (ebd.) analysierten die Prävalenz der Erstsprachnutzung, welche sie als sequenzierte Episoden der Nutzung der L1 operationalisiert haben, wobei eine Episode aus einem Wort bis zu mehreren Turns bestehen kann. Zudem untersuchten sie ebenfalls die Funktionen der Episoden und interviewten die Probanden hinsichtlich ihrer allgemeinen Einstellungen zur Nutzung der L1 in Bildungskontexten und ihrer Einschätzung nützlicher Funktionen der L1 bei der Aufgabenbearbeitung in der Dyade. Sowohl die Gruppenarbeit, die als eine Form informellen PL kategorisiert werden kann, als auch die Interviews wurden mit einem Audiogerät aufgezeichnet.

Vier der sechs Dyaden haben kaum Gebrauch von ihrer geteilten L1 bei der Aufgabenbearbeitung gemacht. Zwei Dyaden mit Mandarin als geteilter L1 nutzten vermehrt ihre L1 bei der Zusammenarbeit, wobei ein Tandem zu fast $50 \%$ bei der Textkompositionsaufgabe sowie zu knapp 30\% bei der Textrekonstruktionsaufgabe und das andere Tandem umgekehrt vermehrt bei der Textrekonstruktionsaufgabe (ca. 50\%) als bei der Textkompositionsaufgabe (ca. 25\%) auf ihre L1 zurückgegriffen haben. Hierbei nutzten beide Dyaden ihre L1 bei der Textkompositionsaufgabe vor allem zum Task-Management und zur Klärung der Aufgabe, während bei der Textrekonstruktionsaufgabe primär die L1 zur Diskussion lexikalischer Entscheidungen und Bedeutungen von Wörtern sowie der Grammatikalität einsetzten.

Als förderlich wurde der Rückgriff auf die L1 zum Diskutieren von Inhalten, zur Überbrückung lexikalischer Lücken und zur Erklärung von Bedeutungen und grammatischen Problemen von den Probanden erachtet. Zudem berichteten zwei Studierende, ihre L1 mental beim inneren Sprechen zu verwenden. Die relativ geringe Prävalenz der Nutzung der L1 im Mittel über alle Dyaden hinweg lässt sich anhand der Interviewdaten rekonstruieren. Am häufigsten wurde von den Studierenden angemerkt, dass der Rückgriff auf die L1 die Bearbeitungsdauer der Aufgabe verlängern würde und dass sie ihre L2 so häufig wie möglich in diesem Kontext verwenden sollten, um ihre Fähigkeiten im Englischen zu verbessern. Allerdings sind acht der zwölf Probanden bezüglich der Frage, ob der Rückgriff auf die L1 bei solchen Aufgaben hilfreich ist, der Ansicht, dass die Nutzung der L1 eine effizientere Aufgabenbearbeitung ermöglicht hätte. Dies gilt insbesondere für die Textkompositionsaufgabe, bei 
der die Interpretation einer Grafik durch die Diskussion dieser in der L1 schneller zu einem adäquaten gemeinschaftlichen Verständnis geführt hätte. Dementsprechend sehen auch Probanden, die keinen oder kaum Gebrauch von der L1 gemacht haben, ein gewisses lernförderliches Potential in ihren erstsprachlichen Fähigkeiten für PL.

Für den Submersionskontext in Deutschland gibt es erste empirische Untersuchungen zu bilingualen Praktiken beim PL, welche primär von den Arbeitsgruppen um Susanne Prediger, Jochen Rehbein und Angelika Redder initiiert und durchgeführt wurden. Rehbein (2011) untersucht die bilinguale Interaktion von vier türkisch-deutschsprachig aufwachsenden SuS der Grundschule beim informellen PL in einer Fallstudie. Die SuS bearbeiten eine Stunde lang außerhalb des Regelunterrichts in einem gesonderten Raum gemeinsam ein Aufgabenblatt, welches sich primär mit dem Thema „Strom“ am Beispiel einer Taschenlampe auseinandersetzt. Hierfür wird der Gruppe eine Taschenlampe als zusätzliches Material zur Verfügung gestellt. Die SuS sollen zunächst den einzelnen Bestandteilen der Taschenlampe die entsprechenden deutschen Begriffe zuordnen und im Anschluss zwei Fragestellungen beantworten, die nach sichtbaren Indikatoren für das Fließen von Strom und Vorsichtsmaßnahmen beim Experimentieren mit Strom fragen. Rehbein (ebd.) untersucht die transkribierte bilinguale Interaktion im Rahmen der Unterscheidung von „Arbeitssprache“ und „Denksprache“, wobei ihn die jeweiligen Zwecke der Nutzung des Türkischen hinsichtlich dieser beiden Diskurstypen interessieren. Während die Arbeitssprache die „organisatorischen Anteile der Kommunikation“ betrifft, bezieht sich die Denksprache auf die ,propositionalen Elemente des Diskurses und die damit verbundene Wissensprozessierung“, was der Unterscheidung in „,nicht-thematischem“ und „thematischem Diskurs“" entspricht (ebd., S. 206). Allerdings sind beide Diskurstypen an Verstehensprozessen beteiligt.

Die SuS verwenden das Türkische bei arbeits- und denksprachlichen Interaktionen. So verwenden sie das Türkische als Arbeitssprache, indem sie türkische Zeigewörter zur Koordination der Aufmerksamkeit der Gruppe auf bestimmte Teile der Taschenlampe einsetzen, Erkenntnisse sowie Verwunderung mit türkischsprachigen Ausrufen markieren und kurze Aufforderungen im Türkischen kommunizieren. Allerdings werden die einzelnen Teile der Taschenlampe stets im Deutschen bezeichnet. Bei eher denksprachlichen Interaktionen wird das Türkische zur Verbalisierung von Wahrnehmungen, Wissen und Erkenntnissen sowie deren Kontrolle verwendet. So werden Vorschläge zur Zuordnung von Einzelteilen zu den jeweiligen deutschsprachigen Bezeichnungen, Kontrollfragen, Repetitionen zur Signalisierung von Einverständnis, Ideen und Erkenntnisse primär im Türkischen kommuniziert. Nichtsdestotrotz ermahnen sich die SuS gegenseitig Deutsch zu sprechen, sobald ein Erwachsener sich in der Nähe ihres abgetrennten Raumes befindet. 
Rehbein (ebd., S. 227f) fasst als wichtigstes Ergebnis der Untersuchung zusammen, dass die SuS in diesem Experiment einen ,mehrsprachig konstituierten Handlungsraum bekommen haben, in dem sie laut nachdenken, sich wechselseitig korrigieren, sich bestätigen, etwas vorschlagen und kommentieren, etwas erläutern und sich gegenseitig evaluieren können in jener Sprache, die ihnen für die Kommunikation die passendste ist, ohne dass sie für den Gebrauch sanktioniert oder korrigiert werden“. Einschränkend gilt darauf hinzuweisen, dass die von Rehbein (2011) beobachteten und interpretierten sprachlichen Handlungen auch stark von der Aufgabe sowie dem Interaktionsstil abhängig sein könnten.

Das Projekt „Sprachenbildung im Mathematikunterricht unter Berücksichtigung der Mehrsprachigkeit" (MuM-Multi) unter der Leitung von Susanne Prediger, Jochen Rehbein und Angelika Redder untersucht in einem experimentellen Design nach dem Regelunterricht eine Intervention zum Bruchrechnen in Kleingruppen in der Sekundarstufe I im Submersionskontext in Deutschland. Hierbei variieren sie die sprachliche Umsetzung der Intervention, wobei die Intervention entweder monolingual in Deutsch oder bilingual in Türkisch und Deutsch vollzogen wird. Die angewandte Methode der im Folgenden darzulegenden Pilotierungsstudien als auch der Hauptstudie des Projekts MuM-Multi kann am ehesten mit informellem PL bezeichnet werden.

In einer Pilotstudie zur Entwicklung geeigneter Designs zur Untersuchung von bilingualen Unterrichtsangeboten führten Meyer und Prediger (2011) Aufgabeninterviews zu textintensiven, offenen, im Türkischen und Deutschen formulierten mathematischen Modellierungsaufgaben mit jeweils zwei oder drei türkisch-deutschsprachigen SuS der vierten bis sechsten Klasse durch. In der ersten Vorstudie traten diverse Probleme bei der Aktivierung bilingualer Interaktion in den Kleingruppen trotz expliziter Ermunterung zur bilingualen Interaktion auf. Bei den Probanden konnte eine allgemeine Zurückhaltung zum Gebrauch des Türkischen beobachtet werden. In der ,freiwilligen Kondition“, bei denen die SuS frei zwischen dem deutsch- oder türkischsprachigen Text sowie der sprachlichen Praktiken der Interaktion wählen konnten, griffen fast alle SuS auf den deutschsprachigen Text zurück und interagierten fast ausschließlich auf Deutsch. Einzelne SuS griffen auf den türkischsprachigen Text mit der Begründung zurück, dass Bruchrechnungen im Türkischen einfacher zu verstehen seien, ansonsten wurde dieser Text nur zur Klärung einzelner Wortbedeutungen verwendet (vgl. ebd., S. 190). In der „forcierten Kondition“ konnte weitestgehend das gleiche Verhalten der SuS beobachtet werden. Diese Kondition zeichnet sich allerdings durch einen zusätzlichen schauspielerischen Kunstgriff aus, bei dem ein türkischsprachiger Hausmeister die SuS explizit nach den Aufgabeninhalten und ihren Lösungen fragt, um den bilingualen Sprachgebrauch zu aktivieren. Zwar erläuterten die SuS die Aufgabeninhalte auch unter Verwendung des Türkischen, allerdings stellt diese Form der Aktivierung eine methodisch schwierig umzusetzende Praxis dar. 
Auf Basis dieser Beobachtungen wurden in einer zweiten Vorstudie die Aktivierungen der bilingualen Bearbeitung der Aufgaben dahingehend modifiziert, dass neben der expliziten Aufforderung zur bilingualen Bearbeitung die $\mathrm{SuS}$ die deutsch- und türkischsprachige Aufgabenformulierung vergleichen und sie zu Beginn der Übungsleitung das Zählen im Türkischen beibringen sollten. Dementsprechend scheint die Übungsleitung nicht über das Türkische zu verfügen, was in dieser Konstellation kritisch zu betrachten ist, da sie die Etablierung eines bilingualen Sprachmodus erschwert. Eine bilinguale Interaktion und Aufgabenbearbeitung der SuS würde die Übungsleitung aus den Prozessen zum Teil ausschließen, was wiederum die Auftretenswahrscheinlichkeit bilingualer Praktiken mindern dürfte.

Bei der Analyse von zwei Fällen dieser Vorstudie identifizierten Meyer und Prediger (ebd., S. 194ff) eine gesteigerte Prävalenz bilingualer Praktiken und einige förderliche Zwecke des Gebrauchs des Türkischen. So wurde ähnlich wie bei Rehbein (2011) das Türkische als Arbeitssprache eingesetzt, mit welcher aber auch propositionale Inhalte wie verschiedene mathematische Mengenrelationen und Vergleiche diskutiert werden, sodass das Türkische auch als Denksprache eingesetzt wurde. Hierbei ist allerdings zu erwarten, dass die Schülerinnen diese arbeits- und denksprachlichen Handlungen auch im Deutschen hätten vollziehen können. Zudem wurden lexikalische Lücken durch den Rückgriff auf das Türkische gefüllt und eine gewisse Privatsphäre geschaffen, in welcher sich die SuS gegenseitig über ihr Verständnis der Aufgabenstellung und Aufgabe selbst rückversichern konnten, ohne dass die Übungsleitung dies verstehen konnte.

In ihrer Hauptstudie führen Schüler-Meyer et al. (2017) in einem quasiexperimentellen Treatment-Kontrollgruppen-Design nach dem Regelunterricht eine Intervention zum Bruchrechnen in der Sekundarstufe I mit insgesamt 128 deutsch-türkischsprachigen SuS der siebten Klasse durch. Die Kleingruppen bestanden aus 3-5 SuS, wobei die Gruppen in der bilingualen Kondition von einer türkisch-deutschsprachigen Übungsleitung unterstützt wurden, das bilinguale Sprachhandeln der bilingualen Übungsleitungen allerdings nicht standardisiert wurde.

Die Verbalisierung von Brüchen weist zwischen dem Deutschen und dem Türkischen einen interessanten Unterschied hinsichtlich der Leserichtung auf. Im Deutschen werden Brüche vom Zähler zum Nenner artikuliert $\left(, 3 / 5^{“}=\right.$ „drei Fünftel“), während im Türkischen die Leserichtung vom Nenner zum Zähler verläuft (,,3/5“ = „,beşte üc“ [,fünf darin drei“]) (vgl. ebd., S. 7). Die türkischsprachige Leserichtung und Formulierung produziert zunächst den Nenner als Ganzes in Kardinalzahlen (fünf) und setzt diesen durch das Morphem ,-te“ sukzessiv in Bezug zum Zähler, der ebenfalls als Kardinalzahl artikuliert wird. Diese Leserichtung und Formulierung akzentuiert den Nenner als Ganzes, von welchem ein bestimmter Anteil fokussiert wird. Diese sprachliche Umsetzung liegt näher am Konzept mathematischer Brüche als die 
deutschsprachige Leserichtung sowie Formulierung und kann durch sprachvergleichende Reflexionen mit dem Deutschen zu einem vertieften Verständnis von mathematischen Brüchen führen (vgl. ebd.).

In der bilingualen Kondition interagierten die SuS im Mittel zu 31\% bilingual, wobei $16 \%$ rein türkischsprachige und $15 \%$ türkisch-deutschsprachig gemischte Äußerungen ausmachen. Schüler-Meyer et al. (ebd.) berichten allerdings kein Streuungsmaß, sodass keine Aussagen zur Heterogenität zwischen den Gruppen gemacht werden können. Hinsichtlich der Lernzuwächse unterscheiden sich die Gruppen der monolingualen und bilingualen Kondition nicht. Allerdings profitieren SuS mit hoher Türkischkompetenz $(d=1.22)$ deskriptiv mehr von dem bilingualen Angebot als SuS mit niedriger Türkischkompetenz $(d=0.63)$. Allerdings sind diese Effektstärken auch mit den Effektstärken der nach Türkischkompetenz differenzierten Untergruppen der monolingualen Kondition vergleichbar $(d=1.23$ und $d=0.86)$. Schüler-Meyer et al. (ebd., S. 14) identifizieren einen signifikanten Interaktionseffekt von Gruppe und Zeit, aber - vermutlich aufgrund der geringen Stichprobengröße - keine signifikanten paarweisen post-hoc Gruppenunterschiede.

Qualitative Analysen der Interaktion der Kleingruppen anhand weniger Fälle (vgl. ebd., S. 14ff) weisen auf temporäre Hürden und Probleme mit der türkischsprachigen Formulierung von Brüchen hin, die primär in Verständnisproblemen der Bedeutung der türkischsprachigen Leserichtung von Brüchen und den damit assoziierten Missverständnissen innerhalb der Kleingruppen liegen. Allerdings können diese Verständnisschwierigkeiten und Missverständnisse überwunden werden, sodass die türkischsprachige Leserichtung und Formulierung im Sprachvergleich zum Deutschen das konzeptuelle Verständnis von mathematischen Brüchen vertieft.

Schüler-Meyer et al. (ebd., S. 18ff) interpretieren die Ergebnisse dahingehend, dass viele Probanden sich trotz der fehlenden Erfahrung im bildungssprachlichen Gebrauch ihrer nicht verkehrssprachlichen L1 an mathematischen Diskursen auf Türkisch oder Türkisch-Deutsch gemischt beteiligen können. Sie beurteilen die vergleichbaren Lernzuwächse in der monolingualen und bilingualen Kondition als Argument für die Implementation von mehrsprachigen Bildungsangeboten im Regelunterricht. Zwar wurden hiermit keine zusätzlichen Effekte auf den Erwerb des Lerninhalts für alle erzeugt, aber das Angebot bilingualer Praktiken beim PL könnte sich durchaus für weitere lernrelevante Phänomene wie Motivation oder Bildungsziele wie Identitätsbildung fruchtbar erweisen. Die Autoren führen als weitere potentielle Gründe für den vergleichbaren Lernzuwachs der beiden Konditionen die Größe der Stichprobe, die Prävalenz der Türkischproduktion der SuS sowie die Kürze der Intervention an.

Abschließend sollen kurz die zentralen Ergebnisse der Studie „Förderung der Deutsch-Lesekompetenz bilingualer Grundschüler durch Peer-Learning“ (BiPeer) vorgestellt werden, welche die Datengrundlage für die vorliegende 
Studie darstellt. Da die Methodik dieser Studie ausführlich in Abschnitt 8.1 erläutert wird, werden hier nur die zentralen methodischen Rahmenbedingungen genannt. Rauch et al. (in Vorb.) untersuchen 168 monolingual deutschsprachig und bilingual türkisch-deutschsprachig aufwachsende SuS der dritten und vierten Klasse beim reziproken Peer-Tutoring in Dyaden mit den Rollen Tutor und Tutand, die nach dem regulären Unterricht innerhalb eines quasiexperimentellen Treatment-Kontrollgruppen Designs Lese- oder Rechenstrategien bearbeiten. Die Probanden sind auf sechs verschiedene Gruppen verteilt, von denen jeweils 3 Gruppen sich inhaltlich mit Lese- (LG, LGD und LGD/T) oder Rechenstrategien (RG, RGD und RGT/D) auseinandersetzen. Innerhalb dieser Lese- oder Rechenstrategiegruppen werden der Sprachhintergrund sowie das Sprachangebot des Trainings systematisch variiert. In den Gruppen LG und RG bilden jeweils ein bilingual türkisch-deutschsprachig aufwachsendes Kind und ein monolingual aufwachsendes Kind eine Dyade. In den Gruppen LGD und LGT/D sowie RGD und RGT/D arbeiten jeweils zwei bilingual türkisch-deutschsprachige Kinder zusammen, wobei in LGD und RGD lediglich das Deutsche zur Interaktion genutzt werden soll. Die Gruppen LGT/D und RGT/D erhalten ein explizit vermitteltes bilinguales Sprachangebot zur türkisch-deutschsprachigen Interaktion.

Die Gruppen mit gemischtem und gleichem Sprachhintergrund unterscheiden sich nicht in ihren Lernzuwächsen, sodass die Effektivität von PL nicht von der Gruppenzusammensetzung der Sprachhintergründe der Dyaden abhängig zu sein scheint. Hinsichtlich der Effektivität des bilingualen Sprachangebots ergibt sich kein eindeutiges Bild. Die Gruppen LGD und LGD/T verzeichnen vergleichbare Zuwächse in den standardisierten und trainingsnahen Testverfahren, sodass keine signifikanten Unterschiede festzustellen sind. Bezüglich der Rechenstrategieintervention weist die Gruppe RGT/D signifikant bessere Lernzuwächse sowohl hinsichtlich der Flexibilität als auch der Adaptivität der Anwendung von halbschriftlichen Rechenstrategien in einem trainingsnahen Rechentest im Vergleich zur Gruppe RGD auf. Dementsprechend exerziert das bilinguale Sprachangebot zusätzliche positive Effekte bei Rechen- allerdings nicht bei Lesestrategien.

\subsubsection{Zusammenfassung}

Zusammengefasst replizieren die eben angeführten Studien einige der in Abschnitt 3.6.5.3 und 3.6.5.4 berichteten Befunde, fügen dem Forschungsstand $\mathrm{zu}$ Bilingualität und Bildung allerdings auch neue Erkenntnisse hinzu. Zunächst gilt es aber zu konstatieren, dass die Studien zu bilingualen Praktiken beim PL eine äußert heterogene Bandbreite an zugrunde liegenden Bildungskontexten und Ländern, Alter, Sprachkombinationen und Sprachkompetenzprofilen der Probanden, angewandten Designs, (in-)formellen PL-Formaten, 
Methoden und Operationalisierungen, untersuchten Inhaltsbereichen sowie distinkten theoretischen Rahmungen zur Interpretation der Ergebnisse aufweisen. Angesichts der relativ bescheidenen Quantität an Studien zu bilingualer Interaktion als Angebot beim PL sowie der eben dargelegten Heterogenität der Studienanlagen kann bislang von keiner robusten empirischen Basis gesprochen werden. Allerdings zeichnen sich in der Gesamtschau erste Hinweise bezüglich der Prävalenz, Funktionen und Effekte von bilingualen Praktiken beim PL ab.

Die Prävalenz bilingualer Praktiken oder des Rückgriffs auf die (weitere) L1 beim PL fällt in den meisten Studien relativ gering aus (vgl. Schüler-Meyer et al., 2017; Storch \& Wigglesworth, 2003; Swain \& Lapkin, 2000) und zeichnet sich durch eine relativ breite Streuung aus (vgl. Storch \& Wigglesworth, 2003; Swain \& Lapkin, 2000). Die Werte übersteigen nur in Einzelfällen ein Drittel der Gesamtäußerungen beim PL. Lediglich die Studie von Planas und Setati (2009) bildet hierbei eine Ausnahme, da sich in der Sozialform von Kleingruppen im Vergleich zum Klassenverbund der Anteil erst- und gemischtsprachlicher Interaktionen umkehrt, sodass diese Äußerungen in den Kleingruppen interindividuell fast ausnahmslos über 85\% liegen. Cooks (2001, S. 405) Beobachtung, ,the L1 creeps back in, however many times you throw it out with a pitchfork" bewahrheitet sich auch für PL, da bilinguale Praktiken ohne vorausgegangene explizite Thematisierung (vgl. Klingner \& Vaughn, 2000) sowie trotz expliziten Verbots (vgl. Hopewell, 2011) von den SuS produziert werden. Allerdings scheint die Aktivierung von bilingualen Praktiken beim PL in der Regel eine Herausforderung darzustellen (vgl. Meyer \& Prediger, 2011), insbesondere bei SuS, die keine oder kaum Erfahrung mit bildungssprachlichen Varietäten ihrer (weiteren) L1 haben. Zudem liefern vereinzelte Studien Hinweise, dass bilinguale SuS mit MH die sprachlichen Normen der Schule und des Unterrichts wahrnehmen (vgl. Planas \& Setati, 2009; Rehbein, 2011). Zudem zeigt die Studie von Rubinstein-Ávila (2003), dass die Sprachwahl als Machtinstrument von den SuS beim Peer-Tutoring eingesetzt werden kann.

Die angeführten Studien identifizieren diverse förderliche Verwendungszwecke bilingualer Praktiken beim PL. So werden bilinguale Praktiken zum Task-Management (vgl. Meyer \& Prediger, 2011; Planas \& Setati, 2009; Rehbein, 2011; Storch \& Wigglesworth, 2003; Swain \& Lapkin, 2000), für metalinguistische Reflexionen (vgl. Hopewell, 2011; Storch \& Wigglesworth, 2003; Swain \& Lapkin, 2000), für Übersetzungen (vgl. Hopewell, 2000; Klingner \& Vaughn, 2000; Storch \& Wigglesworth, 2003; Swain \& Lapkin, 2000) und sowohl bei der gemeinsamen Aufgabenbearbeitung und der Diskussion der Inhalte (Hopewell, 2011; Klingner \& Vaughn, 2000; Planas \& Setati, 2009; Meyer \& Prediger, 2011; Moschkovich, 2007; Schüler-Meyer et al., 2017; Storch \& Wigglesworth; 2003; Swain \& Lapkin, 2000) als auch in mentalen Denkprozessen für sich selbst (vgl. Storch \& Wigglesworth, 2003) verwendet. 
Selbst bilinguale Probanden, die keinen Gebrauch von bilingualer Interkation beim PL machen oder diesem Angebot auf Basis der Time-on-Task Hypothese für den Spracherwerb kritisch gegenüberstehen, können intuitiv diverse förderliche Einsatzmöglichkeiten bilingualer Praktiken beim PL benennen (vgl. ebd.). Hierbei gilt es darauf hinzuweisen, dass das Auftreten bestimmter Zwecke sowie deren Prävalenz von weiteren Variablen beeinflusst zu sein scheint. So unterscheiden sich z. B. eine high- und low-achieving Dyade in der Studie von Swain \& Lapkin (2000) in ihrer Nutzungsweise bilingualer Praktiken, obwohl sie die gleiche Prävalenz bilingualer Praktiken beim PL vorweisen. Diese von weiteren Variablen determinierte Nutzungsweise von bilingualen Praktiken beim PL steht im Einklang sowohl mit den beeinflussenden Faktoren der Sprachmodi (Grosjean, 2008, S. 42ff) als auch mit der Distinktion von García und Kano (2014) in ,dependent“ und ,,independent translanguaging“. Dementsprechend kann nicht davon ausgegangen werden, dass bestimmte Zwecke von bilingualen Praktiken in allen PL-Formaten und bei allen PL-Gruppen in ähnlicher Weise auftreten, sondern sich diese in Abhängigkeit von diversen Individual- und Gruppenmerkmalen unterscheiden.

Bilinguale Praktiken werden beim PL häufig zum Task-Management genutzt (vgl. Meyer \& Prediger, 2011; Planas \& Setati, 2009; Rehbein, 2011; Storch \& Wigglesworth, 2003; Swain \& Lapkin, 2000). Dementsprechend kann eine zentrale Aufgabe beim PL, nämlich die notwendigen Organisationsund Koordinationsprozesse der Gruppe zu vollführen, auch bilingual ausgehandelt werden. Beim PL übernehmen die SuS die Verantwortung zur Strukturierung ihres gemeinschaftlichen Lernens und müssen hierbei Intersubjektivität etablieren, um kooperativ parallel an den gleichen Prozessen beteiligt zu sein sowie auf dasselbe Ziel hinzuarbeiten. Diese Prozesse hängen eng mit den förderlichen Basiselementen der positiven Interdependenz, förderlichen Interaktion und sozialen Kompetenz (vgl. Johnson \& Johnson, 1999) zusammen, welche alle zu einem Teil auf der Wahrnehmung der Gruppe als egalitäre und vertrauenswürdige Einheit beruhen. Da wie in Abschnitt 3.4 dargelegt Sprache und Identität eng zusammenhängen und Sprache ein Marker von Gruppenzugehörigkeiten sein kann, könnte bilinguales Sprachhandeln beim PL ein Vehikel zur Gruppenkonstitution sowie -identifikation darstellen. Hierbei reicht es allerdings nicht, die geteilte Bilingualität als Gruppenmerkmal wahrzunehmen, sondern sie muss durch die Möglichkeit zur Anwendung bilingualer Praktiken handlungsrelevant werden, damit diese als gruppenkonstituierendes Merkmal fungieren kann (vgl. Dirim, 1998, S. 147).

Bei metalinguistischen Reflexionen wird ebenfalls bilingual interagiert, wobei die geteilte (weitere) L1 sprachvergleichend in Beziehung zur L2 oder zur anderen L1 gesetzt wird, was andere Perspektiven auf die Form und Funktion einzelner sprachlicher Phänomene ermöglicht als ein monolingualer Blickwinkel. So können unter Rückgriff auf die (weitere) L1 unter anderem verwandte Wörter erkannt und metalinguistische Analogien auf Wort- und 
Morphemebene gezogen werden (vgl. Hopewell, 2011), womit sich in manchen Fällen auch Wortbedeutungen klären lassen. Bilinguale Praktiken treten auch bei der Diskussion von angemessenem Vokabular (vgl. Swain \& Lapkin, 2000) und von Textkonventionen (vgl. Hopewell, 2011) auf. Diese Funktionen weisen darauf hin, dass Wissen über bildungssprachlich adäquates Vokabular und konzeptionell schriftsprachliche Strukturen der Unterrichtssprache auch im Diskurs in der (weiteren) L1 erworben werden können. Zudem kommunizieren die SuS auch bilingual über grammatische Aspekte (vgl. Swain \& Lapkin, 2000). Metalinguistische Reflexionen in Form bilingualer Interaktion beim PL werden angesichts der bisherigen Evidenz sowohl beim Textverständnis (vgl. Hopewell, 2011) als auch bei der Textproduktion (vgl. Swain \& Lapkin, 2000; Storch \& Wigglesworth, 2003) vollzogen. Diese Befunde zur Funktion von bilingualen Praktiken beim PL zur Initiierung und Umsetzung metalinguistischer Reflexionen sowie die Möglichkeit zu sprachvergleichenden Betrachtungen sprachlicher Phänomene widerspricht der Interpretation der Time-on-Task Hypothese zur Förderung unterrichtssprachlicher Fähigkeiten in zweifacher Weise: 1) Die Interaktion kann in einer anderen Sprache als der Zielsprache stattfinden, um Wissen über bildungssprachliche Formen und Strukturen der Zielsprache zu erzeugen. 2) Der Rückgriff auf die (weitere) L1 kann sprachvergleichende metalinguistische Perspektiven eröffnen und somit ebenfalls sprachliches Wissen über die Zielsprache generieren. Angesichts der im Abschnitt 3.3.2.3 dargelegten Evidenz für eine erhöhte metasprachliche Bewusstheit hinsichtlich des Wortkonzepts sowie des Potentials zu Sprachvergleichen von bilingual aufwachsenden Kindern und Jugendlichen lässt sich bezüglich bilingualer Praktiken durchaus von einer Ressource für metalinguistische Reflexionen beim PL sprechen.

Die in Abschnitt 3.6.5.2 ausgeführte Förderlichkeit von Übersetzungen als Transformationen von Informationen in eine zugängliche, nutzbare mentale Repräsentation mit metakognitiver Komponente des Monitorings (vgl. Kern, 1994) tritt auch beim PL auf. Die Funktion bilingualer Praktiken zum ,language brokering“ (vgl. Klingner \& Vaughn, 2000) repräsentieren die zentralen Aspekte des eben verwendeten Übersetzungsbegriffs nach Kern (1994), da nicht nur lexikalische Lücken gefüllt werden, sondern auch Konzepte sowie mentale Repräsentationen wie z. B. die Funktion von Knorpel bei Knochen (vgl. Klingner \& Vaughn, 2000, S. 88f) durch den Rückgriff auf die (weitere) L1 erläutert werden. Hierbei könnte ein Angebot von bilingualen Praktiken beim PL auch den Zugriff auf lebensweltliche Erfahrungen vereinfachen, die eher im Kontext der (weiteren) L1 als der Unterrichtsprache gemacht wurden, worauf der von Hopewell (2011) identifizierte signifikante Interaktionseffekt einer lebensweltlichen Textgrundlage mit der bilingualen Kondition hinweist, welcher zu einer erhöhten Quantität der schriftlichen Widergabe von Textpropositionen führte. Bilinguale Interaktion kann dementsprechend nicht nur 
den Zugriff auf dieses Wissen womöglich vereinfachen, sondern auch Transferprozesse zur Unterrichtssprache initiieren, sodass das lebensweltliche Wissen auch in (bildungs-)sprachlichen Formen der Unterrichtssprache externalisiert werden könnte.

Bilinguale Praktiken treten beim PL bei der Aufgabenbearbeitung und im Diskurs über den Lerninhalt auf, sodass die bilinguale Interaktion beim PL anscheinend auch als Vehikel für zentrale Lernprozesse und Erkenntnisse im Gruppendialog fungieren kann. Hinsichtlich eines förderlichen Dialogs als Voraussetzung für effektives PL erscheinen insbesondere die durch die Nutzung der (weiteren) L1 sich eröffnenden Möglichkeiten zur Elaboration von Propositionen, ohne diese in der Unterrichtssprache zu wiederholen (vgl. Moschkovich, 2007), sowie für alternative Erklärungen (vgl. Hopewell, 2011; Klingner \& Vaughn, 2000; Schüler-Meyer et al., 2017) als besonders fruchtbare Funktionen bilingualer Praktiken beim PL. Neben der Externalisierung von sich möglichst widersprechenden Assertionen, stellen Elaborationen, Argumente und Erklärungen zentrale Sprachhandlungen für die Effektivität von PL dar (vgl. z. B. Asterhan \& Schwarz, 2009; Howe, 2010; Littleton \& Mercer, 2010). Unter Verwendung bilingualer Praktiken können Elaborationen und Erklärungen vorgenommen werden, die durch die Reformulierung der zu elaborierenden oder erklärenden Propositionen in einer anderen Sprache als der Unterrichtssprache andere Akzente setzen können, die die Propositionen in einer abgeänderten Weise darstellen und/oder differente Aspekte der Proposition fokussieren können. Ein hervorragendes Beispiel hierfür liefern Schüler-Meyer et al. (2017) mit den distinkten Leserichtungen und Formulierungen von mathematischen Brüchen im Deutschen im Gegensatz zum Türkischen, die sich primär in der Fokussierung von Zähler und Nenner unterscheiden. Hierbei akzentuiert die Leserichtung und Formulierung mathematischer Brüche im Türkischen das Konzept des Bruchs aus der Perspektive des Nenners, der als Ganzes die Basis repräsentiert, von welchem ein bestimmter Teil fokussiert wird. Diese sich vom Deutschen unterscheidende Perspektive auf das Konzept mathematischer Brüche kann sprachvergleichend genutzt werden, um eine adäquate mentale Repräsentation mathematischer Brüche aufzubauen und zu festigen.

Der Gebrauch einer Vielzahl verschiedener förderlicher Zwecke bilingualer Praktiken beim PL ist allerdings noch kein Beweis für deren Effektivität, insbesondere angesichts der Tatsache, dass ein Großteil der Funktionen auch monolingual in der (weiteren) L1 oder L2 umgesetzt werden könnten. Intuitiv gibt es aus der Perspektive der empirischen Bildungsforschung zwei übergeordnete Argumentationsansätze hinsichtlich der von bilingualen Praktiken zu erfüllenden Effekte beim PL: 1) Eine starke Position, die verlangt, dass Angebote zur bilingualen Interaktion einen messbaren Mehrwert im Sinne einer höheren Effektstärke als monolinguale Interaktion erzeugen. Mit diesem Argumentationsansatz werden primär kognitive Leistungsmaße oder inhaltliche 
Lernzuwächse des zu erwerbenden Wissens oder der auszubauenden Fähigkeiten des Curriculums in Verbindung gebracht, wobei unter Umständen auch positive Effekte auf motivationale Phänomene wie Lernorientierung, Selbstkonzept, „basic needs“ etc. als Zielkonstrukte dieser Position akzeptiert werden könnten, da diesen bei positiver Ausprägung ein Beitrag zur Lernwirksamkeit zugesprochen wird. 2) Eine schwache Position, die Angebote zur bilingualen Interaktion beim PL legitimiert, sofern diese Angebote keine signifikant niedrigeren Effektstärken hinsichtlich kognitiver und/oder motivationaler Outcomes als monolinguale Interaktion in der Unterrichtssprache erzeugen. Diesem Argumentationsansatz liegt einerseits die Idee zu Grunde, dass bilinguale Interaktionsangebote beim PL nicht zwingend höhere kognitive oder motivationale Effekte als monolinguale Angebote hervorrufen müssen, da das Angebot auf einer anderen Ebene sich positiv auswirken könnte. So könnte ein Angebot zur bilingualen Interaktion beim PL sich auch positiv auf z. B. die Sprachkompetenz in der (weiteren) L1, die Partizipation am PL sowie am Unterricht oder auf das Zusammengehörigkeitsgefühl der Gruppe auswirken. Andererseits stellt sich aus normativer Perspektive die Frage, ob mit solchen Angeboten zwingend messbare Effekte hinsichtlich eines Phänomens erzeugt werden müssen. Aus anerkennungstheoretischer Perspektive wäre bereits die Verhinderung von Missachtung auf den Anerkennungsformen des Rechts und der Solidarität eine Legitimation zur Implementation der (weiteren) L1 bilingualer $\mathrm{SuS}$ in den Regelunterricht.

Zur Unterstützung der eben ausgeführten starken Position trägt der Befund von Rauch et al. (in Vorb.) bei, die in ihrer Untersuchung signifikant höhere Lernzuwächse beim PL in der bilingualen als in der monolingualen Kondition bezüglich Rechenstrategien identifizieren. Die schwache Position wird durch die Evidenz sowohl von Rauch et al. (ebd.) zu Lesestrategien als auch von Schüler-Meyer et al. (2017) zum Bruchrechnen erfüllt. Im Gegensatz hierzu identifizieren Swain und Lapkin (2000) negative Zusammenhänge zwischen der Prävalenz bilingualer Praktiken beim PL und den sprachlichen sowie inhaltlichen Leistungen in den Textkompositionsaufgaben, wobei lediglich die Zusammenhänge beim Aufgabenformat der ,jigsaw task" signifikant sind und hohe Korrelationen erreichen. Diese negativen Zusammenhänge bedeuten aber nicht, dass die Häufigkeit bilingualer Interaktion beim PL sich negativ auf die sprachlichen und inhaltlichen Aspekte von Texten auswirken, sondern können eher als ,dependent translanguaging“ (García \& Kano, 2014) interpretiert werden, die die Dyaden zur Aufgabenbearbeitung benötigen. Gerade SuS mit niedrigen Kompetenzen in der Unterrichtssprache dürften von bilingualen Angeboten beim PL profitieren, da sie sich über die in der Unterrichtsprache zu produzierenden Inhalte in ihrer (weiteren) L1 austauschen können. Weiterhin kann nicht zwingend davon ausgegangen werden, dass bilinguale Interaktionsangebote beim PL zu einer erhöhten Partizipation am Unterrichtsgeschehen 
führen, da trotz erfolgreicher Aufgabenbearbeitung in der Kleingruppe, bilinguale SuS mit MH ihre in der Kleingruppe erarbeiteten Lösungen anschließend nicht im Klassenverbund präsentieren (vgl. Planas \& Setati, 2009).

Abschließend gilt es aber darauf hinzuweisen, dass sowohl die starke als auch die schwache Position für Argumentationsansätze zur Legitimität von Angeboten zur bilingualen Interaktion beim PL methodisch (Gruppen-)Vergleiche zwischen monolingualen und bilingualen Konditionen benötigt, um die Lernwirksamkeit von bilingualen Interaktionsangeboten beim PL empirisch zu ergründen. Empirisch identifizierte positive Effekte ohne Gruppenvergleiche lassen stets die Frage offen, ob diese positiven Effekte nicht auch durch monolinguale Interaktion hätten erzeugt werden können.

In der Gesamtschau kann trotz dieser ersten vorläufigen empirischen Ergebnisse zu Prävalenzen, Funktionen, Effekten sowie Einstellungen zu bilingualen Interaktionsangeboten beim PL von einem unterforschten Bereich bilingualer Angebote beim PL im Bildungskontext gesprochen werden, welcher einige Forschungslücken offen lässt und dessen bisherige Evidenz diverse (methodische) Mängel aufweist.

Zunächst fällt auf, dass der Großteil der Evidenz aus eher explorativ angelegten Fallstudien oder Querschnittsuntersuchungen stammt. Dies hängt sicherlich auch mit dem Umstand zusammen, dass in den meisten Studien informelle PL-Formate implementiert wurden, anstatt formelle PL-Formate evidenzbasiert und didaktisch angemessen zu organisieren und strukturieren. Dies ist angesichts des Befunds, dass ohne vorherige Thematisierung kollaborative, produktive PL-Interaktionen eher nicht zu erwarten sind (vgl. Littleton, 2010, S. 152), zu kritisieren, da den SuS weder förderliche Formen von Interaktionen beim PL noch fruchtbare Möglichkeiten zur Integration von bilingualen Praktiken beim PL explizit bewusst gemacht wurden. Dies stellt insbesondere hinsichtlich des Submersionskontexts ein Problem dar, da die bilingual aufwachsenden SuS kaum Erfahrung im bildungssprachlichen Umgang mit ihrer (weiteren) L1 haben. Dementsprechend fehlt vielen Studien sowohl methodisch als auch bezüglich des PL eine gewisse Kontinuität, in welcher sich die SuS an das bilinguale Interaktionsangebot sowie das PL akklimatisieren können und Zeit sowie Raum zum explorieren und üben erhalten.

Damit einher geht auch der Mangel an Informationen zu den individuellen Voraussetzungen und Eigenschaften der jeweiligen Probanden, sodass nur in einigen wenigen Studien zentrale Konstrukte wie Sprachkompetenzen in den jeweiligen Sprachen erhoben werden und weitere theoretisch bedeutsame Konstrukte wie Spracheinstellungen, die typische Sprachpraxis oder motivationale Phänomene wie Freude an bilingualer Interaktion oder wahrgenommene Schwierigkeit bilingualer Interaktion beim PL nicht berücksichtigt werden. Hierbei lassen auch nur Storch \& Wigglesworth (2003) die Probanden in Form 
von Selbstberichten zu Wort kommen, um einen Eindruck über ihre Einstellungen zu und ihre Erfahrungen mit bilingualer Interaktion beim PL zu erhalten.

Einige Studien kombinieren quantitative und qualitative Methoden in ihren Untersuchungen ohne diese auf der Basis der Forschungsperspektive von Mixed-Methods zu strukturieren, zu legitimieren und zu reflektieren. Zwar werden die quantitativen und qualitativen Befunde im Ergebnis- und Diskussionsteil zum Teil integriert und diskutiert, aber keine der Studien bettet ihre Forschungsfragen und methodischen Herangehensweisen explizit in ein Mixed-Methods Design wie z. B. Sequenz- oder Paralleldesign ein. Dabei stellt die Begründung der Zwecke, potentieller Notwendigkeiten und Vorteile des methodischen Vorgehens ein zentrales Qualitätsmerkmal von Mixed-Methods Forschung dar (vgl. Bryman, 2006). Dieses post-hoc Vorgehen ist insbesondere dann zu kritisieren, wenn qualitative Einblicke in die Daten der Studien gegeben werden, ohne eine bestimmte qualitative Methode wie Inhalts- oder Interaktionsanalyse bewusst hierfür einzusetzen, diese methodisch zu erläutern und anhand methodischer Qualitätskriterien wie Interrater-Übereinstimmung abzusichern.

Ein weiterer zentraler methodischer Mangel der Studien zu bilingualen Praktiken beim PL liegt in der fehlenden Reflexion der Individual- und Gruppenebene als Analyseeinheit. Interaktion ist ein reziprokes, mindestens bidirektionales Phänomen, bei dem nicht nur die individuellen Eigenschaften der Gruppenmitglieder zu berücksichtigen sind, sondern auch deren Komposition in der Gruppe. Sowohl die Modellierung der Sprachmodi nach Grosjean (2008) als auch die empirische Evidenz zu Sprachwechsel- und -mischungsphänomenen (vgl. z. B. Gardner-Chloros, 2009; Myers-Scotton, 2006) akzentuieren ebenfalls die Abhängigkeit von bilingualen Praktiken von der Interdependenz der jeweiligen Gesprächspartner, sodass sich die Frage stellt, ob bilinguale Interaktion überhaupt als Individualmerkmal operationalisiert und erfasst werden kann und sollte.

Die bisherige Befundlage zeichnet sich insgesamt durch eine immense Heterogenität bezüglich der Kontexte, dem Alter der Probanden, der jeweiligen untersuchten Lerninhalte und Kompetenzen, des PL-Formats, der methodischen Anlagen und Operationalisierungen sowie der theoretischen Fundierung aus. Aufgrund dieser Vielfalt und Bandbreite der Untersuchungsanlagen lässt sich kaum von einer robusten, einheitlichen Befundlage sprechen, sodass diesbezüglich weiterhin ein Forschungsdesiderat besteht und auch kommende Untersuchungen abhängig vom jeweiligen Erkenntnisinteresse teilweise explorativ vorgehen müssen. 


\subsection{Fazit}

Formelles PL als systematische, vorher geplante und strukturierte Sozialform zum Erwerb von „knowledge and skill through active helping and supporting among status equals or matched companions" (Topping, 2005, S. 631) stellt eine lernwirksame Methode für verschiedene SuS sowie Lerninhalte dar, mit welcher SuS in einer Gruppe gemeinsam für ihre Lernprozesse verantwortlich sind. Für erfolgreiches PL gilt es neben der Implementation eines strukturierenden Rahmens zur Etablierung einer förderlichen Gruppenkonstitution ebenfalls die notwendigen sprachlichen, kommunikativen und interpersonellen Fähigkeiten der SuS durch Hilfsmittel und Unterstützungsmaterialien zu fördern und zu fordern (vgl. Johnson \& Johnson, 1999). Die Qualität der Kommunikation innerhalb des PL stellt hierbei einen der relevantesten Prädiktoren für die Lernwirksamkeit dar (vgl. z. B. Howe, 2010; Littleton \& Mercer, 2010), die empirisch über bestimmte Sprachhandlungen wie z. B. Assertionen, Argumente und Erklärungen sowie deren Interaktion oder über Ratings des Diskurses operationalisiert wird. Ausgehend von der These, dass Denken eine ,individualized form of interpersonal communication" darstellt, sodass Kommunikation zu ,the very object of learning“ (vgl. Sfard, 2015, S. 249) wird, die sich gut mit Theorien des Konstruktivismus (vgl. de Lisi \& Golbeck, 1999; Hogan \& Tudge, 1999) als auch mit der auf Lerninhalte erweiterte Output-Hypothese von Swain (2000) vereinbaren lassen, akzentuiert gerade PL samt seiner Abhängigkeit von gelungener Interaktion die Förderlichkeit bilingualer Interaktionsangebote für bilingual aufwachsende SuS. Die Subjektentwicklung, das Wissen und die Fähigkeiten des Großteils bilingualer Sprecher konstituieren sich aufgrund ihrer Sozialisation und Lebensrealität in mehr als einer Sprache (vgl. Grosjean, 2010, S. 29ff). Insbesondere Studien zur individuellen mentalen (vgl. z. B. Clarkson, 2007; García \& Kano, 2014; García \& Li Wei, 2014; Hopewell, 2011; Storch \& Wigglesworth, 2003) und interindividuellen interaktionistischen Verwendung beider Sprachen bei Bildungsprozessen (vgl. z. B. Klingner \& Vaughn, 2000; Planas \& Setati, 2009; Rauch et al., in Vorb.; Schüler-Meyer et al., 2017; Swain \& Lapkin, 2000) akzentuieren die Bedeutung beider Sprachen zum Erwerb, zur Konstruktion und zur Vermittlung von Wissen und Fähigkeiten. Selbst in einzelsprachlichen Lernsettings wie der Submersion greifen bilingual aufwachsende SuS abhängig von ihren Sprachkompetenzen auf Praktiken des ,dependent“ und ,independent translanguaging“ (vgl. García \& Kano, 2014) zurück und zwar insbesondere bei herausfordernden Lerninhalten (vgl. z. B. Barwell, 2014; Clarkson, 2007). Zudem weist die Evidenz auf vielfältige lernförderliche Einsatzmöglichkeiten bilingualer Praktiken beim PL (vgl. z. B. Hopewell, 2011; Klingner \& Vaughn, 2000; Storch \& Wigglesworth, 2003; Swain \& Lapkin, 2000) sowie bei Bildungsprozessen im Allgemeinen hin (vgl. z. B. Clarkson, 2007; García \& Kano, 2014; 
García \& Li Wei, 2014) und liefert Evidenz zur Unterstützung der in Abschnitt 5.5.3 angeführten schwachen (vgl. Schüler-Meyer et al., 2017) und starken Position (vgl. Rauch et al., in Vorb.) zur Legitimation bilingualer Interaktionsangebote beim PL über vergleichbare respektive zusätzliche Effekte im Vergleich zu monolingualer Interaktion.

Dementsprechend kann abschließend gefolgert werden, dass die Bilingualismusforschung, die erziehungswissenschaftliche/pädagogische Rezeptionen anerkennungstheoretischer Reflexionen hinsichtlich der Anerkennungsformen des Rechts sowie der Solidarität und lehr-lerntheoretische Grundlagen des Konstruktivismus auf die Förderlichkeit bis Notwendigkeit bilingualer Interaktionsangebote beim PL hinweisen. Da die bisherige Evidenz diverse Forschungslücken, methodische Probleme und Unzulänglichkeiten aufweist, gilt es bilinguale Praktiken beim PL durch weitere empirische Studien zu untersuchen, um eine robuste empirische Befundlage für eine evidenzbasierte Implementation in die Regelschulen des Submersionskontextes zu erzeugen. Hierbei gilt es die Bedingungen des Submersionskontextes sowie die damit verbundenen Voraussetzungen der SuS und Schulen zu berücksichtigen, um die Potentiale und Herausforderungen dieses spezifischen Settings zu identifizieren, die die Grundlage für eine fruchtbare, didaktisch reflektierte Umsetzung in den Regelschulkontext darstellen. Die vorliegende Studie versucht einen Beitrag zu diesem Forschungsdesiderat zu leisten, welches für einen nicht zu unterschätzenden Anteil von bilingual aufwachsenden SuS eine hohe praktische Relevanz aufweist. 


\section{Fragestellungen}

In der vorliegenden Studie werden bilinguale Praktiken beim PL von bilingual türkisch-deutschsprachig aufwachsenden SuS der dritten und vierten Jahrgangsstufe untersucht. Hierbei steht nicht die Prüfung der Effektivität bilingualer Praktiken beim PL auf die Lernwirksamkeit im Fokus (siehe hierfür Rauch et al., in Vorb.), sondern vielmehr das Angebot zur bilingualen Interaktion selbst und dessen Umsetzung durch die Probanden. Dementsprechend werden im Folgenden die Abhängigkeit der Prävalenz bilingualer Interaktion von der Dyade (F1), die Prävalenz bilingualer Praktiken beim PL mit und ohne explizitem Angebot zur bilingualen Interaktion (F2), potentielle Zusammenhänge der individuellen sprachlichen und motivationalen Eigenschaften der Probanden mit der Quantität ihrer bilingualen Sprachproduktion (F3), die von den Probanden selbstberichteten Gründe für die Annahme oder Ablehnung des bilingualen Sprachangebots (F4) sowie die Funktionen bilingualer Praktiken in der Interaktion (F5) untersucht. Der aktuelle Forschungsstand zu bilingualen Praktiken beim PL zeichnet sich - wie in Abschnitt 5.5.3 bereits ausgeführt durch eine relativ geringen Anzahl an Studien, eine gewisse Heterogenität der Untersuchungsanlagen insbesondere angesichts der Vielzahl von Fallstudien, häufig eher als informelles PL zu bezeichnende PL-Formate und teilweise auftretenden methodischen Unzulänglichkeiten aus. Dementsprechend erscheint die bisherige Befundlage nicht sonderlich belastbar, die noch keine robusten Aussagen über bilinguale Praktiken beim PL - insbesondere im Submersionskontext - erlaubt. Dementsprechend weist die vorliegende Studie einen explorativen Charakter auf und formuliert Fragestellungen anstatt Hypothesen.

Zunächst gilt es zu prüfen, ob die Produktion bilingualer Gesprächsbeiträge der Peers abhängig von den anderen Peers derselben Dyade oder Gruppe beim PL ist. Der Begriff „pseudo-unilaterality“ (Duncan, Kanki, Mokros \& Fiske, 1984) bezeichnet den Denkfehler, dass Individualwerte von Individuen einer Dyade nur auf dem jeweiligen Individuum bei dyadischen Messungen beruhen (vgl. Kenny, Kashy \& Cook, 2006, S. 1f). Insbesondere in der Paarforschung wird die Abhängigkeit von bestimmten Individualwerten einer Person von dem jeweiligen Partner berücksichtigt. So ist z. B. die Zuneigung, die ein Individuum X gegenüber seinem Partner Y empfindet, mit sehr hoher Wahrscheinlichkeit in Teilen abhängig von dem Charakter von $\mathrm{Y}$, der Zuneigung $\mathrm{X}$ gegenüber und der Beziehung, die $\mathrm{X}$ und $\mathrm{Y}$ führen. Dementsprechend betrifft die Messung der gegenseitigen Zuneigung sowohl Individuum $\mathrm{X}$ als auch $\mathrm{Y}$ und ist somit dyadisch (vgl. ebd., S. 2). Die Abhängigkeit der jeweiligen Individualwerte in einer Dyade wird als ,nonindependence“ bezeichnet und folgendermaßen definiert: "If the two scores from the two members of the dyad are nonindependent, then those two scores are more similar to (or different) one another than are two scores from two people who are not members of the same 
dyad" (ebd., S. 4). Der Verzicht auf die Kontrolle einer potentiell existierenden „,nonindependence“ führt im Falle der tatsächlichen Existenz dieser zur Nutzung inadäquater statistischer Auswertungsmethoden, die die Abhängigkeit der untersuchten Variable von den Dyadenpartnern nicht berücksichtigen und somit zu verzerrten Ergebnissen führt (vgl. ebd., S. 46ff). Aufgrund der durch die doppelte Negation mit den Morphemen ,non-“ und „-in-“ erzeugten Sperrigkeit des Begriffs „,nonindependence“ wird im Folgenden dieses Phänomen mit „Abhängigkeit von/abhängig sein von der Dyade“ umschrieben.

Die eben angeführte Definition der Abhängigkeit von Individualwerten von der Dyade und die potentiellen Gefahren der Nichtbeachtung dieser legen die Vermutung nahe, dass es sich bezüglich dyadischer Datenanalyse primär um statistisch-methodische Fragen handelt. Dies ist allerdings nicht der Fall, da auch theoretische und inhaltliche Reflexionen in die Entscheidung miteinfließen sollten, ob Methoden der dyadischen Datenanalyse für das zu untersuchende Phänomen angemessen erscheinen (vgl. ebd., S. 46). Bilinguale Praktiken werden in der vorliegenden Studie im Kontext der Interaktion von Peers einer Dyade beim PL produziert. Interaktionen zeichnen sich primär dadurch aus, dass sich die Interaktanten mit ihren Gesprächsbeiträgen abwechselnd aufeinander beziehen und somit Kontinuität im Gesprächsverlauf herstellen (vgl. Deppermann, 2008, S. 68-78). Angesichts dessen sind Interaktionen maßgeblich von den teilnehmenden Individuen abhängig, worauf auch verschiedene Definitionen dieses Phänomens hinweisen. So lässt sich Interaktion als die „wechselseitige Beeinflussung des Handelns mindestens zweier Personen“ (Sarges \& Fricke, 1980, S. 488; zit. nach Nolda, 2000, S. 9) oder als ,die elementare Einheit des sozialen Geschehens, in der Menschen ihr Verhalten aneinander orientieren" (Endruweit \& Trommersdorf, 1989, S. 310; zit. nach Nolda, 2000, S. 9) definieren. Dementsprechend stellt sich die Frage, inwieweit individuelles Handeln in Interaktionen theoretisch und methodisch isoliert von dem Interaktionspartner betrachtet und analysiert werden darf. Zudem wird auch in jedem der in Abschnitt 3.5.1 beschriebenen soziolinguistisch orientierten Modelle von Sprachwahl und -verhalten (vgl. Auer, 1988; Grosjean, 2008; Myers-Scotton, 2006; Sachdev, Giles \& Pauwels, 2013) postuliert, dass die (bilinguale) Sprachwahl und das (bilinguale) Sprachverhalten neben kontextuellen und inhaltlichen Bedingungen maßgeblich von den Eigenschaften der Gesprächspartner sowie ihrer Beziehung zueinander determiniert werden. Auch die Evidenz zu diskursiv-rhetorischen Funktionen von Code-Switching (vgl. z. B. Auer, 2009; Fuller, 2009, S. 120-129; Gardner-Chloros, 2009, S. 67ff; Keim, 2012, S. 157ff; Potowski, 2009; Reyes, 2004; Ritchie \& Bhatia, 2013, S. 384ff) weist auf eine potentielle Abhängigkeit bilingualer Praktiken von der Interaktion und somit auch von der Dyade hin. Nichtsdestotrotz konnte der Autor der vorliegenden Studie bei der Literaturrecherche keine Studien identifizieren, die die Abhängigkeit der Prävalenz bilingualer Praktiken von den Gesprächspartnern empirisch unter Verwendung dyadischer Datenanalyse 
analysiert haben. Als Konsequenz dieser inhaltlichen, theoretischen und statistisch-methodischen Reflexionen gilt es folglich die Abhängigkeit der Prävalenz bilingualer Praktiken der Individuen einer Dyade von der Dyade zu untersuchen, um einerseits dieses Postulat empirisch zu prüfen und um andererseits angemessene Methoden zur Untersuchung der Prävalenz bilingualer Praktiken beim PL für die vorliegende Studie auswählen zu können:

F1: Ist die Prävalenz bilingualer Praktiken eines Individuums beim Peer-Learning abhängig von der Dyade?

Der Gebrauch bilingualer Praktiken beim PL von bilingual aufwachsenden $\mathrm{SuS}$ ist vor dem theoretischen Hintergrund der Sprachmodi nach Grosjean (2008) und weiterer soziolinguistisch orientierter Theorien bilingualen Sprachgebrauchs (vgl. Auer, 1998, Myers-Scotton, 2006; Sachdev, Giles \& Pauwels, 2013), aus der Perspektive der Modellierung von Unterricht als Angebot (vgl. z. B. Helmke, 2017, S. 69-101; Seidel, 2014) sowie vor der empirischen Befundlage zu bilingualen Praktiken beim PL mit explizitem Angebot (vgl. z. B. Planas \& Setati, 2009; Meyer \& Prediger, 2011; Schüler-Meyer et al., 2017; Storch \& Wigglesworth, 2003) nicht zwingend selbstverständlich. In Anlehnung an die Sprachmodi nach Grosjean (2008) gibt es eine Vielzahl gleichzeitig wirkender, dynamischer Faktoren, die die konkrete Sprachwahl und das Sprachverhalten von bilingualen Sprechern beeinflussen. Neben den Gesprächspartnern und ihren Eigenschaften stellen der Kontext und das Thema des Gesprächs zentrale Determinanten für das Auftreten bilingualer Praktiken dar. Die Sprachpraxis der deutschen Regelschule kann als Submersionskontext samt historisch gewachsenem monolingualen Habitus (vgl. Gogolin, 1994) beschrieben werden, in der für gewöhnlich keine Handlungsräume und Angebote zum Gebrauch der migrationsbedingt erworbenen, nicht-verkehrssprachlichen L1 für bilingual aufwachsende SuS bestehen. Die Gegenstände im Unterricht werden typischerweise nur im Deutschen behandelt und unter Verwendung bildungssprachlicher Formen und Funktionen im Unterricht vermittelt, die spezifische sprachliche und handlungsbezogene Anforderungen in der Bearbeitung an die SuS stellen. Da schulische Lerninhalte in der Regel in der Sprache bearbeitet werden, in der diese im Unterricht thematisiert werden (vgl. Clarkson, 2007, S. 194) und bilingual aufwachsende SuS im Submersionskontext nicht zwingend über bildungssprachliche Fähigkeiten in ihrer (weiteren) L1 verfügen, könnten diese Aspekte den Gebrauch bilingualer Praktiken verhindern, selbst wenn bilinguale Praktiken beim PL explizit angeboten werden. Zwar weisen z. B. die Studien von Schüler-Meyer et al. (2017) oder Planas und Setati (2009) auf den Gebrauch bilingualer Praktiken beim PL im Submersionskontext und explizitem Angebot hin, andere Studien wie z. B. Meyer und Prediger (2011) und Storch und Wigglesworth (2003) akzentuieren allerdings 
die Probleme der Aktivierung eines bilingualen Sprachmodus im Submersionskontext trotz expliziten Angebots. Die Studie von Storch und Wigglesworth (ebd.) zeigt hierbei auch, dass die Annahme von Angeboten zur bilingualen Interaktion auch von manchen bilingualen Sprecher bewusst abgelehnt wird, da sie im Rückgriff auf ihre (weitere) L1 eine Vergeudung von Lernmöglichkeiten zur Entwicklung der Fähigkeiten in ihrer anderen Sprache vermuten. Dementsprechend könnte sich auch trotz eines expliziten Angebots zur Nutzung bilingualer Praktiken beim PL ein monolingualer Sprachmodus in der Unterrichtssprache zur Interaktion etablieren, sodass zunächst zu prüfen gilt, ob ein explizites Angebot bilingualer Interaktion beim PL überhaupt zu einer höheren Prävalenz bilingualer Praktiken führt als das Fehlen eines solchen Angebots.

Angesichts des Einflusses des Gesprächsgegenstandes der Kommunikation als Determinante für die Sprachwahl und das Sprachverhalten bilingualer Sprecher (vgl. Auer, 1988; Grosjean, 2008) könnten auch die konkreten Lerninhalte für die Prävalenz bilingualer Praktiken beim PL von Bedeutung sein. Verschiedene Lerninhalte könnten sowohl unterschiedliche sprachliche Voraussetzungen und Anforderungen an die Peers stellen als auch sich potentiell für unterschiedliche Zwecke des Gebrauchs bilingualer Praktiken beim PL eignen. Dementsprechend könnten die konkreten Lerninhalte solche sprachlichen und/oder handlungsbezogenen Anforderungen an die Peers erzeugen, denen sie eventuell nur in einer Sprache gewachsen sind oder die sie ohne den Rückgriff auf ihre (weitere) L1 bewältigen können, sodass monolinguale Interaktion beim PL präferiert wird. Die bisherigen Studien zu bilingualen Praktiken beim PL haben stets lediglich einen Lerngegenstand betrachtet, anstatt zwei verschiedene Domänen wie Lesen und Rechnen miteinander zu vergleichen.

Hierbei könnten nicht nur die Lerninhalte oder Domänen per se, sondern auch die damit einhergehenden einzelnen Phasen und Aufgabenformate für die Prävalenz bilingualer Praktiken beim PL relevant sein. Die soeben angeführte Argumentation hinsichtlich eines potentiellen Einflusses der sich unterscheidenden sprachlichen Voraussetzungen und Anforderungen von Lerninhalten oder Domänen lässt sich auch auf einzelne Phasen und Aufgabenformate eines Lerninhalts oder einer Domäne beziehen. Lediglich die Studie von Swain und Lapkin (2000) prüft inferenzstatistisch den Zusammenhang von Aufgabenformat mit der Prävalenz des Rückgriffs der Probanden auf ihre L1 beim PL. Swain und Lapkin (ebd.) konnten keine signifikanten Unterschiede der Prävalenz bilingualer Praktiken der Dyaden in Abhängigkeit der ,jigsaw“ und „dictogloss task“ identifizieren.

Zusammengefasst stellen sich dementsprechend die Fragen, ob das Sprachangebot, der Lerninhalt sowie die mit dem Lerninhalt verbundenen konkreten Phasen und Aufgaben bedeutsam für die Prävalenz bilingualer Praktiken beim PL sind: 
F2a: Unterscheidet sich die Prävalenz bilingualer Praktiken beim Peer-Learning abhängig vom Angebot dieser?

F2b: Unterscheidet sich die Prävalenz bilingualer Praktiken beim Peer-Learning abhängig vom Lerninhalt?

F2c: Unterscheidet sich die Prävalenz bilingualer Praktiken beim Peer-Learning abhängig von den unterschiedlichen Phasen und Aufgaben des Lerninhalts?

Anschließend soll der potentielle Zusammenhang individueller Merkmale mit der Prävalenz bilingualer Praktiken beim PL untersucht werden. Innerhalb Grosjeans (2008) Sprachmodi und weiteren soziolinguistisch orientierten Theorien bilingualen Sprachgebrauchs (vgl. Auer, 1988; Myers-Scotton, 2006; Sachdev, Giles \& Pauwels, 2013) stellen die Merkmale, Eigenschaften und Motive der Kommunikationspartner sowie ihr Verhältnis zueinander zentrale Einflussgrößen für bilinguale Praktiken dar. Die Prävalenz bilingualer Praktiken ist hierbei abhängig von unter anderem der Sprachkompetenz, den Einstellungen der Sprecher zu ihren Sprachen und ihrer typischen Sprachpraxis (vgl. Grosjean, 2008, S. 42). Eine exklusiv individuelle Perspektive ohne Berücksichtigung des Gesprächspartners erscheint, wie oben bei Fragestellung 1 bereits ausgeführt, bei einem dynamischen, sich durch Reziprozität auszeichnenden Phänomen wie bilingualen Praktiken in der Interaktion verkürzt. Vielmehr dürften die individuellen Eigenschaften beider Gesprächspartner in Bezugnahme aufeinander mit der Prävalenz bilingualer Praktiken zusammenhängen. Wenn z. B. ein Interaktant sich durch eine geringe Sprachkompetenz sowie durch negative Einstellungen zu seiner nicht-verkehrssprachlichen L1 auszeichnet, könnten selbst hohe positive Ausprägungen der eben genannten Variablen des Gesprächspartners nicht die Dispositionen des anderen kompensieren, sodass sich bei einer solchen Konstellation primär eine monolinguale Interaktion im Deutschen etablieren könnte.

Neben individuellen sprachlichen Eigenschaften könnte aus anerkennungstheoretischer Perspektive (vgl. Do Mar Castro Varela \& Mecherill, 2010; Mecherill, 2005; Stojanov, 2006) zudem die Wahrnehmung des bilingualen Sprachangebots eine weitere zentrale Rolle für die Prävalenz bilingualer Praktiken spielen. So könnten z. B. motivationale Bedürfnisse wie Kompetenz- und Autonomieerleben und soziale Eingebundenheit der Self-Determination Theorie (vgl. Ryan \& Deci, 2018) in Zusammenhang mit der Prävalenz bilingualer Praktiken stehen. Stojanov (2006, S. 199) postuliert für Individuen mit MH die kulturell-biographische Anerkennung als Unterform der Anerkennungsform 
der Solidarität, die sowohl die ,partikular- ansozialisierten Selbst- und Wirklichkeitsdeutungsmuster des Einzelnen“ als auch die Fähigkeit, „diese Muster in einem entgrenzten, interperspektivisch strukturierten Raum transformierend zu artikulieren“, umfasst. Zudem stellen nach Stojanov (ebd., S. 168f) „Anerkennungsverhältnisse nicht das Ziel von Bildung“, sondern „vielmehr Voraussetzung und Triebwerk für Bildung" dar. Das bilinguale Sprachangebot könnte hierbei von den SuS als Anerkennung auf den Ebenen des Rechts und/oder der Solidarität wahrgenommen werden, sodass sich in diesem Handlungsraum ein positiver Zusammenhang zwischen motivationalen Variablen wie dem Kompetenz- und Autonomieerleben oder der sozialen Eingebundenheit mit der Prävalenz bilingualer Praktiken bestehen könnte. Aber auch weitere motivationale Variablen wie die empfundene Freude sowie Schwierigkeit, bilingual über Lerninhalte beim PL zu kommunizieren, und der eingeschätzte Nutzen bilingualer Interaktion bei Bildungsprozessen könnten für die Prävalenz bilingualer Praktiken beim PL bedeutsam sein.

Die bisherige Evidenz liefert erste Hinweise dafür, dass sprachliche Eigenschaften wie z. B. Sprachkompetenz, Spracheinstellungen und die gewohnten Sprachpraktiken mit der Prävalenz bilingualer Praktiken in Bildungsprozessen sowohl allgemein (vgl. z. B. Clarkson, 2007; García \& Kano, 2014; García \& Li Wei, 2014) als auch im Kontext des PL (vgl. z. B. Planas \& Setati, 2009; Storch \& Wigglesworth, 2003; Swain \& Lapkin, 2000) zusammenhängen. Hinsichtlich eines Zusammenhangs motivationaler Variablen mit der Prävalenz bilingualer Praktiken in Bildungsprozessen gibt es bisher keine empirischen Erkenntnisse.

Aus theoretischer und/oder empirischer Perspektive könnten positive $\mathrm{Zu}$ sammenhänge zwischen sprachlichen sowie motivationalen Variablen mit der Prävalenz bilingualer Praktiken beim PL bestehen. Bezüglich der sprachlichen Variablen dürfte die potentielle Kausalität der Zusammenhänge so zu modellieren sein, dass die Sprachkompetenz, Spracheinstellungen sowie die typische Sprachpraxis als Prädiktoren für die Prävalenz bilingualer Praktiken fungieren. Hinsichtlich der motivationalen Variablen erscheint eine Modellierung der Kausalität des Zusammenhangs mit der Prävalenz bilingualer Praktiken weniger eindeutig. Einerseits könnte z. B. aus der Perspektive der Sprachmodi nach Grosjean (2008) die erlebte Freude am bilingualen PL die Prävalenz bilingualer Praktiken beeinflussen und somit als Prädiktor der Prävalenz bilingualer Praktiken beim PL operieren. Andererseits ist allerdings auch denkbar, dass z. B. eine hohe Prävalenz bilingualer Praktiken sich positiv auf das Kompetenzund Autonomieerleben der „basic needs“ nach Ryan und Deci (2018) sowie auf weitere motivationale Variablen auswirken könnte, sodass die Prävalenz bilingualer Praktiken als Prädiktor fungiert. 
Da sich die potentielle Kausalität des Zusammenhangs der Prävalenz bilingualer Praktiken mit motivationalen Variablen beim PL theoretisch und inhaltlich nicht klar ableiten lässt, soll der korrelative Zusammenhang anstatt regressive Vorhersagen bilingualer Praktiken mit sprachlichen und motivationalen Eigenschaften der Probanden beim PL untersucht werden. Zudem gilt es auch wie in den vorangegangen Fragestellungen zu berücksichtigen, dass die individuellen Ausprägungen sprachlicher und motivationaler Eigenschaften des einen Gesprächspartners mit der Prävalenz des bilingualen Sprachgebrauchs des anderen und umgekehrt zusammenhängen könnten:

F3a: Besteht ein Zusammenhang zwischen sprachlichen Eigenschaften der Individuen und der Prävalenz bilingualer Praktiken beim Peer-Learning?

F3b: Besteht ein Zusammenhang zwischen motivationalen Eigenschaften der Individuen und der Prävalenz bilingualer Praktiken beim Peer-Learning?

Ebenso zentral für das Erkenntnisinteresse neben der Abhängigkeit der Prävalenz bilingualer Praktiken beim PL von der Dyade (F1), vom bilingualen Angebot, den Lerninhalten und konkreten Aufgaben (F2) sowie dem potentiellen Zusammenhang von individuellen sprachlichen und motivationalen Merkmalen mit der Prävalenz bilingualer Praktiken (F3) sind qualitative Merkmale bilingualer Interaktion beim PL. Sprachliches Handeln kann nicht nur lediglich aus einer quantitativen und/oder formalen Perspektive betrachtet werden, da sich dieses stets durch eine gewisse Funktionalität auszeichnet (vgl. Grice, 1975; Hamers \& Blanc, 2000; Searle, 1969). Gesprächspartner unterstellen sich implizit die Einhaltung gewisser Konversationsmaximen bei der Interaktion (vgl. Grice, 1975). Diese fungieren als eine Orientierung für das gemeinschaftliche Sprachhandeln und verfolgen mit ihren Sprechakten gewisse Absichten, wie z. B. Bitten oder Fragen stellen, Assertionen äußern oder Drohungen aussprechen (vgl. Searle, 1969). Diese Absichten sollen wiederum eine gewisse Wirkung beim Zuhörer erzeugen, wie z. B. diesen zu bestimmten Handlungen oder Antworten zu bewegen, von einer These zu überzeugen oder einzuschüchtern. In manchen Fällen sind die zu Grunde liegenden Motive des Sprachhandelns den Sprechern bewusst zugänglich (vgl. z. B. Myers-Scotton, 2006; Sachdev, Giles \& Pauwels, 2013), sodass sie die mit dem Sprachhandeln zu verfolgenden Ziele auch artikulieren können.

Hinsichtlich des Erkenntnisinteresses der vorliegenden Arbeit weisen zunächst die Einstellungen und Meinungen von bilingual aufwachsenden $\mathrm{SuS} \mathrm{zu}$ bilingualen Interaktionsangeboten beim PL eine hohe Bedeutsamkeit auf. Unterrichtsangebote werden nicht zwingend von SuS angenommen (vgl. Helmke, 
2017, S. 69-101; Seidel, 2014), sodass die Gründe für die Annahme oder Ablehnung bestimmter Unterrichtsangebote von SuS ein hohes Potential bergen, sowohl die wahrgenommene Fruchtbarkeit und Förderlichkeit als auch Herausforderungen und Probleme der SuS bezüglich des jeweiligen Angebots zu identifizieren. Mit Hilfe dieser Einblicke können die Unterrichtsangebote anschließend adaptiert sowie Voraussetzungen bei den SuS geschaffen werden, mit welchen sie das Unterrichtsangebot besser annehmen und umsetzen können. Zwar gibt es bereits einige Befunde zu den selbstberichteten Motiven für bilinguales Sprachhandeln im Bildungskontext bei bilingual aufwachsenden SuS (vgl. z. B. Barwell, 2014; Clarkson, 2007; Culligan, 2015; García \& Kano, 2014), die Gründe von SuS zum Gebrauch oder Verzicht auf bilinguale Interaktion beim PL sind allerdings noch unklar. Lediglich Storch und Wigglesworth (2003) haben ihre Probanden hinsichtlich ihrer Meinung zu bilingualen Praktiken beim PL interviewt. Hierbei wurde der Rückgriff auf die L1 von den Probanden zum Diskutieren von Inhalten, zur Überbrückung lexikalischer Lücken und zur Erklärung von Bedeutungen und grammatischen Problemen als förderlich erachtet. Allerdings identifizierten Storch und Wigglesworth (ebd.) auch einige Herausforderungen und Probleme für die Annahme des bilingualen Interaktionsangebots, da ihre Probanden hinsichtlich des Rückgriffs auf ihre L1 eine Erhöhung der Bearbeitungsdauer bei gleichzeitiger Reduzierung der Lernmöglichkeiten zum Erwerb der L2 vermuten.

Dementsprechend wird in der vorliegenden Untersuchung die Einstellung und Meinung der Probanden zum bilingualen Interaktionsangebot dahingehend untersucht, welche Gründe sie für die Annahme oder Ablehnung des bilingualen Sprachangebots berichten:

F4: Welche Gründe für die Annahme oder Ablehnung des bilingualen Sprachangebots berichten die Probanden?

Abschließend sollen die Funktionen, für die bilinguale Praktiken von den bilingual aufwachsenden SuS beim PL eingesetzt werden, untersucht werden. Hierbei gilt es nicht nur die Bandbreite potentieller Funktionen zu identifizieren, sondern auch die Prävalenzen der einzelnen Funktionen miteinander zu vergleichen. Eine erste übergeordnete Annäherung an das Funktionsspektrum bilingualer Praktiken beim PL stellt die Differenzierung von bilingualen Praktiken bei Diskursen über trainingsrelevante (,on-task") oder trainingsirrelevante Themen (,off-task") dar. Die bisherige Evidenz zu bilingualen Praktiken im Bildungskontext liefert erste Einblicke, für welche lernrelevanten Funktionen bilinguale Praktiken genutzt werden. Hierbei wurden vor allem förderliche „On-Task“-Funktionen wie z. B. Aufgabenbearbeitung und aufgabenorientierte Diskurse (vgl. z. B. Barwell, 2014; Hopewell, 2011; Klingner \& Vaughn, 
2000; Moschkovich, 2007; Planas \& Setati, 2009; Rehbein, 2011), Task-Management (vgl. z. B. Rehbein, 2011; Storch \& Wigglesworth, 2003; Swain \& Lapkin, 2000), metakognitive Prozesse wie Aufmerksamkeitssteuerung (Swain \& Lapkin, 2000), regulative Prozesse wie Monitoring und Kontrolle (Kern, 1994) und metalinguistische Reflexionen (Hopewell, 2011; Storch \& Wigglesworth, 2003) identifiziert. Primär scheinen also bilinguale Praktiken bezüglich „On-Task“-Funktionen zur Aufgabenbearbeitung, zum Task-Management und für metakognitive Prozessen wie z. B. zur (Selbst-)Regulation verwendet zu werden.

Lediglich die Studie von Swain und Lapkin (2000) machen Angaben über Häufigkeiten über aufgabenrelevante und -irrelevante Funktionen bilingualer Praktiken beim PL und deren Prävalenz. Dabei hat die Prävalenz von bilingualen Praktiken für „Off-Task“-Diskurse eine hohe praktische Bedeutung, da Lehrkräfte in der Submersion beim Rückgriff ihrer SuS auf die nicht-verkehrssprachliche L1 während des Unterrichts den Verdacht hegen, die SuS kommunizieren über unterrichtsirrelevante Themen (vgl. Carless, 2008, S. 333). Dies stellt für Lehrkräfte, die nicht über die nicht-verkehrssprachliche L1 ihrer SuS verfügen, einen potentiellen Kontrollverlust dar, der auch ihre moderierende Rolle beim PL erschweren kann. Mit der Analyse der Prävalenz bilingualer Praktiken für „Off-Task“-Zwecke ist allerdings kein Vergleich der Prävalenzen verschiedener Nutzungsweisen bilingualer und monolingualer Praktiken intendiert. Vielmehr soll die Frage fokussiert werden, ob bilinguale Praktiken vermehrt für „On“- oder „Off-Task“-Funktionen eingesetzt werden.

Zudem stellt sich die Frage, ob verschiedene Lerninhalte unterschiedliche Prävalenzen distinkter Zwecke von bilingualen Praktiken erzeugen. Da Lerninhalte hinsichtlich ihrer Zielsetzung und Durchführung sowie Voraussetzungen und Anforderungen divergieren, könnten sich bestimmte Funktionen bilingualer Praktiken für unterschiedliche Lerninhalte beim PL eher eignen als andere:

F5a: Für welche Funktionen werden bilinguale Praktiken beim Peer-Learning eingesetzt?

F5b: Unterscheiden sich die Prävalenzen der Funktionen bilingualer Praktiken beim Peer-Learning zwischen verschiedenen Lerninhalten? 


\section{Methodik}

Die Untersuchung der oben angeführten Fragestellungen erfolgt innerhalb eines sequenziellen Mixed-Methods-Vertiefungsdesign (vgl. Kuckartz, 2014, S. $78 \mathrm{ff}$ ). Ein Vertiefungsdesign ist ein „Zwei-Phasen-Design, in dem zunächst die quantitative Studie durchgeführt und ausgewertet wird. An diese schließt sich die qualitative Studie an“, um ,die Resultate des quantitativen Teils durch die qualitative Vertiefung besser zu verstehen“ oder um ,gezielt die Erklärungslücken zu füllen, die die quantitative Studie hinterlassen hat" (ebd., S. 78). Dementsprechend können je nach Ergebnislage die Ergebnisse der mit den qualitativen Methoden untersuchten Fragestellungen eventuell $\mathrm{zu}$ einem besseren Verständnis der quantitativen Ergebnisse beitragen (vgl. ebd., S.78ff). Greene, Caracelli \& Graham (1989, S. 259) beschreiben diesen Zweck von Mixed-Methods mit dem Begriff ,,complementarity“. Komplementarität „,seeks elaboration, enhancement, illustration, clarification of the results from one method with the results from another" (ebd.). Dies gilt prinzipiell auch für die vorliegende Studie. Allerdings erfüllt die Kombination von quantitativen sowie qualitativen Fragestellungen und Methoden primär den Anspruch an eine dem Untersuchungsgegenstand angemessene Berücksichtigung seiner unterschiedlichen zentralen Aspekte und Komponenten. Eine adäquate Untersuchung bilingualer Praktiken beim PL verlangt die Kombination von quantitativer und qualitativer Methodik, da für das Erkenntnisinteresse der vorliegenden Studie sowohl quantitative Aspekte - wie z. B. Prävalenzen und potentielle Zusammenhänge der Prävalenz bilingualer Praktiken mit individuellen Eigenschaften der Probanden - als auch qualitative Aspekte - wie z. B. zu Grunde liegende Motive für die Annahme und Ablehnung des bilingualen Interaktionsangebots und konkrete Funktionen bilingualer Praktiken beim PL - auszeichnet. Die ausschließliche Betrachtung des Phänomens aus lediglich einer dieser Perspektiven würde den Untersuchungsgegenstand unterspezifizieren und weitere relevante Aspekte übergehen. In der vorliegenden Studie wird dementsprechend auch keine Gewichtung der Relevanz der beiden Phasen beziehungsweise Perspektiven vorgenommen (QUAN $\rightarrow$ QUAL). Diese beiden distinkten Forschungszugänge decken unterschiedliche Aspekte des zu untersuchenden Phänomens ab und tragen somit gemeinsam zum Erkenntnisinteresse bei. Die Datenintegration der quantitativen und qualitativen Ergebnisse erfolgt allerdings erst in der Diskussion (Kapitel 9), da im Ergebnisteil (Kapitel 8) die Ergebnisse einzeln präsentiert werden. Im Zuge der Datenintegration wird ebenfalls das Potential von Mixed-Methods Untersuchungsanlagen zur Triangulation beansprucht. Triangulation lässt sich als ,convergence, corroboration, correspondence of results from different methods" (Greene, Caracelli \& Graham, 1989, S. 259) definieren. Dementsprechend können mit Mixed-Methods auch diesel- 
ben Teilaspekte des Phänomens bilingualer Praktiken beim PL aus der Perspektive verschiedener methodischer Zugänge betrachtet werden. Ein schematischer Überblick über das Design samt den übergeordneten Fragestellungen wird in Abbildung 2 illustriert.

Im quantitativen Teil wird zunächst untersucht, ob die Prävalenz bilingualer Praktiken ein Merkmal darstellt, welches rein vom Individuum determiniert wird oder von der Dyade abhängig ist (F1). Diese Frage trägt zum einen zur Klärung der theoretisch postulierten Bedeutung der Gesprächspartner für die Sprachwahl und das Sprachverhalten bilingualer Sprecher bei, zum anderen liefert sie Informationen für den adäquaten statistisch-methodischen Umgang mit diesem Phänomen. Anschließend wird das Auftreten bilingualer Praktiken im Verhältnis zu monolingual deutschsprachigen Praktiken erfasst und zwischen den Gruppen mit und ohne bilingualem Sprachangebot auf Unterschiede hin untersucht (F2). Danach wird die Existenz potentieller Zusammenhänge individueller Eigenschaften der Probanden mit der Prävalenz bilingualer Praktiken geprüft (F3). Dieses methodisch quantitativ orientierte Vorgehen ist allerdings nicht in der Lage, die jeweiligen zu Grunde liegenden Motive zur Annahme oder Ablehnung des Angebots (F4) sowie die Funktionen bilingualer Praktiken beim PL (F5) zu erfassen. Jedoch ermöglichen erst diese qualitativen Perspektiven Einblicke in die von den Probanden wahrgenommenen Potentiale und Herausforderungen sowie Nutzungsmöglichkeiten bilingualer Praktiken beim PL, sodass die quantitativen und qualitativen Forschungsfragen unterschiedliche Aspekte des Phänomens beleuchten, die für die Einschätzung der Fruchtbarkeit eines solchen potentiellen Sprachangebots notwendig sind.

Aufgrund des vorliegenden Mixed-Methods-Designs ist es notwendig, die Methodik für jede Fragestellung einzeln zu erläutern, da sie sich zum Teil hinsichtlich der verwendeten Stichprobe, Konstrukte, Instrumente sowie Analysemethoden unterscheiden. Bevor die Methodik der einzelnen Fragestellungen dargestellt wird, gilt es allerdings die „BiPeer-Studie“ als Datengrundlage der Studie näher zu beschreiben, insbesondere hinsichtlich des Designs (Abschnitt 7.1.1), der Interventionen (Abschnitt 7.1.2). In Abschnitt 7.1.2.3 werden auch die konkreten halbschriftlichen Rechenstrategien und Globalverfahren der Rechenstrategieintervention der vorliegenden Datengrundlage vorgestellt, die in Abschnitt 5.4.5 ausgespart wurden. 
Abbildung $2{ }^{10}$

Mixed-Methods-Vertiefungsdesign des Forschungsvorhabens

F1: Ist die Prävalenz bilingualer Praktiken abhängig von der Dyade?

F2: Wie hoch ist die Prävalenz bilingualer Praktiken beim PL?

F3: Bestehen Zusammenhänge individueller Eigenschaften mit der Prävalenz bilingualer Praktiken?

F4: Welche selbstberichteten Gründe für die Annahme oder Ablehnung

des bilingualen Sprachangebots nennen die Probanden?

F5: Für welche Funktionen werden bilinguale Praktiken beim PL eingesetzt?
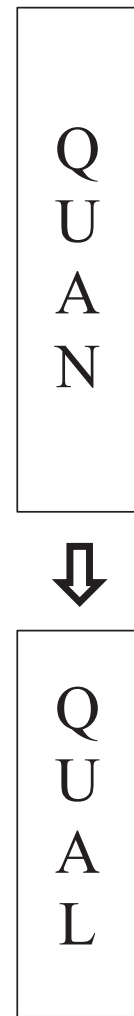

\subsection{BiPeer-Studie}

Die vorliegende Dissertation basiert auf Daten des Projektes „Förderung der Deutsch-Lesekompetenz bilingualer GrundschülerInnen durch Peer-Learning“ (BiPeer), welches in dem Forschungszentrum „Individual Development and Adaptive Education“ (IDeA) am Deutschen Institut für Internationale Pädagogische Forschung (DIPF) durchgeführt wurde. BiPeer wird innerhalb der Förderlinie ,Sprachliche Bildung und Mehrsprachigkeit“" vom Bundesministerium

10 Alle Abbildungen sowie Tabellen ohne expliziten Quellenbeleg sind vom Autor selbst erstellt worden. 
für Bildung und Forschung finanziert und von Frau Jun. Prof. Dominique Rauch und Frau Prof. Dr. Jasmin Decristan geleitet. Die Projektlaufzeit umfasste den Zeitraum von Juli 2014 bis Juli 2017, wobei die Pilotierung von März bis Juni 2015 stattfand und die Feldphase der Hauptstudie des Projektes sich in vier Erhebungsdurchgängen von September 2015 bis Januar 2017 gliederte. Der Autor der vorliegenden Dissertation ist seit Beginn des Projektes in diesem tätig und wirkte in allen zentralen Projektaufgaben wie z. B. der Adaptation der Interventionen, der Erstellung der Diagnostikbatterie und Skalen, der Stichprobenrekrutierung und -pflege, der Schulung und Betreuung von studentischen Hilfskräften und Praktikanten sowie der Datenaufbereitung und -auswertung mit.

\subsubsection{Design der BiPeer-Studie}

BiPeer untersucht in einem quasiexperimentellen Treatment-Kontrollgruppendesign den Einfluss des Sprachhintergrunds und eines bilingualen Sprachangebots auf den Erfolg des PL bei monolingual deutschsprachig und bilingual türkisch-deutschsprachig aufwachsenden SuS der dritten und vierten Klasse. Die Probanden besuchen hierzu eine auf Lese- oder Rechenstrategien fokussierte PL-Intervention, bei welchen sie in Dyaden unter der Anleitung und Aufsicht einer im Vorfeld geschulten, studentischen Übungsleitung zusammenarbeiten (siehe Tabelle 1). Eine der Besonderheiten der Interventionsstudie ist, dass die Variation des Sprachhintergrunds und des Sprachangebots jeweils drei in gleicher Weise manipulierte Lese-(LG) und Rechengruppen (RG) erzeugen, die innerhalb des gleichen Lerninhalts und über diesen hinaus als Kontrollgruppen fungieren können.

Tabelle 1

Interventionsdesign der BiPeer-Studie

\begin{tabular}{lcccccc} 
Gruppe & LG & LGD & LGT/D & RG & RGD & RGT/D \\
\hline Lerninhalt & Lesen & Lesen & Lesen & Rechnen & Rechnen & Rechnen
\end{tabular}

Sprachhinter-

$\begin{array}{lllllll}\text { grund } & \mathrm{M}+\mathrm{B} & \mathrm{B}+\mathrm{B} & \mathrm{B}+\mathrm{B} & \mathrm{M}+\mathrm{B} & \mathrm{B}+\mathrm{B} & \mathrm{B}+\mathrm{B}\end{array}$

(Peer $1+$ Peer 2)

Sprachangebot

$\mathrm{D}$

$\mathrm{D}$

$\mathrm{T} / \mathrm{D}$

$\mathrm{D}$

$\mathrm{D}$

$\mathrm{T} / \mathrm{D}$

Anmerkungen: $\mathrm{LG}=$ Lesegruppe, $\mathrm{RG}=$ Rechengruppe, $\mathrm{D}=$ Deutsch, $\mathrm{T} / \mathrm{D}=$ Türkisch/Deutsch, $\mathrm{M}=$ monolingual Deutsch; $\mathrm{B}=$ bilingual Türkisch/Deutsch 
In den Gruppen LG und RG bilden ein monolingual deutschsprachig und ein bilingual türkisch-deutschsprachig aufwachsendes Kind eine Dyade, wobei die sprachliche Interaktion selbstverständlich auf das Deutsche limitiert ist. Die Gruppen LGD und RGD unterscheiden sich von LG respektive RG dahingehend, dass zwei bilingual türkisch-deutschsprachig aufwachsende Kinder als Dyade zusammenarbeiten. Die sprachliche Interaktion ist hierbei ebenfalls auf das Deutsche restringiert, obwohl die Peers ohne diese Einschränkung theoretisch bilingual miteinander interagieren könnten. Die Gruppen LGT/D und RGT/D bestehen ebenfalls aus Dyaden mit bilingual türkisch-deutschsprachig aufwachsenden SuS. Allerdings erhalten die Dyaden dieser Gruppen explizit das Angebot, auch auf Türkisch oder auf Türkisch und Deutsch gemischt miteinander zu interagieren.

Die Bildung und Gruppenzuteilung der Dyaden erfolgte aufgrund von Restriktionen der Terminkoordination im Nachmittagskontext nach der regulären Unterrichtszeit nicht immer zufällig. Es konnten nur SuS zusammenarbeiten, die zur selben Schule gehörten und an denselben Tagen zu denselben Zeiten die Intervention besuchen konnten, wobei ebenfalls eine Übungsleitung zu diesen Terminen verfügbar sein musste. Diese organisatorischen Parameter bestimmten primär die Bildung der Dyaden. Hierbei wurde versucht, weitestgehend die bisherigen Erkenntnisse zu Gruppenmerkmalen beim PL hinsichtlich des Lernzuwachses zu berücksichtigen (vgl. z. B. Robinson, Schofield \& Steers-Wentzell, 2005). Das heißt, es wurden präferiert Probanden mit dem gleichen Geschlecht sowie der Zugehörigkeit zur gleichen Klassenstufe zu einer Dyade zusammengefasst. Potentielle Leistungsunterschiede der Ausgangsbedingungen im Lesen und Rechnen der Individuen wurden bei der Bildung der Dyaden nicht berücksichtigt. Im nächsten Schritt wurden die gebildeten Dyaden den sechs verschiedenen Gruppen zugeteilt. Die Zuteilung von Dyaden mit monolingual deutschsprachig aufwachsenden Kindern ist aufgrund der Untersuchungsanlage auf eine zufällige Zuteilung der Gruppen LG und RG beschränkt. Dyaden mit türkisch-deutschsprachig aufwachsenden Probanden wurden in Abhängigkeit der Zuteilung einer türkisch-deutschsprachigen studentischen Hilfskraft oder PraktikantIn den Gruppen LGD, RGD, LGT/D oder RGT/D zufällig zugeordnet. Dyaden mit zwei türkisch-deutschsprachig aufwachsenden Probanden, die nicht von einer bilingual türkisch-deutschsprachigen Übungsleitung instruiert werden konnten, konnten nur den Gruppen LGD oder RGD zugewiesen werden. Die verbliebenen Dyaden mit zwei türkischdeutschsprachig aufwachsenden Probanden samt bilingualer Übungsleitung wurden in den ersten beiden Erhebungsdurchgängen anschließend auf die Gruppen LGD, RGD, LGT/D oder RGT/D zufällig aufgeteilt. Um annähernd gleiche Gruppengrößen sowie Ausgangsbedingungen der Gruppen zu schaffen und somit deren Vergleichbarkeit sicherzustellen, wurde die Gruppenzuteilung in den beiden letzten Erhebungsdurchgängen hinsichtlich der folgenden Merkmale auf Individualebene weitestgehend versucht zu balancieren: Geschlecht, 
Jahrgangsstufe, Lese- und Kopfrechenfähigkeiten sowie Sprachkompetenz im Türkischen.

\subsubsection{Interventionen der BiPeer-Studie}

Die Interventionen stellen Adaptionen bereits etablierter PL-Programme dar, wobei vor allem „Peer-Assisted Learning Strategies“ (PALS) von Fuchs et al. (2008) und „Blitzlesen im Team“ von Munser-Kiefer und Kirschhock (2012) als Orientierung und Vorlage dienten. Die Trainings sind durch vorgegebene Abfolgen sowie interaktionssteuernde Elemente mit klar definierten Handlungsspielräumen, Aktivitäten, Routinen und Materialien restringiert, die die Zusammenarbeit der Peers rahmen. Die Kooperation innerhalb der Dyaden wird hinsichtlich der Zuteilung der konkreten Aufgaben durch die reziproken Rollen „Spieler“ und „Trainer“ strukturiert. Der Spieler wendet die Lese- oder Rechenstrategien an und kann bei Unsicherheiten und Problemen den Trainer um Hilfe bitten. Hierbei ist der Spieler aufgefordert, seine Gedanken zu verbalisieren, damit der Trainer an den Prozessen der Strategieanwendung teilhaben kann. Der Trainer hingegen leitet den Spieler an, indem er ihm Anweisungen, Tipps und Hilfestellungen gibt, sein Handeln überwacht und die Güte der Strategieanwendung und Aufgabenbearbeitung prüft. Hierzu gehört auch, dass der Trainer das Fortschreiten im Prozess des Spielers unterbinden kann, wenn der Trainer mit der Strategieanwendung oder Lösung des Spielers unzufrieden ist. Diese Rollen werden regelmäßig gewechselt, sodass jeder Peer die Rolle des Spielers und Trainers übernimmt.

Die Trainings bestehen aus 12 Sitzungen und dauern jeweils 45 Minuten, die zweimal wöchentlich im Nachmittagskontext außerhalb des regulären Unterrichts an der jeweiligen Grundschule der Probanden stattfinden. Zwei Peers bilden zusammen eine Dyade, welche von einer geschulten studentischen Übungsleitung instruiert und begleitet werden. Die Gruppenkonstellation wird durch die ganze Intervention hinweg beibehalten.

Die Erhebung der Implementationsgenauigkeit der BiPeer-Intervention, welche die Umsetzung der Inhalte, des Peer-Learnings und des bilingualen Sprachangebots sowohl der Übungsleitungen als auch der Peers unter Verwendung von Audioaufnahmen der sechsten respektive Transkripten der neunten Sitzungen fokussierte, weist auf eine geglückte Implementation durch sowohl die Probanden als auch die Übungsleitungen hin (vgl. Decristan et al., in Vorb.). 


\subsubsection{Interventionsphasen der BiPeer-Studie}

Der Trainingszeitraum ist in zwei Hälften bestehend aus einer Instruktions(Sitzung 1-6) und einer Übungsphase (Sitzung 7-12) aufgeteilt. Zu Beginn der Instruktionsphase lernen die Peers sich gegenseitig und die Übungsleitung kennen, klären organisatorische Fragen und einigen sich auf Regeln für eine gute Zusammenarbeit. Inhaltlich vermittelt die Übungsleitung - unterstützt durch standardisierte Instruktionsmanuale und Materialien - den Peers die einzelnen Lese- oder Rechenstrategien, die dazugehörigen Handlungsabläufe und rollenspezifischen Aufgaben. Die Sitzungen der Instruktionsphase sind stets gleich strukturiert: Ein kurzes Spiel eröffnet die Sitzung, wonach im Anschluss die Inhalte der letzten Sitzung deklarativ und praktisch von den Peers wiederholt werden. Anschließend wird das Ziel der Sitzung genannt, eine neue Strategie von der Übungsleitung eingeführt sowie modelliert und von den Peers erarbeitet. Die Vermittlungsprozesse der Übungsleitung sind nach allgemeinen Prinzipien der „Conceptual Change Theorie“ (vgl. Hasselhorn \& Mähler, 1998), dem „Cognitive Apprenticeship Approach“ (Collins, Brown \& Newman, 1987) unter sukzessiver Übertragung der Verantwortung für den Lernprozess nach Pearson und Gallagher (1983) organisiert. Zunächst instruiert die Übungsleitung eine Strategie durch direktes Erklären und anschließender Modellierung der Strategieausübung. Danach probieren sich die Peers in der Strategieausübung aus, wobei sie bei Problemen, Fragen und Unklarheiten von der Übungsleitung Unterstützung und Rückmeldung erhalten. Zum Abschluss jeder Instruktionssitzung reflektieren die Peers und die Übungsleitung jeweils die Inhalte und die Zusammenarbeit der abgeschlossenen Sitzung dahingehend, was ihnen (nicht) gefallen hat und was sie beim nächsten Mal besser machen könnten.

Die Übungsphase zeichnet sich durch eine Zunahme der Verantwortung der Peers für den Strategieerwerb aus, da sie in den Sitzungen selbstständig ohne Anleitung und Rückmeldung der Übungsleitung die Materialien bearbeiten und die Strategien anwenden. Die Übungsleitung tritt in den Hintergrund und moderiert die Sitzungen, indem sie die Sitzung eröffnet und schließt sowie die Materialien austeilt und einsammelt. Sie steht für die Peers nur bei dringenden, von der Dyade nicht zu lösenden Fragen und Probleme zur Verfügung. Hierbei ist sie aber angehalten, sich weitestgehend aus den Bearbeitungsprozessen herauszuhalten und kein direktes Feedback in der Sitzung zu geben. Im Gegensatz zur Instruktionsphase beteiligt sich die Übungsleitung auch nicht an den Reflexionsprozessen der Abschlussrunde. Dementsprechend kommt es beim Übergang von der Instruktions- zur Übungsphase zu einer Übertragung der Verantwortung, bei dem die Peers für den Erfolg ihres Lernprozesses voneinander abhängig sind (vgl. ebd.). 
Die Dyade erhält allerdings in der siebten und zehnten Sitzung von der Übungsleitung ein kurzes, prozessorientiertes Feedback. Hierbei werden anhand der Lösungswege, Ergebnisse und Zusammenarbeit aus der sechsten respektive neunten Sitzung der Dyade Stärken und Schwächen in der Ausübung der Strategien sowie der Zusammenarbeit zurückgemeldet und Verbesserungsvorschläge standardisiert vermittelt.

\subsubsection{Lesetraining}

Das übergeordnete Ziel des Lesetrainings ist es, die Lesekompetenz durch eine Steigerung der Leseflüssigkeit und des Leseverstehens zu verbessern. Hierzu werden eine Form des Tandemlesens sowie drei zentrale Lesestrategien vermittelt und angewandt. Die einzelnen Handlungsroutinen und (Teil-)Schritte des Tandemlesens und der Lesestrategien sind als Hilfestellung für die Probanden auf sogenannten „Promptkarten“ (siehe „Anhang 1 Promptkarten des Lesetrainings") schriftlich als Anweisungen formuliert und mit ikonischen Darstellungen illustriert.

$\mathrm{Zu}$ Beginn jeder Sitzung diskutieren die Peers mögliche Textinhalte auf Basis der Überschrift (für einen Beispieltext siehe „Anhang 2 Text „Gagrobatz" (Sitzung 9)"), welche von der Übungsleitung den Probanden vorgelesen wird. Anschließend einigen sich die Peers, wer im ersten Spieler-Trainer Turnus welche Rolle übernimmt. Ein Spieler-Trainer Turnus beginnt, indem ein neuer Textabschnitt im Tandem mit den Rollen Spieler und Trainer gelesen werden. Das Tandemlesen ist der erste Lerninhalt, den die Probanden in der Instruktionsphase vermittelt bekommen und wird in allen Instruktionssitzungen unter der Supervision der Übungsleitung geübt. Beim Tandemlesen liest der Spieler einen Absatz des Textes laut vor, während der Trainer leise mitliest und auf Lesefehler achtet. Als Lesefehler gelten das fehlerhafte Vorlesen eines Wortes (z. B. durch Hinzufügung oder Auslassung von Phonemen oder Silben sowie eine falsche Aussprache), das Vorlesen eines anderen Wortes und das Auslassen von Wörtern. Kommt es zu einem Lesefehler des Spielers, der nicht selbst korrigiert wird, ist der Trainer angehalten, den Spieler in seinem Lesefluss zu unterbrechen und ihn auf seinen Lesefehler aufmerksam zu machen. Hierfür soll der Trainer die auf der Promptkarte „Teamlesen“ vorformulierte Äußerung „Stopp! Lies bitte das Wort und den Satz noch einmal!“ verwenden und dabei auf das falsch gelesene Wort zeigen. Der Spieler muss daraufhin das falsch gelesene Wort korrekt wiederholen und anschließend den Satz, in dem der Fehler auftrat, von vorne vorlesen. Wenn einzelne Wörter oder Phrasen vom Spieler nicht dekodiert werden können, darf der Spieler den Trainer um Hilfe bitten. Beim Tandemlesen wird jeder zu lesende Abschnitt von beiden Tandempartnern gelesen, sodass jedes Kind einmal Spieler und Trainer beim Tandemlesen desselben Abschnittes ist. 
Zur Steigerung des Leseverstehens werden einzelne Textabschnitte mit den drei Lesestrategien „Wörter klären“, „Zusammenfassen“ und „Vorhersagen“ im Tandem in den Rollen Spieler und Trainer bearbeitet. Die drei angeführten Lesestrategien werden in der oben genannten Reihenfolge in der Instruktionsphase in jeweils einer Sitzung vermittelt und sollen sukzessive nacheinander bei jedem zu bearbeitenden Abschnitt in der Übungsphase angewandt werden.

Beim „Wörter klären“ werden nach dem Tandemlesen eines Abschnittes unbekannte oder schwierige Wörter vom Spieler identifiziert, markiert und anschließend in Teilschritten versucht, die Bedeutung der Wörter zu klären und zu notieren. Die Teilschritte werden vom Trainer von der Promptkarte „Wörter klären" vorgelesen und vom Spieler sukzessiv bis zu dem Punkt bearbeitet, bei dem die Bedeutung des Wortes mit Hilfe eines Teilschrittes geklärt wurde. Nach der Klärung der Bedeutung des Wortes wird diese schriftlich auf dem Ergebnisblatt der Sitzung festgehalten. Beim ersten Teilschritt wird versucht, Erklärungen oder Hinweise auf die Bedeutung des zu klärenden Wortes im gelesenen Abschnitt selbst zu finden. Gelingt dies nicht, soll der Spieler sein Vorwissen zu dem im Textabschnitt dargestellten Konzept oder Thema aktivieren und reflektieren, ob dieses zur Klärung des Wortes genutzt werden kann. Führt dies auch nicht zur Klärung der Bedeutung, sollen die Wortbestandteile auf mögliche Hinweise auf die Semantik des zu klärenden Wortes untersucht werden. Der letzte Teilschritt zur Klärung unbekannter oder schwieriger Wörter ist es, den Trainer um einen Tipp zu bitten. Damit der Trainer diesen Teilschritt vollführen kann, darf er ein zum Text gehörendes Kurzlexikon verwenden, in dem die Bedeutungen von im Text enthaltenen schwierigen Wörtern in einer für Grundschulkinder angemessenen Weise erläutert werden (für ein Beispiel siehe „Anhang 3 Kurzlexikon des Textes „Gagrobatz“ (Sitzung 9)“). Wenn die eben angeführten Teilschritte trotz Hilfestellung und Tipps vom Trainer nicht zur Klärung des Wortes führen, wird die Bedeutung des Wortes dem Spieler vom Trainer mit oder ohne Rückgriff auf das Kurzlexikon erklärt. Der Spieler kann so viele Worte des zu bearbeitenden Abschnittes klären, wie er es für nötig hält. Sollte der Spieler bei einem zu bearbeitenden Abschnitt kein Wort zum Klären auswählen, kann der Trainer ihm unbekannte oder schwierige Wörter aus dem Abschnitt nennen, die der Spieler dem Trainer anschließend erklärt. Sollte sich hierbei herausstellen, dass eines oder mehrere dieser Wörter auch dem Spieler nicht bekannt sind, muss der Spieler die Bedeutung dieser Wörter mit Hilfe der oben genannten Teilschritte und Handlungsroutinen klären.

Beim ,Zusammenfassen“ werden in drei vom Trainer angeleiteten Teilschritten die zentrale(n) Proposition(en) des im Tandem gelesenen Abschnittes identifiziert und verschriftlicht. Zunächst nennt der Spieler die in dem Abschnitt zentrale Person, Thema oder Sachverhalt und formuliert mündlich die zentrale(n) Proposition(en) des Abschnittes. Anschließend soll unter Nutzung 
der Erkenntnisse dieser ersten beiden Schritte eine Zusammenfassung des Abschnittes in ein bis zwei Sätzen schriftlich vom Spieler in eigenen Worten und ohne unnötige Details erstellt werden. Abschließend wird die Zusammenfassung gemeinsam auf ihre Qualität geprüft, wobei die zentralen Qualitätskriterien einer Zusammenfassung, das Wichtigste des Abschnittes zu nennen, eigene Worte zu nutzen und auf Details zu verzichten, welche auf einer Promptkarte verschriftlicht sind, herangezogen werden.

Bei der „Vorhersage“ äußert der Spieler auf Basis seines mentalen Textmodells und Weltwissens eine Vermutung, was im nächsten Textabschnitt passieren könnte. Diese wird zunächst mündlich formuliert und nach Zustimmung des Trainers vom Spieler in ein bis zwei Sätzen schriftlich festgehalten. Anschließend wir der vorhergesagte Abschnitt im Tandem gelesen und danach wird die Vorhersage vom Spieler retrospektiv geprüft, indem das (nicht) Eintreten der Vorhersage reflektiert wird.

Den Abschluss eines Spieler-Trainer Turnus bildet nach der Reflexion des Eintretens der Vorhersage eine Frage zu den beiden gelesenen Abschnitten, die auf einer Karte formuliert ist. Bei der Beantwortung der Frage zu den Abschnitten werden die Rollen Spieler und Trainer aufgelöst, sodass beide Peers gleichberechtigt die Frage diskutieren können. Die Fragen zu den Abschnitten eines Spieler-Trainer Turnus sind so konzipiert, dass sie auf Textinferenzen Bezug nehmen und Weltwissen, Meinung oder Lösungsideen der Peers zu einem im Text vorhandenen Sachverhalt oder Problem aktivieren sollen (siehe „Anhang 4 Frage zum Abschnitt am Beispiel des Textes „Gagrobatz“" (Sitzung 9)“).

Dementsprechend besteht ein Spieler-Trainer Turnus aus dem Tandemlesen eines ersten Absatzes, in welchem unbekannte oder schwierige Wörter geklärt, eine Zusammenfassung niedergeschrieben, die Inhalte des nächsten Absatzes vorhergesagt, diese wiederum anschließend durch Tandemlesen geprüft werden und zum Abschluss eine Frage zu den beiden gelesenen Absätzen im Team beantwortet wird. Ein solcher Turnus ist nicht zeitlich limitiert. Die Peers können so viel Zeit für die einzelnen Strategien aufwenden, wie sie benötigen. Dementsprechend kann theoretisch auch kein oder nur ein Wechsel der Rollen in einer Sitzung auftreten. Wird in einer Sitzung nicht der komplette Text bearbeitet, ist die Übungsleitung angehalten, vor der Abschlussrunde die restlichen Abschnitte des Textes den Peers vorzulesen. Zusammengefasst ist eine Sitzung in der Übungsphase des Lesetrainings wie in Abbildung 3 strukturiert: 
Abbildung 3

Struktur einer Sitzung der Übungsphase des Lesetrainings

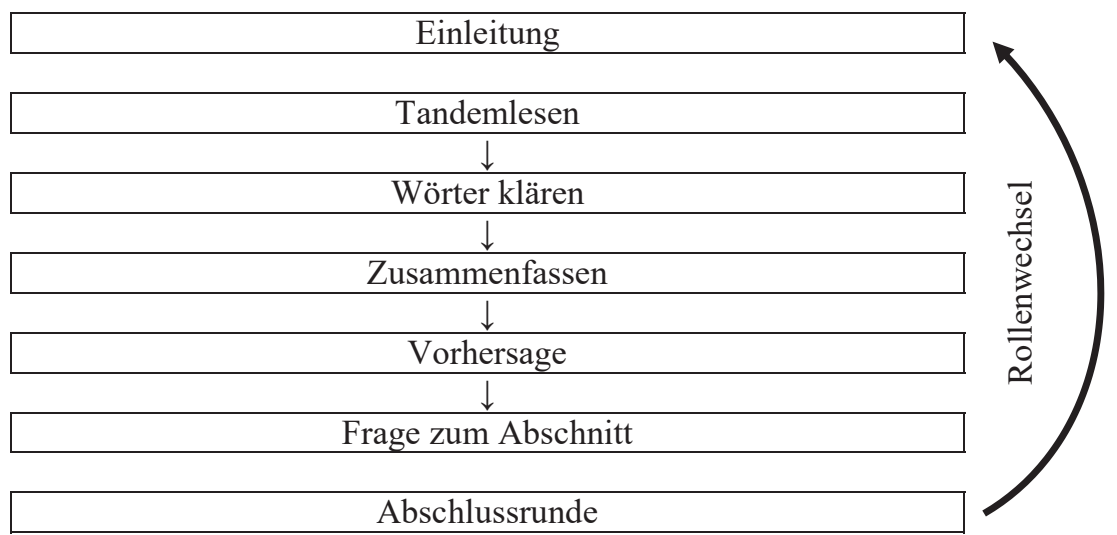

Die Texte für das Lesetraining stammen zum Großteil aus dem Peer-Learning Programm „Blitzelesen im Team“ (Munser-Kiefer \& Kirschhock, 2012). Weiterhin wurden jeweils eine Kindergeschichte aus dem Loewe und eine aus dem Dressler Verlag sowie ein öffentlich zugänglicher Text aus der PIRLS-Studie verwendet. Es wurden sechs Erzähl- als auch Sachtexte genutzt. Die Sachtexte behandeln in kindgerechter Weise Themen wie Tiere, Roboter und Planeten, während die Erzähltexte von Abenteuern, Familie, Schule und Freunden handeln. Alle Texte wurden hinsichtlich der Länge, Abschnittssetzung, der Syntax und des Wortschatzes überprüft und adaptiert. Die Texte der Instruktionsphase sind kürzer als in der Übungsphase und bestehen aus vier bis maximal acht Absätzen. Alle Texte der Übungsphase wurden in acht Absätze unterteilt. Hypotaktische Strukturen mit mehr als einer Subordination wurden versucht zu vermeiden. In den Abschnitten 1, 3, 5, und 7 wurde darauf geachtet, dass sich ein oder zwei Wörter in den Absätzen befinden, die nicht als hochfrequent einzuschätzen sind und sich somit als potentielle Kandidaten für die Strategie „Wörter klären“ eignen könnten. Die adaptierten Texte bewegen sich hinsichtlich ihrer Komplexität nach dem Lesbarkeitsindex „LIX“ (Lenhard \& Lenhard, 2014-2017) in den Kategorien „sehr niedrig“ und „niedrig“.

\subsubsection{Rechentraining}

Die primären Ziele des Rechentrainings sind eine Steigerung der Flexibilität und Adaptivität bei halbschriftlichen Rechenverfahren der Addition und Subtraktion. Hierfür werden neben der Förderung der Kopfrechenfähigkeiten und des Zahlenblicks zwei globale und drei für spezifische Aufgabentypen prädes- 
tinierte Rechenstrategien vermittelt. Wie beim Lesetraining werden die einzelnen Handlungsroutinen und (Teil-)Schritte des Rechentrainings als Hilfestellung für die Probanden auf sogenannten „Promptkarten“ (siehe „Anhang 5 Promptkarten des Rechentrainings") schriftlich als Anweisungen formuliert und mit ikonischen Darstellungen illustriert.

Jede Sitzung beginnt mit 20 Kopfrechenaufgaben, die in einem Zeitraum von zwei Minuten individuell von den Peers gelöst werden und die mit Ausnahme der Sitzungen sechs, neun und zwölf (40 Aufgaben) in der darauf folgenden Sitzung von der Übungsleitung wieder den Probanden korrigiert ausgehändigt werden. In den Instruktionssitzungen werden zunächst die Globalstrategien „Schrittweise Rechnen“ und „Stellenweise Rechnen“ vermittelt, bevor in den nächsten drei Instruktionssitzungen sukzessiv die Rechenstrategien „Kompensation“, „Vereinfachen“ und „Ergänzen“ eingeführt werden.

Die halbschriftlichen Rechenverfahren „Schrittweise Rechnen“ und „Stellenweise Rechnen“"werden als „Globalstrategien“ bezeichnet, da sich mit diesen - mit Ausnahme des Stellenweisen Rechnens bei Subtraktionsaufgaben, bei dem eine Stelle des Subtrahenden die korrespondierende Stelle des Minuenden übersteigt - prinzipiell alle Additions- und Subtraktionsaufgaben in Einzelschritten lösen lassen. Beim Schrittweise Rechnen wird zunächst der erste Summand oder der Minuend mit der größten Stelle des zweiten Summanden oder des Subtrahenden verrechnet, wobei das hieraus resultierende Zwischenergebnis anschließend mit der zweithöchsten Stelle des zweiten Summanden oder des Minuenden addiert respektive subtrahiert wird. Dieses Verfahren wird bis zur letzten Stelle des zweiten Summanden oder Subtrahenden bis zum Endergebnis weitergeführt (siehe Abbildung 4). Dieses Verfahren reduziert die im Rechenprozess $\mathrm{zu}$ vollführenden Operationen und vermeidet weitestgehend Stellenübergänge, was das Arbeitsgedächtnis entlastet.

Beim Stellenweise Rechnen werden sukzessive die Stellen der beiden Summanden oder des Minuenden und Subtrahenden miteinander verrechnet und deren Zwischenergebnisse festgehalten. Diese werden wiederum im letzten Schritt miteinander verrechnet (siehe Abbildung 5). Diese Globalstrategie ist für alle Aufgaben geeignet, außer in Fällen der Subtraktion, bei denen einzelne Stellen des Subtrahenden größer sind als des Minuenden. In diesen Fällen müsste mit Zahlwerten einzelner Stellen im negativen Bereich operiert werden, was keine curriculare Kernkompetenz in der Grundschule darstellt und dementsprechend zu vermeiden ist. 
Abbildung 4

Globalstrategie Schrittweise Rechnen

\begin{tabular}{c|c|c|c}
\hline \multicolumn{2}{c|}{ Addition } & \multicolumn{2}{c}{ Subtraktion } \\
\hline Tausenderraum & Hunderterraum & Tausenderraum & Hunderterraum \\
$\underline{346+258=614}$ & $\underline{33+18=51}$ & $\underline{951-477=474}$ & $\underline{74-35=39}$ \\
$346+200=546$ & $33+10=43$ & $951-400=551$ & $74-30=44$ \\
$546+50=616$ & $43+8=51$ & $551-70=481$ & $44-5=39$ \\
$616+8=614$ & & $481-7=474$ & \\
\hline
\end{tabular}

Abbildung 5

Globalstrategie Stellenweise Rechnen

\begin{tabular}{c|c|r|r}
\hline \multicolumn{2}{c|}{ Addition } & \multicolumn{2}{c}{ Subtraktion } \\
\hline Tausenderraum & Hunderterraum & Tausenderraum & Hunderterraum \\
$\underline{286+137=423}$ & $\underline{56+27=83}$ & $\underline{471-131=340}$ & $\underline{89-63=26}$ \\
$200+100=300$ & $50+20=70$ & $400-100=300$ & $80-60=20$ \\
$80+30=110$ & $6+7=13$ & $70-30=40$ & $9-3=6$ \\
$6+7=13$ & & $1-1=0$ & \\
\hline
\end{tabular}

In der dritten Sitzung wird die Rechenstrategie „Kompensation“ vermittelt, die im Training als „Hilfsaufgabe“ bezeichnet wird. Hierbei wird in einem ersten Schritt einer der Summanden bei der Addition oder der Subtrahend bei der Subtraktion dahingehend manipuliert, dass diese auf den nächst höheren glatten Zehner oder Hunderter gebracht werden. Mit diesem manipulierten Summanden oder Subtrahenden wird die erste Rechenoperation ausgeführt. Abschließend wird das Zwischenergebnis der ersten Rechenoperation um denjenigen Wert der Manipulation kompensiert, sodass die korrekte Summe oder Differenz der ursprünglichen Aufgabe antizipiert wird. Da die Manipulation einen der Summanden oder den Subtrahenden erhöht, gilt es bei der Addition diese Erhöhung vom Zwischenergebnis abzuziehen, während bei der Subtraktion eine Addition in Höhe der Manipulation erfolgt (siehe Abbildung 6). Diese Strategie eignet sich für Additions- und Subtraktionsaufgaben, bei welchen einer der Summanden oder der Subtrahend sich im Tausenderraum nah am nächsten Hunderter und im Hunderterraum nah am nächsten Hunderter oder Zehner befindet. Bei der Vermittlung der Kompensationsstrategie in der BiPeer-Studie wird auf Manipulationen auf den nächst niedrigeren Zehner oder Hunderter verzichtet, da dies sich im Hunderterraum nicht vom Schrittweise Rechnen unterscheiden lässt. Dementsprechend operiert die Kompensationsstrategie in dieser Studie lediglich über die Antizipation des nächst höheren Hunderters oder Zehners. Mit dieser Strategie werden die Anforderungen an die Rechenoperationen sowie an das Arbeitsgedächtnis verringert, da durch 
die Erzeugung von glatten Hundertern oder Zehnern Stellenübergänge weitestgehend vermieden werden.

Abbildung 6

Rechenstrategie Kompensation

\begin{tabular}{c|c|c|c}
\hline \multicolumn{2}{c|}{ Addition } & \multicolumn{2}{c}{ Subtraktion } \\
\hline Tausenderraum & Hunderterraum & Tausenderraum & Hunderterraum \\
$\underline{564+299=863}$ & $\underline{63+28=91}$ & $\underline{735-498=237}$ & $\underline{95-59=36}$ \\
$564+300=864$ & $63+30=93$ & $735-500=235$ & $95-60=35$ \\
$864-1=863$ & $93-2=91$ & $235+2=237$ & $35+1=36$ \\
\hline
\end{tabular}

In der vierten Sitzung der Instruktionsphase lernen die Probanden die Rechenstrategie „Vereinfachen“ kennen. Diese Strategie ähnelt der Rechenstrategie „Kompensation“, allerdings wird hierbei die in der Kompensation nachträgliche Korrektur der Manipulation vermieden, indem beide Summanden oder der Minuend und Subtrahend direkt in einem Schritt in Abhängigkeit voneinander manipuliert werden. Einer der Summanden oder der Subtrahend wird zu einer Zahl mit glattem Zehner oder Hunderter abgeändert. Dies kann durch eine Erhöhung oder Verminderung des ursprünglichen Wertes geschehen. Bei der Addition wird der andere Summand dann diametral um denselben Wert manipuliert, sodass bei der Erhöhung des Wertes eines Summanden der andere Summand um diesen Wert verringert wird und umgekehrt. Bei der Subtraktion hingegen wird der Minuend um denselben Wert verringert oder erhöht wie der Subtrahend (siehe Abbildung 7). Diese Strategie eignet sich für eine Vielzahl von Aufgaben, bei denen die Manipulation Hunderter- und Zehnerübergänge weitestgehend vermeidet. Allerdings stellt diese Strategie hohe Anforderungen an den Zahlenblick, mit welchem spezifische, für die Vereinfachung geeignete Aufgabenmerkmale zunächst identifiziert und anschließend fruchtbar im Sinne einer Vereinfachung manipuliert werden müssen. 
Abbildung 7

Rechenstrategie Vereinfachen

\begin{tabular}{c|c|c|c}
\hline \multicolumn{2}{c|}{ Addition } & \multicolumn{2}{c}{ Subtraktion } \\
\hline Tausenderraum & Hunderterraum & Tausenderraum & Hunderterraum \\
$\underline{583+367=950}$ & $\underline{56+36=92}$ & $\underline{608-225=383}$ & $\underline{83-54=29}$ \\
$600+350=950$ & $52+40=92$ & $583-200=383$ & $89-60=29$ \\
$+17-17$ & $-4+4$ & $-25-25$ & $+6+6$ \\
\hline
\end{tabular}

Die letzte spezifische Rechenstrategie ist das „Ergänzen“, welche in der fünften Sitzung der Instruktionsphase vermittelt wird. Diese Strategie lässt sich nur bei der Subtraktion anwenden. Hierbei wird die Subtraktions- in eine Additionsaufgabe umgewandelt, bei welcher der Subtrahend mit einem Wert addiert werden soll, der den Minuenden zum Ergebnis macht (siehe Abbildung 8). Dieser zu ermittelnde Wert ist das Ergebnis der ursprünglichen Rechenaufgabe. Das Ergänzen eignet sich für Aufgaben, bei denen der Minuend und Subtrahend nah beieinander liegen. Mit dieser Strategie lässt sich sowohl die Anzahl der zu vollführenden Rechenoperationen als auch Stellenübergänge verringern, da in der Regel nur mit den Werten von 1-19 operiert wird.

Abbildung 8

Rechenstrategie Ergänzen

\begin{tabular}{l|c}
\hline \multicolumn{3}{c}{ Subtraktion } \\
\hline Tausenderraum & Hunderterraum \\
$902-897=5$ & $\underline{54-46=8}$ \\
$897+5=902$ & $46+8=54$ \\
\hline
\end{tabular}

In der sechsten Sitzung erhalten die Peers eine Übersicht über alle im Training vermittelten Rechenverfahren, in welchem die Ausführung dieser dargestellt und die Passung zu bestimmten Aufgabentypen erläutert wird (siehe „Anhang 6 Zusammenfassung der halbschriftlichen Rechenverfahren"). Dies ermöglicht den Probanden in der Übungsphase bei Unsicherheiten bezüglich der Rechenstrategien sich selbst zu helfen und verringert somit ihre Abhängigkeit von der Übungsleitung.

Das PL im Rechentraining ist aufgeteilt in das „Teamrechnen“ und die „Rechenkonferenz", welche beide jeweils 20 Minuten dauern. Die Übungsleitung ist für die zeitliche Einhaltung der Phasen des Rechentrainings verantwortlich und instruiert die Phasenwechsel. Durch die zeitliche Limitation ist nicht davon auszugehen, dass in allen Phasen alle angebotenen Aufgaben von den Peers bearbeitet werden. 
Beim Teamrechnen arbeiten die Peers jeweils zehn Minuten in den reziproken Rollen Spieler und Trainer. Die Aufgabentypen beim Teamrechnen unterscheiden sich in Abhängigkeit von der jeweiligen Sitzung. In den Sitzungen neun, zehn und zwölf der Übungsphase bearbeitet der Spieler beim Teamrechnen bis zu acht Rechenaufgaben (für ein Beispiel siehe „Anhang 7 typische Rechenaufgaben des Rechentrainings (Sitzung 9, Tausenderraum)") nach den gleichen vorgegebenen (Teil-)Schritten, die auf der Promptkarte des Teamrechnens verschriftlicht sind. Hierbei muss der Spieler jede zu rechnende Aufgabenstellung zunächst artikulieren, bevor er seine frei zu wählende Rechenstrategie für die Aufgabe nennt. Danach rechnet der Spieler die Aufgabe mit seiner gewählten Rechenstrategie und verbalisiert seine einzelnen Rechenschritte und -operationen, damit der Trainer an diesen Prozessen teilhaben kann. Nach der Lösung der Aufgabe ist der Spieler angehalten zu reflektieren, ob die Lösung richtig ist. Der Trainer prüft anschließend die Korrektheit des Ergebnisses mit Hilfe eines Lösungsblattes, auf dem die Ergebnisse der Aufgaben ohne Rechenwege stehen. Der Trainer ist während des ganzen Prozesses befugt, den Spieler in seinem Handeln zu unterbrechen, wenn er mit der Ausführung eines Teilschrittes, wie z. B. einer antizipierten Rechenstrategie für eine Aufgabe, unzufrieden ist. Die Rollen „Spieler“ und „Trainer“ werden beim Wechsel von „Teamrechnen 1“ zu „Teamrechnen 2“ getauscht.

Die Aufgabenblätter des Teamrechnens zeichnen sich in den Sitzungen sieben, acht und elf hingegen durch „Forced Choice“-Aufgaben und Transferaufgaben aus (für ein Beispiel siehe „Anhang 8 Forced Choice-Aufgaben und Transferaufgaben des Rechentrainings (Sitzung 8, Tausenderraum)“"). In den ersten Aufgaben werden zwei bis drei Rechenwege der gleichen Aufgabe mit kindgerechten Illustrationen gegenübergestellt. Der Spieler ist hierbei angehalten, zunächst die illustrierten Rechenwege dem Trainer zu erläutern und anschließend eine Präferenz für einen dieser Wege samt einer Begründung zu äußern. Diese Aufgaben werden von einem prominent auf dem Arbeitsblatt markierten Hinweis begleitet, dass diese Aufgaben ohne die Promptkarte „Teamrechnen“ bearbeitet werden. Die zweiten Aufgaben sind Forced-Choice Aufgaben, bei denen zwei Rechenaufgaben mit ein oder zwei vorgegebenen Rechenstrategien gelöst werden sollen. Bei den dritten Aufgaben gilt es bis zu drei Rechenaufgaben zu erfinden, die sich für eine bestimmte vorgegebene Strategie eignen. Die beiden letztgenannten Aufgabentypen werden mit den Handlungsroutinen der Promptkarte „Teamrechnen“ und dementsprechend in den Rollen Trainer und Spieler bearbeitet.

Die Rechenkonferenz ist in drei separate Phasen aufgeteilt. In der ersten Phase, der sogenannten „ICH-Phase“, werden zunächst von den Probanden acht Minuten lang die gleichen Aufgaben individuell in ihrem eigenen Tempo bearbeitet. In der zweiten Phase erläutern die Probanden sich zunächst gegenseitig ihre jeweiligen Lösungswege der einzelnen Aufgaben und einigen sich anschließend bei jeder von beiden gelösten Aufgaben auf einen Lösungsweg 
und ein Ergebnis. Diese beiden Teilschritte werden mit den Termini „DU-““ respektive „WIR-Phase“ auf der Promptkarte „Rechenkonferenz" explizit voneinander getrennt und sind gemeinsam auf zehn Minuten zeitlich limitiert ist. In der letzten Phase - der sogenannten „PRÜF-Phase“ - kontrollieren die Peers ihre gemeinsamen Ergebnisse der DU- und WIR-Phase mit Hilfe eines Lösungsblattes. Sollten sie hierbei auf Fehler stoßen, sind sie angehalten, diese gemeinsam zu ergründen und zu korrigieren. Für diese Phase sind zwei Minuten vorgesehen. Die Rechenkonferenz wird ohne die Rollen Spieler und Trainer bearbeitet. Die Aufgabenblätter für die Rechenkonferenz sind immer gleich aufgebaut. Bei der ersten Aufgabe gilt es sechs Rechenaufgaben dahingehend einzuschätzen, ob sie leicht oder schwer zu lösen sind oder sich für spezifische Rechenstrategien eignen. Die sechs Aufgaben werden hierbei nicht ausgerechnet, sondern lediglich kategorisiert. Die zweite Aufgabe enthält acht Rechenaufgaben, die mit freier Rechenstrategiewahl auszurechnen sind. Die Rechenaufgaben sind hierbei in derselben Weise wie die Aufgabenblätter des Teamrechnens der Sitzungen sechs, neun und zwölf konstruiert und enthalten Aufgaben, die sich anhand ihrer Aufgabenmerkmale für bestimmte globale und spezifische Rechenstrategien eignen.

Bei der Abschlussrunde im Rechentraining kommentieren die Peers, was ihnen an den Aufgaben der einzelnen Sitzung (nicht) gefallen hat und was sie in der nächsten Sitzung hinsichtlich ihrer Zusammenarbeit verbessern können. Zusammengefasst ist eine Sitzung in der Übungsphase des Rechentrainings wie in Abbildung 9 strukturiert.

Abbildung 9

Struktur einer Sitzung der Übungsphase des Rechentrainings

\begin{tabular}{|c|}
\hline Einleitung \\
\hline Kopfrechnen \\
$\downarrow \downarrow$ \\
\hline Teamrechnen 1 \\
\hline Teamrechnen 2 \\
\hline$\downarrow$ \\
\hline Rechenkonferenz \\
\hline Abschlussrunde \\
\hline
\end{tabular}




\subsubsection{Implementation des bilingualen Sprachangebots der Gruppen LGT/D und RGT/D}

In der Instruktionsphase erfolgt in den Gruppen LGT/D und RGT/D die Implementation des bilingual deutsch-türkischen Sprachangebots ab der ersten Sitzung. Das Sprachangebot ermöglicht den Dyaden der Gruppen LGT/D und RGT/D die Interaktion in einem bilingualen Sprachmodus zu führen. Sie können hierbei auf Türkisch sowie auf Türkisch und Deutsch gemischt zusammenarbeiten, wobei die Angebotsstruktur ihnen ebenfalls gestattet, auch nur auf Deutsch zu interagieren, wenn sie dies möchten. Aufgrund der Erfahrungen aus der Pilotierung der BiPeer-Studie sowie der bisherigen Empirie zur Problematik der Etablierung eines bilingualen Sprachmodus im Submersionskontext mit Bildungsinhalten (vgl. z. B. Meyer \& Prediger, 2011; Rehbein, 2011) wird auf verschiedene Weise versucht, einen bilingualen Sprachmodus zu aktivieren.

In der ersten Instruktionssitzung werden die bilingualen Tandems der Gruppen LGT/D und RGT/D explizit auf das Angebot zur Nutzung bilingualer Praktiken hingewiesen. Um dieses Angebot zu rahmen, wird zudem eine Fallvignette (siehe „Anhang 9 Fallvignette zur Etablierung bilingualer Interaktion (Sitzung 1)") in der Triade besprochen, in welcher es um zwei türkischdeutschsprachige Kinder in der Grundschule geht, die sich beim Zusammenarbeiten im Unterricht über die Aufgaben und Lösungen auch auf Türkisch oder Türkisch-Deutsch gemischt austauschen. Die Kinder sollen ihre Meinungen zu bilingualer Interaktion und mögliche Gründe für das bilinguale Sprachverhalten der in der Fallvignette dargestellten Kinder äußern. Die Übungsleitungen kommentieren standardisiert positiv das Sprachverhalten der Kinder der Fallvignette und weisen die Probanden ebenfalls darauf hin, dass sie selbst auch Türkisch und Deutsch gemischt sprechen, wenn sie mit türkischsprachigen Freunden gemeinsam für die Universität lernen.

Zudem sprechen die Übungsleitungen der Gruppen LGT/D und RGT/D alle Türkisch sowie Deutsch und sind angehalten, den Smalltalk vor und nach der Sitzung mit ihrem Tandem auf Türkisch oder auf Türkisch-Deutsch gemischt zu halten. Darüber hinaus artikulieren sie bestimmte Instruktionsteile standardisiert in Türkisch oder in Türkisch und Deutsch gemischt. Hierbei wurde vermieden, die standardisierten Instruktionen zu den einzelnen Leseund Rechenstrategien mit türkischsprachigen Anteilen $\mathrm{zu}$ versehen, um die Vergleichbarkeit zwischen den sechs verschiedenen Gruppen zu wahren. Die Promptkarten in den Gruppen LGT/D und RGT/D sind zweisprachig auf Deutsch und Türkisch formuliert, um den offiziellen Status des Türkischen als legitime Sprache des Trainings auch visuell zu repräsentieren.

Im Verlauf der Instruktionssitzungen erhalten die Probanden der Gruppen LGT/D und RGT/D von der Übungsleitung standardisierte Hinweise und Mög- 
lichkeiten, wie das Türkische beim PL und bei den einzelnen Strategien fruchtbar angewandt werden kann. Neben dem Angebot und den oben angeführten Möglichkeiten zur Nutzung beider Sprachen werden in den Instruktionssitzungen bei der Wiederholung der in der letzten Sitzung kennengelernten Leseoder Rechenstrategie stets die Probanden aufgefordert, die Lese- oder Rechenstrategie auf Türkisch oder auf Türkisch und Deutsch gemischt zu erläutern. Hierbei handelt es sich aber um keinen Zwang, sodass Probanden, die die Strategie lieber nur auf Deutsch wiederholen wollen, dies auch machen können.

Als letzte Form der Aktivierung einer zweisprachigen Interaktion erhalten die Kinder am Ende des oben bereits angeführten Feedbacks in den Sitzungen sieben und zehn eine kurze standardisierte Rückmeldung zu ihrer Nutzung des Sprachangebots. Tandems, die miteinander auch auf Türkisch kommunizierten, werden dafür gelobt und aufgefordert, gerne noch mehr miteinander zweisprachig zu kommunizieren. Tandems, die nicht oder kaum zweisprachig miteinander kommunizierten, werden nochmal auf das Angebot mit der Vermutung hingewiesen, dass es dem Tandem sicherlich helfen wird, wenn sie zweisprachig miteinander kommunizieren.

Im Gegensatz hierzu sind die Übungsleitungen aufgefordert, in den Gruppen LGD und RGD zu intervenieren, wenn bilinguale Praktiken auftreten. Die Übungsleitung weist die Dyaden in diesen Fällen darauf hin, dass sie bitte in Deutsch miteinander kommunizieren sollen. Sofern sich die Probanden über diese Sprachregelung hinwegsetzen, soll die Übungsleitung die Dyade wiederholt auf die Unterlassung der bilingualen Interaktion hinweisen. Sollte die Dyade weiterhin die Sprachregelung ignorieren, sind die Übungsleitungen angehalten, die Probanden mit ihrer zweisprachigen Kommunikation gewähren zu lassen. Die türkisch-deutschsprachige Bilingualität der Übungsleitungen war hinsichtlich der Zuteilung zu den Nicht-Angebotsgruppen LGD und RGD keine berücksichtigte Variable, sodass auch monolingual deutschsprachige Übungsleitungen Tandems der Gruppen LGD und RGD trainierten. In diesen Gruppen sollten dementsprechend maximal nur vereinzelt bilinguale Praktiken auftreten, sodass die Nicht-Angebotsgruppen LGD und RGD eine Art Baseline zum Vergleich der Angebotsgruppen LGT/D und RGT/D darstellen.

\subsection{Stichprobe}

Für die vorzunehmenden Analysen der Fragestellungen wird nur auf die Gruppen LGD, LGT/D, RGD und RGT/D der BiPeer-Studie zurückgegriffen. Die für die vorliegende Dissertation genutzte Teilstichprobe besteht aus insgesamt $\mathrm{n}=114$ bilingual türkisch-deutschsprachigen Kindern aus 23 Schulen in Hessen (siehe Tabelle 2). Das Durchschnittsalter der Kinder beträgt 9,7 Jahre. In dieser 306 
Teilstichprobe befinden sich 66 (57,9\%) Mädchen und 75 (65,8\%) Drittklässler. Von dieser Teilstichprobe befinden sich $n=26$ Kinder in LGD, $n=26$ Kinder in LGT/D, n=34 Kinder in RGD und $n=28$ Kinder in RGT/D. Dementsprechend besuchten 52 Kinder das Lese- und 62 Kinder das Rechentraining.

Alle Kinder wachsen türkisch-deutschsprachig auf. Insgesamt 88 Kinder erwerben Deutsch als L2, wovon 80 Kinder mit Türkisch als L1 aufwachsen, sechs das Türkische simultan mit einer weiteren Sprache (Kurdisch, Pomakisch, Aserbaidschan) erwerben und zwei Kinder das Türkische erst spät ab 7 Jahren lernen (L1 Kurdisch). 19 Kinder weisen einen simultanen bilingualen Erwerb des Türkischen und Deutschen auf. Ein Kind erwirbt Kurdisch und Deutsch als simultane Erstsprachen und das Türkische sukzessiv als L2. Für fünf Kinder konnte aufgrund fehlender Angaben zum Erwerbsbeginn kein Erwerbstyp ermittelt werden. In einem Fall war eine Bestimmung des Erwerbstyps mit den Angaben der Eltern nicht möglich. Von diesen 114 Kindern haben insgesamt 72 Kinder schon mal Unterricht im Türkischen erhalten.

Tabelle 2

Zentrale Kennwerte der Stichprobe auf Individualebene

\begin{tabular}{|c|c|c|c|c|c|}
\hline & LGD & $\mathrm{LGD} / \mathrm{T}$ & RGD & RGD/T & Insgesamt \\
\hline $\mathrm{n}$ & 26 & 26 & 34 & 28 & 114 \\
\hline $\begin{array}{c}\varnothing \text { Alter } \\
\text { in Jahren }\end{array}$ & 9,4 & 9,8 & 9,8 & 9,7 & 9,7 \\
\hline Mädchen & $\begin{array}{c}14 \\
(53,8 \%)\end{array}$ & $\begin{array}{c}17 \\
(65,4 \%)\end{array}$ & $\begin{array}{c}19 \\
(55,9 \%)\end{array}$ & $\begin{array}{c}16 \\
(57,1 \%)\end{array}$ & $\begin{array}{c}66 \\
(57,9 \%)\end{array}$ \\
\hline 3. Klasse & $\begin{array}{c}21 \\
(80,8 \%)\end{array}$ & $\begin{array}{c}16 \\
(61,5 \%)\end{array}$ & $\begin{array}{c}21 \\
(61,8 \%)\end{array}$ & $\begin{array}{c}17 \\
(60,7 \%)\end{array}$ & $\begin{array}{c}75 \\
(65,8 \%)\end{array}$ \\
\hline L2a D & $\begin{array}{c}19 \\
(73,1 \%)\end{array}$ & $\begin{array}{c}20 \\
(76,9 \%)\end{array}$ & $\begin{array}{c}23 \\
(67,6 \%)\end{array}$ & $\begin{array}{c}26 \\
(92,9 \%)\end{array}$ & $\begin{array}{c}88 \\
(77,2 \%)\end{array}$ \\
\hline 2L1a T/D & $\begin{array}{c}3 \\
(11,5 \%)\end{array}$ & $\begin{array}{c}6 \\
(23,15 \%)\end{array}$ & $\begin{array}{c}9 \\
(26,5 \%)\end{array}$ & $\begin{array}{c}1 \\
(3,6 \%)\end{array}$ & $\begin{array}{c}19 \\
(16,7 \%)\end{array}$ \\
\hline Türkischunterricht & $\begin{array}{c}18 \\
(69,2 \%)\end{array}$ & $\begin{array}{c}20 \\
(76,9 \%)\end{array}$ & $\begin{array}{c}15 \\
(44,1 \%)\end{array}$ & $\begin{array}{c}19 \\
(67,9 \%)\end{array}$ & $\begin{array}{c}72 \\
(63,2 \%)\end{array}$ \\
\hline
\end{tabular}

Anmerkungen: $\mathrm{D}=$ Deutsch, $\mathrm{T}=$ Türkisch, $\mathrm{T} / \mathrm{D}=$ Türkisch/Deutsch

Eine gleichmäßige Verteilung der Ausprägungen der oben angeführten Variablen ist angesichts der geringen Stichprobengröße sowie dem Fehlen einer echten Zufallsstichprobe nicht zu erwarten. Insbesondere die Gruppengröße 
von RGD, der hohe Anteil an SuS der dritten Klasse in der Gruppe LGD sowie das fast ausnahmslose Vorkommen des Erwerbstyps Deutsch als L2 der Gruppe RGD/T weichen deskriptiv deutlich von den Werten der anderen Gruppen $\mathrm{ab}$.

Aufgrund der Tandemstruktur des PL sind nicht nur die deskriptiven Individualdaten der Stichprobe von Interesse, sondern auch die Dyadenkonstellationen hinsichtlich der Klassenstufen und des Geschlechts. Von den insgesamt 57 Dyaden bestehen 42 Dyaden aus Peers der gleichen Jahrgangsstufe (30 Tandems mit beiden Peers in Jahrgangsstufe 3; 12 Tandems mit beiden Peers in Jahrgangsstufe 4) und 40 Tandems aus gleichgeschlechtlichen Peers (15 Tandems gleichgeschlechtlich männlich; 25 Tandems gleichgeschlechtlich weiblich). Die Verteilung ist in Tabelle 3 illustriert:

Tabelle 3

Zentrale Kennwerte der Dyadenkonstellation der Stichprobe auf Dyadenebene

\begin{tabular}{|c|c|c|c|c|c|}
\hline & LGD & $\mathrm{LGD} / \mathrm{T}$ & RGD & RGD/T & Insgesamt \\
\hline 3. + 3. Klasse & $\begin{array}{c}9 \\
(69,2 \%)\end{array}$ & $\begin{array}{c}6 \\
(46,2 \%)\end{array}$ & $\begin{array}{c}8 \\
(47,1 \%)\end{array}$ & $\begin{array}{c}7 \\
(50 \%)\end{array}$ & $\begin{array}{c}30 \\
(52,6 \%)\end{array}$ \\
\hline 4. + 4. Klasse & $\begin{array}{c}1 \\
(7,7 \%)\end{array}$ & $\begin{array}{c}3 \\
(23,1 \%)\end{array}$ & $\begin{array}{c}4 \\
(23,2 \%)\end{array}$ & $\begin{array}{c}4 \\
(28,6 \%)\end{array}$ & $\begin{array}{c}12 \\
(21,1 \%)\end{array}$ \\
\hline 3. + 4. Klasse & $\begin{array}{c}3 \\
(23,1 \%)\end{array}$ & $\begin{array}{c}4 \\
(30,8 \%)\end{array}$ & $\begin{array}{c}5 \\
(29,4 \%)\end{array}$ & $\begin{array}{c}3 \\
(21,4 \%)\end{array}$ & $\begin{array}{c}15 \\
(26,3 \%)\end{array}$ \\
\hline$q+q$ & $\begin{array}{c}5 \\
(38,5 \%)\end{array}$ & $\begin{array}{c}7 \\
(53,9 \%)\end{array}$ & $\begin{array}{c}8 \\
(47,1 \%)\end{array}$ & $\begin{array}{c}5 \\
(35,7 \%)\end{array}$ & $\begin{array}{c}25 \\
(43,9 \%)\end{array}$ \\
\hline $0+0^{\lambda}$ & $\begin{array}{c}3 \\
(23,1 \%)\end{array}$ & $\begin{array}{c}3 \\
(23,1 \%)\end{array}$ & $\begin{array}{c}6 \\
(35,3 \%)\end{array}$ & $\begin{array}{c}3 \\
(21,4 \%)\end{array}$ & $\begin{array}{c}15 \\
(26,3 \%)\end{array}$ \\
\hline$q+\lambda$ & $\begin{array}{c}5 \\
(38,5 \%)\end{array}$ & $\begin{array}{c}3 \\
(23,1 \%)\end{array}$ & $\begin{array}{c}3 \\
(17,6 \%)\end{array}$ & $\begin{array}{c}6 \\
(42,9 \%)\end{array}$ & $\begin{array}{c}17 \\
(29,8 \%)\end{array}$ \\
\hline
\end{tabular}

Anmerkungen: $q=$ weiblich; $\hat{O}=$ männlich

Auch die Ausprägungen zentraler Kennwerte der Dyadenkonstellation der Stichprobe verteilen sich hinsichtlich der Klassenstufe und des Geschlechts nicht gleichmäßig auf die vier Gruppen. Insbesondere der Anteil an jahrgangsgemischten Dyaden in der Gruppe LGD weicht von den anderen Gruppen ab. Zudem fällt der Anteil an gemischtgeschlechtlichen Dyaden über alle vier Gruppen relativ heterogen aus. 


\subsection{Datengrundlage und Datenaufbereitung bilingualer Praktiken}

Im Folgenden werden die Datengrundlage und Datenaufbereitung des zentralen Konstrukts der vorliegenden Studie näher beschrieben. Bilinguale Praktiken beim PL stellen in ihrer originären Form qualitative Daten dar. Da die Aufbereitung und Auszählung qualitativer Daten einen gewissen Spielraum bei der konkreten Operationalisierung aufweisen, gilt es diese Prozesse der Methodik aus Gründen der Transparenz etwas näher auszuführen.

\subsubsection{Datengrundlage}

Als Datengrundlage für die Erhebung und Analyse bilingualer Praktiken wurde die neunte Sitzung der Intervention ausgewählt, welche die dritte Sitzung der Übungsphase darstellt. Dementsprechend haben die Dyaden zu diesem Zeitpunkt die Instruktionsphase bereits durchlaufen und hatten in den zwei darauf folgenden ersten beiden Sitzungen der Übungsphase die Möglichkeit, die Lese- oder Rechenstrategien eigenverantwortlich im PL zu üben.

Bei den Angebotsgruppen LGT/D und RGT/D wird die komplette Sitzung als Datengrundlage zur Erhebung des Sprachverhaltens verwendet. Bei den Gruppen LGD und RGD werden nur bestimmte Phasen der neunten Sitzung hinsichtlich des Sprachhandelns analysiert, da mit den vorliegenden Ressourcen der BiPeer-Studie keine komplette Transkription sowie Datenaufbereitung der neunten Sitzung dieser Gruppen realisiert werden konnte und somit Kompromisse geschlossen werden mussten. In der Gruppe LGD dienen alle Sequenzen der Lesestrategien „Zusammenfassen“ und „Vorhersagen“ und in RGD die Teamrechenphasen „Teamrechnen 1“ und „Teamrechnen 2“ als Datengrundlage. Die Teamrechenphasen wurden gegenüber der Rechenkonferenz als Datengrundlage präferiert, da diese sich durch eine höhere Quantität an Interaktionen auszeichnet und mehr typische PL-Elemente enthält. Die Lesestrategien „Zusammenfassen“ und „Vorhersagen“ wurden ausgewählt, da die eine Alternative „Tandemlesen“ relativ wenige Interaktionsanlässe bietet und die andere Alternative „Wörter klären“ nicht zwingend von der Dyade bearbeitet werden muss. Wenn Spieler und Trainer der Dyade keine schwierigen oder unbekannten Wörter identifizieren, können sie diese Strategie auch überspringen, was die Vergleichbarkeit der Datengrundlage zwischen den Dyaden schmälert. Die ausgewählten Phasen als Datengrundlage für LGD und RGD sind nicht zwangsläufig zeitgebunden. Die Dauer der Beschäftigung mit den Lesestrategien „Zusammenfassen“ und „Vorhersage“ ist abhängig von der benötigten Zeit des Tandems zur Bearbeitung dieser. So gibt es Tandems, die vier Zusammenfassungen und Vorhersagen in einer Sitzung produzieren und 
andere, die lediglich ein oder zwei Zusammenfassungen und Vorhersagen schaffen. Beim Rechentraining sind das „Teamrechnen 1“ und „Teamrechnen 2 " durch die Instruktion zwar auf jeweils zehn Minuten terminiert, allerdings kann die Bearbeitung dieser auch kürzer ausfallen, wenn die Probanden die sechs oder insgesamt zwölf Aufgaben vor Ablauf des Zeitlimits lösen.

Die neunte Sitzung wird mit einem Audioaufnahmegerät aufgezeichnet, welches am Rande des Arbeitstisches des Tandems platziert ist, um die Salienz dieses möglichst gering zu halten. Die Probanden sind zu diesem Zeitpunkt schon an Aufnahmesituationen mit Diktiergeräten im Projekt BiPeer gewöhnt. Bereits im Prätest wird ihre Leseflüssigkeit mit dem Test „Lernfortschrittsdiagnostik Lesen“(Walter, 2009) geprüft und mit einem Diktiergerät erfasst. Zudem wurde auch die sechste Sitzung komplett aufgezeichnet, sodass alle Probanden in der neunten Sitzung bereits Erfahrungen mit der Aufnahmesituation beim PL machen konnten. Die Aufnahme erfolgt ab dem Beginn der Sitzung direkt nach der Begrüßung und endet mit der Verabschiedung, sodass die komplette Sitzung erfasst wird. Die Dyaden werden zu Beginn der neunten Sitzung explizit mit einer standardisierten Instruktion über die Aufnahmesituation informiert:

\begin{abstract}
„Heute habe ich wieder das Aufnahmegerät mitgebracht. Diesmal zeichne ich das ganze Training auf. Bei BiPeer erforschen wir ja auch, wie Kinder am besten zusammenarbeiten. Deshalb wollen wir uns manche Trainingssitzungen genauer anschauen. Damit wir später auch verstehen, was ihr gesagt habt, ist es wichtig, dass ihr nicht flüstert oder tuschelt. Die Aufnahme kriegt natürlich niemand außer uns von BiPeer zu hören. Also auch nicht eure Eltern oder Lehrer. Ihr bekommt in der nächsten Sitzung auch eine Rückmeldung zu der heutigen Sitzung. Wir sagen euch dann, was ihr schon gut könnt und wo ihr euch noch verbessern könnt. Könnt ihr euren Code kurz einsprechen?“
\end{abstract}

\title{
7.3.2 Sequenzierung der Trainings
}

Zur Transkription der ausgewählten Phasen „Zusammenfassen“ und „Vorhersagen“" sowie „Teamrechnen 1" und „Teamrechnen 2" der Gruppen LGD und RGD gilt es die neunte Sitzung hinsichtlich der verschiedenen Phasen zu sequenzieren. Im Gegensatz hierzu können die Gruppen LGT/D und RGT/D zunächst transkribiert werden, bevor die Sequenzierung des Materials vollzogen wird. Der Vergleich der Prävalenz der bilingualen Praktiken der Nicht-Angebotsgruppen LGD und RGD mit den Angebotsgruppen LGT/D und RGT/D verlangt eine vergleichbare Datenbasis, sodass eine Identifikation der Phasen „Zusammenfassen“ sowie „Vorhersagen“ und „Teamrechnen 1“ und „Teamrechnen 2" bei allen Gruppen notwendig ist. Bezüglich der in F2c vorzunehmenden Analyse der Prävalenz bilingualer Praktiken hinsichtlich verschiedener Phasen innerhalb des Lese- und Rechentrainings müssen darüber hinaus 
alle Phasen für die Gruppen LGT/D und RGT/D nach der Transkription bestimmt werden.

Trotz der sukzessiven Struktur der beiden Trainings samt fester Abfolgen sowie trennscharfer Marker wie z. B. einzelner Schritte der Promptkarten verlief die eindeutige Identifikation der Trainingsphasen nicht komplikationslos. Das zu Grunde liegende Datenmaterial stellt in der Regel Interaktionen von zwei oder drei verschiedenen Interaktanten dar. Zwischen den Interaktanten der Dyade oder Triade besteht nicht immer Intersubjektivität, sodass es zu Divergenzen von fokussierten Themen und Inhalten kommen kann. Die Feststellung, wann beide Peers der Dyade intersubjektiv ein Thema fokussieren und sich somit beide in derselben Phase befinden, ist schwer zu bestimmen und abhängig von der Interpretation des vorliegenden Datenmaterials. Zudem müssen Phasen nicht zwangsläufig direkt aneinander anschließen, sodass es zu Zwischenphasen kommen kann, die sich z. B. durch Stille, Abschweifen vom Lerninhalt, Task-Management zur Vorbereitung der nächsten Phase etc. auszeichnen können. Weiterhin kann sich die Aufgabenbearbeitung einer Phase mit einer anderen überlappen oder kurzzeitig unterbrochen werden. Dementsprechend stellt die Identifikation von Phasen häufig eine Interpretationsleistung dar, insbesondere wenn die Anfänge und/oder Enden nicht durch sprachliche Handlungen kooperativ affirmiert werden.

Die Herausforderungen bei der trennscharfen Identifikation einzelner Phasen soll im Folgenden anhand der Phase „Zusammenfassen“ des Lesetrainings kurz illustriert werden. Der Beginn der Bearbeitungsphase der Lesestrategie „Zusammenfassen“ ist relativ einfach als erste hörbare Handlung zur Strategieausführung wie z. B. in Form einer Aufforderung zur Bestimmung des zentralen Protagonisten durch den Trainer oder durch die Nennung des zentralen Protagonisten durch den Spieler, ohne vorangehende Aufforderung des Trainers, zu bestimmen. Fehlen diese Marker allerdings, gilt es denjenigen Gesprächsbeitrag als Phasenbeginn auszuwählen, der als erster die gemeinsame Orientierung beider Peers zur Phase im Sinne einer intersubjektiven Fokussierung dieser markiert. Das Phasenende der Lesestrategie „Zusammenfassen“ weist gar eine größere Heterogenität potentieller sprachlicher Handlungen auf, mit der das „Zusammenfassen“ abgeschlossen werden kann. Gemäß der Instruktion stellt die Verschriftlichung der Zusammenfassung das Ende der Phase dar. Sollte dies nicht anhand von Verbalisierungen in der Interaktion markiert werden (z. B. Mitsprechen der Wörter beim Aufschreiben oder einer sprachlichen Affirmation der Beendigung der Verschriftlichung), dann gilt es die Äußerungen der Peers am potentiellen Ende dieser Phase zu betrachten und zu entscheiden, welcher Gesprächsbeitrag am ehesten das Ende der Phase markiert. Die folgenden sprachlichen Handlungen werden dann als potentielle Abschlüsse des ,Zusammenfassens" gedeutet und in der folgenden Hierarchie appliziert: 1) Aushandeln des Endes der Phase, 2) Gesprächsbeitrag vor TaskManagement der nächsten Phase oder 3) Gesprächsbeitrag vor Beginn der 
nächsten Phase. Dies bedeutet, dass wenn z. B. weder ein gemeinsames Aushandeln des Endes der Phase durch die Peers noch ein Gesprächsbeitrag vor dem Task-Management der nächsten Phase identifiziert werden kann, der Turn vor Beginn der nächsten Phase das Ende des „Zusammenfassens“" markiert.

Wie am Beispiel der Strategie „Zusammenfassen“ deutlich wird, stellt die trennscharfe Identifikation der einzelnen Phasen eine herausfordernde Interpretationsleitung dar. Zur Erhöhung der Objektivität und Reliabilität der Identifikation der Phasen wurde ein Leitfaden mit allgemeinen Anmerkungen zur Identifikation von Phasen sowie ein Kategoriensystem erstellt, in welchem Definitionen, Ankerbeispiele und Kodierhinweise für die einzelnen Phasen erarbeitet wurden (siehe „Anhang 10 Leitfaden und Kategoriensystem zur Sequenzierung der Phasen"). Die Erfassung der einzelnen Phasen auf Basis von Audiodateien der Gruppen LGD und RGD sowie auf Basis der Transkripte von LGT/D und RGT/D wurden für jedes Tandem von zwei MitarbeiterInnen des Projekts BiPeer vorgenommen. Abweichende Phasenkategorisierungen der MitarbeiterInnen werden anschließend mit dem Ziel besprochen, sich auf eine Lösung zu einigen. Auf eine Berechnung der Interrater-Reliabilität wurde hierbei verzichtet, da diese selbst bei häufig auftretenden Divergenzen der Bestimmung von Anfang und Enden einer Phase sehr hoch ausfallen dürfte. Eine Phase besteht aus einer Vielzahl von Gesprächsbeiträgen, sodass z. B. eine einzelne Phase 100 Gesprächsbeiträge aufweisen kann. Weicht die Kodierung des Anfangs und des Endes einer Phase von den beiden Kodierenden jeweils um einen Gesprächsbeitrag ab, besteht allerdings immer noch eine 98\% Übereinstimmung der beiden Kodierenden bezüglich der restlichen dieser Phase zugeordneten Gesprächsbeiträge, was eine hohe Interrater-Reliabilität sichern würde.

\subsubsection{Transkription der Audioaufnahmen}

Die Transkription der aufgezeichneten Audioaufnahmen erfolgte in Anlehnung an das sequenzielle Transkriptionssystem des GAT 1 (Selting et al., 1998) und GAT 2 (Selting et al., 2009) in Form eines Minimaltranskripts unter Adaptation einiger Konventionen des Basistranskripts sowie unter Berücksichtigung zentraler einführender Literatur zur Transkription von Interaktionen (vgl. z. B. Deppermann, 2008; Dittmar, 2009; Karbach \& Fuß, 2014) (siehe "Anhang 11 Transkriptionssystem“). Da der Umgang mit zweisprachigen Transkripten in den oben angeführten Quellen kaum thematisiert wird, wurden eigene Richtlinien diesbezüglich entwickelt. Hierzu gehören neben Konventionen zur Übersetzung sowie Markierung grammatischer und semantischer Fehler auch die Erläuterung von Redewendungen sowie von kulturell/religiös geprägten Ausdrücken. Zur Unterstützung der automatisierten Erfassung der 
bilingualen Praktiken auf der Ebene von Gesprächsbeiträgen wurden diese bereits im Transkriptionsprozess mit „\#Ü“ sowie roter Schriftfarbe markiert. Die Transkriptionsregeln wurden in Transkriptionsmodulen zusammengefasst. Die Transkription wurde von türkisch-deutschsprachigen studentischen Hilfskräften durchgeführt, die zuvor eine zweitägige Schulung mit theoretischen und praktischen Teilen zur Transkription absolvierten. Die Audiodateien werden in das Programm MAXQDA 12 importiert und unter Verwendung dessen Transkriptionsfunktionen transkribiert. Die Audioaufnahmen der verschiedenen Dyaden werden einzelnen studentischen Hilfskräften zugeteilt und jedes Transkript durchläuft nach der Fertigstellung ein Überprüfungsverfahren durch eine weitere studentische Hilfskraft, um potentielle Fehltranskriptionen zu minimieren.

Das Einsprechen der pseudonymisierten Probanden-ID von den Probanden zu Beginn der neunten Sitzung dient auch dazu, zu Beginn der Sitzung eine Referenz für die Stimmen der Probanden zu erhalten. Diese Referenz soll die Zuordnung der einzelnen Gesprächsbeiträge zu den jeweiligen Sprechern vereinfachen und somit die Transkription der Sitzung erleichtern. Sofern diese Referenz nicht ausreichen sollte, wurden die Transkribierenden darauf hingewiesen, sich längere Stimmproben unter Verwendung der Audioaufnahmen der Testung mit der „Lernfortschrittsdiagnostik Lesen“ (Walter, 2009) anzuhören.

\subsubsection{Operationalisierung bilingualer Praktiken}

Das zentrale Konstrukt der Dissertation stellen bilinguale Praktiken beim PL dar. Bilinguale Praktiken werden in der vorliegenden Dissertation als alle vokalsprachlichen Handlungen mit mindestens einem türkischsprachigen Element operationalisiert, die nicht ausschließlich in der typischen Unterrichtssprache Deutsch kommuniziert werden. Hierzu zählen sowohl bilingual deutsch-türkischsprachige als auch monolingual türkischsprachige Gesprächsbeiträge.

Sprachliche Handlungen werden in der vorliegenden Dissertation auf der Ebene von sogenannten „Turns“ operationalisiert, welche eine häufig verwendete Analyseeinheit bei der Untersuchung von Interaktionen darstellt (vgl. z. B. Deppermann, 2008; Kallmeyer \& Schütze, 1976; Krummheuer, 2011). Turns sind Gesprächsbeiträge eines Sprechers und umfassen „was ein Gesprächsteilnehmer inhaltlich sagt, wenn er an der Reihe ist" (Brinker \& Sager, 2010, S. 109). Dementsprechend können Turns z. B. aus lediglich einem Wort oder gar einem Morphem bestehen, aber auch ausschweifende Monologe umfassen. Turns sind hierbei auch nicht zwangsläufig an Sprecherwechsel gebunden, sondern können sukzessive vom gleichen Sprecher erfolgen, z. B. wenn 
der Gesprächspartner auf sein Rederecht verzichtet oder dem vorangegangenen Turn nichts hinzuzufügen hat.

Turns, die mindestens ein türkischsprachiges Element enthalten, werden als bilinguale Praktiken gewertet und in ein Verhältnis zu rein deutschsprachigen Turns gesetzt. Hierbei ist es unerheblich, in welchem quantitativen Verhältnis das Türkische zum Deutschen innerhalb eines Turns selbst steht und was für ein türkischsprachiges Element auftritt, sei es eine Phrase, ein Wort oder ein Morphem. Eine solche Operationalisierung umfasst unterschiedliche Sprachwechsel- und -mischungsphänomene wie Code-Switching, -Mixing oder Borrowings. Dementsprechend werden lediglich Turns, die rein deutschsprachige Äußerungen aufweisen, als deutschsprachige Turns kodiert.

Zwar erscheinen Turns als recht grobe, heterogene Analyseeinheit, aber weitere mögliche, feinkörnigere Analyseeinheiten wie Wörter oder gar Morpheme sind mit diversen Problemen verbunden, die die quantitative Vergleichbarkeit des Deutschen und Türkischen auf Wort- und Morphemebene betreffen. Das Auszählen türkischer und deutscher Wörter sowie deutsch-türkischer Code-Mixings auf der Wortebene stellt mit technischen Hilfsmitteln der Textverarbeitung zwar kein Problem dar, allerdings ist das Türkische und Deutsche auf der Wortebene quantitativ kaum vergleichbar, da das Türkische den agglutinierenden und das Deutsche den flektierenden Sprachen zuzuordnen ist. In agglutinierenden Sprachen werden formal „grammatische und lexikalische Morpheme mit einfachen Bedeutungen aneinandergereiht", während in flektierenden Sprachen „Wörter sich nicht ohne weiteres in einzelne Morpheme mit einfachen Bedeutungen segmentieren lassen“ (Bußmann, 2008, S. 664), sondern sich benachbarte Morpheme gegenseitig beeinflussen (vgl. ebd., S. 193). Dies führt dazu, dass im Türkischen Wörter mehr grammatische und lexikalische Informationen inkorporieren als im Deutschen, sodass z. B. ein türkischsprachiges Wort im Deutschen aus mehreren Wörtern besteht (siehe Beispiel 1):

Beispiel 1:

(D1) Ich schaue im Lexikon nach und gebe dir einen Tipp!

(T1) Ben sözlüğe bakacagım ve sana bir ipucu vereceğim!

Während im Türkischen der Satz aus lediglich acht Wörtern besteht (T1), enthält das deutsche Äquivalent (D1) zehn Wörter. Würden bilinguale Praktiken anhand der Wortebene analysiert werden, würde der Anteil bilingualer Praktiken zur Kommunikation im Verhältnis zu rein deutschsprachigen Wörtern systematisch unterschätzt werden.

Eine Analyse auf Morphemebene würde andererseits zu systematischen Verzerrungen in die entgegengesetzte Richtung führen. Während z. B. im Deutschen grammatische Morpheme zur Markierung der Kongruenz von Sub- 
jekt und Verb Informationen zu Person, Numerus und Tempus enthalten, müssen diese grammatischen Kategorien im Türkischen in der Regel mit eigenen Morphemen realisiert werden (vgl. Schroeder \& Şimşek, 2014, S. 123ff). Dieser Unterschied ist in Beispiel 2 illustriert:

Beispiel 2:

(D2) ich frier-e

(T2) (ben) üşü-yor-um

Während im Deutschen das Affix „-e“ die 1. Person Singular und das Präsens markiert, wird im Türkischen Präsens durch das Affix ,-yor" und die erste Person Singular durch ,-um“ markiert. Dementsprechend würde eine Analyse auf Morphemebene zu systematischen Überschätzungen des Anteils bilingualer Praktiken zur Kommunikation im Verhältnis zu rein deutschsprachigen Äußerungen führen.

Neben der Kategorisierung der Gesprächsbeiträge hinsichtlich bilingualer Praktiken und rein deutschsprachigen Turns existieren weitere Arten von Turns, die für eine exakte Bestimmung der Prävalenzen berücksichtigt werden müssen. „Nicht vokalsprachliche Turns“ enthalten keine vokalsprachlichen Elemente und bestehen aus paraverbalen Lautäußerungen wie gähnen, seufzen, lachen etc., die in Interaktionen diverse Zwecke erfüllen. Da solche Turns keiner Sprache zugeordnet werden können, müssen diese gesondert erfasst werden, um Verzerrungen der Prävalenzen zu vermeiden. Dies gilt auch für Turns, die sprachliche Elemente aus anderen Sprachen als dem Deutschen und Türkischen enthalten. Sobald ein Turn ein sprachliches Element enthält das weder dem Türkischen noch dem Deutschen zugeordnet werden kann, wird dieser Turn als „Anderssprachiger Turn“ kategorisiert. Ausnahmen stellen hierbei Anglizismen dar, die in das Deutsche aufgenommen wurden. Hierzu zählen eingedeutschte, umgangssprachliche Anglizismen wie z. B. „,sorry“, „Smartphone“, „ok“, „Bro“, „,cool“, ,nice“, „Loser“, „Chips“, „wack“" etc. und interventionsgebundene Anglizismen wie „Prompt“. Zudem werden Turns, die aus nicht bestimmbaren sprachlichen Äußerungen oder lediglich aus der Äußerung eines Namens bestehen, als „Nicht kategorisierbare Turns“ erfasst.

Angesichts dieser verschiedenen Kategorien von Turns stellt sich die Frage, wie mit Turns umgegangen wird, die potentiell mehrere verschiedene Arten von Turns inkorporieren. So könnte zum Beispiel ein Turn sowohl paraverbale Äußerungen, englischsprachige Lexeme und bilinguale Praktiken enthalten. Mehrfachkodierungen eines Turns sind zu vermeiden, sodass ein Turn immer nur einer Kategorie zugeordnet werden kann. Hierfür wird eine Hierarchie implementiert, bei der das Vorkommen eines definitorischen Elementes der angeführten verschiedenen Kategorisierungen die Zuordnung dieses regelt. Die Hierarchie ist folgendermaßen angeordnet: „Anderssprachige Turns“ > „Bilinguale Praktiken“ > „Deutschsprachige Turns" > „Nicht vokalsprachliche 
Turns“ > „Nicht kategorisierbare Turns“. Wenn z. B. ein Turn bilinguale Praktiken, Lachen sowie Seufzen und ein nicht eingedeutschtes englischsprachiges Element enthält, gilt es diesen als „Anderssprachigen Turn“ zu kategorisieren. Wäre in diesem Turn das englischsprachige Element nicht enthalten, würde dieser Turn als „bilinguale Praktik“ zu erfassen sein. Der Leitfaden sowie das Kategoriensystem zur Bestimmung der Turns kann im Anhang nachgesehen werden (siehe „Anhang 12 Kategoriensystem zur Bestimmung von Turns“).

Eine weitere Herausforderung bezüglich der Kategorisierung der Turns stellen Interjektionen und Planungsäußerungen dar. Interjektionen sind Wörter, ,die zum Ausdruck von Empfindungen, Flüchen und Verwünschungen sowie zur Kontaktaufnahme dienen (Au! Verflixt! Hallo!)“ (Bußmann, 2008, S. 302). Planungsäußerungen wie „ehm“ oder „hmm“ hingegen stellen Äußerungen dar, die zur Überbrückung von Planungsphasen in der Sprachproduktion eingesetzt werden, um die Weiterführung der Äußerung durch den Sprecher zu markieren. Planungsäußerungen und Interjektionen sind größtenteils sprachgebunden, sodass diese hinsichtlich des Türkischen und Deutschen zu differenzieren sind. Eine Aufzählung von türkischsprachigen Interjektionen wurde in Anlehnung an Ersen-Rasch (2001, S. 117) und Planungsäußerungen in Anlehnung an Furman \& Özyürek (2007) für das Kodiervorgehen erstellt und kann im Anhang nachgesehen werden (siehe „Anhang 12 Kategoriensystem zur Bestimmung von Turns"). Die Aufzählung enthält ebenfalls Interjektionen wie z. B. „oh“ oder „ei“, die lautlich nicht zwischen dem Deutschen und dem Türkischen differenziert werden können. Diese Elemente werden bei der Kategorisierung der Turns nicht berücksichtigt. Alle weiteren Interjektionen oder Planungsäußerungen, die nicht in der im Anhang angeführten Liste enthalten sind, werden wie deutschsprachige Elemente behandelt. Es gelten dementsprechend für Planungsäußerungen und Interjektionen die gleichen Kategorisierungsvorgaben wie für alle anderen türkisch-oder deutschsprachigen Elemente mit Ausnahme von Interjektionen, die lautsprachlich nicht zwischen dem Deutschen und Türkischen differenziert werden können.

Das Verhältnis der bilingualen Praktiken zu rein deutschsprachigen Äußerungen wird sowohl auf der Dyaden- und auf der Individualebene erfasst, da bilinguale Praktiken bei der Interaktion nicht zwingend unabhängig von den am Gespräch partizipierenden Individuen auftreten könnten. Die Analyse auf Dyaden- oder Gruppenebene wird theoretisch durch die Sprachmodi nach Grosjean (2008) sowie sozialpsychologischen Theorien bilingualer Praktiken (vgl. Myers-Scotton, 2006; Sachdev \& Giles \& Pauwels, 2013;) gestützt, empirisch durch Forschungsarbeiten zu Bedingungen und Zwecken von CodeSwitching und -Mixing (vgl. z. B. Auer, 2009; Gardner-Chloros, 2009; Keim, 2007, 2012) legitimiert und stellt letztlich das typische Analysevorgehen in Studien dar, die die Quantität von bilingualen Praktiken beim PL erheben (vgl. z. B. Hopewell, 2011; Swain \& Lapkin, 2000). Aus der Perspektive der Datenaufbereitung ermöglicht die Dyadenebene zudem eine validere Auswertung als 
die Individualebene, da sich bei der Transkription der Interaktion der Peers nicht immer jeder Turn eindeutig einem bestimmten Peer zuordnen lässt. Dementsprechend erhält in der vorliegenden Studie jedes Tandem einen Wert, der sich aus der Aggregation der bilingualen Praktiken, der rein deutschsprachigen Turns der beiden Peers sowie der nicht einem Peer zuzuordnenden Turns zusammensetzt. Nichtsdestotrotz wird auch die individuelle Prävalenz bilingualer Praktiken der einzelnen Probanden erfasst, da mit Methoden der dyadischen Datenanalyse (vgl. z. B. Kenny, Kashy \& Cook, 2006) die Individualwerte in Abhängigkeit der Dyade ausgewertet werden können.

\subsubsection{Auszählung bilingualer Praktiken mit MAXQDA}

Die Auszählung der Turns wurde mit MAXQDA unter Verwendung der lexikalischen Suchfunktion des Programms vorgenommen. Bei der Transkription der Turns wurde die Zuordnung des Turns zu einem Probanden und der Übungsleitung mit den Abkürzungen „P1:“”, „P2:““ oder „ÜL:“ am Zeilenbeginn markiert. Wenn ein Turn von einem Peer oder von einer Person der Triade geäußert wurde, aber keine eindeutige Zuordnung vorgenommen werden konnte, wurde der Turn mit „P?:“" respektive „G?:“ am Zeilenbeginn gekennzeichnet. Diese Kennzeichnungen am Zeilenbeginn der jeweiligen Transkriptionen der Turns ermöglichen die personengebundene automatische Erfassung aller Turns unter Verwendung der lexikalischen Suchfunktion von MAXQDA.

Zunächst werden die Turns undifferenziert den jeweiligen Sprechern dieser zugeordnet. Hinsichtlich der beiden Probanden erfolgt daraufhin eine Kategorisierung aller Turns abhängig von der Art der Turns. Das folgende Vorgehen hat sich als zeitökonomisch und effizient erwiesen: Nach der Erfassung aller Turns eines Sprechers werden mit Hilfe des Suchbegriffs „\#Ü“, mit dem bereits während der Transkription Sprecherbeiträge mit bilingualen Praktiken markiert wurden, Turns mit bilingualen Praktiken herausgefiltert und kategorisiert. Anschließend werden alle übrig gebliebenen Gesprächsbeiträge des Sprechers vorerst als rein deutschsprachige Turns kategorisiert. Diese Turns werden danach sukzessive dahingehend geprüft, ob sie nicht doch einer anderen Kategorie angehören. Sollte dieser Fall auftreten, wird der Turn der anderen ihm entsprechende Kategorie zugeordnet und die rein deutschsprachige Kategorisierung gelöscht. Durch dieses Vorgehen werden auch bilinguale Praktiken identifiziert, deren Markierung mit „\#Ü“ im Transkriptionsprozess übersehen oder vergessen wurden. Diese Prozedur wird für beide Peers sowie für die nicht zuordnungsbaren Turns auf Dyaden- und Triadenebene durchgeführt.

Zur Überprüfung der Reliabilität der Kategorisierungen und des Kodiervorgehens wurde die Interrater-Reliabilität mit Hilfe einer studentischen Hilfskraft ermittelt. Die studentische Hilfskraft kodierte alle Turns eines zufällig 
ausgewählten Drittels der Stichprobe unter Verwendung des Leitfadens und des Kategoriensystems zur Bestimmung der Sprecherbeiträge. Cohens Kappa wurde mit Hilfe der Funktion „Intercoder-Übereinstimmung“ von MAXQDA sowie Excel ermittelt und beträgt $\kappa=.99$. Diese fast vollständige Beurteilerübereinstimmung ist nicht verwunderlich, da das Kodiervorgehen mit Hilfe des Programms MAXQDA zum Großteil automatisiert abläuft und die verschiedenen Turntypen aufgrund ihrer trennscharfen sowie salienten Oberflächenmerkmale in der Regel eindeutig und ohne großen Interpretationsaufwand zu kategorisieren sind.

\subsection{Quantitative Datenanalyse}

Die quantitative Datenanalyse ${ }^{11}$ ist der qualitativen vorangestellt und fokussiert deskriptiv als auch inferenzstatistisch primär die Prävalenz bilingualer Praktiken der Gruppen LGT/D und RGT/D sowie potentielle Zusammenhänge individueller sprachlicher und motivationaler Eigenschaften mit der Prävalenz bilingualer Praktiken. Bevor allerdings Fragestellung F2 und F3 analysiert werden können, gilt es die Prävalenz bilingualer Praktiken hinsichtlich ihrer „Nonindependence“ von der Dyade zu prüfen (vgl. ebd.), um die adäquate Analyseebene für diese Daten zu identifizieren. Dieses in erster Linie methodische Anliegen hat allerdings ebenfalls Relevanz für die theoretische Modellierung bilingualer Praktiken, da in Modellen der Sprachwahl die Gesprächspartner stets als eine zentrale Einflussgröße der Sprachwahl und Sprachpraxis bilingualer Sprecher postuliert werden (vgl. z. B. Grosjean, 2008; Myers-Scotton, 2006; Sachdev, Giles \& Pauwels, 2013). Der Autor der vorliegenden Studie konnte trotz intensiver Recherche keine quantitativen Studien unter Verwendung dyadischer Datenanalyse zur Prüfung der Abhängigkeit bilingualer Sprachpraxis von bilingualen Gesprächspartnern anhand des konkreten Sprachverhaltens innerhalb einer Dyade identifizieren. Die Untersuchung dieses Phänomens stellt zum einen eine Voraussetzung zur Wahl adäquater Methoden, zum anderen allerdings ebenfalls eine Prüfung des Postulats der Abhängigkeit bilingualer Praktiken von den Gesprächspartnern dar.

10 Die quantitative Datenanalyse einiger der vorliegenden Fragestellungen und Daten lässt hinsichtlich der Wahl zwischen parametrischen und non-parametrischen Verfahren zur Datenanalyse gewisse Spielräume frei. Die Auswahl an potentiellen Analysemethoden sowie die vom Autor gewählten Präferenzen werden in diesem Abschnitt für die hiervon betroffenen Fragestellungen erläutert. Vorweg kann allerdings darauf hingewiesen werden, dass der Autor der vorliegenden Studie für die betroffenen Fragestellungen sowohl parametrische als auch non-parametrische Verfahren zur Analyse ausprobiert hat, um mögliche Unterschiede abhängig vom gewählten Verfahren zu prüfen. Die Ergebnisse aller Fragestellungen sind allerdings so robust, dass sie unabhängig von der gewählten Methode die gleichen Resultate liefern. 


\subsubsection{Prüfung der Abhängigkeit bilingualer Praktiken von der Dyade beim Peer-Learning}

Zentral für die Auswahl einer adäquaten Methode zur Prüfung der Existenz einer potentiellen Abhängigkeit eines Konstrukts von Dyaden ist die Bestimmung des Typus der Dyade. Dyaden unterscheiden sich dahingehend, ob die jeweiligen Individuen aller Dyaden ein bestimmtes Merkmal aufweisen, anhand dessen sich die Dyadenpartner sinnvoll differenzieren lassen. Dementsprechend wird zwischen „,distinguishable“ und ,indistinguishable dyads“ unterschieden: „Dyad members are considered distinguishable if there is a meaningful way to order the two scores" (Kenny, Kashy \& Cook, 2006, S. 6). So stellen z. B. das Geschlecht bei Untersuchungen mit heterosexuellen Paaren oder das Alter bei der Untersuchung von Geschwistern - mit Ausnahme von Zwillingen - Merkmale dar, anhand derer die beiden Dyadenpartner in allen Dyaden differenziert werden können, sodass diese Dyaden unterscheidbare Dyaden darstellen. Hinsichtlich homosexueller Paare und Zwillingen sind das Geschlecht respektive das Alter keine Merkmale, anhand derer die Dyadenpartner sich unterscheiden, sodass in diesen Fällen ununterscheidbare Dyaden vorliegen. Unbedeutende Differenzierungen von Dyadenpartnern in ununterscheidbaren Dyaden wie z. B. durch die Verwendung pseudonymisierter Probanden-IDs als Variable zur Ordnung der Dyadenpartner fügt den Daten eine weitere arbiträre Komponente hinzu, die wiederum zu statistischen Verzerrungen führen kann und dementsprechend zu vermeiden ist (vgl. ebd.).

\subsubsection{Stichprobe}

Zur Prüfung einer potentiellen Abhängigkeit der Prävalenz bilingualer Praktiken von der Dyade werden nur die Daten von Probanden der Angebotsgruppen LGT/D und RGT/D verwendet. Die Gruppen LGD und RGD werden hierbei exkludiert, da eine Berücksichtigung dieser die Ergebnisse angesichts des fehlenden Angebots zur bilingualen Interaktion verzerren würde. In diesen Gruppen ist kaum bilinguale Interaktion zu erwarten, sodass beide Peers der Dyaden mit hoher Wahrscheinlichkeit geringe Prävalenzen bilingualer Praktiken aufweisen, was sich in einer hohen Korrelation dieser Werte niederschlagen würde. Die Inklusion der Gruppen LGD und RGD würde dementsprechend die Wahrscheinlichkeit einer Abhängigkeit der Prävalenz bilingualer Praktiken von der Dyade deutlich erhöhen.

\subsubsection{Datengrundlage}

Als Datengrundlage dienen alle sequenzierten Phasen der Transkripte der Gruppen LGT/D und RGT/D. 


\subsubsection{Analysemethode}

Es gibt kein Merkmal in der vorliegenden Stichprobe, anhand derer sich die Dyadenpartner der jeweiligen Dyaden konsequent differenzieren lassen. Typische Variablen zur Zusammenstellung von Dyaden in PL-Studien wie z. B. die Kompetenz bezüglich des zu lernenden Inhalts oder die Klassenstufe (vgl. Robinson, Schofield \& Steers-Wentzell, 2005) wurden in der Dyadenzusammenstellung zwar versucht zu berücksichtigen, stellen allerdings keine systematische, konsequent in jeder Dyade applizierte Gruppierungsvariable dar. Dementsprechend handelt es sich bei den Dyaden der Stichprobe um ununterscheidbare Dyaden.

Zur Prüfung der Abhängigkeit einer Variable von nicht unterscheidbaren Dyaden gibt es verschiedene methodische Möglichkeiten wie z. B. die Verwendung von Intraklassenkorrelation, ANOVA, Mehrebenenanalyse oder „pairwise correlation“ (vgl. Kenny, Kashy \& Cook, 2006, S. 32-39). Diese Methoden setzen alle mindestens Intervallskalenniveau und eine Normalverteilung der Daten voraus. Eine Normalverteilung der Prävalenz bilingualer Praktiken ist angesichts der Stichprobengröße sowie der Optionalität des bilingualen Sprachangebots nicht zwingend für die Prävalenz bilingualer Praktiken zu erwarten. Sollte keine Normalverteilung der Prävalenz bilingualer Praktiken vorliegen, hätte dies Konsequenzen für die Prüfung der potentiell existierenden Abhängigkeit der Prävalenz bilingualer Praktiken von der Dyade, da diese den z-Wert zur Prüfung signifikanter Korrelationen verwendet, welcher von einer Normalverteilung ausgeht. Eine etablierte non-parametrische Methode zur Prüfung der Abhängigkeit einer Variable bei ununterscheidbaren Dyaden ohne eine arbiträre Zuordnung der Peers gibt es nach Kenny, Kashy \& Cook (ebd., S. 42f) nicht. Im Falle einer fehlenden Normalverteilung wird auf Datentransformation durch einen Logarithmus zurückgegriffen, um durch diese Transformation eventuell eine Normalverteilung zu erhalten. Logtransformationen stellen einen in der Sozialforschung akzeptierten und etablierten Umgang mit nicht normalverteilten Daten dar, auch wenn diese mit diversen methodischen Problemen verbunden sein können (vgl. z. B. Feng et al., 2014).

Die Abhängigkeit der Prävalenz bilingualer Praktiken von der Dyade wird für die vorliegenden Daten mit der „pairwise correlation“ geprüft (vgl. ebd., S. 37-39), welche von Griffin \& Gonzalez (1995) in die psychologische Literatur eingeführt wurde (vgl. Kenny, Kashy \& Cook, 2006, S. 37). Zur Berechnung der ,pairwise correlation“ benötigt es einen paarweise strukturierten Datensatz (vgl. ebd., S. 16). Jede Dyade erhält zwei Zeilen, in denen die Daten der Dyadenpartner enthalten sind. Hierbei enthält allerdings jede Zeile eines Probanden der Dyade auch die Daten des Dyadenpartners, was schematisch in der Abbildung 10 illustriert ist. Kenny, Kashy und Cook (ebd., S. 38) beschreiben die Datenstruktur folgendermaßen: „In this method, one of the persons is designated as $X$, and the other $Y$. Then the data are doubled, but the two persons" 
scores are flip-flopped, making each $X$ a $Y$ and each $Y$ an $X$." So kann z. B. mit einer solchen Datenstruktur unter Verwendung der Pearson Produkt-MomentKorrelation von $\mathrm{X}_{1}$ und $\mathrm{X}_{2}$ (siehe Abbildung 10) sowie der z-Statistik geprüft werden, ob die zu prüfende Variable abhängig von der Dyade ist oder nicht. $\mathrm{X}_{1}$ repräsentiert hierbei den Wert der Variable X von Person 1 und $\mathrm{X}_{2}$ den Wert der Variable $X$ von Person 2. Die paarweise Korrelation gilt es abschließend mit der Quadratwurzel der Anzahl der Dyaden zu multiplizieren und den resultierenden Wert als z-Wert zu interpretieren.

Abbildung 10

Beispiel für einen paarweise strukturierten Datensatz

\begin{tabular}{cccccc}
\hline Dyade & Person & Variable $\mathrm{X}_{1}$ & Variable $\mathrm{Y}_{1}$ & Variable $\mathrm{X}_{2}$ & Variable $\mathrm{Y}_{2}$ \\
\hline 1 & 1 & 3 & 9 & 5 & 7 \\
1 & 2 & 5 & 7 & 3 & 9 \\
2 & 1 & 8 & 6 & 1 & 2 \\
2 & 2 & 1 & 2 & 8 & 6 \\
\hline
\end{tabular}

\subsubsection{Prävalenz bilingualer Praktiken mit und ohne explizitem bilingualen Interaktionsangebot beim Peer-Learning}

Die Prävalenz bilingualer Praktiken mit und ohne explizitem Angebot beim PL soll zunächst deskriptiv betrachtet werden und anschließend hinsichtlich des expliziten Sprachangebots (F2a), der unterschiedlichen Lerninhalte Lese- und Rechenstrategien (F2b) sowie der verschiedenen Phasen innerhalb des Leseund Rechentrainings (F2c) inferenzstatistisch auf signifikante Unterschiede untersucht werden. Zuvor gilt es allerdings zu prüfen, ob die vier Gruppen LGD, RGD, LGT/D und RGT/D sich bezüglich theoretisch zentraler, potentiell die Prävalenz bilingualer Praktiken beeinflussender Faktoren wie Sprachkompetenz, Einstellungen zur Bilingualität und bilinguale Sprachpraxis in ihren Ausgangsbedingungen gleichen.

\subsubsection{Stichprobe}

Für die Fragestellung F2a werden alle Probanden der Gruppen berücksichtigt, da hierbei der Effekt des bilingualen Sprachangebots auf die Prävalenz bilingualer Praktiken der Gruppen LGT/D und RGT/D mit den Gruppen LGD und RGD, die kein bilinguales Sprachangebot erhalten haben, verglichen werden soll. Die Angebots- und Nicht-Angebotsgruppen werden für diesen Vergleich 
jeweils aggregiert. Bei Fragestellung F2b und F2c werden lediglich die Angebotsgruppen LGT/D und RGT/D geprüft, da ohne ein explizites Angebot zur bilingualen Interaktion keine Unterschiede zwischen den Lerninhalten sowie den einzelnen Trainingsphasen zu erwarten ist.

\subsubsection{Konstrukte und Instrumente}

Die Sprachkompetenz im Deutschen und Türkischen wird über den „Wortschatz- und Wortfindungstest für 6- bis 10-Jährige“ (Glück, 2011) - kurz WWT 6-10 - erhoben. Der WWT 6-10 ist ein Wortschatztest, der anhand von Bildauswahl den rezeptiven und durch Bildbenennung den produktiven Wortschatz im Deutschen und Türkischen mit den gleichen Items erhebt. Neben der Bildauswahl und -benennung zentraler grammatischer Kategorien wie Nomen, Verben und Adjektiven testet der WWT 6-10 auch Hyperonyme (Oberbegriffe wie z. B. „Jahreszeiten“, „Gemüse“ oder „Möbel“) sowie Antonyme (Wörter mit entgegengesetzter Bedeutung wie z. B. „schwarz“/,weiß“ oder „groß“/,klein“) und beansprucht dementsprechend auch sprachliche Fähigkeiten, die eher dem bildungssprachlichen Spektrum zuzuordnen sind (vgl. ebd., S. 28-35).

Im Projekt BiPeer wurde nur der produktive Wortschatz im Türkischen und Deutschen mit der Kurzform bestehend aus den für die beiden Sprachen gleichen 40 Items erhoben, sodass ein Vergleich des produktiven Wortschatzes im Deutschen und Türkischen möglich ist. Hierbei wurde die Testform für fünfeinhalb bis sechseinhalb Jahre alte Kinder verwendet, was nicht der Altersspanne der vorliegenden Stichprobe entspricht. Die Entscheidung für die Verwendung dieser nicht altersgerechten Testform beruht auf den folgenden drei Gründen: 1) Bei der Pilotierung mit der altersadäquaten Testform traten geringe Varianzen und zum Teil Bodeneffekte beim Türkischen auf. Angesichts der diametralen Entwicklung bilingual aufwachsender SuS in Deutschland bezüglich ihrer sprachlichen Fähigkeiten im Deutschen und Türkischen ab der Grundschule zu Gunsten des Deutschen (vgl. Reich, 2009) sind geringere Leistungen im Türkischen als im Deutschen zu erwarten. Um eine geringe Varianz sowie Bodeneffekte im Türkischen zu vermeiden, wurde auf die Testform für die jüngste Population des WWT 6-10 zurückgegriffen. Potentielle Deckeneffekte im Deutschen als Konsequenz der nicht altersgerechten Testform für die jüngste Population wurden hierbei bewusst in Kauf genommen. 2) Zudem weist die türkischsprachige Version des WWT 6-10 keine eigenständige Normierung auf (vgl. Glück, 2011, S. 112ff), sodass Vergleiche mit einer Eichstichprobe von vornherein nicht möglich wären. 3) Die Altersspanne der Probanden umfasst zwei verschiedene Testformen mit distinkten Itemsets, sodass die Verwendung der für die jeweiligen Altersbereiche vorgeschlagenen Testformen auf Kosten der Vergleichbarkeit der Werte zwischen jüngeren und älteren Kindern der Stichprobe gehen würde. 
Eine weitere Adaptation des Instrumentes durch die BiPeer-Studie besteht in der Hinzufügung zusätzlicher plausibler Antwortmöglichkeiten der türkischsprachigen Items. Bei der Pilotierung der türkischsprachigen Version fiel auf, dass korrekte Benennungsalternativen einzelner Items nicht in der Auswertung des WWT 6-10 auftraten. Dementsprechend wurden bei den jeweiligen Items weitere passende Lösungen in die Auswertung hinzugefügt. Eine letzte Adaptation des Instruments bestand im Bearbeitungsmodus. Während der WWT 6-10 die türkische Testversion nur auf dem PC anbietet, wurde für in der BiPeer-Studie der produktive türkische und deutsche Wortschatz in der „Paper and Pencil" Version erhoben.

Dementsprechend erfolgt die Messung der Sprachkompetenz im Türkischen über den produktiven Wortschatz. Dies stellt natürlich eine radikale Vereinfachung unter Auslassung zentraler weiterer sprachlicher Fähigkeiten als Operationalisierung von Sprachkompetenz dar. Geeignete Sprachstandsdiagnostikinstrumente im Türkischen für den Altersbereich der Probanden konnten allerdings nicht identifiziert werden (vgl. Rauch, Schastak \& Richter, 2016; Reitenbach, Schastak \& Rauch, 2018). Zudem ist der Wortschatz eng mit allgemeineren Fähigkeiten der Sprachkompetenz verknüpft (vgl. z. B. Limbird et al., 2014, Marx \& Roick, 2012; Marx \& Stanat, 2012), sodass dieser als proximales Maß für allgemeine Sprachfähigkeiten genutzt werden kann.

Die affektiven sowie bildungsbezogenen Einstellungen zum Türkischen und die Sprachpraxis werden über Fragebogenskalen erhoben. Da zu diesen Konstrukten für den vorliegenden Altersbereich keine geeigneten Skalen in der bisherigen Forschung identifiziert werden konnten, wurden die verwendeten Skalen selbst entwickelt. Alle Items konnten auf einer Likert-Skala von $1=$ „stimmt nicht“ bis $4=$,stimmt genau“ beantwortet werden. Die interne Konsistenz der Skalen wurde auf Basis der in der BiPeer-Studie vorhandenen Gesamtstichprobe bilingualer deutsch-türkischsprachiger Probanden berechnet und können alle als ,gut" bezeichnet werden. Die Skala „Türkischfreude“ (vier Items, z. B. „Türkisch sprechen macht mir Spaß“, Cronbachs $\alpha=.85$ ) erfasst die affektive Einstellung zum Türkischen. Bildungsbezogene Einstellungen und Praktiken werden mit der Skala „Türkisch als Ressource“ (fünf Items, z. B. „Türkisch hilft mir beim Lernen für die Schule“; Cronbachs $\alpha=.86$ ) respektive „Türkisch bei Denkprozessen im Lesen/Rechnen“ (vier Items, z. B. „Wenn ich über den Inhalt eines Texts/einen Rechenweg nachdenke, denke ich in Türkisch", Cronbachs $\alpha=.83$ ) erhoben. Die Sprachpraxis in der Schulpause und im Unterricht wird jeweils mit einem Item erhoben („In der Pause/ Im Unterricht unterhalte ich mich mit meinen Türkisch sprechenden Mitschülern auch auf Türkisch"). 


\subsubsection{Datengrundlage und Durchführung}

Die Datengrundlage für den Vergleich der Angebots- und Nicht-Angebotsgruppen hinsichtlich der Prävalenz bilingualer Praktiken (F2a) beschränkt sich auf die Phasen „Zusammenfassen“ und „Vorhersage“ sowie „Teamrechnen 1“ und „Teamrechnen 2“, da aufgrund limitierter Ressourcen der BiPeer-Studie nur diese Phasen in den Gruppen LGD und RGD transkribiert wurden. Die Relevanz der Größe der Datengrundlage wird durch das Skalenniveau der Operationalisierung bilingualer Praktiken beschränkt, da bilinguale Praktiken im Verhältnis zu monolingual deutschsprachigen Turns quantifiziert werden. Es könnten lediglich Probleme im Vergleich der Angebots- und Nicht-Angebotsgruppen auftreten, wenn genau die ausgewählten Phasen als Datengrundlage verwendet werden, in denen bilinguale Praktiken signifikant seltener oder häufiger als in anderen Phasen auftreten. Eine Prüfung der Eignung der ausgewählten Datengrundlage für den Vergleich der Prävalenz bilingualer Praktiken zwischen den Angebots- und Nicht-Angebotsgruppen erfolgt indirekt auch in Fragestellung F2c, in der potentielle Unterschiede in der Prävalenz abhängig von den verschiedenen Trainingsphasen geprüft werden. Für die Fragestellungen F2b und F2c werden fast alle Phasen der Angebotsgruppen berücksichtigt. LGT/D wird hinsichtlich der insgesamt sechs verschiedenen Phasen „Tandemlesen“, „Wörter Klären“, „Zusammenfassen“, „Vorhersagen“, „Frage zu den Abschnitten“ sowie „Abschlussrunde Lesen“ und RGT/D bezüglich „Teamrechnen 1“, „Teamrechnen 2“, „Rechenkonferenz“ sowie „Abschlussrunde Rechnen“ analysiert. Zudem werden für LGT/D sowie RGT/D die Interaktionen, die zwischen den Phasen liegen und keiner dieser spezifisch zugeordnet werden können, unter der Kategorie „Zwischenphasen“" ebenfalls analysiert. Lediglich die Phase „Kopfrechnen“ des Rechentrainings wird ausgeschlossen, da sich diese Phase durch die individuelle Aufgabenbearbeitung unter hohem Zeitdruck auszeichnet und diese sich somit durch ihre fehlenden Interaktionsmöglichkeiten von den anderen Phasen unterscheidet.

Der WWT 6-10 wird im Prätest in einer Einzeltestung durch bilinguale deutsch-türkischsprachige studentische Hilfskräfte administriert, die zuvor in der Anwendung aller Testinstrumente und Erhebungen im Projekt BiPeer theoretisch und praktisch geschult wurden. Die Testung durch bilingual deutschtürkischsprachige studentische Hilfskräfte war notwendig, da zum einen ein bilingualer Sprachmodus für die Testsituation etabliert werden sollte und zum anderen sowohl die Instruktion als auch die Auswertung deutsch- und türkischsprachige Fähigkeiten benötigen. Nach der standardisierten Instruktion werden zunächst vier Probeitems zur Eingewöhnung und anschließend die 40 Testitems bearbeitet. Die Erhebung des deutschen und türkischen produktiven Wortschatzes erfolgt parallel für die einzelnen Testitems in der Reihenfolge Deutsch-Türkisch. Dementsprechend wird das vorgelegte Testitem zunächst im Deutschen und anschließend im Türkischen benannt, bevor das nächste 
Testitem bearbeitet wird. Die Auswertung erfolgt direkt in der Erhebung. Allerdings wurden Benennungen, die nicht direkt und eindeutig als richtig oder falsch gewertet werden können, vorerst notiert und später ausgewertet.

Die Fragebogenskalen wurden wie der WWT 6-10 in einer Einzeltestung im Prätest von einer bilingual deutsch-türkischsprachigen studentischen Hilfskraft erhoben. Um das Verständnis der Items zu sichern, wurden die Items von der studentischen Hilfskraft vorgelesen, bevor die Probanden ihre Antwort ankreuzten.

\subsubsection{Analysemethode}

Zunächst werden die vier einzelnen Gruppen LGD, RGD, LGT/D und RGT/D hinsichtlich der Sprachkompetenz, den Einstellungen zum Türkischen und der Sprachpraxis auf gleiche Ausgangsbedingungen auf der Individual- und Tandemebene geprüft. Hinsichtlich der Sprachkompetenz werden die Gesamtwerte des WWT 6-10 im Deutschen und Türkischen verwendet. Die Einstellungen zum Türkischen werden mit den Skalen „Türkischfreude“, „Türkisch als Ressource“ sowie „Türkisch bei Denkprozessen im Lesen/Rechnen“ und die typische Sprachpraxis mit jeweils einem Item zum Sprachverhalten in der Schulpause und im Unterricht geprüft. Potentielle Unterschiede in den Ausgangsbedingungen für die oben angeführten Variablen zwischen den Gruppen werden mit Hilfe von Mittelwertsunterschieden sowohl auf Basis der Individualwerte der Probanden als auch auf Dyadenebene durch aggregierte Mittelwerte der Dyadenpartner zwischen den vier Gruppen geprüft.

Abhängig von der Verteilung der Prävalenz bilingualer Praktiken wird auf entweder auf parametrische oder non-parametrische Verfahren zurückgegriffen. Angesichts der Optionalität des bilingualen Sprachangebots in den Gruppen LGT/D und RGT/D sowie des standardisierten Eingriffs der Übungsleitungen in den Gruppen LGD und RGD, bei bilingualer Interaktion die Dyade zur monolingualen Interaktion aufzufordern, dürften sich die Prävalenzen bilingualer Praktiken wahrscheinlich nicht normalverteilen. Die Fragestellung F2a wird dementsprechend entweder mit dem t-Test für unabhängige Stichproben oder mit dem non-parametrischen Mann-Whitney-U-Test auf signifikante Unterschiede untersucht. Um der potentiellen Abhängigkeit der Prävalenz bilingualer Praktiken gerecht zu werden, gilt es bei non-parametrischen Analysemethoden auf die Dyaden- statt Individualwerte der Dyaden zurückzugreifen. Abhängig von der Wahl eines parametrischen oder non-parametrischen Verfahrens zur Analyse der Fragestellung F2a wird für die Fragestellungen F2b und F2c die gleiche Analysestrategie beibehalten. Dementsprechend würde die Wahl des Mann-Whitney-U-Tests für die Fragestellung F2a die Nutzung non-parametrischer Methoden auch für die Fragestellungen F2b und F2c implizieren, obwohl bei diesen theoretisch auch - abhängig von der Verteilung 
und weiteren typischen Voraussetzungen - auf parametrische Verfahren zurückgegriffen werden könnte. Allerdings stellen sowohl Fragestellung F2b als auch F2c weiterführende, indirekte Legitimationen der in F2a angewandten Methodik dar. In Fragestellung F2b wird indirekt geprüft, ob die in F2a vorgenommene Aggregation der Angebotsgruppen LGT/D und RGT/D auch hinsichtlich der kompletten Datengrundlage anstatt der ausgewählten Phasen vertretbar ist, da bei signifikanten Unterschieden zwischen diesen Gruppen eine Aggregation der Gruppen LGT/D und RGT/D zu hinterfragen wäre. In Fragestellung F2c wiederum wird indirekt geprüft, ob sich die ausgewählten Phasen für den in F2a vollzogenen Vergleich der Angebots- und Nicht-Angebotsgruppen eignen. Sollte sich zeigen, dass in den ausgewählten Phasen signifikant häufiger oder seltener bilinguale Praktiken produziert werden, müsste die Eignung der selektierten Phasen reflektiert und die Auswahl unter Umständen revidiert werden. Aufgrund dieser indirekten Prüfungen sollten vergleichbare Methoden für alle Fragestellungen von F2 gewählt werden. Dementsprechend wird Fragestellung F2b ebenfalls entweder mit Hilfe des t-Tests für unabhängige Stichproben oder dem Mann-Whitney-U-Tests auf potentielle Unterschiede der Nutzungshäufigkeit bilingualer Praktiken beim PL bezüglich der verschiedenen Lerninhalte Lese- und Rechenstrategien analysiert. Zur Prüfung von Fragestellung F2c wird entweder auf die einfaktorielle Varianzanalyse mit Messwiederholung oder auf den Friedman-Test für abhängige Stichproben zurückgegriffen.

\subsubsection{Der Zusammenhang sprachlicher und motivationaler Eigenschaften mit der Prävalenz bilingualer Praktiken beim Peer-Learning}

Bei Fragestellung F3 stehen mögliche Zusammenhänge von sprachlichen und motivationalen Eigenschaften der Probanden mit ihrer Produktion bilingualer Praktiken beim PL im Fokus. Hierbei werden anhand von Prätestdaten potentielle Zusammenhänge von sprachlichen Variablen wie Sprachkompetenz, einstellungen und -praxis (F3a) und anhand von motivationalen Variablen aus der achten Sitzung wie z. B. „basic needs“ (vgl. Ryan \& Deci, 2018) oder Freude am Türkischen im Training (F3b) mit der Prävalenz bilingualer Praktiken beim PL unter Verwendung dyadischer Datenanalyse untersucht.

\subsubsection{Stichprobe}

Für die Fragestellungen F3a und F3b werden nur Probanden aus den Angebotsgruppen LGT/D und RGT/D untersucht, da - wie in Fragestellung F1 eine Berücksichtigung der Probanden aus den Gruppen LGD und RGD aufgrund des fehlenden bilingualen Sprachangebots keine adäquate Stichprobe 
zur Prüfung der potentiellen Zusammenhänge darstellt. Sofern sich in Fragestellung F2b herausstellen sollte, dass sich die Prävalenzen bilingualer Praktiken nicht zwischen den Gruppen LGT/D und RGT/D signifikant unterscheiden, erscheint es legitim, diese beiden Gruppen für die Analysen der Fragestellungen F3a und F3b zu aggregieren. Somit kann auch die geringe Power der kleinen Stichprobengrößen zumindest etwas erhöht werden.

\subsubsection{Konstrukte und Instrumente}

Es werden hinsichtlich sprachlicher Variablen die gleichen Konstrukte und Instrumente genutzt, die zur Überprüfung gleicher Ausgangsbedingungen der Gruppen LGD, RGD, LGT/D und RGT/D bei Fragestellung F2 verwendet wurden. Bezüglich der Prüfung eines potentiellen Zusammenhangs motivationaler Variablen mit der Prävalenz bilingualer Praktiken werden insgesamt sieben verschiedene Konstrukte geprüft. Die Konstrukte werden mithilfe von Likert-Skalen erhoben. Die einzelnen Items der Skalen können auf einer vierstufigen Skala von $1=$,stimmt nicht" bis $4=$,stimmt genau“ beantwortet werden.

Die „basic needs“ nach Ryan und Deci (2018) werden mit Skalen zum Autonomie- und Kompetenzerleben sowie der sozialen Eingebundenheit erhoben, welche alle nach Kunter (2005) und Prenzel et al. (1996) adaptiert wurden. Die interne Konsistenz der Skalen wurde auf Basis der in der BiPeer-Studie vorhandenen Gesamtstichprobe berechnet, wobei die Skalen Autonomie- und Kompetenzerleben hinsichtlich des Lese- und Rechentraining einzeln ausgewertet wurden, da sich die Referenz einiger Items dieser „basic needs"-Skalen zwischen den Interventionen unterscheidet. Die Skala „Autonomieerleben“ besteht aus sieben Items (z. B. „Im Lese-/Rechentraining konnte ich Aufgaben auf meine Art erledigen“, Lesetraining: Cronbachs $\alpha=0,64$; Rechentraining: Cronbachs $\alpha=0,58$ ). Die Skalen „Kompetenzerleben“ (z. B. „Im Lese-/Rechentraining konnte ich zeigen, was ich schon alles weiß", Lesetraining: Cronbachs $\alpha=0,46$; Rechentraining: Cronbachs $\alpha=0,55)$ und ,soziale Eingebundenheit“" (z. B. „Mein BiPeer-Partner hilft mir, wenn es nötig ist“ Cronbachs $\alpha$ $=0,84$ ) enthalten jeweils vier Items. Die interne Konsistenz der Skalen muss mit Ausnahme von der Skala „,soziale Eingebundenheit“ allerdings als „fragwürdig“, „schlecht" und im Falle der Skala „Kompetenzerleben“ im Lesetraining gar als ,inakzeptabel“ bezeichnet werden. Alternative Daten, mit denen Komponenten der „,basic needs“ der Probanden erfasst werden, liegen leider im Datensatz nicht vor, sodass auf diese Skalen zurückgegriffen werden muss.

Die Skalen zur Erhebung der empfundenen Freude, der eingeschätzten Schwierigkeit und dem allgemeinen sowie strategiespezifischen Nutzen des Türkischen in der PL-Intervention sind Eigenkonstruktionen des Teams der BiPeer-Studie. Da diese Skalen nur von den Probanden der Angebotsgruppen LGT/D und RGT/D bearbeitet wurden, erfolgt die Berechnung der internen 
Konsistenz auf Basis dieser Probanden. Auch hier wurden die Skaleneigenschaften abhängig von der Intervention differenziert ausgewertet. Die Skala „Türkischfreude im Training“ (z. B „Im Lese-/Rechentraining habe ich gerne Türkisch gesprochen", Lesetraining: Cronbachs $\alpha=0,95$; Rechentraining: Cronbachs $\alpha=0,91$ ) und ,Schwierigkeit der Türkischnutzung im Training“ (z. B. „Im Lese-/Rechentraining ist es mir leicht gefallen, Türkisch zu sprechen.“, Lesetraining: Cronbachs $\alpha=0,64$; Rechentraining: Cronbachs $\alpha=0,78)$ enthalten jeweils fünf Items. Die Skalen zur Einschätzung des allgemeinen Nutzens des Türkischen für die PL-Intervention (z. B. „Beim Lese-/Rechentraining hat mir Türkisch geholfen, mit meinem Teampartner zusammenzuarbeiten“, Lesetraining: Cronbachs $\alpha=0,92$; Rechentraining: Cronbachs $\alpha=0,76$ ) bestehen aus jeweils 4 Items. Hinsichtlich des strategiespezifischen Nutzens enthält die Skala im Lesetraining vier (z. B. „Beim Lesetraining hat mir Türkisch für das Zusammenfassen von Abschnitten geholfen“, Cronbachs $\alpha=$ 0,93 ) und im Rechentraining sieben Items (z.B. „Beim Rechentraining hat mir Türkisch geholfen, Aufgaben zu vereinfachen“", Cronbachs $\alpha=0,88$ ). Die interne Konsistenz der einzelnen Skalen ist zum Großteil als „exzellent“ und „gut" zu bezeichnen, wobei die Skala „Schwierigkeit der Türkischnutzung im Training“ im Lesetraining „fragwürdig“, aber im Rechentraining ,akzeptabel“ erscheint.

\subsubsection{Datengrundlage und Durchführung}

Hinsichtlich der Prävalenz bilingualer Praktiken werden alle Phasen der Gruppen LGT/D und RGT/D berücksichtigt. Die motivationalen Variablen wurden im Gegensatz zu den sprachlichen Variablen nicht im Prätest, sondern zu Beginn der achten Sitzung erhoben. Es handelt sich bei dieser Erhebung auch nicht um eine Einzeltestung, da die Fragebögen von den beiden Probanden der Dyade parallel ausgefüllt wurden. Die einzelnen Items der motivationalen Fragebogenskalen wurden wieder zur Verständnissicherung von der anwesenden studentischen Übungsleitung bei der Erhebung vorgelesen.

\subsubsection{Analysemethode}

Die Fragestellungen F3a und F3b werden auch unter Verwendung der in F1 bereits eingesetzten paarweisen Korrelation für ununterscheidbare Dyaden (vgl. Griffin \& Gonzalez, 1995; Kenny, Kashy \& Cook, 2006, S. 37ff, 136ff) analysiert, sofern sich bilinguale Praktiken in F1 als abhängig von der Dyade herausstellen sollten. Sollte eine solche Abhängigkeit nicht bestehen, wird auf die Pearson Produkt-Moment-Korrelation oder bei Verletzung dessen Voraussetzungen auf die Rangkorrelation nach Spearman zurückgegriffen. Denn angesichts der Stichprobengröße sowie der inhaltlichen Ausrichtung der Skalen der sprachlichen und motivationalen Variablen könnten sich diese eventuell 
nicht normal verteilen und durch potentielle Deckeneffekte auch die Voraussetzung der Linearität verletzen. Dies würde ein Problem für die Adäquatheit des Einsatzes der Pearson Produkt-Moment-Korrelation als Analysemethode sowie für die Prüfung von Zusammenhängen auf Signifikanz darstellen, da diese unter Verwendung des z-Wertes erfolgt, welcher auf der Normalverteilung basiert. Sofern bilinguale Praktiken aber abhängig von der Dyade sind, wäre die Nutzung der non-parametrischen Rangkorrelation nach Spearman unter Verwendung der aggregierten Werte der Dyadenpartner zur Berücksichtigung der dyadischen Abhängigkeit mit diversen anderen Problemen behaftet. Aus den Individualwerten aller zu untersuchenden Variablen müssten die entsprechenden Dyadenwerte erzeugt werden, auch wenn diese Variablen nicht zwingend abhängig von der Dyade sind. Ein weiterer methodischer Nachteil eines solchen Analysevorgehens liegt in der Reduktion der Stichprobengröße um die Hälfte der Fälle, was angesichts der bereits auf Individualebene als klein zu bezeichnenden Stichprobe die Power der Tests noch weiter reduzieren würde, sodass nur äußerst starke Effekte identifiziert werden könnten. Angesichts der Vielzahl möglicher, simultan einflussnehmender Faktoren sowie der dynamischen Modellierung der Sprachmodi nach Grosjean (2008) sind theoretisch allerdings keine großen Effekte der zu untersuchenden Variablen zu erwarten. Zudem dürfte dieses Vorgehen auch nicht das potentielle Problem einer fehlenden Linearität der Zusammenhänge bei einigen zu prüfenden Variablen beheben können.

Sollten die Voraussetzungen der Pearson Produkt-Moment-Korrelation bezüglich einzelner zu untersuchender Variablen verletzt werden und bilinguale Praktiken sich in F1 als eine von der Dyade abhängige Variable darstellen, werden nichtsdestotrotz aus Ermangelung an methodischen Alternativen die paarweisen Korrelationen dyadischer Daten auf der Individualebene berechnet. Bei der Beschreibung und Interpretation der Ergebnisse wird dies berücksichtigt, indem mittlere und hohe Zusammenhänge sowie potentiell signifikante Korrelationen äußerst kritisch reflektiert werden, insbesondere bei Zusammenhängen mit Skalen, die sich durch eine suboptimale interne Konsistenz auszeichnen.

In Anlehnung an Griffin \& Gonzalez (1995, S. 431f) werden für jeden zu prüfenden Zusammenhang zwei Korrelationen berechnet, nämlich die „overall correlation“ $\left(\mathrm{r}_{\mathrm{xy}}\right)$ und die ,cross-intraclass correlation“ $\left(\mathrm{r}_{\mathrm{xy}}{ }^{\prime}\right)$. Insgesamt sind bei Korrelationsanalysen dyadischer Daten sechs mögliche paarweise Korrelation zweier Variablen denkbar (siehe Abbildung 11): 
Abbildung 11

Mögliche paarweise Korrelationen zweier Variablen bei ununterscheidbaren Dyaden nach Griffin \& Gonzalez (1995, S. 432)

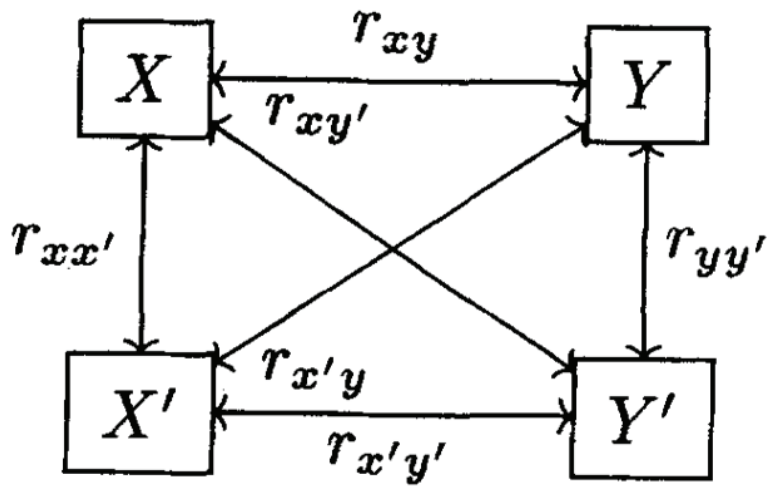

Die Korrelationen $r_{x y}$ und $r_{x^{\prime} y^{\prime}}$ sowie $r_{x y^{\prime}}$ und $r_{x^{\prime} y}$ sind hierbei allerdings identisch (vgl. ebd., S. 432f), da es sich um ununterscheidbare Dyaden mit „flipflopped" Werten (vgl. Kenny, Kashy \& Cook, 2006, S. 38) und somit letztlich um dieselben Werte nur in umgedrehter Reihenfolge handelt. Der Zusammenhang $\mathrm{r}_{\mathrm{xx}} \cdot$ wurde bereits in F1 untersucht und eine eigenständige Prüfung von $\mathrm{r}_{\mathrm{yy}}$ ist für das vorliegende Erkenntnisinteresse nicht zentral, auch wenn sich die motivationalen Variablen unter Umständen auch als abhängig von der Dyade herausstellen könnten. Zur Prüfung der Signifikanz der beiden Korrelationen wird der z-Wert herangezogen (vgl. Griffin \& Gonzalez, 1995, S. 432). Der $z$-Wert der „overall correlation“ $\left(r_{x y}\right)$ berechnet sich über $Z=r_{x y}$ $\sqrt{N_{1}}{ }^{*}$, wobei $\mathrm{N}_{1} *=2 \mathrm{~N} /\left(1+\mathrm{r}_{\mathrm{xx}} \mathrm{r}_{\mathrm{yy}}{ }^{*}+\mathrm{r}_{\mathrm{xy}}^{2}\right)$ ist (vgl. ebd.) Der z-Wert der ,crossintraclass correlation" $\left(\mathrm{r}_{\mathrm{xy}}\right)$ berechnet sich über $\mathrm{Z}=\mathrm{r}_{\mathrm{xy}} \cdot \sqrt{\mathrm{N}_{2}} *$, wobei $\mathrm{N}_{2} *=2 \mathrm{~N}$ $/\left(1+r_{x x} \cdot r_{y y^{c}}+r^{2}{ }_{x y}\right)$ ist (vgl. ebd.).

\subsection{Qualitative Datenanalyse}

Im Anschluss an die in 7.4 dargelegten quantitativen Analysen folgen qualitative Untersuchungen der selbstberichteten Motive für die Annahme oder Ablehnung des Sprachangebots (F4) und der Funktionen bilingualer Praktiken (F5). Hierfür wird für beide Fragestellungen auf die Qualitative Inhaltsanalyse nach Mayring (2015) zurückgegriffen. 


\subsubsection{Selbstberichte des Sprachgebrauchs im Training und dessen Begründung}

Zur Erfassung von zu Grunde liegenden Motiven für ein bestimmtes Verhalten sowie für Handlungen allgemein sind offene Antwortformate geeignet (vgl. Moosbrugger \& Kelava, 2007, S. 41). Während hierfür in der bildungsorientierten Bilingualismusforschung typischerweise verschiedene Formen von Interviewmethoden - häufig mit Hilfe von durch Videosequenzen initiierter ,stimulated recalls" - verwendet werden (vgl. z. B. Barwell, 2014; Clarkson, 2007; Culligan, 2015; García \& Kano, 2014; Storch \& Wigglesworth, 2003; Wlossek \& Rost-Roth, 2016), wurde für das vorliegende Forschungsvorhaben aufgrund der bereits umfangreichen, aufbereitungs- und auswertungsintensiven Studienanlage auf ein äußerst schlankes, zeitökonomisches Verfahren in Form eines kurzen, schriftlichen Selbstberichts zurückgegriffen.

\subsubsection{Stichprobe}

Für die Fragestellung F4 werden nur Probanden aus den Angebotsgruppen LGT/D und RGT/D befragt. Angesichts der zum Teil auch in den Nicht-Angebotsgruppen LGD und RGD auftretenden bilingualen Praktiken wären Informationen zu den hierfür zu Grunde liegenden Ursachen äußerst interessant. Allerdings wurde hierauf in der achten Sitzung verzichtet, um die systematische Variation des Sprachangebots zwischen den Angebots- und Nicht-Angebotsgruppen nicht zu gefährden, da ein solcher Impuls in den Fragebogenitems unter Umständen Probanden der Gruppen LGD und RGD zu bilingualer Interaktion hätte motivieren können. Von dieser Befürchtung hätte für den Posttest abgesehen werden können, da zu diesem Zeitpunkt die Intervention beendet war. Die Ergebnisse wären dann für diesen einen Messzeitpunkt für potentielle Vergleiche der Angebots- und Nicht-Angebotsgruppen möglich gewesen. Diese Option wurde allerdings nicht ergriffen, sodass diesbezüglich keine Daten vorliegen.

Eine Dyade sowie ein weiterer Proband eines Tandems der Gruppe RGT/D wurden aus den Analysen exkludiert, da sie nur an einem der beiden Erhebungszeitpunkte teilgenommen haben. Dementsprechend liegen für die Fragestellung F4 Daten von jeweils 13 Dyaden der Gruppen LGT/D und RGT/D vor, wobei insgesamt aber nur 51 Probanden $(L G T / D n=26 ; R G T / D n=25)$ die Stichprobe bilden.

\subsubsection{Instrumente und Durchführung}

Sowohl während (Sitzung 8) als auch am Ende des Trainings (Posttest) wurden mithilfe eines schriftlichen Fragebogens die selbstberichtete Annahme und 
Ablehnung des bilingualen Sprachangebots in den Gruppen LGT/D und RGT/D erfragt. Zusätzlich wurden die Kinder gebeten, ihr (bilinguales) Sprachverhalten während der Intervention in Form eines kurzen Selbstberichts schriftlich zu begründen. Die konkrete Formulierung des ersten Items lautete: „Hast du während des Trainings Türkisch gesprochen?“. Das Antwortformat dieses Items ist geschlossen, sodass die Probanden lediglich „Ja“ oder „Nein“ ankreuzen konnten. Neben diesen Antwortoptionen befand sich jeweils eine freie Zeile in der Breite eines DIN A4 Blattes, mit welchem das zweite Item durch den schriftlichen Impuls „warum:“ eingeleitet wurde. Die Probanden sollten dementsprechend bei dem zweiten Item ihre Antwort auf das Item im geschlossenen Format begründen. Hierbei wurden den Probanden weder Einschränkungen zu der Anzahl an potentiell zu liefernden Begründungen noch zur Detailliertheit der Ausführungen dieser auferlegt.

Neben dem Vorteil einer zeitökonomischen, ressourcenschonenden Erfassung der Ursachen für das jeweilige sprachliche Interaktionsverhalten weist dieses schlanke Format zwei weitere Vorteile im Gegensatz zu Interviewmethoden auf. Zum einen verfügt ein solches Verfahren über eine höhere Objektivität in der Auswertung (vgl. Moosbrugger und Kelava 2007, S. 42), da die Kürze und die einheitliche linguistische Form der Antworten (zum Großteil mit ,weil" eingeleitete Nebensätze als Folge des Impulses ,warum:“) die Analyse deutlich vereinfacht. Zum anderen kann davon ausgegangen werden, dass mit diesem Verfahren die präsentesten und relevantesten Gründe elizitiert werden, die den Probanden bewusst sind, was aus testtheoretischer Perspektive die Validität der Antworten stärkt.

\subsubsection{Analysemethode und Datenauswertung}

Zur Analyse des geschlossenen Antwortformats werden die jeweiligen Antworten ausgezählt, deskriptiv dargestellt und inferenzstatistisch unter Verwendung des Binomialtests auf signifikante Unterschiede hin geprüft. Zudem werden potentielle Unterschiede und Entwicklungen hinsichtlich der beiden Erhebungszeitpunkte deskriptiv betrachtet.

Die selbstberichteten Gründe der Probanden werden mit Hilfe der qualitativen Inhaltsanalyse nach Mayring (2015) ausgewertet. Die qualitative Inhaltsanalyse zeichnet sich durch die Analyse „fixierter“ Kommunikation aus, die systematisch, explizit regel- und theoriegeleitet vorgeht und mit welcher sich Rückschlüsse auf die analysierte Kommunikation beziehungsweise das sprachliche Material ziehen lassen (vgl. ebd., S. 12f). Innerhalb der qualitativen Inhaltsanalyse gibt es verschiedene Grundtechniken (vgl. ebd., S. 13-16) und Interpretationsvorgänge (vgl. ebd., S. 67), die als Leitlinien zur Analyse herangezogen werden können. Hierbei ist es wichtig zu erwähnen, dass die Inhaltsanalyse kein Standardinstrument darstellt, ,das immer gleich aussieht; sie muss an den konkreten Gegenstand, das Material angepasst sein und auf 
die spezifische Fragestellung hin konstruiert werden“ (vgl. ebd., S. 51). Für das vorliegende Datenmaterial und Erkenntnisinteresse empfiehlt sich die Technik der „Strukturierung“ (vgl. ebd., S. 67, 97-99) und zwar in Form der „,inhaltlichen Strukturierung“, die „Material zu bestimmten Themen, zu bestimmten Inhaltsbereichen extrahieren und zusammenfassen" (ebd., S. 99) will.

In Anlehnung an Mayring (ebd., S. 97-99) wird bei der vorzunehmenden Strukturierung zunächst die Analyseeinheit definiert, welche in diesem Fall die auf den Impuls ,warum:“ folgenden schriftlich verfassten Begründungen darstellen. Diese Selbstberichte werden bei Vorhandensein mehrerer Begründungen in ihre einzelnen Propositionen zerlegt, anstatt alle angegebenen Begründungen eines Selbstberichts als eine Einheit auszuwerten. Die Propositionen lassen sich bei diesem Format leicht voneinander trennen, da diese von den Probanden in der Regel selbst unter Verwendung der Konjunktion ,und“ markiert werden.

Anschließend gilt es die Strukturierungsdimensionen zu bestimmen. Hierfür werden ausgehend von der Fragestellung, der zu Grunde liegenden Theorie(n) und bisherigen empirischen Erkenntnissen des zu untersuchenden Phänomens Definitionen der Strukturierungsdimensionen erzeugt. Diese definierten Strukturierungsdimensionen werden in übergeordnete deduktive Hauptkategorien umgewandelt. Die Strukturierungsdimensionen und daraus resultierenden Hauptkategorien wurden in Anlehnung an die Sprachmodi nach Grosjean (2008) sowie potentielle Einflussfaktoren auf Sprachwechsel sowie -mischungen (vgl. Gardner-Chloros, 2009, S. 42-43; Grosjean, 2008, S. 42ff; Ritchie \& Bhatia, 2013, S. 378ff, 386), bildungsinstitutionellen Reflexionen der mono- bzw. multilingualen Schule (vgl. z. B. Cummins, 2000; García \& Kano, 2014; García \& Li Wei, 2014; Gogolin, 1994) sowie empirischer Evidenz hinsichtlich bilingualer Praktiken beim PL (vgl. z. B. Hopewell, 2011; Klingner \& Vaughn, 2000; Storch \& Wigglesworth, 2003; Swain \& Lapkin, 2000) konstruiert. Hierbei wurden zunächst die Strukturierungsdimensionen „Individuum“, „Gesprächspartner“" und „Kontext" als übergeordnete Ebenen konzipiert, um die Begründungen des sprachlichen Verhaltens hinsichtlich ihrer primären Bezugnahme zu kategorisieren.

Anschließend gilt es diese Hauptkategorien weiter auszudifferenzieren, indem sie in einzelne Unterkategorien aufgeteilt werden, die für das Erkenntnisinteresse relevante Teilaspekte und Nuancen der interessierenden Konstrukte erfassen. Daraufhin wurde eine Vielzahl deduktiver Kategorien wie z. B. Sprachkompetenz, Einstellungen, Identität, typische Sprachpraxis, Vereinfachung der Bearbeitung der Aufgaben, Vereinfachung der Kommunikation, Rücksichtnahme auf Dyadenpartner, monolingualer Habitus der Schule etc. getrennt für die Annahme und Ablehnung des bilingualen Sprachangebots erzeugt. 
Damit hieraus ein anwendbares Kategoriensystem entsteht, müssen abschließend die Haupt- und Unterkategorien trennscharf operationalisiert werden. Hierzu werden die Kategorien definiert, Abgrenzungen zwischen den Kategorien mit Hilfe von Kodierhinweisen verdeutlicht und mit möglichst eindeutigen und repräsentativen „Ankerbeispielen“ versehen.

Dieses deduktiv erzeugte Kategoriensystem wird anschließend einem Testlauf mit einem reduzierten Datenmaterial unterzogen (vgl. Mayring, 2015, S. 99). Hierfür wurde ca. ein Viertel des Materials verwendet. Nach diesem Probedurchlauf kommt es in der Regel zu einer Überarbeitung des Kategoriensystems (vgl. ebd.), in der nicht nur die bereits bestehenden deduktiven Kategorien modifiziert und angepasst werden, sondern auch weitere, aus dem Datenmaterial inspirierte induktive Kategorien gebildet werden. Der erste Testdurchlauf führte sowohl zum Ausschluss (z. B. „monolingualer Habitus der deutschen Schule“, „Identität") als auch zur Zusammenfassung einiger deduktiver Kategorien (z. B. „Sprachkompetenz“ und „Vereinfachung der Bearbeitung der Aufgaben“ zu „Kompetenz“ auf der Individualebene) sowie zur Erzeugung einer neuen Ebene (z. B. „Keine Begründung“) und induktiver Kategorien (z. B. ,Aufforderung“ auf Kontextebene, Restkategorien „Sonstige“ auf verschiedenen Ebenen).

In die Auswertung wurden nur Probanden aufgenommen, die an beiden Erhebungszeitpunkten teilgenommen haben und auf das geschlossene Antwortformat maximal ein Missing oder eine Mehrfachantwort (,ja“ und „nein“ im geschlossenen Format angekreuzt) lieferten. Aufgrund dessen mussten drei Probanden aus den Analysen exkludiert werden, was zu einer ungeraden Anzahl der Probanden trotz Dyadenstruktur des Trainings für diese Fragestellung führt. Weder das geschlossene noch offene Antwortformat wurden bei Mehrfachantworten (,ja“" und ,nein“ im geschlossenen Format angekreuzt) ausgewertet. Zunächst werden die Begründungen auf übergeordneter Ebene kategorisiert und anschließend hinsichtlich der jeweiligen Kategorie zugeordnet. Wenn das geschlossene Antwortformat beantwortet, aber die Zeile zur Begründung der Antwort frei gelassen wurde, wurde das offene Antwortformat als „keine Begründung“ kategorisiert.

Die Begründungen für die Annahme oder Ablehnung des bilingualen Interaktionsangebots wurden von zwei Personen unabhängig voneinander in ihrem originalen Wortlaut ohne sprachliche Glättung und Fehlerkorrektur innerhalb der SPSS-Datenmaske kategorisiert. Der Autor der vorliegenden Dissertation kategorisierte alle vorliegenden Selbstberichte und eine studentische Praktikantin wertete 33\% des Materials aus. Vor dem Rating des Materials durch eine studentische Praktikantin wurden Begründungen, die Namen des Tandempartners oder der Übungsleitung enthielten, anonymisiert, wobei festgehalten wurde, ob sich die Antwort auf den Tandempartner oder die Übungsleitung bezieht. Sowohl für die Ebenen $(\mathrm{K}=.851)$ als auch für die Kategorien 
$(\mathrm{K}=.846)$ kann von einer fast vollständigen Beurteilerübereinstimmung anhand Cohens Kappa gesprochen werden, welche unter Verwendung des Programms SPSS errechnet wurden. Die Fälle, in denen keine Übereinstimmung vorlag, wurden im Anschluss an das Rating besprochen, sodass eine Einigung für die Kategorisierung gefunden und im Hauptdatensatz dementsprechend korrigiert wurde.

\subsubsection{Kategoriensystem zur Kodierung der selbstberichtetenGründe für die Annahme oder Ablehnung des Angebots zur bilingualen Interaktion}

Das hierdurch entstandene Kategoriensystem besteht letztlich aus den vier übergeordneten Ebenen „Individuum“, „Gesprächspartner“, „Kontext" und „Keine Begründung“, wobei die drei erstgenannten Ebenen jeweils mit drei weiteren Kategorien ausdifferenziert werden. Diese Ebenen können gleichermaßen zur Einordnung der Gründe für die Annahme sowie für die Ablehnung des Sprachangebots angewandt werden. Unter der Individuumsebene werden alle Gründe für die Annahme und Ablehnung des Angebots kategorisiert, die die eigene Person und/oder ihre Eigenschaften und Einstellungen zur Begründung des sprachlichen Verhaltens anführen. Der Gesprächspartnerebene entsprechen alle Antworten zur Annahme und Ablehnung des Angebots, die die Gesprächspartner - den Tandempartner, das Tandem, die Übungsleitung oder die komplette Triade - in ihrer Begründung fokussieren. Der Ebene „Kontext“ werden diejenigen Fälle zugeordnet, die die Intervention selbst oder deren kontextuelle Rahmenbedingungen als Gründe für die Annahme oder Ablehnung des Angebots nennen. Dementsprechend werden Antworten, die zwar die Übungsleitung adressieren, sich aber eigentlich auf die Intervention beziehen, da die Übungsleitung die Bestandteile der Intervention lediglich umsetzt, auf der Kontextebene kategorisiert (z. B. ,weil unsere Trainingsleitung gesagt hat, dass wir auch Türkisch reden können"). Zudem gibt es einige Fälle, die keine Gründe für die berichtete Sprachpraxis nennen, aber das geschlossene Antwortformat bearbeitet haben. Solche Antworten entsprechen der Ebene „Keine Begründung“".

Auch die drei Unterkategoiren der Individuumsebene (IE), Gesprächspartnerebene (GE) und Kontextebene (KE) lassen sich sowohl zur Begründung der Annahme als auch der Ablehnung des bilingualen Interaktionsangebots verwenden, da die zu Grunde liegenden Argumentationen auf denselben Konstrukten operieren, sich allerdings in ihrer Richtung unterscheiden. Auf der Individuumsebene werden Kompetenz, Affinität und sonstige, auf das Selbst bezogene Gründe zur Legitimation des Sprachhandelns verwendet. Die Kategorie „IE Kompetenz" umfasst alle Begründungen für die Annahme oder Ablehnung des Angebots, die individuelle sprachliche und aufgabenbezogene Kompetenzen anführen. Dementsprechend fallen Antworten, die vorhandene oder fehlende Kompetenzen im Türkischen oder im Deutschen, Sprachasymmetrien 
zugunsten des Türkischen oder des Deutschen sowie eine Erleichterung oder eine zusätzliche Belastung zur Aufgabenbewältigung durch das bilinguale Interaktionsangebot adressieren, unter die Kategorie „IE Kompetenz“. Die Kategorie „IE Affinität" umfasst emotionale Gründe sowie positive und negative Einstellungen zu den jeweiligen Sprachen bezüglich der Annahme oder Ablehnung des Sprachangebots. Hierzu gehören Antworten, die auf positive sowie negative Empfindungen und Gefühlszustände oder eine emotionale Favorisierung des Türkischen oder des Deutschen hinweisen. Gründe für die Annahme oder Ablehnung des Angebots, die auf der Individuumsebene anzusiedeln sind, allerdings andere Argumente neben Kompetenz und Affinität zur Legitimation der Sprachpraxis anführen - wie z. B. Identität - werden der Restkategorie „IE Sonstige“ zugeordnet.

Begründungen auf der Gesprächspartnerebene lassen sich primär zwischen Bezugnahmen auf den Tandempartner oder die Dyade selbst sowie auf die Übungsleitung oder die komplette Triade differenzieren, wobei auch auf dieser Ebene eine Restkategorie eingerichtet werden musste. Gründe für die Annahme oder Ablehnung des Angebots, die den Tandempartner oder die Dyade beziehungsweise das sprachliche Verhalten des Tandempartners oder der Dyade anführen, werden unter die Kategorie „GE Peertandem“ zusammengefasst. Beziehen die Legitimationen sich allerdings primär auf die Übungsleitung beziehungsweise auf ihr sprachliches Verhalten, gilt es solche Antworten der Kategorie „GE Übungsleitung“ zuzuordnen. Gründe für die Annahme oder Ablehnung des Angebots, die auf der Gesprächspartnerebene anzusiedeln sind, aber nicht explizit den Peerpartner, die Dyade oder die Übungsleitung fokussieren, sodass z. B. die Gruppe als Ganzes beziehungsweise das sprachliche Verhalten der Gruppe adressiert wird, sind als „GE Sonstige“ zu kategorisieren.

Bezüglich der Kontextebene können zwei sich diametral entgegengesetzte Begründungslogiken identifiziert werden, da die Probanden die explizite Erlaubnis zur bilingualen Interaktion zum Teil als Angebot, allerdings ebenfalls als obligatorisches, zu erfüllendes Merkmal der Intervention interpretierten. Antworten, die auf den Angebotscharakter beziehungsweise auf die Erlaubnis zum Sprechen des Türkischen hinweisen, werden der Kategorie „KE Angebot“ zugeordnet, während Antworten als „KE Aufforderung" kategorisiert werden, die das Angebot zum Sprechen des Türkischen als einen zu erfüllenden Teil der Intervention darstellen, die von dem Training selbst oder von der Übungsleitung gefordert wurde. Die Restkategorie „KE Sonstige“ umfasst Gründe für die Annahme und Ablehnung des Angebots, die andere Faktoren neben Aufforderung und Angebot wie z. B. die Örtlichkeit oder das Material als Legitimation anführen.

Die Entscheidung für nach den verschiedenen Ebenen zu spezifizierenden Restkategorien erscheint als fruchtbarer Ansatz im Gegensatz zu einer überge- 
ordneten Restkategorie, da die zu Grunde liegende Bezugsebene hierbei mitberücksichtigt wird, sodass die einzelnen Antworten in den Restkategorien gehaltvoller zuzuordnen und zu interpretieren sind. Die Ebene „Keine Begründung" wird nicht weiter ausdifferenziert, da der propositionale Gehalt von fehlenden Antworten oder Begründungen wie ,weiß nicht" keinen weiteren Erkenntnisgewinn ermöglicht. Das vollständig aufgeschlüsselte Kategoriensystem samt Definitionen, Beispielen und Kodierhinweisen ist im Anhang unter „Anhang 13 Kategoriensystem der selbstberichteten Motive für die Annahme oder Ablehnung des bilingualen Sprachangebots" dargestellt.

\subsubsection{Funktionen bilingualer Praktiken beim Peer-Learning}

Ein weiterer fruchtbarer Schritt hin zu einer qualitativen Untersuchung bilingualer Praktiken als Angebot beim PL stellt die Kategorisierung der auftretenden türkischsprachigen und türkisch-deutschsprachig gemischten Turns hinsichtlich ihrer Funktionen dar. Hierbei sind allerdings weder quantitative Vergleiche mit den monolingual deutschsprachigen Turns der jeweiligen Dyaden noch mit den monolingual interagierenden Gruppen LGD und RGD intendiert. Ob ein anderes Verhältnis von On- und Off-Task Turns zwischen den deutschund türkischsprachigen Turns der Gruppen mit bilingualem Interaktionsangebot besteht oder ob das bilinguale Sprachangebot zu einem anderen Verhältnis von On- und Off-Task Turns im Vergleich zu den Nicht-Angebotsgruppen LGD und RGD führt, sind für das vorliegende Erkenntnisinteresse nicht zentral. Stattdessen haben die Funktionen, für die bilinguale Praktiken im PL eingesetzt werden, eine hohe theoretische aber vor allem praktische Relevanz. Die Ermittlung der Zwecke bilingualer Praktiken beim PL sowie deren Prävalenzen kann Einblicke hinsichtlich der Chancen, Grenzen und Herausforderungen bei der Implementation bilingualer Praktiken in Bildungsprozessen ermöglichen, die zur Modifikation von Instruktionsdesigns und zur Organisation sowie Strukturierung bilingualer Interaktionsangebote beim PL beitragen können. Eine solche Erfassung und Auszählung der Funktionen bilingualer Praktiken beim PL kann z. B. dahingehend Erkenntnisse liefern, ob bilinguale Praktiken für verschiedene Zwecke ungefähr gleich häufig eingesetzt werden, ob bestimmte Verwendungsweisen präferiert mit bilingualen Praktiken umgesetzt werden und ob sich diese in Abhängigkeit vom konkreten Lerninhalt unterscheiden.

Die qualitative Untersuchung des Materials in F5 erfolgt in Anlehnung an ein von Deppermann (2008, S. 37) vorgeschlagenes Vorgehen in drei Schritten: 1) Zunächst empfiehlt es sich, „eine erschöpfende Erfassung aller Vorkommen eines Phänomens“ beziehungsweise „eine Kollektion aller Segmente" anzulegen und zwar insbesondere, ,wenn sehr oberflächennahe Merk- 
male untersucht werden“ (ebd.). Diese Aufbereitung des Materials erfolgte bereits im quantitativen Teil der Analysen. 2) In einem folgenden Analyseschritt kann anschließend die vorliegende Kollektion ,in Subklassen unterteilt werden" (ebd.), was in der vorliegenden Studie anhand eines Überblicks über das Spektrum der Funktionen bilingualer Praktiken beim PL in F5 erfolgt. 3) Auf Basis dessen schließt sich eine möglichst repräsentative Fallauswahl an (ebd.), welche durch eine feinkörnige Analyse der organisationalen sowie handlungsund bedeutungskonstituierenden Aspekte bilingualer Praktiken untersucht wird. Dieser letzte Schritt nach Deppermann (ebd.) wird in der vorliegenden Arbeit allerdings nicht als Interaktionsanalyse samt repräsentativer Fallauswahl erfolgen, sondern sich in einer vertieften deskriptiven Beschreibung der verschiedenen Funktionen innerhalb der jeweiligen Haupt- und Unterkategorien der Funktionen bilingualer Praktiken beim PL anhand ausgewählter Beispiele erschöpfen.

\subsubsection{Stichprobe}

Hinsichtlich Fragestellung F5 werden alle bilingualen Praktiken der Tandems der Gruppen LGT/D und RGT/D berücksichtigt und ausgewertet. In LGT/D gibt es ein Tandem, das keine bilingualen Praktiken produziert hat, sodass diese Dyade aus der Analyse exkludiert wird. Weiterhin gibt es ebenfalls jeweils ein Tandem der Gruppe LGT/D und der Gruppe RGT/D, welche aufgrund ihrer äußerst hohen Prävalenz bilingualer Praktiken aus den Analysen ausgeschlossen werden. Diese beiden Dyaden produzierten 399 respektive 156 Turns mit bilingualen Praktiken. Dementsprechend übersteigen sie zusammen die Summe aller bilingualen Praktiken der restlichen Tandems der Gruppen LGT/D und RGT/D, welche bei 471 Turns mit bilingualen Praktiken liegt. Eine Auswertung der 555 Turns mit bilingualen Praktiken der zwei auszuschließenden Tandems würde zu einer separierten Analyse dieser zwingen, da die Ergebnisse in der Gesamtschau durch diese verzerrt werden würden. Diese beiden Tandems eignen sich zudem vielmehr für Fallanalysen und eine feinkörnige interaktionsanalytische Untersuchung ihrer bilingualen Interaktion beim PL, was nicht der übergeordneten methodischen und erkenntnistheoretischen Anlage der vorliegenden Arbeit entspricht. Auf eine Analyse des funktionalen Gebrauchs bilingualer Praktiken beim PL dieser beiden Dyaden wird dementsprechend im Folgenden verzichtet. Innerhalb der Gruppe LGT/D haben drei Probanden und innerhalb der Gruppe RGT/D ein Individuum keine bilingualen Praktiken produziert, obwohl ihre Dyadenpartner bilingual interagierten. Dementsprechend liegen Werte zum funktionalen Gebrauch bilingualer Praktiken beim PL für elf Dyaden der Gruppe LGT/D und 13 Dyaden der Gruppe RGT/D vor, wobei allerdings nur 19 respektive 25 Probanden in den jeweiligen Gruppen hinsichtlich ihres funktionalen Gebrauchs bilingualer Praktiken beim PL analysiert werden können. 


\subsubsection{Analysemethode}

Für die Identifikation der Funktionen bilingualer Praktiken beim PL wird wie bei F4 auf die Methodik der Qualitativen Inhaltsanalyse nach Mayring (2015) zurückgegriffen, wobei sich ebenfalls die ,inhaltliche Strukturierung“ (vgl. ebd., S. 99ff) am besten für das zugrundeliegende Datenmaterial eignet. Das Datenmaterial stellen die aufbereiteten Transkripte der Gruppen LGT/D und RGT/D dar. Hierbei wird auf die für die quantitativen Fragestellungen bereits erfassten rein türkischsprachigen und bilingual türkisch-deutschsprachigen Turns zurückgegriffen. Die Analyseeinheit stellen dementsprechend rein türkischsprachige und bilingual türkisch-deutschsprachige Turns dar.

Wie bei Fragestellung F4 entstand das Kategoriensystem zur Erfassung der Funktionen bilingualer Praktiken zunächst durch die Prüfung der deduktiv aus der bisherigen Theorie und Empirie abgeleiteten Kategorien an einem Teil des Datenmaterials. Die Strukturierungsdimensionen sind auf einer übergeordneten Ebene an der Distinktion von „On-“ und „Off-Task“ (vgl. z. B. Carroll, 1963; Slavin, 1994) und typischen PL Phänomenen wie z. B. förderliche Sprachhandlungen (Assertionen, Argumente, Erklärungen, Ko-Konstruktionen), Organisation der Zusammenarbeit sowie der Bearbeitung der Aufgaben, metakognitive Regulationshandlungen etc. (vgl. z. B. Asterhan \& Schwarz, 2009; Chi et al., 2001; De Backer et al., 2016; Littleton \& Mercer, 2010; Topping, 2005; Whitebread et al., 2009) unter Berücksichtigung der Trainings- und Rollenstruktur der BiPeer-Intervention sowie bisheriger Empirie zur Nutzung bilingualer Praktiken beim PL (vgl. z. B. Hopewell, 2011; Klingner \& Vaughn, 2000; Rehbein, 2011; Swain \& Lapkin, 2000) orientiert. Die trainingsbezogenen Funktionen sind nicht hinsichtlich des Lerninhalts Lese- und Rechenstrategien spezifiziert, sodass die Kategorien auf beide Lerninhalte angewandt werden können.

Bei der deduktiven Kategorienerstellung wurden zunächst die übergeordneten Hauptkategorien „On-Task“ und „Off-Task“ als typische Strukturierungsdimensionen der empirischen Unterrichtsforschung abgeleitet sowie die Hauptkategorie „Nicht Kategorisierbar“ zum Umgang mit nicht interpretierbaren Turns erzeugt. Da eine Ausdifferenzierung der Kategorien „Off-Task“ und „Nicht Kategorisierbar" keinen Mehrwert für das Erkenntnisinteresse dieser Arbeit liefert, wurde lediglich die Hauptkategorie „On-Task“ mit Unterkategorien versehen. Hierbei wurden zunächst die Unterkategorien „Aufgabenbearbeitung“, „Task-Management“, „Metakognition und (Selbst-)Regulation“ sowie eine Restkategorie „On-Task Sonstige“ gebildet. Die Unterkategorie „Aufgabenbearbeitung" wiederum wurde angesichts der Bedeutung eines förderlichen (bilingualen) Dialogs für die Lernwirksamkeit von PL nochmal weiter ausdifferenziert, sodass die weiteren Subkategorien „Assertion“, „Argument“, „Erklärung“ und „Ko-Konstruktion“ gebildet wurden. Auf weitere un- 
tergeordnete Abstufungen der Kategorien „Task-Management“ und „Metakognition und (Selbst-)Regulation" wurde verzichtet, da eine Untergliederung dieser für das vorliegende Erkenntnisinteresse keinen nennenswerten Mehrwert hinsichtlich bilingualer Interaktion beim PL verspricht.

Bei dem ersten Testlauf an einem Teil des Datenmaterials wurde allerdings deutlich, dass die weiteren Subkategorien der Unterkategorie „Aufgabenbearbeitung " für das vorliegende Datenmaterial ungeeignet waren. Die zentrale Ursache hierfür war die relativ geringe Prävalenz von bilingualen Praktiken zur Aufgabenbearbeitung. Dementsprechend wurden alle Unterkategorien der Hauptkategorie „On-Task“ nicht weiter ausdifferenziert. Weiterhin fiel bei diesem ersten Testlauf ebenfalls auf, dass eine isolierte, lediglich auf den Turn selbst fokussierte Kategorisierung von verschiedenen Fällen der „On-Task“Unterkategorien nur selten möglich war, da für eine Bestimmung des konkreten Falles in der Regel die vorangegangene und nachfolgende Interaktion berücksichtigt werden muss. Diese Notwendigkeit für eine adäquate Zuordnung der jeweiligen Turns zu den entsprechenden Kategorien führt eine interaktionsanalytische Komponente in die Qualitative Inhaltsanalyse ein, die zu einer Steigerung der Komplexität und des Aufwands der Kodierung führt. Eine solche feinkörnige, detaillierte Analyse wird für einzelne Formen einer Unterkategorie mit den entsprechenden Ressourcen durchaus in der PL-Forschung umgesetzt, allerdings werden hierbei nur einzelne Phänomene wie z. B. ,metakognitive Regulation“ (vgl. z. B. de Backer et al., 2016) oder „Erklärungen“ (vgl. z. B. Adl-Amini, 2018; Asterhan \& Schwarz, 2009) fokussiert. Ein weiteres mögliches Vorgehen für tiefschürfende, ausdifferenzierte Kategorisierungen stellt das Kodieren vorher selektierter, als bedeutsam für die vorliegende Fragestellung eingeschätzter, einzelner Turns oder Interaktionen dar (vgl. z. B. Chi et al., 2001).

Aufgrund des explorativen Charakters der Studienanlage liegt das vorliegende Erkenntnissinteresse allerdings primär in der Bandbreite und Prävalenz auftretender Funktionen bilingualer Praktiken PL von bilingualen SuS der dritten und vierten Klasse im Submersionskontext zu Lese- oder Rechenstrategien. Zudem würde der Aufwand einer detaillierten Analyse aller auftretenden Funktionen bilingualer Praktiken hinsichtlich der Aufgabenbearbeitung, des Task-Managements und der Metakognition sowie (Selbst-)Regulation als eine von insgesamt fünf Fragestellungen den Rahmen eines Dissertationsprojektes deutlich sprengen. Dementsprechend wird von einer solchen feinkörnig ausdifferenzierten Konzeption der Kategorien sowie Zuordnung der bilingualen Praktiken zu diesen abgesehen. Stattdessen werden die den jeweiligen Unterkategorien der Hauptkategorie „On-Task“ entsprechenden Turns diesen zugeordnet und anschließend bezüglich hierbei häufig auftretender oder auffälliger spezifischer Funktionen näher betrachtet. Dementsprechend besteht das für die Untersuchung verwendete Kategoriensystem aus den drei Hauptkategorien „On-Task“, „Off-Task“ und „Nicht Kategorisierbar“, wobei die erstgenannte 
Hauptkategorie in die eher oberflächlich konzeptionierten Unterkategorien „Aufgabenbearbeitung“, „Task-Management“, „Metakognition und (Selbst)Regulation“ sowie „Sonstige“ untergliedert wird. Mithilfe dieser Kategorien wird ,eine erschöpfende Erfassung aller Vorkommen eines Phänomens" (Deppermann, 2008, S. 37) angestrebt. Die Haupt- und Unterkategorien werden im Folgenden näher ausgeführt.

\subsubsection{Kategoriensystem zur Kodierung der Funktionen bilingualer Praktiken}

„On-Task“ wird in der empirischen Bildungsforschung vornehmlich umschrieben als ,actively engaged in learning” (Carroll, 1963, S. 723) oder ,participation in relevant learning activities" (Slavin, 1994, S. 149). Dementsprechend werden bilinguale Praktiken, die als aktive Auseinandersetzung mit relevanten Lernaktivitäten oder als Partizipation an Lernprozessen interpretiert werden können, der Hauptkategorie „On-Task“ zugeordnet. Im Gegensatz hierzu entsprechen Turns mit bilingualen Praktiken, die sich nicht auf relevante Lernaktivitäten beziehen und somit keine aktive Auseinandersetzung mit oder Partizipation an Lernprozessen aufweisen, der Hauptkategorie „Off-Task“. Hierzu gehören bilinguale Praktiken, die z. B. trainings- und aufgabenirrelevante Themen wie Weltgeschehen, Freizeit, Familie, Mitschüler, Lehrer etc. betreffen und mit denen allgemein Unfug wie z. B. das verbale Ärgern des Dyadenpartners oder der Übungsleitung betrieben wird.

Die Hauptkategorie „Nicht Kategorisierbar“ dient der Exklusion von Turns mit bilingualen Praktiken, die aufgrund fehlender semantischer Klarheit nicht hinsichtlich ihrer konkreten Funktion interpretiert werden können. Hierzu gehören vor allem unvollständige Sätze, unverständliche Äußerungen sowie Ambiguitäten. Bilinguale Praktiken, die nicht eindeutig den Kategorien „On-“ oder „Off-Task“ zugeordnet werden können, fallen ebenfalls unter diese Hauptkategorie.

Unterkategorien werden, wie oben bereits begründet, nur für die Hauptkategorie „On-Task“ definiert. Diese wird in die Unterkategorien „Aufgabenbearbeitung“, „Task-Management“, „Metakognition und (Selbst-)Regulation“ und „Sonstige“" unterteilt. Die Unterkategorie „Aufgabenbearbeitung“ erfasst bilinguale Praktiken, die die inhaltliche Bearbeitung der Lerninhalte und Aufgaben betreffen. Dementsprechend fallen unter diese Kategorie alle bilingualen Praktiken, die sich auf trainings- und aufgabenrelevante Themen und Handlungen beziehen. Hierzu gehören unter anderem die inhaltliche Aufgabenbearbeitung, Strategieanwendungen, interaktionsförderliche Sprachhandlungen wie Assertionen, Erläuterungen, Erklärungen, Ko-Konstruktionen und Aushandlungsprozesse. Hinsichtlich der Strukturierung großer Teile des Trainings in Rollen dürfte diese Kategorie vornehmlich beim Handeln in der Spie- 
lerrolle auftreten, da dieser primär für die Bearbeitung der Aufgaben und Anwendung der Strategien verantwortlich ist, während die Trainerrolle sich stärker durch ihre organisatorische und regulierende Funktion auszeichnet.

Die Unterkategorie „Task-Management“ umfasst alle bilingualen Praktiken, die die Organisation der inhaltlichen Bearbeitung der Lerninhalte, Aufgaben und der Zusammenarbeit betreffen. Hierzu gehören unter anderem die Zuteilung von Rollen und Aufgaben, die Nennung von Arbeitsschritten und -phasen, das Vorlesen von Bearbeitungsschritten der Promptkarten, die Beschaffung von benötigtem Material und ähnliche Sprachhandlungen zur Organisation des PL. Zudem werden Prozesse der Planung der Aufgabenbearbeitung und Zusammenarbeit ebenfalls unter dieser Kategorie statt als metakognitive Planungsprozesse kategorisiert, da die PL-Struktur sowie die Aufgabenbearbeitung in der Lese- und Rechenintervention relativ strikt nach zu absolvierenden Interaktionsmustern respektive Bearbeitungsschritten vorgegeben sind, sodass genuine, originäre Planungsideen nicht benötigt werden und somit auch eher nicht zu erwarten sind.

Die Unterkategorie „Metakognition und (Selbst-)Regulation“ erfasst alle bilingualen Praktiken, die sowohl metakognitives Wissen als auch emotionale, motivationale sowie metakognitive (Selbst-)Regulationen in Form von Monitoring, Kontrolle oder Bewertung betreffen. Dementsprechend können hierunter auch bilinguale Praktiken fallen, die auf den Lerninhalt, die Aufgaben oder die Zusammenarbeit bezogen sind, aber primär regulatorische Funktionen des Monitorings, der Kontrolle und der Bewertung hinsichtlich Emotion, Motivation oder Metakognition (und dementsprechend auch metakognitiven Wissens) bezüglich sich selbst, des Lernpartners oder der Dyade adressieren. Hinsichtlich metakognitiver (Selbst-)Regulation erfasst diese Kategorie unter anderem Aufmerksamkeitssteuerung, Hilfeverhalten wie Fragen stellen, Tipps geben oder auf alternative Lösungswege und inhaltliche oder organisatorische Fehler hinweisen, das Wechseln zu anderen Lösungsansätzen, Selbstkorrekturen, die Bewertung von Ergebnissen, Strategien und Prozessen etc. Bezüglich emotionaler und motivationaler (Selbst-)Regulation entsprechen dieser Kategorie bilinguale Praktiken, die emotionale und motivationale Zustände artikulieren oder konstatieren.

Die Kategorie „On-Task Sonstige“ fungiert als Restkategorie, der alle bilingualen Praktiken zugeordnet werden können, die sich auf das PL beziehen, aber keiner der oben angeführten „On-Task“-Unterkategorien zugeordnet werden können. Weiterhin werden hiermit ambige, nicht eindeutig zu interpretierende Turns bilingualer Praktiken kategorisiert, die aufgrund fehlender Spezifizierung theoretisch mehreren „On-Task“-Unterkategorien zugeordnet werden können. Zudem werden Korrekturen von linguistischen Fehlern von türkischsprachigen Produktionen unter diese Kategorie statt der Unterkategorie „Metakognition und (Selbst-)Regulation“ zugeordnet, da diese Korrekturen sich nicht auf das PL und/oder die zu vermittelnden Inhalte beziehen. 
Zur Überprüfung der Reliabilität des Kategoriensystems wird die Interrater-Reliabilität unter Verwendung von Cohens Kappa für die Kategorien ermittelt. Der Autor der vorliegenden Studie hat hierfür das komplette Datenmaterial und eine studentische Praktikantin eine Zufallsauswahl von ca. 33\% ebenfalls kodiert. Eine Zufallsauswahl von einem Drittel der in der Stichprobe von F5 vorhandenen Dyaden könnte zu Verzerrungen führen, da nicht alle Tandems vergleichbare Prävalenzen bilingualer Praktiken vorweisen und somit unter Umständen weniger oder mehr als 33\% des Datenmaterials kodiert werden könnten. Dementsprechend gilt es die zu kodierende Menge für die Interrater-Reliabilität an der Prävalenz der bilingualen Praktiken zu orientieren, sodass aus allen identifizierten bilingualen Praktiken der Angebotsgruppen LGT/D und RGT/D eine Zufallsstichprobe von einem Drittel des Datenmaterials zur Bestimmung der Interrater-Reliabilität gezogen wird. Hierfür werden sukzessive zufällig Tandems aus den Gruppen LGT/D sowie RGT/D gezogen und deren Prävalenz der bilingualen Praktiken erfasst. Dieses Vorgehen wird solange vollzogen, bis die Summe der bilingualen Praktiken der zufällig ausgewählten Tandems 33\% des kompletten Datenmaterials erreicht.

Innerhalb dieses Verfahrens wurden insgesamt zehn verschiedene Dyaden in die Zufallsstichprobe aufgenommen und insgesamt 181 Turns mit bilingualen Praktiken durch die studentische Praktikantin kodiert, was ca. 38\% des Datenmaterials entspricht. Cohens Kappa als Maß für die Interrater-Reliabilität wurde unter Verwendung der MAXQDA-Funktion „Intercoder-Übereinstimmung" sowie Excel berechnet und liegt bei $\mathrm{K}=.79$, was einer starken Übereinstimmung entspricht. Nach der Berechnung der Interrater-Reliabilität mit Cohens Kappa wurden die Fälle mit fehlender Übereinstimmung der beiden Rater besprochen und sich in all diesen Fällen auf eine Kodierung geeinigt. Diese Änderungen wurden dann in den Hauptdatensatz übernommen.

Das soeben dargelegte Kategoriensystem kann im Anhang unter „Anhang 14 Kategoriensystem der Funktionen bilingualer Praktiken beim Peer-Learning“ samt Definitionen, Ankerbeispielen und Kodierhinweisen eingesehen werden. Das Kodiervorgehen wird im nächsten Abschnitt näher erläutert, da zum einen ein Turn mehrere Funktionen haben kann und zum anderen die deutschsprachigen Anteile in gemischtsprachlichen türkisch-deutschsprachigen Äußerungen zur Semantik und Pragmatik des Turns beitragen können, sodass konkrete Handlungsanweisungen im Umgang mit diesen Besonderheiten bei der Kategorisierung zu formulieren sind. Zudem wird hierbei ebenfalls die beim Kodieren zu berücksichtigende Abhängigkeit einzelner Turns von der sie umgebenden Interaktionssequenz kurz konkretisiert. 


\subsubsection{Kodiervorgehen}

Aufgrund der potentiellen Abhängigkeit der Funktion eines Turns von der Interaktionssequenz dürfte es in einigen Fällen notwendig sein, die vorangegangene und unter Umständen die sich anschließende Interaktion zu berücksichtigen. Hierbei soll allerdings keine feinkörnige Analyse des Interaktionsgeschehens vorgenommen werden, sondern sich lediglich ein Eindruck über den unmittelbaren Interaktionskontext verschafft werden, um die Turns hinsichtlich ihrer Funktion bewerten zu können. Hierbei gilt es ebenfalls zu berücksichtigen, dass die Aufgabenstruktur des Trainings und die rollenbezogenen Handlungen in Abhängigkeit von den jeweiligen Zwischenschritten der Strategieanwendung bestimmte darauf folgende Handlungen implizieren oder zumindest nahe legen. So führt z. B. der Impuls „Helfen dir die Wortteile?“ bei der Lesestrategie „Wörter klären“ mit hoher Wahrscheinlichkeit zu einer metakognitiven Reflexion, ob die Wortbestandteile sich als hilfreich für den Spieler beim Klären des Wortes erweisen. Im Gegensatz hierzu impliziert der darauf folgende Impuls „Soll ich dir einen Tipp geben?“ eher eine Antwort hinsichtlich der weiteren gemeinsamen Aufgabenbearbeitung, da diese die konkrete Form der unmittelbar folgenden Zusammenarbeit betrifft. Eine Antwort hierauf würde dementsprechend eher als Task-Management kategorisiert werden. Das Gleiche gilt auch für das Teamrechnen bei der Rechenintervention. Während die Aufforderung „Löse die Aufgabe und denke dabei laut“" eher eine Bearbeitung der mathematischen Aufgabe initiiert, legt der Schritt „Bist du dir sicher mit der Lösung und warum?" eher eine metakognitive Reflexion nahe. Dementsprechend gilt es die zu kategorisierenden Turns in manchen Fällen nicht nur hinsichtlich ihrer Rolle innerhalb der übergeordneten Interaktionssequenz einzuordnen, sondern auch in Abhängigkeit von dem (durch die Intervention vorgegebenen) vorausgegangenen Impuls einzuschätzen.

Da innerhalb eines Turns mehrere der im Kategoriensystem angeführten Funktionen konstituiert werden können, sind Mehrfachkodierungen eines Turns möglich. Dies ist ein typisches Phänomen von Interaktionen, da im Prozess des „Turn-Takings“ Kontinuität und Progress hergestellt werden. So initiiert z. B. eine Referenz in Form einer negativen Bewertung eines Ergebnisses einen neuen Impuls für das Gespräch, woraufhin die Bewertung zunächst kommentiert und auf Basis dieser weitere (Sprach-)Handlungen wie z. B. eine wiederholte Aufgabenbearbeitung oder eine Modifikation des Ergebnisses im selben Turn folgen können, die den Gesprächstopik aktualisieren und somit den Fortschritt des Gesprächs sichern. Angesichts des Auftretens von Mehrfachkodierungen wäre eine trennscharfe Markierung derjenigen Äußerungsanteile eines Turns, die sich auf die zu kodierende inhaltliche Dimension beziehen, das präziseste Vorgehen. Allerdings würde dies zu aufwendigen Analysen der Syntax und Semantik der Äußerung führen, was wiederrum einen gewissen 
Interpretationsspielraum erzeugt, da zu klären wäre, welche Äußerungsbestandteile zur Semantik der zu kodierenden Dimension beitragen und welche nicht. Dies wäre ein mühseliges Unterfangen, insbesondere angesichts der Agglutination des Türkischen, die eine semantische Analyse der einzelnen Morpheme verlangen würde. Eine solche feinkörnige Analyse würde zudem hinsichtlich des Erkenntnisinteresses der Funktionen bilingualer Praktiken keinen zusätzlichen Nutzen liefern. Dementsprechend werden nicht die der Kategorie entsprechenden Bestandteile des Turns exklusiv kodiert, sondern der ganze Turn. Mehrfachkodierungen werden allerdings nur vorgenommen, wenn innerhalb eines Turns mindestens zwei verschiedene Kategorien durch bilinguale Praktiken bedient werden. Enthält ein Turn z. B. zwei Funktionen, die beide als „Task-Management“ $\mathrm{zu}$ interpretieren sind, wird dieser Code nur einmal vergeben.

Bei der Kodierung bilingualer Praktiken mit Code-Switching müssen in vereinzelten Fällen womöglich auch die auftretenden deutschsprachigen Anteile berücksichtigt werden. Die Funktion eines Turns kann sich auch erst auf satzsemantischer Ebene ergeben, sodass sich diese eventuell nur unter Einbezug der deutschsprachigen Anteile bestimmen lässt. Wenn allerdings bei bilingualen Praktiken mit Code-Switching die türkischsprachigen Anteile des Turns eine andere Funktion als die deutschsprachigen betreffen, wird nur die Funktion kodiert, die durch den türkischsprachigen Äußerungsanteil konstituiert wurde. 


\section{Ergebnisse}

Im Folgenden werden die Ergebnisse der Fragestellungen F1 bis F5 vorgestellt. Die Präsentation der Ergebnisse erfolgt sukzessiv und weitestgehend unabhängig voneinander. Lediglich die Unterfragestellungen werden, sofern dies notwendig erscheint, teilweise in Beziehung zueinander gesetzt. Die Verbindung der Ergebnisse untereinander sowie die Datenintegration der quantitativen und qualitativen Ergebnisse erfolgt erst in Abschnitt 9 bei der Diskussion der Ergebnisse.

\subsection{Prüfung der Abhängigkeit der Prävalenz bilingualer Praktiken von der Dyade}

Fragestellung F1 prüft die Existenz einer potentiellen Abhängigkeit der Prävalenz bilingualer Praktiken von der Dyade. Bevor die Abhängigkeit der Prävalenz bilingualer Praktiken von der Dyade geprüft wird, sollen allerdings zunächst die Werte der Prävalenz bilingualer Praktiken aggregiert über die Gruppen LGT/D und RGT/D auf Individualebene visuell unter Verwendung eines Histogramms inspiziert werden, um einen ersten Eindruck über die Quantität und Verteilung der Prävalenz bilingualer Praktiken zu erhalten (siehe Abbildung 12). Wie in Abschnitt 7.3.4 bereits erläutert, handelt es sich bei den vorliegenden Werten um Verhältniswerte von Turns mit bilingualen Praktiken zu rein deutschsprachigen Turns, sodass die Werte nicht als absolute, sondern als Prozentwerte zu interpretieren sind. Dementsprechend sind im Folgenden mit „Turns" nur diejenigen Sprecherbeiträge gemeint, die zur Summe aller Turns mit bilingualen Praktiken oder rein deutschsprachigen Turns gehören.

Das in Abbildung 12 dargestellte Histogramm der Prävalenz bilingualer Praktiken der aggregierten Gruppen LGT/D und RGT/D deutet auf eine rechtsschiefe, steilgipflige Verteilung hin, die keiner Normalverteilung entspricht. Am häufigsten scheinen die Prävalenzen bilingualer Praktiken der Probanden unter fünf Prozent zu liegen, sodass für die meisten Dyaden der Großteil der Interaktion monolingual auf Deutsch erfolgt. Deutlich seltener treten Prävalenzen bilingualer Praktiken über fünf Prozent auf, wobei der Großteil der Probanden kaum den Wert von 15\% übersteigt. Allerdings gibt es vereinzelte Probanden, deren Prävalenz bilingualer Praktiken mehr als ein Drittel der gesamten Turns ausmachen. Zwei Probanden interagieren häufiger bilingual als monolingual innerhalb ihrer Dyade, die angesichts der Verteilung wohl als Ausreißer zu bezeichnen sind. 
Abbildung 12

Histogramm der Prävalenz bilingualer Praktiken der aggregierten Angebotsgruppen $L G T / D \& R G T / D$ auf Individualebene

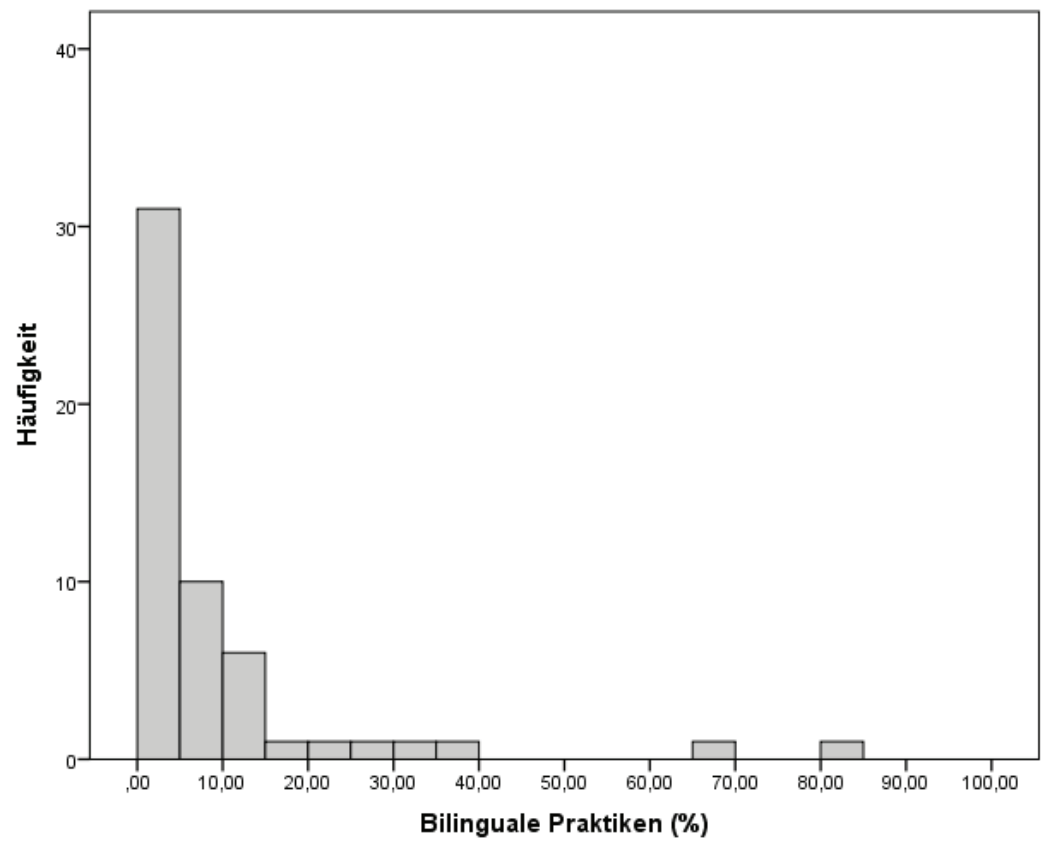

In Tabelle 4 sind die zentralen deskriptiven Werte der Prävalenz bilingualer Praktiken der Gruppen LGT/D und RGT/D aggregiert dargestellt:

Tabelle 4

Deskriptive Werte der Prävalenz bilingualer Praktiken der aggregierten Angebotsgruppen LGT/D \& RGT/D auf Individualebene

\begin{tabular}{lcccccccc}
\hline & $\mathrm{n}$ & Min & Max & MW & S & Median & Schiefe & Kurtosis \\
\hline $\begin{array}{l}\text { LGT/D \& } \\
\text { RGT/D }\end{array}$ & 54 & 0,00 & 80,30 & 8,99 & 15,37 & 4,33 & 3,34 & 12,15 \\
\hline
\end{tabular}

Die deskriptiven Werte erhärten die ersten Einschätzungen zur Verteilung der Prävalenz bilingualer Praktiken auf Basis der visuellen Inspektion des Histogramms. Im Mittel produzierten die Probanden der Gruppen mit bilingualem Sprachangebot knapp neun Prozent aller Gesprächsbeiträge unter Verwendung bilingualer Praktiken. Sowohl die Range als auch die Standardabweichung 
weisen auf eine deutliche Heterogenität der Probanden hinsichtlich der Produktion bilingualer Praktiken hin. Unter Berücksichtigung des Medians von 4,33\% scheint die Standardabweichung durch sehr hohe Prävalenzen bilingualer Praktiken einzelner Probanden zu entstehen. Dementsprechend sollten sich auch einige Ausreißer in der Verteilung finden. Diese Interpretation wird auch durch die rechtschiefe Verteilung und positive Kurtosis gestützt.

Eine Prüfung auf Normalverteilung unter Verwendung des Shapiro-WilkTests, welcher nach Yap und Sim (2011) für asymmetrische Verteilungen anderen Tests zur Prüfung auf Normalverteilung vorzuziehen ist, bestätigt, dass die Prävalenz bilingualer Praktiken in der vorliegenden Stichprobe nicht normalverteilt ist ( $W=.569, d f=54, p<.001)$. Da es für ununterscheidbare Dyaden keine etablierten non-parametrischen Verfahren ohne eine arbiträre Zuordnung der Probanden zur Prüfung der Unabhängigkeit der Prävalenz bilingualer Praktiken von der Dyade gibt (vgl. Kenny, Kashy \& Cook, 2006, S. 42f), werden die vorliegenden Daten logarithmiert.

Die Transformation der Werte der bilingualen Praktiken erfolgt mit dem dekadischen Logarithmus „ $\log _{10}(\mathrm{x}+1)^{\text {“ }}$, da im vorliegenden Datensatz auch Nullwerte vorhanden sind. Nach der Transformation liegen die folgenden deskriptiven Werte vor (siehe Tabelle 5), die sich wie in Abbildung 13 verteilen:

Tabelle 5

Logtransformierte deskriptive Werte der Prävalenz bilingualer Praktiken der aggregierten Angebotsgruppen LGT/D \& RGT/D auf Individualebene

\begin{tabular}{lcccccccc}
\hline & $\mathrm{n}$ & Min & Max & MW & S & Median & Schiefe & Kurtosis \\
\hline LGT/D \& & 54 & 0,00 & 1,91 & 0,70 & 0,49 & 0,73 & 0,41 & 0,30 \\
RGT/D & & & & & & & & \\
\hline
\end{tabular}

Die Verteilung der Prävalenz der logtransformierten bilingualen Praktiken erscheint angesichts des Histogramms nicht normalverteilt zu sein. Eine Prüfung auf Normalverteilung unter Verwendung des Shapiro-Wilk-Tests bestätigt allerdings, dass die Prävalenz bilingualer Praktiken nach der Logtransformation normalverteilt ist ( $W=.958, d f=54, p=.059)$, auch wenn der $\mathrm{p}$-Wert sehr nah am kritischen Wert liegt. Durch die Transformation sind auch keine Ausreißer mehr in der Stichprobe vorhanden, sodass die Daten aller Probanden der Gruppen LGT/D und RGT/D in der folgenden Prüfung einer potentiellen Abhängigkeit der Prävalenz bilingualer Praktiken von der Dyade berücksichtigt werden können. 


\section{Abbildung 13}

Histogramm der logtransformierten Werte der Prävalenz bilingualer Praktiken der aggregierten Angebotsgruppen $L G T / D \& R G T / D$ auf Individualebene

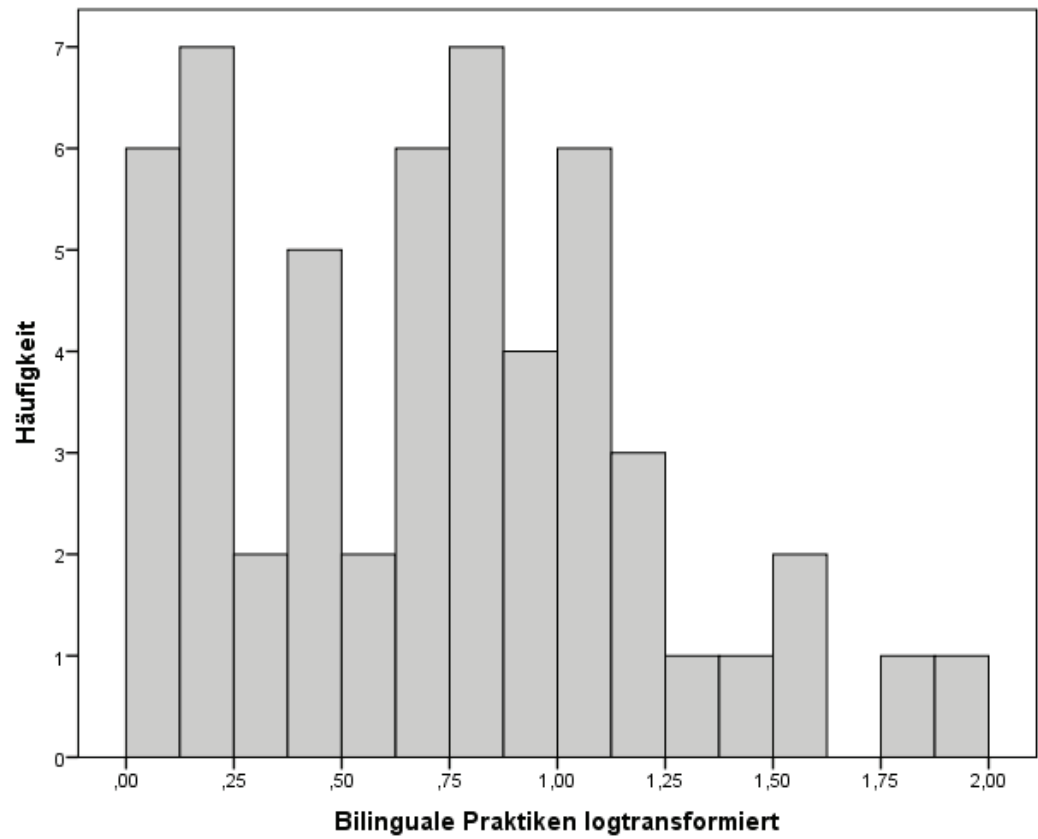

Um einen ersten Eindruck bezüglich der potentiellen dyadischen Abhängigkeit der Prävalenz bilingualer Praktiken zu erhalten, empfiehlt sich zunächst eine visuelle Inspektion des Zusammenhangs der Prävalenz bilingualer Praktiken von den Dyadenpartnern anhand eines Streudiagramms (siehe Abbildung 14). Die Symmetrie der Streuung der Datenpunkte entsteht durch die in Abschnitt 7.4.1.3 beschriebene paarweise Datenstruktur, bei der die Werte der ununterscheidbaren Dyadenpartner „flip-flopped“ (Kenny, Kashy \& Cook, 2006, S. 38) in zweifacher Ausführung in verschiedener Reihenfolge repräsentiert werden. Das Streudiagramm weist einen positiven linearen Trend auf, der eine positive Korrelation der Prävalenz bilingualer Praktiken der Dyadenpartner vermuten lässt. Zudem lässt sich erkennen, dass in manchen Dyaden nur ein Peer Turns mit bilingualen Praktiken produziert, während der dazugehörige Dyadenpartner nur monolingual in Deutsch kommuniziert.

Die paarweise Korrelation unter Verwendung des Korrelationskoeffizienten nach Pearson ist $r_{p}=.690$. Der resultierende $z$-Wert beträgt $z=3,5905$, was einem Signifikanzwert von $p$ (zweiseitig) $<0.001$ entspricht. Dies bedeutet, dass die Prävalenz bilingualer Praktiken abhängig vom Dyadenpartner ist. 
Dementsprechend gilt es die Prävalenz bilingualer Praktiken als dyadische Variable zu analysieren.

Abbildung 14

Streudiagramm der logtransformierten deskriptiven Werte der Prävalenz bilingualer Praktiken der Probanden der aggregierten Angebotsgruppen LGT/D $\& R G T / D$

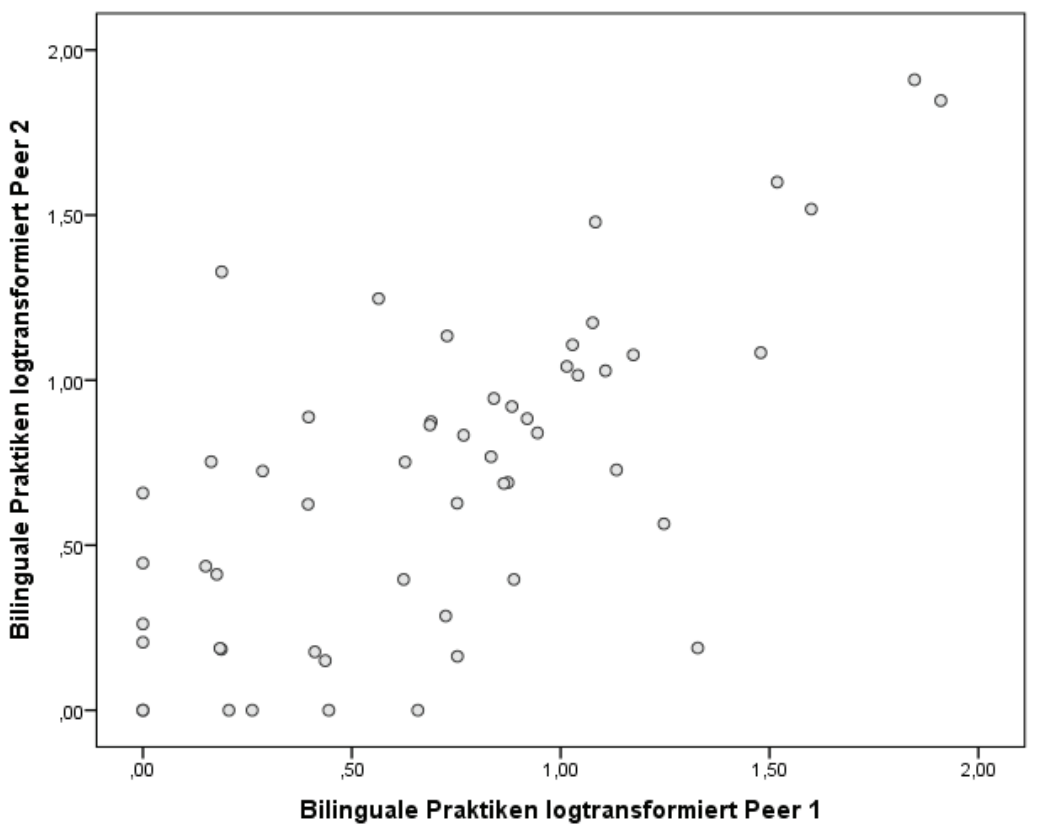




\subsection{Prävalenz bilingualer Praktiken mit und ohne explizitem bilingualen Interaktionsangebot beim Peer-Learning}

Im Folgenden werden die Ergebnisse zur Prävalenz bilingualer Praktiken mit und ohne explizitem, bilingualem Sprachangebot beim PL präsentiert. Zunächst werden die Ergebnisse der Prävalenz bilingualer Praktiken der aggregierten Angebots- mit den Nicht-Angebotsgruppen (F2a) hinsichtlich signifikanter Unterschiede betrachtet. Anschließend werden die Ergebnisse des Vergleichs der Prävalenz bilingualer Praktiken zwischen den unterschiedlichen Lerninhalten Lese- und Rechenstrategien (F2b) sowie zwischen den verschiedenen Phasen innerhalb des Lese- und Rechentrainings (F2c) präsentiert, wobei hinsichtlich dieser beiden letztgenannten Fragestellungen lediglich die Gruppen LGT/D und RGT/D untersucht werden. Vor den Analysen der Fragestellung F2a-F2c werden allerdings die sprachlichen Ausgangsbedingungen der vier Gruppen LGD, RGD, LGT/D und RGT/D dargestellt, um deren Vergleichbarkeit zu prüfen.

Die deskriptiven Werte der Sprachkompetenz, sprachbezogener Einstellungen sowie der Sprachpraxis auf Individualebene scheinen zwischen den Gruppen vergleichbar zu sein (siehe Tabelle 6). In jeder Gruppe herrscht eine Sprachasymmetrie zu Gunsten des Deutschen vor, da im Mittel ungefähr ein doppelt so hoher Score beim deutschsprachigen als beim türkischsprachigen produktiven Wortschatz erzielt wurde. Berücksichtigt man die Streuung sowie die Minima des Wortschatzes im Türkischen in allen Gruppen, so fällt auf, dass die Probanden zum Teil über äußerst geringe produktive Fähigkeiten im Türkischen verfügen. Zudem treten keine Deckeneffekte weder im deutsch- noch im türkischsprachigen produktiven Wortschatz auf, obwohl auf die Version für die jüngste Testpopulation des WWT 6-10 zurückgegriffen wurde. Die Einstellungen zum Türkischen und die Sprachpraxis weisen eine gemischte Befundlage auf. Während in allen Gruppen die Probanden im Mittel eher Freude am Türkischen haben und sie mit türkischsprachigen Freunden in den Schulpausen durchaus auch auf Türkisch kommunizieren, stellt das Türkische für die Probanden allerdings eher keine Ressource zum Lernen dar, wird eher nicht in mentalen Denkprozessen beim Lesen und Rechnen und auch eher nicht zur Kommunikation mit türkischsprachigen Freunden im Unterricht verwendet 


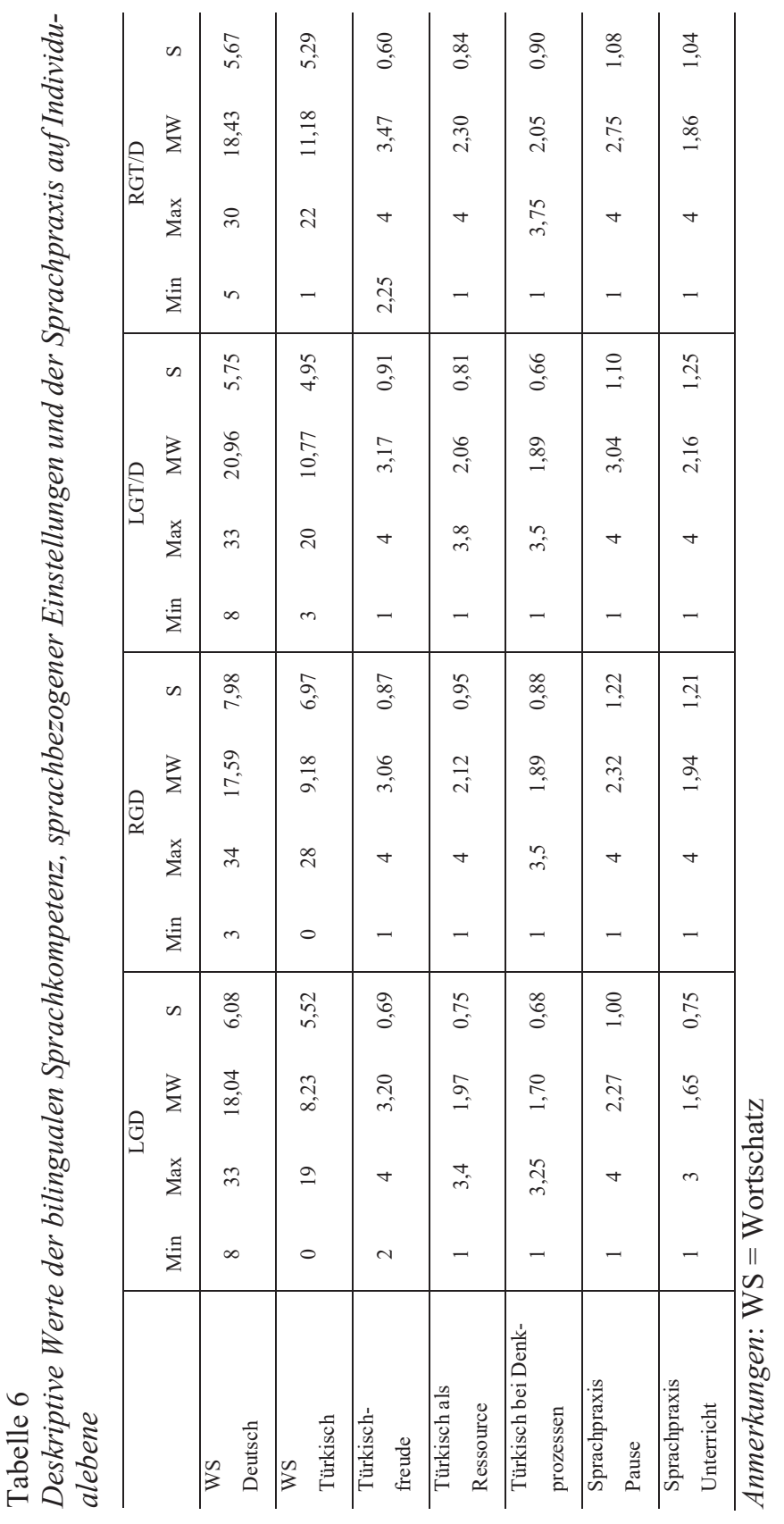


Die Prüfung der Testwerte, Skalen und Items auf Normalverteilung mit dem Shapiro-Wilk Test ergibt, dass mit Ausnahme des produktiven Wortschatzes im Deutschen $(W=.960-.980, d f=25-30, p=.421-.860)$ alle sonstigen Variablen in mindestens einer Gruppe keine Normalverteilung aufweisen. Um die Vergleichbarkeit der Ausgangsbedingungen zu prüfen, werden dementsprechend potentielle Gruppenunterschiede für alle Variablen mit Hilfe des verteilungsfreien Kruskal-Wallis Tests geprüft. Mit Ausnahme des Items „Sprachpraxis Pause" unterscheiden sich alle Tests, Skalen und Variablen nicht signifikant zwischen den Gruppen. Anschließend durchgeführte Dunn-BonferroniTests zeigen, dass sich das Item „Sprachpraxis Pause“ zwischen den Gruppen LGD $($ Median $=2)$ und LGT/D $($ Median $=3)$ signifikant unterscheidet $(z=$ $\left.2,529, p_{\text {angepasst }}(z w e i s e i t i g)=.035\right)$. Dementsprechend lässt sich schließen, dass - mit Ausnahme des Items „Sprachpraxis Pause“ - die vier Gruppen sich in ihren sprachlichen Ausgangsbedingungen nicht unterscheiden.

Die deskriptiven Werte der Sprachkompetenz, sprachbezogener Einstellungen sowie der Sprachpraxis auf Dyadenebene weichen hinsichtlich der Mittelwerte kaum von den Werten auf Individualebene ab (siehe Tabelle 7), da die Operationalisierung der Tandemwerte auch über die Mittelwerte der Aggregationen der Werte der Dyadenpartner vorgenommen wurde. Dementsprechend wird trotz der Fokussierung der Dyadenebene für die Fragestellungen F2a-F2c zur Vermeidung von Redundanz auf eine Beschreibung der deskriptiven Werte auf Dyadenebene verzichtet. Auch die inferenzstatische Prüfung potentieller Unterschiede zwischen den Gruppen auf Dyadenebene dürfte dieselben Ergebnisse wie auf Individualebene liefern, da durch die Reduktion der Stichprobengrößen um die Hälfte die Teststärke deutlich verringert wird, was die Wahrscheinlichkeit der Existenz signifikanter Unterscheide zwischen den Gruppen mindert. 


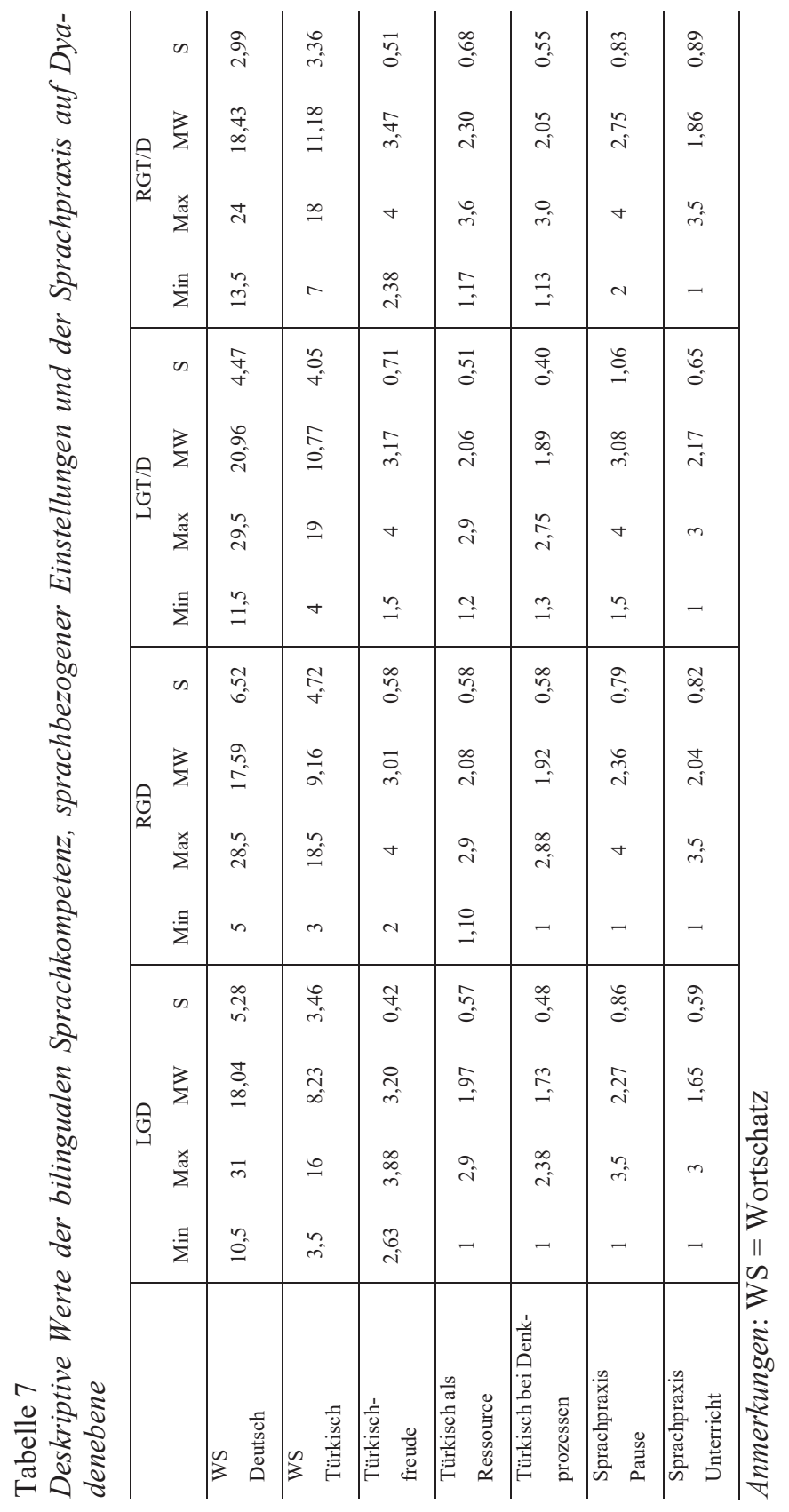




\subsubsection{Vergleich der Prävalenz bilingualer Praktiken zwischen den Angebots- und Nichtangebotsgruppen}

Um die Abhängigkeit der Prävalenz bilingualer Praktiken von der Dyade auch bei non-parametrischen Verfahren zu berücksichtigen, erfolgen die Analysen der Fragestellungen F2a-F2c auf Dyadenebene. Bevor die Angebots- und Nicht-Angebotsgruppen auf signifikante Unterschiede hinsichtlich der Prävalenz bilingualer Praktiken geprüft werden, lohnt sich ein Blick auf die Quantität der Prävalenz bilingualer Praktiken der aggregierten Nicht-Angebotsgruppen LGD und RGD auf Dyadenebene. Zudem werden für die in F2a vorzunehmende Analyse lediglich die Phasen „Zusammenfassen“ und „Vorhersagen“ sowie „Teamrechnen 1" und „Teamrechnen 2" berücksichtigt, sodass die deskriptiven Werte der Gruppen LGT/D und RGT/D für diese Phasen ebenfalls dargestellt werden (siehe Tabelle 8).

Tabelle 8

Deskriptive Werte der Prävalenz bilingualer Praktiken der aggregierten Angebotsgruppen LGT/D und RGT/D sowie der aggregierten Nicht-Angebotsgruppen $L G D$ und RGD auf Dyadenebene

\begin{tabular}{c|cccccccc}
\hline & $\mathrm{n}$ & Min & Max & MW & S & Median & Schiefe & Kurtosis \\
\hline LGD \& RGD & 30 & 0 & 10,55 & 0,68 & 1,99 & 0 & 4,55 & 22,50 \\
\hline LGT/D \& RGT/D & 27 & 0 & 66,00 & 10,48 & 14,36 & 5,79 & 2,67 & 8,36 \\
\hline
\end{tabular}

Der Mittelwert und die Standardabweichung zeigen, dass es auch ohne explizites bilinguales Sprachangebot zur Produktion von bilingualen Praktiken beim PL kommt. Angesichts der Größenordnung der Werte und dem Median kann allerdings für den Großteil der Dyaden der Gruppen LGD und RGD konstatiert werden, dass diese beim PL in einem monolingualen Sprachmodus interagierten. Allerdings haben sich einige Dyaden über diese Beschränkung hinweggesetzt. So verwendet z. B. eine Dyade in fast $11 \%$ aller Gesprächsbeiträge bilinguale Praktiken, was vergleichbar mit dem Mittelwert bilingualer Praktiken der aggregierten Angebotsgruppen LGT/D und RGT/D auf Dyadenebene ist. Die Prävalenz bilingualer Praktiken der Gruppen LGT/D und RGT/D auf Basis der für den Vergleich mit den Gruppen LGD und RGD ausgewählten Phasen „Zusammenfassen“ und „Vorhersagen“ sowie „Teamrechnen 1“ und „Teamrechnen 2" unterscheidet sich kaum von ihrer Prävalenz bilingualer Praktiken auf Basis aller Phasen.

In Anbetracht des Medians, der Schiefe und Kurtosis der bilingualen Praktiken der aggregierten Gruppen LGD und RGD dürfte auch keine Normalver- 
teilung vorliegen, was durch den Shapiro-Wilk-Test auf Normalverteilung bestätigt wird $(W=.383, d f=30, p<.001)$. Selbst eine Logtransformation mit dem dekadischen Logarithmus „, $\log _{10}(\mathrm{x}+1)$ “ führt zu keiner Normalverteilung ( $W=.595, d f=30, p<.001$ ). Dementsprechend wird für die Fragestellung F2a auf den non-parametrischen Mann-Whitney-U-Test zur Prüfung signifikanter Unterschiede der Prävalenz bilingualer Praktiken zwischen den Angebots- und Nicht-Angebotsgruppen auf Dyadenebene zurückgegriffen.

Die Prävalenz bilingualer Praktiken unterscheidet sich unter Verwendung des Mann-Whitney-U-Tests signifikant zwischen den aggregierten Angebots$($ Median $=5,79)$ und Nichtangebotsgruppen (Median = 0; Mann-Whitney-UTest $U=107, z=-4,915, p$ (zweiseitig) <.001). Das bilinguale Sprachangebot führt zu einer signifikant höheren Prävalenz bilingualer Praktiken im Vergleich zu keinem expliziten Angebot bilingualer Praktiken. Die Stärke des Effekts liegt bei $r=0.66$, was einem großen Effekt entspricht.

Die Aggregation der Angebots- und Nichtangebotsgruppen hinsichtlich der ausgewählten Phasen „Zusammenfassen“ und „Vorhersagen“ sowie „Teamrechnen 1" und „Teamrechnen 2" erscheint methodisch legitim, da sich sowohl die Nicht-Angebotsgruppen LGD $($ Median $=0)$ und RGD (Median =0, MannWhitney-U-Test $U=91, z=-, 973, p($ zweiseitig $)=.432)$ als auch die Angebotsgruppen LGT/D (Median $=3,74$ ) und RGT/D (Median $=7,68$, MannWhitney-U-Test $U=67,50, z=-1,142, p$ (zweiseitig) $=.259$ ) nicht signifikant hinsichtlich der Prävalenz bilingualer Praktiken in den für den Vergleich selektierten Phasen unterscheiden.

\subsubsection{Vergleich der Prävalenz bilingualer Praktiken zwischen den Angebotsgruppen LGT/D und RGT/D}

Im Folgenden wird geprüft, ob sich die Prävalenzen bilingualer Praktiken der Angebotsgruppen LGT/D und RGT/D unterscheiden, wobei auf die komplette Datengrundlage unter Berücksichtigung aller Phasen - mit Ausnahme der Phase „Kopfrechnen“ im Rechenstrategietraining aufgrund des fehlenden Interaktionspotentials dieser Phase (siehe Abschnitt 7.4.1.3) - zurückgegriffen wird. Die Prävalenzen bilingualer Praktiken der Angebotsgruppen LGT/D und RGT/D weichen im Mittel deskriptiv deutlich voneinander ab (siehe Tabelle 9). Die Gruppe RGT/D weist im Mittel eine höhere Prävalenz und Standardabweichung bilingualer Praktiken als die Gruppe LGT/D auf. Diese Tendenz spiegelt auch der Median der Gruppe RGT/D wider. 
Tabelle 9

Deskriptive Werte der Prävalenz bilingualer Praktiken der Angebotsgruppen $L G T / D \& R G T / D$ auf Dyadenebene

\begin{tabular}{ccccccccc}
\hline & $\mathrm{n}$ & Min & Max & MW & S & Median & Schiefe & Kurtosis \\
\hline LGT/D & 13 & 0 & 35,27 & 6,62 & 9,50 & 3,92 & 2,63 & 7,78 \\
\hline RGT/D & 14 & 0,40 & 74,86 & 11,39 & 19,12 & 5,96 & 3,22 & 11,08 \\
\hline
\end{tabular}

Den deutlichen deskriptiven Unterschieden zum Trotz lassen sich keine signifikanten Unterschiede hinsichtlich der Prävalenz bilingualer Praktiken zwischen den Gruppen LGT/D (Median $=3,92)$ und RGT/D (Median = 5,96, Mann-Whitney-U-Test $U=72, z=-, 922, p$ (zweiseitig) $=.375$ ) feststellen. Dementsprechend scheint sich die Prävalenz von bilingualen Praktiken beim expliziten Angebot dieser zwischen den verschiedenen Lerninhalten Lese- und Rechenstrategien nicht zu unterscheiden.

\subsubsection{Prävalenz bilingualer Praktiken innerhalb der verschiedenen Phasen der Gruppen LGT/D und RGT/D}

Im Folgenden wird geprüft, ob sich die Prävalenzen bilingualer Praktiken innerhalb der verschiedenen Phasen der Angebotsgruppen LGT/D und RGT/D unterscheiden. Die deskriptiven Werte der Prävalenz bilingualer Praktiken der einzelnen Phasen des Lesestrategie- und Rechenstrategietrainings auf Dyadenebene sind in den Tabellen 10 und 11 dargestellt. Im Lesestrategietraining produzieren die Dyaden im Mittel deskriptiv mehr bilinguale Praktiken in den Phasen, in denen die Lesestrategien „Wörter klären“, „Zusammenfassen“ und „Vorhersagen“ bearbeitet werden, als in den restlichen Phasen. Beim „Tandemlesen", der Beantwortung von Fragen zu den gelesenen Abschnitten (,Frage zum Abschnitt") sowie in den „Zwischenphasen“ und der „Abschlussrunde" treten kaum bilinguale Praktiken innerhalb der Dyaden der Gruppe LGT/D auf. Die Prävalenz bilingualer Praktiken dürfte auch in den einzelnen Phasen keine Normalverteilung aufweisen. Die Werte der Mediane, Schiefe und Kurtosis weisen auf eine fehlende Normalverteilung der Daten hin, was sich mit dem Shapiro-Wilk Test für alle Phasen bestätigen lässt ( $W=.416$ $.818, d f=13, p=.011-\leq .001)$. 
Tabelle 10

Deskriptive Werte der Prävalenz bilingualer Praktiken innerhalb der verschiedenen Phasen der Gruppe LGT/D auf Dyadenebene

\begin{tabular}{lcccccccc}
\hline & $\mathrm{n}$ & Min & Max & MW & S & Median & Schiefe & Kurtosis \\
\hline $\begin{array}{l}\text { Tandem- } \\
\text { lesen }\end{array}$ & 13 & 0 & 2,72 & 0,51 & 0,77 & 0,17 & 2,19 & 5,60 \\
\hline $\begin{array}{l}\text { Wörter } \\
\text { klären }\end{array}$ & 13 & 0 & 9,98 & 1,76 & 2,79 & 0,46 & 2,51 & 6,67 \\
\hline $\begin{array}{l}\text { Zusammen- } \\
\text { fassen }\end{array}$ & 13 & 0 & 11,79 & 1,62 & 3,15 & 0,95 & 3,26 & 11,11 \\
\hline $\begin{array}{l}\text { Vorher- } \\
\text { sagen }\end{array}$ & 13 & 0 & 5,90 & 1,69 & 2,00 & 0,84 & 1,20 & 0,46 \\
\hline $\begin{array}{l}\text { Frage zum } \\
\text { Abschnitt }\end{array}$ & 13 & 0 & 2,49 & 0,25 & 0,68 & 0,00 & 3,45 & 12,17 \\
\hline $\begin{array}{l}\text { Zwischen- } \\
\text { phase }\end{array}$ & 13 & 0 & 2,50 & 0,56 & 0,86 & 0,35 & 1,84 & 2,32 \\
\hline $\begin{array}{l}\text { Abschluss- } \\
\text { runde }\end{array}$ & 13 & 0 & 1,40 & 0,25 & 0,42 & 0,00 & 2,05 & 4,23 \\
\hline
\end{tabular}

Hinsichtlich der Phasen des Rechenstrategietrainings ergibt sich ein ähnliches Bild. Im „Teamrechnen 1“, „Teamrechnen 2“ sowie der „Rechenkonferenz“, in denen sowohl die Bearbeitung von Rechenaufgaben als auch der Diskurs über Rechenwege und -ergebnisse im Fokus stehen, treten deskriptiv mehr bilinguale Praktiken auf als in den Zwischenphasen und der Abschlussrunde. Die Prävalenz bilingualer Praktiken dürfte auch in den einzelnen Phasen keine Normalverteilung aufweisen. Die Werte der Mediane, Schiefe und Kurtosis weisen auf eine fehlende Normalverteilung der Daten hin, was sich mit dem Shapiro-Wilk Test für alle Phasen bestätigen lässt $(W=.391-.865, d f=14, p=$ $.035-<.001$ ). 
Tabelle 11

Deskriptive Werte der Prävalenz bilingualer Praktiken innerhalb der verschiedenen Phasen der Gruppe RGT/D auf Dyadenebene

\begin{tabular}{lcccccccc}
\hline & $\mathrm{n}$ & Min & Max & MW & SD & Median & Schiefe & Kurtosis \\
\hline $\begin{array}{l}\text { Team- } \\
\text { rechnen 1 }\end{array}$ & 14 & 0 & 10,49 & 2,93 & 3,04 & 1,94 & 1,36 & 1,74 \\
\hline $\begin{array}{l}\text { Team- } \\
\text { rechnen 2 }\end{array}$ & 14 & 0 & 23,64 & 3,72 & 6,53 & 1,28 & 2,63 & 7,10 \\
\hline $\begin{array}{l}\text { Rechen- } \\
\text { konferenz }\end{array}$ & 14 & 0 & 33,02 & 3,06 & 8,70 & 0,42 & 3,62 & 13,35 \\
\hline $\begin{array}{l}\text { Zwischen- } \\
\text { phase }\end{array}$ & 14 & 0 & 8,82 & 1,12 & 2,37 & 0,23 & 3,05 & 9,85 \\
\hline $\begin{array}{l}\text { Abschluss- } \\
\text { runde }\end{array}$ & 14 & 0 & 1,70 & 0,48 & 0,54 & 0,36 & 1,05 & 0,34 \\
\hline
\end{tabular}

Mithilfe des Friedman-Tests sowie Post-Hoc-Analysen unter Verwendung des Dunn-Bonferroni-Tests lassen sich die nicht normalverteilten Prävalenzen bilingualer Praktiken der verschiedenen Phasen untereinander auf signifikante Unterschiede prüfen. Die Dunn-Bonferroni-Tests identifizieren paarweise signifikante Unterschiede hinsichtlich der Phase „Frage zum Abschnitt“ (Median $=0,00$ ) und den Phasen „Wörter klären“ (Median $=0,46, z=3,404$, $p_{\text {ange- }}$ ${ }_{\text {passt }}($ zweiseitig $\left.)=0,014\right)$, ,Zusammenfassen“ (Median $=0,95, z=3,132, p_{\text {ange- }}$ passt $($ zweiseitig $)=0,036)$ und „Vorhersagen“ (Median $=0,84, z=3,132$, $p_{\text {ange- }}$ passt $($ zweiseitig $)=0,036)$. Die restlichen paarweisen Vergleiche weisen keine signifikanten Unterschiede auf.

Die Phasen im Rechentraining unterscheiden sich nicht signifikant bezüglich der Prävalenz bilingualer Praktiken. Unter Verwendung des Dunn-Bonferroni-Tests weist kein paarweiser Vergleich der verschiedenen Phasen im Rechentraining signifikante Unterschiede in der Prävalenz bilingualer Praktiken auf.

\subsection{Zusammenhänge sprachlicher und motivationaler Merkmale mit der Prävalenz bilingualer Praktiken}

Im Folgenden werden potentielle Zusammenhänge von sprachlichen (F3a) und motivationalen Variablen mit der Prävalenz bilingualer Praktiken (F3b) geprüft. Zunächst werden die deskriptiven Werte der in der achten Sitzung erhobenen motivationalen Variablen für die Gruppen LGT/D und RGT/D berichtet sowie deren Verteilungen geprüft. Die Darstellung der deskriptiven Werte der 
im Prätest erhobenen sprachlichen Variablen der Gruppen erfolgte bereits in Abschnitt 8.2.1 beim Vergleich der Ausgangsbedingungen der vier Gruppen LGD, RGD, LGT/D und RGT/D. Anschließend werden die Ergebnisse der paarweisen Korrelationen $r_{x y}$ und $r_{x y}$ für die sprachlichen und motivationalen Variablen mit der Prävalenz bilingualer Praktiken berichtet.

Die deskriptiven Werte der motivationalen Variablen der Gruppen LGT/D und RGT/D sind in Tabelle 12 dargestellt. Die einzelnen Werte der motivationalen Variablen unterscheiden sich deskriptiv kaum zwischen den Gruppen LGT/D und RGT/D. Während in der Gruppe RGT/D die mittleren Tendenzen hinsichtlich der „basic needs“ - mit Ausnahme von Autonomieerleben - etwas geringer im Vergleich zur Gruppe LGT/D ausfallen, kehrt sich dieses Verhältnis für die restlichen, auf die Wahrnehmung der Nutzung des Türkischen im Training bezogenen Skalen um. Die Gruppe RGT/D weist bei diesen Skalen im Mittel deskriptiv etwas höhere Werte im Vergleich zur Gruppe LGT/D auf. Im Mittel stimmen die Probanden eher zu, Autonomie, Kompetenz und soziale Eingebundenheit während des Trainings zu erleben. Die Probanden stimmen im Mittel auch eher zu, Freude am Gebrauch des Türkischen im Training zu finden und dass die Nutzung des Türkischen im Training ihnen eher leicht fiel. Hinsichtlich des allgemeinen Nutzens des Türkischen ergibt sich keine klare Tendenz, aber hinsichtlich des trainingsspezifischen Nutzens stimmen die Probanden eher nicht zu, dass das Türkische ihnen bei der Anwendung der Leseund Rechenstrategien geholfen hat.

Keine der oben angeführten motivationalen Variablen für die aggregierten Gruppen LGT/D und RGT/D weist nach dem Shapiro-Wilk-Test eine Normalverteilung auf $(\mathrm{W}=.826-.938, \mathrm{df}=51-54, \mathrm{p}=.000-.008)$. Zudem scheinen einige der zu prüfenden Variablen unter visueller Inspektion der Streudiagramme keine linearen Zusammenhänge mit der logtransformierten Prävalenz bilingualer Praktiken aufzuweisen. Dies lässt sich z. B. an der „overall correlation“ der sprachlichen Variable „Türkischfreude“ (siehe Abbildung 15) und der motivationalen Variable „Basic Needs soziale Eingebundenheit“ (siehe Abbildung 16) mit der logtransformierten Prävalenz bilingualer Praktiken illustrieren: 
Tabelle 12

Deskriptive Werte der motivationalen Variablen der Gruppen LGT/D und $R G T / D$ auf Individualebene

\begin{tabular}{|c|c|c|c|c|c|c|c|c|}
\hline & \multicolumn{4}{|c|}{ LGT/D } & \multicolumn{4}{|c|}{ RGT/D } \\
\hline & Min & Max & MW & $\mathrm{S}$ & Min & Max & MW & S \\
\hline $\begin{array}{l}\text { BN } \\
\text { Autonomie }\end{array}$ & 1,29 & 3,86 & 2,90 & 0,58 & 1,86 & 3,71 & 2,95 & 0,59 \\
\hline $\begin{array}{l}\text { BN } \\
\text { Kompetenz }\end{array}$ & 1,75 & 4 & 3,37 & 0,51 & 1,50 & 4 & 3,25 & 0,73 \\
\hline $\begin{array}{l}\text { BN soz. } \\
\text { Eingebun- } \\
\text { denheit }\end{array}$ & 1 & 4 & 3,30 & 0,82 & 1,50 & 4 & 3,05 & 0,85 \\
\hline $\begin{array}{l}\text { TN Train. } \\
\text { Freude }\end{array}$ & 1 & 4 & 2,91 & 1,18 & 1 & 4 & 3,12 & 0,97 \\
\hline $\begin{array}{l}\text { TNTrain. } \\
\text { Schwierigkeit }\end{array}$ & 1 & 4 & 2,99 & 0,76 & 1 & 4 & 3,03 & 0,75 \\
\hline $\begin{array}{l}\text { Allgemeiner } \\
\text { Nutzen } \\
\text { Türkisch }\end{array}$ & 1 & 4 & 2,36 & 1,15 & 1 & 4 & 2,74 & 0,83 \\
\hline $\begin{array}{l}\text { Spezifischer } \\
\text { Nutzen } \\
\text { Türkisch }\end{array}$ & 1 & 4 & 2,26 & 1,08 & 1 & 4 & 2,31 & 0,87 \\
\hline
\end{tabular}

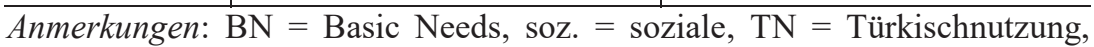
Train. $=$ Training 
Abbildung 15

„Overall correlation“ der Skala ,Türkischfreude“ mit der logtransformierten Prävalenz bilingualer Praktiken

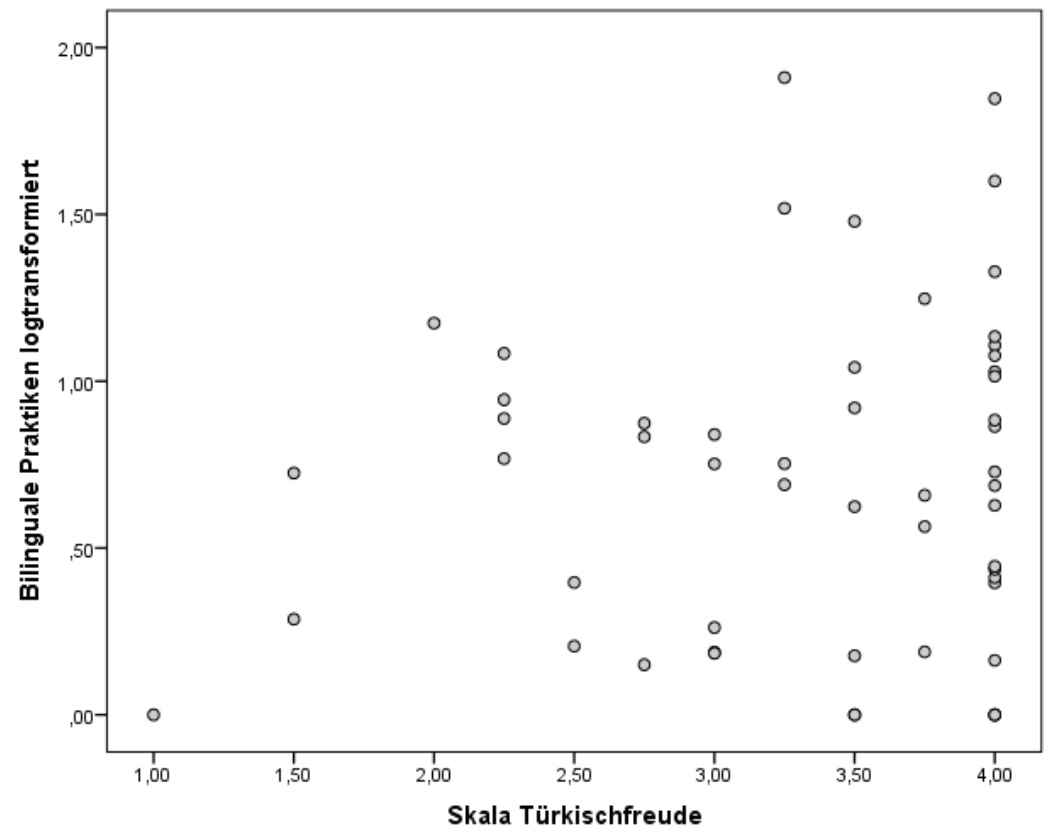


Abbildung 16

„Overall correlation“ der Skala „Basic Needs soziale Eingebundenheit“ mit der logtransformierten Prävalenz bilingualer Praktiken

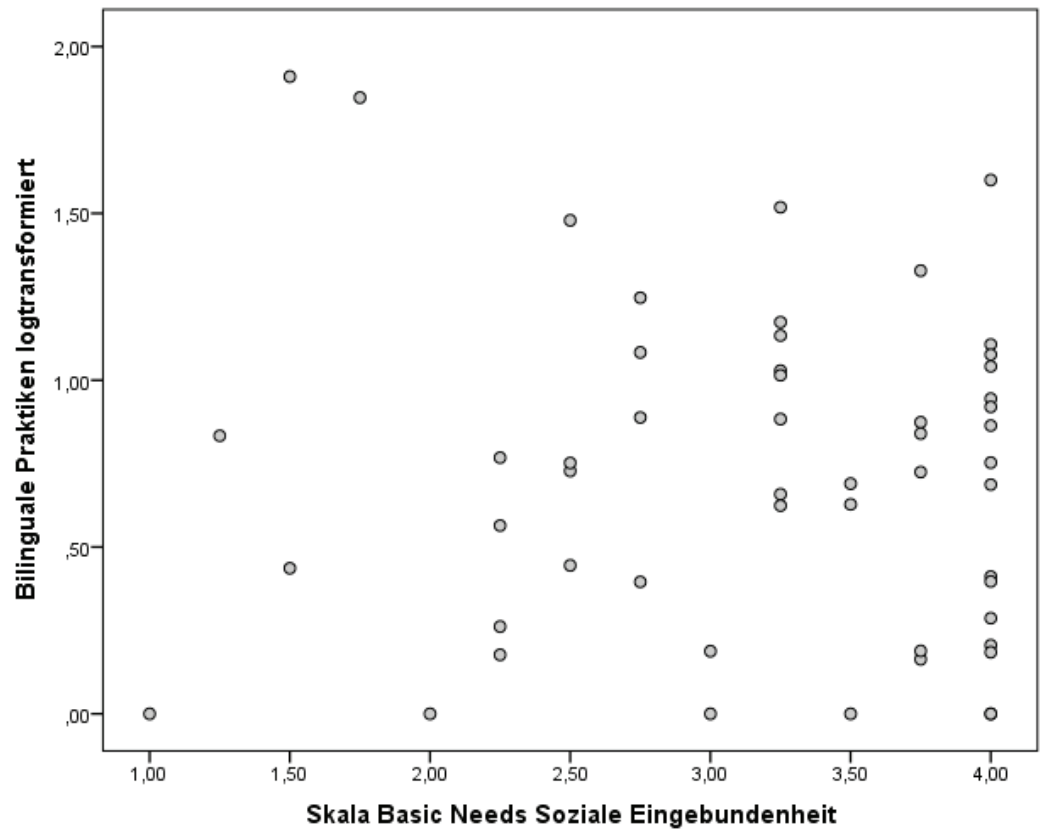

Auch unter Verwendung von Transformationen der Variablen lassen sich weder Normalverteilungen noch eher als linear zu bezeichnende Zusammenhänge erzeugen. Nichtsdestotrotz werden - wie bereits in Abschnitt 7.4.2.4 diskutiert - auf paarweise Korrelation für ununterscheidbare Dyaden (vgl. Griffin \& Gonzalez, 1995; Kenny, Kashy \& Cook, 2006, S. 37ff, 136ff) als Analysemethode für die Fragestellungen F3a und F3b zurückgegriffen.

Unter Verwendung des verteilungsfreien Kruskal-Wallis-Tests unterscheiden sich die einzelnen motivationalen Variablen nicht signifikant zwischen den Gruppen LGT/D und RGT/D. Dementsprechend können die Gruppen LGT/D und RGT/D aufgrund fehlender signifikanter Unterschiede hinsichtlich der sprachlichen (siehe Abschnitt 8.2) und motivationalen Variablen für die folgenden Analysen aggregiert werden. In Tabelle 13 sind die Ergebnisse der paarweisen Korrelationen der sprachlichen und in Tabelle 14 der motivationalen Variablen mit der Prävalenz bilingualer Praktiken der aggregierten Gruppen LGT/D und RGT/D dargestellt. Es werden für jeden Zusammenhang die vorhandene Stichprobengröße (n), die „overall correlation“ $\left(\mathrm{r}_{\mathrm{xy}}\right)$ und ,crossintraclass correlation" $\left(\mathrm{r}_{\mathrm{xy}}\right.$ ) sowie deren jeweilige $\mathrm{z}$-Werte aufgeführt. Die von 
der Gesamtstichprobe $(n=54)$ abweichenden geringeren Stichprobenumfänge entstehen durch Missings.

Zunächst fällt auf, dass die „overall correlations“ alle relativ gering ausfallen und keine mittleren Korrelationen auftreten. Der Großteil dieser Korrelationen weist verschwindend geringe Zusammenhänge auf, sodass auch die zWerte sich nicht dem kritischen Wert nähern und dementsprechend kein $\mathrm{Zu}$ sammenhang signifikant ist. Auch die Zusammenhänge der „cross-intraclass correlations" fallen äußerst gering aus, wobei wie im Falle der Korrelationen der Variablen „Wortschatz Türkisch“ und „Türkisch bei Denkprozessen“ die „cross-intraclass correlations“ die „overall correlations“ deskriptiv übersteigen.

Für die Zusammenhänge der motivationalen Variablen mit der logtransformierten Prävalenz bilingualer Praktiken ergibt sich ein ähnliches Bild wie bei den sprachlichen Variablen des Prätests. Die „overall correlation“ und „crossintraclass correlation“ der Zusammenhänge fallen eher gering aus, sodass die z-Werte sich nicht dem kritischen Wert nähern und dementsprechend kein $\mathrm{Zu}-$ sammenhang signifikant ist. Auch bei diesen Analysen treten Fälle auf, bei denen die „cross-intraclass correlations“ die „overall correlations“ deskriptiv übersteigen.

Die theoretischen und methodischen Probleme der Analysen von F3a und F3b werden in der Diskussion wieder aufgegriffen. Eventuell können die Ergebnisse der qualitativen Analysen einen Beitrag zur Erklärung der fehlenden signifikanten Zusammenhänge sprachlicher und motivationaler Variablen leisten, welche im Folgenden berichtet werden. 
Tabelle 13

„Overall correlation “ und ,,cross-intraclass correlation “ sprachlicher Variablen mit der logtransformierten Prävalenz bilingualer Praktiken der aggregierten Gruppen LGT/D und RGT/D auf Individualebene

\begin{tabular}{|c|c|c|c|c|c|}
\hline & $\mathrm{n}$ & $r_{x y}$ & z & $r_{x y}$ & z \\
\hline $\begin{array}{l}\text { WS } \\
\text { Türkisch }\end{array}$ & 54 & .031 & 0,227 & .053 & 0,388 \\
\hline $\begin{array}{l}\text { Türkisch- } \\
\text { freude }\end{array}$ & 54 & .118 & 0,795 & .060 & 0,043 \\
\hline $\begin{array}{l}\text { Türkisch } \\
\text { als } \\
\text { Ressource }\end{array}$ & 54 & -.060 & 0,420 & -.016 & 0,112 \\
\hline $\begin{array}{l}\text { Türkisch } \\
\text { bei Denk- } \\
\text { prozessen }\end{array}$ & 54 & .018 & 0,121 & -.031 & 0,209 \\
\hline $\begin{array}{l}\text { Sprach- } \\
\text { praxis } \\
\text { Pause }\end{array}$ & 53 & -.073 & 0,505 & .007 & 0,048 \\
\hline $\begin{array}{l}\text { Sprach- } \\
\text { praxis } \\
\text { Unterricht }\end{array}$ & 53 & -.202 & 1,467 & .018 & 0,128 \\
\hline
\end{tabular}

Anmerkungen: $\mathrm{WS}=$ Wortschatz 
Tabelle 14

„Overall correlation“ und „,cross-intraclass correlation“ motivationaler Variablen mit der logtransformierten Prävalenz bilingualer Praktiken der aggregierten Gruppen LGT/D und RGT/D auf Individualebene

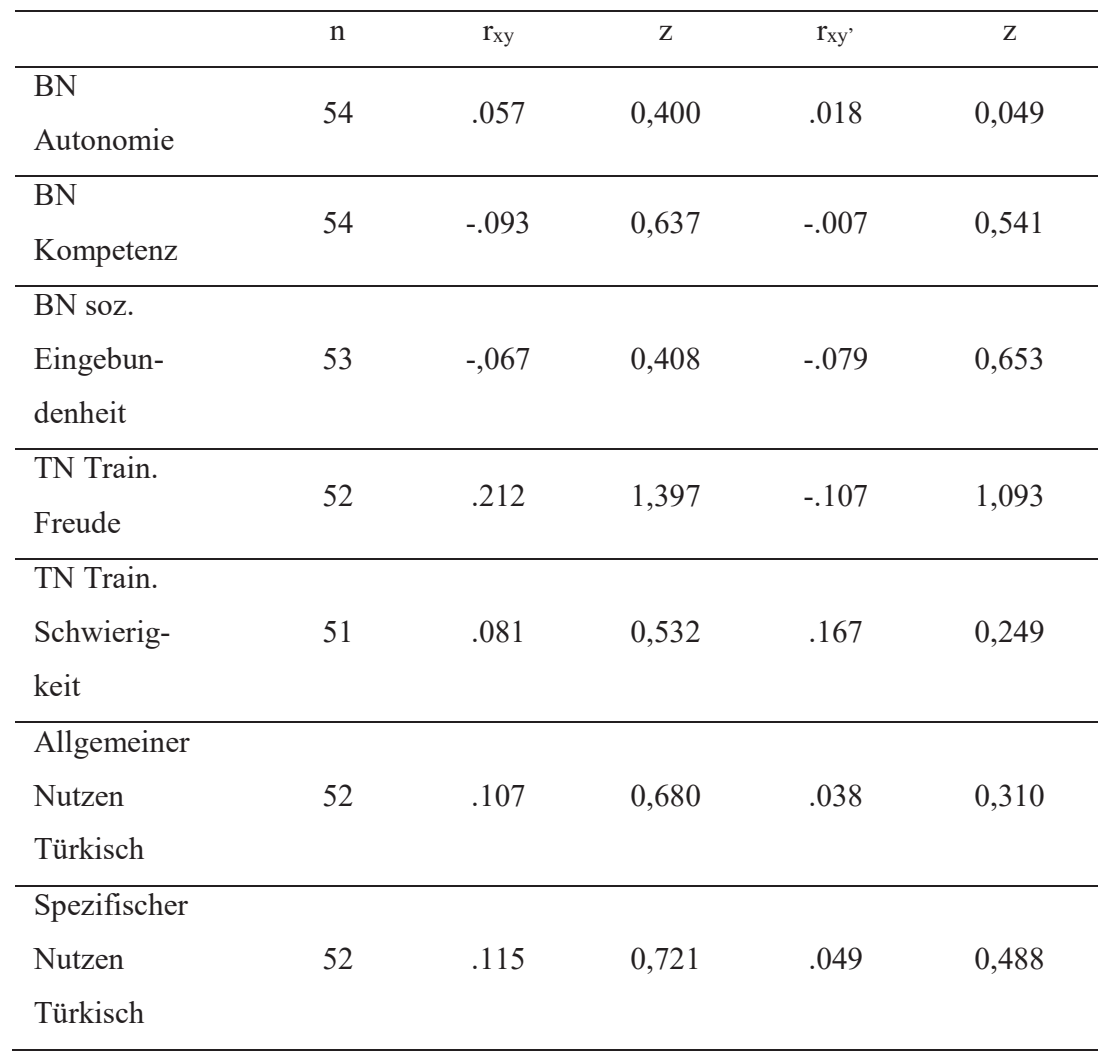

Anmerkungen $: \mathrm{BN}=$ Basic Needs, soz. $=$ soziale, $\mathrm{TN}=$ Türkischnutzung, Train. $=$ Training 


\subsection{Ergebnisse der Selbstberichte für die Annahme und Ab- lehnung des bilingualen Sprachangebots}

Zunächst werden die Ergebnisse des geschlossenen Antwortformats zur selbstberichteten Annahme und Ablehnung des Angebots, bei der Intervention auch auf Türkisch miteinander zu kommunizieren, betrachtet. Anschließend werden dann die Ergebnisse der Qualitativen Inhaltsanalyse der Begründungen für die Annahme und Ablehnung des bilingualen Sprachangebots getrennt vorgestellt. Hierbei wird anhand von Beispielantworten auch die Vielfalt der Argumente innerhalb der Kategorien beleuchtet, um die komplette Spannbreite der zu Grunde liegenden Motive für die Annahme und Ablehnung des bilingualen Sprachangebots zu präsentieren.

\subsubsection{Ergebnisse des geschlossenen Formats der Selbstberichte für die} Annahme und Ablehnung des bilingualen Sprachangebots

Tabelle 15 fasst für die jeweiligen Erhebungszeitpunkte sowie Interventionsgruppen LGT/D und RGT/D zusammen, wie häufig die Probanden berichteten, vom bilingualen Sprachangebot (nicht) Gebrauch gemacht zu haben:

Tabelle 15

Selbstberichtete Annahme und Ablehnung des Sprachangebots im geschlossenen Format

\begin{tabular}{l|c|c|c|c}
\hline \multicolumn{2}{c}{} & \multicolumn{1}{c}{ EZP 1 } & EZP 2 & EZP \\
\multicolumn{1}{l}{} & \multicolumn{2}{c}{$1+2$} \\
\multicolumn{1}{l}{} & \multicolumn{2}{c}{$\mathrm{n}$} & $\mathrm{n}$ \\
\hline Annahme & LGT/D & 16 & 21 & 37 \\
& RGT/D & 19 & 14 & 33 \\
& Gesamt & 35 & 35 & 70 \\
\hline Ablehnung & LGT/D & 8 & 5 & 13 \\
& RGT/D & 6 & 10 & 16 \\
& Gesamt & 14 & 15 & 29 \\
\hline Ungültig & - & 2 & 1 & 3 \\
\hline
\end{tabular}

Anmerkungen: $\mathrm{EZP}=$ Erhebungszeitpunkt 
Anhand der deskriptiven Daten zeigt sich, dass insgesamt mehr Probanden widergeben, Türkisch beim PL gesprochen zu haben als das Angebot abgelehnt zu haben. Über die Gruppen LGT/D und RGT/D hinweg unterscheiden sich die Häufigkeiten zwischen Erhebungszeitpunkt 1 und 2 kaum. Unter Verwendung des exakten Binomialtests zeigt sich, dass die Probanden der aggregierten Gruppen LGT/D und RGT/D insgesamt signifikant häufiger berichten, dass sie das Angebot angenommen haben und zwar sowohl für Erhebungszeitpunkt 1 $(p($ zweiseitig $)=.004, \mathrm{n}=49)$ als auch für Erhebungszeitpunkt 2 ( $p$ (zweiseitig) $=.007, \mathrm{n}=50)$.

Allerdings lässt sich deskriptiv eine diametrale Entwicklung der Gruppen LGT/D und RGT/D von Erhebungszeitpunkt $1 \mathrm{zu} 2$ konstatieren, da bei der Gruppe LGT/D die berichtete Annahme zunimmt, während diese bei RGT/D abnimmt. Diesbezüglich wurde die (In-)Konsequenz der Antworten über beide Erhebungszeitpunkte betrachtet. Der Großteil der Probanden antwortete konsequent über beide Erhebungszeitpunkte hinweg, wobei 27 Probanden das geschlossene Format zu beiden Erhebungszeitpunkten bejahten, während acht dies konsequent verneinten. Inkonsequent über beide Erhebungszeitpunkte hinweg antworteten 13 Probanden. Sieben dieser Probanden berichteten eine Annahme zum ersten aber nicht zum zweiten Erhebungszeitpunkt, wovon fünf Probanden zur Gruppe RGT/D angehören. Sechs der 13 inkonsequent antwortenden Probanden wiesen das umgekehrte Antwortmuster auf, wovon fünf aus der Gruppe LGT/D stammen. Dementsprechend scheint die Zunahme und Abnahme der Selbstberichte zur Annahme des bilingualen Interaktionsangebots über die Erhebungszeitpunkte 1 und 2 der Gruppen LGT/D respektive RGT/D zum Großteil durch das Antwortverhalten der eben angeführten inkonsequent antwortenden Probanden bedingt zu sein.

\subsubsection{Ergebnisse des offenen Formats der Selbstberichte für die Annahme und Ablehnung des bilingualen Sprachangebots}

Deutlich interessanter und aufschlussreicher sind allerdings die von den Probanden angegebenen Gründe für die Annahme und Ablehnung des Angebots. Insgesamt wurden über beide Erhebungszeitpunkte hinweg 102 Begründungen für die verwendete Sprachpraxis identifiziert. Zum ersten Erhebungszeitpunkt wurden 52 und zum zweiten 50 Gründe angegeben, wobei fast jeder Proband einen Grund berichtet. Lediglich drei Probanden nennen beim ersten Erhebungszeitpunkt zwei Gründe für ihr sprachliches Interaktionsverhalten.

In Tabelle 16 sind die Häufigkeiten der Antworten pro Ebene abhängig von der Annahme und Ablehnung des Sprachangebots angegeben. Die am häufigsten adressierte Ebene über beide Erhebungszeitpunkte hinweg ist sowohl bei der Annahme als auch bei der Ablehnung des Angebots die Individuumsebene. Die Gesprächspartner spielen für die Argumentation der Probanden angesichts 
der geringen Prävalenzen lediglich eine untergeordnete Rolle. Gründe auf der Kontextebene werden bei der Annahme des Angebots häufig, bei der Ablehnung des Angebots kaum berichtet. Das jeweilige sprachliche Verhalten kann nicht von jedem Proband legitimiert werden, da insgesamt achtmal kein Grund angegeben wurde. Hinsichtlich der verschiedenen Erhebungszeitpunkte fällt auf, dass die Begründungen bei der Annahme und Ablehnung des Angebots vom ersten zum zweiten Erhebungszeitpunkt hinsichtlich der Individuumsebene abnehmen. Im Gegensatz hierzu verdoppelt sich bei der Annahme des Angebots die Prävalenz der Begründungen auf der Kontextebene.

Tabelle 16

Häufigkeiten der Begründungsebenen nach Annahme und Ablehnung sowie nach Erhebungszeitpunkten

EZP $1 \quad$ EZP $2 \quad$ EZP $1+2$

$\mathrm{n} \quad \mathrm{n} \quad \mathrm{n}$

\begin{tabular}{l|l|c|c|c}
\hline Annahme & Individuumsebene & 23 & 13 & 36 \\
& Gesprächspartnerebene & 4 & 5 & 9 \\
& Kontextebene & 7 & 14 & 21 \\
& Keine Begründung & 3 & 3 & 6 \\
& Gesamt & 37 & 35 & 72 \\
\hline Ablehnung & Individuumsebene & 14 & 9 & 23 \\
& Gesprächspartnerebene & 1 & 3 & 4 \\
& Kontextebene & - & 1 & 2 \\
& Keine Begründung & - & 2 & 30 \\
\hline
\end{tabular}

Anmerkungen: EZP = Erhebungszeitpunkt

Die Prävalenzen der Kategorien bei der Annahme des Sprachangebots sind in Tabelle 17 getrennt nach Erhebungszeitpunkten und Gruppen dargestellt. Zunächst sollen die aggregierten Prävalenzen über beide Erhebungszeitpunkte und beide Gruppen betrachtet werden, bevor potentielle Unterschiede im Antwortverhalten zwischen den Gruppen LGT/D und RGT/D deskriptiv inspiziert werden. Anschließend wird ein vergleichender Blick auf die zwei Erhebungszeitpunkte geworfen und abschließend werden ausgewählte Kategorien unter Verwendung von Beispielantworten der Probanden näher beschrieben, um einen besseren Eindruck über die Bandbreite der Legitimationen der bilingualen Interaktion der Probanden auch innerhalb einer Kategorie zu erhalten. 


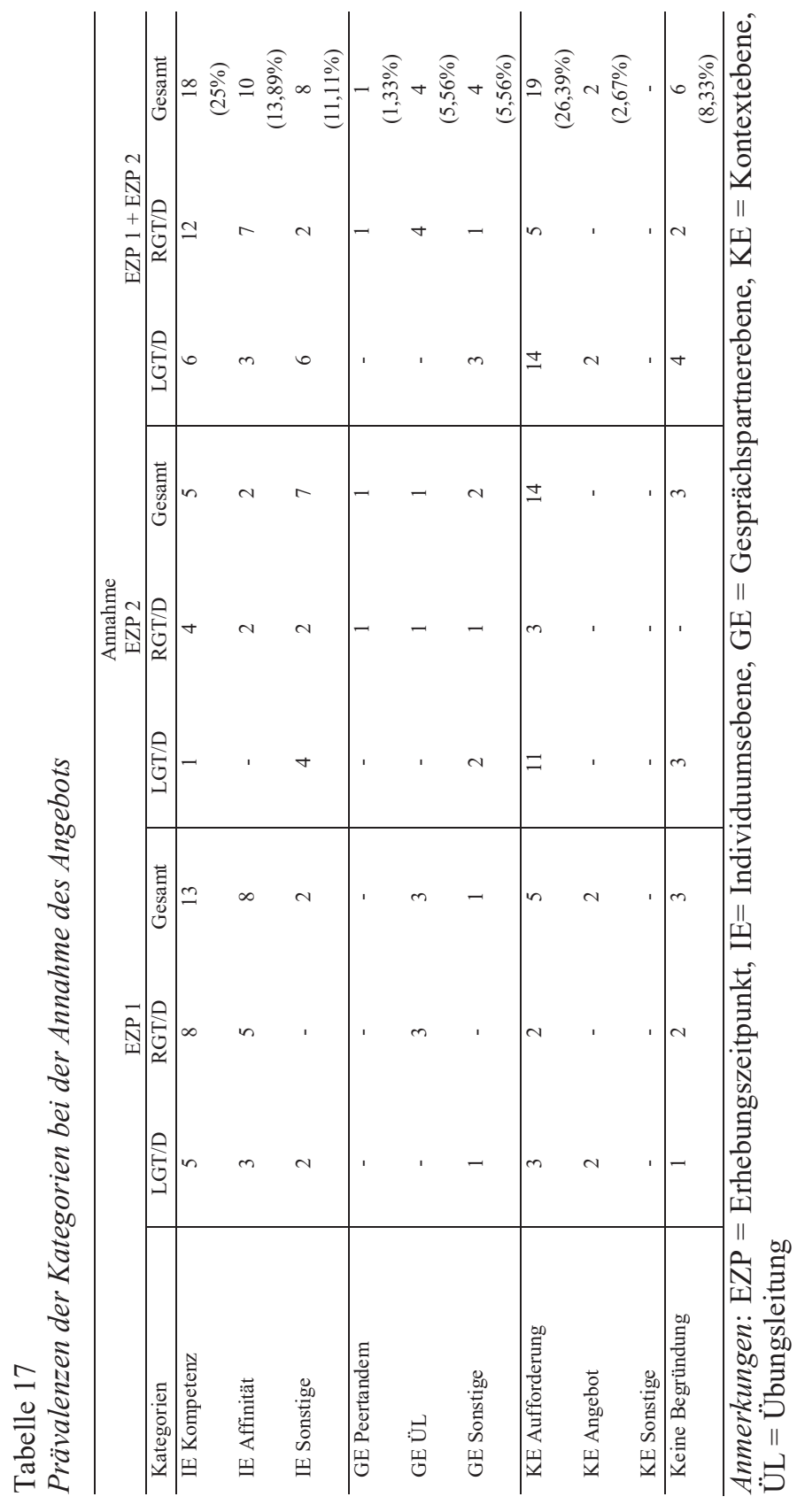


Mit Ausnahme der Kategorie „KE Sonstige“ werden bei der Annahme des Sprachangebots alle im Prozess der Deduktion und Induktion erzeugten Kategorien durch die Antworten der Probanden bedient. Mit deutlichem Abstand weisen die Kategorien „IE Kompetenz“ und „KE Aufforderung“ die höchsten Prävalenzen auf und bilden zusammen ein wenig mehr als die Hälfte aller Antworten hinsichtlich der Begründung der Annahme ab. Die Kategorien „IE Affinität“ und „IE Sonstige“ stellen mit zehn respektive acht Fällen ebenfalls häufig von den Probanden genutzte Legitimationen dar, sodass etwas mehr als 75\% aller Antworten zur Annahme des Angebots durch die eben genannten vier verschiedenen Kategorien repräsentiert werden. Im Gegensatz zu diesen Kategorien spielen alle Kategorien der Ebene „Gesprächspartner“ als auch die Kategorie „KE Angebot“" kaum eine Rolle in den Argumentationshaushalten der Probanden.

Die Legitimationen der Sprachpraxis weisen zum Teil nennenswerte deskriptive Unterschiede zwischen den Gruppen LGT/D und RGT/D auf. So stammen zwei Drittel aller kompetenzbezogenen Begründen aus der Gruppe RGT/D, während etwas weniger als drei Viertel aller Antworten der Kategorie „KE Aufforderung“ von den Probanden aus der Gruppe LGT/D gegeben wurden. Bezüglich der Kategorien „IE Affinität“ und „IE Sonstige“ weisen die beiden Gruppen ebenfalls unterschiedliche Präferenzen auf, da die erstgenannte Kategorie primär von Probanden der Gruppe RGT/D besetzt wird, während die letztgenannte eher von den Probanden der Gruppe LGT/D adressiert wird. Zudem stammen drei Viertel der Gründe auf Gesprächspartnerebene aus der Gruppe RGT/D und die Kategorie „KE Angebot“" wurde nur von zwei Antworten aus der Gruppe LGT/D belegt. Bezüglich der verschiedenen Erhebungszeitpunkte fällt auf, dass kompetenz- und affinitätsbezogene Begründungen von Erhebungszeitpunkt $1 \mathrm{zu} 2$ abnehmen, während Legitimationen der Sprachpraxis als zu erfüllender Bestandteil der Intervention deutlich zunehmen.

Angesichts der vorliegenden Prävalenzen sollen die drei Kategorien der Individuumsebene sowie die Kategorie „KE Aufforderung“ anhand von Beispielantworten näher betrachtet werden, da diese zusammen etwas mehr als $75 \%$ aller Begründungen ausmachen und zum Teil innerhalb der jeweiligen Kategorien verschiedene Argumente anführen. Um einen besseren Eindruck der Form der Antworten zu erhalten und keine versehentlichen Verfälschungen der artikulierten Propositionen vorzunehmen, werden die Beispielantworten der Probanden ohne sprachliche Glättung oder Korrektur berichtet.

Die einzelnen Antworten der Kategorie „KE Aufforderung“ ähneln sich alle in ihrer konkret verschriftlichten Proposition. Häufig wird das Angebot zur bilingualen Interaktion als eine (zumindest temporär zu erfüllende) Pflicht in den Antworten der Probanden dargestellt, was an der häufigen Nutzung der Modalverben ,müssen“ und ,sollen“ deutlich wird, die beide eine Notwendigkeit implizieren (z. B. „weil wir türkisch sprechen müssen“; „weil man bei 
bipeer gemischt sprechen muss“; „Weil wir das mussten“; „weil wir es mussten“; „weil wir auch ein bisschen reden sollten.“; ,weil wir Türkisch sprechen sollen"). Die Übungsleitung wird hierbei zum Teil ebenfalls adressiert (z. B. „weil unsere Tränerin es gesagt. hat.“; „Ja, weil unsere Trainerin es so wollte.“), allerdings werden diese Antworten nicht der Kategorie „GE Übungsleitung" zugeordnet, da die Übungsleitung für die Umsetzung der Intervention zuständig ist und nicht aus eigenem Antrieb die explizite Erlaubnis zur bilingualen Interaktion implementierte. Im Gegensatz hierzu akzentuieren lediglich zwei Antworten das Angebot selbst als Begründung für die Annahme des Sprachangebots (,weil unsere Trainingsleitung gesagt hat das wir auch Türkisch reden können“; „weil wir das machen dürfen“).

Die Kategorie „IE Kompetenz“ zeichnet sich im Gegensatz zu der eben näher betrachteten Kategorie „KE Aufforderung“ durch eine gewisse Heterogenität aus. Viele Antworten innerhalb der Kategorie „IE Kompetenz“ weisen in sehr allgemeiner und nicht näher spezifizierter Form auf eine Erleichterung oder Vereinfachung durch die Annahme des bilingualen Sprachangebots hin (z. B. ,,weil es mir einfacher ist“; ,Weil es so einfach war“; , ,warm mirs leichter"; ,Weil ich Schwierigkeiten habe“; ,,weil es besser ist"). Drei Begründungen artikulieren konkrete Defizite im Sprachverständnis oder in der Sprachproduktion im Deutschen, welche die Probanden zur bilingualen Interaktion bewegt haben (,weil ich manche Wörter auf deutsch nicht verstehe!“; ,weil ich manche Sachen nicht sagen konnte“; ,weil ich nicht alles auf deutsch sagen kann"). Interessanterweise treten auch drei Argumentationen auf, die das Angebot zur bilingualen Interaktion als Möglichkeit sehen, die sprachlichen Fähigkeiten im Türkischen zu verbessern (,weil ich bisschen mehr türkisch lernen will“; , „Weil ich mehr Türkisch lernen will.“; ,Ja weil ich dann mehr Türkisch gelernt habe."). Zwei Antworten legitimieren ihre bilingualen Praktiken mit einem nicht näher spezifizierten Lernzuwachs (,damit ich was lerne“; „[...] dass ich meistens gelernt habe was"). Lediglich ein Fall führt die Annahme des Angebots direkt auf die individuell vorhandene Türkischkompetenz zurück (,weil ich gut Türkisch kann“).

Die Argumente der Kategorien „IE Affinität“ und „IE Sonstige“ zeichnen sich durch eine gewisse Homogenität aus. Sieben der zehn Antworten der Unterkategorie „IE Affinität“ begründen die Annahme des Angebots konkret mit dem Erleben von Spaß (z. B. ,das hat mir Spaß gemacht“; ,,weil türkisch macht mir spaß“; „weil es mir Spaß gemacht hat"). Die anderen drei affinitätsbezogenen Argumentationen drücken in eher unspezifischer Weise Sympathie für das Sprechen von Türkisch aus (,weil ich es mag“; ,,weil ich türkisch sprechen mag“; , „Weil mir Türkisch sprechen gefallen hat und [...]"). Der Großteil der Antworten der Kategorie „IE Sonstige“ zeichnet sich durch die Verwendung des Modalverbs, ,wollen“ aus, welches eine Präferenz, einen Wunsch, eine Absicht etc. impliziert (z. B. „Weil ich es wollte.“; , ,weil ich es so will!“; , ,[...] und ich wollte!"). Der übrig gebliebene Fall dieser Kategorie legitimiert die 
Annahme des Angebots mit einem national geprägten Identitätsverständnis (z. B. „Weil ich Türke bin“).

Die Prävalenzen der Kategorien bei der Ablehnung des Sprachangebots sind in Tabelle 18 getrennt nach Erhebungszeitpunkten sowie Gruppen dargestellt und werden in ähnlicher Weise wie die Ergebnisse der Annahme näher betrachtet. Bei der Ablehnung des Angebots treten deskriptiv weniger Kategorien im Vergleich zur Annahme des Sprachangebots auf, da die Unterkategorien „GE Sonstige“, „KE Aufforderung“ und „KE Angebot" nicht bedient werden. Der Großteil der Argumente zur Ablehnung des Angebots stammt aus den Kategorien „IE Kompetenz" und „IE Affinität“, die zusammen 70\% aller Antworten ausmachen. Auch bei der Ablehnung des Angebots spielen die Kategorien der Gesprächspartnerebene kaum eine Rolle und nicht immer kann die berichtete Sprachpraxis mit einem Grund legitimiert werden.

Hinsichtlich Gruppenunterschieden zwischen LGT/D und RGT/D sticht lediglich die Kategorie „IE Affinität" heraus, die primär von der Gruppe LGT/D adressiert wird. Wie bei der Annahme kann ebenso bei der Ablehnung des Angebots beobachtet werden, dass die Prävalenz der Antworten der Kategorie „IE Kompetenz" vom ersten zum zweiten Zeitpunkt deutlich abnehmen.

Da die Kategorien „IE Kompetenz“ und „IE Affinität“ zusammen 70\% der Legitimationen für die Ablehnung des Sprachangebots ausmachen und beide sich durch eine gewisse Heterogenität auszeichnen, sollen diese im Folgenden etwas näher betrachtet werden. Bezüglich der Kategorie „IE Kompetenz“ akzentuieren sechs Antworten konkret eine Asymmetrie der sprachlichen Fähigkeiten zu Gunsten des Deutschen (,weil mir deutsch leichter fällt"; „Weil Deutsch leichter ist“; „Weil ich besser Deutsch reden kann“; „Weil ich besser Deutsch als Türkisch kann“; ,Weil ich in deutsch besser bin“; ,Weil ich besser deutsch sprechen kann."). Die Begründung ,[...] und mehr Deutsch spreche“ könnte ebenfalls als Sprachasymmetrie gewertet werden, aber prinzipiell auch im Sinne der alltäglichen Sprachpraxis als ein höherer Gebrauch des Deutschen als des Türkischen interpretiert werden. Drei Antworten der Kategorie „IE Kompetenz" weisen allgemein auf eine geringe Kompetenz im Türkischen hin (,weil ich nicht so gut Türkisch reden kann“; ,,weil ich nicht gut Türkisch kann“; „Ich kann nicht so gut türkick") und eine fokussiert die Schwierigkeit Türkisch zu sprechen („Weil Türkisch sprechen ist schwer.“). Zwei Gründe akzentuieren spezifische, fehlende sprachliche Kompetenzen, die die Annahme des Sprachangebots beim Rechenstrategietraining verhinderten (,weil ich türkische Zahlen nicht zählen kann“; ,,weil ich die Zahlen bis zehn nur kann auf Türkisch“ 


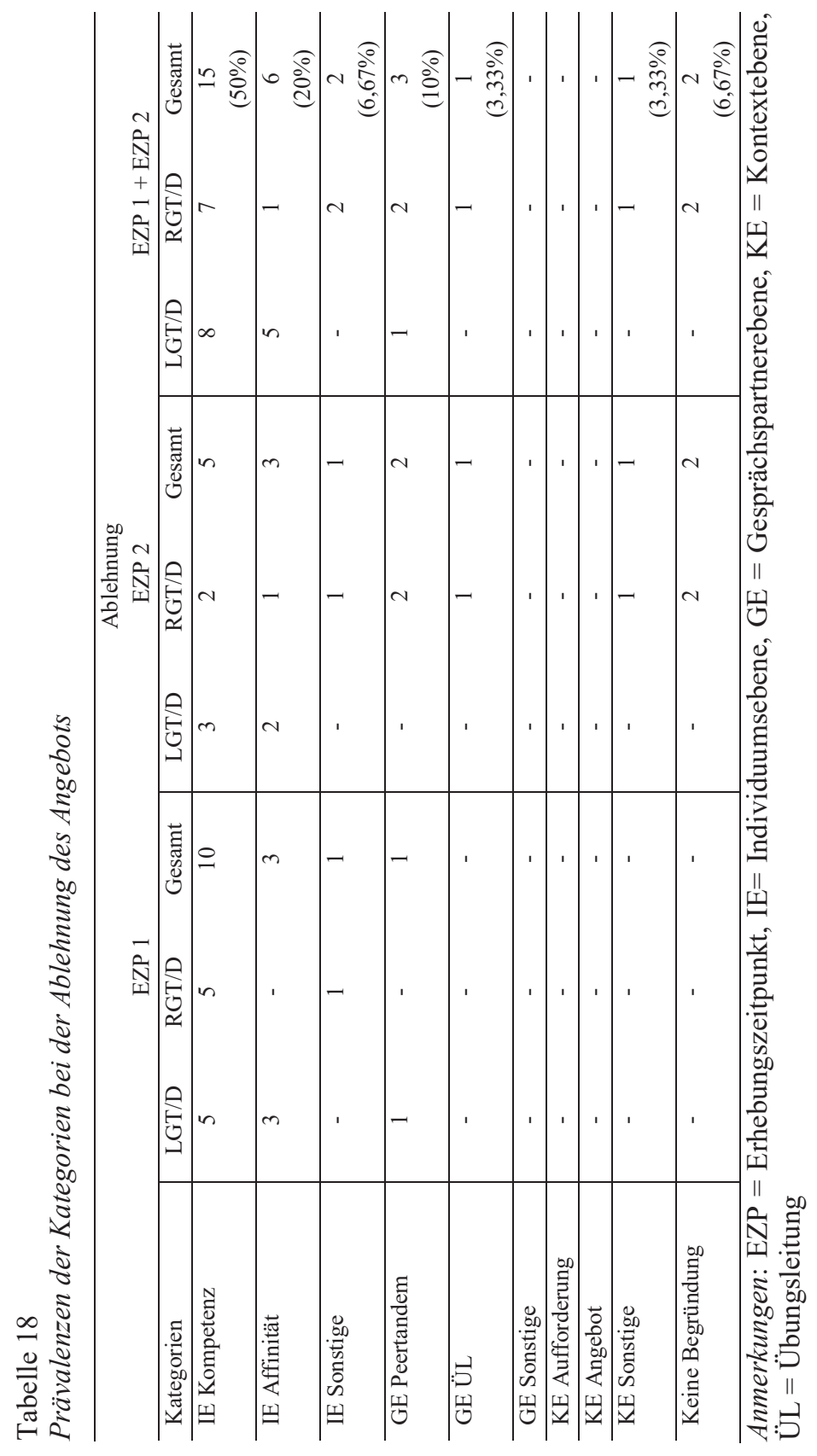


In einem Fall wird die Ablehnung des Angebots damit begründet, dass beim Rechnen nicht an das Türkische gedacht und darüber gesprochen werden kann (,Weil wir rechnen und ich nicht an türkisch denken kann. Wir rechnen ja und reden nicht über türkisch."). Eine andere Antwort legitimiert die Ablehnung des Angebots mit der Notwendigkeit Deutsch zu lernen („Weil ich Deutsch lernen mus").

Vier Fälle innerhalb der Kategorie „IE Affinität“ begründen die Ablehnung des Angebots mit Unlust oder gar einer Antipathie zum Sprechen des Türkischen in der Intervention (,,weil ich keinen Bock drauf habe“; „Weil türkisch zu sprechen langweilig ist"; „Keine Lust"; , weil ich es scheiße finde“) und zwei Fälle führen eine positiv besetzte Bereitwilligkeit zum Sprechen des Deutschen an („,weil ich gerne Deutsch spreche“; „Weil ich gerne [...] Deutsch spreche").

\subsection{Ergebnisse der Funktionsanalyse bilingualer Praktiken beim Peer-Learning}

Die Prävalenzen der verschiedenen Funktionen bilingualer Praktiken beim PL werden im Folgenden zunächst sowohl über beide Angebotsgruppen hinweg als auch getrennt für LGT/D und RGT/D deskriptiv summativ und hinsichtlich ihrer zentralen Tendenzen dargestellt (F5a). Anschließend werden potentielle signifikante Unterschiede der Prävalenzen der Haupt- und Unterkategorien zwischen der Lese- und Rechenstrategieintervention mit Hilfe des Mann-Whitney-U-Tests geprüft (F5b). Abschließend wird ein vertiefender Blick auf die verschiedenen Funktionen innerhalb der Unterkategorien der Hauptkategorie „On-Task“ geworfen, wobei diese unter Verwendung von Beispielen näher ausgeführt werden.

\subsubsection{Summative Ergebnisse der Haupt- und Unterkategorien der Funktionsanalyse bilingualer Praktiken beim Peer-Learning}

Insgesamt wurden 491 Fälle auf Basis der insgesamt 471 Turns mit bilingualen Praktiken für die Funktionsanalyse identifiziert. Dementsprechend wiesen 20 Turns bilingualer Praktiken mehr als eine Funktion auf. Von den 491 Fällen stammen 226 Fälle aus der Gruppe LGT/D und 265 aus der Gruppe RGT/D. Diese verteilen sich getrennt nach und aggregiert über die Gruppen LGT/D und RGT/D auf die Hauptkategorien wie in Tabelle 19 dargestellt: 
Tabelle 19

Prävalenzen der Hauptkategorien der Funktionen bilingualer Praktiken beim Peer-Learning

\begin{tabular}{|c|c|c|c|c|c|}
\hline \multicolumn{6}{|c|}{ Hauptkategorien } \\
\hline \multicolumn{2}{|c|}{ On-Task } & \multicolumn{2}{|c|}{ Off-Task } & \multicolumn{2}{|c|}{ Nicht Kategorisierbar } \\
\hline \multicolumn{2}{|c|}{ LGT/D + RGT/D } & \multicolumn{2}{|c|}{$\mathrm{LGT} / \mathrm{D}+\mathrm{RGT} / \mathrm{D}$} & \multicolumn{2}{|c|}{ LGT/D + RGT/D } \\
\hline \multicolumn{2}{|c|}{431} & \multicolumn{2}{|c|}{51} & \multicolumn{2}{|c|}{9} \\
\hline \multicolumn{2}{|c|}{$(87,78 \%)$} & \multicolumn{2}{|c|}{$(10,39 \%)$} & \multicolumn{2}{|c|}{$(1,83 \%)$} \\
\hline $\mathrm{LGT} / \mathrm{D}$ & RGT/D & $\mathrm{LGT} / \mathrm{D}$ & RGT/D & $\mathrm{LGT} / \mathrm{D}$ & $\mathrm{RGT} / \mathrm{D}$ \\
\hline 192 & 239 & 31 & 20 & 3 & 6 \\
\hline$(84,96 \%)$ & $(90,19 \%)$ & $(13,72 \%)$ & $(7,55 \%)$ & $(1,33 \%)$ & $(2,26 \%)$ \\
\hline
\end{tabular}

431 Fälle bilingualer Praktiken können der Hauptkategorie „On-Task“ zugeordnet werden, während bilinguale Praktiken zur Kommunikation von „OffTask“-Gesprächsbeiträgen lediglich in 51 Fällen identifiziert werden konnten. Dementsprechend werden bilinguale Praktiken beim PL zu fast $88 \%$ zur aktiven Auseinandersetzung mit dem Lerninhalt eingesetzt und nur zu knapp 10\% für interventions- und lernirrelevante Sprachhandlungen. Zwei Prozent der bilingualen Praktiken konnten aufgrund fehlender semantischer Klarheit nicht bezüglich ihrer Funktion interpretiert werden. Die Gruppen LGT/D und RGT/D unterscheiden sich bezüglich der Verteilung der Fälle auf die drei Hauptkategorien kaum, wobei deskriptiv die Gruppe LGT/D im Vergleich zur Gruppe RGT/D einen höheren Anteil bilingualer Praktiken für „Off-Task“Funktionen und dementsprechend einen geringeren Anteil für "On-Task“Funktionen aufweist.

Die Ausdifferenzierung der Hauptkategorie „On-Task“ weist klare Tendenzen in den Prävalenzen der dazugehörigen Unterkategorien auf, die in Tabelle 20 sowohl aggregiert als auch getrennt nach den Gruppen LGT/D und RGT/D summativ dargestellt sind: 
Tabelle 20

Prävalenzen der On-Task-Unterkategorien der Funktionen bilingualer Praktiken beim Peer-Learning

On-Task-Unterkategorien

\begin{tabular}{|c|c|c|c|c|c|c|}
\hline $\begin{array}{c}\text { Aufgaben- bearbei- } \\
\text { tung }\end{array}$ & Task-M & agement & $\begin{array}{l}\text { Metako } \\
\text { (Selbst-) }\end{array}$ & $\begin{array}{l}\text { ition und } \\
\text { egulation }\end{array}$ & Sor & tige \\
\hline LGT/D + RGT/D & LGT/D & RGT/D & LGT/D & RGT/D & $\mathrm{LGT} / \mathrm{D}$ & RGT/D \\
\hline 14 & & & & & & \\
\hline$(3,25 \%)$ & (61 & $2 \%)$ & $(30$ & $6 \%)$ & $(4$, & $\%)$ \\
\hline RGT/D & LGT/D & RGT/D & LGT/D & RGT/D & LGT/D & RGT/D \\
\hline 9 & 104 & 159 & 65 & 68 & 14 & 7 \\
\hline$(4,69 \%) \quad(2,09 \%)$ & $(54,17 \%)$ & $(66,53 \%)$ & $(33,85 \%)$ & $(28,45 \%)$ & $(7,29 \%)$ & $(2,93 \%)$ \\
\hline
\end{tabular}

Bilinguale Praktiken werden in der Auseinandersetzung mit relevanten Lernaktivitäten beim PL primär zum Task-Management sowie für metakognitive Zwecke produziert, wobei die erstgenannte Unterkategorie knapp 61\% und die zweitgenannte fast $31 \%$ der Hauptkategorie „On-Task“" ausmachen. Allerdings werden bilinguale Praktiken nur äußerst selten für die Aufgabenbearbeitung selbst eingesetzt und zwar lediglich zu knapp 3\%. Weitere, nicht durch die eben angeführten Unterkategorien abgedeckte Funktionen treten in fast 5\% der Fälle auf. Die jeweiligen Anteile der Gruppen LGT/D und RGT/D bezüglich der Unterkategorien der Hauptkategorie „On-Task“ unterscheiden sich deskriptiv. Die Gruppe LGT/D weist höhere Anteile hinsichtlich der Unterkategorien „Aufgabenbearbeitung“, „Metakognition und (Selbst-)Regulation“ und „Sonstige“ im Vergleich zur Gruppe RGT/D auf, wobei die letztgenannte Gruppe folglich einen höheren Anteil bezüglich der Unterkategorie „Task-Management" besitzt.

\subsubsection{Zentrale Kennwerte der Haupt- und Unterkategorien der Funktionsanalyse bilingualer Praktiken beim Peer-Learning}

Diese Summenwerte sagen allerdings nichts über die zentralen Tendenzen und Varianzen der kompletten Stichprobe der Gruppen LGT/D und RGT/D aus. In Tabelle 21 sind die zentralen deskriptiven Kennwerte für die Hauptkategorien der Gruppen LGT/D und RGT/D getrennt und aggregiert dargestellt. Die Dyade sowie die insgesamt vier Probanden, die keine bilingualen Praktiken 
produziert haben, werden aus diesen Analysen ausgeschlossen, da diese zu einer Verzerrung der zentralen deskriptiven statistischen Kennwerte führen würden.

Tabelle 21

Zentrale Kennwerte der Hauptkategorien der Funktionen bilingualer Praktiken beim Peer-Learning

\begin{tabular}{lcccccc}
\hline & \multicolumn{2}{c}{ On-Task } & \multicolumn{2}{c}{ Off-Task } & \multicolumn{2}{c}{ Nicht } \\
& MW & S & MW & S & MW & S \\
\hline LGT/D & 10,11 & 7,48 & 1,63 & 2,09 & 0,16 & 0,38 \\
\hline RGT/D & 9,56 & 10,81 & 0,8 & 1,32 & 0,24 & 0,44 \\
\hline LGT/D + & 9,80 & 9,42 & 1,16 & 1,73 & 0,20 & 0,41 \\
RGT/D & & & & & & \\
\hline
\end{tabular}

Im Mittel produziert jeder Proband der Stichprobe für Fragestellung F5 fast zehn bilinguale Praktiken zu relevanten Lernaktivitäten, während im Mittel lediglich knapp eine lern- und interventionsirrelevante bilinguale Praktik geäußert wird. Hierbei gilt allerdings zu berücksichtigen, dass die Standardabweichungen angesichts der Größe der korrespondierenden Mittelwerte relativ groß ausfallen, sodass von einer starken interindividuellen Heterogenität auszugehen ist.

Die Gruppen LGT/D und RGT/D weisen hinsichtlich dieser Kennwerte kaum deskriptive Differenzen im Vergleich zueinander auf. Da die Werte der verschiedenen Funktionen der Hauptkategorien sich nach der Prüfung mit dem Shapiro-Wilk-Test nicht normalverteilen ( $W=.496-.833, d f=44, p<.001)$, werden potentielle signifikante Unterschiede mit dem non-parametrischen Mann-Whitney-U-Test geprüft. Weder hinsichtlich der Hauptkategorie „OnTask" (Median LGT/D = 9, Median RGT/D = 7, $U=206,500, p$ (zweiseitig) = .461) noch bezüglich der Hauptkategorie „Off-Task“ (Median LGT/D = 1, Median $\mathrm{RGT} / \mathrm{D}=0, U=167, p$ (zweiseitig) $=.072$ ) unterscheiden sich die Werte zwischen den Gruppen LGT/D und RGT/D signifikant. Dementsprechend unterscheiden sich die Gruppen LGT/D und RGT/D nicht hinsichtlich der Quantität von aufgaben- und lernrelevanten als auch aufgaben- und lernirrelevanten bilingualen Praktiken.

In Tabelle 22 sind die zentralen deskriptiven Kennwerte für die Unterkategorien der Hauptkategorie „On-Task“ der Gruppen LGT/D und RGT/D aggregiert und getrennt dargestellt: 
Tabelle 22

Zentrale Kennwerte der Unterkategorien der Hauptkategorie „On-Task“ der Funktionen bilingualer Praktiken beim Peer-Learning

\begin{tabular}{ccccccccc}
\hline & $\begin{array}{c}\text { Aufgaben- } \\
\text { bearbeitung }\end{array}$ & \multicolumn{2}{c}{$\begin{array}{c}\text { Task- } \\
\text { Management }\end{array}$} & \multicolumn{2}{c}{$\begin{array}{c}\text { Metakognition \& } \\
\text { (Selbst-)Regulation }\end{array}$} & \multicolumn{2}{c}{ Sonstige } \\
\hline MW & S & MW & S & MW & S & MW & S \\
\hline LGT/D & 0,47 & 0,84 & 5,47 & 4,81 & 3,42 & 2,95 & 0,74 & 0,87 \\
\hline RGT/D & 0,20 & 2,19 & 6,36 & 9,11 & 2,72 & 3,9 & 0,28 & 0,61 \\
\hline LGT/D & & & & & & & & \\
+ & 0,32 & 0,67 & 5,98 & 7,50 & 3,02 & 3,50 & 0,48 & 0,76 \\
RGT/D & & & & & & & & \\
\hline
\end{tabular}

Im Mittel produziert jeder Proband fast sechs und knapp drei bilinguale Praktiken, die die Organisation der Aufgabenbearbeitung sowie der Zusammenarbeit betreffen respektive metakognitives Wissen repräsentieren und/oder regulative Funktionen erfüllen. Im Gegensatz hierzu werden bilinguale Praktiken kaum zur Aufgabenbearbeitung oder für sonstige On-Task-Funktionen eingesetzt, sodass diese Funktionen im Mittel nicht von jedem Probanden umgesetzt werden. Auch hinsichtlich der Unterkategorien der Hauptkategorie „On-Task“ kann eine relativ große interindividuelle Heterogenität in Anbetracht der Standardabweichungen konstatiert werden.

Die Gruppen LGT/D und RGT/D unterscheiden sich hinsichtlich der Mittelwerte der Unterkategorien kaum. Da die Werte der verschiedenen Funktionen der Hauptkategorien sich nach der Prüfung mit dem Shapiro-Wilk-Test nicht normalverteilen ( $W=.539-.787, d f=44, p<.001)$, werden potentielle signifikante Unterschiede mit dem non-parametrischen Mann-Whitney-U-Test geprüft. Lediglich bezüglich der Unterkategorie „Sonstige“ unterscheiden sich die Gruppen LGT/D $($ Median $=1)$ und RGT/D $($ Median $=0)$ signifikant $(U=$ 161,50, $p$ (zweiseitig) $=.032$ ).

\subsubsection{Nähere Beschreibung der Nutzungsweise bilingualer Praktiken für die vier Unterkategorien der Hauptkategorie „, On-Task“}

Im Folgenden werden die vier Unterkategorien der Hauptkategorie „On-Task“ bezüglich ihrer jeweiligen Bandbreite an auftretenden unterschiedlichen Zwecken der übergeordneten Funktionen der Aufgabenbearbeitung, des Task-Managements, der Metakognition und (Selbst-)Regulation sowie hinsichtlich sonstiger, den eben angeführten Funktionen nicht zuordnungsbaren Nutzungsweisen näher ausgeführt. Hierbei werden Beispiele präsentiert, die die konkrete Form der jeweiligen Funktionen und Zwecke illustrieren sollen. 
Bilinguale Praktiken werden nur sehr selten sowie lediglich von vereinzelten Dyaden für die Aufgabenbearbeitung eingesetzt. So werden manche Aufgabenstellungen samt der hierfür benötigten Kardinalzahlen im Türkischen artikuliert (,ehm 375 eh artı artı 200 e-e---elli-ellibeş. “ [375 eh plus plus $200 \mathrm{f}$ f---fünfzig- fünfundfünzig.]), allerdings anschließend nicht türkischsprachig ausgerechnet. Zudem werden kurze, obligatorische Handlungen zur Aufgabenbearbeitung, die sprachlich keine großen Anforderungen stellen, in äußerst seltenen Fällen mit bilingualen Praktiken umgesetzt. Im Gegensatz hierzu nutzte allerdings eine Dyade der Lesestrategieintervention bilinguale Praktiken zur Formulierung der Vorhersage des nächsten Abschnitts. Zwei Vorhersagen dieses Tandems sollen zur Illustration in der sie umgebenden Interaktionssequenz dargestellt werden, allerdings ohne diese feinkörnig interaktionsanalytisch zu analysieren:

P1: ,ser-servis sonra da onun içine binecek ehm sonra da diyecek herkes ih ih bu ne? bu ne? herşey her tarafi iğrenç sonra da o sonra da gine (yine?) ye gine (yine?) o yerine geldiğinde arkadaşlarının yanına gidecek orada da onlarlaN (onlarla?) yemek yiyecek." [Schulbus und dann werden alle da einsteigen und dann sagen: ,ih ih Was ist das? Was ist das?“ Alles überall ist es ekelhaft und dann nochmal und nochmal wenn er an seinen Platz kommt, geht er zu seinen Freunde und isst dort mit ihnen Essen.]

P2: was?

$\mathrm{P} 1$ : weil er langwei-

P2: der ist doch ein monster.

P1: ja und der hat doch monsterfreunde.

P2: achso die essen die ---

Der Proband P1 formuliert auf Türkisch eine Vorhersage zu dem nächsten zu lesenden Abschnitt. Der Dyadenpartner P2 versteht diese Vorhersage nicht ganz und fragt mit einem unspezifischen, im Deutschen formulierten „was?“ implizit nach einer Erläuterung dieser. P1 versucht daraufhin P2 klar zu machen, dass das Monster in der Geschichte sicherlich andere Monsterfreunde hat, woraufhin P2 schließt, dass das Monster der Geschichte mit seinen Monsterfreunden die Schulkinder verspeisen möchte. Dementsprechend wurde die mit bilingualen Praktiken formulierte Vorhersage anschließend im Deutschen erläutert und gerechtfertigt.

Diese Dyade animiert sich gegenseitig zur Verwendung bilingualer Praktiken für die Vorhersage, obwohl zumindest einer der Peers Türkisch nicht so gut zu verstehen scheint: 
P2: „ich denke, dass er da das --- tahminim sonrada ge- sonra biriler insanlar gelcek onu bulmaya çalışacak." [Meine Vermutung und danach, danach werden welche, Menschen kommen und versuchen ihn zu finden.]

P1: $\quad$ "|_(flüstert) türkisch“"

P1: , könntest du es einmal auch auf deutsch sagen? “

P2: „sonra da onu ehm öldürmek için yiyecekler." [Und dann ehm werden sie ihn essen, um ihn zu töten.]

P1: ,ja, das geht aber [Name von P2], könntest du es auch einmal auf deutsch sagen, denn ich habe es nicht ganz so verstanden."

Proband P2 formuliert seine Vorhersage zunächst auf Deutsch, bis P1 diesem flüsternd ins Wort fällt, was das Transkriptionszeichen ,|_ “ sowie dessen Positionierung ausdrückt, und P2 mit der Äußerung ,türkisch“ darauf hinweist, die Vorhersage auf Türkisch zu formulieren. Daraufhin pausiert P2 kurz, was das Transkriptionszeichen ,---“" markiert, und formuliert eine Vorhersage auf Türkisch. P1 bittet P2 anschließend, diese Vorhersage nochmal im Deutschen zu wiederholen, allerdings führt P2 seine Vorhersage um neue propositionale Elemente weiter auf Türkisch aus. P1 stimmt P2 zunächst zu, dass dies eine mögliche Vorhersage sein könnte, bittet P2 allerdings wiederholt, die Vorhersage auf Deutsch zu wiederholen, da er diese nicht ganz verstanden hat.

Anhand der kategorisierten Fälle des Task-Managements können bezüglich dieser Kategorie zwei übergeordnete Zwecke bilingualer Praktiken identifiziert werden: Zum einen werden hiermit von der Intervention vorgegebene Aufforderungen zur Bearbeitung der nächsten Teilschritte ausgedrückt und zum anderen wird die allgemeine Zusammenarbeit in der Dyade organisiert. Der Großteil der bilingualen Praktiken zum Task-Management wird zum Zwecke der erstgenannten Funktion eingesetzt und zwar zur Nennung der nächsten zu vollziehenden Arbeitsschritte der einzelnen Lesestrategien und des Teamrechnens sowie der Rechenkonferenz. Nach der Struktur der Intervention stellen diese Sprachhandlungen eine zentrale Aufgabe der Trainerrolle dar. Hierbei kann der Trainer auf die Promptkarten zurückgreifen, auf welchen die chronologische Reihenfolge der zu vollziehenden Teilschritte der einzelnen Lesestrategien und des Teamrechnens sowie der Rechenkonferenz vorgegeben sind, die in LGT/D und RGT/D sowohl im Deutschen als auch im Türkischen auf diesen Karten verschriftlicht sind. Diese auf den Promptkarten verschriftlichten Aufforderungen werden in unterschiedlicher Weise in Form bilingualer Praktiken umgesetzt. So werden die nächsten Arbeitsschritte zunächst im Deutschen und anschließend im Türkischen ausformuliert (,,wie willst du die aufgaben lösen? ödevi nasıl çözmek istiyorsun? " [[...] Wie willst du die Aufgabe lösen?]), aber auch nur türkischsprachige Prompts kommuniziert (,,ah lüt- 
fen kelimeyi ve cümleyi tekrar oku!" [Lies bitte das Wort und den Satz nochmal!]). Hierbei weichen die konkreten Formulierungen zum Teil von den Vorlagen der Promptkarten ab (,[...] tahmin ediyorsun?" [Was vermutest du?]; „devamını açıkla.“ [Begründe die Fortsetzung.]) und weisen manchmal auch grammatische Fehler und lexikalische Abweichungen auf (,kişileYE [kişiyle] ve ne- neyle neyli ilgili ö-önemli şey neledir?" [Was sind die wichtigsten Dinge über das Wer und Was?]).

Zudem werden bilinguale Praktiken zur allgemeinen Organisation der $\mathrm{Zu}$ sammenarbeit verwendet. So werden z. B. die zu bearbeitenden Strategien und Phasen angekündigt, wobei ebenfalls diese sukzessiv im Deutschen und Türkischen (,vorhersage, tahmin. bei einem film gibt es auch özet. die zeigen das auch bei paramparça meistens." [Vorhersage. [...] Zusammenfassung [...] „Paramparça“: Türkische TV-Serie]), aber auch nur im Türkischen artikuliert werden (,„özet, özet. “ [Zusammenfassung, Zusammenfassung.]. Die notwendigen Materialien für die zu bearbeitenden Aufgaben werden ebenfalls durch bilinguale Praktiken eingefordert (,ver!“ [gib!]), geklärt (,öbür taraf.“ [Andere Seite.]; ,ah hier böyle taktın ya buraya." [du hast das ja hier so dran gemacht.]), administriert (,ok, koy oraya.“ [leg sie dahin.]; „dur ben tutayım.“ [Warte ich halte es.]) und vergeben (,ben vereyim." [ich gebe es.]). Weiterhin werden auch Absprachen (,tamam. --- kartı çekiceğim, sana vericem, tamam?“" [Okay. --- Ich werde die Karte ziehen und dir geben, okay?]; „öbür sayfayamı geçicez?" [Gehen wir zur anderen Seite über?]) und Fragen (,zamanımız geçti?" [Ist unsere Zeit abgelaufen?]; ,[...]. lösungswegi mi yapmak zorunda miyız?" [Müssen wir den Lösungsweg machen?]) zum aktuellen Stand sowie zum Fortfahren bilingual kommuniziert. Hierzu gehören auch die Kommunikation des Beginnens mit (,,ben daha hazır değilim." [Ich bin noch nicht bereit.]) sowie das Anzeigen des Beendens einer Aufgabe oder eines Teilschritts („400 (räuspert sich) tüh--- miinus 395 ist gleich fünf. bitti.“ [Mist [...] [Ist fertig]; , ,alles alle kinder sind ausgestiegen --- und der bus --- kaputt --- bitti.“ [Fertig.]; ,bittik.“ [Wir sind fertig.]).

Hinsichtlich der Kategorie „Metakognition und (Selbst-)Regulation“ werden bilinguale Praktiken primär für regulatorische Zwecke und zum Teil zum Ausdruck von metakognitivem Wissen eingesetzt. Die bilingualen Praktiken zur Regulation richten sich zum Großteil an den Dyadenpartner, während Selbst-Regulationen sowie geteilte Gruppenregulation seltener auftreten. Hierbei werden die regulatorischen Funktionen der Kontrolle, des Monitoring sowie der Bewertung bedient, die sich nicht nur auf die Aufgabenbearbeitung sowie Zusammenarbeit beziehen, sondern auch emotionale und motivationale Zustände widergeben.

Die mit bilingualen Praktiken ausgedrückten Selbst-Regulationen beziehen sich vor allem auf die Bewertung der eigenen Aufgabenbearbeitung (,,nein, 
weil meine vorhersage ist falsch, çünkü ben dedim eh die kl-okulsinıf şey eh" [[...], weil ich gesagt habe eh die kl- die Schulklasse Dings eh]) oder der Zusammenarbeit (,ehm das hat mir gefallen auch und bunlar bana çok hoşuma gitti also güzeldi bugün" [Diese haben mir sehr gefallen also es war heute schön.]). Seltener treten Fälle von Monitoring auf, bei denen die Aufgabenbearbeitung im Prozess selbst überwacht wird („,...??) \#00:23:25\# beş ah sechs ich hab falsch. okay. häkchen. --- minus minus“ [[...] fünf [...]]; „warte ich habe das falsch gemacht. ich dussel --- allah ya." [[...] Oh Gott.]). Allerdings werden auch die eigenen emotionalen sowie motivationalen Zustände manchmal unter Verwendung von bilingualen Praktiken adressiert (,,sinirlerim ta şuraya çıktı başımın tepesine." [Meine Nerven, mir steht es bis zum Hals]). Mit bilingualen Praktiken werden nur sehr selten geteilte Regulationen ausgedrückt (,ok bunu da yazmamıza gerek yok. [...]“ "Es ist nicht nötig, dass wir das auch noch schreiben.]).

Am häufigsten richten sich die mit bilingualen Praktiken artikulierten Regulationen allerdings an den Dyadenpartner. Hinsichtlich des Monitorings wird der Dyadenpartner hierbei unter anderem aufgefordert, mit der Aufgabenbearbeitung endlich zu beginnen (,yap!“ [Mach!]; „du musst lesen. hadi!“" [[...], los!]) oder diese zu unterbrechen (,dur!“ [...] [Halt!]; ,dur bakayım. ja.“ [Warte mal. [...]]), sein Arbeitsverhalten zu verändern (,sus be.“ [Sei still man.]; , üf --- sinir ediyon beni ha. [Oh---du nervst mich, ey];) und auf potentiell falsche Lösungsansätze hingewiesen (,ya sen napıyon, du musst 280-276. was ist daran so schwer?" [Oh was machst du, [...]]). Kontroll- und Bewertungsprozesse werden häufig innerhalb eines Turns vollzogen, wobei mit bilingualen Praktiken primär die Bewertung ausgedrückt wird (,,doğru. null fehler gerade. mach." [Richtig. [...]]; ,ah sen de yaptın ha (pause) tamam ich habe monster." [du hast es auch gemacht (pause) okay [...]]; ,,nein, ja, doğru da hättest du ehm 53-3 gemacht zuerst. --- egal. yok." [[...] richtig [...] [Nein.]).

Mit bilingualen Praktiken drücken die Probanden auch metakognitives (Un-)Wissen aus. Ein beträchtlicher Anteil dieser Fälle wird durch bestimmte Teilschritte der Lesestrategie „Wörter klären“ initiiert, die metakognitives Wissen mit Teilschritten wie ,Steht eine Erklärung im Text?", „Weißt du selbst etwas darüber?" und „Helfen dir die Wortteile?" abfragen, welche für gewöhnlich mit ,hayir" beantwortet und dementsprechend verneint werden. Weiterhin werden bilinguale Praktiken von den Peers verwendet, um sich gegenseitig transparent zu machen, ob sie über benötigtes oder zu erarbeitendes Wissen bei einer Aufgabe verfügen (,biliyom.“ [Ich weiß.]; „,bilmem“. [Weiß nicht.]; ,ich weiss, ben biliyom ne diceğimi. “ [[...] ich weiß nicht, was ich sagen soll.]). Zudem werden Ausdrücke der Verwunderung (,„̧üş.“ [Oha]; ,allah.“ [Oh Gott.]) mit bilingualen Praktiken markiert. 
Die meisten Fälle der Unterkategorie „Sonstige“ lassen sich zwar der Hauptkategorie „On-Task“ zuordnen, können aber aufgrund fehlender semantischer Klarheit nicht eindeutig einer anderen Unterkategorie zugeordnet werden. Allerdings treten in dieser Kategorie auch einige Korrekturen (,,bul! nicht bol!"“ [Finde! Nicht reichlich!]) und Reflexionen türkischsprachiger Äußerungen auf. Anstatt wie zuvor vor allem einzelne Turns zur Illustration zu berichten, soll hierfür eine kurze Interaktionssequenzen dargestellt, allerdings nicht näher interaktionsanalytisch analysiert werden, in der die adäquate Lexik beziehungsweise grammatisch korrekte Verbalform ko-konstruktiv erörtert wird:

P1: „also ich, ben tahmin ediyorum ehm o şimdi o okul okul (...?) \#00:41:04\# o okula hää“[ich vermute ehm er/sie/es jetzt diese Schule, Schule zu dieser Schule]

P2: „otobüsü.“ [Den Bus.]

P1: „o onun için. es gab es noch extra noch ein wort dafür, weil “- [Der ist dafür.]

P2: „-schulbus.“

$\mathrm{P} 1:$,nein, aber auf türkisch. in der türkei. servis midir nedir. service oder so“ [Schulbus oder so]

P2: „okul servisi.“ [Schulbus.]

Diese kurze Interaktionssequenz stammt aus der Anwendung der Lesestrategie „Vorhersage“ einer Dyade, bei der P1 eine Vorhersage für den nächsten Abschnitt formulieren möchte. Hierbei scheint P1 Formulierungsprobleme im Türkischen zu haben, die sein Dyadenpartner P2 als lexikalische Lücke für „Bus“ beziehungsweise „den Bus“ interpretiert. Die Intention von P2 war richtig, allerdings sucht P1 das spezifische türkische Lexem für Schulbus, welchem sich P1 mit ,servis midir nedir“ annähert und die Vermutung äußert, dass „service“ ein Bestandteil des gesuchten Lexems darstellt. P2 kann daraufhin das gesuchte Wort als „okul servisi“ identifizieren. 


\section{Diskussion}

Die Diskussion wird aufgrund der Vielzahl an Ergebnissen sowie der Datenintegration der quantitativen und qualitativen Resultate folgendermaßen strukturiert: Zunächst werden die einzelnen Ergebnisse vor dem theoretischen Hintergrund und der bisherigen empirischen Befundlage diskutiert (Abschnitt 9.1). Danach werden die quantitativen und qualitativen Ergebnisse im Sinne der Datenintegration bei Mixed-Methods Designs (vgl. Kuckartz, 2017) miteinander in Beziehung gesetzt sowie die zentralen Erkenntnisse kurz zusammengefasst (Abschnitt 9.2). Anschließend werden Implikationen für die Praxis auf Basis der Diskussion und Integration der Ergebnisse abgeleitet (Abschnitt 9.3). Abschließend werden die Limitationen der Studie kritisch diskutiert und Forschungsdesiderate angesichts des aktuellen Erkenntnisstandes reflektiert (Abschnitt 9.4).

\subsection{Diskussion der einzelnen Ergebnisse der Studie}

Das Ergebnis der Fragestellung F1 weist eine hohe sowie signifikante paarweise Korrelation der Prävalenz bilingualer Praktiken der Dyadenpartner auf, was die Abhängigkeit der Prävalenz bilingualer Praktiken von der Dyade empirisch bestätigt. Dementsprechend handelt es sich bei der Prävalenz bilingualer Praktiken beim PL mit explizitem bilingualen Interaktionsangebot um eine dyadische „mixed variable“, die zwar für jeden Peer derselben Dyade unterschiedlich ausfallen kann, deren Werte sich allerdings zwischen Dyadenpartnern stärker ähneln als Werte von Peers aus zwei unterschiedlichen Dyaden (vgl. Kenny, Kashy \& Cook, 2006, S. 4ff).

Die Abhängigkeit der Prävalenz bilingualer Praktiken von der Dyade beim PL ist angesichts theoretischer Reflexionen zum Phänomen der Interaktion sowie zu potentiellen Einflussfaktoren auf die Sprachwahl und das Sprachverhalten bilingualer Sprecher zu erwarten. Interaktionen - verstanden als die „wechselseitige Beeinflussung des Handelns mindestens zweier Personen“ (Sarges \& Fricke, 1980, S. 488; zit. nach Nolda, 2000, S. 9) oder als ,die elementare Einheit des sozialen Geschehens, in der Menschen ihr Verhalten aneinander orientieren" (Endruweit \& Trommersdorf, 1989, S. 310; zit. nach ebd.) - werden qua Definition maßgeblich von den Wechselwirkungen der miteinander kommunizierenden Individuen determiniert. Die Abhängigkeit der Prävalenz bilingualer Praktiken von der Dyade beim PL kann als Bestätigung zentraler theoretischer Prämissen soziolinguistisch orientierter Modelle der Sprachwahl und der Sprachpraxis von bilingualen Sprechern (vgl. z. B. Auer, 1988; Grosjean, 2008; Myers-Scotton, 2006; Sachdev, Giles \& Pauwels, 2013) 
interpretiert werden. Grosjeans (2008) Sprachmodi akzentuieren neben individuellen, kontextuellen und inhaltlichen Einflussfaktoren auch die Beziehung und Intimität der Gesprächspartner als Determinanten des Sprachmodus, was eine individuelle Perspektive auf bilinguales Sprachverhalten zu Gunsten eines holistischeren Blicks auf die Dyade der Gesprächspartner abschwächt. Mit Ausnahme der individuellen Eigenschaften gelten zum Großteil die gleichen kontextuellen und inhaltlichen Bedingungen für die beiden Gesprächspartner, sodass diese sich in ihrem Sprachverhalten eher ähneln sollten. Lediglich in Fällen, in denen ein Gesprächspartner sich vom anderen distanzieren möchte, um Divergenz zwischen den Gesprächspartnern zu markieren (vgl. Sachdev, Giles \& Pauwels, 2013), dürften die Interaktanten ein sich unterscheidendes bilinguales Sprachverhalten exerzieren. Da sich PL als Sozialform allerdings durch Kollaboration und Kooperation auszeichnet, auch wenn Divergenzen wie z. B. in Form von Widersprüchen äußerst lernwirksam sind (vgl. Howe, 2010), dürften die Gesprächspartner eher Konvergenz als Ziel anvisieren und sich dementsprechend stärker in ihrem Sprachverhalten annähern. Myers-Scotton (2006) modelliert bilinguale Sprachpraxis wie Sachdev, Giles und Pauwels (2013) ebenfalls als bewusste Sprachwahl und Sprachpraxis zur Erreichung eines Kommunikationsziels, allerdings nicht auf Basis zwischenmenschlicher Beziehungen, sondern auf normativer Ebene der Einhaltung von oder des Bruchs mit gesellschaftlichen Konventionen. Da das bilinguale Sprachangebot in den Trainings der vorliegenden Studie den Status einer offiziellen Unterrichtssprache erhält, kann bilinguale Interaktion als temporär gültige Norm im Rahmen der Lese- und Rechenstrategieintervention verstanden werden. Aufgrund der durch das PL strukturierten Interdependenz der Dyadenpartner sollten sich beide Peers an einer ähnlichen Auslegung der Sprachnorm orientieren, um das übergeordnete Ziel der Konvergenz zu wahren. Auer (1988) hingegen akzentuiert die Interaktion sowie den Diskurs selbst als zentrale Einflussgröße für die Sprachwahl und das Sprachverhalten, was auch durch die empirische Befundlage zu diskursiv-rhetorischen Zwecken bilingualer Praktiken (vgl. z. B. Auer, 2009; Fuller, 2009, S. 120-129; Gardner-Chloros, 2009, S. 67ff; Keim, 2012, S. 157ff; Potowski, 2009; Reyes, 2004; Ritchie \& Bhatia, 2013, S. 384ff) gestützt wird. Da die Gesprächspartner sich in derselben Interaktion befinden und den Diskurs gemeinsam gestalten, sollten sich die Interaktanten in ihrer Sprachpraxis auch hinsichtlich Auers (1988) Modell annähern.

Nichtsdestotrotz scheint die Abhängigkeit des bilingualen Sprachverhaltens von den Gesprächspartnern in der Bilingualismusforschung bisher primär eine nicht hinterfragte theoretische Prämisse darzustellen, die mit Hilfe von diversen Studien mit unterschiedlichen Untersuchungsanlagen (vgl. z. B. Grosjean, 1997; Poplack, 1981; Treffers-Daller, 1998) zwar empirisch untermauert wurde, allerdings - zumindest nach den Recherchen des Autors der vorliegenden Studie - bisher nicht explizit mit Methoden dyadischer Datenanalyse anhand der Prävalenz bilingualer Praktiken in der Interaktion geprüft 
wurde. In Studien, die bilinguale Praktiken beim PL auf der Dyadenebene quantifizieren (vgl. Schüler-Meyer et al., 2017; Storch \& Wigglesworth, 2003; Swain \& Lapkin, 2000), könnte eine Berücksichtigung der Abhängigkeit der Prävalenz von bilingualen Praktiken von der Dyade vermutet werden. Allerdings erwähnen die soeben aufgezählten Studien die Abhängigkeit der Prävalenz bilingualer Praktiken von der Dyade beim PL mit explizitem bilingualem Interaktionsangebot nicht, weder auf theoretischer und empirischer noch auf statistisch-methodischer Ebene. Auch Studien, die die Quantität von bilingualen Praktiken auf Individualebene erfassen (vgl. z. B. Planas \& Setati, 2009), nehmen keine korrelativen Analysen der individuellen Prävalenzen der Gruppenmitglieder vor.

Insgesamt scheint die Abhängigkeit der Prävalenz von bilingualen Praktiken von der Dyade beim PL in der Bilingualismusforschung bisher nicht als eine empirisch zu prüfende Hypothese wahrgenommen worden zu sein, sondern als ein allgemeines theoretisches Postulat. Die in der vorliegenden Arbeit explizit identifizierte Abhängigkeit der Prävalenz bilingualer Praktiken von der Dyade beim PL ist nicht nur von theoretischer, empirischer und praktischer Bedeutung, sondern weist auch für die statistische Auswertung der Daten eine hohe Relevanz auf. Die Prävalenz bilingualer Praktiken gilt es für eine adäquate Analyse auf Dyaden- oder Gruppenebene zu analysieren, da eine Missachtung der Abhängigkeit der Daten von der Dyade zu statistischen Verzerrungen führen würde (vgl. Kenny, Kashy \& Cook, 2006, S. 46ff). Dementsprechend konnte die vorliegende Studie unter Verwendung dyadischer Datenanalyse empirisch demonstrieren, dass die Prävalenz bilingualer Praktiken beim PL mit explizitem bilingualen Interaktionsangebot abhängig von der Dyade ist.

Wie das Ergebnis von F2a deutlich zeigt, führt das explizite bilinguale Interaktionsangebot mit einer hohen Effektstärke zu einer signifikant höheren Prävalenz bilingualer Praktiken beim PL als bei fehlendem Angebot. Zwar treten bilinguale Praktiken auch im Kontext eines fehlenden Angebots samt maximal zwei Aufforderungen der Übungsleitung zur Unterlassung bilingualer Kommunikation auf, allerdings sind die Prävalenzen bilingualer Praktiken in diesem Kontext gering und erfolgen auch nur in einzelnen Dyaden. Nichtsdestotrotz weist dieser Befund auf einen gewissen Grad an Unvermeidlichkeit von bilingualer Interaktion bei bilingualen Sprechern im Bildungskontext hin (vgl. z. B. Cook, 2001, S. 405; García \& Kano, 2014), was das Postulat der permanenten Aktivierung beider Sprachen der Sprachmodi nach Grosjean (2008) stützt. Nur die Studien von Schüler-Meyer et al. (2017) und Storch und Wigglesworth (2003) alternieren systematisch zwischen einem expliziten Angebot und keinem Angebot bilingualer Praktiken beim PL. Allerdings thematisieren weder Schüler-Meyer et al. (2017) noch Storch und Wigglesworth (2003) potentielle Gruppenunterschiede, da sie keine Quantitäten bilingualer Interaktion beim fehlenden Angebot für bilinguale Praktiken benennen. Insbesondere an- 
gesichts des bei Schüler-Meyer et al. (2017) antizipierten Ziels der Identifikation der Lernwirksamkeit des bilingualen Sprachangebots hinsichtlich Bruchrechnen müsste das Sprachverhalten der Nicht-Angebotsgruppen kontrolliert werden, um die Einhaltung der Gruppenvariation bilingualer und monolingualer Interaktion zu überprüfen. Denkbar wäre, dass auch in diesen Gruppen bilinguale Praktiken auftreten, wie es auch in der vorliegenden Untersuchung der Fall war.

Angesichts der Prävalenzwerte bilingualer Praktiken beim PL von nicht weniger als $85 \%$ (Planas \& Setati, 2009), von 31\% (Schüler-Meyer et al., 2017) sowie abhängig von der jeweiligen Aufgabe von 30\% bis 50\% (Storch \& Wigglesworth, 2003) und $21 \%$ bis $29 \%$ (Swain \& Lapkin, 2000) fällt die Quantität bilingualer Interaktion in der vorliegenden Studie mit einem Mittelwert von ungefähr zehn Prozent deutlich geringer aus. Die Probanden der Untersuchungen von Planas und Setati (2009), Storch und Wigglesworth (2003) sowie sehr wahrscheinlich auch von Swain und Lapkin (2000) weisen eine Sprachasymmetrie zu Gunsten der L1 auf, was die hohen Prävalenzen bilingualer Praktiken beim PL zum Teil erklären könnte. Eventuell könnte es sich hierbei um Formen des „dependent translanguaging“ (vgl. García \& Kano, 2014) handeln, sodass die Probanden zum Teil auf den Rückgriff auf ihre L1 angewiesen sind, um die Aufgabe bearbeiten zu können. Im Gegensatz hierzu zeichnen sich die Probanden der Studie von Schüler-Meyer et al. (2017) anhand ihrer Werte in C-Tests des Türkischen und Deutschen im Mittel zumindest deskriptiv durch eine Dominanz des Deutschen aus, was aber inferenzstatistisch von den Autoren nicht auf Signifikanz überprüft wurde. Allerdings lagen in dieser Studie alle Materialien in den Gruppen mit explizitem bilingualen Sprachangebot in zweisprachiger Ausführung vor und in der Intervention wurden auch konkrete sprach- und konzeptvergleichende Aufgaben der türkischund deutschsprachigen Realisierung von Brüchen bearbeitet (vgl. ebd.), was beides die Prävalenz bilingualer Praktiken steigern könnte. Die Stichprobe der vorliegenden Untersuchung zeichnet sich durch eine Asymmetrie zu Gunsten der Verkehrssprache Deutsch aus und die Intervention verzichtet mit Ausnahme der Promptkarten der Gruppen LGT/D und RGT/D auf zweisprachige Materialien. Dementsprechend könnten sowohl Sprachasymmetrien zu Gunsten der nicht-verkehrssprachlichen L1 als auch spezifische zweisprachige Materialien samt sprachvergleichenden, das zu lernende Konzept betreffende Aufgaben die Prävalenz bilingualer Praktiken beim PL erhöhen.

Angesichts des Ergebnisses von F2b haben die Domänen sowie die konkreten Lerninhalte keine große Bedeutung für die Prävalenz bilingualer Praktiken beim PL, da sich die Prävalenzen bilingualer Praktiken der Gruppe der Lesestrategieintervention nicht von der Gruppe der Rechenstrategieintervention signifikant unterscheiden. Nicht nur hinsichtlich der Domänen unterscheiden sich Lese- und Rechenstrategien voneinander, sondern auch in ihrer Spezifizität sowie der Vielfältigkeit der Ziele. Einige Lesestrategien wie z. B. das 
Zusammenfassen zeichnen sich durch eine geringe Spezifizität und vielfältige potentiell zu verfolgende Ziele aus, während Halbschriftliche Rechenstrategien stets auf eine Vereinfachung des Rechenprozesses abzielen und eine sehr hohe Spezifizität aufweisen, da sie sich nur für bestimmte Aufgaben eignen. Nichtsdestotrotz werden bilinguale Praktiken in zu vergleichender Quantität für diese beiden Domänen und Lerninhalte eingesetzt. Zwar weisen die Mittelwerte der Gruppen LGT/D und RGT/D deskriptiv einen deutlichen Unterschied auf, der allerdings angesichts der hohen Standardabweichungen relativiert wird. Zudem weist der Vergleich insgesamt eine geringe Stichprobengröße auf, was die Wahrscheinlichkeit der Identifikation eines signifikanten Unterschieds zusätzlich vermindert.

Hinsichtlich der Untersuchung der Bedeutung bilingualer Praktiken in Bildungsprozessen allgemein und beim PL wird häufig auf Lesen oder Rechnen als Domänen zurückgegriffen. So gibt es diverse Untersuchungen, die die Fruchtbarkeit bilingualer Praktiken für die Domäne des Lesens (vgl. z. B. García \& Kano, 2014; Hopewell, 2011; Klingner \& Vaughn, 2000; Swain \& Lapkin, 2000; Storch \& Wigglesworth, 2003) sowie des Rechnens (Barwell, 2014; Clarkson, 2007; Moschkovich, 2007; Planas \& Setati, 2009; SchülerMeyer et al., 2017) bestätigen. Allerdings hat keine Studie bisher zwei verschiedene Domänen hinsichtlich der Prävalenz bilingualer Praktiken beim PL verglichen, sondern lediglich verschiedene Aufgaben (vgl. z. B. Storch \& Wigglesworth, 2003; Swain \& Lapkin, 2000) oder Textgrundlagen (vgl. z. B. Hopewell, 2011) derselben Domäne variiert. Zudem liegen auch für Bildungsprozesse im Allgemeinen keine Studien vor, die die Prävalenz und/oder Bedeutung von bilingualen Praktiken in Bildungsprozessen für verschiedene Lerninhalte miteinander kontrastieren. Anhand der vorliegenden Ergebnisse lässt sich für die Annahme des Angebots und deren quantitative Umsetzung schließen, dass bilinguale Sprachangebote für die verschiedenen Domänen Lesen und Rechen als auch für die Lerninhalte Lese- und Rechenstrategien eingesetzt werden können.

Mit der Fragestellung F2c wurde die Prävalenz bilingualer Praktiken hinsichtlich ihrer Abhängigkeit von bestimmten Phasen und Strategien innerhalb der Lese- und Rechenstrategieintervention geprüft, wobei sich lediglich für einzelne Phasen des Lesetrainings signifikante Unterschiede ergeben haben. So ist die Prävalenz bilingualer Praktiken bei den Lesestrategien „Wörter Klären“, „Zusammenfassen“ und „Vorhersagen“ signifikant größer als die der Phase „Frage zum Abschnitt“. Dementsprechend können bilinguale Praktiken bei explizitem Angebot dieser beim PL auch für zentrale Inhalte des Curriculums wie Lesestrategien verwendet werden. Insbesondere die Strategie „Wörter Klären" ermöglicht theoretisch die fruchtbare Integration bilingualer Praktiken bei Lesestrategien durch den Rückgriff auf die (weitere) L1 zum Füllen lexikalischer Lücken, was speziell SuS mit einer Sprachasymmetrie zu Gunsten der nicht-verkehrssprachlichen L1 unterstützen könnte. 
Die geringe Prävalenz bilingualer Praktiken bei der Phase „Frage zum Abschnitt" könnte auf dem deutschsprachigen Input dieser Phase beruhen. Die Einzelschritte der Lesestrategien können mit oder ohne Verwendung der zweisprachigen Promptkarten auch im Türkischen kommuniziert werden. Eine türkischsprachig formulierte Aufforderung zur Ausführung eines Einzelschrittes erhöht die Wahrscheinlichkeit, dass der Peerpartner die Interaktion in der gleichen Sprache fortführt. Die Frage zum Abschnitt ist stets im Deutschen formuliert, sodass die Beantwortung dieser ebenfalls eher im Deutschen erfolgen könnte.

Im Rechenstrategietraining hingegen unterscheiden sich die Prävalenzen nicht signifikant zwischen den verschiedenen Phasen. Die zwei zentralen Phasen des Teamrechnens und der Rechenkonferenz weisen zwar strukturelle und handlungsbezogene Unterschiede auf wie z. B. das Interagieren mit und ohne Rollen oder das Bearbeiten von Rechenaufgaben mit oder ohne Monitoring dieses Prozesses durch einen Peer. Prinzipiell liegt der Schwerpunkt der beiden Phasen allerdings auf der flexiblen Selektion geeigneter Rechenstrategien zur Reduktion der Anforderungen der Rechenprozesse sowie zur Steigerung der korrekten Lösungswahrscheinlichkeit. Dementsprechend ist es nicht verwunderlich, dass sich die beiden Phasen bezüglich der Prävalenz bilingualer Praktiken nicht unterscheiden, da sie dieselben Inhalte und Prozesse fokussieren.

Hinsichtlich der bisherigen empirischen Befundlage zur Prävalenz bilingualer Praktiken beim PL in Abhängigkeit der Phasen und Aufgaben gibt es kaum vergleichbare Studien, die herangezogen werden könnten, da diese Fragestellungen zum Großteil innerhalb von „Between-Subjects-Designs“ untersucht werden und eher verschiedene Aufgabenformate oder Materialgrundlagen variieren, anstatt distinkte Phasen und/oder kognitive Lernstrategien zu fokussieren (vgl. z. B. Storch \& Wigglesworth, 2003; Swain \& Lapkin, 2000). Diese Studien relativieren allerdings die Abhängigkeit der Prävalenz bilingualer Praktiken von der Aufgabe oder dem Material, da Swain und Lapkin (2000) keine signifikanten Unterschiede identifizieren und Storch und Wigglesworth (2003) aufgrund des Fallstudiencharakters nur deskriptive Werte berichten. Die Untersuchung von Planas und Setati (2009) und auch von Hopewell (2011) operieren zwar mit „Within-Subjects-Designs“, allerdings vergleicht die erstgenannte Studie die Prävalenz bilingualer Praktiken zwischen verschiedenen Sozialformen des Unterrichts (PL und Diskussionen im Klassenverbund) und die zuletzt genannte differierende Textgrundlagen abhängig von ihrem Lebensweltbezug. Dementsprechend lassen sich kaum fruchtbare Vergleiche sowie Einordnungen der vorliegenden Befunde in den bisherigen Forschungsstand vollziehen. Allerdings liefert die vorliegende Untersuchung Hinweise darauf, dass sich - zumindest für die Domäne Lesen - innerhalb verschiedener Phasen und Strategien beim PL die Prävalenz bilingualer Praktiken unterscheiden kann, was die Dynamik bilingualer Interaktion auf einem Kontinuum eines bilingualen bis monolingualen Sprachmodus akzentuiert (vgl. Grosjean, 2008). 
Die Fragestellungen F3a und F3b fokussieren potentielle Zusammenhänge individueller sprachlicher Eigenschaften respektive motivationaler Merkmale der Peers mit der Prävalenz bilingualer Praktiken. Allerdings konnten in der vorliegenden Studie keine signifikanten Zusammenhänge weder für die sprachlichen noch motivationalen Variablen mit der Prävalenz bilingualer Praktiken identifiziert werden. Dies dürfte vor allem an statistisch bedeutsamen Faktoren wie der Stichprobengröße, der geringen Varianz samt Deckeneffekten und teils fragwürdigen bis inakzeptablen internen Konsistenzen einzelner Fragebogenskalen liegen. Da eine Vielzahl inter- aber auch intraindividueller Faktoren das Sprachverhalten potentiell beeinflussen kann, dürften die Effektstärken der einzelnen Faktoren nicht besonders hoch ausfallen. Die Stichprobengröße der vorliegenden Studie verfügt nur über eine eingeschränkte Power, sodass die als klein einzuschätzenden Effekte der einzelnen Faktoren mit dieser Stichprobengröße eher nicht identifiziert werden können. Hierzu kommt noch die geringe Varianz samt Deckeneffekten einzelner sprachlicher und motivationaler Skalen, was ein Auffinden signifikanter Zusammenhänge zunehmend erschwert, insbesondere wenn einige Skalen aufgrund ihrer eher schlecht ausgeprägten internen Konsistenz die operationalisierten Konstrukte in ihren einzelnen Facetten nicht gut repräsentieren.

Die bisherigen Studien zu bilingualen Praktiken beim PL nehmen keine Zusammenhangsanalysen hinsichtlich der Prävalenz bilingualer Praktiken beim PL mit sprachlichen und/oder motivationalen Eigenschaften der Probanden vor. Auf theoretischer Ebene sprechen allerdings alle soziolinguistisch orientierten Modelle der Sprachwahl und des Sprachverhaltens bilingualer Sprecher sprachlichen Merkmalen der interagierenden Individuen eine gewisse Relevanz zu (vgl. z. B. Auer, 1988; Grosjean, 2008; Myers-Scotton, 2006; Sachdev, Giles \& Pauwels, 2013), auch wenn sie eigentlich andere Konstrukte wie Konvergenz und Divergenz, Einhaltung oder Bruch mit Normen oder den Diskurs selbst akzentuieren. Aus anerkennungstheoretischer Perspektive (vgl. z. B. Mecherill, 2005; Stojanov, 2006, 2011) sowie der „Self-DeterminationTheory“ von Ryan und Deci (2018) sind Zusammenhänge motivationaler Variablen mit der Prävalenz bilingualer Praktiken ebenfalls denkbar. Dementsprechend steht die empirische Konfirmierung eines Zusammenhangs sprachlicher und motivationaler Eigenschaften von Individuen mit ihrer Prävalenz bilingualer Praktiken beim PL noch aus.

Die qualitativen Fragestellungen fokussieren nicht die Prävalenz bilingualer Praktiken beim PL, sondern von den Probanden selbstberichtete Begründungen zur Annahme oder Ablehnung des Sprachangebots (F4) und Funktionen bilingualer Praktiken beim PL (F5). Die in F4 analysierten Selbstberichte der Probanden zur Annahme oder Ablehnung des Sprachangebots sowie deren Begründungen erlauben trotz ihrer Kürze sowie der häufigen Nennung eines einzelnen Grundes zur Legitimation ihres Sprachverhalten fruchtbare Einblicke in Chancen und Herausforderungen bilingualer Praktiken beim PL. Die 
Probanden berichten für beide Erhebungszeitpunkte signifikant häufiger, das Angebot angenommen statt abgelehnt zu haben, wobei die meisten Probanden konsequent in eine Richtung über beide Erhebungszeitpunkte antworten. Die inkonsequenten Antwortmuster lassen sich mit der Dynamik der Sprachmodi nach Grosjean (2008, S. 45) erklären, da bilinguale Gesprächspartner auf dem Kontinuum zwischen den verschiedenen Sprachmodi wechseln können. So könnten die inkonsequent antwortenden Probanden im Verlauf des Trainings zwischen dem monolingualen, gemischtsprachlichen oder bilingualen Sprachmodus abhängig von sich ändernden Einflussfaktoren diffundieren, sodass sie zu den verschiedenen Erhebungszeitpunkten unterschiedliche Antworten liefern.

Dieses geschlossene, dichotome Format sollte in seiner Bedeutung allerdings nicht überschätzt werden, da es in seiner Interpretierbarkeit limitiert ist. Zum einen ist unklar, welche Menge oder Qualität bilingualer Praktiken die einzelnen Probanden als Cut-Off zu Grunde legen, um den Impuls „Hast du im Training Türkisch gesprochen?" zu affirmieren oder zu negieren. Zum anderen lässt sich ebenfalls nicht schließen, auf welchen Zeitraum sich die Antworten zu Erhebungszeitpunkt 1 und 2 beziehen. So lässt sich zwar vermuten, dass die Probanden zu Erhebungszeitpunkt 1 die ersten sieben Sitzungen in ihrer Antwort berücksichtigen, aber nicht eindeutig klären, ob sie zu Erhebungszeitpunkt 2 alle Sitzungen oder nur die Sitzungen acht bis zwölf in Betracht ziehen.

Die selbstberichteten Argumente für die Annahme und Ablehnung des bilingualen Interaktionsangebots fokussieren deskriptiv am häufigsten Eigenschaften, Wahrnehmungen, Einstellungen sowie Ziele des Individuums. Dies liegt eventuell auch in der Formulierung des Items begründet, welches mit dem Impuls „Hast du im Training Türkisch gesprochen? Warum?“ das Individuum direkt adressiert. Denkbar wäre, dass diese Formulierung die Ebene des Individuums salient macht, sodass die Probanden primär die Gründe für die Annahme oder Ablehnung des bilingualen Sprachangebots bei sich selbst verorten. Im Gegensatz hierzu spielt die Ebene des Peertandems zur Legitimation des Sprachverhaltens keine große Rolle. Lediglich 13 von insgesamt 102 Antworten können der Gesprächspartnerebene zur Begründung des bilingualen Sprachverhaltens zugeordnet werden. Dies ist verwunderlich, da die Forschung zu Einflussfaktoren der Sprachwahl und des Sprachverhaltens bilingualer Sprecher stets auch das Verhältnis und somit reziproke Wechselwirkungen der Gesprächspartner als Determinante bilingualen Interaktionsverhalten akzentuiert (vgl. Grosjean, 2008; Myers-Scotton, 2006; Sachdev, Giles \& Pauwels, 2013). Während das Individuum und die Gesprächspartner in den Selbstberichten der Probanden als Argumentationsebenen sowohl für die Annahme als auch Ablehnung des expliziten Sprachangebots fungieren, ist dies bei der Kontextebene nicht der Fall. Zwar weist diese Ebene bei der Annahme des Angebots hohe Fallzahlen auf, sie spielt allerdings bei der Ablehnung des An- 
gebots mit lediglich einem Fall keine Rolle. Dabei wären Antworten, die implizit den monolingualen Habitus der deutschen Regelschule oder allgemein den Submersionskontext als Argumente zur Ablehnung des Sprachangebots adressieren, aufgrund ihrer zentralen Bedeutung für sprachliches Verhalten in der Schule theoretisch zu erwarten gewesen. In acht Fällen konnte die Annahme oder Ablehnung des Sprachangebots nicht begründet werden, obwohl das geschlossene Format des Items beantwortet wurde. Dies weist auf das theoretische Postulat hin, dass bilinguale Sprecher sich nicht immer zwingend über die zu Grunde liegenden Determinanten ihres Sprachverhaltens bewusst sind (vgl. Grosjean, 2008, S. 45). So kann z. B. ein Zusammenspiel mehrerer Faktoren das bilinguale Sprachverhalten beeinflussen, was die Identifikation einer konkreten Begründung aufgrund der Interaktion dieser Faktoren obstruieren könnte. Zudem können die einzunehmenden Positionen auf dem Kontinuum der Sprachmodi sich dynamisch verändern (vgl. ebd.), sodass zu unterschiedlichen Zeitpunkten des Trainings die Salienz einzelner Faktoren sowie deren Konstellationen nicht stabil sind, was z. B. durch einen Wechsel der Trainerrollen (vgl. Rubinstein-Ávila, 2003), der Gesprächsgegenstände oder der Trainingsphasen initiiert werden könnte und eine Benennung konkreter Argumente für das jeweilige Sprachverhalten erschwert.

Die Kategorie „KE Aufforderung“ der Kontextebene ist mit 19 Fällen die am häufigsten adressierte Kategorie, sodass fast ein Fünftel aller Selbstberichte dieser Kategorie entsprechen. Das Sprachangebot wird dementsprechend von einem gewissen Teil der Probanden als ein obligatorischer Bestandteil der Intervention interpretiert, den es zu erfüllen gilt, obwohl es eigentlich explizit als Angebot instruiert wurde. Dies könnte einerseits an der Vielzahl und Vielfältigkeit der Aktivierungen eines bilingualen Sprachmodus über Fallvignetten, Aufforderungen zur Erläuterung der in den Instruktionssitzungen erarbeiteten Lese- oder Rechenstrategien auf Türkisch oder Türkisch und Deutsch gemischt, zweisprachigen Promptkarten, wiederholtes Verweisen auf das bilinguale Interaktionsangebot während des Feedbacks der Sitzungen sieben und zehn etc. liegen. Insbesondere die wiederholte Erinnerung an das bilinguale Sprachangebot sowie Aufforderungen zur Produktion bilingualer Praktiken bei der Erläuterung von Lese- und Rechenstrategien könnten den Angebotscharakter der bilingualen Interaktion überschreiben, sodass der Eindruck einer obligatorisch zu erfüllenden Bedingung entsteht. Andererseits könnten die Aktivierungen zum Teil als zusätzliche Belastung empfunden worden sein, da den Probanden Erfahrung im Umgang mit ihren beiden Sprachen bei Bildungsprozessen fehlt. Das bilinguale Sprachangebot könnte innerhalb eines vom monolingualen Habitus sowie der Sprachasymmetrie zu Gunsten des Deutschen geprägten Schulkontextes von den Probanden zunächst nicht als eine Form rechtlicher sowie solidarischer Anerkennung oder gar als fruchtbare Hilfestellung für Bildungsprozesse interpretiert werden, sondern als weitere zu erfüllende 
Aufgabe, die neben den Lese- und Rechenstrategien ebenfalls kognitive Ressourcen bindet. Dies könnte darauf hinwiesen, dass entweder den bilingual aufwachsenden Probanden die Restriktionen des monolingualen Habitus nicht bewusst sind oder sie diese nicht als kritikwürdig einstufen. Die Sozialisation der Probanden innerhalb einer ,assimilation ideology“ (vgl. Bourhis, 2001) könnte zu einer Verinnerlichung des monolingualen Habitus führen, sodass bilinguale Interaktion in ihrer Wahrnehmung keine naheliegende und/oder legitime Unterrichtspraxis darstellt. Es gibt empirische Hinweise für solche Entwicklungen bei migrationsbedingter Mehrsprachigkeit, da z. B. Gardner-Chloros, McEntee-Atalianis und Finnis (2005) negative Zusammenhänge von Aufenthaltsdauer sowie Bildungsgrad mit der Einstellung zu Code-Switching bei bilingualen Sprechern mit MH identifizierten oder Krumm (2009) bei ca. einem Viertel der Sprachenportraits bilingual aufwachsender Kinder und Jugendlicher im Submersionskontext Formen der Geheimhaltung oder Negation der nicht-verkehrssprachlichen L1 auffindet.

Im Gegensatz hierzu akzentuieren lediglich zwei Fälle das Angebot selbst als Grund zur Annahme des bilingualen Sprachangebots. Die Verwendung der Modalverben „,ürfen“ und „können“ in den Selbstberichten drücken eine Erlaubnis oder eine Berechtigung aus, was zumindest als eine Kontrastierung zum typischen monolingualen Sprachverhalten oder gar als implizite Kritik am monolingualen Habitus der Regelschule interpretiert werden könnte. Zudem wird in nur drei Fällen das bilinguale Interaktionsangebot als eine Chance betrachtet, die sprachlichen Fähigkeiten im Türkischen auszubauen. Dementsprechend lassen sich lediglich fünf Selbstberichte als eine indirekte Adressierung der Restriktionen des monolingualen Habitus interpretieren.

Die Fälle in den Kategorien „IE Kompetenz“ und „IE Affinität“" der Individuumsebene weisen auf die interindividuell unterschiedliche Wahrnehmung des Angebots hin, die fruchtbare Einblicke in die von den Probanden wahrgenommenen Chancen und Herausforderungen bilingualer Praktiken beim PL ermöglichen. So akzentuieren etwas mehr als ein Viertel aller Fälle die positiven Effekte des bilingualen Sprachgebrauchs, indem sie mit diesem eine Vereinfachung des PLs oder das Empfinden von Spaß assoziieren. Die wahrgenommene, nicht genau spezifizierte Vereinfachung durch das Sprachangebot könnte als ein geringerer „,ognitive load“ beim PL interpretiert werden. Die normalerweise exklusiv in der Verkehrssprache zu exerzierenden Interaktionen und Aushandlungen der Zusammenarbeit und Lerninhalte beim PL können nach Hopewell (2011, S. 607) als ,learning burden“ bezeichnet werden, die zu einer Steigerung der Anforderungen an die Verarbeitung der Lerninhalte bei den bilingual aufwachsenden SuS durch das Verbot des Rückgriffs auf ihr komplettes sprachliches Repertoire führt. Durch das bilinguale Interaktionsangebot wird die ,learning burden“ (ebd.) aufgehoben, sodass die hierdurch freigesetzten kognitiven Ressourcen für die Aufgabenbearbeitung eingesetzt werden können. Drei Antworten der Kompetenzkategorie bei der Annahme des 
Sprachangebots weisen indirekt auf das „complementary principle“ (Grosjean, 2010, S. 29ff) und auf Praktiken des „dependent translanguaging“ (García \& Kano, 2014) hin, indem sie Limitierungen der deutschsprachigen Fähigkeiten in Produktion und Rezeption akzentuieren. Diese Fälle legitimieren ihren bilingualen Sprachgebrauch mit ihrer fehlenden Kompetenz, alles auf Deutsch ausdrücken oder verstehen zu können, sodass sie auf ihre Fähigkeiten in der nicht-verkehrssprachlichen L1 zurückgreifen, um diese Problematik zu lösen. Diese Antworten erscheinen angesichts der allgemeinen Sprachasymmetrie der Stichprobe zu Gunsten des Deutschen, welche auch häufig bei der Ablehnung des Sprachangebots thematisiert wird, zunächst zu überraschen. Da sich allerdings verschiedene sprachliche Fähigkeiten aufgrund der zweisprachigen Lebensrealität komplementär über verschiedene Lebensbereiche bei bilingualen Sprechern verteilen können, sodass der Rückgriff auf die nicht-verkehrssprachliche L1 in manchen Fällen gar notwendig wird, lassen sich diese drei Antworten dementsprechend im Sinne des „complementary principle“ (Grosjean, 2010, S. 29) und „dependent translanguaging“ (García \& Kano, 2014) einordnen.

Die positiven Emotionen, die zehn Fälle mit dem bilingualen Sprachgebrauch verbinden und häufig als Erleben von Spaß deklarieren, weisen auf das motivationale Potential eines bilingualen Interaktionsangebots hin. Auf Basis der Kürze der Antworten lassen sich allerdings nicht die zu Grunde liegenden Mechanismen der positiven Affinität zum bilingualen Sprachangebot ergründen. So bleibt unklar, ob die SuS z. B. durch die rechtliche und/oder solidarische Anerkennung positive Emotionen empfinden, das Sprachangebot mit der familiären Lebensrealität assoziieren oder dieses ihre Identität bestätigt, beim Sprachgebrauch des Türkischen Befriedigung der Subdimensionen der „basic needs" (vgl. Ryan \& Deci, 2018) erfahren oder andere Prozesse für die Affinität zum bilingualen Sprachangebot verantwortlich sind.

Ungefähr ein Fünftel der Fälle begründen ihre Ablehnung des Sprachangebots mit einer Invertierung der eben diskutierten Argumente der Kompetenzund Affinitätskategorien bei der Annahme des bilingualen Interaktionsangebots. So führen ungefähr ein Zehntel aller Antworten eine Sprachasymmetrie $\mathrm{zu}$ Gunsten des Deutschen, allgemein geringe sprachliche oder spezifische fehlende Kompetenzen im Türkischen als Gründe für die Ablehnung des Sprachangebots an. Dies deckt sich zum einen mit der Beobachtung von Reich (2009), dass sich die sprachlichen Fähigkeiten des Türkischen und Deutschen diametral im Verlauf der Kindheit mit MH im Submersionskontext entwickeln, wobei die sprachlichen Fähigkeiten im Deutschen im Verlauf der Grundschule die Sprachkompetenz im Türkischen übersteigen. Zum anderen weisen einige der Antworten auf das Fehlen spezifischer bildungssprachlicher Fähigkeiten in der L1 hin, was spätestens seit der Postulierung von Cummins (2000) Thresholdund Interdependenzhypothese in den späten 1970er Jahren einen robusten em- 
pirischen Befund im Submersionskontext darstellt. So verfügen einige Probanden nicht über das Kardinalzahlensystem im Türkischen im Hunderter- und Tausenderraum, was eine bildungssprachliche Voraussetzung zur bilingualen Interaktion über die Rechenprozesse in der Rechenstrategieintervention darstellt. Zudem akzentuiert ein Fall die zusätzliche Belastung, die das Sprachangebot zu den Rechenprozessen exerziert, was als eine Erhöhung des „,cognitive load" durch das Sprachangebot interpretiert werden kann. Dementsprechend sind diese Probanden ihrer eigenen Ansicht nach nicht in der Lage, das bilinguale Sprachangebot furchtbar umzusetzen, sodass sie dieses ablehnen.

Ein Fall begründet die Ablehnung des Sprachangebots mit dem Lernziel, Deutsch lernen zu müssen. Diese Antwort könnte im Sinne der Time-on-TaskHypothese (Carroll, 1963) interpretiert werden, dass der Erwerb einer Sprache abhängig von deren Lerngelegenheiten ist, was auch in diversen anderen Untersuchungen (vgl. Culligan, 2015; Storch \& Wigglesworth, 2003) von den SuS hinsichtlich des Rückgriffs auf die (weitere) L1 erwähnt wird. Angesichts der Threshold- und Interdependenzhypothese von Cummins (2000) und empirischen Erkenntnissen zu kritischen Schwellenwerten eines reduzierten Inputs bei bilingual aufwachsenden Sprechern, der allerdings zu vergleichbaren morphosyntaktischen und lexikalischen Fähigkeiten wie bei monolingual aufwachsende Sprecher führt (vgl. z. B. vgl. Gathercole, 2016, S. 130; Unsworth, 2016, S. 111), erscheint der von der Time-on-Task-Hypothese postulierte Zusammenhang von Sprachgebrauch und Spracherwerb inadäquat zur Erklärung von bildungsbezogenen Sprachlernprozessen zu sein.

Zudem weisen insgesamt sechs Fälle auf negative Emotionen gegenüber dem Türkischen hin, die allerdings aufgrund ihrer Kürze nichts über die zugrunde liegenden Mechanismen dieser negativen Affinität offenbaren. So lässt sich lediglich wie oben bereits auch für den monolingualen Habitus vermuten, dass die Sozialisation innerhalb einer ,assimilation ideology“ (vgl. Bourhis, 2001) in der Lage ist, negative Einstellungen zur Bilingualität oder ihrer nichtverkehrssprachlichen L1 bei bilingualen Sprechern zu erzeugen, worauf auch Gardner-Chloros (2009, S. 81f) sowie Ritchie und Bhatia (2013, S. 389) hinweisen. Krumm (2009, S. 239ff) bezeichnet solche Einstellungen als „Konflikt-Zweisprachigkeit", welche in diesen Fällen sich in einer Negation des Türkischen ausdrückt. Für diese Probanden ist das bilinguale Sprachangebot jedenfalls mit negativen Emotionen verbunden.

Begründungen des Sprachverhaltens auf Basis der Identität treten kaum in den Selbstberichten der Probanden auf, obwohl Identität und Sprache in enger, reziproker Beziehung zueinander stehen (vgl. Hu, 2007). Lediglich eine Antwort in der Kategorie „IE Sonstige“ legitimiert die Annahme des Angebots mit der nationalen Identität als „Türke“. Der Großteil der restlichen Fälle in dieser Kategorie besteht aus äußerst unspezifischen Begründungen der Annahme des bilingualen Sprachangebots. Die mit dem Modalverb „wollen“ gebildeten Ant- 
worten der Kategorie „IE Sonstige“ können zwar als eine bewusste, absichtsvolle Entscheidung für die Annahme des Sprachangebots gedeutet werden, allerdings können anhand dieser Antworten keine Aussagen über die tatsächlichen zugrunde liegenden Mechanismen und Motive getroffen werden, die letztlich zur Nutzung des bilingualen Sprachgebrauchs führen. Auch diese Antworten weisen auf das Postulat hin, dass die Motive für die Sprachwahl und das Sprachverhalten nicht zwingend den bilingualen Sprechern bewusst sein müssen, was die Notwendigkeit der Reflexion bilingualer Praktiken beim PL im Kontext der Submersion unterstreicht.

Angesichts der konträren Begründungen für die Annahme und Ablehnung des Sprachangebots der Kompetenz- und Affinitätskategorien kann nicht davon ausgegangen werden, dass alle bilingual aufwachsenden SuS in der Submersion ein solches bilinguales Interaktionsangebot auch umsetzen. So akzentuieren die Selbstberichte der Probanden primär sowohl fehlende sprachliche Fähigkeiten in der nicht-verkehrssprachlichen L1 als auch die Präferenz der Verkehrssprache aufgrund der höheren Sprachkompetenz als Begründungen für die Ablehnung des bilingualen Sprachangebots. Zudem scheinen negative Einstellungen zur nicht-verkehrssprachlichen L1 ebenfalls Hürden für die Implementation bilingualer Praktiken beim PL darzustellen. Nichtsdestotrotz weisen insbesondere die Antworten der Annahme des Sprachangebots in den Kategorien „IE Kompetenz“, „IE Affinität“ und „IE Sonstige“ sowohl auf die Bereitschaft der bilingual aufwachsenden SuS zur Nutzung bilingualer Praktiken beim PL als auch auf die Chancen und Potentiale eines solchen bilingualen Interaktionsangebots für bilingual aufwachsende SuS im Submersionskontext hin. Dieser Befund steht in Einklang mit den Ergebnissen von Studien wie z. B. von Barwell (2014), Clarkson (2007), Culligan (2015) sowie García und Kano (2014) bei welchen die SuS der Nutzung der L1 in Bildungsprozessen ebenfalls diverse fruchtbare Bildungspotentiale zusprechen.

Bei der Analyse der Fragestellung F5 konnten eine Vielzahl verschiedener Zwecke bilingualer Praktiken beim PL mit klaren quantitativen Tendenzen identifiziert werden. So werden bilinguale Praktiken beim PL von den Probanden deutlich häufiger für aufgabenrelevante $(87,78 \%)$ statt für -irrelevante Handlungen (10,39\%) verwendet, was vergleichbar mit den Prävalenzen bilingualer Praktiken für „Off-Task“-Zwecke der Studie von Swain \& Lapkin (2000) mit 11\% und 12\% ist. Allerdings werden bilinguale Praktiken von den Probanden nur sehr selten für die konkrete Aufgabenbearbeitung verwendet. Dabei akzentuieren eine Vielzahl der Studien zu Funktionen bilingualer Praktiken beim PL den Einsatz bilingualer Praktiken für die Erarbeitung des Lerninhalts und die Aufgabenbearbeitung, auch wenn sie keine Prävalenzen für diese Funktion berichten (vgl. z. B. Hopewell, 2011; Klingner \& Vaughn, 2000; Moschkovich, 2007; Planas \& Setati, 2009; Schüler-Meyer et al., 2017). Hierbei fällt auch auf, dass insbesondere als äußerst lernförderlich betrachtete Sprachhandlungen wie Übersetzungen, alternative Erklärungen im Türkischen 
sowie Code-Switching ins Türkische zur Elaboration einer bereits in der anderen Sprache realisierten Aussage in der vorliegenden Studie kaum oder gar nicht auftreten. Dabei stellen genau diese drei Zwecke exklusive Funktionen bilingualen Sprachhandelns dar, die nicht einsprachig in der Verkehrssprache umgesetzt werden können, was ihr besonderes Potential für Bildungsprozesse ausmacht. Dies könnte an der fehlenden Erfahrung von bilingual aufwachsenden SuS in der Submersion im bildungssprachlichen Umgang mit bilingualen Praktiken zusammenhängen. So dürften die Probanden es nicht gewohnt sein, strategiebezogene Leseaufgaben wie Zusammenfassungen und Vorhersagen sowie Halbschriftliche Rechenprozesse im Hunderter- und Tausenderraum im Türkischen zu realisieren, sodass sie diesbezüglich eher auf die Verkehrssprache zurückgreifen. Hierbei könnte auch die Beobachtung von Clarkson (2007, S. 194) einen Beitrag zur Erklärung leisten, da SuS primär die Sprache zur Bearbeitung von Aufgaben verwenden, die sie aus dem Unterricht gewohnt sind und in der sie den Lerninhalt aufbereitet sowie präsentiert bekommen haben. In der BiPeer-Intervention wurden alle Instruktionen zum PL selbst als auch zu den einzelnen Lese- und Rechenstrategien von den Übungsleitungen stets im Deutschen artikuliert, damit die Vergleichbarkeit zu den Gruppen LG und RG gegeben ist, in denen alle Dyaden aus einem mono- und einem bilingualen Probanden bestehen. Dementsprechend wurden die von der Übungsleitung modellierten Sprachhandlungen und -prozesse der Lese- und Rechenstrategien stets im Deutschen ausgeführt, sodass den SuS ein konkretes Modell zur Umsetzung dieser Handlungen und Prozesse im Türkischen fehlt. Neben der fehlenden Erfahrung sind sicherlich auch die fehlenden bildungssprachlichen Kompetenzen im Türkischen als auch die Sprachasymmetrie zu Gunsten des Deutschen für die spärliche Nutzung bilingualer Praktiken für die Aufgabenbearbeitung von Bedeutung. Insbesondere die Rechenprozesse in der Rechenstrategieintervention verlangen bestimmte bildungssprachliche lexikalische Elemente des Türkischen wie Kardinalzahlen, mathematischen Operatoren sowie spezifische Verben zum Ausdruck der verschiedenen vorzunehmenden Manipulation, um die Rechenproesse zu kommunizieren. In der Lesestrategieintervention sind die für die Anwendung der Lesestrategien notwendigen lexikalischen Elemente nicht eindeutig zu bestimmen, da diese von der Textgrundlage abhängen. Der geringere produktive Wortschatz im Türkischen als im Deutschen und die deutschsprachige Textgrundlage dürften allerdings einen Beitrag zur Präferenz der Anwendung der Lesestrategien im Deutschen geleistet haben.

Am häufigsten wurden bilinguale Praktiken zum Zwecke des Task-Managements produziert. Die Probanden nutzen bilinguale Praktiken vor allem zur Ansage der nächsten Phasen und Schritte, zum Besorgen von Materialien, zur Klärung des weiteren Ablaufs etc., wobei diese sowohl in spontaner, kreativer Form als auch in enger Anlehnung an die zweisprachigen Promptkarten arti- 
kuliert werden. Ein fruchtbares Task-Management stellt eine zentrale Bedingung für die Lernwirksamkeit von PL dar (vgl. Johnson \& Johnson, 1999), bei welchem gemeinsame Ziele vereinbart, Intersubjektivität erzeugt, die Verteilung von Aufgaben und Verantwortlichkeiten organisiert werden müssen, um die Prozesse der Gruppe effizient zu koordinieren. Dementsprechend werden mit bilingualen Praktiken notwendige und äußerst relevante Handlungen zur Organisation und Strukturierung der Zusammenarbeit vorgenommen. Zum Task-Management sind auch keine elaborierten sprachlichen Fähigkeiten der nicht-verkehrssprachlichen L1 notwendig, da das Task-Management auch mit BICS zu bewältigen ist und nicht zwingend bildungssprachliche Fähigkeiten benötigt.

Eine Vielzahl von Studien weist diese Funktion bilingualer Praktiken beim PL empirisch nach (vgl. Meyer \& Prediger, 2011; Planas \& Setati, 2009; Rehbein, 2011; Storch \& Wigglesworth, 2003; Swain \& Lapkin, 2000), wovon aber nur Storch \& Wigglesworth (2003) sowie Swain \& Lapkin (2000) konkrete Prävalenzen benennen. Bei der Studie von Swain und Lapkin (2000) werden abhängig von der konkreten Aufgabe im Mittel 43\% und 35\% aller bilingualen Praktiken zum Task-Management verwendet, während es bei Storch und Wigglesworth (2003) zu deutlicheren Schwankungen der Prävalenz von Task-Management abhängig von der Aufgabe bei den drei Dyaden kam. So weisen die drei Dyaden bei der Textkompositionsaufgabe Prävalenzen des Task-Managements mit bilingualen Praktiken von ca. 56\%, 37\% und 100\% und bei der der Textrekonstruktionsaufgabe Prävalenzen von ca. 13\%, 8\% und 9\% auf. Dementsprechend unterscheidet sich die Prävalenz des Task-Managements mit bilingualen Praktiken deutlich zwischen den Aufgaben innerhalb eines Tandems. Die Prävalenz des Task-Managements mit bilingualen Praktiken in der vorliegenden Studie mit knapp 61\% bewegt sich dementsprechend in vergleichbaren Bereichen wie bei der Studie von Swain und Lapkin (2000) und bei der Textkompositionsaufgabe von Storch und Wigglesworth (2003).

Bilinguale Praktiken werden in der vorliegenden Studie ebenfalls relativ häufig mit fast $31 \%$ für metakognitive und regulatorische Zwecke eingesetzt, wobei die Regulation den Großteil der Fälle in dieser Kategorie ausmacht. Diese werden vermehrt in der Trainerrolle produziert, da das Monitoring, die Kontrolle sowie die Bewertung der Handlungen des Spielers von der PL-Intervention vorgegebene zentrale Aufgaben der Trainerrolle darstellen. Dementsprechend werden Regulationen mit bilingualen Praktiken vor allem beim Dyadenpartner vorgenommen und seltener zur eigenen Regulation oder der Regulation der ganzen Gruppe eingesetzt. Die Regulation des Dyadenpartners benötigt ähnlich zum Task-Management auch keine elaborierten bildungssprachlichen Fähigkeiten in der nicht-verkehrssprachlichen L1, sondern kann mit alltagssprachlichen, hochfrequenten Ausdrücken wie „yapmak" („machen“), „hadi“ (,,los“), ,dur“ (,,stop“), ,sus“ (,pssst“), „doğru“ (,,richtig“) etc. oder mit gemischtsprachlichen Turns realisiert werden. 
Obwohl metakognitive Regulation als bedeutsames Phänomen für PL zunehmend Beachtung in der PL-Forschung findet (vgl. Whitebread et al., 2009; DeBacker et al., 2016; Iiskala, Vauras \& Lehtinen, 2004), wird diese Funktion in den bisherigen Studien zur Bedeutung bilingualer Praktiken für Bildungsprozesse allgemein als auch beim PL weitestgehend ignoriert. Dabei stellen regulative Prozesse eine zentrale Aufgabe für alle PL-Formate dar, welche insbesondere aufgrund der asymmetrischen Rollenverteilung beim Peer-Tutoring relevant sind.

Innerhalb der Kategorie „On-Task Sonstige“ befinden sich einige Fälle, die metalinguistische Reflexionen türkischsprachiger Elemente adressieren. Hierbei korrigieren die Dyadenpartner gegenseitig ihre türkischsprachigen Äußerungen oder erörtern gemeinsam angemessene türkischsprachige Lexeme und Ausdrücke. Metalinguistische Reflexionen werden als förderliche Funktion bilingualer Praktiken bei Bildungsprozessen und beim PL stets akzentuiert sowie empirisch identifiziert (vgl. Hopewell, 2011; Schüler-Meyer et al., 2017; Storch \& Wigglesworth, 2003; Swain \& Lapkin, 2000), wobei sich diese Funktion nicht nur zum Erwerb linguistischen Wissens eignet, sondern auch in bestimmten Fällen dem Konzepterwerb dienen kann (vgl. Schüler-Meyer et al., 2017). In der vorliegenden Studie treten allerdings nur selten metalinguistische Reflexionen auf. Dies könnte zum einen daran liegen, dass die Lerninhalte Lese- und Rechenstrategien nicht zwingend metalinguistische Reflexionen initiieren oder benötigen. Sofern es bei den Rechenstrategien keine Unterschiede in der sprachlichen Realisierung gibt, die wie z. B. beim Bruchrechnen unterschiedliche Propositionen der Konzepte akzentuieren (vgl. ebd.), scheinen metalinguistische Reflexionen für Rechenstrategien kaum fruchtbare Erkenntnisse zu liefern. Hinsichtlich der Lesestrategien können metalinguistische Reflexionen zwar fruchtbare Beiträge zum Textverständnis, zum Klären von Wörtern sowie zum Verfassen von Zusammenfassungen und Vorhersagen leisten, allerdings scheinen die Probanden der vorliegenden Untersuchung hiervon kaum Gebrauch gemacht zu haben. Zudem waren - außer den Promptkarten keine türkischsprachigen Materialien wie Texte und/oder Aufgabenstellungen in den Gruppen LGT/D und RGT/D vorhanden, sodass auch keine Sprachvergleichsbasis vorlag, die produktiv hätte genutzt werden können.

Die Prävalenzen der bilingualen Praktiken der einzelnen Unterkategorien der Hauptkategorie „On-Task“ unterscheiden sich mit Ausnahme der Kategorie „On-Task Sonstige“ nicht zwischen dem Lese- und Rechentraining. Da die Kategorie „On-Task Sonstige“ häufig metalinguistische Reflexionen und Korrekturen türkischsprachiger Äußerungen enthält, kann mit Vorsicht geschlossen werden, dass Lesestrategien eher metalinguistische Reflexionen initiieren oder benötigen als Rechenstrategien. Allerdings sollte die Bedeutung dieses signifikanten Unterschieds zwischen den Gruppen nicht überschätzt werden, da es sich letztlich um eine Restkategorie handelt, in welcher noch weitere, nicht metalinguistisch orientierte Funktionen enthalten sind. Die restlichen 
Unterkategorien unterscheiden sich nicht in ihren Prävalenzen, was auch in der Studie von Swain und Lapkin (2000) zu beobachten ist, die abhängig von der Aufgabe keine signifikanten Unterschiede in den Prävalenzen der verschiedenen Funktionen bilingualer Praktiken beim PL identifizieren.

\subsection{Datenintegration der quantitativen und qualitativen Ergebnisse der Studie}

Während soeben die einzelnen Ergebnisse sukzessiv hinsichtlich des theoretischen Hintergrundes sowie der bisherigen empirischen Evidenz zu bilingualen Praktiken beim PL sowie zum Teil für Bildungsprozesse allgemein diskutiert wurden, sollen nun die Ergebnisse der vorliegenden Studie in Beziehung zueinander gesetzt werden. Hierbei soll in Anlehnung an das Mixed-Methods Vertiefungsdesign primär das Ziel verfolgt werden, ,die Resultate des quantitativen Teils durch die qualitative Vertiefung besser zu verstehen“, wobei man „nicht einfach nur Ergänzendes erfahren, sondern ganz gezielt die Erklärungslücken füllen" möchte, ,die die quantitative Studie hinterlassen hat" (vgl. Kuckartz, 2014, S. 78). Dementsprechend gilt es mit den distinkten methodischen Zugängen nicht nur lediglich die verschiedenen quantitativen und qualitativen Aspekte des untersuchten Phänomens zu analysieren, um das Konstrukt in seiner Multidimensionalität adäquat zu erfassen, sondern diese so aufeinander zu beziehen, sodass neue Erkenntnisse entstehen. Dies schließt nicht nur die von Kuckartz (ebd.) akzentuierte gegenseitige Ergänzung der Ergebnisse durch Komplementarität ein, sondern auch potentielle zu klärende Widersprüche. In Anlehnung an Max Weber beschreibt Kuckartz (ebd.) die zu Grunde liegende Prämisse dieses Forschungsmotivs dahingehend, „dass bloße statistische Zusammenhänge erst dann für SozialforscherInnen und GesellschaftstheoretikerInnen interessant und verstehbar werden, wenn sie mit dem subjektiv dahinter erscheinenden Sinn verknüpft werden können“". Dementsprechend werden die Ergebnisse der Prävalenzen bilingualer Praktiken mit den zu Grunde liegenden Motiven des mono- oder bilingualen Sprachhandelns sowie dem funktionalen Gebrauch bilingualer Praktiken der Probanden in Beziehung zueinander gesetzt und vor dem bisherigen theoretischen Hintergrund sowie der empirischen Befundlage diskutiert.

Während die in F1 ermittelte paarweise Korrelation deutlich die Abhängigkeit der Prävalenz bilingualer Praktiken der Dyadenpartner empirisch bestätigt, spielt dieser Aspekt in den Selbstberichten der Probanden nur eine untergeordnete Rolle. Dies könnte zum einen - wie oben bereits angeführt - durch die Fragestellung bedingt sein, die womöglich individuelle Begründungen akzentuiert und somit auch als Prime fungieren könnte. Die hohen Fallzahlen auf der 
Kontextebene bei der Annahme des Angebots könnten aufgrund der Prominenz des bilingualen Sprachangebots erzeugt worden sein, welches nicht nur durch die Vielzahl von standardisierten Aktivierungen eine gesteigerte Salienz erfährt, sondern auch eine neue, den Probanden eher unbekannte Praxis darstellt, die angesichts des monolingualen Habitus des Submersionskontextes der deutschen Regelschule ihren bisherigen Erfahrungen in schulischen Bildungsprozessen nicht entspricht. Da die zu Grunde liegenden Einflussfaktoren für bilinguale Praktiken den Probanden nicht zwingend bewusst sind, dürften insbesondere diejenigen Determinanten von den Probanden als Motive für ihr Sprachhandeln genannt werden, die durch die äußeren Bedingungen der Intervention akzentuiert werden. Zwar stellen die Peers beim PL ebenfalls einen relevanten Bestandteil der Intervention dar, allerdings erscheinen diese den Probanden womöglich als relativ unbedeutende Faktoren für ihr individuelles Sprachhandeln. Lediglich drei Fälle adressieren direkt den Dyadenpartner für die Begründung der Annahme oder Ablehnung des bilingualen Sprachengebots. Von diesen drei Fällen begründet eine Antwort die Annahme des Angebots mit der Unterstützung des Dyadenpartners (,Weil das manchmal [Name des Trainingspartners] geholfen hat") und zwei Antworten die Ablehnung des Sprachangebots äußerst unspezifisch mit dem Verweis auf den Dyadenpartner (,wegen [Name des Trainingspartners]"). Dementsprechend ist es fraglich, ob dem Großteil der Probanden der Einfluss des Dyadenpartners auf ihr Sprachverhalten in ihrer subjektiven Wahrnehmung des bilingualen Sprachangebots bewusst wird sowie überhaupt vorstellbar erscheint. Diese sich aufgrund der unterschiedlichen Perspektive widersprechenden Befunde der Fragestellungen F1 und F4 weisen auch auf die Fruchtbarkeit der Triangulation hin. Triangulation beschreibt ein methodisches Vorgehen, bei welchem dieselben Phänomene mit verschiedenen Methoden oder Operationalisierungen beziehungsweise aus verschiedenen Blickwinkeln betrachtet werden (vgl. Greene, Caracelli \& Graham, 1989, S. 259). Wäre nur ein methodischer Zugang gewählt worden, hätte der gegenseitige Einfluss der Gesprächspartner auf ihr bilinguales Sprachverhalten nicht in seiner unterschiedlichen Bedeutung abhängig von der gewählten Perspektive reflektiert werden können.

Die Abhängigkeit der Prävalenz bilingualer Praktiken zeigt sich auch partiell in den kurzen beispielhaften Einblicken in die Interaktion bei der in Abschnitt 8.5.3 präsentierten Funktionsanalyse bilingualer Praktiken beim PL. So motiviert sich z. B. eine Dyade gegenseitig zum Gebrauch bilingualer Praktiken, indem vor allem der Peer in der Trainerrolle den Peer in der Spielerrolle auf die Nutzung von bilingualen Praktiken auch bei der Aufgabenbearbeitung hinweist, obwohl der Trainer selbst über keine hohe Sprachkompetenz im Türkischen verfügt, sodass dieser den Spieler zur Wiederholung der Äußerung im Deutschen bittet und anschließend wieder vermehrt in Deutsch über die Inhalte interagiert wird. Dementsprechend könnte eine geringe Produktions- und Rezeptionsfähigkeit des einen Peers zu einer geringeren Prävalenz bilingualer 
Praktiken des anderen Peers führen, da die Herstellung von Intersubjektivität in der Dyade in für beide Peers zugänglicher Weise erzeugt werden muss. Dieselbe Dyade engagiert sich auch in metalinguistischen Reflexionen über das türkischsprachige Äquivalent zu „Schulbus“, was ebenfalls eine Episode darstellt, in der die bilingualen Praktiken eines Peers das bilinguale Sprachhandeln des anderen beeinflusst.

Die Fragestellung F2a weist auf einen großen Effekt des bilingualen Sprachangebots auf die Prävalenz bilingualer Praktiken hin, die sich signifikant von den Quantitäten beim fehlenden expliziten Angebot unterscheidet. In den Selbstberichten geben deutlich mehr Probanden an, das Angebot zumindest teilweise angenommen als abgelehnt zu haben. Dementsprechend konvergieren hinsichtlich der Wahrnehmung des bilingualen Sprachangebots die beiden verschiedenen methodischen Perspektiven. Angesichts der hohen Fallzahlen der Kontextebene bei der Annahme des Angebots scheint das explizite Angebot eine hohe Prominenz in der Wahrnehmung der Lese- und Rechenstrategieintervention der Probanden aufzuweisen. Diese beiden Befunde akzentuieren nicht nur die Relevanz expliziter Angebote zur Initiation bilingualer Interaktion, sondern auch die Salienz eines solchen Angebots im Submersionskontext, welches diametral zu den Erfahrungen der Probanden in einem vom monolingualen Habitus geprägten Unterricht steht.

Hinsichtlich der Prävalenz bilingualer Praktiken lässt sich kein Unterschied zwischen der Lese- und Rechenintervention identifizieren, was - mit Ausnahme der Unterkategorie „On-Task Sonstige“ - auch hinsichtlich der Prävalenzen der Funktionen bilingualer Praktiken beim PL zwischen der Leseund Rechenstrategieintervention gilt. Bilinguale Praktiken werden in der BiPeer-Intervention vornehmlich zum Task-Management und zur metakognitiven Regulation genutzt. Diese Funktionen unterscheiden sich kaum in den benötigten Voraussetzungen zur Umsetzung und der tatsächlichen Ausführung zwischen der Lese- und Rechenstrategieintervention, da sie zentrale Mittel zur Erzeugung von Intersubjektivität darstellen und zur Koordination der Dyadenpartner beim PL beitragen. Zudem werden sowohl das Task-Management als auch die metakognitive Regulation durch ähnliche Strukturen der TrainerSpieler Interaktion sowie der Materialien von beiden Interventionen unterstützt. Dementsprechend spielen die spezifischen Eigenschaften der Domänen Lesen und Rechnen sowie die Unterschiede zwischen Lese- und Rechenstrategien angesichts der Präferenz des Gebrauchs bilingualer Praktiken für das Task-Management und die metakognitive Regulation keine große Rolle, sodass sie keinen Einfluss auf eine unterschiedliche Prävalenz sowie Nutzung bilingualer Praktiken ausüben.

Hinsichtlich potentieller Unterschiede der Prävalenz bilingualer Praktiken abhängig von den Phasen der Lese- und Rechenstrategieintervention weisen nur die Phasen „Wörter klären“, „Zusammenfassen“ und „Vorhersage“ signifikant höhere Quantitäten als die Phase „Frage zum Abschnitt auf“. Die in F5 
unternommene Funktionsanalyse kann diesbezüglich ebenfalls einen potentiellen Beitrag zur Aufklärung der diesem Ergebnis zugrunde liegende Mechanismen leisten. Die drei Lesestrategien zeichnen sich durch strukturierte Trainer-Spieler Interaktionen mit unterstützendem Material aus, in denen bereits Prozesse des Task-Managements und der metakognitiven Regulation durch die Teilschritte der Lesestrategien festgeschrieben sind. Die drei Lesestrategien haben mehr zu koordinierende Teilschritte, die mehr Task-Management benötigen, aber auch mehr Anlässe zur metakognitiven Regulation bieten, da die Teilschritte der Lesestrategien diverse Monitoring- und Kontrollprozesse beinhalten sowie einzeln bewertet werden können. Im Gegensatz hierzu enthält die Phase „Frage zum Abschnitt" keine Teilschritte und stellt insgesamt eine strukturell äußerst offene Phase ohne Rollenbelegung dar, in der die Peers liberal die Frage zum Abschnitt gemeinsam beantworten. Dementsprechend könnte die niedrige Prävalenz bilingualer Praktiken in dieser Phase auch mit der geringeren Notwendigkeit zur Koordination und Regulation der Bearbeitung dieser zusammenhängen.

Bei Fragestellung F3 konnten keine signifikanten Zusammenhänge von der Prävalenz bilingualer Praktiken weder mit sprachlichen noch motivationalen Eigenschaften und Merkmalen der Probanden unter Berücksichtigung der dyadischen Abhängigkeit identifiziert werden. Neben den oben bereits diskutierten potentiellen statistisch-methodischen Erklärungen auf Basis der geringen Power der Stichprobe, der zum Teil geringen Varianz samt Deckeneffekten und fragwürdigen bis inakzeptablen internen Konsistenzen einiger Skalen können die qualitativen Erkenntnisse ebenfalls zur Erklärung dieses Befundes beitragen. Wie an den Ergebnissen von F4 zu sehen ist, interpretiert ein beträchtlicher Anteil der Stichprobe das bilinguale Interaktionsangebot nicht als optionalen Aspekt, sondern als obligatorisch zu erfüllenden Bestandteil der Intervention. Eine solche Wahrnehmung des Sprachangebots könnte einen anderen Umgang mit dem Angebot erzeugen als die intendierte freiwillige Perspektive. Bei der intendierten Perspektive auf das Angebot sollten die Probanden das Angebot in einer Quantität und Weise verwenden, die den individuellen Fähigkeiten und den Bedürfnissen der Dyade entspricht. Die nicht intendierte Perspektive eines obligatorischen Bestandteils der Intervention hingegen erzeugt einen gewissen Druck zur Erfüllung dieses Bestandteils, was eher Bewältigungsstrategien bei den Probanden hervorrufen könnte. Hierbei könnte das Angebot von den Probanden in seiner Effektivität und seinem Nutzen gering eingeschätzt oder gar als zusätzliche Belastung empfunden werden, welches aus der nicht intendierten Perspektive eines obligatorischen Bestandteils des Trainings dazu führen könnte, dieses möglichst ressourcenschonend und voraussetzungsfrei $\mathrm{zu}$ bedienen. Insbesondere das Task-Management unter Verwendung der Promptkarten oder einfache organisatorische Handlungen wie das Ansagen der nächsten Phase stellen leicht zu bewältigende Formen und Funktionen bilingualer Praktiken dar, mit welchen sich die Nutzung des 
bilingualen Sprachangebots leicht umsetzen lässt. Auch metakognitive Regulationen benötigen nicht zwingend elaborierte rezeptive und produktive türkischsprachige Fähigkeiten, sodass auch mit dieser Funktion, die an diversen Stellen der Intervention eingebracht werden kann, das Sprachangebot erfüllt werden könnte. Angesichts der hohen Prävalenz der Funktionen „Task-Management" und „Metakognition und (Selbst-)Regulation“ könnte vermutet werden, dass ein nicht zu unterschätzender Anteil der Probanden womöglich diese Formen und Funktionen bilingualer Praktiken angewandt haben, um möglichst ressourcenschonend und voraussetzungsfrei das bilinguale Sprachangebot umzusetzen. Dementsprechend ist es nicht verwunderlich, dass keine signifikanten Zusammenhänge mit sprachlichen und motivationalen Variablen bestehen, da solche Formen und Funktionen der Umsetzung des bilingualen Sprachangebots beim PL keine hohen Voraussetzungen an sprachliche und motivationale Eigenschaften stellen, sodass unabhängig von diesen Eigenschaften der Individuen bilingual interagiert wird. Vielmehr kann eine solche Umsetzung des bilingualen Sprachangebots als die Erfüllung eines bestimmten Teils des durch die Intervention definierten „Schülerjobs“ (vgl. Breidenstein, 2006) interpretiert werden. In diesem Sinne würden einige Probanden teilweise bilingual interagieren, um den vermeintlichen obligatorischen Bestandteil des Trainings zu erfüllen und nicht weil sie hiervon profitieren.

Zusammenfassend lässt sich aus der Diskussion der einzelnen Ergebnisse der vorliegenden Untersuchung sowie deren Verbindung untereinander im Sinne der Datenintegration folgendes schließen: Bilinguale Praktiken beim PL von türkisch-deutschsprachig aufwachsenden SuS der dritten und vierten Jahrgangsstufe im Submersionskontext sind abhängig von der Dyade. Dieser Befund unterstützt das Postulat diverser soziolinguistisch orientierter Theorien zur Sprachwahl und zum Sprachverhalten (vgl. z. B. Grosjean, 2008; Sachdev, Giles \& Pauwels, 2013), dass sich miteinander interagierende bilinguale Sprecher hinsichtlich ihres bilingualen Sprachhandelns gegenseitig beeinflussen. Bilinguale Praktiken beim PL sollten dementsprechend mit Methoden der dyadischen Datenanalyse (vgl. Kenny, Kashy \& Cook, 2006) untersucht werden. Bilinguale Praktiken treten signifikant häufiger auf, wenn die SuS ein explizit formuliertes Angebot zur bilingualen Interaktion beim PL erhalten. Dieser Befund weist auf die Wirkmächtigkeit des monolingualen Habitus des Submersionskontexts der deutschen Regelschule (vgl. Gogolin, 1994) hin. Das bilinguale Interaktionsangebot der vorliegenden Studie schwächt diesen ab, indem es dem Türkischen den Status einer weiteren offiziellen Sprache neben dem Deutschen für die Bearbeitung von Lerninhalten zuspricht, die die SuS optional und nach ihren Bedürfnissen in der Dyade einsetzen können. Die Nutzungshäufigkeit bilingualer Praktiken beim PL unterscheidet sich hierbei nicht signifikant zwischen den verschiedenen Lerninhalten Lese- und Rechenstrategien. Zwar stellen Lese- und Rechenstrategien bei deren Applikation unterschiedliche 
sprachliche Anforderungen an das Handeln der SuS, allerdings werden bilinguale Praktiken in der vorliegenden Studie „on-task“ in etwas mehr als 90\% der Fälle zum Task-Management sowie zur metakognitiven Regulation verwendet. Sowohl Task-Management als auch metakognitive Regulation sind beim PL neben der Bearbeitung des konkreten Lerninhalts essenziell, da mit diesen primär die Zusammenarbeit organisiert sowie koordiniert wird, und können mit alltagssprachlichen Mitteln umgesetzt werden, was die vergleichbaren Prävalenzen bilingualer Praktiken im Lese- und Rechentraining erklären könnte. Auch die signifikant höheren Prävalenzen bilingualer Praktiken bei den drei Lesestrategien „Wörter klären“, „Zusammenfassen“ und „Vorhersagen“ im Gegensatz zur Phase „Frage zum Abschnitt" im Lesetraining lassen sich sehr wahrscheinlich mit der Präferenz der Nutzung bilingualer Praktiken zum Task-Management sowie zur metakognitiven Regulation erklären, anstatt mit unterschiedlichen sprachlichen oder konzeptionellen Anforderungen der Lesestrategien und Phasen.

Die Prävalenz bilingualer Praktiken weist keine signifikanten Zusammenhänge mit zentralen sprachlichen als auch motivationalen Variablen unter Berücksichtigung der Abhängigkeit der Prävalenz bilingualer Praktiken von der Dyade auf, obwohl in Anlehnung an Grosjeans (2008) Sprachmodi, anerkennungstheoretischen Reflexionen (vgl. Mecherill, 2005; Stojanov, 2006, 2011) und motivationalen Bedürfnissen in Bildungsprozessen (vgl. Ryan \& Deci, 2018) solche Zusammenhänge theoretisch zu erwarten gewesen wären. Neben den oben bereits erwähnten potentiellen methodisch-statistischen Ursachen scheint die Wahrnehmung des bilingualen Sprachangebots zur Erklärung dieses Ergebnisses beizutragen. Die Ergebnisse von F4 zeigen, dass ein nicht zu unterschätzender Anteil der Probanden der Gruppen LGT/D und RGT/D das bilinguale Interaktionsangebot als einen obligatorisch zu erfüllenden Bestandteil der Intervention interpretiert haben. Eine solche von der Intervention nicht intendierte Wahrnehmung des bilingualen Sprachangebots könnte dessen Bedeutung eines optionalen, nach den Bedürfnissen der Dyade einsetzbaren Hilfsmittels zur Interaktion zu einer zusätzlichen Anforderung umdeuten, die es wie andere Elemente des PL umzusetzen gilt. Eine solche Wahrnehmung des Sprachangebots könnte wiederum Bewältigungsstrategien zur Erfüllung dieser vermeintlichen Anforderung hervorrufen, welche sich durch eine möglichst ressourcenschonende, voraussetzungsfreie sowie materialgestützte Produktion bilingualer Praktiken zum Zwecke des Task-Managements und der metakognitiven Regulation auszeichnen, die nicht mit den jeweiligen sprachlichen und motivationalen Dispositionen der Probanden zusammenhängen.

Allerdings gilt eine solche Wahrnehmung und Umsetzung des bilingualen Interaktionsangebots nicht für alle Probanden. Insbesondere die in F4 untersuchten selbstberichteten Gründe für die Annahme des Angebots der Kategorien „IE Kompetenz“, „IE Affinität“" und „KE Angebot" weisen auf das förder- 
liche Potential bilingualer Praktiken beim PL hin. So führt das bilinguale Interaktionsangebot nach der Ansicht eines ebenfalls nicht zu unterschätzenden Anteils der Probanden zu einer Vereinfachung des PL. Es hebelt die Konsequenzen des „,complementary principle“ (Grosjean, 2010, S. 29ff) der bilingualen Lebenswelt aus, ruft positive Emotionen wie das Empfinden von Spaß hervor und stellt für einige Probanden eine Möglichkeit zur Steigerung der sprachlichen Fähigkeiten im Türkischen dar. Andererseits gilt es allerdings auch die Voraussetzungen und Hürden eines bilingualen Sprachangebots beim PL ernst zu nehmen und zu berücksichtigen, da diverse Probanden ihre (bildungs-)sprachlichen Fähigkeiten im Türkischen als ungenügend zur Umsetzung des bilingualen Sprachangebots einschätzen und/oder bereits negative Einstellungen zum Türkischen entwickelt haben. Zudem können einige Probanden nicht nachvollziehen, warum sie bilingual interagieren sollen, wenn ihre sprachlichen Fähigkeiten im Deutschen besser als im Türkischen sind.

Angesichts der Prävalenzen bilingualer Praktiken beim PL mit explizitem bilingualen Sprachangebot und den Selbstberichten der Probanden der vorliegenden Studie kann geschlossen werden, dass nicht alle bilingual aufwachsenden SuS ein solches Angebot beim PL im Unterricht der Regelschule unverzüglich umsetzen würden. Vielmehr gilt es bestimmte sprachliche Voraussetzungen abhängig vom jeweiligen Lerninhalt zu schaffen und eine gewisse Entwicklungszeit für eine fruchtbare Umsetzung des bilingualen Sprachangebots bereitzustellen. Im Folgenden werden praktische Implikationen der vorliegenden Studie für eine Umsetzung bilingualer Interaktionsangebote beim PL im Regelunterricht reflektiert.

\subsection{Praktische Implikationen der Studie}

Auch wenn die Intervention in einem quasiexperimentellen Setting von einer Übungsleitung und zwei SuS nach dem regulären Unterricht durchgeführt wurde und somit über eine eingeschränkte ökologische Validität verfügt, lassen sich auf Basis der Ergebnisse der vorliegenden Studie vor dem aktuellen theoretischen Hintergrund sowie der empirischen Befundlage einige praktische Implikationen für die Implementation eines bilingualen Interaktionsangebots beim PL im Regelunterricht des Submersionskontextes ableiten. Bilinguale Interaktion beim PL sollte als explizites Angebot implementiert werden, bei welchem die (weitere) L1 der SuS den Status einer offiziellen Unterrichtssprache beim PL zugesprochen bekommt. Ein solches explizites Sprachangebot verleiht der (weiteren) L1 der SuS auf den Anerkennungsformen des Rechts sowie der Solidarität die rechtliche Gleichstellung mit dem Deutschen respektive soziale Wertschätzung zumindest während der Sozialform des PL. 
Die Angebotsstruktur der bilingualen Interaktion überlässt hierbei allerdings den SuS die Entscheidung, in welchem Sprachmodus sie miteinander beim PL kommunizieren möchten. Diese Optionalität und Freiwilligkeit ist aus verschiedenen Gründen relevant: 1) Zunächst wird mit der Freiwilligkeit des bilingualen Interaktionsangebots einer Segregation durch die produktive Seite von Anerkennungsprozessen vorgebeugt, da somit einer Etikettierung der bilingual aufwachsenden SuS als sich von der Mehrheitsgesellschaft unterscheidende Individuen entgegengetreten wird. Zudem hebelt der Angebotscharakter auch den monolingualen Habitus während der Sozialform des PL aus, sodass die SuS weder zur monolingualen noch zur bilingualen Interaktion gezwungen werden, sondern sie ihren Sprachmodus selbst bestimmen können. 2) Weiterhin können die SuS ihr Sprachverhalten beim PL mit ihrer Identität in Einklang bringen. Somit kann das sprachliche Verhalten zur Markierung der jeweiligen Identitäten und Gruppenzugehörigkeiten fungieren, welches sowohl hybride Identitäten als auch stärker an der Minder- oder Mehrheitsgesellschaft orientierte Identitäten repräsentieren kann. 3) Neben diesen primär an Anerkennung und Identität orientierten Motiven für den Angebotscharakter bilingualer Interaktion beim PL stellen selbstverständlich auch bildungs- und lerntheoretische Aspekte zentrale Argumente für die Freiwilligkeit eines solchen Angebots dar. Durch den Angebotscharakter werden die Chancen und Herausforderungen des Gebrauchs bilingualer Praktiken beim PL für die jeweiligen bilingual aufwachsenden Individuen berücksichtigt. SuS, die in ihrer (weiteren) L1 kaum über bildungssprachliche Fähigkeiten verfügen und/oder negative Einstellungen gegenüber dieser vertreten, können bilinguale Praktiken beim PL als zusätzliche Belastung empfinden. Eine Implementation bilingualer Interaktion als Angebot ermöglicht solchen SuS, autonom über ihr sprachliches Verhalten zu entscheiden. Sie können somit bilinguale Praktiken in der Weise einsetzen, die sie und ihre Gruppe beim PL für notwendig und/oder förderlich erachten. Dementsprechend erzeugt die Freiwilligkeit des Angebots zur bilingualen Interaktion beim PL einen kontinuierlichen Handlungsraum, in welchem die bilingual aufwachsenden SuS ihre bilinguale Handlungsdisposition selbstbestimmt verwenden sowie entwickeln können.

Die Angebotsstruktur bilingualer Praktiken beim PL darf hierbei allerdings nicht lediglich aus einem explizit formulierten Angebot bestehen, sondern sollte Zeit, Instruktionen und Materialien für die sprachliche Entwicklung in der (weiteren) L1 beim PL bereit halten. Mit Hilfe diverser Aktivierungen, Unterstützungsformen und -materialien können bilinguale Praktiken beim PL kontinuierlich exploriert, eingeübt, transferiert sowie reflektiert werden. Hierbei gilt es zusätzliche Angebote systematisch didaktisch aufzubereiten, bei welchen die $\mathrm{SuS}$ sich die bildungssprachlichen Grundlagen für bestimmte Lerninhalte erarbeiten können. So benötigt die bilinguale Interaktion bei der Aufgabenbearbeitung arithmetischer Probleme mit Halbschriftlichen Re- 
chenstrategien sprachliches Wissen in der (weiteren) L1 über das Kardinalzahlensystem sowie mathematische Operatoren und Operationen. Die Lehrkraft muss hierbei nicht über die Erstsprachen ihrer SuS verfügen, sondern die SuS können sich diese sprachlichen Voraussetzungen beim PL gemeinsam erarbeiten und gegenseitig vermitteln. Allerdings müsste die Lehrkraft diesbezüglich zumindest eine Selektion von geeignetem zusätzlichem Material wie z. B. zweisprachige oder selbsterklärende Lernmaterialien in der L1 der SuS bereitstellen. Mittlerweile gibt es eine Vielzahl fruchtbarer zweisprachiger Praxismaterialien wie Sprachbeschreibungen, Sprachvergleiche, Unterrichtsentwürfe und -einheiten (vgl. z. B. ProDaZ), zweisprachige Alphabetisierungsprogramme (vgl. z. B. Ayten, 2016) oder sprachensensible Family Literacy Programme (vgl. z. B. FLY), die für PL adaptiert werden können. Angesichts der zunehmenden Verbreitung von Europaschulen auch in Deutschland dürfte sowohl die Quantität als auch die Qualität von zwei- oder mehrsprachigen Materialien für schulische Bildungsprozesse kontinuierlich steigen. Zudem können sich die Lehrkräfte hinsichtlich geeigneter Materialien sowie deren didaktischer Einführung auch an die Lehrkräfte des HSU wenden oder gar im Sinne eines fächerübergreifenden Unterrichts längerfristig mit diesen kooperieren, sofern der HSU an ihren Schulen etabliert ist.

Die ausgewählten Materialien sollten speziell förderliche Funktionen bilingualer Praktiken fokussieren, wofür sich z. B. Fallvignetten zu bilingualer Interaktion eignen könnten. Hierbei sollten die bildungsförderlichen, metalinguistischen und sprachvergleichenden Potentiale bilingualen Sprachverhaltens beim PL adressiert werden. Lehrkräfte können sich bezüglich geeigneter Aufgabenformate zur Initiation dieser Prozesse von didaktischer Literatur zu Translanguaging sowie von empirischen Studien zu bilingualem Sprachhandeln bei Bildungsprozessen (vgl. z. B. García \& Kano, 2014; García \& Li Wei, 2014; Hopewell, 2011; Klingner \& Vaughn, 2000; Moschkovich, 2007; Schüler-Meyer et al., 2017; Worthy et al., 2013) inspirieren lassen. So könnten z. B. SuS eine Aufgabe oder ein Konzept erst in der Verkehrssprache erarbeiten und anschließend in der nicht-verkehrssprachlichen L1 widergeben, Konzepte in beiden Sprachen erläutern oder linguistische Eigenschaften ihrer beiden Sprachen sprachvergleichend betrachten. Hierbei sollten vor allem die förderlichen Funktionen bilingualer Praktiken beim PL akzentuiert werden, die nicht auch einsprachig zu vollziehen sind. Diesbezüglich sind insbesondere die Möglichkeiten zur Elaboration von Propositionen unter Verwendung von Code-Switching und Code-Mixing (vgl. Moschkovich, 2007), sprachvergleichende metalinguistische Reflexionen (vgl. z. B. Hopewell, 2011; Klingner \& Vaughn, 2000) sowie das intensivere Verarbeiten von Lerninhalten durch eine zweisprachige Durchdringung (vgl. Baker, 2011, S. 288ff) zu nennen. Abhängig vom jeweiligen Lerngegenstand können auch potentielle Unterschiede in der bildungssprachlichen Umsetzung der Konzepte in beiden Sprachen akzen- 
tuiert werden, wie z. B. hinsichtlich der sprachlichen Realisierung von mathematischen Brüchen (Schüler-Meyer et al., 2017) oder bezüglich unterschiedlicher Rechenschritte und Notationsverfahren beim schriftlichen Rechnen (vgl. Gonas et al., 2018) im Deutschen und Türkischen. Auch verschiedene Aufgabenkulturen, die sich nur in der Fokussierung anderer Zahlenmerkmale wie beim „Türkischen Einmaleins“ (vgl. Müller \& Wittmann, 2014, S. 3) oder lediglich anderer Darstellungskonventionen wie bei „Türkischen Zahlenraupen“ (vgl. ebd.) von typischen Aufgaben im deutschen Bildungswesen unterscheiden, können produktiv kontrastierend eingesetzt werden. Sprachvergleichende, metalinguistische Aufgaben eignen sich insbesondere beim Schriftspracherwerb, bei welchem die Lautsprache als Analysegegenstand betrachtet wird und innerhalb einer größtenteils systematischen Graphematik visuell fixiert wird. So würden sich sprachvergleichende Angebote zur Kontrastierung der deutschen und türkischen Schriftsprache zum Erwerb von Phonem-Graphem-Beziehungen, zur Reflexion formaler schriftsprachlicher Konventionen wie Groß-und-Kleinschreibung sowie silbischen und morphematischen Schreibungen eignen, insbesondere angesichts der sprachstrukturellen Unterschiede der Fusionssprache Deutsch und der Agglutinationssprache Türkisch.

Bei der Erstellung des didaktischen Rahmens für bilinguales PL gilt es den Angebotscharakter des bilingualen Interaktionsangebots beim PL den SuS wiederholt bewusst zu machen. Die vielfältigen Aktivierungs- und Unterstützungsmaterialen sowie die systematische Implementation eines solchen Angebots kann den Eindruck eines obligatorischen, zu erfüllenden Bestandteils erwecken, was kontraproduktiv zur eigentlichen Intention des Sprachangebots operiert. So könnte z. B. die Optionalität der Sprachwahl und des Sprachverhaltens in der Interaktion in verschriftlichten Arbeitsaufträgen für das PL adressiert werden, visuelle Erinnerungen in Form kleiner Schilder am Arbeitsplatz der Gruppe angebracht werden oder die Freiwilligkeit von den Lehrkräften „on the fly“ bei der Moderation des PL wiederholt akzentuiert werden. Andererseits dürften einige SuS von sich selbst aus beim PL nicht bilingual interagieren, sodass diese eventuell explizite Aufforderungen hierzu benötigen würden, damit sie das Angebot zumindest phasenweise ausprobieren. Diesbezüglich gilt es von der Lehrkraft einen Spagat zwischen der Freiwilligkeit des Angebots einerseits sowie Aktivierungen und Aufforderungen andererseits zu vollführen, die initiale Blockaden hinsichtlich des bilingualen Angebots auflockern.

Eine der größten Herausforderungen der Implementation bilingualer Sprachangebote beim PL in den Regelunterricht stellt die Gruppenzusammensetzung dar. Neben der Zusammensetzung möglichst gleichgeschlechtlicher und leistungsheterogener Gruppen beim PL (vgl. z. B. Robinson, Schofield \& Steers-Wentzell, 2005; Rohrbeck et al., 2003) sollte die Gruppenzusammensetzung auch hinsichtlich der Möglichkeiten des bilingualen Sprachangebots 
für den konkret vorliegenden Klassenverbund reflektiert werden. Als Voraussetzung zur Implementation eines solchen Angebots müssen mindestens zwei SuS vorhanden sein, die über die gleichen Sprachprofile verfügen. In städtischen Ballungsgebieten des Westens Deutschlands dürften sich hinsichtlich der größten Gruppen mit MH in Deutschland verschiedene Möglichkeiten zur Gruppenzusammensetzung ergeben, da in diesen Kontexten eine höhere Wahrscheinlichkeit existiert, dass mehr als eine zweisprachig aufwachsende SchülerIn mit der (weiteren) L1 Türkisch, Russisch, Polnisch und Italienisch in der Klasse vorhanden ist. Im ländlichen Raum sowie im Osten Deutschlands, in welchen der Anteil von Bürgern mit MH geringer als in städtischen Ballungsgebieten respektive im Westen Deutschlands ist, dürften die Lehrkräfte wohl weniger Gestaltungsmöglichkeiten bei der Gruppenzusammensetzung in Abhängigkeit der Sprachprofile ihrer SuS haben. Zudem ist ein bilinguales Interaktionsangebot für weitere Gruppen mit $\mathrm{MH}$ in Deutschland nur schwerlich umzusetzen, da sich nicht für jedes Sprachprofil stets zwei SuS zur Gruppierung finden lassen werden. Dementsprechend sind die Gestaltungsmöglichkeiten der Gruppenzusammensetzung primär von den Gegebenheiten der jeweiligen Klasse abhängig.

Zudem gilt es die produktive Seite von Anerkennungsprozessen als $\mathrm{Zu}-$ schreibung möglichst zu vermeiden, sodass die Gruppen nicht nur ausgehend von den Sprachprofilen der SuS zusammengesetzt werden und auch nicht zwingend dauerhaft bestehen bleiben sollten. Ein dauerhafte Gruppierung primär nach Sprachprofilen würde eine Segregation der Klasse anhand sprachlich-biographischer Merkmale vornehmen, die bilingual aufwachsenden SuS die Kategorie des „Anderen“(vgl. Mecherill, 2005, S. 323f) oktroyieren würde und auch missverständlich als Gruppierung nach nationalen Kategorien verstanden werden könnte. So könnten zunächst diejenigen bilingual aufwachsenden SuS einer Klasse, die zu PL-Gruppen zusammengesetzt werden können, hinsichtlich ihrer Einstellungen zu bilingualer Interaktion beim PL von der Lehrkraft in kindgerechter Weise befragt werden. Ausgehend von diesen Informationen können zunächst Gruppen mit und ohne exklusive Besetzung von SuS mit denselben Sprachen erzeugt werden. Bei PL-Formaten mit einer Gruppengröße mit mindestens vier SuS könnte auch zeitweise in zwei Zweiergruppen mit verschiedenen Aufgaben und Materialien zusammengearbeitet werden, die sich zum Schluss wieder in der Gesamtgruppe einfinden und ihre Ergebnisse besprechen. In solchen Gruppen könnte auch ein bilinguales Sprachangebot implementiert werden, sofern zwei der Gruppenmitglieder dieselben Sprachen teilen.

Nach einigen Sitzungen mit der Sozialform des PL können die Gruppen neu zusammengestellt werden, sodass auch bilinguale SuS die vorher in bilingual interagierenden Gruppen waren, in monolingual interagierende Gruppen wechseln. Hierdurch können die bilingual aufwachsenden SuS auch die poten- 
tiellen Unterschiede ihrer Partizipation und Leistung beim PL in einem monolingualen und bilingualen Sprachmodus miteinander vergleichen. Solche changierenden Gruppenzusammensetzungen ermöglichen es auch bilingual aufwachsenden SuS, die das bilinguale Interaktionsangebot strikt ablehnen, temporär mit anderen bilingual aufwachsenden SuS zusammenarbeiten, die quantitativ einen hohen Gebrauch von bilingualen Praktiken beim PL machen. Da das bilinguale Interaktionsverhalten beim PL abhängig von der Dyade oder Gruppe ist, könnte sich ein solches Setting eignen, um Peers mit einer ablehnenden Einstellung gegenüber bilingualer Interaktion in Bildungsprozessen mit dieser zu konfrontieren. In den sich anschließenden Reflexionsphasen können die Chancen und Herausforderungen bilingualer Praktiken für die einzelnen SuS identifiziert und reflektiert werden, die in Zukunft bei der Gruppenzusammensetzung adaptiv berücksichtigt werden können.

Zusammengefasst erfordert die Umsetzung eines bilingualen Interaktionsangebots beim PL in den Unterricht der Regelschule die Berücksichtigung weiterer organisatorischer Aspekte sowie zusätzlicher Aktivierungen und Materialien, die es bei der Schaffung von notwendigen sprachlichen Voraussetzungen sowie der Planung und Strukturierung des konkreten PL-Formats zu berücksichtigen gilt. Die eben angesprochene Problematik, ein bilinguales Sprachangebot in Abhängigkeit der Sprachprofile der jeweiligen Klasse sowie den Einstellungen und Präferenzen der SuS zu implementieren, weist auf die Notwendigkeit der Langfristigkeit solcher Angebote hin. Die bilingual aufwachsenden SuS benötigen eine Vielzahl von Lerngelegenheiten in unterschiedlichen Gruppenkonstellationen, um die individuelle Förderlichkeit sowie die Herausforderungen eines solchen Sprachangebots für sich selbst auszuprobieren. Zudem sollten sie die Chance erhalten, über einen längeren Zeitraum ihre sprachlichen Kompetenzen in beiden Sprachen in dieser Sozialform weiter zu entwickeln. Dementsprechend sollten solche Angebote nicht als kurzfristige Sozialformen in einem bestimmten Fach oder für einen bestimmten Lerninhalt strukturiert werden, sondern bereits ab der ersten Klasse über mehrere Jahrgangsstufen und Fächer angeboten werden, wobei auch die Grundlagen der Sozialform des PL selbst langfristig und schrittweise während der Schullaufbahn erarbeitet werden sollten. Mit einer Implementation des bilingualen Interaktionsangebots ab der ersten Klasse lässt sich auch das Potential von Transferprozessen im Sinne der Interdependenzhypothese (vgl. Cummins, 2000) steigern, da die SuS durch zweisprachiges Handeln ihre eventuell exklusiv in der nichtverkehrssprachlichen L1 gemachten vorschulischen Literacyerfahrungen in der Familie in den Unterricht einbringen könnten. 


\subsection{Limitationen der Studie und Forschungsdesiderata}

Die Forschungsanlage der BiPeer-Studie weist als Datengrundlage angesichts des komplexen, den Lerninhalt, den Sprachhintergrund sowie das Sprachangebot systematisch variierenden quasi-experimentellen Treatment-Kontrollgruppen-Designs ein großes Potential zur Untersuchung der Bedeutung des Sprachhintergrunds sowie des Sprachangebots für die curriculumsrelevanten Phänomene Lese- und Rechenstrategien beim PL auf. Allerdings sind mit diesem komplexen Design sowie der begrenzten Ressourcen drittmittelfinanzierter Interventionsstudien auch einige methodisch-statistische Herausforderungen verbunden, die sich auch in der vorliegenden Studie widerspiegeln. Das spezifische Probandenprofil bilingual deutsch-türkischsprachig aufwachsender SuS der dritten und vierten Klasse sowie die organisatorischen Bedingungen der Dyadenzusammenstellung abhängig von der Zugehörigkeit zur selben Schule und übereinstimmenden Trainingszeiten verminderten nicht nur die Menge potentieller Probanden, sondern schränkten auch die Möglichkeiten der Gruppierung der Peers zu Dyaden ein. Hinzu kommt die dyadische Abhängigkeit der Prävalenz bilingualer Praktiken beim PL, die Verfahren zur dyadischen Datenanalyse oder eine Aggregation der Individualdaten auf Dyadenebene verlangt. Diese organisatorischen und statistisch-methodischen Limitierungen gepaart mit restringierten Ressourcen und einem komplexen quasi-experimentellen Treatment-Kontrollgruppen-Design mit insgesamt sechs Gruppen führt zu einer als klein zu bezeichnende Stichprobengröße, die über eine geringe Power zur inferenzstatistischen Identifikation von signifikanten Effekten, Unterschieden sowie Zusammenhängen verfügt, hinsichtlich einiger zentraler Variablen keine Normalverteilung aufweist und letztlich keine echte Zufallsstichprobe darstellt. Dementsprechend lassen sich geringe Effektstärken mit der vorliegenden Stichprobe kaum identifizieren, was sicherlich zu den ausbleibenden signifikanten Zusammenhängen der Fragestellung F3 beigetragen hat. Die fehlende Normalverteilung zentraler Variablen wie der Prävalenz bilingualer Praktiken zwingt zur Transformationen der Datengrundlage wie bei Fragestellung F1, zur teilweisen Nutzung non-parametrischer Analyseverfahren auf Dyadenebene wie bei Fragestellung F2 und zum Gebrauch bestimmter, die dyadische Abhängigkeit berücksichtigender Analyseverfahren trotz der Verletzung von statistischen Voraussetzungen wie bei Fragestellung F3. Zwar sind die Ergebnisse robust genug, sodass deren Tendenzen auch unter Alternation der Analyseverfahren unverändert bleiben. Nichtsdestotrotz kann die Stichprobengröße der Studienanlage sowie der einzelnen Gruppen als suboptimal bezeichnet werden.

Das quasi-experimentelle, konsequenzfreie „low stakes setting“ der Intervention samt äußerst hohem Betreuungsschlüssel von eins zu zwei nach dem 
regulärem Unterricht lässt sich hinsichtlich seiner ökologischen Validität kritisieren. Die Intervention der vorliegenden Untersuchung spiegelt kaum die Bedingungen des typischen Regelschul- und Regelunterrichtskontexts wider, was die Übertragbarkeit der Ergebnisse in die Praxis vermindert. Insbesondere die mit den Aktivierungen des bilingualen Sprachangebots verbundenen Aspekte wie z. B. die türkisch-deutschsprachigen bilingualen Übungsleitungen in den Gruppen LGT/D und RGT/D und deren standardisierte bilinguale Instruktionen stellen Bestandteile der Trainings dar, deren Implementation im Kontext des Regelunterrichts aufgrund fehlender personeller Ressourcen kaum umzusetzen ist. Die ökologische Validität der vorliegenden Studie wird durch die Fokussierung lediglich einer Sprachgruppe der bilingual türkisch-deutschsprachig aufwachsenden SuS sowie eines bestimmten PL-Formats weiterhin eingeschränkt. Es ist nicht zwingend davon auszugehen, dass andere Sprachgruppen und auch liberalere PL-Formate mit mehr als zwei Gruppenmitgliedern in ähnlicher Weise das bilinguale Sprachangebot umsetzen. Dementsprechend kann auf Basis der bisherigen und in dieser Studie vorliegenden empirischen Befundlage nicht zwingend von ähnlichen Prävalenzen bilingualer Praktiken, Einstellungen zum Sprachangebot sowie Funktionen bilingualer Interaktion im Regelschulkontext ausgegangen werden. Für eine praktische Implementation eines solchen bilingualen Sprachangebots beim PL in die Praxis gilt es - wie oben in Abschnitt 9. 3 dargestellt - Adaptionen vorzunehmen, die den Voraussetzungen und Bedingungen der Regelschule gerecht werden, damit sich bilinguale Interaktionsangebote beim PL auch flächendeckend etablieren können.

Die Intervention kann angesichts der Dauer von zwölf Sitzungen je $45 \mathrm{Mi}-$ nuten als ein relativ kurzes Training beschrieben werden. Zwar weisen die Ergebnisse von Meta-Analysen auf die Unabhängigkeit der Sozialform des PL von der Dauer bezüglich der Lernwirksamkeit hin (vgl. z. B. Robinson, Schofield \& Steers-Wentzell, 2005; Rohrbeck et al., 2003), allerdings erscheinen zwölf Sitzungen zur Umsetzung bilingualer Praktiken im Bildungskontext und zur Entwicklung bildungssprachlicher Fähigkeiten zur Interaktion beim PL als äußerst kurzer Zeitraum, insbesondere in Anbetracht der fehlenden Erfahrung im bildungssprachlichen Gebrauch der nicht-verkehrssprachlichen L1 innerhalb eines vom monolingualen Habitus geprägten Submersionskontextes. Zudem enthielt die Intervention nur relativ wenige, exklusiv auf das bilinguale Sprachangebot fokussierte Phasen, in denen der Umgang, potentielle Vorteile sowie Anwendungsmöglichkeiten bilingualer Praktiken beim PL instruiert und geübt wurden, da die Lerninhalte Lese- und Rechenstrategien selbst viel Zeit in Anspruch genommen haben. Hierbei handelt es sich trotz der verschiedenen Erhebungszeitpunkte auch nicht wirklich um ein Längsschnittdesign, sodass anhand der vorliegenden Daten keine validen Aussagen über Entwicklungstendenzen des bilingualen Sprachverhaltens der Probanden gemacht werden können. 
Ein in dieser Studie nicht umgesetztes Potential zur Erweiterung des Forschungsfeldes sowie zur Vertiefung des Verständnisses der subjektiven Wahrnehmung von bilingual aufwachsenden SuS hinsichtlich bilingualer Interaktionsangebote stellt zum einen der fehlende empirische Einbezug von identitätstheoretisch und anerkennungstheoretisch bedeutsamen Phänomenen durch die Operationalisierung von Identitätstypen und Anerkennungs- sowie Missachtungserfahrungen unter Verwendung von Fragebogenskalen oder Interviews dar. Mit solchen Variablen hätten die Einstellungen sowie Wahrnehmungen von bilingual aufwachsenden SuS bezüglich des empfundenen Zusammenhangs ihrer Identität mit ihrem Sprachprofil und -verhalten sowie der rechtlichen Anerkennung und sozialen Wertschätzung ihrer bilingualen Disposition in der Regelschule fruchtbarer ergründet werden können. Solche Einblicke sind nicht nur für die praktische Implementation bilingualer Sprachangebote relevant, sondern bergen auch ein gewisses Potential zur Verwendung anerkennungstheoretischer Perspektiven als Analyseinstrumente in pädagogisch/erziehungswissenschaftlichen Kontexten (vgl. Balzer \& Ricken, 2010, S. 43). Zum anderen hätten Interviews mit ausgewählten Probanden zu ihrer subjektiven Wahrnehmung des Sprachangebots im Anschluss an die Intervention tiefere Einblicke ermöglicht als das in der vorliegenden Studie kurze Selbstberichtsformat.

Angesichts der vorliegenden Ergebnisse unter Berücksichtigung der bisherigen Befundlage würden sich Längsschnittstudien zur Implementation fächerübergreifender bilingualer Interaktionsangebote beim PL in regulären Klassen des Submersionskontextes ab der Einschulung über einen die Grundschule überspannenden Zeitraum empfehlen. Eine solche Untersuchungsanlage in einem ökologisch validen Kontext mit verschiedenen Sprachgruppen und -profilen, eingeschränkten zeitlichen, personellen und materiellen Ressourcen, Lehrkräften mit heterogenen Wissensbeständen, Einstellungen und Überzeugungen zu mehrsprachigen Unterrichtsangeboten etc. ermöglicht die Überprüfung der Fruchtbarkeit bilingualer Praktiken beim PL in der praktischen Umsetzung unter der Gewährung eines angemessenen Zeitraums zur Entfaltung des Entwicklungspotentials der bilingualen Disposition. Insbesondere die Herausforderungen der begrenzten und suboptimalen Ressourcen der Regelschule sowie der Alternierung der Gruppenzusammensetzungen über einen längeren Zeitraum könnten in einer solchen Forschungsanlage hinsichtlich ihrer Bedeutung für PL mit bilingualen Interaktionsangeboten untersucht werden. Eine solche Untersuchungsanlage würde eine regelmäßige, konsequente und fächerübergreifende Implementation der Sozialform des PL in den jeweiligen Schulen verlangen, sodass diese ebenfalls eine längsschnittliche Untersuchung der Sozialform des PL selbst ermöglichen würde. Diesbezüglich wären voranzustellende Lehrerfortbildungen mit Praxisphasen zur fruchtbaren Umsetzung von PL für den eigenen Klassenkontext eine vielversprechende, wenn nicht gar notwendige Voraussetzung. 
Das PL müsste mit dem Schulbeginn unter Berücksichtigung zentraler Erkenntnisse des bisherigen Forschungsstandes zu lernförderlichen Merkmalen und zentralen Determinanten von PL (vgl. Howe, 2010; Johnson \& Johnson, 1999; Iiskala, Vauras \& Lehtinen, 2004; Littleton \& Mercer, 2010; Robinson, Schofield \& Steers-Wentzell, 2005; Rohrbeck et al., 2003) systematisch geplant und sukzessive eingeführt werden, wobei auch Zeit und Ressourcen für förderliche Gruppeninteraktionen und -prozesse zur Verfügung gestellt und curriculumsrelevante Inhalte beim PL erworben werden müssten. Insbesondere hinsichtlich des Schriftspracherwerbs und dem Erwerb arithmetischer Grundlagen könnten die nicht zwingend in der verkehrssprachlichen L1 gemachten Bildungserfahrungen innerhalb der familiären Sozialisation durch Transferprozesse in die Regelschule übermittelt werden. Zudem können die sprachvergleichenden, metalinguistischen Fähigkeiten bilingual aufwachsender SuS sowie potentielle Unterschiede in der (schrift-)sprachlichen Realisierung derselben Konzepte in verschiedenen Sprachen in lernförderlicher Weise in PL-Formate integriert werden. Ein solch aufwendiges und langfristiges Forschungsvorhaben könnte dementsprechend zur Untersuchung einer Vielzahl weiterer aktueller Forschungsdesiderate der Sozialform PL wie z. B. fruchtbare Implementationsstrategien für PL, geeignete Lerninhalte für PL oder gegenseitige und geteilte metakognitive Regulation beim PL genutzt werden.

Für ein solches Forschungsvorhaben würde sich eine Treatment-Kontrollgruppen Studienanlage mit Mixed-Methods Paralleldesign anbieten, bei welchem sowohl quantitative als auch qualitative Aspekte des PL mit bilingualem Sprachangebots anhand der gleichen Stichprobe simultan untersucht werden. Die Kontrollgruppe könnte hierbei aus Klassen von Regelschulen bestehen, die Formen des PL nicht kontinuierlich und geplant umsetzen. Aus quantitativer Perspektive würden sich Mehrebenenmodelle mit Gruppenunterschieds-, Zusammenhangs- und Regressionsanalysen empfehlen, um potentielle Unterschiede zwischen Experimental- und Kontrollgruppe, Zusammenhänge von individuellen Merkmalen und der Lernwirksamkeit des PL mit bilingualem Interaktionsangebot sowie Prädiktoren für gelingendes PL zu identifizieren. Mehrebenenmodelle können hierbei den Einfluss der Schul-, Klassen und auch der Gruppenebene kontrollieren. Aus qualitativer Perspektive dürften sich insbesondere Interaktionsanalysen auf Basis von Audio- oder Videodateien selektierter Unterrichtseinheiten, Interviews mit „stimulated recall“ mit ausgewählten SuS sowie regelmäßige, kurze schriftsprachliche Selbstberichte zu diversen Aspekten des PL mit bilingualem Sprachangebot empfehlen. Neben typischen bildungsbezogenen sowie motivationalen Aspekten sollten zudem auch verstärkt quantitative und qualitative Operationalisierungen von identitäts- und anerkennungstheoretischen Phänomenen erfolgen, um eine stärker sozio-kulturell geprägte Perspektive auf die zu untersuchenden Aspekte zu erhalten. 
Ein solches Forschungsvorhaben wäre in der Lage, die in Abschnitt 5.5.3 adressierten Forschungslücken und -unzulänglichkeiten wie die hohe Prävalenz von Fallstudien und informeller PL-Formate, die fehlende Konsequenz einer Vielzahl von Mixed-Methods Untersuchungsanlagen sowie der Ignoranz gegenüber der dyadischen Abhängigkeit bilingualer Praktiken beim PL zu adressieren. Das tatsächliche Potential der bedingten bilingualen Disposition bilingual aufwachsender SuS im Submersionskontext für PL lässt sich nur durch längsschnittliche Untersuchungsanlagen ergründen, die personale sowie materielle Ressourcen und Zeit zur Verfügung stellen, um PL mit bilingualem Interaktionsangebot langfristig und fächerübergreifend zu implementieren. 


\section{Fazit}

Die vorliegende Studie hat anhand der Aufbereitung des theoretischen Hintergrunds sowie der aktuellen empirischen Befundlage hinsichtlich Bilingualität, Anerkennungstheorie und PL einerseits das Potential von bilingualen Praktiken für Bildungsprozesse - insbesondere bezüglich der Sozialform des PL dargestellt und andererseits die Missachtung der bilingualen Disposition von SuS mit MH auf der Anerkennungsform des Rechts sowie der Solidarität durch die Regelschule in Deutschland herausgearbeitet.

Bilingual aufwachsende SuS unterscheiden sich in ihrer Sprachsozialisation und -entwicklung von monolingualen SuS primär aufgrund des ,complementary principle“ (vgl. Grosjean, 2010, S. 29ff), welches ihre Lerngelegenheiten zum Spracherwerb in ihren beiden Einzelsprachen determiniert. Auch wenn die (meta-)linguistischen und (meta-)kognitiven Gemeinsamkeiten hinsichtlich des Spracherwerbs und damit einhergehend auch der Sprachkompetenzen zwischen bilingual und monolingual aufwachsenden SuS überwiegen, werden die (temporären) Unterschiede dieser Gruppen durch den monolingualen Habitus (vgl. Gogolin, 1994) des Submersionskontexts der deutschen Regelschule akzentuiert. Das Lernen sowie die Interaktion erfolgen stets monolingual im Deutschen, wobei monolingual aufwachsende SuS die soziale Bezugsnorm darstellen (vgl. ebd.). Dabei weist eine Vielzahl empirischer Studien auf das fruchtbare Potential von bilingualen Praktiken für Bildungsprozesse sowohl im Allgemeinen (vgl. z. B. Barwell, 2014; Clarkson, 2007; Dirim, 1998; García \& Kano, 2014; García \& Li Wei, 2014; Worthy et al, 2013) als auch beim PL (vgl. z. B. Hopewell, 2011; Klingner \& Vaughn, 2000; Moschkovich, 2007; Planas \& Setati, 2009; Rehbein, 2011; Schüler-Meyer et al., 2017; Storch \& Wigglesworth, 2003; Swain \& Lapkin, 2000) hin. Insbesondere diejenigen Potentiale von bilingualen Praktiken für Bildungsprozesse, die nicht unter Verwendung lediglich einer Sprache erzeugt werden können, stellen äußerst fruchtbare und vielversprechende Lernmöglichkeiten dar. Diesbezüglich wären vor allem die folgenden Potentiale zu nennen: die tiefere Verarbeitung von Lerninhalten durch ein zweisprachige Durchdringung dieser (vgl. Baker, 2011, S. 288), metalinguistische Reflexionen und Sprachvergleiche (vgl. z. B. Hopewell, 2011; Storch \& Wigglesworth, 2003; Swain \& Lapkin, 2000), Übersetzungen (vgl. Kern, 1994), Transferprozesse (Cummins, 2000), alternative Erklärungen durch bilinguales Sprachhandeln (vgl. z. B. Hopewell, 2011; Klingner \& Vaughn, 2000), Elaboration von Propositionen ohne Wiederholung des zuvor Gesagten durch Code-Switching (vgl. Moschkovich, 2007) und die produktive Nutzung potentieller Unterschiede in der sprachlichen Realisierung von zu lernenden Konzepten, die distinkte Aspekte des Konzepts akzentuieren wie z. B. bei der Artikulation von mathematischen Brüchen im Türkischen und Deutschen (vgl. Schüler-Meyer et al., 2017). 
Wenn Denken als ,an individualized form of interpersonal communication“ (Sfard, 2015, S. 249) sowie Kommunikation und Lernen als „two sides of the same coin" interpretiert werden können, dann spielt Kommunikation keine sekundäre Rolle als Vermittler von Lernprozessen, sondern ist ,the very object of learning“ (ebd.). Wissen ist in Form von Propositionen, Schemata und mentalen Modellen sprachlich enkodiert (vgl. Bialystok, 2013, S. 624). Wird die Nutzung der (weiteren) L1 von bilingual aufwachsenden SuS als kognitives Werkzeug (vgl. Hamers \& Blanc, 2000) für Lernprozesse vom monolingualen Habitus (vgl. Gogolin, 1994) des Submersionskontexts unterbunden, sodass die SuS nur unter Verwendung der Verkehrssprache lernen dürfen, kann dies einerseits zu einer Reduktion ihrer ,thinking power“ führen oder gar mit ,asking the child to fail“" gleichgesetzt werden (vgl. Baker, 2011, S. 289). Die zentrale Bedeutung von Sprache für Bildungsprozesse wird auch durch empirische Befunde zur Lernwirksamkeit förderlicher Kommunikation beim PL unterstützt (vgl. z. B. Johnson \& Johnson, 1999; Howe, 2010; Littleton \& Mercer, 2010). Gerade diejenigen fruchtbaren Sprachhandlungen beim PL wie z. B. Assertionen/Propositionen (vgl. Howe et al., 2007), Widersprüche (Howe, 2010), Erklärungen und Argumente (vgl. Asterhan \& Schwarz, 2007; Chinn \& Clark, 2013) können durch bilinguale Praktiken fruchtbar beim PL umgesetzt werden. So kann bilinguales Sprachhandeln prinzipiell die Partizipation sowie Teilhabe und dementsprechend auch die Externalisierung von Assertionen beim PL erhöhen (vgl. Baker, 2011, S. 289f; Meyer \& Prediger, 2011). Die Markierung von Widersprüchen durch Code-Switching stellt eine zentrale diskursiv-rhetorische Funktion von Sprachwechseln dar (vgl. z. B. Keim, 2012, S. 167ff). Durch Code-Switching können auch alternative Erklärungen ausgedrückt (vgl. z. B. Hopewell, 2011; Klingner \& Vaughn, 2000) und Elaborationen von Propositionen ohne Wiederholung des Gesagten erzeugt werden (vgl. Moschkovich, 2007). Die potentielle unterschiedliche sprachliche Realisierung von zu lernenden Konzepten samt der eventuell damit einhergehenden Akzentuierung distinkter Aspekte des Konzepts durch die Verwendung von zwei Sprachen (vgl. Schüler-Meyer et al., 2017) kann ebenfalls zur alternativen Erklärung von Sachverhalten, aber auch zur Unterstützung und Stärkung der jeweiligen Argumentation verwendet werden.

Zudem darf nicht vergessen werden, dass die Konstrukte Identität, Kultur und Sprache eng miteinander verwoben sind (vgl. z. B. Hu, 2007) und Bilingualität beziehungsweise die (weitere) L1 von bilingual aufwachsenden Sprechern einen Marker der Identität sowie der Gruppenzugehörigkeit darstellen kann (vgl. z. B. Gogolin, 1994, S. 14; Hamers \& Blanc, 2000, S. 202; Keim, 2007, S. 186): „Migrantinnen und Migranten selbst, Erwachsene wie Kinder, sehen, verstehen und gebrauchen ihre Sprachen [...] vor allem im Rahmen ihrer Identitätsbewusstwerdung und ihrer Familienbindung" (Krumm, 2009, S. 245). Bilingual aufwachsende Sprecher mit MH können hierbei hybride Identitäten (vgl. z. B. Bohnsack \& Nohl, 2001; Hu, 2007; Keim, 2007; Stojanov, 
2006; Tracy, 2011) ausbilden, „die im Übergang zwischen verschiedenen Positionen schweben, die zur gleichen Zeit auf verschiedene kulturelle Traditionen zurückgreifen und die das Resultat komplizierter Kreuzungen und kultureller Verbindungen sind“ (Hall, 1994, S. 218). Werden diese hybriden Identitäten sowie allgemein die bilinguale Disposition durch Sprachideologien wie der ,assimilation ideology“ oder der „ethnist ideology“ (vgl. Bourhis, 2011) unterdrückt und/oder zu einem vermeintlichen Problem für Integration, Identitätsbildung (vgl. Kersten et al., 2011) und die schulische Bildung (vgl. z. B. Gomolla \& Radtke, 2009; Stojanov, 2011) - insbesondere in Form eines Konkurrenzverhältnisses zwischen der (weiteren) L1 und der Verkehrssprache (vgl. z. B. Esser, 2009; Wlossek \& Rost-Roth, 2016) - stilisiert, kann sich dies negativ auf das Selbstkonzept auswirken: „It is not just the child's home language that is deprecated. The identity of the child, the parents, grandparents, the home, community, religion and culture appear to be deprecated [...]. It also denies or denounces what they hold most sacred: self-esteem, identity, relationships, roots, religion and sometimes race." (Baker, 2011, S. 213).

Die vorliegende Studie charakterisiert auf Basis erziehungswissenschaftlich/pädagogisch orientierter anerkennungstheoretischer Reflexionen und Perspektiven (vgl. z. B. Balzer \& Ricken, 2010; Mecherill, 2005; Stojanov, 2006, 2011) den durch den monolingualen Habitus geprägten Umgang der deutschen Regelschule mit der migrationsbedingten bilingualen Disposition eines Teils ihrer Schülerschaft als eine Missachtung auf den Anerkennungsformen des Rechts sowie der Solidarität. Die flexibel eingesetzte institutionelle Diskriminierung auf Basis ideologischer, vermeintlich auf sprachlich-kultureller Differenz beruhender Begründungslogiken, die fehlende oder positiv diskriminierende Berücksichtigung der zweisprachigen Lebensrealität (vgl. Gomolla \& Radtke, 2009) und die Negation der (weiteren) L1 als Ressource für Bildungsprozesse bei bilingual aufwachsenden SuS mit MH weisen in der Terminologie Honneths (2003) Formen der Ausschließung auf. Die implizite Sprachenhierarchie der deutschen Regelschule, die durch den $\mathrm{MH}$ vorhandene, natürlich erworbene Sprachen ihrer Schülerschaft samt ihrer typischen bilingualen Praktiken aus einer Defizitperspektive problematisiert, während dieses Narrativ für bestimmte andere (weitere) Erstsprachen und/oder Personengruppen nicht gilt, kann in Anlehnung an Honneth (ebd.) als eine Entwürdigung interpretiert werden. Die Identifikation dieser Missachtungen kann als eine Form von Bildungsungerechtigkeit bezeichnet werden, insbesondere angesichts der Charakterisierung von Anerkennung durch Stojanov (2006, S. 168) als „Voraussetzung und Triebwerk von Bildung". Zudem unterstützt diese Analyse auch die These von Gomolla \& Radtke (2009, S. 20-21), dass ein „nicht unbedeutender Teil der Ungleichheit in der Bildungsbeteiligung“von SuS ohne im Vergleich zu SuS mit MH nicht auf den Eigenschaften der SuS mit MH und ihren migrationsbedingten Ausgangsvoraussetzungen beruhen, ,,sondern [...] in der Organisation Schule selbst erzeugt" wird. 
Angesichts dieser Diskrepanz zwischen den Bildungspotentialen von Bilingualität und bilingualem Sprachhandeln und dem Umgang der deutschen Regelschule mit migrationsbedingter Mehrsprachigkeit hat die vorliegende Studie unter Verwendung eines Mixed-Methods Vertiefungsdesigns untersucht, ob ein optionales, explizit vermitteltes bilinguales Interaktionsangebot beim PL eine potentielle Möglichkeit zur temporären Implementation bilingualer Praktiken in den Regelschulkontext darstellt. Die Ergebnisse dieser Untersuchung unterstützen zum Teil Befunde aus der bisherigen Forschung, fügen dem Forschungsstand aber auch neue Evidenz hinzu. Das Ergebnis der Fragestellung F1 akzentuiert die Notwendigkeit der Operationalisierung der Prävalenz bilingualer Praktiken als ,,mixed variable“ in Abhängigkeit von der Dyade oder Gruppe (vgl. Kenny, Kashy \& Cook, 2006). Für einen adäquaten methodisch-statistischen Umgang mit der Prävalenz bilingualer Praktiken beim PL benötigt es dementsprechend Verfahren der dyadischen Datenanalyse (vgl. ebd.) oder der Mehrebenenanalyse. Die Ergebnisse von F2 weisen einen hohen Effekt eines expliziten, mit diversen Aktivierungen versehenen bilingualen Interaktionsangebots auf die Prävalenz bilingualer Praktiken auf (F2a). Zudem deuten die Ergebnisse auf die Robustheit der Prävalenz bilingualer Praktiken vom konkreten Lerninhalt (F2b) sowie von den Phasen und Aufgaben innerhalb einer Domäne hin (F2c), auch wenn sich diesbezüglich eine Phase im Lesetraining signifikant von den Lesestrategien unterschieden hat. Bei bilingualen Interaktionsangeboten beim PL gilt es aber darauf zu achten, dass die SuS das optionale Interaktionsangebot nicht als obligatorisch zu erfüllenden Bestandteil des PL missverstehen, was angesichts der Ergebnisse von F4 von einem Teil der Probanden der Stichprobe der vorliegenden Studie in dieser Weise fehlinterpretiert wurde. Dementsprechend scheint die Etablierung einer angemessenen Balance zwischen der Charakterisierung der bilingualen Interaktion beim PL als optionales Angebot und Aktivierungen zu bilingualem Sprachhandeln eine herausfordernde didaktische Aufgabe darzustellen. Wie in Abschnitt 9.2 ausgeführt, könnten die fehlenden signifikanten Zusammenhänge von sprachlichen und motivationalen Variablen mit der Prävalenz bilingualer Praktiken (F3) hierdurch ebenfalls beeinflusst worden sein, wobei die statistischen Limitationen der Datengrundlage sicherlich einen relevanten Beitrag zu diesen Ergebnissen geleistet haben dürften. Angesichts soziolinguistisch orientierter Theorien der Sprachwahl und des Sprachverhaltens bilingualer Sprecher (vgl. Grosjean, 2008; Myers-Scotton, 2006, Sachdev, Giles \& Pauwels, 2013) sowie anerkennungstheoretischer Reflexionen (vgl. Mecherill, 2005; Stojanov, 2006, 2011) sollten sich solche Zusammenhänge mit einer geeigneteren Datengrundlage unter Verwendung dyadischer Datenanalyse finden lassen. Die in F4 untersuchten Motive für die Annahme und Ablehnung des bilingualen Sprachangebots stellen für die praktische Umsetzung eines solchen Angebots höchstrelevante Datenquellen zu Chancen und Herausforderungen bilingualer Praktiken beim PL aus der Perspektive der SuS dar. So scheint das 
bilinguale Interaktionsangebot einerseits für einen Teil der Stichprobe zu einer Vereinfachung des PL sowie Erweiterung ihres sprachlichen Handlungsraums, zu Lerngelegenheiten ihrer (weiteren) L1 und positiven Emotionen wie dem Empfinden von Spaß zu führen. Andererseits stellt das Angebot für andere Probanden eine zusätzliche Belastung dar, der sie aufgrund fehlender Sprachkompetenzen in der (weiteren) L1 nicht nachkommen können oder angesichts ihrer Sprachasymmetrie zu Gunsten des Deutschen nicht nachkommen wollen. Zudem scheint die (weitere) L1 für einige Probanden negativ konnotiert zu sein, was ebenfalls eine Hürde zur Implementation eines bilingualen Sprachangebots beim PL darstellt. Die Ergebnisse von F5 zeigen, dass die Probanden bilinguale Praktiken vor allem ,on-task“ statt „off-task“ einsetzen (F5a) und sich nicht zwischen den Lerninhalten Lesen und Rechnen signifikant unterscheiden (F5b). Bilinguale Praktiken werden von den Probanden nur selten zur direkten Aufgabenbearbeitung, dafür aber vor allem zum Task-Management und zur metakognitiven Regulation verwendet, welche beide zentrale Aufgaben beim PL darstellen und zur effizienten Koordinierung der Zusammenarbeit und zur Erzeugung von Intersubjektivität beitragen. Zwar ist hierbei auch unklar, ob die hohen Prävalenzen dieser Funktionen zum Teil auch durch die Fehlinterpretation des bilingualen Sprachangebots als obligatorischer Bestandteil der Intervention determiniert wurden. Nichtsdestotrotz stellen Task-Management und metakognitive Regulation Funktionen dar, die trotz geringer sprachlicher Kompetenzen in der (weiteren) L1 umgesetzt werden können, was sie insbesondere zu Beginn von bilingualem PL für eine Annäherung an den Gebrauch bilingualer Praktiken prädestiniert.

Insgesamt lassen sich die Ergebnisse der vorliegenden Studie in die bisherigen Befunde zum Bildungspotential von Bilingualität beziehungsweise von bilingualen Praktiken beim PL einreihen. Letztlich haben diese Ergebnisse allerdings aufgrund des in Abschnitt 9.4 kritisch reflektierten quasi-experimentellen, konsequenzfreien „low stakes“ Kontext der Intervention samt äußerst hohem Betreuungsschlüssel von eins zu zwei nach dem regulären Unterricht eine eingeschränkte ökologische Validität. Viele der in Abschnitt 9.3 diskutierten weiteren Hürden und Herausforderungen einer Implementation von PL mit bilingualem Interaktionsangebot konnten mit der Studienanlage der vorliegenden Arbeit nicht untersucht werden. Insbesondere zentrale Faktoren für die Implementation eines solchen Angebots wie z. B. alternierende Gruppenzusammensetzungen beim PL und die Unterstützung bilingualer Interaktion durch Instruktionen und Materialien einer monolingualen Lehrkraft, die die Lernwirksamkeit und Annahme eines solchen Angebots stark beeinflussen sollten, konnten in der vorliegenden Studie nicht berücksichtigt werden. Erst dauerhaft angelegte Mixed-Methods Längsschnittuntersuchungen im Regelschulkontext unter enger Kooperation von praktizierenden Lehrkräften und wissenschaftlicher Forschung können die Chancen und Herausforderungen eines bilingualen Interaktionsangebots beim PL ökologisch valide untersuchen. 
Eventuell kann bereits das Nachfolgeprojekt der BiPeer-Studie „Förderung der Deutsch-Lesekompetenz durch mehrsprachigkeitssensibles Reziprokes Lehren im Grundschulunterricht“ - kurz „MerLe“ - unter der Leitung von Frau Jun. Prof. Dr. Dominique Rauch und Prof. Dr. Jasmin Decristan einen Beitrag zu dieser Forschungslücke leisten. Auch wenn die Studienanlage von „MerLe“ auch kein Längsschnittdesign darstellt, wird in diesem Forschungsprojekt PL mit bilingualem Interaktionsangebot von zuvor geschulten Grundschullehrkräften im regulären Unterrichtskontext des Fachs Deutsch implementiert. Mit dieser Forschungsanlage können sicherlich einige der in Abschnitt 9.3 diskutierten praktischen Implikationen sowie Chancen und Herausforderungen von PL mit bilingualem Interaktionsangebot im Regelschulkontext untersucht werden.

Es bleibt zu hoffen, dass sich in Zukunft weitere an Bildungsgerechtigkeit interessierte Forscher der Erziehungswissenschaft, empirischen Bildungsforschung, Linguistik, Didaktik und Psychologie sowie Lehrkräfte mit dem interdisziplinären Themenkomplex (migrationsbedingter) Bilingualität und Bildung evidenzbasiert auseinandersetzen. Nur durch eine überzeugende empirische Befundlage und den Einbezug von praktizierenden Lehrkräften kann ein fruchtbarer Transfer der Erkenntnisse der Forschung in die pädagogische Praxis gelingen. Die Missachtung der migrationsbedingten bilingualen Disposition von SuS mit MH durch die Regelschule in der Bundesrepublik Deutschland gilt es in Zukunft zu überwinden, damit diese Schülerschaft Anerkennung für ihre potentiell lernförderliche Ressource der Bilingualität erhält, die ihnen aus wissenschaftlicher, evidenzbasierter Perspektive zusteht. 


\section{Literaturverzeichnis}

Adesope, O. O., Lavin, T., Thompson, T. \& Ungerleider, C. (2011). Pedagogical Strategies for Teaching Literacy to ESL Immigrant Students: A MetaAnalysis. British Journal of Educational Psychology, 81 (4), 629-653.

Adi-Japha, E., Berberich-Artzi, J. \& Libnawi, A. (2010). Cognitive Flexibility in Drawings of Bilingual Children. Child Development, 81, 1356-1366.

Adl-Amini, K. (2018). Tutorielles Lernen im naturwissenschaftlichen Sachunterricht der Grundschule. Umsetzung und Wirkung. Münster: Waxmann.

Altarriba, J. \& Isurin, L. (Hrsg.) (2012). Memory, Language, and Bilingualism. Theoretical and Applied Approaches. Cambridge: Cambridge University Press.

Arends-Tóth, J. \& van de Vijver, F. (2006). Issues in the Conceptualization and Assessment of Acculturation. In M. H. Bornstein \& L. R. Cote (Hrsg.), Acculturation and Parent-Child Relationships. Measurement and Development (S. 32-66). Mahwah, NJ: Lawrence Erlbaum Associates.

Aronin, L. \& Singleton, D. M. (2012). Multilingualism. Amsterdam: John Benjamins.

Artelt, C. (2006). Lernstrategien in der Schule. In H. Mandl \& H. Friedrich (Hrsg.), Handbuch Lernstrategien (S. 337-351). Göttingen: Hogrefe.

Artelt, C., Schneider, W. \& Schiefele, U. \& Stanat, P. (2002). Ländervergleich zur Lesekompetenz. In J. Baumert, C. Artelt, E. Klieme, M. Neubrand, M. Prenzel, U. Schiefele, W. Schneider, K.-J. Tillmann \& M. Weiß (Hrsg.), PISA 2000. Die Länder der Bundesrepublik Deutschland im Vergleich: Deutsches PISA-Konsortium (S. 55-94). Opladen: Leske + Budrich.

Asterhan, C. S. C. \& Schwarz, B. B. (2009). Argumentation and Explanation in Conceptual Change. Indications From Protocol Analyses of Peer-toPeer Dialog. Cognitive Science, 33 (3), 374-400.

Athanasopoulos, P. (2009). Cognitive Representation of Colour in Bilinguals. The Case of Greek Blues. Bilingualism: Language and Cognition, 12 (1), 83-95.

Athanasopoulos, P., Damjanovic, L., Krajciova, A. \& Sasaki, M. (2011). Representation of Colour Concepts in Bilingual Cognition. The Case of Japanese Blues. Bilingualism: Language and Cognition, 14 (1), 9-17.

Athanasopouloulos, P. \& Aveledo, F. (2012). Linguistic Relativity and Bilingualism. In J. Altarriba \& L. Isurin (Hrsg.), Memory, Language, and Bilingualism. Theoretical and Applied Approaches (S. 236-255). Cambridge: Cambridge University Press.

Auer, P. (1988). A Conversation Analytic Approach to Code-Switching and Transfer. In M. Heller (Hrsg.), Codeswitching. Anthropological and Sociolinguistic Perspectives (S. 187-211). Berlin: De Gruyter Mouton. 
Auer, P. (2009). Competence in Performance: Code-Switching und andere Formen bilingualen Sprechens. In I. Gogolin \& U. Neumann (Hrsg.), Streitfall Zweisprachigkeit-The Bilingualism Controversy (S. 91-110). Wiesbaden: VS Verlag für Sozialwissenschaften.

Auerbach, E. R. (1993). Reexamining English Only in the ESL Classroom. TESOL Quarterly, 27 (1), 9-32.

Ayten, A. (2016). KOALA - koordinierte Alphabetisierung im Anfangsunterricht. Die Grundschulzeitschrift, 30, 46-47.

Baker, C. (2011). Foundations of Bilingual Education and Bilingualism. Bristol: Multilingual Matters.

Balzer, N. \& Ricken, N. (2010). Anerkennung als pädagogisches Problem. Markierungen im erziehungswissenschaftlichen Diskurs. In A. Schäfer \& C. Thompson (Hrsg.), Anerkennung (S. 35-87). Paderborn: Schöningh.

Barkley, R. A. (2012). Executive Functions. What They are, How they Work, and Why they Evolved. New York: Guilford.

Bartolotti, J. \& Marian, V. (2012). Bilingual Memory: Structure, Access, and Processing. In J. Altarriba \& L. Isurin (Hrsg.), Memory, Language, and Bilingualism. Theoretical and Applied Approaches (S. 7-47). Cambridge: Cambridge University Press.

Barwell, R. (2014). Centripetal and Centrifugal Language Forces in one Elementary School Second Language Mathematics Classroom. ZDM, 46 (6), 911-922.

Barwell, R. (Hrsg.). (2009). Multilingualism in Mathematics Classrooms: Global Perspectives. Bristol, UK: Multilingual Matters.

Bauer, L. (1998). Schriftliches Rechnen nach Normalverfahren - wertloses Auslaufmodell oder überdauernde Relevanz? Journal für Mathematikdidaktik, 19 (2/3), 179-200.

Baumert, J. \& Schümer, G. (2001). Familiäre Lebensverhältnisse, Bildungsbeteiligung und Kompetenzerwerb. In J. Baumert, E. Klieme, M. Neubrand, M. Prenzel, U. Schiefele, W. Schneider, P. Stanat, K.-J. Tillmann \& M. Weiß (Hrsg.), PISA 2000. Basiskompetenzen von Schülerinnen und Schülern im internationalen Vergleich (S. 323-407). Wiesbaden: VS Verlag für Sozialwissenschaften.

Baumert, J., Klieme, E., Neubrand, M., Prenzel, M., Schiefele, U., Schneider, W., Stanat, P., Tillmann, K.-J. \& Weiß, M. (Hrsg.) (2001). PISA 2000. Basiskompetenzen von Schülerinnen und Schülern im internationalen Vergleich. Wiesbaden: VS Verlag für Sozialwissenschaften.

Bhabha, H. K. (1990). Nation and Narration. London: Routledge.

Bhatia, T. K. \& Ritchie, W. C. (Hrsg.) (2013). The Handbook of Bilingualism and Multilingualism. Hoboken, NJ: John Wiley \& Sons Inc.

Bialystok, E. (1986). Children's Concept of Word. Journal of Psycholinguistic Research, 15, 13-32. 
Bialystok, E. (1988). Levels of Bilingualism and Levels of Linguistic Awareness. Developmental Psychology, 24 560-567.

Bialystok, E. (2001). Bilingualism in Development. Language, Literacy, and Cognition. Cambridge, UK: Cambridge University Press.

Bialystok, E. (2009). Effects of Bilingualism on Cognitive and Linguistic Performance across Lifespan. In I. Gogolin \& U. Neumann (Hrsg.), Streitfall Zweisprachigkeit-The Bilingualism Controversy (S. 53-68). Wiesbaden: VS Verlag für Sozialwissenschaften.

Bialystok, E. (2013). The Impact of Bilingualism on Language and Literacy Development. In T. K. Bhatia \& W. C. Ritchie (Hrsg.), The Handbook of Bilingualism and Multilingualism (S. 624-648). Hoboken, NJ: John Wiley \& Sons, Inc.

Bialystok, E. (2017). The Bilingual Adaptation: How Minds Accomodate Experience. Psychological Bulletin, 143 (3), 233-262.

Bialystok, E. \& Majumder, S. (1998). The Relationship Between Bilingualism and the Development of Cognitive Processes in Problem-Solving. Applied Psycholinguistics, 19, 69-85.

Bialystok, E., Luk, G. \& Kwan, E. (2005). Bilingualism, Biliteracy, and Learning to Read: Interactions among Languages and Writing Systems. Scientific Studies of Reading, 9, 43-61.

Bialystok, E., Luk, G., Peets, K. F. \& Yang, S. (2010). Receptive Vocabulary Differences in Monolingual and Bilingual Children. Bilingualism: Language and Cognition, 13 (4), 525-531.

Bialystok, E., Craik, F. I. M. \& Luk, G. (2012). Bilingualism. Consequences for Mind and Brain. Trends in Cognitive Sciences, 16 (4), 240-250.

Bien-Miller, L., Akbulut, M., Wildemann, A. \& Reich, H. H. (2017). Zusammenhänge zwischen mehrsprachigen Sprachkompetenzen und Sprachbewusstheit bei Grundschulkindern. Zeitschrift für Erziehungswissenschaft, 20 (2), 193-211.

Bohnsack, R. \& Nohl, A.-M. (2001). Ethnisierung und Differenzerfahrung: Fremdheit als alltägliches und als methodologisches Problem. Zeitschrift für qualitative Bildungs-, Beratungs- und Sozialforschung, 2 (1), 15-36.

Bohrnan, T. M., Bedore, L. M., Peña, E. D., Mendez-Perez, A. \& Gillam, R. B. (2010). What you Hear and What you Say: Language Performance in Spanish-English Bilinguals. International Journal of Bilingual Education and Bilingualism, 13, 325-344.

Bos, W., Klieme, E. \& Köller, O. (Hrsg.). (2010). Schulische Lerngelegenheiten und Kompetenzentwicklung. Festschrift für Jürgen Baumert. Münster: Waxmann.

Bourdieu, P. (1982). Ce que Parler Veut Dire. Paris: Fayard. 
Bourhis, R. (2001). Acculturation, Language Maintenance and Language Shift. In J. Klatter-Folmer \& P. van Avermaet (Hrsg.), Theories on Maintenance and Loss of Minority Languages. Towards a More Integrated Explanatory Framework (S. 5-37). Münster: Waxmann.

Brandenburg, M. (2006). Family Literacy in Deutschland. Die Alphabetisierungsdekade der Vereinten Nationen (UNLD) und ihre Auswirkungen auf die Alphabetisierungsarbeit in Deutschland. Hamburg: Kovač.

Bräuer, C. (2010). Könnerschaft und Kompetenz in der Leseausbildung. Theoretische und empirische Perspektiven. Weinheim [u.a.]: Juventa-Verlag.

Breidenstein, G. (2006). Teilnahme am Unterricht. Ethnographische Studien zum Schülerjob. Wiesbaden: VS Verlag für Sozialwissenschaften.

Brinck, C. (07. 04. 2003). Ohne Ausländer wird es nicht besser. Die Welt. Verfügbar unter: https://www.welt.de/print-welt/article629778/Ohne-Auslaender-wird-es-nicht-besser.html. Letzter Zugriff: 21. 10. 2018.

Brinker, K. \& Sager, S. F. (2010). Linguistische Gesprächsanalyse. Eine Einführung. Berlin: Erich Schmidt Verlag.

Britz, L. (2006). Bildungsbe(nach)teiligung von MigrantInnen. In L. Reiberg (Hrsg.), Berufliche Integration in der multikulturellen Gesellschaft (S. 1832). Bonn: Bundeszentrale für politische Bildung.

Brown, A. L., \& Palincsar, A. S. (1982). Inducing Strategic Learning from Texts by Means of Informed, Self-Control Training. Topics in Learning \& Learning Disabilities, 2 (1), 1-17.

Brügelmann, H. \& Brinkmann, E. (2001). Die Schrift erfinden. Beobachtungshilfen und methodische Ideen für einen offenen Anfangsunterricht im Lesen und Schreiben (Überarbeitete und erweiterte Neuauflage). Lengwil am Bodensee: Libelle Verlag.

Bruner, J. S. (1975). Language as an Instrument of Thought. In A. Davies (Hrsg.), Problems of Language and Learning (S. 61-88). London: Heinemann.

Bryman, A. (2006). Integrating Quantitative and Qualitative Research. How is it Done? Qualitative Research, 6 (1), 97-113.

Bußmann, H. (2008). Lexikon der Sprachwissenschaft. Stuttgart: Kröner.

Butler, J. (2001). Psyche der Macht. Das Subjekt der Unterwerfung. Frankfurt am Main: Suhrkamp.

Butler, Y. G. (2013). Bilingualism/Multilingualism and Second-Language Acquisition. In T. K. Bhatia \& W. C. Ritchie (Hrsg.), The Handbook of Bilingualism and Multilingualism (S. 109-136). Hoboken, NJ: John Wiley \& Sons, Inc.

Byers-Heinlein, K. \& Werker, J. F. (2013). Lexicon Structure and the Disambiguation of Novel Words: Evidence from Bilingual Infants. Cognition, $128,407-416$. 
California State Department of Education (1985). Case Studies in Bilingual Education: First Year Report. Sacramento, CA: California State Department of Education.

Canale, M., Frenette, N. \& Bélanger, M. (1987). Evaluation of Minority Students Writing in First and Second Languages. In J. Fine (Hrsg.), Second Language Discourse. A Textbook of Current Research (S. 147-166). Norwood, N. J.: Ablex.

Cantone, K. J. (2007). Code-Switching in Bilingual Children. Netherlands: Springer.

Carless, D. (2008). Student Use of the Mother Tongue in the Task Based Classroom. ELT Journal, 62 (4), 331-338.

Carroll, J. B. (1963). A Model of School Learning. Teachers College Record, 64 (8), 723-733.

Casasanto, D. (2008). Who's Afraid of the Big Bad Whorf? Cross-Linguistic Differences in Temporal Language and Thought. Language Learning, 58, 63-79.

Cattani, A., Abbot-Smith, K., Farag, R., Kratt, A., Arreckx, F., Dennis, I. \& Floccia, C. (2014). How much Exposure to English is Necessary for a Bilingual Toddler to Perform like a Monolingual Peer in Language Tests? International Journal of Language \& Communication Disorders, 49, 649671.

Chambers, I. (1996). Migration, Kultur, Identität. Tübingen: Stauffenburg.

Chamot, A. U. \& Kupper, L. (1989). Learning Strategies in Foreign Language Instruction. Foreign Language Annals, 22, 13-24.

Chi, M. T. H., Siler, S. A., Jeong, H., Yamauchi, T. \& Hausmann, R. G. (2001). Learning from Human Tutoring. Cognitive Science, 25 (4), 471-533.

Chiat, S., Armon-Lotem, S., Marinis, T., Polišenská, K., Roy, P. \& Seeff-Gabriel, B. (2013). Assessment of Language Abilities in Sequential Bilingual Children: The Potential of Sentence Imitation Tasks. In V. C. M. Gathercole (Hrsg.), lssues in the Assessment of Bilinguals (S. 56-89). Bristol: Multilingual Matters.

Chin, N. B. \& Wigglesworth, G. (2007). Bilingualism. An Advanced Resource Book. London [u.a.]: Routledge.

Chinn, C. \& Clark, D. (2013). Learning Through Collaborative Argumentation. In C. E. Hmelo-Silver (Hrsg.), The International Handbook of Collaborative Learning (S. 314-332). New York: Routledge.

Chomsky, N. (2000). New Horizons in the Study of Language and Mind. Cambridge: Cambridge University Press.

Clark, E. V. (1993). The Lexicon in Acquisition. Cambridge: Cambridge University Press.

Clarkson, P. C. (2007). Australian Vietnamese Students Learning Mathematics. High Ability Bilinguals and Their Use of Their Languages. Educational Studies in Mathematics, 64 (2), 191-215. 
Collier, V. P. (1992). A Synthesis of Studies Examining Long-Term Language Minority Student Data on Academic Achievement. Bilingual Research Journal, 16, 187-212.

Collins, A., Brown, J. S. \& Newman, S. E. (1987). Cognitive Apprenticeship: Teaching the Craft of Reading, Writing and Mathmatics. BBN Labratories, Centre for the Study of Reading. Verfügbar unter: https://www.ideals.illinois.edu/bitstream/handle/2142/17958/ctrstreadtechrepv01987i00403_opt.pdf?sequence. Letzter Zugriff: 04. 04. 2017.

Coltheart, M., Rastle, K., Perry, C., Langdon, R. \& Ziegler, J. (2001). DRC. A Dual Route Cascaded Model of Visual Word Recognition and Reading Aloud. Psychological Review, 108 (1), 204-256.

Cook, V. (2001). Using the First Language in the Classroom. Canadian Modern Language Review, 57 (3), 402-423.

Costa, A., Santesteban, M. \& Ivanova, I. (2006). How do Highly Proficient Bilinguals Control their Lexicalization Process? Inhibitory and LanguageSpecific Selection Mechanisms are Both Functional. Journal of Experimental Psychology. Learning, Memory, and Cognition, 32 (5), 1057-1074.

Creemers, B. P. M. \& Kyriakides, L. (2008). The Dynamics of Educational Effectiveness. A Contribution to Policy, Practice and Theory in Contemporary Schools. London: Routledge.

Cromdal, J. (1999). Childhood Bilingualism and Metalinguistic Skills: Analysis and Control in Young Swedish-English Bilinguals. Applied Psycholinguistics, 20, 1-20.

Crystal, D. (2008). A Dictionary of Linguistics and Phonetics. Boston, MA: Blackwell Publishing.

Culligan, K. (2015). Student and Teacher Perceptions of First Language Use in Secondary French Immersion Mathematics Classrooms. Alberta Journal of Educational Research, 61 (1), 1-19.

Cumming, A. (1989). Writing Expertise and Second-Language Proficiency. Language Learning, 39 (1), 81-141.

Cummins, J. (1978). Bilingualism and the Development of Metalinguistic Awareness. Journal of Cross-Cultural Psychology, 9, 131-149.

Cummins, J. (1979). Cognitive/Academic Language Proficiency, Linguistic Interdependence, the Optimum Age Question and Some Other Matters. Working Papers on Bilingualism, 19, 198-205.

Cummins, J. (1981). Age on arrival and immigrant second language learning in Canada: A reassessment. Applied Linguistics, 1, 132-149.

Cummins, J. (1991). Conversational and Academic Language Proficiency in Bilingual Contexts. In J.H. Hulstijn \& J.F. Matter (Hrsg.), Reading in Two Languages (S. 75-89). Amsterdam: AILA. 
Cummins, J. (2000). The Threshold and Interdependence Hypothesis Revisisted. In J. Cummins (Hrsg.), Language, Power, and Pedagogy. Bilingual Children in the Crossfire (S. 173-200). Clevedon, ENG: Multilingual Matters.

Cummins, J. (2008). BICS and CALP: Empirical and Theoretical Status of the Distinction. Empirical and Theoretical Status of the Distinction. In B. Street \& N. H. Hornberger (Hrsg.), Encyclopedia of Language and Education, $2^{\text {nd }}$ Edition, Volume 2: Literacy. (S. 71-83). New York: Springer Science + Business Media LLC.

Dabla-Norris, E., Kochhar, K., Suphaphiphat, N., Ricka, F. \& Tsounta, E. (2015). Causes and Consequences of Income Inequality. A Global Perspective. Washington, D.C.: International Monetary Fund.

Darcy, N. T. (1953). A Review of the Literature on the Effects of Bilingualism upon the Measurement of Intelligence. Journal of Genetic Psychology, 82, 21-57.

Davidson, R. G., Kline, S. B. \& Snow, C. E. (1986). Definitions and Definite Noun Phrases. Indicators of Children's Decontextualized Language Skills. Journal of Research in Childhood Education, 1 (1), 37-48.

Davison, C. \& Williams, A. (2001). Integrating Language and Content: Unresolved Issues. In B. Mohan, C, Leung \& C. Davison (Hrsg.), English as a Second Language in the Mainstream: Teaching, Learning and Identity (S. 51-70). Harlow: Longman.

De Backer, L., van Keer, H. \& Valcke, M. (2015). Exploring Evolutions in Reciprocal Peer Tutoring Groups' Socially Shared Metacognitive Regulation and Identifying its Metacognitive Correlates. Learning and Instruction, 38, 63-78.

De Backer, L., van Keer, H., Moerkerke, B. \& Valcke, M. (2016). Examining Evolutions in the Adoption of Metacognitive Regulation in Reciprocal Peer Tutoring Groups. Metacognition and Learning, 11 (2), 187-213.

De Lisi, R. \& Golbeck, S. (2009). The Implications of Piagetian Theory for Peer Learning. In A. M. O'Donnell \& A. King (Hrsg.), Cognitive Perspectives on Peer Learning (S. 3-37). New York: Routledge.

Decristan, J., Rauch, D. P., Reitenbach, V. \& Schastak, M. (in Vorb.). Implementation Check of the „BiPeer“-Study: Language Background and Bilingual Communication in Peer-Learning.

Deppermann, A. (2008). Gespräche Analysieren. Eine Einführung. Opladen: Leske + Budrich.

Dewey, J. (1916). Democracy and Education: An Introduction to the Philosophy of Education. New York: The Macimilllian Company.

Dirim, I. (1998). „Var mı lan Marmelade?“. Türkisch-deutscher Sprachkontakt in einer Grundschulklasse. Münster: Waxmann.

Dittmann, J. (2006). Der Spracherwerb des Kindes. Verlauf und Störungen. München: C. H. Beck. 
Dittmar, N. (2009). Transkription. Ein Leitfaden mit Aufgaben für Studenten, Forscher und Laien. Wiesbaden: Verlag für Sozialwissenschaften.

Do Mar Castro Varela, M. \& Mecherill, P. (2010). Anerkennung als erziehungswissenschaftliche Referenz? Herrschaftskritische und identitätsskeptische Anmerkungen. In A. Schäfer \& C. Thompson (Hrsg.), Anerkennung (S. 89-118). Paderborn: Schöningh.

Driessen, G., van der Slik, F. \& de Bot, K. (2002). Home Language and Language Proficiency: A Large-Scale Longitudinal Study in Dutch PrimarySchools. Journal of Multilingual and Multicultural Development, 23, 175194.

Duncan, S., Kanki, B., Mokros, H. \& Fiske, D. (1984). Pseudo-Unilaterality, Simple-Rate Variables, and Other Ills to which Interaction Research is Heir. Journal of Personality and Social Psychology, 46, 1335-1348.

Durgunoğlu, A. Y. (1998). Acquiring Literacy in English and Spanish in the United States. In A. Y. Durgunoğlu \& L. Verhoeven (Hrsg.), Literacy Development in a Multilingual Context. Cross-Cultural Perspectives (S. 135145). Mahwah, NJ: Erlbaum.

Eastman, C. M. (1992). Codeswitching. Clevedon: Multilingual Matters.

Edwards, D. \& Christophersen, H. (1988). Bilingualism, Literacy, and Metalinguistic Awareness in Preschool Children. Journal of Developmental Psychology, 6, 235-244.

Edwards, J. (2013). Bilingualism and Multilingualism: Some Central Concepts. In T. K. Bhatia \& W. C. Ritchie (Hrsg.), The Handbook of Bilingualism and Multilingualism (S. 5-25). Hoboken, NJ: John Wiley \& Sons, Inc.

Eichler, W. \& Nold, G. (2008). Sprachbewusstheit. In E. Klieme, W. Eichler, A. Helmke, R. Lehmann, G. Nold, G. Rolff, K. Schröder, G. Thomé \& H. Willenberg (Hrsg.), Unterricht und Kompetenzerwerb in Deutsch und Englisch. Ergebnisse der DESI-Studie (S. 63-82). Weinheim: Beltz.

Ellis, R. (2004). The Definition and Measurement of L2 Explicit Knowledge. Language Learning, 54 (2), 227-275.

Engle, R. W. (2002). Working Memory Capacity as Executive Attention. Current Directions in Psychological Science, 11 (1), 19-23.

Ersen-Rasch, M. (2001). Türkische Grammatik: für Anfänger und Fortgeschrittene. Ismaning: Max Huber.

Esser, H. (2009). Der Streit um die Zweisprachigkeit: Was bringt die Bilingualität? In I. Gogolin \& U. Neumann (Hrsg.), Streitfall ZweisprachigkeitThe Bilingualism Controversy (S. 69-90). Wiesbaden: VS Verlag für Sozialwissenschaften

Eviatar, Z. \& Ibrahim, R. (2000). Bilingualism is as Bilingual Does: Metalinguistic Abilites of Arabic-Speaking Children. Applied Psycholinguistics, 21, 451-571. 
Feng, C., Wang, H., Lu, N., Chen, T., He, H., Lu, Y. et al. (2014). Log-Transformation and its Implications for Data Analysis. Shanghai Archives of Psychiatry, 26 (2), 105-109.

Fischer, P., Jander, K. \& Krueger, J. I. (2018). Sozialpsychologie für Bachelor. Berlin: Springer.

Flecken, M. (2011). Event Conceptualization by Early Dutch-German Bilinguals: Insights from Linguistic and Eye-Tracking Data. Bilingualism: Language and Cognition, 14, 61-77.

FLY. Materialien rund um das Projekt FLY. Landesinstitut für Lehrerbildung und Schulentwicklung Hamburg. Verfügbar unter https://li.hamburg.de/family-literacy/4553200/artikel-family-literacy/. Letzter Zugriff: 04.12.2018.

Friedrich, H. \& Mandl, H. (2006). Lernstrategien: Zur Strukturierung des Forschungsfeldes. In H. Mandl \& H. Friedrich (Hrsg.), Handbuch Lernstrategien (S. 1-26). Göttingen: Hogrefe.

Fuchs, D., Fuchs, L. S., Mathes, P. G. \& Simmons, D. C. (1997). Peer-Assisted Learning Strategies: Making Classrooms More Responsive to Diversity. American Educational Research Journal, 34 (1), 174-206.

Fuchs, D., Fuchs, L. S., Simmons, D. C. \& Mathes, P. G. (2008). Peer Assisted Learning Strategies Reading: Methods for Grades 2-6. Nashville: Vanderbilt University.

Fuller, J. M. (2009). How Bilingual Children Talk: Strategic Codeswitching Among Children in Dual Language Programs. In M. Turnbull \& J. DaileyO'Cain (Hrsg.), First Language Use in Second and Foreign Language Learning (S. 115-130). Clevedon, UK: Multilingual Matters.

Furman, R. \& Özyürek, A. (2007). Development of Interactional Discourse Markers. Insights from Turkish Childrens' and Adults' Oral Narratives. Journal of Pragmatics, 39, 1742-1757.

Fuß, S. \& Karbach, U. (2014). Grundlagen der Transkription. Eine praktische Einführung. Leverkusen: UTB \& Barbara Budrich.

Füssenich, I. (2012). Gibt es Vorläuferfertigkeiten beim Schrifterwerb? Vom Sprechen zur Schrift beim Übergang von der Kita in die Schule. MitSprache (3), 5-16.

Gámez, P. B. \& Levine, S. C. (2013). Oral Language Skills of Spanish-Speaking English Language Learners: The Impact of High-Quality Native Language Exposure. Applied Psycholinguistics, 34, 673-696.

Gantefort, C. \& Roth, H.-J. (2010). Sprachdiagnostische Grundlagen für die Förderung bildungssprachlicher Fähigkeiten. Zeitschrift für Erziehungswissenschaft, 13 (4), 573-591.

García, O., Makar, C., Starcevic, M. \& Terry, A. (2011). Translanguaging of Latino Kindergarteners. In K. Potowski \& J. Rothman (Hrsg.), Bilingual Youth. Spanish in English-Speaking Societies (S. 35-55). Amsterdam: John Benjamins. 
García, O. \& Kano, N (2014). Translanguaging as Process and Pedagogy: Developing the English Writing of Japanese Students in the US. In J. Conteh \& G. Meier (Hrsg.), The Multilingual Turn in Languages Education. Opportunities and Challenges (S. 258-277). Bristol: Multilingual Matters.

García, O. \& Li Wei. (2014). Translanguaging. Language, Bilingualism and Education. Basingstoke: Palgrave Macmillan.

Gardner-Chloros, P. (2009). Code-Switching. Cambridge, UK: Cambridge University Press.

Gardner-Chloros, P., McEntee-Atalianis, L. \& Finnis, K. 2005. (2005). Language Attitudes and Use in a Transplanted Setting: Greek Cypriots in London. 52-80. International Journal of Multilingualism, 2, 51-80.

Gathercole, V. C. M. (1997). The Linguistic Mass / Count Distinction as an Indicator of Referent Categorization in Monolingual and Bilingual Children. Child Development, 68, 832-842.

Gathercole, V. C. M. (2016). Factors Moderating Proficiency in Bilingual Speakers. In E. Nicoladis \& S. Montanari (Hrsg.), Bilingualism Across the Lifespan. Factors Moderating Language Proficiency (S. 123-140). Berlin: De Gruyter Mouton.

Gathercole, V. C. M. \& Montes, C. (1997). That-Trace Effects in Spanish- and English-Speaking Monolinguals and Bilinguals. In A. T. Pérez Leroux \& W. R. Glass (Hrsg.), Contemporary Perspectives on the Acquisition of Spanish (S. 75-95). Somerville: Cascadilla Press.

Gathercole, V. \& Thomas, E. M. (2009). Bilingual First-Language Development: Dominant Language Takeover, Threatened Minority Language Take-up. Bilingualism: Language and Cognition, 12, 213-237.

Genesee, F. (1979). Acquisition of Reading Skills in Immersion Programs. Foreign Language Annals, 12, 71-77.

Gennari, S., Sloman, S., Malt, B. \& Fitch, T. (2002). Motion Events in Language and Cognition. Cognition, 83, 49-79.

Giesinger, J. (2008). Fairer Wettbewerb und demokratische Gleichheit. Zum Problem der Bildungsgerechtigkeit. Zeitschrift für Berufs- und Wirtschaftspädagogik, 104 (4), 556-572.

Giesinger, J. (2015). Bildungsgerechtigkeit und die sozialen Funktionen der Schule. Zu den normativen Grundlagen des Chancenspiegels. In V. Manitius, B. Hermstein, N. Berkemeyer \& W. Bos (Hrsg.), Zur Gerechtigkeit von Schule. Theorien, Konzepte, Analysen (S. 150-164). Münster, New York: Waxmann.

Ginsburg-Block, M. D., Rohrbeck, C. A. \& Fantuzzo, J. W. (2006). A MetaAnalytic Review of Social, Self-Concept, and Behavioral Outcomes of Peer-Assisted Learning. Journal of Educational Psychology, 98 (4), 732749. 
Gleitman, L. \& Papafragou, A. (2005). Language and Thought. In K. J. Holyoak \& R. G. Morrison (Hrsg.), The Cambridge Handbook of Thinking and Reasoning (S. 633-662). New York: Cambridge University Press.

Glück, C. W. (2011). Wortschatz- und Wortfindungstest für 6- bis 10-Jährige. WWT 6-10. München: Elsevier Urban \& Fischer.

Gnutzmann, C. (1997). Language Awareness. Geschichte, Grundlagen, Anwendung. Praxis des neusprachlichen Unterrichts, 44 (3), 227-236.

Gogolin, I. (1994). Der monolinguale Habitus der multilingualen Schule. Münster: Waxmann.

Gogolin, I. (2015). Vervielfältigung von sprachlicher Vielfalt. Beobachtungen und Forschungsergebnisse zur sprachlichen Lage in Deutschland. Migration und Soziale Arbeit, 37 (4), 292-298.

Gogolin, I. \& Neumann, U. (1991). Sprachliches Handeln in der Grundschule. Die Grundschulzeitschrift, 43, 6-13.

Gogolin, I. \& Neumann, U. (Hrsg.). (2009). Streitfall Zweisprachigkeit-The Bilingualism Controversy. Wiesbaden: VS Verlag für Sozialwissenschaften / GWV Fachverlage GmbH Wiesbaden.

Goldthorpe, J. (1996). Problems of Meritocracy. In R. Erikson (Hrsg.), Can Education be Equalized? The Swedish Case in Comparative Perspective (S. 255-287). Boulder [u.a.]: Westview Press.

Gomolla, M. \& Radtke, F.-O. (2009). Institutionelle Diskriminierung. Die Herstellung ethnischer Differenz in der Schule. Wiesbaden: VS Verlag für Sozialwissenschaften / GWV Fachverlage GmbH.

Gonas, G. Gürsoy, E., Handt, C. \& Weis, I. (2018). Schriftliche Rechenverfahren international. Verfügbar unter https://www.uni-due.de/impe$\mathrm{ria} / \mathrm{md} /$ content/prodaz/schriftliche_rechenverfahren_international_2018.pdf. Letzter Zugriff: 04. 12. 2018.

González, L. A. (1986). The Effects of First Language Education on the Second Language and Academic Achievement of Mexican Immigrant Elementary School Children in the United States. Doctoral Dissertation. University of Illinois at Urbana-Champaign.

González, L. A. (1989). Native Language Education: The Key to English Literacy Skills. In D. J. Bixler-Márquez, G. K. Green \& J. L. Ornstein-Galicia (Hrsg.), Mexican-American Spanish in its Societal and Cultural Contexts. (S. 209-224). Brownsville: Pan American University.

Green, D. W. (1998). Mental Control of the Bilingual Lexico-Semantic System. Bilingualism: Language and Cognition, 1 (2), 67-81.

Green, D. W. \& Abutalebi, J. (2013). Language Control in Bilinguals. The Adaptive Control Hypothesis. Journal of Cognitive Psychology, 25 (5), 515-530.

Greene, J. (1998). A Meta-Analysis of the Effectiveness of Bilingual Education. Claremont, CA: Tomas Rivera Policy Institute. 
Greene, J. C., Caracelli, V. J. \& Graham, W. F. (1989). Toward a Conceptual Framework for Mixed-Method Evaluation Designs. Educational Evaluation and Policy Analysis, 11 (3), 255-274.

Grewendorf, G., Hamm, F. \& Sternefeld, W. (1987). Sprachliches Wissen. Eine Einführung in moderne Theorien der grammatischen Beschreibung. Frankfurt am Main: Suhrkamp.

Grice, H. P. (1975). Logic and Conversation. In P. Cole \& J. L. Morgan (Hrsg.), Syntax and Semantics (S. 41-58). Orlando: Academic Press.

Griffin, D. \& Gonzalez, R. (1995). Correlational Analysis of Dyad-Level Data in the Exchangeable Case. Psychological Bulletin, 118 (3), 430-439.

Grimm, A., Müller, A., Hamann, C. \& Ruigendijk, E. (Hrsg.) (2011). Production-Comprehension Asymmetries in Child Language. Proceedings der AG 3 der 31. Jahrestagung der DGFS in Berlin. New York: De Gruyter.

Grimm, A. \& Schulz, P. (2016). Warum man bei mehrsprachigen Kindern dreimal nach dem Alter fragen sollte: Sprachfähigkeiten simultan-bilingualer Lerner im Vergleich mit monolingualen und frühen Zweitsprachlernern. Diskurs Kindheits- und Jugendforschung, 11 (1), 27-42.

Grosjean, F. (1982). Life with two Languages: An Introduction to Bilingualism. Cambridge: Harvard University Press.

Grosjean, F. (1997). Processing Mixed Language: Issues, Findings, and Models. In A. M. B. de Groot \& J. F. Kroll (Hrsg.), Tutorials in Bilingualism. Psycholinguistic Perspectives (S. 225-254). New York: Psychology Press. Grosjean, F. (2008). Studying Bilinguals. Oxford: Oxford University Press.

Grosjean, F. (2010). Bilingual. Life and Reality. Cambridge: Harvard University Press.

Grosjean, F. \& Li, P. (2013). The Psycholinguistics of Bilingualism. Chichester: Wiley-Blackwell.

Gutmann, M. (1998). Der Begriff der Kultur. Präliminarien zu einer methodischen Phänomenologie der Kultur in systematischer Absicht. In D. Hartmann (Hrsg.), Die kulturalistische Wende. Zur Orientierung des philosophischen Selbstverständnisses (S. 269-332). Frankfurt am Main: Suhrkamp.

Haag, N., Böhme, K. \& Stanat, P. (2012). Zuwanderungsbezogene Disparitäten. In P. Stanat, H. A. Pant, K. Böhme \& D. Richter (Hrsg.), Kompetenzen von Schülerinnen und Schülern am Ende der vierten Jahrgangsstufe in den Fächern Deutsch und Mathematik. Ergebnisse des IQB-Ländervergleichs 2011 (S. 209-235). Münster: Waxmann.

Haag, N., Böhme, K., Rjosk, C. \& Stanat, P. (2016). Zuwanderungsbezogene Disparitäten. In P. Stanat, K. Böhme, S. Schipolowski \& N. Haag (Hrsg.), IQB-Bildungstrend 2015. Sprachliche Kompetenzen am Ende der 9. Jahrgangsstufe im zweiten Ländervergleich (S. 431-480). Münster: Waxmann. 
Haarmann, H. (1996). Identität. In H. Goebl (Hrsg.), Kontaktlinguistik. Ein internationales Handbuch zeitgenössischer Forschung (S. 218-233). Berlin: De Gruyter.

Haberzettl, S. (2006). Verbstellung in der Zweitsprache Deutsch. Forum Logopädie, 20 (6), 6-11.

Hacker, Douglas J., Dunlosky, J. \& Graesser, A. (2009). Handbook of Metacognition in Education. New York [u.a.]: Routledge.

Halbig, C. \& Kohl, T. (2004). Zur Rechtfertigung von Anerkennungsforderungen. In C. Halbig, M. Quante \& A. Honneth (Hrsg.), Axel Honneth. Sozialphilosophie zwischen Kritik und Anerkennung (S. 67-72). Münster: LIT Verlag.

Hall, S. (1994). Rassismus und kulturelle Identität. Hamburg: Argument.

Hamers, J. F. \& Blanc, M. (2000). Bilinguality and Bilingualism. Cambridge: Cambridge University Press.

Hammer, S., Fischer, N. \& Koch-Priewe, B. (2016). Überzeugungen von Lehramtsstudierenden zu Mehrsprachigkeit in der Schule. In B. Koch-Priewe \& M. Krüger-Potratz (Hrsg.) Qualifizierung für sprachliche Bildung (S. 147-171). Münster: Waxmann.

Hasselhorn, M. \& Mähler, C. (1998). Wissen, auf das Wissen baut: Entwicklungspsychologische Erkenntnisse zum Wissenserwerb und zum Erschließen von Wirklichkeit im Grundschulalter. In J. Kahlert (Hrsg.), Wissenserwerb in der Grundschule. Perspektiven erfahren, vergleichen, gestalten (S. 73-90). Bad Heilbrunn: Klinkhardt.

Hasselhorn, M. \& Gold, A. (2017). Pädagogische Psychologie. Erfolgreiches Lernen und Lehren: Kohlhammer Verlag.

Hattie, J. (2014). Lernen sichtbar machen. Überarbeitete deutschsprachige Ausgabe von „Visible Learning“ besorgt von Wolfgang Beywl und Klaus Zierer. London [u.a.]: Routledge / Taylor and Francis.

Hegel, G. W. F. (1986). Grundlinien der Philosophie des Rechts oder Naturrecht und Staatswissenschaft im Grundrisse. Frankfurt am Main: Suhrkamp.

Helmke, A. (2017). Unterrichtsqualität und Lehrerprofessionalität. Diagnose, Evaluation und Verbesserung des Unterrichts: Franz Emanuel Weinert gewidmet. Seelze-Velber: Klett/Kallmeyer.

Hélot, C. (2006). Bridging the Gap between Prestigious Bilingualism and the Bilingualism of Minorities: Towards an Integrated Perspective of Multilingualism in the French Education Context. In M. Ó Laoire (Hrsg.), Multilingualism in Educational Settings (S. 49-72). Baltmannsweiler: Schneider Verlag Hohengehren.

Hennon, E., Hirsh-Pasek, K. \& Golinkoff, R. M. (2000). Die besondere Reise vom Fötus zum spracherwerbenden Kind. In N. Birbaumer, C. F. Graumann \& H. Grimm (Hrsg.), Sprachentwicklung (S. 41-60). Göttingen [u.a.]: Hogrefe. 
Heppt, B., Stanat, P., Dragon, N., Berendes, K. \& Weinert, S. (2014). Bildungssprachliche Anforderungen und Hörverstehen bei Kindern mit deutscher und nicht-deutscher Familiensprache. Zeitschrift für Pädagogische Psychologie, 28 (3), 139-149.

Herdina, P. \& Jessner, U. (2002). A Dynamic Model of Multilingualism: Perspectives of Change in Psycholinguistics. Clevedon: Multilingual Matters Limited.

Hessisches Kultusministerium (2018). Hessisches Schulgesetz. Verfügbar unter https://kultusministerium.hessen.de/sites/default/files/media/hkm/lesefassung schulgesetz mit inhaltsverzeichnis zweispaltig_stand_30.05.2018.pdf. Letzter Zugriff: 30. 12. 2018.

Hoff, E., Core, C., Place, S., Rumiche, R., Señor, M. \& Parra, M. (2012). Dual Language Exposure and Early Bilingual Development. Journal of Child Language, 39, 1-27.

Hogan, D. \& Tudge, J. (2009). Implications of Vygotsky's Theory for Peer Learning. In A. M. O’Donnell \& A. King (Hrsg.), Cognitive Perspectives on Peer Learning (S. 39-65). New York: Routledge.

Holler-Zittlau, I., Dux, W. \& Berger, R. (2004). Evaluation der Sprachentwicklung 4-41/2-jähriger Kinder in Hessen. Wiesbaden: Hessisches Sozialministerium.

Honneth, A. (2003). Kampf um Anerkennung: Zur moralischen Grammatik sozialer Konflikte. Berlin: Suhrkamp.

Hoover, W. A. \& Gough, P. B. (1990). The Simple View of Reading. Reading and Writing: An Interdisciplinary Journal, 2, 127-160.

Hopewell, S. (2011). Leveraging Bilingualism to Accelerate English Reading Comprehension. International Journal of Bilingual Education and Bilingualism, 14 (5), 603-620.

Hopp, H. C. (2007). Ultimate Attainment at the Interfaces in Second Language Acquisition. Grammar and Processing. Groningen: Groningen Dissertations in Linguistics.

Hörning, K. H. \& Winter, R. (1999). Widerspenstige Kulturen. Cultural Studies als Herausforderung. Frankfurt: Suhrkamp.

Horster, D. (2015). Bildungsgerechtigkeit aus sozialphilosophischer Sicht. In V. Manitius, B. Hermstein, N. Berkemeyer \& W. Bos (Hrsg.), Zur Gerechtigkeit von Schule. Theorien, Konzepte, Analysen (S. 42-50). Münster/New York: Waxmann.

Howe, C. (2010). Peer Dialogue and Cognitive Development. A Two-Way Relationship? In K. Littleton \& C. Howe (Hrsg.), Educational Dialogues. Understanding and Promoting Productive Interaction (S. 32-47). London: Routledge.

Howe, C., Tolmie, A., Thurston, A., Topping, K., Christie, D., Livingston, K., Jessiman, E. \& Donaldson, C. (2007). Group Work in Elementary Science. 
Towards Organisational Principles for Supporting Pupil Learning. Learning and Instruction, 17 (5), 549-563

$\mathrm{Hu}$, A. (2007). Mehrsprachigkeitsforschung, Identitäts- und Kulturtheorie: Tendenzen der Konvergenz. In A. Hu \& I. De Florio-Hansen (Hrsg.), Plurilingualität und Identität. Zur Selbst-und Fremdwahrnehmung mehrsprachiger Menschen (S. 1-24). Tübingen: Stauffenburg.

Humboldt, W. (1905). Über das vergleichende Sprachstudium in Beziehung auf die verschiedenen Epochen der Sprachentwicklung. In W. v. Humboldts (Hrsg. von der Königlich Preußischen Akademie der Wissenschaften), Gesammelte Schriften (S. 1-34). Berlin: B. Behr's.

Hurrelmann, B. (2004). Informelle Sozialisationsinstanz Familie. In N. Groeben \& B. Hurrelmann (Hrsg.), Lesesozialisation in der Mediengesellschaft. Ein Forschungsüberblick (S. 169-201). Weinheim: Juventa.

Iiskala, T., Vauras, M. \& Lehtinen, E. (2004). Socially-Shared Metacognition in Peer-Learning? Hellenic Journal of Psychology, 1, 147-178.

Ikaheimo, H., Laitinen, A. \& Quante, M. (2004). Leistungsgerechtigkeit: Ein Prinzip der Anerkennung für kulturelle Besonderheiten? In C. Halbig, M. Quante \& A. Honneth (Hrsg.), Axel Honneth. Sozialphilosophie zwischen Kritik und Anerkennung (S. 81-85). Münster: Lit.

Jeon, K.-N., Moon, S. M. \& French, B. (2011). Differential Effects of Divergent Thinking, Domain Knowledge, and Interest on Creative Performance in Art and Math. Creativity Research Journal, 23 (1), 60-71.

Jeuk, S. (2003). Erste Schritte in der Zweitsprache Deutsch. Eine empirische Untersuchung zum Zweitspracherwerb türkischer Migrantenkinder in Kindertageseinrichtungen. Freiburg im Breisgau: Fillibach-Verlag.

Jeuk, S. (2009). Probleme der Sprachstandserhebung bei mehrsprachigen Kindern. Zeitschrift für Soziologie der Erziehung und Sozialisation, 29 (2), 141-156.

Johnson, D. W. \& Johnson, R. T. (1999). Making Cooperative Learning Work. Theory Into Practice, 38 (2), 67-73.

Johnson, J. (1989). Factors Related to Cross-Language Transfer and Metaphor Interpretation in Bilingual Children. Applied Psycholinguistics, 10, 157117.

Johnson, J. (1991). Constructive Processes in Bilingualism and their Cognitive Growth. In E. Bialystok (Hrsg.), Language Processing in Bilingual Children (S. 193-221). Cambridge: Cambridge University Press.

Johnson, J. S. \& Newport, E. L. (1989). Critical Period Effects in Second Language Learning. The Influence of Maturational State on the Acquisition of English as a Second Language. Cognitive Psychology, 21 (1), 60-99.

Johnstone, R. (2002). Immersion in a Second or Additional Language at School: A Review of the International Research. Stirling: Scottish Centre for Information on Language Teaching. 
Jude, N. (2008). Zur Struktur von Sprachkompetenz. Unveröffentlichte Dissertation. Johann Wolfgang Goethe-Universität, Frankfurt am Main.

Kallmeyer, W. \& Schütze, F. (1976). Konversationsanalyse. Studium Linguistik, 1, 1-28.

Kany, W. \& Schöler, H. (2007). Fokus: Sprachdiagnostik. Leitfaden zur Sprachstandsbestimmung im Kindergarten. Berlin: Cornelsen Scriptor.

Karasu, I. (1995). Bilinguale Wortschatzentwicklung türkischer Migrantenkinder vom Vor- bis ins Grundschulalter in der Bundesrepublik Deutschland . Frankfurt am Main [u. a.]: Peter Lang.

Karmiloff-Smith, A. (1992). Beyond Modularity. A Developmental Perspective on Cognitive Science. Cambridge, MA [u.a.]: MIT Press.

Kauschke, C. (2012). Kindlicher Spracherwerb im Deutschen. Verläufe, Forschungsmethoden, Erklärungsansätze. Berlin [u.a.]: De Gruyter.

Keim, I. (2007). Die „türkischen Powergirls“. Lebenswelt und kommunikativer Stil einer Migrantinnengruppe in Mannheim. Tübingen: Narr.

Keim, I. (2012). Mehrsprachige Lebenswelten. Sprechen und Schreiben der türkischstämmigen Kinder und Jugendlichen. Tübingen: Narr Francke Attempto.

Kempert, S., Edele, A., Rauch, D. P., Wolf, K., Paetsch, J., Darsow, A., Maluch, J. \& Stanat, P. (2016). Die Rolle der Sprache für zuwanderungsbezogene Ungleichheiten im Bildungserfolg. In C. Diehl, C. Hunkler \& C. Kristen (Hrsg.), Ethnische Ungleichheiten im Bildungsverlauf. Mechanismen, Befunde, Debatten (S. 157-241). Wiesbaden: Springer VS.

Kenny, D. A., Kashy, D. A. \& Cook, W. L. (2006). Dyadic Data Analysis. New York: Guilford Press.

Kerby, A. P. (1991). Narrative and the Self. Bloomington: Indiana University Press.

Kern, R. (1994). The Role of Mental Translation in Second Language Reading. Studies in Second Language Acquisition, 16, 441-460.

Kersten, A., Geist, B. \& Voet Cornelli, B. \& Schulz, P. (2011). Mehrsprachigkeit: Mythen und was dahinter steckt. KiTa HRS, 4, 89-91.

Kessels, U. \& Hannover, B. (2009). Gleichaltrige. In E. Wild \& J. Möller (Hrsg.), Pädagogische Psychologie (Springer-Lehrbuch, S. 283-304). Berlin/Heidelberg: Springer.

Kessler, T. \& Fritsche, I. (2018). Sozialpsychologie. Wiesbaden: Springer Fachmedien Wiesbaden.

Kharkurin, A. (2007). The Role of Cross-Linguistic and Cross-Cultural Experiences in Bilinguals' Divergent Thinking. In Kecskés, I. \& Albertazzi, L. (Hrsg.), Cognitive Aspects of Bilingualism (S. 175-210). Dordrecht: Springer.

Kharkurin, A. (2009). The Role of Bilingualism in Creative Performance on Divergent Thinking and Invented Alien Creatures Tests. The Journal of Creative Behavior, 43 (1), 59-71. 
Kharkurin, A. (2010). Bilingual Verbal and Nonverbal Creative Behavior. International Journal of Bilingualism, 14 (2), 211-226.

Kharkurin, A. (2017). Language Mediated Concept Activation in Bilingual Memory Facilitates Cognitive Flexibility. Frontiers in Psychology, 8, 1067.

Klieme, E., Schümer, G., \& Knoll, S. (2001). Mathematikunterricht in der Sekundarstufe I: „Aufgaben-kultur“" und Unterrichtsgestaltung im internationalen Vergleich. In Klieme, E. \& Baumert, J. (Hrsg.), TIMSS - Impulse für Schule und Unterricht (S. 43-57). Bonn: BMBF.

Klieme, E., Pauli, C., \& Reusser, K. (2009). The Pythagoras Study: Investigating Effects of Teaching and Learning in Swiss and German Mathematics Classroom. In T. Janik \& T. Seidel (Hrsg.), The Power of Video Studies in Investigating Teaching and Learning in the Classroom (S. 137-160). Münster: Waxmann.

Klingner, J. \& Vaughn, S. (2000). The Helping Behaviors of Fifth Graders While Using Collaborative Strategic Reading During ESL Content Classes. Teachers of English to Speakers of Other Languages (TESOL), 34 (1), 69-98.

Knapp, W. (1999). Verdeckte Sprachschwierigkeiten. Die Grundschule, 99 (5), 30-33.

Koalitionsvertrag zwischen CDU, CSU und FDP (2009). Wachstum. Bildung. Zusammenhalt. Verfügbar unter https://www.csu.de/common/_migrated/csucontent/091026_koalitionsvertrag_02.pdf. Letzter Zugriff: 21. 10. 2018.

Koch, P. \& Oesterreicher, W. (1985). Sprache der Nähe - Sprache der Distanz. Mündlichkeit und im Spannungsfeld von Sprachtheorie und Sprachgeschichte. Romanistisches Jahrbuch, 36, 15-43.

Kostandyan, M. E. \& Ledovaya, Y. A. (2013). How the Age of Language Acquisition Relates to Creativity. Procedia-Social and Behavioral Sciences, 86, 140-145.

Kratzmann, J., Jahreiß, S., Frank, M., Ertanir, B. \& Sachse, S. (2017). Einstellungen pädagogischer Fachkräfte in Kindertageseinrichtungen zur Mehrsprachigkeit. Zeitschrift für Erziehungswissenschaft, 20 (2), S. 237-258.

Krebs, A. (2000). Einleitung: Die neue Egalitarismuskritik im Überblick. In A. Krebs (Hrsg.), Gleichheit oder Gerechtigkeit. Texte der neuen Egalitarismuskritik (S. 7-37). Frankfurt am Main: Suhrkamp.

Kroffke, S. \& Rothweiler, M. (2004). Sprachmodi im kindlichen Zweitspracherwerb. Sprachlicher Kontext und seine Bedeutung für die sprachpädagogische Diagnostik. Die Sprachheilarbeit, 49 (1), 18-24.

Kroll, J. F., Dussias, P. E., Bice, K. \& Perrotti, L. (2015). Bilingualism, Mind, and Brain. Annual Review of Linguistics, 1, 377-394.

Krumm, H.-J. \& Jenkins, E.-M. (Hrsg.). (2001). Kinder und ihre Sprachen lebendige Mehrsprachigkeit. Sprachenporträts. Wien: Eviva. 
Krumm, H.-J. (2009). Die Bedeutung der Mehrsprachigkeit in den Identitätskonzepten von Migrantinnen und Migranten. In I. Gogolin \& U. Neumann (Hrsg.), Streitfall Zweisprachigkeit-The Bilingualism Controversy (S. 233247). Wiesbaden: VS Verlag für Sozialwissenschaften.

Krummheuer, G. (2011). Die Interaktionsanalyse. Fallarchiv Schulpädgogik der Universität Kassel. Verfügbar unter: http://www.fallarchiv.uni-kassel.de/wp-content/uploads/2010/07/krummheuer_inhaltsanalyse.pdf.

Letzter Zugriff: 27. 11. 2018.

Kuckartz, U. (2014). Mixed Methods. Methodologie, Forschungsdesigns und Analyseverfahren. Wiesbaden: Springer VS.

Kuckartz, U. (2017). Datenanalyse in der Mixed-Methods-Forschung. Kölner Zeitschrift für Soziologie und Sozialpsychologie, 69 (S2), 157-183.

Kultusministerium (2002). PISA 2000 - zentrale Handlungsfelder. Zusammenfassende Darstellung der laufenden und geplanten Maßnahmen in den Ländern. Verfügbar unter: https://www.kmk.org/fileadmin/Dateien/veroeffentlichungen beschluesse/2002/2002 10_07-Pisa-2000-ZentraleHandlungsfelder.pdf. Letzter Zugriff: 21. 10. 2018.

Kunter, M. (2005). Multiple Ziele im Mathematikunterricht. Münster: Waxmann.

Lambert, W. E. (1974). Culture and Language as Factors in Learning and Education. In F. E. Aboud \& R. D. Meade (Hrsg.), Cultural Factors in Learning and Education. Bellingham: Western Washington State University.

Lasagabaster, D. (1998). The Threshold Hypothesis Applied to Three Languages in Contact at School. International Journal of Bilingual Education and Bilingualism, 1 (2), 119-133.

Lee, H. \& Kim, K. H. (2010). Relationships Between Bilingualism and Adaptive Creative Style, Innovative Creative Style, and Creative Strengths Among Korean American Students. Creativity Research Journal, 22 (4), 402-407.

Lee, H. \& Kim, K. H. (2011). Can speaking more languages enhance your creativity? Relationship Between Bilingualism and Creative Potential among Korean American Students with Multicultural Link. Personality and Individual Differences, 50 (8), 1186-1190.

Lehtonen, M., Soveri, A., Laine, A., Järvenpää, J., Bruin, A. de \& Antfolk, J. (2018). Is Bilingualism Associated with Enhanced Executive Functioning in Adults? A Meta-Analytic Review. Psychological Bulletin, 144 (4), $394-$ 425.

Leikin, M. (2012). The Effect of Bilingualism on Creativity. Developmental and Educational Perspectives. International Journal of Bilingualism, 17 (4), 431-447.

Leikin, M. \& Tovli, E. (2014). Bilingualism and Creativity in Early Childhood. Creativity Research Journal, 26 (4), 411-417. 
Lenhard, W. \& Lenhard, A. (2014-2017). Berechnung des Lesbarkeitsindex LIX nach Björnson. Verfügbar unter: https://www.psychometrica.de/lix.html. Letzter Zugriff: 27. 11. 2018.

Lenzen, D. (2015). Stichwort: Gerechtigkeit und Erziehung. In V. Manitius, B. Hermstein, N. Berkemeyer \& W. Bos (Hrsg.), Zur Gerechtigkeit von Schule. Theorien, Konzepte, Analysen (S. 111-130). Münster, New York: Waxmann.

Lewis, G., Jones, B. \& Baker, C. (2012). Translanguaging. Origins and Development from School to Street and Beyond. Educational Research and Evaluation, 18 (7), 641-654.

Lewis, M. P., Simons, G. F. \& Fennig, C. D. (2015). Ethnologue: Languages of the World. Dallas: SIL International. Verfügbar unter: https://www.ethnologue.com/. Letzter Zugriff: 18. 12. 2018.

Li Wei (2011). Moment Analysis and Translanguaging Space: Discursive Construction of Identities by Multilingual Chinese Youth in Britain. Journal of Pragmatics, 43, 1222-1235.

Li, P. \& Farkas, I. (2002). A Self-Organizing Connectionist Model of Bilingual Processing. In R. Heredia \& J. Altarriba (Hrsg.), Bilingual Sentence Processing (S. 59-85). North-Holland: Elsevier Science Publisher.

Limbird, C. K., Maluch, J. T., Rjosk, C., Stanat, P. \& Merkens, H. (2014). Differential Growth Patterns in Emerging Reading Skills of Turkish-German Bilingual and German Monolingual Primary School Students. Reading and Writing, 27 (5), 945-968.

Littleton, K. (2010). Social Interaction and Learning. In B. MacGaw, E. Baker \& P. Petersson (Hrsg.), International Encyclopedia of Education (S. 149161). Amsterdam: Elsevier.

Littleton, K. \& Mercer, N. (2010). The Significance of Educational Dialogues Between Primary School Children. In K. Littleton \& C. Howe (Hrsg.), Educational Dialogues. Understanding and Promoting Productive Interaction (S. 272-288). London: Routledge.

Lohmann, I. (2007). Die „gute Regierung“ des Bildungswesens: Bertelsmann Stiftung. In J. Wernicke (Hrsg.), Netzwerk der Macht - Bertelsmann. Der medial-politische Komplex aus Gütersloh (S. 155-171). Marburg: BdWiVerlag.

Lüdtke, U. \& Kallmeyer, K. (2007a). Kritische Analyse ausgewählter Sprachstandserhebungsverfahren für Kinder vor Schuleintritt aus Sicht der Linguistik, Diagnostik und Mehrsprachigkeitsforschung. Die Sprachheilarbeit, 52 (6), 261-278.

Lüdtke, U. \& Kallmeyer, K. (2007b). Vorschulische Maßnahmen zur Sprachstandserhebung und Sprachförderung in den deutschen Bundesländern:. Wissenschaftliche Vorschläge zur Optimierung bildungspolitischer Initiativen. Die Sprachheilarbeit, 52 (6), 244-260. 
MacSwan, J. (2013). Code-Switching and Grammatical Theory. In T. K. Bhatia \& W. C. Ritchie (Hrsg.), The Handbook of Bilingualism and Multilingualism (S. 323-350). Chichester: Wiley-Blackwell.

MacSwan, J. (2017). A Multilingual Perspective on Translanguaging. American Educational Research Journal, 54 (1), 167-201.

Makoni, S. \& Pennycook, A. (2007). Disinventing and Reconstituting Languages. Clevedon: Multilingual Matters.

Mandl, H. \& Friedrich, H. (Hrsg.). (2006). Handbuch Lernstrategien. Göttingen: Hogrefe.

Marfels, G. (2011). Von der Ehre zur Anerkennung? Die Bedeutung sozialphilosophischer Anerkennungstheorien für den strafrechtlichen Ehrbegriff. Bochum, Univ. Diss. Baden-Baden: Nomos.

Marinova-Todd. (2012). "Corplum is a Core from a Plum": The Advantage of Bilingual Children in the Analyses of Word Meaning. Bilingualism: Language and Cognition, 15, 117-127.

Martin, P.-Y. \& Nicolaisen, T. (2015). Einführung und Grundlagen. In Martin, Pierre-Yves \& Nicolaisen, T. (Hrsg.), Lernstrategien fördern. Modelle und Praxisszenarien (S. 9-69). Weinheim: Beltz Juventa.

Martin, P.-Y. (2014). Auswertung t1 der Studie „Wirkung des Zertifikatslehrgangs Lerncoaching der PHTG im schulischen Alltag (Zwischenbericht). Kreuzlingen: Pädagogische Hochschule Thurgau.

Marx, A. \& Roick, T. (2012). Prädiktoren des Hörverstehens bei Jugendlichen deutscher und Jugendlichen nichtdeutscher Herkunftssprache. Zeitschrift für Pädagogische Psychologie, 26 (2), 121-134.

Marx, A. \& Stanat, P. (2012). Reading Comprehension of Immigrant Students in Germany: Research Evidence on Determinants and Target Points for Intervention. Reading and Writing, 25 (8), 1929-1945.

McClure, E. (1981). Formal and Functional Aspects of Codeswitched Discourse of Bilingual Children. In R. P. Durán (Hrsg.), Latino language and communicative behavior (S. 69-94). Norwood, N. J.: Ablex.

McDowell, J. H. (1996). Mind and World: With a New Introduction. Cambridge/London: Harvard University Press.

McMaster, K. L., Fuchs, D. \& Fuchs, L. S. (2006). Research on Peer-Assisted Learning Strategies. The Promise and Limitations of Peer-Mediated Instruction. Reading \& Writing Quarterly, 22 (1), 5-25.

McMillan, B. \& Turnbull, M. (2009). Teachers' Use of the First Language in French Immersion: Revisiting a Core Concept. In M. Turnbull \& J. DaileyO'Cain (Hrsg.), First Language Use in Second and Foreign Language Learning (15-34). Clevedon: Multilingual Matters.

Mead, G. H. (1973). Geist, Identität und Gesellschaft. Aus der Sicht des Sozialbehaviorismus. Frankfurt am Main: Suhrkamp.

Mecherill, P. (2003). Prekäre Verhältnisse. Über natio-ethno-kulturelle (Mehrfach-)Zugehörigkeit. Münster: Waxmann Verlag GmbH. 
Mecherill, P. (2005). Pädagogik der Anerkennung. Eine programmatische Kritik. In F. Hamburger, T. Badawia \& M. Hummrich (Hrsg.), Migration und Bildung. Über das Verhältnis von Anerkennung und Zumutung in der Einwanderungsgesellschaft (S. 311-328). Wiesbaden: VS Verlag für Sozialwissenschaften.

Menken, K. (2009). No Child Left Behind and its Effects on Language Policy. Annual Review of Applied Linguistics, 29, 103-117.

Menken, K. \& Kleyn, T. (2010). The Long-Term Impact of Subtractive Schooling in the Educational Experiences of Secondary English Language Learners. International Journal of Bilingual Education and Bilingualism, 13, 399-417.

Meyer, M. \& Prediger, S. (2011). Vom Nutzen der Erstsprache beim Mathematiklernen. Fallstudien zu Chancen und Grenzen erstsprachlich gestützter mathematischer Arbeitsprozesse bei Lernenden mit Erstsprache Türkisch. In S. Prediger \& E. Özdil (Hrsg.), Mathematiklernen unter den Bedingungen der Mehrsprachigkeit. Stand und Perspektiven der Forschung und Entwicklung in Deutschland (S. 185-204). Münster: Waxmann.

Micus-Loos, C. (2012). Anerkennung des Anderen als Herausforderung in Bildungsprozessen. Zeitschrift für Pädagogik, 58 (3), 302-320.

Miyake, A., Friedman, N. P., Emerson, M. J., Witzki, A. H., Howerter, A. \& Wager, T. D. (2000). The Unity and Diversity of Executive Functions and Their Contributions to Complex "Frontal Lobe" Tasks. A Latent Variable Analysis. Cognitive Psychology, 41 (1), 49-100.

Miyake, A. \& Friedman, N. P. (2012). The Nature and Organization of Individual Differences in Executive Functions. Four General Conclusions. Current Directions in Psychological Science, 21 (1), 8-14.

Möller, J., Hohenstein, F., Fleckenstein, J., Köller, O. \& Baumert, J. (2017). Erfolgreich integrieren. Die Staatliche Europa-Schule Berlin. Münster: Waxmann.

Moschkovich, J. (2007). Using Two Languages When Learning Mathematics. Educational Studies in Mathematics, 64, 121-144.

Müller, G. \& Wittmann, E. (2014). Das Zahlenbuch 3. Leipzig: Klett-Grundschulverlag.

Munser-Kiefer, M. \& Kirschhock, E.-M. (2014). 25 komplette Trainingseinheiten - ausgezeichnet vom BLLV. Donauwörth: Auer.

Muter, V. \& Diethelm, K. (2001). The Contribution of Phonological Skills and Letter Konowledge to Early Reading Development in a Multilingual Population. Language Learning, 51, 187-219.

Myers-Scotton, C. (1993). Duelling Languages. Oxford: Oxford University Press.

Myers-Scotton, C. (2006). Multiple Voices. An Introduction to Bilingualism. Malden [u. a.]: Blackwell Publishing. 
Neumann, K., Holler-Zittlau, I., von Minnen, S., Sick, U. \& Euler, H. A. (2009). Das Kinder-Sprach-Screening (KiSS) - das hessische Verfahren zur Feststellung des Sprachstandes vierjähriger Kinder. http://www.unikassel.de/fb4/psychologie/personal/euler/Sprachscreening.pdf. Letzter Zugriff: 20. 11. 2018.

Nickel, S. (2004). Family Literacy - Familienorientierte Zugänge zu Schrift. In A. Panagiotopoulou (Hrsg.), Sprachentwicklung und Schriftspracherwerb. Beobachtungs- und Fördermöglichkeiten in Familie, Kindergarten und Grundschule (S. 71-83). Baltmannsweiler: Schneider-Verlag Hohengehren.

Nickel, S. (2007). Family Literacy in Deutschland: Stand der Entwicklung und Gedanken zur konzeptionellen Weiterentwicklung. In M. Elfert \& G. Rabkin (Hrsg.), Gemeinsam in der Sprache baden: Family Literacy. Internationale Konzepte zur familienorientierten Schriftsprachförderung (S. 6584). Barcelona: Ernst-Klett Sprachen.

Nicoladis, E. \& Foursha-Stevenson, C. (2012). Language and Culture Effects on Gender Classification of Objects. Journal of Cross-Cultural Psychology, 43, 1095-1119.

Nicoladis, E. (2016). Bilingual Speakers' Cognitive Development in Childhood. In E. Nicoladis \& S. Montanari (Hrsg.), Bilingualism Across the Lifespan. Factors Moderating Language Proficiency (S. 269-284). Berlin: De Gruyter Mouton.

Nix, D. (2011). Förderung der Leseflüssigkeit. Theoretische Fundierung und empirische Überprüfung eines kooperativen Lautlese-Verfahrens im Deutschunterricht. Weinheim: Juventa.

Noack, P. \& Brändl, S. (2007). Förderung der phonologischen Bewusstheit bei Kindergartenkindern. Trainingseffekte und Prüfung möglicher Moderatoren. Diskurs Kindheits- und Jugendforschung, 2, 311-321.

Nolda, S. (2000). Interaktion in pädagogischen Institutionen: Wiesbaden: VS Verlag für Sozialwissenschaften.

Nussbaum, M. C. (2010). Die Grenzen der Gerechtigkeit. Behinderung, Nationalität und Spezieszugehörigkeit. Berlin: Suhrkamp.

Oerter, R. \& Montada, L. (2002). Entwicklungspsychologie. Weinheim: Beltz, PVU.

Oller, D. K. \& Eilers, R. E. (2002). Language and Literacy in Bilingual Children. Clevedon: Multilingual Matters.

O’Malley, J. M., Chamot, A. U., Stewner-Manzanares, G., Kupper, L. \& Russo, R. P. (1985). Learning Strategies Used by Beginning and Intermediate ESL Students. Language Learning, 35, 21-46.

Özdil, E. (2010). Codeswitching im zweisprachigen Handeln. Sprachpsychologische Aspekte verbalen Planens in türkisch-deutscher Kommunikation. Münster [u.a.]: Waxmann. 
Padberg, F. \& Benz, C. (2011). Didaktik der Arithmetik. Für Lehrerausbildung Und Lehrerfortbildung: Spektrum Akademischer Verlag GmbH.

Palermo, F., Mikulski, A. M., Fabes, R. A., Hanish, L. D., Martin, C. L. \& Starget, L. E. (2014). English Exposure in the Home and Classroom: Predictions to Spanish-Speaking Preschoolers' English Vocabulary Skills. Applied Psycholinguistics, 35, 1163-1187.

Palincsar, A. S. \& Brown, A. L. (1984). Reciprocal Teaching of Comprehension-Fostering and Comprehension-Monitoring Activities. Cognition and Instruction, 1 (2), 117-175.

Paradis, J., Genesee, F. \& Crago, M. B. (2011). Dual Language Development and Disorders. A Handbook on Bilingualism and Second Language Learning. Baltimore: Paul H. Brookes Publication Company.

Peal, E. \& Lambert E. W. (1962). The Relation of Bilingualism to Intelligence. Psychological Monographs, 76, 1-23.

Pearson, P. D. \& Gallagher, M. C. (1983). The Instruction of Reading Comprehension. Contemporary Educational Psychology, 8 (3), 317-344.

Philipp, M. \& Schilcher, A. (2012). Selbstreguliertes Lesen. Ein Überblick über wirksame Leseförderansätze. Seelze: Friedrich.

Philipp, M. (2015). Lesestrategien. Bedeutung, Formen und Vermittlung. Weinheim : Beltz Juventa.

Phillipson, R. (1992). Linguistic Imperialism. Oxford: University Press.

Place, S. \& Hoff, E. (2011). Properties of Dual Language Exposure That Influence 2-Year-Olds' Bilingual Proficiency. Child Development, 82, 1834-1849.

Planas, N. \& Setati, M. (2009). Bilingual Students Using their Languages in the Learning of Mathematics. Mathematics Education Research Journal, 21 (3), 36-59.

Poplack, S. (1980). Sometimes I'll Start a Sentence in Spanish Y TERMINO EN ESPANOL: Toward a Typology of Code-Switching. Linguistics, 18 (7/8), 581-618.

Potowski, K. (2009). Forms and Functions of Codeswitching by Dual Immersion Students: A Comparison of Heritage Speaker and L2 Children. In M. Turnbull \& J. Dailey-O'Cain (Hrsg.), First Language Use in Second and Foreign Language Learning (S. 87-114). Clevedon, UK: Multilingual Matters.

Prengel, A. (1993). Pädagogik der Vielfalt. Verschiedenheit und Gleichberechtigung in interkultureller, feministischer und integrativer Pädagogik. Opladen: Leske + Budrich.

Prenzel, M., Kristen, A., Dengler, P., Ettle, R. \& Beer, T. (1996). Selbstbestimmt motiviertes und interessiertes Lernen in der kaufmännischen Erstausbildung. Zeitschrift für Berufs- und Wirtschaftspädagogik, 13, 108127. 
ProDaZ. Sprachbeschreibungen, Sprachvergleiche und Unterrichtsentwürfe. Institut für Deutsch als Zweit- und Fremdsprache an der Universität Duisburg-Essen Verfügbar unter https://www.uni-due.de/prodaz/veroeffentlichungen. Letzter Zugriff: 04. 12. 2018.

Ramírez, J. D. \& Yuen, S. D. \& Ramey, D. R. (1991). Executive Summary, Final Report: Longitudinal Study of Structured English Immersion Strategy, Early-Exit and Late-Exit Transitional Bilingual Education Programs for Language-Minority Children. San Mateo: Aguirre International.

Ramírez, J. D. (1992). Executive Summary. Bilingual Research Journal, 16 (1 \& 2), 1-62.

Ratgheb-Schnierer, E. (2014). Flexibel Rechnen lernen. Die Grundschulzeitschrift, 28, 28-33.

Rauch, D. P., Decristan, J., Reitenbach, V. \& Schastak, M. (in Vorb.). Language Background and Bilingual Communication in Peer-Learning.

Rauch, D. P., Mang, J., Härtig, H. \& Haag, N. (2016). Naturwissenschaftliche Kompetenz von Schülerinnen und Schülern mit Zuwanderungshintergrund. In K. Reiss, C. Sälzer, A. Schiepe-Tiska, E. Klieme \& O. Köller (Hrsg.), PISA 2015. Eine Studie zwischen Kontinuität und Innovation (S. 317-347). Münster: Waxmann.

Rauch, D. P., Schastak, M. \& Richter, K. (2016). Diagnostik sprachlicher Kompetenzen bei Personen mit Migrationshintergrund. In D. B. Maehler \& H. U. Brinkmann (Hrsg.), Methoden der Migrationsforschung. Ein interdisziplinärer Forschungsleitfaden (S. 97-133). Wiesbaden: Springer VS.

Rawls, J. (1971). Gerechtigkeit als Fairneß. Ein Neuentwurf. Frankfurt am Main: Suhrkamp.

Rehbein, J. (2011). „Arbeitssprache“ Türkisch im mathematisch-naturwissenschaftlichen Unterricht der deutschen Schule - ein Plädoyer. In S. Prediger \& E. Özdil (Hrsg.), Mathematiklernen unter den Bedingungen der Mehrsprachigkeit. Stand und Perspektiven der Forschung und Entwicklung in Deutschland (S. 205-232). Münster: Waxmann.

Reich, H. H. (2009). Zweisprachige Kinder. Sprachenaneignung und sprachliche Fortschritte im Kindergartenalter. Münster: Waxmann.

Reich, H. H. \& Hienz-De Albentiis, M. (1998). Der Herkunftssprachenunterricht. Erlaßlage und statistische Entwicklung in den alten Bundesländern. Deutsch lernen, 1, 3-45.

Reimann, A. (06. 12. 2014). Die CSU spielt Migranten-Polizei. Verfügbar unter http://www.spiegel.de/politik/deutschland/csu-will-dass-migrantenzuhause-deutsch-sprechen-a-1006932.html. Letzter Zugriff: 17.12.2018.

Reitenbach, V., Schastak, M. \& Rauch, D. P. (2018). Sprachstandsdiagnostik. In D. B. Maehler, A. Shajek \& H. U. Brinkmann (Hrsg.), Diagnostik bei Migrantinnen und Migranten. Ein Handbuch (S. 95-149). Göttingen: Hogrefe. 
Reyes, I. (2004). Functions of Code Switching in Schoolchildren's Conversations. Bilingual Research Journal, 28 (1), 77-98.

Ricciardelli, L. A. (1992). Bilingualism and Cognitive Development in Relation to Threshold Theory. Journal of Psycholinguistic Research, 21, 301316.

Ricciardelli, L. A. (1993). An Investigation of the Cognitive Development of Italian-English Bilinguals and Italian Monolinguals from Rome. Journal of Multilingual and Multicultural Development, 14 (4), 345-346.

Ricoeur, P. (1985). History as Narrative and Practice. Philosophy Today, 29 (3), 213-228.

Ritchie, W. C. \& Bhatia, T. K. (2013). Social and Psychological Factors in Language Mixing. In T. K. Bhatia \& W. C. Ritchie (Hrsg.), The Handbook of Bilingualism and Multilingualism (S. 375-389). Hoboken: John Wiley $\&$ Sons, Inc.

Robinson, D. R., Schofield, J. W. \& Steers-Wentzell, K. L. (2005). Peer and Cross-Age Tutoring in Math: Outcomes and Their Design Implications. Educational Psychology Review, 17 (4), 327-362.

Rohrbeck, C. A., Ginsburg-Block, M. D., Fantuzzo, J. W. \& Miller, T. R. (2003). Peer-Assisted Learning Interventions with Elementary School Students. A Meta-Analytic Review. Journal of Educational Psychology, 95 (2), 240-257.

Rolstad, K., Mahony, K. S. \& Glass, G. V. (2005). Weighing the Evidence: A Meta Analysis of Bilingual Education in Arizona. Bilingual Research Journal, 29 (1), 43-67.

Rosebrock, C. (2007). Anforderungen von Sach- und Informationstexten, Anforderungen literarischer Texte. In A. Bertschi-Kaufmann (Hrsg.), Lesekompetenz, Leseleistung, Leseförderung. Grundlagen, Modelle und Materialien (S. 50-65). Seelze-Velber: Klett/Kallmeyer [u.a.].

Rosebrock, C. \& Nix, D. (2008). Grundlagen der Lesedidaktik und der systematischen schulischen Leseförderung. Baltmannsweiler: Schneider-Verlag Hohengehren.

Rosenshine, B. \& Meister, C. (1994). Reciprocal Teaching. A Review of the Research. Review of Educational Research, 64 (4), 479-530.

Rothweiler, M. (2007). Bilingualer Spracherwerb und Zweitspracherwerb. In M. Steinbach (Hrsg.), Schnittstellen der germanistischen Linguistik (S. 103-135). Stuttgart: Metzler.

Rothweiler, M. \& Kauschke, C. (2007). Lexikalischer Erwerb. In H. Schöler \& A. Welling (Hrsg.), Sonderpädagogik der Sprache (S. 42-57). Göttingen: Hogrefe.

Rubinstein-Avila. (2003). Negotiating Power and Redefining Literacy Expertise: Buddy Reading in a Dual-Immersion Programme. Journal of Research in Reading, 26 (1), 83-97. 
Ryan, R. \& Deci, E. (2018). Self-Determination-Theory. Basic Psychological Needs in Motivation, Development, and Wellness. New York: Guilford.

Sachdev, I., Giles, H. \& Pauwels, A. (2013). Accommodating Multilinguality. In T. K. Bhatia \& W. C. Ritchie (Hrsg.), The Handbook of Bilingualism and Multilingualism (S. 391-414). Hoboken: John Wiley \& Sons, Inc.

Sarris, V. \& Reiss, S. (2005). Kurzer Leitfaden der Experimentalpsychologie. München: Pearson Studium.

Schäfer, A. \& Thompson, C. (2010). Anerkennung - eine Einleitung. In A. Schäfer \& C. Thompson (Hrsg.), Anerkennung (S. 7-34). Paderborn: Schöningh.

Schleppegrell, M. J. (2004). The Language of Schooling. A Functional Linguistics Perspective. Mahwah: Lawrence Erlbaum Associates Publishers.

Schmöe, F. (2016a). Internationalismen. In. H. Glück \& M. Rödel. Metzler Lexikon Sprache (S. 304). Stuttgart: J.B. Metzler.

Schmöe, F. (2016b). Transferleistung. In. H. Glück \& M. Rödel. Metzler Lexikon Sprache (S. 720). Stuttgart: J.B. Metzler.

Schrader, F.-W. (2006). Kognitive Voraussetzungen. In K.-H. Arnold, U. Sandfuchs \& J. Wiechmann (Hrsg.), Handbuch Unterricht (S. 569-574). Bad Heilbrunn: Julius Klinkhardt.

Schröder, C. \& Şimşek, Y. (2014). Das Türkische. In M. Krifka, J. Blaszczak, A. Leßmöllmann, A. Meinunger, B. Stiebels, R. Tracy \& H. Truckenbrod (Hrsg.), Das mehrsprachige Klassenzimmer. Über die Muttersprachen unserer Schüler (S. 115-133). Berlin und Heidelberg: Springer VS.

Schröder, C. \& Küppers, A. (2016). Türkischunterricht im deutschen Schulsystem. Bestandsaufnahme und Perspektiven. In A. Küppers, B. Pusch \& P. Uyan Semerci (Hrsg.), Bildung in transnationalen Räumen. Education in Transnational Spaces (S. 191-212). Wiesbaden: Springer VS.

Schuler, T. (2010). Bertelsmannrepublik Deutschland. Eine Stiftung macht Politik. Frankfurt am Main: Campus.

Schüler-Meyer, A., Prediger, S., Kuzu, T., Wessel, L. \& Redder, A. (2017). Is Formal Language Proficiency in the Home Language Required to Profit from a Bilingual Teaching Intervention in Mathematics? A Mixed Methods Study on Fostering Multilingual Students' Conceptual Understanding. International Journal of Science and Mathematics Education, 34 (1), 123.

Schulz, P. (2007). Erstspracherwerb Deutsch: Sprachliche Fähigkeiten von Eins bis Zehn. In U. Graf \& E. Moser Opitz (Hrsg.), Diagnostik und Förderung im Elementarbereich und Grundschulunterricht. Lernprozesse wahrnehmen, deuten und begleiten (S. 67-86). Baltmannsweiler: Schneider Verlag Hohengehren. 
Schulz, P. \& Grimm, A. (2012). Spracherwerb. In H. Drügh, S. Komfort-Hein, A. Kraß, C. Meier, G. Rohowski, R. Seidel \& H. Weiß (Hrsg.), Germanistik: Sprachwissenschaft - Literaturwissenschaft - Schlüsselkompezenten (S. 155-172). Stuttgart: J. B. Metzler.

Schulz, P. \& Tracy, R. (2011). Linguistische Sprachstandserhebung. Deutsch als Zweitsprache (LiSe-DaZ). Göttingen: Hogrefe Verlag.

Schwabe, J., Grüßing, M., Heinze, A. \& Lipowsky, F. (2014) Instruktionsstrategien zur Förderung der individuellen Kompetenz zur adaptiven Wahl von Additions- und Subtraktionsstrategien im Zahlenraum bis 1000. Ergebnisbericht (Stand März 2014). Verfügbar unter: https://www.ipn.unikiel.de/de/das-ipn/abteilungen/didaktik-der-mathematik/dokumente/ergebnisbericht-tiger/at_download/file. Letzter Zugriff: 11. 11. 2018

Schwarz, B. B. \& Linchevski, L. (2007). The Role of Task Design and Argumentation in Cognitive Development During Peer-Interaction. The Case of Proportional Reasoning. Learning and Instruction, 17 (5), 510-531.

Schwarz, M. (1996). Einführung in die kognitive Linguistik. Tübingen, Basel: Francke.

Schwippert, K. \& Klinger, T. (2008). Das Evaluationskonzept von FöRMIG: Anlage und Durchführung - eine Zwischenbilanz. In T. Klinger (Hrsg.), Evaluation im Modellprogramm FörMig. Planung und Realisierung eines Evaluationskonzepts (S. 11-28). Münster: Waxmann.

Searle, J. R. (1969). Speech Acts. An Essay in the Philosophy of Language. Cambridge: Cambridge Univ. Press.

Seibel, A. (19. 06. 2016). Deutsch-Pflicht auf dem Schulhof? Selbstverständlich! Verfügbar unter https://www.welt.de/debatte/kommentare/article158891627/Deutsch-Pflicht-auf-dem-Schulhof-Selbstverstaendlich.html. Letzter Zugriff: 17. 12. 2018.

Seidel, T. (2014). Angebots-Nutzungs-Modelle in der Unterrichtspsychologie. Integration von Struktur- und Prozessparadigma. Zeitschrift für Pädagogik, 60 (6), 850-866.

Selter, C. (2009). Creativity, Flexibility, Adaptivity, and Strategy Use in Mathematics. ZDM, 41 (5), 619-625.

Selting, M., Auer, P., Barden, B., Bergmann, J., Couper-Kuhlen, E., Günthner, S., Meier, C., Quasthoff, U., Schlobinski, P. \& Uhmann, S. (1998). Gesprächsanalytisches Transkriptionssystem (GAT). Linguistische Berichte, 173, 91-122.

Selting, M., Auer, P., Barth-Weingarten, D., Jörg Bergmann, J., Bergmann, P., Birkner, K., Couper-Kuhlen, E., Deppermann, A., Giles, P., Günthner, S., Hartung, M., Kern, F., Mertzlufft, C., Meyer, C., Morek, M., Oberzaucher, F., Peters, J., Quasthoff, U., Schütte, W., Stukenbrock, A. \& Uhmann, S. (2009). Gesprächsanalytisches Transkriptionssystem 2 (GAT 2). Gesprächsforschung - Online-Zeitschrift zur verbalen Interaktion, 10, 353402. 
Sfard, A. (2015). Why All This Talk About Talking Classrooms? Theorizing the Relation Between Talking and Learning. In L. B. Resnick, C. S. C. Asterhan \& S. N. Clarke (Hrsg.), Socializing Intelligence Through Academic Talk and Dialogue (S. 245-255). Washington: American Educational Research Association.

Shipstead, Z., Harrison, T. L. \& Engle, R. W. (2015). Working Memory Capacity and the Scope and Control of Attention. Attention, Perception \& Psychophysics, 77 (6), 1863-1880.

Shook, A, \& Marian, V. (2013). The Bilingual Language Interaction Network for Comprehension of Speech. Bilingualism: Language and Cognition, 16 (2), 304-324.

Simonton, D. (2008). Bilingualism and Creativity. In Altarriba, J. \& Heredia, R. (Hrsg.), An Introduction to Bilingualism. Principles and Practies (S. 147-166). New York: Lawrence Erlbaum Associates.

Singleton, D. M. \& Ryan, L. (2004). Language Acquisition. The Age Factor. Clevedon: Multilingual Matters.

Sirim, E. (2009). Monolingualer Erwerb des Türkischen - Erträge der Forschung. In U. Neumann \& H. H. Reich (Hrsg.), Erwerb des Türkischen in einsprachigen und mehrsprachigen Situationen (S. 11-38). Münster: Waxmann.

Slavin, R. E. (1994). Educational Psychology. Theory and Practice. Boston: Pearson/Allyn \& Bacon.

Slavin, R. E. \& Cheung, A. (2005). A Synthesis of Research on Language of Reading Instruction for English Language Learners. Review of Educational Research, 75 (2), 247-284.

Snow, C. E., Cancino, H., Gonzalez, P. \& Shriberg, E. (1989). Giving Formal Definitions: An Oral Language Correlate of School Literacy. In D. Bloome (Hrsg.), Classrooms and Literacy (S. 233-249). Norwood: Ablex.

Solga, H. (2008). „Meritokratie“ - die moderne Legitimation ungleicher Bildungschancen. In P. A. Berger (Hrsg.), Institutionalisierte Ungleichheiten. Wie das Bildungswesen Chancen blockiert (S. 19-38). Weinheim: Juventa-Verlag.

Spearman, C. (1904). "General Intelligence". Objectively Determined and Measured. American Journal of Psychology, 15, 201-293.

Spörer, N., Brunstein, J. C. \& Kieschke, U. (2009). Improving Students' Reading Comprehension Skills: Effects of Strategy Instruction and Reciprocal Teaching. Learning and Instruction, 19 (3), 272-286.

Spörer, N., Koch, H., Schünemann, N. \& Völlinger, V. A. (2016). Das Lesetraining mit Käpt' $n$ Carlo für 4. und 5. Klassen. Ein Lehrermanual mit Unterrichtsmaterialien zur Förderung des verstehenden und motivierten Lesens. Göttingen: Hogrefe.

Stadthagen-González, H., Gathercole, V. C. M., Perez-Tattam, R. \& Yavas, F. (2013). Vocabulary Assessment of Bilingual Adults: To Cognate or not to 
Cognate. In V. C. M Gathercole (Hrsg.), Solutions for the Assessment of Bilinguals (S. 125-145). Bristol: Multilingual Matters.

Stojanov, K. (2006). Bildung und Anerkennung. Soziale Voraussetzungen von Selbst-Entwicklung und Welt-Erschließung. Wiesbaden: VS Verlag für Sozialwissenschaften.

Stojanov, K. (2011). Bildungsgerechtigkeit. Rekonstruktionen eines umkämpften Begriffs. Wiesbaden: VS Verlag für Sozialwissenschaften.

Storch, N. \& Wigglesworth, G. (2003). Is There a Role for the Use of the L1 in an L2 Setting? Teachers of English to Speakers of Other Languages, 37 (4), 760-770.

Streblow, L. \& Schiefele, U. (2006). Lernstrategien im Studium. In H. Mandl \& H. Friedrich (Hrsg.), Handbuch Lernstrategien (S. 352-364). Göttingen: Hogrefe.

Swain, M. (1985). Communicative Competence: Some Roles of Comprehensible Input and Comprehensible Output in its Development. In S. Gass \& C. Madden (Hrsg.), Input in Second Language acquisition (S. 235-253), Rowley, MA: Newbury House.

Swain, M. (2000). The Output Hypothesis and Beyond: Mediating Acquisition Through Collaborative Dialogue. In J. P. Lantolf (Hrsg.), Sociocultural Theory and Second Language Learning (S. 97-114). Oxford: Oxford University Press.

Swain, M. \& Lapkin, S. (2000). Task-Based Second Language Learning: The Uses of the First Language. Language Teaching Research, 4 (3), 251-274.

Szagun, G. (2016). Sprachentwicklung beim Kind. Weinheim: Beltz.

Tajfel, H. (1974). Social Identity and Intergroup Behaviour. Social Science Information, 13, 65-93.

Tajfel, H. (1981). Human Groups and Social Categories: Studies in Social Psychology. Cambridge: Cambridge University Press.

Tarelli, I., Schwippert, K. \& Stubbe, T. C. (2012). Mathematische und naturwissenschaftliche Kompetenzen von Schülerinnen und Schülern mit Migrationshintergrund. In W. Bos, H. Wendt, O. Köller \& C. Selter (Hrsg.), TIMSS 2011. Mathematische und naturwissenschaftliche Kompetenzen von Grundschulkindern in Deutschland im internationalen Vergleich (S. 247-267). Münster: Waxmann.

Tenorth, H.-E. (2007). Bildung. In H.-E. Tenorth \& R. Tippelt (Hrsg.), Beltz Lexikon Pädagogik (S. 92-95). Weinheim: Beltz.

Thierry, G. \& Wu, Y. (2004). Electrophysiological Evidence for Language Interference in Late Bilinguals. Neuroreport, 15, 1555-1558.

Thierry, G. \& Wu, Y. J. (2007). Brain Potentials Reveal Unconscious Translation During Foreign-Language Comprehension. Proceedings of the $\mathrm{Na}$ tional Academy of Sciences of the United States of America, 104 (30), 12530-12535. 
Thomas, E. M., Williams, N., Jones, L. A., Davies, S. \& Binks, H. (2014). Acquiring Complex Structures Under Minority Language Conditions: Bilingual Acquisition of Plural Morphology in Welsh. Bilingualism: Language and Cognition, 17, 478-494.

Thomas, W. P. \& Collier, V. P. (2002). A National Study of School Effectiveness for Language Minority Students' Long-Term Academic Achievement. Final Report. Washington: Center for Research on Education, Diversity \& Excellence.

Thordardottir, E. (2011). The Relationship Between Bilingual Exposure and Vocabulary Development. The International Journal of Bilingualism, 426445.

Thordardottir, E. (2015). The Relationship Between Bilingual Exposure and Morphosyntactic Development. International Journal of Speech-Language Pathology, 17, 97-114.

Thordardottir, E. (2011). Towards Evidence-Based Practice in Language Intervention for Bilingual Children. Journal of Communication Disorders, 2011 (43), 523-537.

Threlfall, J. (2009). Strategies and Flexibility in Mental Calculations. ZDM, 41 (5), 541-555.

Thurston, A., van de Keere, K., Topping, K. J., Kosack, W., Gatt, S., Marchal, J., Mestdagh, N., Schmeinck, D., Sidor, W. \& Donnert, K. (2005). Peer Learning in Primary School Science: Theoretical Perspectives and Implications for Classroom Practice. Electronic Journal of Research in Educational Psychology, 13, 477-496.

Topping, K. J. (2005). Trends in Peer Learning. Educational Psychology, 25 (6), 631-645.

Topping, K. J. \& Whiteley, M. (1993). Sex Differences in the Effectiveness of Peer Tutoring. School Psychology International, 14, 57-67.

Torbeyns, J., Smedt, B., Ghesquière, P. \& Verschaffel, L. (2009). Jump or compensate? Strategy Flexibility in the Number Domain up to 100. ZDM, 41 (5), 581-590.

Tracy, R. (2008). Wie Kinder Sprachen lernen. Und wie wir sie dabei unterstützen können. Tübingen: Narr Francke Attempto Verlag.

Tracy, R. (2011). Mehrsprachigkeit: Realität, Irrtümer, Visionen. In L. M. Eichinger (Hrsg.), Sprache und Integration. Über Mehrsprachigkeit und Migration (S. 69-100). Tübingen: Narr Francke Attempto Verlag.

Tracy, R. (2014). Mehrsprachigkeit. Vom Störfall zum Glücksfall. In M. Krifka, J. Blaszczak, A. Leßmöllmann, A. Meinunger, B. Stiebels, R. Tracy \& H. Truckenbrod (Hrsg.), Das mehrsprachige Klassenzimmer. Über die Muttersprachen unserer Schüler (S. 13-33). Berlin \& Heidelberg: Springer VS.

Trautmann, M. (2005). Überzeugungen von Englischlehrern. Zeitschrift für Erziehungswissenschaft, 8 (1), 38-52. 
Treffers-Daller, J. (1998). Variability in Code-Switching Styles: Turkish-German Code-Switching Patterns. In R. Jacobson (Hrsg.), Code-Switching Worldwide. (S. 177-197). Berlin: Mouton de Gruyter.

Turnbull, M. \& Dailey-O'Cain, J. (Hrsg.). (2009). First Language Use in Second and Foreign Language Learning. Clevedon: Multilingual Matters.

Unsworth, S. (2015). Amount of Exposure as a Proxy for Dominance in Bilingual Language Acquisition. In Silva-Corvalan, C. \& Treffers-Daller, J. (Hrsg.), Language Dominance in Bilinguals: lssues of Measurement and Operationalization (S. 156-173). Cambridge: Cambridge University Press.

Unsworth, S. (2016). Quantity and Quality of Language Input in Bilingual Language Development. In E. Nicoladis \& S. Montanari (Hrsg.), Bilingualism Across the Lifespan. Factors Moderating Language Proficiency (S. 103-121). Berlin: De Gruyter Mouton.

Valdés, G. \& Figueroá, R. A. (1994). Bilingualism and Testing. A Special Case of Bias. Norwood: Ablex.

Van Dijk, M., Kroesbergen, E. H., Blom, E. \& Leseman, P. P. M. (2018). Bilingualism and Creativity. Towards a Situated Cognition Approach. The Journal of Creative Behavior, 7, 1-11.

Van Heuven, W. J. B., Schriefers, H., Dijkstra, T. \& Hagoort, P. (2008). Language Conflict in the Bilingual Brain. Cerebral Cortex, 18 (11), 27062716.

Veenman, M. V. J., van Hout-Wolters, B. H. A. M. \& Afflerbach, P. (2006). Metacognition and Learning. Conceptual and Methodological Considerations. Metacognition and Learning, 1 (1), 3-14.

Vereinigung Bayerischer Wirtschaft (vbw) (2007). Bildungsgerechtigkeit. Jahresgutachten 2007. Wiesbaden: VS Verlag für Sozialwissenschaften / GWV Fachverlage GmbH.

Vereinigung der Bayerischen Wirtschaft (vbw) (2018). Mitglieder der Vereinigung Bayerischer Wirtschaft. Verfügbar unter: https://www.vbw-bayern.de/vbw/\%C3\%9Cber-uns/Mitglieder/index.jsp. Letzter Zugriff: 25. 12. 2018.

Viernickel, S. \& Schwarz, S. (2009). Schlüssel zu guter Bildung, Erziehung und Betreuung. Wissenschaftliche Parameter zur Bestimmung der pädagogischen Fachkraft-Kind-Relation. Berlin: Der Paritätische Gesamtverband Diakonie GEW.

Vygotskij, L. S. (1962). Thought and Language. Cambridge: MIT Press.

Wagner, R. \& Torgesen, J. (1987). The Nature of Phonological Processing and Its Causal Role in the Acquisition of Reading Skills. Psychological Bulletin, 10, 192-212.

Walter, J. (2009). Lernfortschrittsdiagnostik Lesen (LDL). Göttingen: Hogrefe. Watson, L. (1979). Der unbewusste Mensch. Frankfurt am Main: Umschau. 
Watson, R. (2003). Literacy and Oral Language: Implications for Early Literacy Acquisition. In D. K. Dickinson \& S. B. Neuman (Hrsg.), Handbook of Early Literacy Research (S. 43-53). New York: Guilford Press.

Webb, N. M., Franke, M. L., De, T., Chan, A. G., Freund, D., Shein, P. \& Melkonian, D. K. (2009). Explain to your Partner. Teachers' Instructional Practices and Students' Dialogue in Small Groups. Cambridge Journal of Education, 39 (1), 49-70.

Wegener, H. (1995). Die Nominalflexion des Deutschen - verstanden als Lerngegenstand. Tübingen: Niemeyer.

Weinert, R. (2004). Relative Clauses in Spoken English and German - Their Structure and Function. Linguistische Berichte, 197, 3-51.

Wendt, H. \& Schwippert, K. (2017). Lesekompetenzen von Schülerinnen und Schülern mit und ohne Migrationshintergrund. In A. Hußmann, H. Wendt, W. Bos, A. Bremerich-Vos, D. Kasper, E.-M. Lankes, N. McElvany, T. Stubbe \& R. Valtin (Hrsg.), IGLU 2016. Lesekompetenzen von Grundschulkindern in Deutschland im internationalen Vergleich (S. 219-234). Münster: Waxmann.

Whitebread, D., Coltman, P., Pasternak, D. P., Sangster, C., Grau, V., Bingham, S., Almeqdad, Q. \& Demetra Demetriou, D. (2009). The Development of Two Observational Tools for Assessing Metacognition and SelfRegulated Learning in Young Children. Metacognition and Learning, 4 (1), 63-85.

Whitehurst, G. \& Lonigan, C. (2003). Emergent Literacy: Development from Prereaders to Readers. In D. K. Dickinson \& S. B. Neuman (Hrsg.), Handbook of Early Literacy Research (S. 11-29). New York: Guilford Press.

Whorf, B. L. (1956). Language, Thought, and Reality. Selected Writings of Benjamin Lee Whorf. Mansfield Centre: Martino Publishing.

Wiese, H., Schröder, C., Zimmermann, M., Krifka, M., Gabriel, C., Gogolin, I. Klein, W. \& Tomasello, M. (2010). Die sogenannte „Doppelte Halbsprachigkeit". Eine sprachwissenschaftliche Stellungnahme. Verfügbar unter: http://www.zas.gwz-berlin.de/fileadmin/material/presse/pressemitteilungen/pressemitteilung doppelte-halbsprachigkeit_dez2010.pdf. Letzter Zugriff: 05. 12. 2018.

Wigger, L. (2015). Bildung und Gerechtigkeit - Eine Kritik des Diskurses um Bildungsgerechtigkeit aus bildungstheoretischer Sicht. In V. Manitius, B. Hermstein, N. Berkemeyer \& W. Bos (Hrsg.), Zur Gerechtigkeit von Schule. Theorien, Konzepte, Analysen (S. 72-92). Münster/New York: Waxmann.

Wikipedia. (2018). Jim Cummins. Verfügbar unter https://de.wikipedia.org/wiki/Jim_Cummins. Letzter Zugriff: 23.12.2018.

Wikipedia. (2018). Threshold Hypotheses Verfügbar unter https://en.wikipedia.org/wiki/Threshold_hypothesis. Letzter Zugriff: 23.12.2018. 
Williams, C. (1994). Arfarniad o Ddulliau Dysgu ac Addysgu yng Nghyddestun Addysg Uwchradd Ddwyieithog. Unveröffentlichte Dissertationsschrift. Bangor: University of Wales.

Williams, C. (1996). Secondary Education: Teaching in the Bilingual Situation. In C. Williams, G. Lewis \& C. Baker (Hrsg.), The Language Policy: Taking Stock (S. 39-78). Llangefni: CAI.

Willig, A. C. (1985). A Meta-Analysis of Selected Studies on the Effectiveness of Bilingual Education. Review of Educational Research, 55 (3), 269-317.

Winnicott, D. W. (1984). Reifungsprozesse und fördernde Umwelt. Frankfurt am Main: Fischer-Taschenbuch-Verlag.

Winnicott, D. W. (1989). Vom Spiel zur Kreativität. Stuttgart: Klett-Cotta.

Wlossek, I. \& Rost-Roth, M. (2016). Sprache/n als Ressource im Klassenzimmer? Erfahrungen und Einschätzungen von Lehrkräften in Regel- und Übergangsklassen. In V. Schurt, W. Waburg, V. Mehringer \& J. Strasser (Hrsg.), Heterogenität in Bildung und Sozialisation (S. 105-124). Opladen: Verlag Barbara Budrich.

Worthy, J., Durán, L., Hikida, M., Pruitt, A. \& Peterson, K. (2013). Spaces for Dynamic Bilingualism in Read-Aloud Discussions. Developing and Strengthening Bilingual and Academic Skills. Bilingual Research Journal, $36(3), 311-328$.

Yağmur, K. (2016). The Differences Between Policy and Practice of Multilingualism in the European Context. In A. Küppers, B. Pusch \& P. Uyan Semerci (Hrsg.), Bildung in transnationalen Räumen. Education in Transnational Spaces (S. 91-107). Wiesbaden: Springer VS.

Yap, B. W. \& Sim, C. H. (2011). Comparisons of Various Types of Normality Tests. Journal of Statistical Computation and Simulation, 81 (12), 21412155.

Zentella, A. C. (1997). Growing up Bilingual. Puerto Rican Children in New York. New York: Applause Books. 


\section{Wissenschaftlicher Werdegang des Autors}

Martin Schastak studierte von 2008 bis 2013 Germanistik, Pädagogik und Psychologie an der Goethe-Universität in Frankfurt und schloss sein Magister Artium mit Auszeichnung ab. Anschließend arbeitete er zwei Semester als wissenschaftlicher Mitarbeiter am Lehrstuhl für „Deutsch als Zweitsprache - Theorie und Didaktik des Zweitspracherwerbs“ "bei Frau Prof. Dr. Petra Schulz am Institut für Psycholinguistik und Didaktik der deutschen Sprache an der Goethe-Universität in Frankfurt, bevor er von 2014 bis 2019 als wissenschaftlicher Mitarbeiter zur Promotion in der Abteilung „Bildungsqualität und Evaluation“ bei Herrn Prof. Dr. Eckhard Klieme am DIPF | Leibniz Institut für Bildungsforschung und Bildungsinformation tätig war. In dieser Zeit arbeitete er in der Interventionsstudie „BiPeer - Förderung der Deutsch-Lesekompetenz bilingualer Grundschüler durch Peer-Learning“, welche die Datengrundlage seiner hier vorliegenden Dissertation stellt, und absolvierte zudem von 2016 bis 2018 ein Zusatzstudium im Fachbereich Erziehungswissenschaften an der GoetheUniversität in Frankfurt. Die Dissertation wurde im Juni 2019 erfolgreich verteidigt und insgesamt mit „magna cum laude“ bewertet. Seit dem August 2019 arbeitet Martin Schastak als wissenschaftlicher Mitarbeiter in der Abteilung „Bildung und Entwicklung“ bei Herrn Prof. Dr. Markus Hasselhorn am DIPF | Leibniz Institut für Bildungsforschung und Bildungsinformation. $\mathrm{Zu}$ seinen Forschungsschwerpunkten zählen Mehrsprachigkeit und Bildung, Translanguaging, Peer-Learning, Mixed Methods und Anerkennungstheorie. 


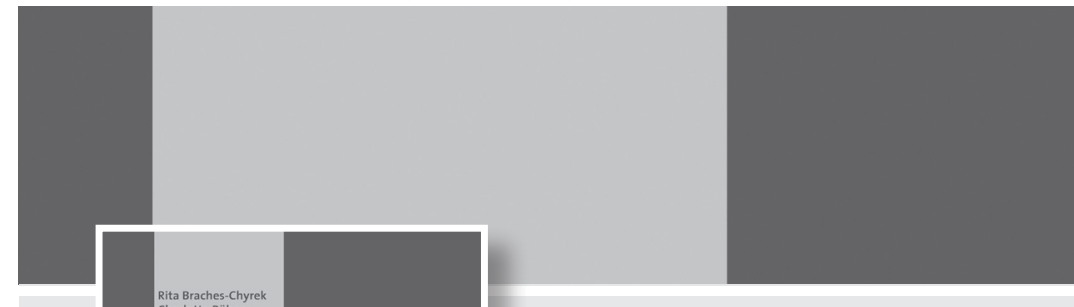

Rita Braches-Chyrek

Michaela Hopf (Hrsg)

Handbuch

Rita Braches-Chyrek

Frühe Kindheit

Charlotte Röhner

2., aktualisierte und

enveiterte Auflage

Heinz Sünker

Michaela Hopf (Hrsg.)

Handbuch Frühe Kindheit

2., aktualisierte und erweiteterte Auflage 2020.

849 Seiten • Hardcover • 94,90€ (D) • 97,60€ (A)

ISBN 978-3-8474-0688-4 • eISBN 978-3-8474-0832-1

Die zweite erweiterte Auflage des Handbuchs Frühe Kindheit gibt den aktuellen Stand der Forschung wieder. Es werden dabei sowohl theoretische als auch empirische Forschungsfelder und deren Ergebnisse vorgestellt sowie zusammengefasst. Die verschiedenen Beiträge des Handbuchs ermöglichen einen interdisziplinären Blick auf die Forschungs- und Handlungsfelder der frühen Kindheit und der Kindheitsforschung. 


\section{Mehrsprachigkeit und Bildung}

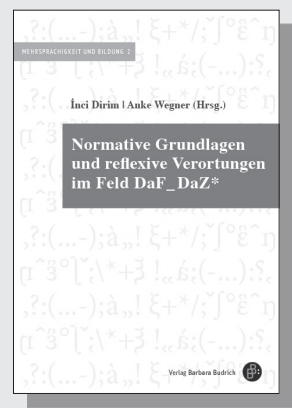

Band 2

$2018 \cdot 462$ Seiten $\cdot$ Kart. $49,90 €(D) \cdot 51,30 €(A)$

ISBN 978-3-8474-0558-0

eISBN 978-3-8474-0814-7

Deutschlernen ist nicht gleich Deutschlernen. Ob als Fremdsprache in aller Welt oder als Zweitsprache in den amtlich deutschsprachigen Regionen: Die spezifischen Beteiligten und der gesellschaftliche Kontext sind von essenzieller Bedeutung für Deutsch als Fremd- und Zweitsprache.

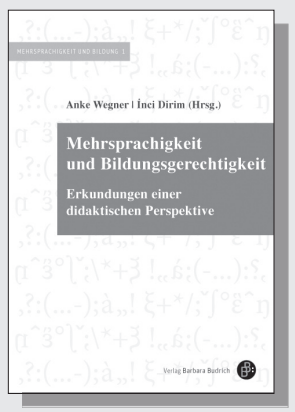

\section{Band 1}

2016 322 Seiten Kart. $39,90 €(D) \cdot 41,10 €(A)$

ISBN 978-3-8474-0669-3

eISBN 978-3-8474-0952-6

Die Schule in den amtlich deutschsprachigen Staaten ist von dem Spannungsverhältnis der konzeptionellen Einsprachigkeit im Deutschen und der faktischen Mehrsprachigkeit der Schülerlnnen gekennzeichnet, die sich immer weiter ausdifferenziert. 
Inklusionsforschung im

Spannungsfeld von

Erziehungswissenschaft

und Bildungspolitik

Jurgen Budde, Andrea Dlugosch, Petra Herzmann.

Lisa Rosen, Argyro Panagioto, Tanja S turm,

Schriftenreine

der AG Inklusionsforschung der Deutschen Gesellschaft fur Erzie hungswissenschaft (DGFE)

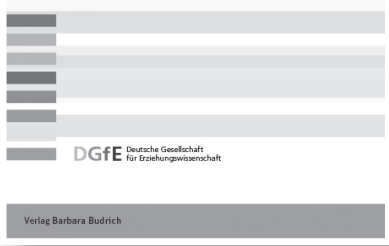

Jürgen Budde u.a.

Inklusionsforschung im Spannungsfeld von Erziehungswissenschaft und Bildungspolitik

Schriftenreihe der AG Inklusion der Deutschen Gesellschaft für

Erziehungswissenschaft (DGfE)

2019. 204 Seiten $\cdot$ Kart. $\cdot 28,00 €(D) \cdot 28,80 €(A)$

ISBN 978-3-8474-2201-3 • eISBN 978-3-8474-1227-4

Der Band reflektiert Inklusion als Gegenstand bildungstheoretischer und sozialwissenschaftlicher Forschung. Darüber hinaus konturiert er die Inklusionsforschung als eigenes erziehungswissenschaftliches Feld. Gegenwärtige Inklusionsforschung realisiert sich damit in Spannungsfeldern, die in unterschiedlichen Bereichen der Erziehungswissenschaft in verschiedener Weise verhandelt werden.

www.shop.budrich.de 
Die vorliegende Studie versucht einen Beitrag zur Erforschung von Implementationsmöglichkeiten des bilingualen Sprachvermögens von Schüler*innen mit Migrationshintergrund für den Regelschulkontext zu leisten, indem ein bilinguales Interaktionsangebot beim Peer-Learning für türkisch-deutschsprachig aufwachsende Schüler*innen der dritten und vierten Klasse in einem quasi-experimentellen Setting unter Verwendung von Mixed Methods untersucht wird.

Der Autor:

Martin Schastak, wissenschaftlicher Mitarbeiter, DIPF Leibniz-Institut für Bildungsforschung und Bildungsinformation 